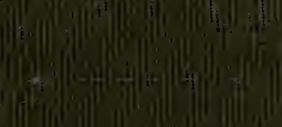

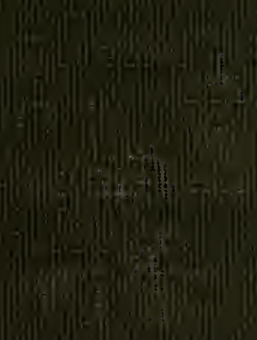

$$
\text { (1) }
$$

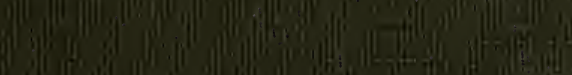

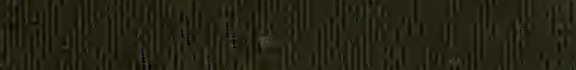

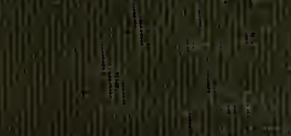

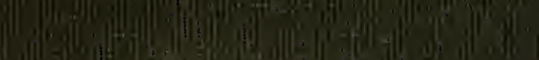
(1)

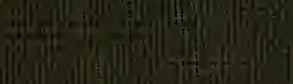

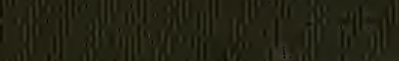




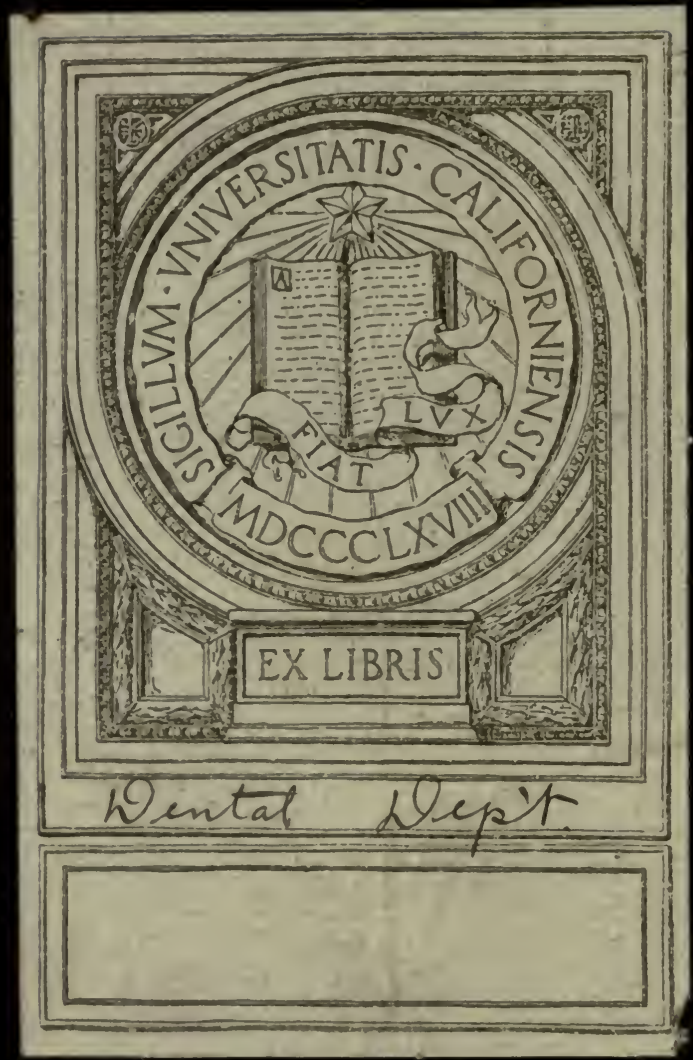



Digitized by the Internet Archive in 2007 with funding from Microsoft Corporation 




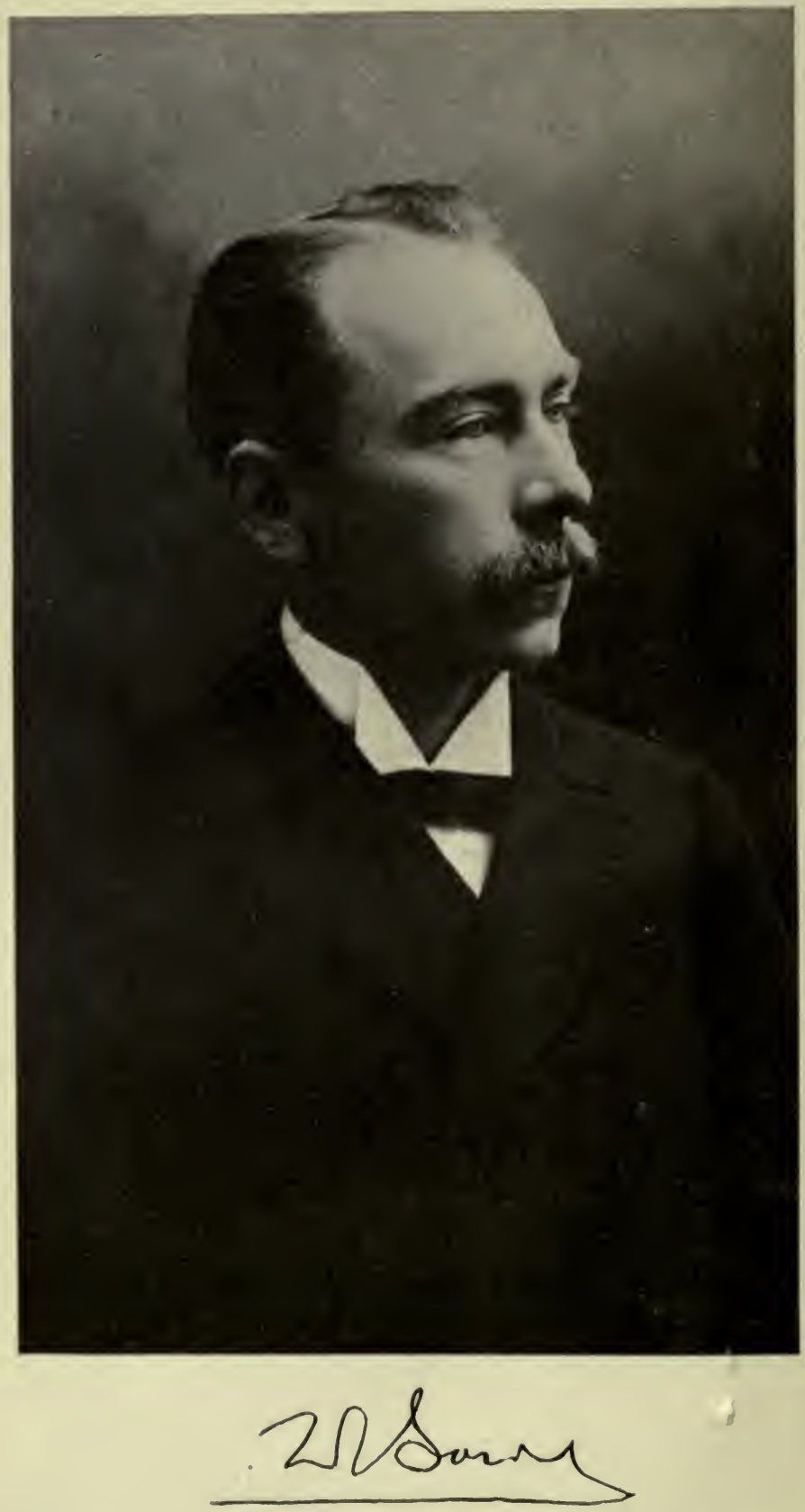

Frontispiece. 


\title{
STUDIES IN IMMUNITY
}

\author{
BY 48 ही० \\ Professor JULES BORDET \\ PROFESSOR OF BACTERIOLOGY AT THE UNIVERSITY OF BRUSSELS \\ DIRECTOR OF THE PASTEUR INSTITUTE OF BRABANT
}

AND HIS COLLABORATORS

COLLECTED AND TRANSLATED

BY

FREDERICK P. GAY, A.B., M.D.

INSTRUCTOR IN PATHOLOGY, HARVARD MEdicAL SCHOOL

INCLUDING A CHAPTER WRITTEN EXPRESSLY FOR THIS PUBLICATION BY PROFESSOR BORDET

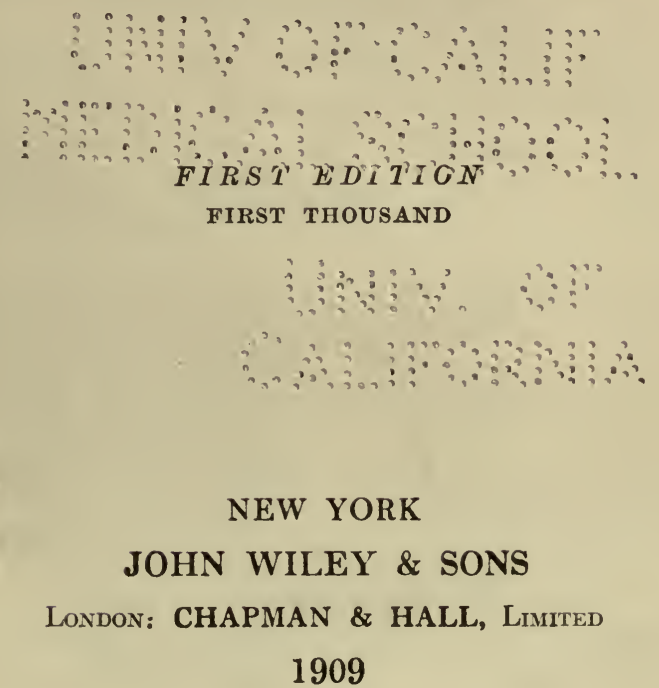


Copyeight, 1909, вт

FREDERICK P. GAY

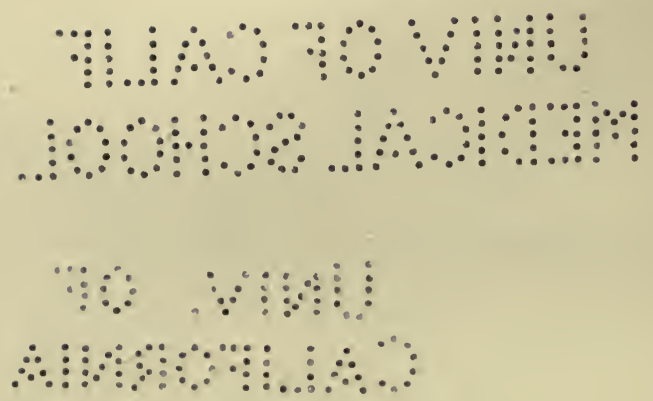

Stanbope Press

F. H. GILSON COMPANY

BOSTON, U.S.A. 


\section{Acuesion No. 1330}

\section{PREFACE}

THE reader of this book will understand from its contents, and particularly from its concluding chapter, why it has remained for one of Bordet's pupils to present his work as a whole to the scientific world. Although for more than fifteen years a protagonist in the modern development of Immunity, Bordet has continued an investigator instead of becoming a generalizer; he has been led by thoroughness of observation and brilliancy of inductive reason to the collection of successive significant facts rather than to the assimilation of scattered data in support of a preconceived theory. Whereas others have been willing to venture more fully explanatory theories of Immunity, Bordet, although contributing a disproportionate number of important facts, has contented himself with the hypotheses which bridge from one experiment to another, and has fully realized the necessarily fragmentary nature of our present knowledge in this field of science.

This compilation was undertaken, not so much owing to the apparent demand for similar examples of monographic collection, as from a sincere conviction of the fundamental importance of each contribution which Jules Bordet has directly or indirectly given us. The somewhat extensive task has been welcomed as a means of expressing gratitude for a personal inspiration in scientific thought and method.

It would seem of particular importance, in view of the overGermanizing of American science, that this collection of monographs should appear in this country.

The volume includes all of Bordet's scientific contributions with a few exceptions. Among the latter may be mentioned the article with Danysz on the method of combined active and passive immunization against Rinderpest, a series of four articles on Coagulation of Blood (with Gengou), and a note on the Spirocheta pallida, 
which organism Bordet and Gengou were incidentally, the first to see. The articles have in general been arranged in chronological order; an exception, however, was made in some of the latter chapters (XXIV to XXX) which deal rather with the application of more theoretical studies which precede.

I am indebted to Professor Bordet, not only for consenting to the collection of these articles, but also for furnishing the concluding chapter and for certain suggestions and corrections. Doctor Gengou was so kind as to abstract one of his articles for this publication. Thanks are also due to the publishers of the various journals in which these articles appeared for permission to translate or to reprint them.

Boston, August, 1909

F. P. G. 


\section{TABLE OF CONTENTS.}

(From the Laboratory of Professor Errera, Botanical Institute, Brussels.)

Chapter

I. The Adaptive Changes of Bacterial Cultures in the Body of Immunized Animals.......................... BORDET.

(From the Laboratory of Professor Metchnikoff, Pasteur Institute, Paris.)

II. Studies on the Serum of Vaccinated Animals. The Relation of

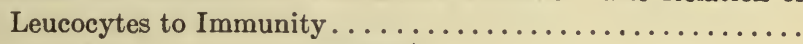
BORDET.

III. Studies on the Serum of Vaccinated Animals. Pfeiffer's

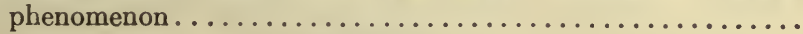

BORDET.

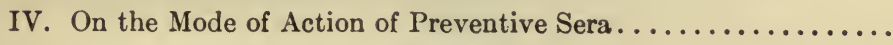

BORDET.

V. A Contribution to the Study of Antistreptococcus Serum...... BORDET.

VI. On the Agglutination and Dissolution of Red Blood Cells by the Serum of Animals Injected with Defibrinated Blood.......... BORDET.

VII. The Mechanism of Agglutination.

BORDET.

VIII. The Agglutination and Dissolution of Red Blood Cells by Serum (Second Memoir)

BORDET.

IX. Hemolytic Sera and Their Antitoxins; and Theories Concerning Cytolytic Sera in General....................... 
Chapter
X. On the Existence of Sensitizing Substances in the Majority of

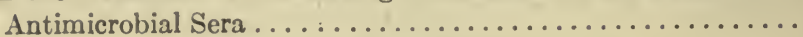
PAGE Bordet and Gengou.

XI. On the Mode of Action of Cytolytic Sera; and on the Unity of the

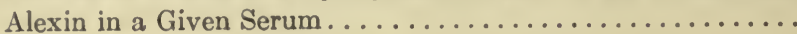
228 BORDET.

(From the Pasteur Institute of Brabant, Brussels.)

XII. On the Sensitizers of Sera Active against Albuminous Substances Gengou.

XIII. On the Mode of Action of Antitoxins on Toxins........... 259 BORDET.

XIV. The Properties of Antisensitizers and the Chemical Theories of Immunity . . . . . . . . . . . . . . . . . . . 280

\section{BORDET.}

XV. Researches on the Agglutination of Red Blood Cells by Chemical Precipitates, and on the Suspension of Such Precipitates in

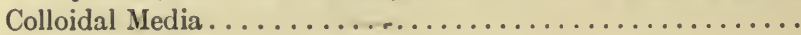

Gengou.

XVI. Observations on the Single Nature of Hemolytic Immune Bodies and on the Existence of So-called "Complementoids" ....... GaY.

XVII. The Fixation of Alexins by Specific Serum Precipitates GaY.

XVIII. Deviation of the Alexin in Hemolysis. ................. GaY.

IX. On the Relations of Sensitizers to Alexin

Bordet and GaY.

XX. On the Nature of Opsonins

SleeswiJK.

XXI. Alexin Absorption and the Antagonistic Property of Normal Sera Bordet and GaY.

XXII. A Contribution to the Study of Molecular Adhesion, with a consideration of its Function in Various Biological Phenomena.. 
Chapter

XXIII. The Phenomena of Adsorption and the Conglutinin of Bovine Serum.............................. 440 Bordet And Streng.

XXIV. Sensitizers for the Tubercle Bacillus.............. 462 BORDET AND GENGOU.

XXV. New Contribution to the Study of Sensitizers for Tubercle Bacilli 464 Gengou.

XXVI. A Contribution to Our Knowledge of Antituberculous Sensitizers 467 Gengou.

XXVII. The Bacillus of Whooping Cough . . Bordet AND Gengou.

XXVIII. An Additional Note on the Whooping-Cough Bacillus........ 482 Bordet ANd GENGou.

XXIX. The Endotoxin of the Whooping-Cough Bacillus 488 BORDET.

XXX. Researches on Avian Diphtheria 492 BORDET.

XXXI. A General Résumé of Immunity 496 BORDET.

Index of Authorities quoted........................... 531

Index of Subjects................................. 535 



\section{STUDIES IN IMMUNITY.}

\section{THE ADAPTIVE CHANGES OF BACTERIAL CULTURES IN THE BODY OF IMMUNIZED ANIMALS.*}

\section{BY JULES BORDET, STUDENT OF MEDICINE.}

BACTERIA are highly adaptable. They frequently change both morphologically and functionally. Their virulence is also an essentially fluctuating property, that increases or diminishes according to the conditions to which the pathogenic organism is subjected.

The vibrio Metchnikovi, as described by Gamaleia, is very virulent for certain animals. A small amount of culture suffices to kill guinea-pigs. Sterilized bouillon in which the organism has been grown is also very toxic for these animals and kills in a mean dose of 2 c.c. to 100 grams of body weight.

Guinea-pigs, although they succumb to this micro-organism so easily, become immunized when they have received one or two injections of sufficient quantities 0.5 to 1 c.c. per 100 grams) of a culture freed from living bacteria by filtration or by autoclaving. The protection obtained in this manner is constant.

Metchnikoff has shown us, however, that, when a guinea-pig protected in this manner is given a small amount of a virulent culture of living organisms, the latter are not immediately destroyed. They disappear only after a relatively long period, 60 to 90 hours if injected subcutaneously, or 6 to 7 days when injected into the anterior chamber of the eye. There is, however, a much more notable collection of leucocytes at the point of inoculation than in an animal that has not been protected against the disease. Not only do the vibrios remain alive, but they lose none of their harmful

* Adaptation des virus aux organismes vaccinés. Annales de l'Institut Pasteur, VI, 1892, 28. From the laboratory of Prof. Errera. Botanical Institute, Brussels. 
properties; on the contrary, their virulence is to a considerable extent increased. With an organism modified in this manner, Metchnikoff has succeeded in killing a guinea-pig in from 6 to 7 hours, whereas the ordinary culture takes about 20 hours.

What is the cause of this marked increase in pathogenicity? By what modification has the virus acquired this powerful activity? This point we have attempted to determine.

Let us immunize a guinea-pig of 550 grams weight by injecting 3.5 c.c. of a sterilized culture of V. Metchnikovi, followed 8 days later by 0.5 c.c. of a living culture of the organism. Ten days later, when the animal has returned to a normal condition, we introduce 1 c.c. of a vigorous culture under the skin of the belly. Twenty hours later a small amount of the exudate which has been formed is withdrawn and grown in veal bouillon containing peptone.

When this culture has grown out, a cubic centimeter of it is taken and injected subcutaneously in another similarly vaccinated guineapig. Twenty-seven hours after inoculation, a drop of the exudate is withdrawn as from the first animal and likewise grown in bouillon.

This second guinea-pig, although previously in good condition, is much sicker than the first vaccinated animal. There is a large area of necrosis about the point of inoculation; the eyes are half closed, the animal inert, and prostration manifest. This is in harmony with the experiments of Metchnikoff, who demonstrated that the vibrio that had remained under the skin of a vaccinated guinea-pig for 20 hours became more pathogenic.

We have now three different cultures of the vibrio Metchnikovi: a culture of the organism modified by a single passage through an immunized animal; a culture of the same organism modified by two passages; and a culture of the vibrio grown on agar in successive generations during several months. We may obtain a fourth culture by passing the vibrio through a non-vaccinated guinea-pig. This last constitutes a culture of normal vibrios whose virulence has undergone no modification through prolonged growth on artificial media.

The increase in virulence of cultures which we call "modified," or very virulent, may be due, according to our present ideas, to one of two causes: either their chemiotactic power toward leucocytes has diminished, which would allow them to escape more readily 
the destructive action of these cells and consequently develop and multiply with greater security; or, indeed, they may secrete more abundant or more dangerous toxic products than before.

These two causes may operate simultaneously. It is necessary then to compare, first, the chemiotactic influence of the original or normal organism with that of the "modified" organism, and second, the toxic properties of the substances formed by these two strains of vibrios.

Experiment 1. The four different organisms were grown in a special fluid that offers a good culture medium which has already been used by J. Massart and Ch. Bordet; this medium has in itself not the slightest chemiotactic effect on leucocytes.

After three days of growth at $32^{\circ} \mathrm{C}$., capillary tubes are filled with these virulent cultures and placed in lots of a dozen in the peritoneal cavity of a normal guinea-pig. They are removed 8 hours later, when it is found that the columns of leucocytes that have entered the tubes vary in length. In the tube containing the normal vibrio, cultivated for several months on agar, the length of this column is the same as with the normal vibrio recently isolated from the tissues of an unvaccinated guinea-pig. With the vibrio modified by a single passage through a vaccinated guinea-pig the column is about half that of the preceding. With the organism modified by two passages it is slightly less than half.

The same experiment was performed with cultures sterilized at $115^{\circ} \mathrm{C}$. No difference in the influx of leucocytes was noted.

It is evident, then, that the attracting power for leucocytes is notably less in organisms grown in immunized guinea-pigs than in the two normal organisms; one that was passed through a normal guinea-pig and the other grown for a long time on artificial media.

It is rather interesting to note that there is no appreciable difference between these last two strains of vibrio. The chemiotactic properties evidently have not been altered by growing on agar.

What is more, it may be mentioned that we have found that the vibrio Metchnikovi in old cultures exposed to the air is little, if at all attenuated; a culture bearing the date Dec. 16, 1891, and inoculated March 29, 1892, in a dose of 0.5 c.c., killed guinea-pigs in 20 hours. As is well known, many pathogenic organisms attenuate more rapidly. 
These experiments show us, moreover, that a vibrio that has undergone two passages is only slightly less attracting than one that has undergone a single passage.

Let us now consider whether the toxicity of the bacterial secretions has changed in a manner similar to that of the chemiotactic property.

Experiment 2. A healthy guinea-pig, weighing 620 grams, was given an inoculation of 6 c.c. of a very virulent, modified, sterilized culture. Although this amount was rather small, it killed the animal in 60 hours. At autopsy no lesions other than intestinal congestion were noted.

Another guinea-pig, weighing 450 grams, received 5.5 c.c. of the ordinary vibrio Metchnikovi, which in proportion to the weight of the animal was slightly more than was given the preceding animal. This animal showed no effect, however, and remained in good condition.

It is to be noted that an increase of toxicity here accompanies a lowering in positive chemiotactic power.

To what is this weakness in attraction due? Are we to regard it simply as a quantitative decrease in the attracting substance that the normal vibrio produces so abundantly? Or is there formed, on the contrary, a repelling substance of which little or none is formed by the original vibrio? An attempt to reply to these questions follows.

EXPERIMENT 3. We placed in the peritoneal cavity of a normal guinea-pig capillary tubes containing the following vibrios, prepared on the same culture media as in experiment 1:

(a) Normal sterilized culture of vibrio Metchnikovi.

(b) Sterilized culture of the vibrio Metchnikovi from the exudate withdrawn from the second immunized guinea-pig. (c) A mixture of equal parts of the two first fluids. (d) A mixture of equal parts of fluid " $b$ " and sterile culture medium, identical with that on which the organisms had been grown.

These tubes were left in the animal body for 8 hours. On examination the columns of leucocytes in tubes containing liquids " $a$," " $c$," and " $d$ " were seen to be equal. In the " $b$ " tubes the length of the column of leucocytes was less than half this.

A consideration of tube " $d$ " shows us that dilution with an inert 
fluid increases the attracting power of the "modified" vibrio Metchnikovi and renders it quite as energetic as the normal, organism.

We may then put aside the hypothesis that the modified vibrio has lost a part of its attracting properties owing to a smaller amount of positive chemiotactic substance. There is evidently a negative chemiotactic influence which lessens to a considerable degree the effect of the attractive substances. Experiment shows us that dilution weakens the activity of the first more than that of the second.

There is nothing to prove that these substances, acting in an opposite manner on white corpuscles, are distinct. It may be that there are not two different chemical substances, but that one and the same substance may attract leucocytes when diluted and repel them when concentrated. This last hypothesis is far from difficult to accept: we know, indeed, the profound influence which the concentration of various substances has on their power to affect susceptible cells. This is not a supposition, however, that we have been able to prove.

Whatever the substance may be to which the repelling action is due, it is present and acts energetically in the modified vibrio, but it is also manifest to a less degree in the ordinary organism. As a matter of fact, the attracting power of the secretions of this latter organism increases with dilution, as demonstrated by a capillary tube experiment.

The vibrio that has been grown in an immunized guinea-pig does not long retain its intense repelling property when grown on artificial media.

EXPERIMENT 4. A comparison was made as regards chemiotaxis, by the same method of capillary tubes, between the vibrio Metchnikovi cultivated for a long time on agar tubes and a vibrio marked "very virulent" that had been transplanted every four days for nearly half a month in veal bouillon containing 1 per cent peptone. The leucocytes filled equal lengths of the tubes of each strain.

We have seen that residence in the body of a vaccinated animal lends certain modifications to the vibrio Metchnikovi; it becomes more toxic and less attracting for leucocytes. Let us now consider how these modifications are produced. 
When we inject vibrios subcutaneously in a vaccinated guineapig an emigration of leucocytes rapidly takes place. The first white corpuscles to arrive at the point of inoculation find a considerable number of adversaries of which they can destroy only a few. It would be sufficient for a few of these vibrios to be endowed with a slightly more intense power of attraction than their fellows, to cause the phagocytes to direct themselves by preference toward these organisms and to take them up. Very slight, almost inappreciable differences will consequently predestine certain bacteria to rapid phagocytosis. In the same way an inferiority in secretion of toxic products, however slight, will cause a predisposition to rapid destruction.

In a word, leucocytes kill first those organisms that are less resistant, and the culture inoculated will be freed first of those individuals which either form less poison than their fellows or attract the leucocytes more.

In the meantime the vibrios that have been left alone will divide and produce new organisms which in turn will be exposed to the attack of phagocytes. These latter will again suppress those individuals that are most poorly armed for the struggle, and will leave only those that possess in highest degree the two characters just noted. Thanks to this process of selection, new generations of organisms, like those represented by the cultures used in our experiments, will be derived for the most part from those bacteria which have been endowed with certain advantages.

For the production of this new race it is necessary:

First: that leucocytes come up continually. If they do not come to the point of inoculation, no selection occurs and the organisms do not gain in pathogenicity. So, when virulent vibrios are injected subcutaneously in a normal guinea-pig, the influx of leucocytes is very slight and the organism obtained by this passage has no particular characteristics. If, on the contrary, a normal guinea-pig is given an inoculation of an attenuated vibrio Metchnikovi, which has a strong attraction for leucocytes, the selection is brought about, and the organism increases in virulence. The leucocytes are the active agents in selection, and it is they that, by eliminating the least resistant organisms and sparing the others for a while so that they may multiply, cause the increase in virulence that has been noted. 
Second: that the influx of leucocytes take place with a certain regularity and in a gradual and progressive manner. Selection requires a certain time. It is in those places in an immunized animal where the influx of leucocytes occurs slowly, for example, in the anterior chamber of the eye, that the vibrio Metchnikovi acquires the most extreme virulence. It has been noted in referring to Metchnikoff's work that the vibrio obtained in this manner is the most active, killing the guinea-pig in 6 hours.

Another fact of Metchnikoff's is very similar. Anthrax is more deadly for the pigeon when injected into the aqueous humor than elsewhere. It will be recalled also that with some organisms inoculation into the circulation is much less dangerous than into the connective tissue.

This ends our account of the vibrio Metchnikovi for the present; we shall later return to it:

Not all organisms, as, for example, the bacillus of swine plague (rouget du porc), acquire the same virulence when passed through vaccinated or more or less refractory animals.

New studies to determine whether a culture subjected to various conditions becomes modified in respect to its chemiotactic power and the toxicity of its secretions are in progress. 


\section{STUDIES ON THE SERUM OF VACCINATED ANIMALS.*}

BY DR. JULES BORDET.

The subject of immunity has expanded in the last few years. The fundamental problem, to the solution of which a considerable number of researches have been devoted, is to explain how animals, either on account of a natural refractory condition or owing to an artificial immunization, resist the invasion of a given micro-organism and overcome an infection by destroying the bacterium or the poison which it secretes. But there are other facts which also demand explanation. Animals which have been well immunized against a given infection either by repeated injections of bacterial products or of living cultures may be distinguished from normal animals not only by the fact that they henceforth resist this infection, but also, in numerous instances, by the fact that their body fluids, particularly the blood serum, possess properties which are not observed in the non-vaccinated animal. The serum of vaccinated animals is often preventive and at times bactericidal or antitoxic. It is not sufficient for theories of immunity to explain how an animal that has been vaccinated is fitted to overcome an infection; they must also explain how these new properties, the study of which has so greatly interested bacteriologists, have been formed in the body fluids. The study of the serum of immunized animals forms a new chapter in the history of the struggle between the animal and infective agents, under which heading practical results of the highest importance are already inscribed. Any explanation of the phenomena is, however, still far from complete.

The substances which are present in the serum of vaccinated animals and which endow them with their particular property are

* Contribution à l'étude du serum chez les animaux vaccinés. Annales de la Societé royale des sciences médicales et nat. de Bruxelles, IV, 1895. 
unknown to us. But apart from their chemical constitution, concerning which hypotheses only may be offered, these substances should be studied from various points of view, and, indeed, innumerable questions concerning their functions and their origin at once suggest themselves. Where are these materials formed? By what cells are they elaborated? Are they uniformly distributed and dissolved in the body fluids; or are they concentrated in certain individual regions? What is their function in acquired immunity? To what extent does bactericidal power contribute to protect the organism? By what mechanism do preventive substances in sera insure immunity in animals in which they have been injected? Certain of these problems have already occupied the attention of experimenters; certain of them have been attacked with more or less success and treated more or less completely, and others have only been touched on. Without any premature hope of ultimate explanation, one may extend our knowledge by offering new facts, or contributing a detail.

\section{LEUCOCYTES AND IMMUNITY.}

\section{Phagocytosis in General. Phagocytosis in Cholera.}

The studies which are to be detailed in the present article lead us to attribute to the leucocytes an important function in the elaboration of those substances which endow sera with their activity. It has been known for some time, thanks to the persevering efforts of the creator of the existing theory of immunity, Metchnikoff, that body cells take an energetic part in dissipating an infection and so aid in a very important manner in the cure of infectious diseases. Leucocytes respond to stimuli in several ways and are capable of reacting in several different manners. These different reactions are often all exercised in the course of a given phenomenon. They are often all necessary for the accomplishment of a single vital act. The study of these properties forms, then, a collective whole which may not be dissociated, and for this reason it seems useful to review at the beginning whatever is known concerning the participation of the white blood corpuscles in the struggle against bacterial parasites.

The fundamental facts of the phagocytic theory have been so 
frequently determined by individual researches, so frequently demonstrated in articles dealing with the general problem of immunity, that it would scarcely be necessary to repeat the history of its development and to enumerate the data on which it has been founded. We may be permitted, however, to recall the most demonstrative facts which may be cited in confirmation of this doctrine.

The phagocytic function belongs essentially to the polynuclear amphophiles,* to the large mononuclear leucocytes and to certain endothelial cells of the capillaries of the liver and the spleen (their parenchymatous cells may also in certain cases manifest phagocytic properties). Phagocytosis is found throughout the animal kingdom. It occurs in all the contagious diseases that have been well studied, not only in those of a particularly infectious nature, but also in those that are preëminently toxic and in which bacterial invasion is extremely limited. The tetanus bacillus and the diphtheria bacillus, for example, are taken up and destroyed by phagocytes. Phagocytes also play an essential rôle in chronic bacterial infections, as the constitution of the tubercle indicates.

Phagocytes do not limit their activities to the taking up of living or dead microbes. They may also capture spores and prevent their germination. Their activity may also be utilized against other foreign bodies and against the other cells of the same animal when they have become useless or even harmful and are destined to disappear. The protoplasm of phagocytes likewise takes up soluble substances introduced into the body, as was shown by the researches of Samoiloff $\nmid$ and of Lipski, $\ddagger$ (Pharmacological Institute of Dorpat) on the distribution of iron and of silver as soluble compounds in the animal body.

What proves the very fundamental function of phagocytes in the destruction of bacteria is the surprising rapidity with which the engulfing of organisms may be accomplished, as several observers,

* Eosinophilic leucoeytes only rarely take up bacteria. Mesnil (Annales de l'Institut Pasteur, May, 1895) has noted, in the frog, eosinophilic eells in the act of engulfing anthrax bacilli. We have still more recently noted the taking up of the diphtheria bacillus by the eosinophiles of a guinea-pig immunized by a strong dose of anti-diphtheria serum.

$\dagger$ Samoiloff, Arbeiten des pharmakologischen Institutes zu Dorpat, IX, 1893.

$\ddagger$ Lipski, Ibid. 
and Werigo * in particular, have shown. We shall have occasion to consider the taking up by phagocytes of cholera vibrios injected intravenously. We have already noted the almost instantaneous occurrence of this phenomenon.

The intensity with which phagocytosis occurs in infected animals is always proportionate to the resistance of the animal; in the majority of cases it may serve in the course of an infection as an indication of the outcome of the struggle between the bacterium and the animal. So, for instance, it is more active in vaccinated animals than in normal animals, whether the refractory stage be obtained as a result of the injection of attenuated or sterilized cultures or by means of a preventive serum.

The existence of a peculiar reaction on the part of leucocytes to chemical substances secreted by bacteria offers important evidence of the part which these cells take in defense of the body. $\dagger$ As soon as the bacteria penetrate the tissue their presence is evident from the fact that their diffusible products cause a phenomenon of chemiotaxis on the part of the phagocytes. The tactile reaction of these cells permits them, when chemiotaxis has been manifested and when they have approached the infecting bacteria to the point of contact, to send elongations about them and to engulf them. The rapidity with which these various phenomena follow each other, particularly in vaccinated animals or when dealing with slightly virulent organisms, shows how active phagocytic intervention is.

The relation between the quality, method of reaction, and number of phagocytes on the one hand and the efficiency of the defense on the other is shown by a series of phenomena. Leucocytes are little attracted, and may even be repulsed by very virulent microbes.

An attempt has recently been made to discredit the importance of chemiotaxis in immunity. Werigo, who of course recognizes the existence and the purpose of positive chemiotaxis, has certain doubts as to the existence of a negative chemiotaxis. Woronin goes even further. He attributes to chemiotaxis, whether positive or negative, an almost negligible function in the defense of the

* Werigo, Les globules blancs protecteurs du sang: Annales de l'Institut Pasteur, 1892.

$\dagger$ J. Massart et Ch. Bordet, Journal de la Societé royale des sciences naturelles et médicales de Bruxelles, 1890. 
organism against infection. It is certain that positive chemiotaxis of leucocytes has been proved in an exact manner by demonstrative experiments that cannot be controverted. Bacterial cultures attract leucocytes, and this attraction is often very marked. This is an established fact, the significance of which from the standpoint of immunity appears quite evident. Let us hasten to add that Woronin does not deny chemiotaxis; but he thinks that this reaction on the part of the leucocytes is not indispensable nor even particularly useful to them. Phagocytes may, according to this author, fulfil their function simply by means of their tactile reaction. This investigator attempts to show anew the already well known fact that leucocytes do react to contact, that they voluntarily enter porous bodies, openings in tissues, etc. He recognizes that the - reaction to contact is very highly developed and concludes that the engulfing of bacteria may be due simply to this reaction. No one doubts that the tactile reaction is a valuable property in leucocytes, as is made clear by those authors who have studied chemiotaxis.* It appears quite certain that phagocytes, when they open up on contact with inert non-attractive particles (charcoal, for example), may ingest them in their protoplasm; this ingestion is due entirely to the tactile sensitiveness of the cells. But how does the importance of the leucocytic reaction to contact diminish the reaction to chemical substances? How much must the taking-up of living or dead particles be facilitated if these particles diffuse substances that attract leucocytes and cause them to move toward the particles? If we admit the intensity of positive chemiotaxis, which is easily determined, we cannot deny to these phenomena their evident function. Werigo's objections $†$ to the arguments which tend to admit a negative chemiotaxis of leucocytes are better founded. Werigo does not think it has been shown that there are substances capable of producing a retraction of leucocytes. We have been accustomed to regard lactic acid, for example, as possessing such a repellent action from the following experiment: if we mix a small quantity of lactic acid with substances which are very attractive (bacterial products), it is noted that the mixture no longer attracts

* J. Massart and C. Bordet., loc. cit.

† Werigo, Développement du charbon chez le lapin. Annales de l'Institut Pasteur, 1894. 
leucocytes, and from this fact we may assume that lactic acid is endowed with properties that are antagonistic to those of bacterial products; in other words, one may attribute to it a negative chemiotactic power.

Werigo does not agree with this interpretation. It seems to him that lactic acid inhibits the attraction of leucocytes not by exercising on them a truly repelling action, but by harming them or paralyzing them, and so preventing them from feeling the attracting influence of bacterial products. Nor does Werigo believe in the repelling action of bacterial secretions any more than he does in the same action on the part of lactic acid. In serious infections where no phagocytosis is present the non-intervention of leucocytes seems to him due not to the fact that they are repelled, but that they are simply not attracted. In other words, there is an absence of positive chemiotaxis without any actual negative chemiotaxis. In brief, "we find," says Werigo, " that a negative chemiotaxis for leucocytes has not yet been proved in a satisfactory manner. The question must be left to further experiments."

Although Werigo's objections have some foundation, we believe that a negative chemiotaxis of leucocytes does exist and that it may be proved experimentally.

EXPERIMENT 1. A normal guinea-pig was given an injection in the peritoneal cavity of 1 c.c. of a two-day culture of streptococcus. (The culture medium is a mixture of bouillon and serum suitable for maintaining the virulence of the organism.) The streptococcus employed was a very virulent organism kindly furnished us by our friend Marmorek.

Before injection the peritoneal cavity was found to contain a few leucocytes largely of the mononuclear type and a few eosinophiles; there were very few polynuclear microphages.

After injection a small amount of exudate was removed at intervals and stained preparations were made.

Within a few minutes some organisms were found within mononuclear leucocytes. But as these leucocytes are few in number a majority of the organisms remain free.

After an hour the polynuclear leucocytes are to be found, but they are still few in number; it is to be noted, however, that the majority of them have taken up organisms. The number of these leucocytes 
then increases rapidly. After 3 hours, for example, it is found that the exudate is very rich in polynuclears. These cells are far more than are required to take up all the organisms present; as a matter of fact, only a relatively small number of cocci were injected and these have had no opportunity to increase. But no matter how. numerous the phagocytes are, free organisms which have escaped the destructive phagocytic action are still found in the surrounding fluid. Only a certain number of them have become the prey of the white corpuscles.

Then a second phase of the infection begins. The number of microphages continues to increase. At the same time the organisms that have not been taken up multiply and give rise to new individuals. At the end of 6 hours the exudate is seen to contain very large numbers of phagocytes and at the same time a considerable number of organisms. But these phagocytes are empty. The phagocytosis of the coccus noted at first is no longer present. This fact is still more surprising since the organisms and the phagocytes must still be in contact; it would seem as if the tactile reaction which the cells usually show would suffice to produce phagocytosis.

It must be that some active influence prevents the phagocytes from taking up those organisms scattered about them. What is this influence? Two explanations occur: (a) either the leucocytes are paralyzed by the toxin that the streptococcus secretes in multiplying; or, (b) the leucocytes, while retaining all their motility and facility for taking up organisms, may have been subjected to a negative chemiotactic influence from the streptococcus that prevents them from taking up the bacteria. We shall see that the first of these hypotheses is incorrect. The second is the true explanation.

Eight hours after injection, when the animal appears to be very sick and the peritoneal exudate is alive with organisms and very rich in phagocytes, we give a second injection. We inoculate 1 c.c. of a rich bouillon culture of Proteus vulgaris ( 24 hours old); this organism is only slightly virulent and is easily destroyed by phagocytes. A few moments later, if we take out a little exudate, we find that the cells which refuse to take up the streptococcus have eagerly taken hold of the new organism offered them. Within a half hour almost the entire culture is within phagocytes. It is extraordinary how these phagocytes have chosen between the two 
varieties of bacteria. With greatest delicacy they have reacted to a new chemical substance and each one takes up numerous Proteus organisms, recognizable by their rod shape, but still refuses the streptococci which remain scattered throughout the preparation outside the cells. The protoplasm of the phagocytes acts rapidly on the bodies of the bacteria that have been engulfed. These Proteus bacilli soon manifest changes in their reaction to dyes: a preparation from the exudate taken a short time after the second injection or kept for a few hours in a moist chamber shows, with a contrast stain of eosin and methylene blue, phagocytes containing reddish rods (Proteus); outside the cells are seen numerous streptococci stained blue. The organisms that have been taken up, then, take an acid stain instead of the usual methylene blue.

We may conclude, then, that the leucocytes of the peritoneal exudate have been neither killed nor paralyzed by a toxin from the streptococcus. Their faculty of engulfing has remained intact, but they refuse to enter in contact with the streptococcus owing to the fact that they receive a negative chemical stimulation from this organism.

It may be clearly seen from this experiment that phagocytes are capable of choosing with great delicacy between organisms offered them on account of their reaction to chemical substances. It is then very probable that when an animal is inoculated with a given bacterium the phagocytes first take hold of those individual organisms that are most attracting, that is to say, the least dangerous. From this standpoint it is easy to understand that the increase in virulence by passage through the animal body is due at least in part to selection on the part of the phagocytes. The evolution of the infection which has just been described gives evidence of this fact. The injection of the relatively scanty culture of streptococcus caused a gathering of leucocytes (it is known that the injection of simple bouillon without the presence of bacterial products, also causes this phenomenon). Leucocytes in the beginning take up a certain number of bacteria, but they fail, although present in relatively larger numbers than the bacteria, to absorb certain individuals particularly endowed with repelling properties. These more virulent organisms may multiply, and furnish new individuals of like virulence, and similarly insusceptible to phagocytic action. 
We believe, then, that we are justified in repeating what we wrote three years ago on the increase in virulence of bacterial infective agents:* "When we inject vibrios subcutaneously in a vaccinated guinea-pig an emigration of leucocytes rapidly takes place. The first white corpuscles to arrive at the point of inoculation find a considerable number of adversaries, of which they can destroy only a few. It would be sufficient for a few of these vibrios to be endowed with a slightly more intense power of attraction than their fellows, to cause the phagocytes to direct themselves by preference toward these organisms and to take them up. Very slight, almost inappreciable differences will consequently predestine certain bacteria to rapid phagocytosis. In the same way an inferiority in secretion of toxic products, however slight, will cause a predisposition to rapid destruction.

"In a word, leucocytes kill first those organisms that are less resistant, and the culture inoculated will be freed first of those individuals which either form less poison than their fellows or attract the leucocytes more.

"In the meantime the vibrios that have been left alone will divide and produce new organisms which, in turn, will be exposed to the attack of phagocytes. These latter will again suppress those individuals that are most poorly armed for the struggle, and will leave only those that possess in highest degree the two characters just noted. Thanks to this process of selection, new generations of organisms, like those represented by the cultures used in our experiments, will be derived for the most part from those bacteria which have been endowed with certain advantages."

It has already been well established that virulent strains of an organism attract leucocytes less strongly than do attenuated strains.

This phenomenon of selection certainly comes into play in mixed infections. Phagocytes may direct their efforts particularly toward one of the invaders and so neglect the other.

A diversion would thus be caused which would favor the development of the more dangerous infection. As we have given a very clear experimental example of this fact, it may be assumed that phenomena of the same sort occur under natural conditions.

* See "Adaptative changes of bacterial cultures, etc.," p. 6. 
In animals that have been vaccinated by the injection of cultures or by a preventive serum, the chemiotactic response of leucocytes for the bacterium with which the immunization has been brought about has been increased. The number of phagocytes is in general larger in immunized animals than in normal animals: the army of defenders is increased. If phagocytes are filled up by injecting inert powders into the blood stream, or if the animal has been weakened by fasting, cold, bleeding, or over-heating, an infection may develop which under normal conditions might be overcome. The objections that have been directed against the phagocytic theory by partisans of the "bactericidal theory of the body fluids" have been gradually disposed of. The body fluids do not always appear to be of importance in defending the animal body. It has already been noted that bacteria which have been inoculated in sera may suffer simply from the sudden change of medium to which they have been subjected. This is particularly true when they have become unaccustomed to living in body fluids on account of their culture on artificial media.

Besides, there is no constant or necessary parallel in normal animals, any more than in vaccinated animals, between the antiseptic properties of serum and a refractory state. It is true that the serum of animals vaccinated against the cholera vibrio, or against the vibrio Metchnikovi, is endowed with an energetic bactericidal property against its respective organism, while the serum of normal animals has only very weak destructive properties for these bacteria. But the existence of a strong bactericidal property in the serum of vaccinated animals is exceptional rather than usual. Animals well immunized against pneumonia, tetanus, hog-cholera, diphtheria, etc., have sera endowed with preventive or curative properties, which show no bactericidal property. The destruction of bacteria in such animals is always brought about by phagocytes.

The theory of the "attenuation of bacteria by the body fluids" is no more general in application than is the theory of the "bactericidal property of the body fluids." Organisms cultivated in the serum of vaccinated animals generally become accustomed to this medium and suffer no diminution in their pathogenic power. No diminution, moreover, is produced by passage through a vaccinated animal; on the contrary, it is frequently to be noted under 
these conditions that an increase in virulence occurs, due apparently to selection by the phagocytes.* Bacteria that develop in the tissues of refractory animals have little attraction for leucocytes, which is the proof of their pathogenic power. The experiments of Charrin and Roger on the attenuation of Bacillus pyocyaneus are too open to criticism to furnish any reasonable data for the doctrines of an attentuation by body fluids.

Since the intervention of phagocytes is regularly observed in animals that defend themselves against invading organisms, the phagocytic theory is fully capable of explaining the problem of immunity. But although the fact that phagocytes are the usual factors in immunity is undeniable, their importance in certain special cases may be limited. These limitations have been found particularly in infections caused by organisms belonging to the group of vibrios; and it is particularly in regard to the cholera vibrio that the discussion between the phagocytic doctrine and the "humoral" theory has grown very active. There is, in fact, in these special cases an evident correlation between the appearance of a bactericidal power in serum and the beginning of the refractory condition. This destructive property, though very slight in normal animals, becomes very marked in vaccinated animals, and if it should be shown in such animals that the destructive properties of the serum are present during life, the intervention of phagocytes would no longer appear necessary in dissipating the infection; this point, however, is one that has never been demonstrated. The researches of various observers, and particularly of Metchnikoff, tend to convince us that the tissue fluids possess during life no bactericidal property comparable to that shown by the serum in vitro. $\dagger$

One of the principal objects of the present study is to determine whether these bactericidal substances are not present in cells in the living body. It is to be determined whether it is the leucocytes particularly that contain the bactericidal substances, whether the elaboration of these substances is a special manifestation of their protective activity, and whether this activity is brought about

* See p. 4.

† See Metchnikoff, Études sur l'immunité, 4 e memoire. Ann. de l'Institut Pasteur, 1891. 
by an adaptation that occurs in them owing to vaccination. If such were the origin of these substances, the appearance of a bactericidal power in the serum of vaccinated animals would be indicative only of the degree of perfection which phagocytosis has reached.

Whatever may be the degree of bactericidal activity in animals vaccinated against cholera, phagocytosis can no longer be considered as a phenomenon of secondary importance in the destruction of this organism.* It will be found, to be sure, as Pfeiffer $†$ has recently shown, that vibrios injected into the peritoneal cavity of a vaccinated animal (where leucocytes are always present) may be for the most part destroyed by the fluid without having been taken up by phagocytes, but it is none the less true that many of them are taken up in the manner that Metchnikoff has demonstrated. Metchnikoff injected a culture of cholera into the peritoneal cavity of a vaccinated animal, and then.withdrew after a certain time a small amount of exudate, in which phagocytes containing vibrios are to be seen. If the exudate is placed in a moist chamber at body temperature, it is found that phagocytes removed in this manner from the animal body, and subjected to unfavorable conditions, no longer successfully oppose the development of the living organisms that they have taken up; their growth goes on inside the cells, which soon become veritable sacs stuffed with vibrios that finally burst and let the organisms escape into the surrounding fluid.

Pfeiffer does not consider these facts of great importance. Let us take his point of view for a moment and admit that the phenomena of phagocytosis have only a secondary function and that the destruction of the vibrio in the vaccinated animal is due primarily to the activity of the body fluids apart from cellular activity; it would be reasonable to conclude, then, that this humoral action would occur with greater intensity in those parts of the body most richly endowed with bactericidal substances irrespective of the presence of phagocytes. If the blood of a rabbit or guinea-pig immunized against cholera contains more of the destructive substances than the peritoneal exudate, it is reasonable to conclude that it is in the blood

* See Cantacuzène, Recherches sur le mode de déstruction du vibrion cholérique dans l'organisme, 1894.

$\dagger$ Pfeiffer, Zeitschrift für Hygiene, 1894, XVIII, p. 1. 
that the least phagocytosis takes place and that here especially the vibrio will be killed and disappear entirely, apart from any intervention of the cellular protoplasm.

It may indeed be shown that the serum of a rabbit vaccinated against cholera is more bactericidal than its peritoneal exudate.

Before considering the experiments that we have performed on this subject, it might be well to recall briefly the method of determining the relative bactericidal power of body fluids and the means of ascertaining which of two fluids - or of two sera, to be exact is the more destructive for a given micro-organism.

Small carefully measured amounts of the fluids to be compared (1 c.c. for example) are placed in separate test tubes. The growth of the organism, which, let us say, is a culture of cholera on agar, is suspended in a few cubic centimeters of salt solution ( 0.6 per cent) or of bouillon. The first of the sera is inoculated with a platinum loop of this culture dilution. The loop is agitated in the fluid with extreme precaution to distribute uniformly all the bacteria introduced; this serum has then become a homogeneous medium containing the organisms. A small amount of this inoculated serum is then carefully withdrawn by means of the loop and carried over into a tube containing fluid gelatin at body temperature. The gelatin is then shaken to scatter the inoculated organisms, and poured into a Petri dish. (First culture.)

The number of colonies that develop indicates relatively exactly the number of bacteria contained in a loop of the inoculated serum. Since the operation has been carried out rapidly, themicro-organisms carried over to the gelatin have been in contact with the serum for a short time only, and whatever bactericidal power the serum may possess has not had sufficient time to affect them. After a certain time the same amount of serum, measured accurately by means of the same loop, is again withdrawn. A second gelatin plate is inoculated. (Second culture.) If the serum is bactericidal, this second plate should contain either less colonies than the first, or perhaps none at all. If we repeat successively at intervals the same procedure, we shall have gelatin plates that will give us an indication of the number of micro-organisms remaining alive in the serum.

If the same set of manœuvres is performed in an identical manner 
at the same time with two more or less completely bactericidal liquids, a comparison may be drawn as to their bactericidal value according to the number of colonies that grow on the respective plates. It is obvious that the same emulsion of organisms is used for each series of tubes, and the same platinum loop is used so as to render the quantities of liquids strictly comparable. A table may be made indicating the number of colonies which have developed on each plate and the time after inoculation at which the transplantations were made. With this explanation we offer the protocol of an experiment made for the purpose of comparing the bactericidal properties of the blood and of the peritoneal exudate from an animal vaccinated against cholera.

Experiment 2. A rabbit weighing 1720 grams had been well vaccinated against the vibrio of Massaouah; two weeks after the last injection a small amount of blood was drawn, from which was obtained serum I; two sponges previously washed and boiled in water, sterilized, and dried (June 14, 1894), were then placed in the peritoneal cavity. They were removed the next day and found to be filled with fluid that was squeezed into two sterile vessels. (Exudates I and II.) The leucocytes in this fluid were infrequent (about 500 to the c. mm.) and quite a number of them adhered to the sponge. A second specimen of blood was then taken (serum II) which was found to contain 8200 leucocytes. One cubic centimeter of each of the samples of serum and of the peritoneal exudates was placed in a tube and their respective bactericidal powers against the vibrio of Massaouah determined by the plate method. Since it had already been determined that the serum was rather strongly bactericidal, a relatively large amount of this culture was planted each time (a loop of a vigorous agar culture, 24 hours old, suspended in 5 c.c. of salt solution of 0.6 per cent). The following plates were made at intervals indicated in the table:

NUMBER OF COLONIES.

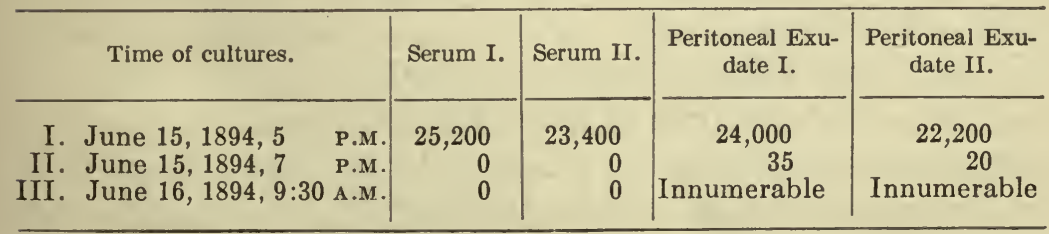


The fluid portion of the blood contains, then, more bactericidal substance than an equal amount of the peritoneal exudate. Besides this, the total volume of blood is far greater than that of the peritoneal fluid and consequently contains very much more of this destructive substance. Vibrios introduced directly into the circulation and disseminated, are therefore exposed to the maximum bactericidal action. If the phagocytes are not indispensable agents in the destruction of the vibrio inoculated into the peritoneum they must be still less important factors in the destruction of organisms injected into the circulation. Phagocytosis, however, under these latter conditions occurs energetically and rapidly.

Experiment 3. (a) A guinea-pig weighing 420 grams had been well vaccinated against the true cholera vibrio (a culture labelled Eastern Prussia). One-third of a 24-hour agar culture of this organism suspended in 0.6 per cent salt solution was injected into the jugular vein. At intervals a drop of blood was taken either from the paw or from the ear and spread on slides. After a quarter of an hour the animal was killed and preparations were made from the liver, spleen and heart's blood. The preparations were fixed by 5 per cent carbolic acid solution instead of heat, and stained by Ehrlich's method (eosin and methylene blue).

Preparations of blood taken at 4, 5, 9 and 13 minutes after injection show phagocytes containing vibrios. These phagocytes are particularly numerous in specimens taken very soon ( 4 or 5 minutes); one can see well stained and perfectly intact vibrios within the protoplasm. In the specimens taken 9 to 13 minutes after injections, and particularly in the smears from the liver and kidneys, the phagocytes contain, in addition to the normal vibrios, incompletely or irregularly stained organisms showing definite granules. A culture from the heart's blood on agar gave a few colonies. Cultures from the liver and kidney contained a great many more colonies.

(b) A well vaccinated guinea-pig of 530 grams weight (V. choleræ, Eastern Prussia) was given a third of a culture into the jugular. The animal was killed one half hour after injection; the number of leucocytes had fallen from 16,500 to 8000 . Phagocytosis is evident in blood drawn 5, 8, and 12 minutes after injection. Many polynuclears in smears from the spleen and lung. The leucocytes of the 
spleen contain irregular vibrios which are stained in places, and of a reddish or violet tinge; this evidences the chemical alterations that these vibrios have undergone. In the heart's blood half an hour after injection there are a few phagocytes containing intact and well colored vibrios. A culture from the heart on agar showed a few colonies; cultures from the liver and especially from the spleen were rich in organisms.

(c) A half culture injected into the jugular of an immunized guineapig (480 grams). The dose was so large that the animal died in 4 minutes with symptoms of asphyxia. It is well shown, however, in preparations from the blood taken at death that phagocytosis was already present.

When the number of bacteria injected into the circulation is not too large the blood soon becomes sterile, but the organs still contain living vibrios that grow well on agar.* The number of leucocytes in the blood is decreased during this same period. The phenomena following injection of cholera vibrios into the circulation are quite similar, then, to those obtained under the same conditions with other pathogenic micro-organisms; the infecting organisms are taken up by the phagocytes and carried by them to the internal organs, where they multiply. Immunity against cholera is dependent, then, on the same mechanism as immunity against other infections.

Phagocytic activity is apparently not easily inhibited, since the accumulation of the organism in the internal organs and the disappearance of leucocytes from the blood may be noted even in chloroformed animals. Chloroform anesthesia then, sufficient to deaden the nervous centers, has no effect on the activity of phagocytes.

Experiment 4. Vaccinated guinea-pig "A," weight 400 grams; the animal was well anesthetized, so that all reflexes were lost. Five minutes later one-sixth of a culture of the vibrio from Eastern Prussia was injected into the jugular. The anesthetic was continued; the animal died after 20 minutes, as too much chloroform was given. The leucocyte count had fallen from 13,000 to 4300 . The heart's blood was sterile, whereas the extract from liver, spleen and lungs gave positive cultures.

* Vibrios that have been in contact with bactericidal substances frequently fail to grow in gelatin, although they still grow in agar. 
A control guinea-pig "B," weighing 400 grams, had been vaccinated in the same manner as "A." One-sixth of a culture was given this animal without chloroform. In 20 minutes the leucocyte count had fallen from 9500 to 5000 . At this time the blood was sterile; liver, lung and spleen gave positive cultures.

\section{Leucocytes and the Bactericidal Power of Serum.}

Phagocytosis is not of secondary importance in the mechanism of cholera immunity. Leucocytes take up vibrios which soon show alterations in form and reaction to dyes within the protoplasm, which indicates that they have been affected by some harmful substance. Is it not possible that the bactericidal substance in serum comes from the leucocytes? Metchnikoff has already offered the suggestion (1887) that the bactericidal substances of serum might be of leucocytic origin. In 1899 he wrote:* "I might add that those who have asserted that the action of serum against bacteria is independent of leucocytes have not taken into consideration those substances liberated in the serum as the result of the destruction of leucocytes. It has been repeatedly noticed that these cells when removed from their normal surroundings break up and liberate their contents into the surrounding fluid." Hankin $\dagger$ and an English experimenter, Kanthack, later attributed to the eosinophiles the secretion of bactericidal substances. According to this conception these latter cells may be broken up in the blood stream and so affect the micro-organisms that have entered the body. Bacteria, killed or attenuated by contact with these substances, might later on be taken up by phagocytes. According to this conception also it is the phagocytes which form the bactericidal substances. The engulfing of bacteria by living cells instead of being a phenomenon essential to the defense of the organism would be rather an accessory phenomenon subsequent to the destruction of the bacteria by bactericidal substances dissolved in the plasma.

* Annales de l'Institut Pasteur, December, 1889.

$\dagger$ Hankin, Ueber den Ursprung und Vorkommen von Alexinen im Organismus (Centralblatt für Bakteriologie, 12, Nos. 22 et 23, December, 1892); Ueber die Theorie der Alexocyten (Centralblatt für Bakteriologie, 14, No. 25, December, 1893). 
Hankin's theory, although resembling the bactericidal theory of body fluids, is not open to the objections that may be offered to the latter.

The two essential points in these doctrines are the following: 1. There is a bactericidal substance in solution in the body fluids, and bacteria are taken up by the phagocytes only after having been subjected to the influence of this substance. But, far from being subsequent to a preparing action on the part of the bactericidal substance, phagocytosis takes place with surprising rapidity; it begins, indeed, immediately after inoculation of the organisms. In the case of cholera particularly we have just seen that shortly after the intravenous injection of cholera vibrios a number of them are found inside of phagocytes. And, what is more, it has frequently been proved by Metchnikoff that cells can take up living bacteria.

2. Bactericidal substances are elaborated by eosinophilic leucocytes. It is known, however, that these cells are of no great importance in defense of the organism. Phagocytosis and the destruction of bacteria, moreover, goes on very well in invertebrates, although they have no eosinophiles. As we shall see later on in our experiments on "phagocytosis in vitro," various bacteria when mixed with an exudate rich in phagocytes, but containing no true eosinophiles, are rapidly taken up and modified in their form and staining reaction.

Denys * and his pupils think that leucocytes play an important part in the elaboration of bactericidal substances; and they endeavor to reconcile the phagocytic and the humoral theories. Buchner $\dagger$ is inclined to admit that leucocytes are of great importance in the formation of bactericidal substances.

There are two phases to the problem; first: do the leucocytes form the bactericidal substances found in serum? And, secondly: if the leucocytes really form these bactericidal substances, do they retain them during life or do they excrete them into the surrounding plasma?

It is perfectly evident that in order to draw conclusions from experiments which are of any value to our knowledge of immunity, we must study the bactericidal property in its simplest aspects and

* La Cellule, 1893 et 1894.

† Buchner, Münchener medicinische Wochenschrift, 1894. 
when it bears some definite relation to a condition of resistance. It often happens, as we know, that the serum of an animal is bactericidal for a given micro-organism, although the animal itself is quite susceptible to infection by that very organism. For example, rabbit serum is bactericidal for the anthrax bacillus, although the rabbit is highly susceptible to this bacterium.*

This is simply an example of the spontaneous bactericidal power found in normal animals; its significance from the standpoint of immunity is not clearly established, and its importance is doubtless exaggerated.

We shall consider then, more particularly the strong bactericidal property in animals immunized against certain infections, for example, against cholera. The bactericidal property of the serum of normal animals against the cholera vibrio is insignificant. Following vaccination the bactericidal property becomes very distinct. Since it is very evident during the stage when the animal is insusceptible to infection, its relation to immunity is unmistakable.

* The aqueous humor of the rabbit is bactericidal for anthrax. This fluid contains few or no leucocytes; and it would seem, therefore, difficult to attribute the bactericidal property to cells. But it is to be noted that bacteriolysis occurs in this instance in a highly susceptible animal, and obviously, therefore, bears no relation to a condition of resistance. It differs, also essentially, from the bacteriolysis that occurs in certain instances of immunity, as the property in this instance resists heating, to 60 degrees for an hour. The bactericidal property would seem to be more like that shown by such liquids as bouillon.

It is evident from the following table that the aqueous humor of the rabbit even after heating for an hour to $60^{\circ} \mathrm{C}$. is quite bactericidal for B. anthracis.

EXPERIMENT 4. Aqueous humor was taken from a rabbit. Part of it was heated to 60 degrees for an hour, and the rest was not heated. Equal amounts of each fluid were placed in tubes. A culture of non-spore-forming anthrax bacilli, 24 hours old, was suspended in 0.6 per cent $\mathrm{NaCl}$ solution and planted in these tubes. Gelatin plates were made at intervals.

Number of Colonies.

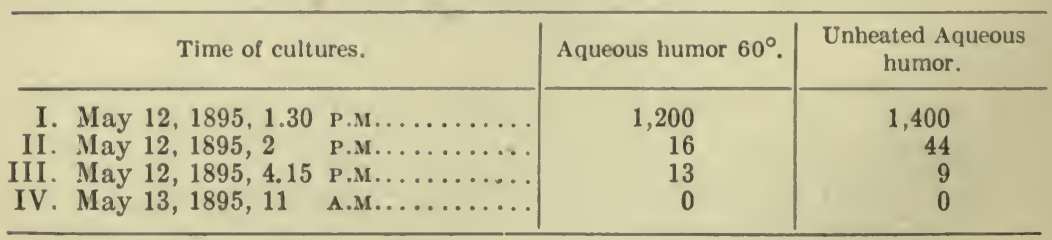


Our experiments have been carried out largely with two cholera vibrios: the vibrio of Massaouah, isolated by Pasquale, and the vibrio from Eastern Prussia. We shall not consider here whether or not the vibrio of Massaouah is of the same variety as the typical Koch vibrio. The researches of Pfeiffer would indicate that there are characteristic differences between cholera vibrios from different sources, which would lead us to think that they do not all belong to the same strain of bacteria. It would appear that there are several varieties of cholera vibrios and that one of these varieties which is widely distributed and is of great importance is represented by Koch's vibrio. This organism, according to Pasquale, is the sole cause of epidemics. However that may be, animals immunized against the vibrio of Massaouah have energetic specific preventive and bactericidal properties in their sera and therefore fulfill the conditions we desire. What is more, this vibrio has the advantage of being highly virulent and relatively constant in its pathogenicity.

We have tried to effect a separation between the blood fluid and the blood cells in an animal vaccinated against cholera. For this purpose it was necessary to obtain a more or less cell-free plasma, and we succeeded in doing this by causing an edema of the leg or ear by venous compression. The edema fluid represents blood plasma filtered under pressure and almost completely deprived of cells. The walls of blood vessels are not sufficiently impermeable to hold back all the cells, and the fluid obtained in this manner contains a few red blood cells and very infrequent leucocytes. A comparison may be made between fluid obtained in this manner and the serum of the same animal as regards their respective bactericidal powers. The serum is obtained by spontaneous coagulation of the whole blood containing many cells. If during life bactericidal substances remain within the cells and appear in the blood fluid only after removal from the body and coagulation, a distinct difference should appear between these two fluids.

EXPERIMENT 5. A rabbit weighing 1790 grams was vaccinated against the Vibrio Massaouah. The serum of this rabbit was tested for bactericidal and preventive properties and found to be markedly bactericidal. One-fifth of a cubic centimeter moreover protected 
against $\frac{1}{13}$ of a 24 -hour culture, a fatal dose, and even against $\frac{1}{10}$ of a culture.

A few days later an edema was produced in this rabbit by constricting the root of the ear with a rubber ring (edema fluid " $A$ ").

One of the fore legs received the same treatment, and, on tapping, a clear liquid, edema fluid "B," was obtained, which was slightly pinkish but contained very few leucocytes (one to two hundred per cubic mm.). A few cubic centimeters of blood were then taken and found to contain 3600 leucocytes. The serum of a normal rabbit was used as a control. One cubic centimeter of each of these fluids was put in a separate tube. A platinum loop from a 24-hour culture of the organism suspended in 5 c.c. of salt solution was placed in each tube.

\section{EXPERIMEN'T WITH EDEMA "A" (FROM EAR).}

\begin{tabular}{|c|c|c|c|}
\hline Time of cultures. & $\begin{array}{l}\text { Serum of vac- } \\
\text { cinated ani- } \\
\text { mal. }\end{array}$ & $\begin{array}{c}\text { Edema of vac- } \\
\text { cinated ani- } \\
\text { mal. }\end{array}$ & $\begin{array}{l}\text { Serum of } \\
\text { normal rab- } \\
\text { bit. }\end{array}$ \\
\hline 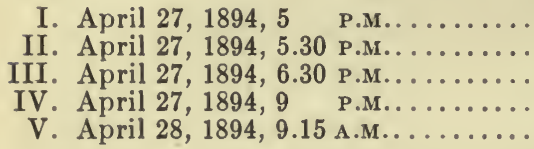 & $\begin{array}{r}15,000 \\
1 \\
0 \\
0 \\
0\end{array}$ & $\begin{array}{c}14,000 \\
6 \\
3 \\
0 \\
\text { Innumerable }\end{array}$ & $\begin{array}{c}12,900 \\
840 \\
180 \\
420 \\
\text { Innumerable }\end{array}$ \\
\hline
\end{tabular}

EXPERIMENT WITH EDEMA "B" (FROM LEG).

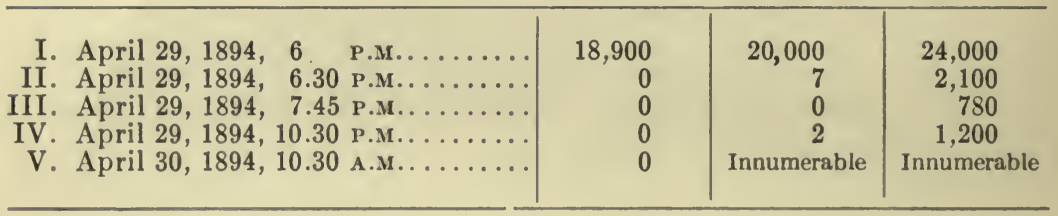

The day following inoculation the tubes containing normal rabbit serum and edema fluid were both cloudy and filled with vibrios; the serum of the vaccinated rabbit was perfectly clear. Two days later a culture made on agar from this serum showed that it had remained sterile. The difference in bactericidal power between the edema and the serum of the vaccinated animal is therefore evident. 
Although the cholera vibrio grew well in the edema fluid; this fluid showed, during the first few hours, a very distinctive bactericidal property, although less than that of serum. The normal serum also possessed a certain bactericidal property. It is to be noted that the gelatin plate made three or four hours after inoculation from the edema fluid was sterile. It is evident, however, that not all the organisms in this fluid were killed, since the cultures made on the following day showed numerous colonies. It should be remarked in this connection that gelatin is not a very favorable medium on which to grow the cholera vibrio. Vibrios that are slightly weakened but are still alive may fail to grow on gelatin when they grow very well on agar. We have often noted this fact, and a growth on agar, then, must be considered the necessary criterion of the sterility of a sample of inoculated serum.

Experiment 6. A rubber ring was placed about the fore leg of a guinea-pig vaccinated by sterilized and living cultures of the cholera vibrio from Eastern Prussia. An edema was formed which was withdrawn and found to be slightly reddish in color and to contain a few leucocytes. A specimen of blood was taken containing 11,000 leucocytes per cubic centimeter. The serum and the edema fluid were inoculated with a 24 -hour culture of the vibrio (Oriental Prussia).

NUMBER OF COLONIES.

\begin{tabular}{ccc|r|r}
\hline \multicolumn{2}{c|}{ Time of cultures. } & Serum. & Edema. \\
\hline I. February 23, 1895, 11 & A.M...... & 15,600 & 15,000 \\
II. February 23, 1895, 1.30 P.M..... & 0 & 0 \\
III. February 23, 1895, 6 & P.M...... & 0 & 660 \\
IV. February 24, 1895, 10 & A.M..... & 0 & Innumerable \\
\hline
\end{tabular}

The edema fluid was cloudy the next day, and under the microscope an enormous number of vibrios were found in it; the serum was clear and contained no bacteria. The edema fluid, however, had sufficient activity to weaken the vibrios during the first few hours and to prevent growth on gelatin. The serum gave no growth on agar.

The production of edema is the best means of separating the cells from the blood plasma, but there is another method which may be 
used to study the effect of a variation in leucocytes on the bactericidal property of the blood. This method consists in artificially lowering the number of leucocytes in the circulating blood and testing the bactericidal property of the blood before and after.

The most efficient method of lowering the number of leucocytes is to inject bacteria, but the method could not be used in this work, as the introduction of bacterial substances might affect the bactericidal property of the blood in some manner. Instead of bacteria, then, an emulsion of some fine inert powder such as carmin must be used. It has been asserted that cooling an animal in water brings about a fall in the number of leucocytes, but we have had no success with this method. The injection of carmin generally produces a rapid hypoleucocytosis. In certain cases, however, the fall in white corpuscles does not follow. It is probable that the taking up of grains of carmin by phagocytes depends on the tactile reaction of these cells. It is important to determine that the fluid in which the carmin is suspended has no positive chemiotactic influence on leucocytes, as shown by an experiment with capillary tubes.

Experiment 7. A guinea-pig weighing 355 grams had been vaccinated against the vibrio of Massaouah. A small amount of blood was taken from the animal and found to contain 11,000 leucocytes. One-tenth of a cubic centimeter of a rather thick emulsion of carmin in salt solution (50 centigrams of carmin to 10 c.c. of fluid) was slowly injected into the jugular vein. Two hours later the leucocyte count had fallen to 3000. Another small amount of blood was taken. One cubic centimeter of serum from each blood specimen was then placed in a tube and inoculated with a 24-hour culture of Massaouah (1 loop of a culture suspended in 10 c.c. of normal salt solution).

NUMBER OF COLONIES.

\begin{tabular}{|c|c|c|}
\hline Time of cultures. & $\begin{array}{l}\text { Serum containing } \\
11,000 \text { leucocytes. }\end{array}$ & $\begin{array}{l}\text { Serum containing } \\
3,000 \text { leucocytes }\end{array}$ \\
\hline $\begin{array}{r}\text { I. July } 5,1894,5 \text { Р.м. } \ldots \ldots \ldots \ldots \ldots \\
\text { II. July } 5,1894,7 \text { Р.м.... } \\
\text { III. July } 6,1894,11 \text { А.м.... } \ldots \ldots \ldots \ldots\end{array}$ & $\begin{array}{r}9,600 \\
0 \\
0\end{array}$ & $\begin{array}{l}10,000 \\
2 \\
\text { Innumerable }\end{array}$ \\
\hline
\end{tabular}


Frequently, as already mentioned, the injection of carmin, even though repeated, gives no evident hypoleucocytosis. Under these conditions there is no difference in the bactericidal property of the blood before and after injection. Carmin itself has no effect on the bactericidal substances, as may be shown by adding it to serum.

The injection into normal animals of a rather large quantity of serum from an unvaccinated animal brings about a distinct increase in the bactericidal property of the serum of the animal. The increased bactericidal property under these conditions, however, is not to be compared with that caused by injecting the serum of vaccinated animals and, moreover, is not specific. The effect of injecting normal serum is to increase slightly the normal bactericidal property which is to be found in non-immunized animals, and this property is by no means specific. We have tried to determine whether the bactericidal property following these injections is more evident in a fluid rich in leucocytes than in one containing only a few.

Experiment 8. A small amount of blood was drawn from a guinea-pig (leucocytes, 8000). From this blood was obtained serum "A"; the same guinea-pig received, in the peritoneal cavity, 3 c.c. of serum from another normal guinea-pig. On the following day a rubber ring was placed about the paw of this animal. Three hours later the edema was removed and a small amount of blood was also taken. This blood contained 6000 leucocytes and the serum from it was labelled serum "B." Equal amounts of these fluids were then inoculated with a 24-hour culture of cholera (Oriental Prussia).

\begin{tabular}{|c|c|c|c|c|}
\hline \multicolumn{2}{|c|}{ Time of cultures. } & Serum “A." & Edema. & Serum "B." \\
\hline $\begin{array}{r}\text { I. March 7, } 1895,6 \\
\text { II. March 7, 1895, } 8 \\
\text { III. March 7, 1895, } 10.30\end{array}$ & 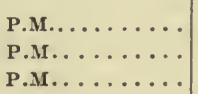 & $\begin{array}{l}5,700 \\
5,520 \\
6,420\end{array}$ & $\begin{array}{c}5,400 \\
12,000 \\
\text { Innumerable }\end{array}$ & $\begin{array}{r}6,480 \\
600 \\
840\end{array}$ \\
\hline
\end{tabular}

The edema fluid removed at practically the same time as the blood that formed serum "B" may be properly compared with this serum. After $4 \frac{1}{2}$ hours at body temperature there was no increase in vibrios in serum " $\mathrm{A}$ "; there was a very marked decrease in serum "B," but in the edema fluid there is an immediate and unrestricted 
increase of the organisms. Preparations from these fluids are even more striking than the plates; they show .a large number of organisms in the edema fluid, very few in serum "A," and still fewer in serum "B." It is also to be noted that the colonies grow more rapidly on the plates inoculated with edema fluid than on the other plates.

The increase in bactericidal property in the blood following the injection of normal serum would appear to be due to a reaction on the part of the leucocytes and not to an increase in the number of cells in the blood, as it is not accompanied by a hyperleucocytosis; on the contrary, the number of white corpuscles is found to be slightly decreased. Another similar experiment was performed with bouillon in place of normal serum. The same result was obtained, and in the same way there was no increase in leucocytes noted in the blood ( 8400 before the injection and 7400, 24 hours after injection).

There are certain undoubted conclusions that may be drawn from these experiments. If the number of leucocytes in the blood is diminished during life, the blood is found to be less bactericidal. The blood plasma, more or less completely separated from the cellular elements within the body, has less bactericidal property than the whole blood. The separation of the fluid from the cellular part of the blood outside the body by coagulation does not bring about the same result. The serum of vaccinated animals although deprived of cells is energetically bactericidal. We shall take occasion to elaborate these results later. It seems reasonable to admit not only that bactericidal substances are present in leucocytes, but that when the blood has been removed from the body the white corpuscles soon discharge into the serum those bactericidal substances which under normal conditions they retain within themselves. This would explain why the bactericidal property of body fluids which is so marked in vitro is much less evident during life. This latter fact has been particularly well brought out by Metchnikoff, and indeed it is notorious that results obtained in vitro do not always exactly indicate what goes on within the animal body. Phagocytes have an efficient and rapid means of destroying the bacteria they have taken hold of. The evidence of the effect of phagocytic secretions on many organisms, particularly on cholera vibrios, is 
well shown in their change in shape and reaction to dyes within the body of the phagocyte. We shall consider in the following section the nature of these changes within the phagocyte. There is a simple technical method that has permitted us to study with accuracy and facility a good number of bacteria from this standpoint.

\section{Phagocytosis in Vitro. The Bactericidal Property}

\section{of Phagocytes.}

Phagocytosis may take place not only in the animal body, but also in vitro. The phenomenon of phagocytosis occurs rapidly in a mixture of an exudate rich in phagocytes and a bacterial emulsion, if it be placed in a moist chamber at a temperature of 35 degrees.

A good way to obtain an exudate containing a large number of leucocytes is to inject a few cubic centimeters of peptone bouillon into the peritoneal cavity of an animal. For example, if a guineapig is given 3 c.c. of bouillon into the peritoneal cavity, the exudate on the following day is found to contain large numbers of white corpuscles, as Issaeff * pointed out. In an exudate obtained in this manner there are found large numbers of polynuclear amphophiles, considerably fewer mononuclear leucocytes and a very few true eosinophiles (that is to say, leucocytes with large round eosinophilic granules); these latter are in such small numbers that they are frequently not to be found on a single slide. The exudate may be drawn from the animal by puncturing the belly wall with the point of a finely drawn out pipette. As soon as the peritoneal cavity is reached the exudate rises in the tube to considerable height.

A drop of this exudate is placed on a cover slip. A platinum loop full of the culture fluid, in which the organism to be studied has grown, is then added to this small drop and mixed with it. The cover slip is then placed on a hollow ground slide and sealed with vaseline. After incubation for a while, stained preparations are made from this hanging drop. Preparations stained with eosin and then methylene blue (Ehrlich) are very satisfactory. The exudate is spread out on the slide and, after drying, is fixed either by heat (Ehrlich's method) or, better still, by a 5 per cent solution of carbolic acid or a saturated solution of picric acid. The latter

* Issaeff, Zeitschrift für Hygiene, t. XVI, 1894. 
method is particularly to be recommended for preparations of exudates or any body fluids containing albumin. The eosin used is an alcoholic solution (0.5 grams of eosin to 100 c.c. of 60 per cent alcohol) and the methylene blue is in saturated aqueous solution.

In general, it is best to leave the inoculated drop of exudate in the incubator for about 4 hours. By this time the bacteria will not have increased to an unreasonable extent, but phagocytosis and the changes caused in the bacteria by the secretion of leucocytes are already quite manifest. It may be noted that in a quarter of an hour, at a temperature of 35 degrees, or in even less time, leucocytes may show a very distinct phagocytic activity.

The exudate employed was obtained from guinea-pigs that had never received an injection of bacteria, but had simply been given bouillon. A list of the bacteria subjected to the action of leucocytes, with the resulting phenomena that we have noted, follows:

V. cholerce (culture from Eastern Prussia).-Polynuclear leucocytes have taken up a large number of the vibrios. The microorganisms outside the cells have retained intact their form and their reaction to dyes. Within the phagocytes there are vibrios of normal appearance colored a good blue, and also numerous organisms that show distinct granulations. These granulations take different shades of blue, light pink and deep pink. They are quite like those granules that Pfeiffer noted in the peritoneal cavity of immunized animals after injecting a culture of vibrios. These oval or rounded granulations are simply vibrios that have contracted in response to the harmful secretion from the leucocytes. The fact that none of these granulations are found outside the cells shows very clearly the superior bactericidal power of the protoplasm of the phagocytes over that of the surrounding fluid. We shall later consider these granulations more carefully and also the influences that produce them.

Some of the vibrios within the leucocytes, whether transformed into granules or not, show marked changes in reaction to dyes. Instead of staining with methylene blue, the basic color, they stain with eosin. Metchnikoff has already observed vibrios taken up in the animal body that stain with eosin; he also noted the same fact 
with the spirillum of recurrent fever.* Mesnil noted a similar fact with the Bacillus anthracis. $\dagger$

$V$. cholerce (culture from Constantinople).-Same result.

$B$. typhosus. - There are many organisms within leucocytes of which the majority have been transformed into rounded or oval granules which stain shades varying from blue to pink. The change is similar to that noted in the cholera vibrio. There are a few intact bacteria within cells that stain with eosin. The bacilli that have not been taken up by phagocytes have a normal appearance.

$B$. coli. - The results are similar to those with B. typhosus. The same intracellular granulation is to be noted due to the contraction of the organism. There are also some bacteria within the cells that appear swollen. Stains are all the way from blue to red. No change in the organism outside the cells.

Bacillus of Hog Cholera. - The results are also similar to those noted under cholera and typhoid. Within the phagocytes, in addition to normal bacteria, granules stained either with blue or with eosin are noted. The organisms outside leucocytes are intact.

Bacillus of Danysz. - This organism was discovered by Danysz in an epidemic among rats, and when injected into these animals causes a contagious and fatal enteritis. The organism is nonpathogenic for rabbits and birds, and kills only rats, mice, and fieldmice, so that it is actually being used in agricultural circles for the purpose of destroying these harmful animals. It is a cocco-bacillus that does not stain by Gram and resembles the colon bacillus in general. When subjected to the leucocytes of the guinea-pig it is taken up with extraordinary avidity. The phagocytes take up so many bacteria that each one appears to be stuffed with them. The organisms that have been taken up, when examined after the usual period, are found colored violet and at times red.

Bacillus diphtheria. - There is a very marked phagocytosis. The shape of the organisms is not changed (at least in 4 hours), but the reaction to dyes is extraordinarily modified. The majority of diphtheria bacilli taken up by phagocytes are colored a deep red or violet. Outside the leucocytes, on the contrary, the organism shows

* Cantacuzène, Mode de destruction du vibrion cholérique dans l'organisme, 1894.

$†$ Mesnil, Sur le mode de résistance des vertébrés inférieurs aux invasions microbiennes. Annales de l'Institut Pasteur, May, 1895. 
its normal reaction for the basic stain. Mononuclear leucocytes also have a very distinct affinity for diphtheria bacilli and take up a good number of them, but the organisms taken up by the mononuclears show fewer color changes than those taken up by the polynuclears.

Bacillus proteus vulgaris. - The change to a red staining bacillus is still more striking with Proteus vulgaris than with the diphtheria bacillus. After contact for quarter of an hour with a rich leucocytic exudate, eosin-stained bacteria may be found within the phagocytes. It is evident that the protoplasm of leucocytes has a very marked action on Proteus. After a few hours' contact it is very hard to find any organisms in the phagocytes that stain blue. But although these bacteria are very much changed in their chemical constitution, they show little morphological alteration. The bacteria scattered in the surrounding fluid are normal in appearance, with the exception of a few individuals that stain red. Proteus is one of the best organisms to demonstrate phagocytosis in vitro, and shows most clearly the effect of leucocytic fluid.

$B$. anthracis. - The engulfing of the anthrax bacillus by phagocytes in vitro is easily demonstrable. The polynuclears take up one or two rods; when the chain of bacteria is long, it frequently happens that several phagocytes join together about the chain and unite in absorbing it. The filaments that have been taken up stain red or a shade between red and blue. There are to be noted, it is true, in the surrounding fluid also bacteria in which changes are quite evident, and it must be admitted that the fluid of the exudate seems to show bactericidal effects on this organism. But a careful study of smears shows clearly that these substances in the surrounding fluid have come by diffusion from the leucocytes. It is perfectly evident that the red-stained bacteria are more numerous within the phagocytes than without them; and, what is more, it is not unusual to find long threads of anthrax stained blue throughout their length, with the exception of those spots in contact with the protoplasm of a leucocyte. Such a picture shows well the phagocytic origin of the soluble substances that are harmful for the anthrax bacillus.

B. muscoïdes. - Abundant phagocytosis. Extracellular organisms normal. Within the phagocytes reddish or bluish rods.

B. vermicularis. - Same result. 
B. pyocyaneus. - Distinct phagocytosis. Some of the organisms within the phagocytes are colored a faint pink.

B. ramosa and the red bacillus of Kiel. - Similar phenomena.

Streptococcus. - Polynuclear leucocytes capture large numbers of streptococci. Within 4 hours the majority of the cocci taken up still stain blue. There are to be noted, however, in some phagocytes, chains of red streptococci; at times one or two of the cocci in a chain remain blue or violet.

It is evident that the same phenomena may be observed in vivo as in vitro. For example, we have injected the diphtheria bacillus (1 c.c. of a 24-hour bouillon culture) into two guinea-pigs, one of which had received the day before 3 c.c. of bouillon in the peritoneal cavity, and the other of which had received 3 c.c. of anti-diphtheria serum. The injection of bouillon produced a rich leucocytic exudate composed largely of polynuclears, but also containing a few mononuclears. In the animal injected with preventive serum, the exudate contained many polynuclears, but, in addition, a number of mononuclears (macrophages). In both animals phagocytosis is noted after injection of the culture, and, as is usual, the polynuclears seem to take the greater part in it. In the case of the guinea-pig vaccinated with serum, however, the macrophages are found to have taken up considerable numbers of organisms. After an hour there are numerous intraphagocytic bacilli staining red both in the animal that had received bouillon and in the one immunized with serum. Although the taking up and destruction of the organisms appears to go on without any difficulty, there remain certain resistant bacteria, so that positive cultures were obtained from the exudates of both animals 18 hours after injection.

The uniformity with which phagocytosis occurs even when leucocytes have been taken out of the animal body and are no longer in their normal condition is amply proved by these experiments.* Changes of the bacteria in cells are always noted. One

* The activity of phagocytes against bacteria in vitro gives a means of studying the effect of different poisons, toxins or drugs on these cells. Active substances may be added to leucocytes and then, after varying periods of time, bacteria may also be added. We are at present engaged in studies along this line. We are rather surprised to find that diphtheria toxin, which kills the guinea-pig in a dose of 0.2 to 0.1 of a cubic centimeter, produces no effect on the phagocytic activity of the leucocytes of this animal. 
of the most interesting of these changes, as we have already observed, is the ease with which certain bacteria gain the power of absorbing eosin instead of methylene blue. On account of the regularity with which this phenomenon occurs, one is inclined to attribute the pseudo-eosinophilic granules in macrophages to bacterial origin. As for the morphological changes in engulfed bacteria, it is to be noted that there are present, in leucocytes of normal animals that have received an injection of cholera vibrios, the same sort of granules (transformed vibrios) as in the peritoneal fluid of highly vaccinated animals. In the normal animals, however, the bactericidal property, as evidenced by the transformation of the vibrio into granules, is never so energetic, and brings about the change only where the bactericidal substance is highly concentrated, that is to say, within the phagocytes.

\section{Leucocytes and the Preventive Power of Serum.}

It is well known that the blood of animals that have been vaccinated several times with a culture of living vibrios killed by heat, has not only bactericidal but preventive properties. The injection of serum from such vaccinated animals into healthy animals permits the latter to withstand a dose of culture that, under ordinary conditions, is certainly fatal. There are preventive cholera sera which are so very powerful that they act in doses as small as two or three milligrams.

Will facts offered in explanation of the bactericidal property of serum also explain the preventive power? Are the substances that endow the serum of vaccinated animals with their preventive properties present in the body fluids of the living animal? Or are they, on the contrary, more or less retained by the white blood corpuscles during life? The experimental procedures already described are also applicable to this problem.

It is perfectly evident that the relative preventive value of serum and of edema fluid from the same vaccinated animal may be compared. We may also compare two specimens of blood from the same animal containing different numbers of leucocytes, and this method we have employed. In these experiments we used the vibrio of Massaouah, which is an advantageous culture for two reasons: its virulence is relatively constant, and, secondly, it is so 
pathogenic that there is no necessity for injecting large amounts of the culture into the animals, which might be toxic apart from any increase in organisms that may take place. It frequently happens in using a culture of cholera vibrios of low pathogenic power, but of high toxicity (for example, the culture from Eastern Prussia), that well vaccinated guinea-pigs die on receiving a sub-lethal dose, and die, not of infection, but from intoxication; it is well known, indeed, that protection by means of serum, or active immunization by means of bacteria, does not decrease the sensitivity of the animal to the toxin. It is evident, then, that such cultures should not be used in comparing the preventive value of two fluids. The Massaouah vibrio employed kills guinea-pigs in a dose of $\frac{1}{15}$ of a 24 -hour agar culture intraperitoneally.

The preventive fluids were injected into the peritoneum 24 hours before the intraperitoneal injection of the culture. The agar culture used for injection was suspended in a definite amount of salt solution ( 0.6 per cent) and formed a homogeneous emulsion that could be easily measured.

ExPERIMENT 9. Rabbit " A" had been vaccinated against the Massaouah culture and the blood and edema fluid had been studied in respect to their bactericidal properties. 0.25 of a cubic centimeter of each liquid was injected into the peritoneal cavity of a guinea-pig. The one receiving the serum weighed 330 grams, the one that received edema fluid, 345 grams. Twenty-fours hours later these two guinea-pigs, as well as a control normal guinea-pig (400 grams), received each $\frac{1}{15}$ of a culture. The control died rapidly. The two guinea-pigs that had received protective injections recovered.

In this experiment both the edema fluid and the serum were shown to contain preventive properties. But it was found by increasing the amount of culture and diminishing slightly the amounts of the preventive fluids injected that the properties are not present in equal amounts in the two fluids.

EXPERIMENT 10. A guinea-pig weighing 500 grams received 0.2 of a cubic centimeter of serum from the vaccinated rabbit. Another guinea-pig ( 525 grams) received 0.2 of a cubic centimeter of edema fluid. A control guinea-pig (535 grams) received 0.2 of a cubic centimeter of normal rabbit serum. Twenty-five hours later 
each guinea-pig received $\frac{1}{10}$ of a culture. The next morning the guinea-pigs that had received the edema fluid and the normal rabbit serum respectively were found dead; the guinea-pig that had received preventive serum was slightly sick, but soon recovered. There were a number of leucocytes in the peritoneal exudate of the guinea-pig that had received the edema fluid, but very few in the control that had received normal rabbit serum.

Even when the dose of culture used is very large or the guineapigs are very small, so that the immunity conferred by the serum is not perfect, there is always to be noted a distinct delay in the death of the animal receiving the preventive serum over the other two. For example:

Experiment 11. Guinea-pig "A" (245 grams) received 0.2 of a cubic centimeter of serum; guinea-pig "B" (255 grams), 0.2 of a cubic centimeter of edema fluid. The following day these animals and a control "C" (339 grams) were given each $\frac{1}{10}$ of a culture. The following day the guinea-pig injected with edema and the control were found dead; the guinea-pig injected with preventive serum did not die until the following day, which is a considerable delay when one considers the usual rapid evolution of the peritonitis caused by the cholera vibrio. In such instances an examination of the peritoneal exudate is interesting. The exudate in the control is always found to be poor in leucocytes and over-running with vibrios. The exudate from the guinea-pig that has received edema fluid always contains considerably more cells than the control, but very much fewer than does the exudate of the animal that has received serum; and in this latter animal, too, the vibrios are very much fewer in number. The proportion of leucocytes in the exudate indicates the relative resistance of the individual.

ExPERIMENT 12. A rabbit had been well vaccinated against the Massaouah organism. Eighteen days after the last injection an edema was produced. The blood contained 5000 leucocytes per c.m.m.

(a) Rather large guinea-pigs were used. No. 1 (455 grams) received 0.3 of a cubic centimeter of edema fluid; No. 2 (430 grams), 0.3 of a cubic centimeter of serum; on the following day these two animals and a control, No. 3 (450 grams), received each $\frac{1}{10}$ of a culture. The control was the only one to die. 
(b) Smaller guinea-pigs. No. 1 (240 grams) received 0.2 of a cubic centimeter of serum; No. 2 ( 265 grams), 0.2 cubic centimeters of edema. These two animals and a control, No. 3 (300 grams), were given $\frac{1}{10}$ of a culture on the following day. The control (No. 3 ) and the guinea-pig that had received edema fluid were found dead the following day. The guinea-pig that had received serum withstood the infection.

(c) A guinea-pig (325 grams) received 0.2 of a cubic centimeter of serum. Guinea-pig No. 2 (350 grams), 0.2 of a cubic centimeter of edema. Control guinea-pig (340 grams). Each received $\frac{1}{10}$ of a culture the evening of the same day. The following morning the control and the animal that had received edema fluid were found dead. The guinea-pig that had received serum died two days later, probably of intoxication, since the heart's blood was sterile; the peritoneal exudate was found to contain a rather large number of leucocytes, which were very infrequent in the exudates from the other two animals.

Let us now consider the preventive value of two samples of serum from the same animal, containing different numbers of leucocytes.

ExPERIMENT 13. A guinea-pig that had been well vaccinated against Massaouah was given an injection of carmin. A specimen of blood was taken before and after injection (serum "A" before, number of leucocytes 11,000 ; serum " $B$ " after injection, number of leucocytes 3000). The bactericidal properties of the two sera were studied by the method already described.

(a) Guinea-pig No. 1 (360 grams) received 0.3 of a cubic centimeter of serum "A." Guinea-pig No. 2 (390 grams) received 0.3 of a cubic centimeter of serum "B." Control guinea-pig (455 grams). The following day each animal received $\frac{1}{15}$ of a Massaouah culture. The control alone died.

(b) Guinea-pig No. 1 (345 grams) received 0.2 of a cubic centimeter of serum "A"; guinea-pig No. 2 (375 grams), 0.2 of a cubic centimeter of serum "B." Control (440 grams). The following day each animal received $\frac{2}{10}$ of a culture. The control and the animal that received serum " $\mathrm{B}$ " (containing the smaller number of leucocytes) were found dead the following morning. The guineapig that received serum "A" died the same day in the afternoon; the peritoneal exudate from this animal was the richest in leucocytes. 
(c) Guinea-pig No. 1 (340 grams) received 0.2 of a cubic centimeter of serum "A"; guinea-pig No. 2 (350 grams), 0.2 of a cubic centimeter of serum "B"; control guinea-pig (355 grams). Animals given $\frac{1}{10}$ of a culture. Guinea-pig No. 2 and the control died the following afternoon. Guinea-pig No. 1, that had received serum "A," survived.

It is evident, then, that serum has a higher preventive power than plasma, or, rather, than a transudate caused by venous compression and containing fewer leucocytes than serum. Serum containing the normal number of leucocytes is more preventive than serum in which the leucocytes have been artificially diminished. Although the edema fluid has, to be sure, a distinct preventive property, the data given indicate very clearly the importance of leucocytes in respect to the preventive properties of serum. It is quite possible that during life, under normal conditions, a certain diffusion of preventive substances from the leucocytes may take place, although there is no certainty that the edema produced by a rubber band corresponds at all exactly to the plasma of the blood.

\section{Specificity of the Bactericidal Substance in the Serum of Vaccinated Animals.}

In the group of vibrios there are several bacterial species having similar characters. It is very difficult either by morphology or by cultural characteristics to distinguish the cholera vibrio from the vibrio of Massaouah, the vibrio of Deneke or the vibrio of Finkler. The great importance of finding some means of distinction between these organisms accounts for the careful study that has been made of them. On account of its pathogenic importance and for the value that a positive diagnosis of its presence would have, the cholera vibrio has been particularly well studied in order to determine such characteristics as could be used to separate it from other similar micro-organisms. Most of the attempts at separation of this organism have failed, and it was found that certain properties which were first supposed to be specific were also present in other vibrios. The comma bacillus presents frequent confusing changes; it changes morphologically; it does not always liquefy gelatin with the same rapidity; and its virulence is very changeable and easily lost. The Finkler organism or the Deneke organism, which are 
usually considered non-pathogenic, may, after a few passages, infect animals quite as well as the typical cholera vibrio. The cholera red reaction is no certain method of diagnosis. Morphologically and culturally, then, the separation of Koch's bacillus from other vibrios is impossible.

The question arises as to whether these vibrios, which are so much alike, always react in the same manner to the bactericidal sera of vaccinated animals. Is the bactericidal property of the serum of a vaccinated animal active only against the species of vibrio used to vaccinate the animal, or is it, on the contrary, a more general property and active against the majority of organisms belonging to the same group? Pfeiffer states that the bactericidal property in the sera of vaccinated animals is specific. We have made a few experiments along this line. The sera of several guinea-pigs and rabbits, each of which had been immunized against a certain vibrio, were tested for their bactericidal property against several different vibrios.

I. Serum of a rabbit immunized against the Massaouah Vibrio.

NUMBER OF COLONIES.

\begin{tabular}{|c|c|c|c|c|c|c|}
\hline Time of cultures. & 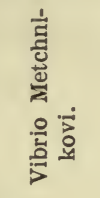 & 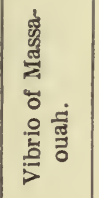 & 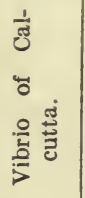 & 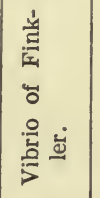 & 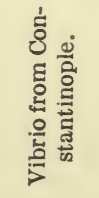 & 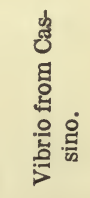 \\
\hline $\begin{array}{l}\text { I. Apr. } 30,1894,4.30 \text { P.м. } \\
\text { II. Apr. } 30,1894,6.15 \text { P.M. } \\
\text { III. May 1,1894, } 11.15 \text { A.M. }\end{array}$ & $\begin{array}{c}16,800 \\
16,200 \\
*\end{array}$ & $\begin{array}{r}13,000 \\
8 \\
0\end{array}$ & $\begin{array}{r}7,800 \\
0 \\
0\end{array}$ & $\begin{array}{r}13,500 \\
70 \\
120\end{array}$ & $\begin{array}{l}17,400 \\
16,800 \\
*\end{array}$ & $\begin{array}{r}12,000 \\
\quad 30 \\
*\end{array}$ \\
\hline
\end{tabular}

II. Serum of a guinea-pig immunized against the Massaouah Vibrio.

\begin{tabular}{|c|c|c|c|c|c|}
\hline Time of cultures. & 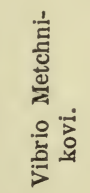 & 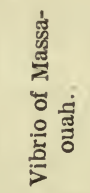 & 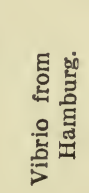 & 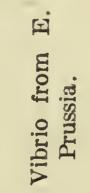 & 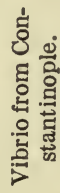 \\
\hline 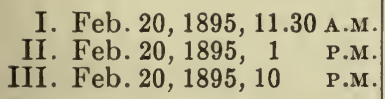 & $\begin{array}{c}1,150 \\
420 \\
*\end{array}$ & $\begin{array}{r}1,200 \\
0 \\
0\end{array}$ & $\begin{array}{l}1,380 \\
210 \\
*\end{array}$ & $\begin{array}{c}1,280 \\
240 \\
*\end{array}$ & $\begin{array}{l}600 \\
420 \\
*\end{array}$ \\
\hline
\end{tabular}

* Innumerable. 
III. Serum of a guinea-pig immunized against the Vibrio Metchnikovi.

\begin{tabular}{|c|c|c|c|c|c|}
\hline Time of Cultures. & 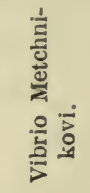 & 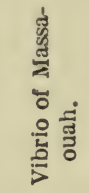 & 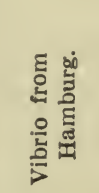 & 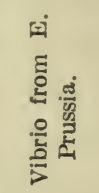 & 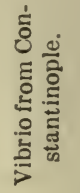 \\
\hline $\begin{array}{l}\text { I. March 2, 1895, } 2 \text { P.м. } \\
\text { II. March 2, 1895, } 5 \text { P.M. } \\
\text { III. March 3, 1895, } 10 \text { А.M. }\end{array}$ & $\begin{array}{r}1,200 \\
0 \\
0\end{array}$ & $\begin{array}{r}6,600 \\
90 \\
240\end{array}$ & $\begin{array}{c}12,420 \\
4,320 \\
*\end{array}$ & $\begin{array}{c}12,900 \\
4,800 \\
*\end{array}$ & $\begin{array}{l}9,000 \\
5,700 \\
*\end{array}$ \\
\hline
\end{tabular}

IV. Serum of a guinea-pig vaccinated against the Cholera Vibrio. (Eastern Prussia.)

\begin{tabular}{rrr|r|r|r|r}
\hline I. Mar. 6, 1895, 4 & P.M. & 15,200 & 12,400 & 16,000 & 14,300 & 16,800 \\
II. Mar. 6, 1895, 6 & P.M. & 6,200 & 950 & 0 & 0 & 0 \\
III. Mar. 7, 1895, 10.30 A.M. & $*$ & $*$ & 0 & 0 & 0 \\
\hline
\end{tabular}

* Innumerable.

We have not considered it necessary to carry these experiments farther because the results agree wholly with those recently obtained by various observers. In fact, we have a method which is much easier to employ than the gelatin plate method to detect whether a given vibrio is susceptible to a given immune serum. This method, which we shall later consider, consists in estimating the susceptibility of the vibrio to granular transformation (Pfeiffer's phenomenon) which occurs when the organism is mixed either with the specific immune serum or the immune serum plus a small amount of fresh normal serum.* It will be seen in the tables which have been given that the bactericidal property of a serum is always more active against the vibrio which has served to vaccinate the animal furnishing the serum, or for those few organisms that are closely allied to the immunizing organism. There is no doubt that the bactericidal effect is highly specialized, since, however similar organisms may be morphologically and culturally, certain of them are wholly unaffected by a serum fatal to others. It is to be noted

* As will be seen later, the immune serum must have been recently obtained in order to effect a granular transformation of an organism without the addition of fresh normal serum. 
that there are several differences of this sort in the type organisms of the species "cholera vibrio." For example, the vibrio of Massaouah does not act as do the cholera vibrios of Eastern Prussia, Constantinople, or Hamburg. This does not at all mean that it has not the same pathogenic properties as these latter. Immune serum gives a delicate reaction, but we do not know whether the distinctions it draws between vibrios are always fundamental ones. It is certainly proper to collect all the organisms that react in the same way to a given serum into one distinct group and to separate this group from all the others, provided that the criterion of separation is distinctly noted. The grouping of a given species is not always the same if some other criterion is used. There is no means of proving that a classification based on a reaction to serum is the same as a classification according to pathogenic power. There is no reason for saying, for example, that the vibrio of Massaouah cannot produce cases or epidemics of cholera from the simple fact that it is not affected by the serum of animals immunized against the cholera vibrio from Eastern Prussia.

\section{The Nature of the Bactericidal Substance in Vacci- nated Animals. Its Identity with the Bactericidal Substances in Normal Animals.}

The immunized animal is remarkably well equipped for combating the organism against which it has been vaccinated. In its phagocytes it has a bactericidal power which would seem to direct itself exclusively against that bacterial species against which it has been immunized. But even the normal unvaccinated animal has a certain amount of protective power against vibrios. When vibrios are inoculated in normal serum there is generally noted in the beginning a decrease in their number. Although this destructive power is not distinctly marked against virulent vibrios, it is more marked, as Pfeiffer has recently shown, against such vibrios as have been grown for some time on artificial media and have therefore become unaccustomed to the body fluids and lost their virulence. It is well known, moreover, that this normal bactericidal power may be increased by injecting into the animal a few cubic centimeters of bouillon or of normal serum.

There are certain pioperties in common between the bactericidal 
substance in normal animals and the specific bactericidal substance in immunized animals. They are both destroyed at about $60^{\circ} \mathrm{C}$., and sera heated to this temperature become excellent culture media. In both instances the power is lodged within the phagocytes. But these two substances may be sharply distinguished from one another by the fact that the less active substance of normal serum is in no sense specific and attacks vibrios indifferently, while the other, in vaccinated animals, is very powerful and is highly specific. The normal bactericidal substance is harmful for attenuated vibrios, whatever may be their origin, but the specific bactericidal substance is fatal only for a definite race of vibrios. The two substances would appear, then, to be quite different.

What is more, the bactericidal substance in the serum of immunized animals differs from the preventive substance in the same serum. The researches of C. Frankel and Sobernheim* have shown that the preventive substance resists a prolonged heating to 70 degrees, whereas the bactericidal substance is entirely destroyed at this temperature. Serum heated to 70 degrees is quite as capable as fresh serum of conferring passive immunity; in other words, the preventive substance is quite unaltered by this temperature. It may be noted that the essential property of this preventive substance lies in causing an intense bactericidal property in the treated animals, as was well established by Frankel and Sobernheim in their important study. These experimenters injected serum from animals vaccinated against cholera into guinea-pigs, and noted on the following day that the serum of these guinea-pigs had become energetically bactericidal for Koch's vibrio. The same result was observed in animals given injections of serum heated to 70 degrees and thereby deprived of its own bactericidal property. For the moment, the fact of importance to us is that the preventive substance which immunizes animals differs from the bactericidal substance.

It would seem, then, that we must consider at least three important active substances in immunity: two different bactericidal substances, and a preventive substance. But, as a matter of fact, the matter is not so complicated. It would seem that the bactericidal substances of normal and of immunized animals which differ in the matter of specificity are, in reality, quite identical. The feeble bac-

* Fraenkel and Sobernheim, Hygienische Rundschan, Nos. 3 et 4, 1894. 
tericidal substance in the normal serum acts energetically in the immune serum owing to the action of the preventive substance that accompanies it, increases its power, and lends to it its specific character.

As a matter of fact it suffices to add a small amount of anticholera serum, either fresh or previously heated to 60 degrees and so deprived of its bactericidal property, to normal serum to endow the latter with a very strong bactericidal property. Two liquids then, neither of which is markedly bactericidal, form a mixture that is strongly bactericidal. As we shall later see this bactericidal property is specific and evidenced only toward that race of bacteria used to vaccinate the animal from which the preventive serum has been obtained. It makes no difference in the bactericidal property if the serum is quite clear and without cells or contains only a few corpuscles. This fact may be expressed in another way by saying that after the preventive serum has been heated its intense and specific property may be restored by adding normal serum. This is brought out by the following experiment:

Experiment 14. Normal guinea-pig serum recently obtained was allowed to settle and the upper non-cellular clear fluid was taken off; the lower part of the fluid contained red and white corpuscles. Tubes were made up as follows: No. 1, 12 drops of serum from a goat immunized against cholera (Eastern Prussia) (this serum had been heated for one hour at 58 degrees). No. 2, 12 drops of normal guinea-pig serum. No. 3, 8 drops of guinea-pig serum plus 4 drops of goat serum, 58 degrees. No. 4, identical with No. 3 except that the cellular part of the normal guinea-pig serum was used.

These tubes were then inoculated with a 24-hour culture of the vibrio suspended in 10 c.c. of normal salt solution, and gelatin plates were made at intervals.

\section{NUMBER OF COLONIES.}

\begin{tabular}{rrr|c|c|r|r}
\hline \multicolumn{3}{c|}{ Time of cultures. } & No. 1. & No. 2. & No. 3. & No. 4. \\
\cline { 1 - 2 } \cline { 5 - 6 } I. April 22, 1895, 6 & P.M. . & 8,640 & 9,600 & 10,200 & 9,120 \\
II. April 22, 1895, 7.30 P.M. . & 4,320 & 2,160 & 0 & 0 \\
III. April 22, 1895, 10 & P.M. .. & 6,480 & 3,600 & 0 & 0 \\
IV. April 23, 1895, 10 & A.M. . & Innumerable & Innumerable & 0 & 0 \\
\hline
\end{tabular}


It is evident from this experiment that normal serum may be made strongly bactericidal by the addition of a small amount of preventive serum, as shown by the plate method. This fact may also be demonstrated by adding a small drop of a suspension of vibrios to a drop of the serum mixture, when it will be found that the organisms are rapidly transformed into granules. Pfeiffer's phenomenon, then, may be produced in vitro as we shall presently consider more in detail. In the same way that a small amount of preventive serum vaccinates animals and gives them a bactericidal property against the vibrio, so it would seem to "vaccinate" normal serum as evidenced by the intense bactericidal property which this serum acquires.

\section{The Specificity of the Preventive Substance in the Serum of Immunized Animals.}

The serum of animals well immunized against a vibrio contains a preventive substance. This preventive substance is separate from the bactericidal substance also present in the serum. Is the preventive substance likewise specific? In other words will the serum of animals vaccinated against a vibrio other than Koch's, protect against infection with the true cholera organism? The question is still in dispute. Certain observers assert that the serum of animals vaccinated against the vibrio Massaouah protects animals from true cholera; but this is denied by other observers. It is easy enough to perform experiments to elucidate this question, but the interpretations of such experiments are confusing. The studies of Issaeff * have shown us that the injection of certain fluids with no distinct protective power in animals may cause a certain degree of immunity against cholera infection.

Cholera peritonitis develops rapidly. Guinea-pigs that receive injections of the vibrio are either rapidly killed or else recover rapidly, the struggle between the animal and the parasite being very short. When a sub-lethal dose is given the rapidity of the cure shows that the animal defense is adequate for a time; it has been shown, moreover, that vibrios are quickly transformed within the phagocytes. On the other hand the vibrio multiplies and secretes poisons, and consequently if the animal does not defend

* Issaeff, Zeitschrift für Hygiene, XVI, 1894, 283. 
itself immediately after inoculation it will soon find itself opposed by a large number of invaders and resistance will be useless. The rapidity of defense then is of considerable importance in the cure; the slightest influences that hasten the reaction against an infection may decide the issue of the combat. Injections of bouillon and of normal serum are preventive only by increasing slightly the bactericidal property and by attracting leucocytes. These slight preventive properties suffice to cause the animal defense to begin immediately and so protect the animal. A cure which in itself is of vital importance may be effected by agents in themselves insignificant. There is no comparison to be drawn between the preventive power of these inert fluids and that of a specific serum, and it is unsafe to judge of the preventive power of a fluid simply by the results which it produces. For example, the immune serum against the vibrio Metchnikovi has an energetic attraction for guinea-pig leucocytes. If this serum is injected into the peritoneal cavity it may consequently attract leucocytes and thus prepare the animal for a defense against any vibrio that may subsequently be injected. The most important result caused by such an injection, however, is the genesis of an energetic bactericidal power against the vibrio Metchnikovi which endows the leucocytes with a strong destructive power for this vibrio. But this latter bactericidal power, which is so effectual against the vibrio Metchnikovi, is useless against the cholera vibrio.

EXPERIMENT 15. A small amount of blood was taken from a normal guinea-pig (serum I). . The animal was then given intraperitoneally 1 c.c. of serum from a guinea-pig vaccinated against the vibrio Metchnikovi; 24 hours later a small amount of blood was again withdrawn (serum II). The bactericidal properties of these two sera against the vibrio Metchnikovi and against the cholera vibrio (Eastern Prussia) were then determined by the plate method.

\begin{tabular}{|c|c|c|c|c|c|c|}
\hline \multirow{2}{*}{\multicolumn{3}{|c|}{ Time of cultures. }} & \multicolumn{2}{|c|}{ Serum I. } & \multicolumn{2}{|c|}{ Serum II. } \\
\hline & & & $\begin{array}{l}\text { Vibrio Metch- } \\
\text { nikovi. }\end{array}$ & $\begin{array}{l}\text { Vibrio chol- } \\
\text { eræ. }\end{array}$ & $\begin{array}{l}\text { Vibrio Metch- } \\
\text { nikovi. }\end{array}$ & $\begin{array}{l}\text { Vibrio chol- } \\
\text { eræ. }\end{array}$ \\
\hline $\begin{array}{l}\text { I. } \\
\text { II. } \\
\text { IIII. }\end{array}$ & $\begin{array}{l}\text { March } 28,4 \\
\text { March 28, } 7 \\
\text { March 28, } 11.30\end{array}$ & $\begin{array}{l}\text { P.M. } \\
\text { P.M. } \\
\text { P.M. }\end{array}$ & $\begin{array}{c}4 ; 500 \\
12,000 \\
\text { Innumerable }\end{array}$ & $\begin{array}{r}2,040 \\
180 \\
7,800\end{array}$ & $\begin{array}{r}4,800 \\
0 \\
0\end{array}$ & $\begin{array}{r}1,800 \\
420 \\
7,050\end{array}$ \\
\hline
\end{tabular}


As is seen by this table the serum of the guinea-pig before injection of immune serum was not strongly bactericidal either for the vibrio Metchnikovi or the cholera vibrio. After injecting the preventive serum, the serum of the guinea-pig became distinctly bactericidal for the vibrio Metchnikovi, but not at all so for the cholera vibrio.

The preventive value of a given serum against the vibrio Metchnikovi and against the cholera organism may be compared. The animal treated with serum acquired a means of defense solely against the vibrio Metchnikovi. It is not proper then to call a serum preventive against such and such a vibrio unless inoculation of this serum into an animal endows the latter with a marked bactericidal property for the organism in question. The experiment offered gives an example of the specificity of the preventive power. It by no means proves that there is no cholera vibrio or never will be a cholera vibrio similar enough to the vibrio Metchnikovi, so that an infection by such an organism might not be prevented by a serum active against the vibrio Metchnikovi. It indicates simply that the preventive substance formed by the animal body bears a distinct relation to the bacterium which has formerly attacked the animal and against which it has become immunized.

We know that the bactericidal activity of immune serum is directed only against that species of vibrio used for immunization. To what is this specificity in bactericidal power due? We have already seen that the bactericidal substance in the serum of immunized animals does not differ from the weak non-specific substance in normal animals. The specificity and strength of the bactericidal substance in the serum of immunized animals must be due to the preventive substance. The specificity of the bactericidal substance indicates the specificity of the preventive substance, and the preventive property is specific to the same degree that the bactericidal power is.

\section{ViII. Some Characteristics of the Preventive Substance.}

The preventive substances present in the sera of animals immunized against different organisms are not identical. The fact that an infection produced by one vibrio cannot be prevented by the serum of an animal immunized against another vibrio is an evidence of 
this difference. There is distinct correlation between the preventive substances and the bactericidal substances employed during the process of immunization. It seems hard to realize $a$ priori how an animal can be so delicately constructed from the chemical standpoint that according to necessity it may build up from its own elements substances which are preventive, now against one vibrio and now against another. It seems, therefore, quite reasonable that the preventive substance may be a simple bactericidal substance more or less transformed and changed. This was the hypothesis that Buchner offered in such infections as diphtheria and tetanus, on the ground of certain analogies between the properties of diphtheria toxin and antitoxin. Buchner thought that antitoxin was derived from toxin since the preventive power of a serum depends on the amount of toxin that has been injected rather than on the real resistance of the animal.*

We do not know the nature of the preventive substance in cholera serum and we have no knowledge concerning the toxin this micro-organism forms. We do not know whether the substance that gives rise in the immunized animal to the preventive substance is the toxin or some other vaccinating substance in the culture. In the extremely vague position in which we are at the present time it is best to review the various properties which the preventive sera and the bacterial products are known to possess, and to note whatever conditions and analogies occur in comparing these properties.

The culture products from the cholera vibrio have an attraction for leucocytes. Does preventive serum have the same property? And does it differ from normal serum in this respect?

We already know that inoculation of preventive serum into the peritoneal cavity causes an increase in the number of leucocytes there. It may certainly be concluded from this fact that the influx of leucocytes is due to some attraction on the part of the serum. Chemiotaxis, however, is not the sole influence to which the influx of leucocytes is due. It is quite possible that it may be one of the causes that produces this collection of leucocytes, but there is cer-

* This fact has been established not only in tetanus and diphtheria, where it is a question of toxin and antitoxin, but also in instances where this combination does not occur, as, for example, in the serum of animals vaccinated against the hog-cholera bacillus studied by Metchnikoff. 
tainly another factor that affects it, namely, the condition of the capillary walls.* Issaeff has already shown that a substance with no chemiotactic influence, namely, normal salt solution, when injected into the peritoneal cavity, increases the number of cells there.

Definite information then may be obtained by placing the substance to be studied within capillary tubes closed at one end and leaving these tubes in the peritoneal cavity for several hours.

If the fluid in such tubes is a good culture medium, careful asepsis must be practised. After the tubes are examined under the microscope their contents may be taken out and spread on a slide. With suitable stains the types of leucocytes present may be noted and also the presence of any micro-organism. The phenomena of attraction which might be attributed to the serum may be due to bacterial secretion, and so it is well when testing the chemiotactic property of a given fluid to place in the peritoneal cavity, at the same time, tubes containing a fluid of which the chemiotactic properties have already been determined.

ExPERIMENT 16. (a) Four small bundles of tubes were placed in the peritoneal cavity of a normal mouse at 6 P.M., and withdrawn 18 hours later. Ether anesthesia was administered during the opening of the peritoneum and placing the tubes. The following table shows the effect of the fluids employed on the leucocytes.

No. 1. Twenty-four hour agar culture of the Massaouah vibrio suspended in salt solution and sterilized at 100 degrees. Strongly attracting for leucocytes.

No. 2. Serum of a rabbit vaccinated against the vibrio Massaouah; this serum is powerfully preventive and bactericidal. Distinct attraction for leucocytes.

No. 3. The same serum heated for an hour to 70 degrees (no longer bactericidal but still preventive). Distinct attraction for leucocytes.

No. 4. Salt solution of 0.65 per cent. No attraction for leucocytes.

In the tubes containing the sterile cholera culture the leucocytes form a thick plug not extending very far into the tube. In the tubes

* It has already been shown that leucocytes approach certain substances owing to a reaction to chemical substances, but it has not been proved that this chemiotactic influence is sufficient to affect leucocytes through the vessel wall and cause their emigration. It may be that chemiotaxis is of importance only after the leucocytes have passed through the wall of the capillary and into the tissues. 
containing serum the white corpuscles penetrate more deeply and are more scattered. This difference in appearance is probably due to the fact that in the serum the leucocytes find a suitable non-toxic medium that allows them to remain motile for a longer period.

(b) Tubes were placed in the peritoneal cavity of a normal guinea-pig and withdrawn after 12 hours.

No. 1. Agar culture of Massaouah suspended in 10 c.c. of salt solution and sterilized. Strong attraction for leucocytes.

No. 2. Serum of a rabbit immunized against Massaouah. Marked attraction.

No. 3. Serum of a normal rabbit. Very slight attraction.

No. 4. Salt solution of 0.65 per cent. No attraction.

These experiments show that preventive serum has a much more marked chemiotactic influence than normal serum. It may be asked whether the leucocytes of a vaccinated animal are attracted by its own serum. That such an attraction takes place is shown by the following experiment:

(c) A specimen of blood was taken from a guinea-pig weighing 420 grams that had been well vaccinated against the vibrio of Massaouah. Two days later the serum was put in tubes which were placed in the peritoneal cavity of the same animal.

No. 1. Agar culture of the vibrio Massaouah suspended in salt solution. Strong attraction for the leucocytes.

No. 2. Serum from a normal guinea-pig. Faint attraction.

No. 3. Serum from the animal used in the experiment. Very distinct attraction.

The following experiment also shows that cultures of cholera vibrios will attract leucocytes even when they have been suspended in a rather large volume of salt solution.

(d) A normal guinea-pig was used.

No. 1. Massaouah culture on agar 24 hours old suspended in 5 c.c. of salt solution. Strong attraction.

No. 2. The same culture suspended in 100 c.c. of the same solution. Strong attraction.

No: 3. Same culture suspended in 200 c.c. of the solution. Very distinct attraction.

Considering these experiments collectively it is to be noted that the serum of a normal rabbit or guinea-pig has only slight attraction 
for the leucocytes of the mouse, of normal guinea-pigs, or of guineapigs vaccinated against cholera. The attraction of the serum of immunized rabbits or guinea-pigs for leucocytes is very much more marked. It will be noted also that cultures of the cholera vibrio suspended in a large amount of salt solution still attract leucocytes strongly. Preventive serum, even when heated for an hour to 70 degrees, still attracts leucocytes.

The leucocytes present in largest numbers in these experiments are the polynuclear amphophiles.

Are there other points of resemblance between the substances in cultures and those materials characteristic of preventive serum? One of the most noteworthy properties of this serum is that it will immunize with great rapidity even in a small dose. It also may be shown that very small amounts of a sterilized culture of cholera when injected into the peritoneal cavity 24 hours before inoculation of the culture will protect guinea-pigs. For example, 1/200 of a twenty-four hour agar culture of Massaouah was suspended in salt solution, sterilized at 100 degrees, and injected. Controls that had received a corresponding dose of salt solution containing no bacteria succumbed to infection, whereas the guinea-pigs which had received the tiny doses of culture resisted. An even smaller dose of an older culture was equally successful. When mixed in equal volume with a solution containing 0.6 per cent of $\mathrm{NaCl}$ and 0.5 per cent of $\mathrm{K}_{2} \mathrm{CO}_{3}$ and then heated to 100 degrees these small amounts of culture still vaccinate. In the same way serum from immunized rabbits mixed in equal parts with this solution and heated to 100 degrees, does not coagulate and preserves for the most part its preventive properties.

In view of these facts the hypothesis that the preventive properties of the serum are due to a conservation of certain vaccinating substances from the immunizing culture cannot be thrown aside. But if we study the nature of the immunity produced by small injections of culture more attentively it appears to be in no respects similar to the immunity afforded by preventive serum. Following the injection of preventive serum, the serum of the animal inoculated acquires a strong bactericidal power, but no such thing happens after the administration of small amounts of killed vibrio. 
Experiment 17. A small amount of blood was taken from a normal guinea-pig (serum I). One twentieth of a cubic centimeter of an old culture of cholera vibrio (Eastern Prussia) was then injected into the peritoneal cavity. Twenty-four hours later another specimen of blood was taken (serum II). The bactericidal property of the two sera against the vibrio was then determined by gelatin plates.

NUMBER OF COLONIES.

\begin{tabular}{|c|c|c|}
\hline Time of cultures. & Serum I. & Serum II. \\
\hline 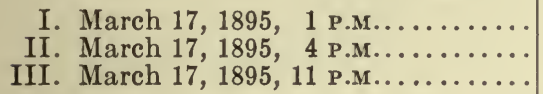 & $\begin{array}{c}3,500 \\
420 \\
\text { Innumerable }\end{array}$ & $\begin{array}{c}4,000 \\
510 \\
\text { Innumerable }\end{array}$ \\
\hline
\end{tabular}

As is seen from this experiment the bactericidal power is not increased. It is probable that the injection of small amounts of culture produces a relative immunity due to the attraction such substances have for leucocytes. If preventive substances owe their origin to bacterial products, as their specificity would indicate, it is not owing to any direct relation between the two, but on account of some elaboration on the part of the animal body. 


\section{STUDIES ON THE SERUM OF VACCINATED ANIMALS.*}

BY DR. JULES BORDET.

\section{Pfeiffer's Phenomenon (The Extracellular Granular Transformation of Vibrios).}

\section{Interpretations given to Pfeiffer's Phenomenon.}

Pfeiffer $\dagger$ found that if a certain amount of cholera vibrios suspended in bouillon is injected into the peritoneal cavity of a rabbit or guinea-pig well immunized against cholera, that a certain number of these organisms undergo within a short time an interesting change. They lose their motility and then contract into globules which at first are oval and then rounded. These globules resemble cocci. They subsequently become easily stained and according to Pfeiffer they finally break up, and in this way the culture introduced is rapidly destroyed.

The most important part of Pfeiffer's discovery lies in the fact that this retrograde transformation of the vibrio takes place for the most part outside the cells. As may easily be imagined Pfeiffer has drawn from these observations conclusions which would seem unfavorable to the phagocytic theory. This eminent bacteriologist has still further noted that the same phenomenon -occurs if the vibrio is given to a normal animal, provided a small amount of preventive serum is injected simultaneously. The dose of this serum varies naturally with its activity, but if very strong the dose is very small. The serum causes the same phenomenon when deprived of all bactericidal property by heating to 60 degrees or 70 degrees. According to Pfeiffer the phenomena cannot occur without the coöperation of the living animal. According to this author

* See p. 8.

$\dagger$ Pfeiffer, Zeit. für Hygiene XVIII, 1894, 1. 
the metamorphosis of vibrios is quite apart from any leucocytic intervention and due to the activity of the endothelial cells of the peritoneum. When a normal guinea-pig is given a mixture of vibrios and preventive serum the endothelial cells are stimulated and react by rapidly secreting harmful substances which produce morphological changes in the vibrios and then destroy them.

The transformation of the vibrio, which precedes its destruction, gives evidence of the harm that the bactericidal substances in the animal have done. But these substances as we have already seen belong to the leucocytes and it is quite reasonable to imagine, contrary to the opinion of Pfeiffer, that the bacteria are modified because in the presence of vibrios the leucocytes of the peritoneal cavity liberate into the surrounding fluid the substances they have formed and which they normally retain. Leucocytes indeed show the effects of this sudden introduction of the culture fluid; many cells become motionless and swollen or broken up.* Some of them collect in clumps.

Metchnikoff has just proved that this latter reasonable hypothesis represents the true state of affairs. He was able to produce Pfeiffer's phenomenon in vitro by adding to a mixture of preventive serum and culture, leucocytic extract from the peritoneal cavity of a normal guinea-pig. This experiment evidently rules out the function which Pfeiffer has attributed to the endothelial cells.

We have pointed out in our experiments on phagocytosis in vitrot that vibrios that have been taken up by normal guinea-pig phagocytes may undergo a granular transformation identical with the one described by Pfeiffer. Since the normal guinea-pig has only a faint bactericidal power the transformation of vibrios goes on only where the destructive substance is most concentrated, that is within the phagocyte. But if the preventive serum is added, as Metchnikoff has done, the bactericidal power becomes much more marked and metamorphosis of the organism may be noted not only in the phagocyte but also in the surrounding fluid.

Whatever may be the ultimate conclusions, Peiffer has made a great contribution to the study of immunity. By this visible and microscopically detectable alteration the vibrio shows the effect

* Elie Metchnikoff, Annales de l'Institut Pasteur, June, 1895.

$\dagger$ See p. 33. 
that body fluids have upon it and acts therefore as an index of bactericidal properties of these fluids.

We have done a number of experiments with Pfeiffer's phenomenon for various purposes which we shall consider collectively for the purpose of exposition. The method employed was uniform and may be detailed once for all.

\section{The Production of Pfeiffer's Phenomenon in Vitro through the Combined Action of Preventive Serum and Normal Serum.}

Metchnikoff produced Pfeiffer's phenomenon in vitro by mixing the cholera vibrio with preventive serum and then adding leucocytes drawn from the peritoneal cavity to this mixture. These leucocytes may, however, come from another source. We have found that the cholera vibrio (Oriental Prussia) is rapidly changed into rounded granules when placed in a mixture of preventive serum and defibrinated blood of a normal guinea-pig. The control of defibrinated blood without preventive serum gave a negative result. When the specific serum is absent the great majority of the vibrios remain quite motile and extracellular changes only rarely occur. The leucocytes of the defibrinated blood, to be sure, take up a few vibrios that become transformed within the protoplasm of the leucocyte, but the preventive serum is necessary to produce transformation in the surrounding fluid.

Metamorphosis takes place under these conditions quite as well as in the animal body, but more rapidly at body temperature than at room temperature. The defibrinated blood used in the experiment may be obtained from various animals; guinea-pig, rat, rabbit, or goat, but human or guinea-pig blood gives the most distinctive and demonstrable granulations. In preparations with rat or rabbit blood it is rather difficult to find the vibrios, as the bactericidal effect is apparently so intense that the granules rapidly lose their staining reaction.

The preventive serum used may have been kept for some time, but the defibrinated blood from the normal animal must have been obtained recently. When kept for a few days, even if protected from strong light, it loses some of its properties. 
The amount of preventive serum necessary in the experiment may be very small. If the serum is very powerful the slightest amount brings about the phenomenon very well.

Defibrinated blood is a mixture of serum and cells. Are the cells necessary to produce Pfeiffer's phenomenon? If we mix in a hanging drop a small amount of preventive serum and of perfectly clear normal serum, free from cells, and then add a suspension of cholera vibrios, it is found that the organisms soon give a complete transformation phenomenon. It is evident, then, that the phenomenon may occur without the presence of the cellular portion of the blood. We have already noted* that the addition of a small amount of preventive serum, even when heated to 60 degrees or 70 degrees and so deprived of bactericidal power, endows normal serum with strong bactericidal property for the vibrio. We were able to demonstrate this property by the gelatin plate method; we shall also show that it may be demonstrated by the phenomenon of granular transformation. Later on we shall return to a discussion of the relation of this experiment to immunity. Let us consider for the present its practical application as a means of diagnosis of the cholera vibrio.

\section{iII. Diagnosis of the Cholera Vibrio by Pfeiffer's Phenomenon in Vitro.}

It is well known how difficult it is to determine the exact properties of the cholera vibrio and consequently how difficult it is to distinguish it from other similar vibrios. The diagnosis of the cholera vibrio in the dejecta of patients is not of great importance during a declared epidemic of typical cholera. It is quite another matter in isolated cases of choleriform enteritis. It is very important for hygienic reasons under these conditions to be able to say positively whether or not the dangerous organism is present.

Pfeiffer and Issaeff $\dagger$ claim a specificity in the bactericidal and preventive power of the serum of animals vaccinated against cholera, and the experiments that we have mentioned previously led us to corroborate these conclusions.

The German scientists have used a serum from animals immunized

* See p. 47.

$\dagger$ Pfeiffer et Issaeff, Deutsche medicinische Wochenschrift, No. 13, 1894. 
against an undoubted cholera vibrio. By injecting this serum they produce a passive immunity in guinea-pigs against the cholera vibrio. If a pathogenic vibrio from a suspected case of enteritis is subsequently injected into these immunized animals they do not succumb if the vibrio is a true cholera organism. If, on the other hand, the animal does succumb, they conclude that the culture is not the authentic Koch vibrio.

Pfeiffer* has recently modified and simplified this diagnostic procedure. He inoculates a normal guinea-pig with a bouillon culture of the suspected organism to which a small amount of anticholera serum has been added. If the organism in question is a true cholera vibrio, granular transformation takes place.

Granular transformation is indicative of a bactericidal effect by the surrounding body fluid on the vibrio. This bactericidal power is specific and affects only those vibrios which are identical with the one used to immunize the animal from which the serum is taken. In addition to Pfeiffer, Dunbar has more recently shown by numerous experiments that those organisms which resemble Koch's vibrio culturally may be considered to be true cholera vibrios if they undergo granular degeneration when injected with cholera serum into the peritoneal cavity of a normal guinea-pig. This means of diagnosis does not require much serum, but is costly on account of the animals used and the time consumed if there are a large number of vibrios to examine, or if there is no well-equipped laboratory at hand. It would therefore be very desirable to use for diagnosis the method indicated, which consists essentially in the production of Pfeiffer's phenomenon in vitro by means of the combined action of preventive serum and blood or serum from a normal animal. This method is very easy and economical and requires only small amounts of preventive serum and fresh blood. A few drops of blood obtained by puncture and taken in a capillary tube will give a drop or two of serum which is sufficient to examine several specimens.

Let us consider first whether the granular transformation in vitro with our serum occurs with vibrios belonging to species other than that of the Koch vibrio as defined by Pfeiffer.

Each of the vibrios found in the following table was placed with * Pfeiffer, Deutsche medicinische Wochenschrift, 1894. 
a mixture of the two sera. The sign + indicates that a positive granular transformation took place.

\begin{tabular}{|c|c|c|c|}
\hline Vibrios. & 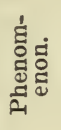 & Vibrios. & 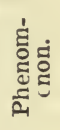 \\
\hline 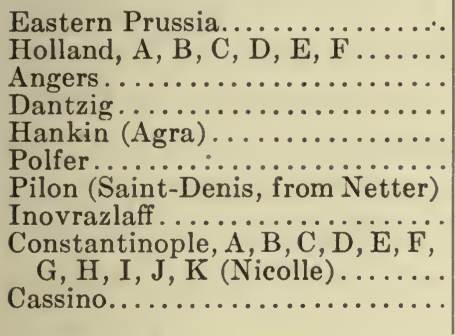 & $\begin{array}{l}+ \\
+ \\
+ \\
+ \\
+ \\
+ \\
+ \\
+ \\
+\end{array}$ & 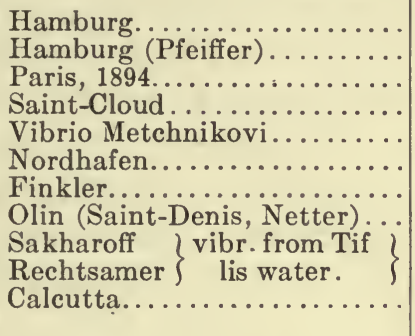 & $\begin{array}{l}+ \\
+ \\
+ \\
+ \\
0 \\
0 \\
0 \\
0 \\
0 \\
0\end{array}$ \\
\hline
\end{tabular}

Certain of these vibrios that give Pfeiffer's phenomenon will also show it to a certain extent in normal serum without the addition of cholera serum. These are the slightly virulent vibrios which are very susceptible to the normal protective agents of the animal body. The most remarkable of these organisms in this respect is vibrio "G." from Constantinople. It should be noted, however, that this granular transformation of vibrios by normal serum is always rather limited and is rarely comparable to that caused by the normal serum plus cholera serum. As a general rule the addition of a very small amount of preventive serum suffices to produce the phenomenon in vitro. The technique that we usually employ is the following: A drop of 24-hour culture suspended in 6 c.c. of salt solution is placed on a slide; to this drop is added a loop of anticholera serum. To one drop of this mixture is added a similar drop of normal serum. A slightly larger amount of serum is necessary to cause the phenomenon in Pfeiffer's organism or in the vibrio from Saint-Cloud. It is not surprising, however, that certain varieties should resist better than others.

Vibrios that do not give the phenomenon in vitro also fail to give it when injected with cholera serum into the peritoneal cavity of animals. There is therefore complete correspondence between these in vitro experiments and experiments in the living animal. This method, then, may certainly be utilized as simpler and less expen- 
sive than Pfeiffer's method of diagnosis. Certain general rules of technique should be followed to insure uniformity:

A 24-hour culture of the vibrio to be examined is suspended in from 5 to 7 c.c. of bouillon or normal salt solution. - Two drops of this suspension are then dropped from a fine capillary tube on a slide or in a watch glass; a drop of preventive cholera serum is then added (a serum supposedly as strong as our own); as a matter of fact, this dose is considerably more than is necessary, but it is well to use as much as this on the chance of dealing with a very resistant vibrio. A small platinum loop of this mixture is then taken and placed on a cover slip, and a small drop of fresh normal serum is added by means of the same loop after sterilizing it. Defibrinated blood, of course, may be used in place of serum or simply a freshly drawn drop of human blood. The drop of normal serum is mixed with the other drop and the cover slip applied to a hollow ground slide and sealed with vaseline. The slide is then placed for two hours in the incubator, which time is quite sufficient to obtain a complete transformation. We emphasize these details because the three factors - vibrio, normal serum and preventive serum - should be used in rather exact proportions. It is well to make two control preparations at the same time and by the same method. One of these should contain a typical Koch vibrio that is known to give the phenomenon rapidly with active serum. From this control we learn whether enough of each serum has been used. A second control is made with the vibrio to be examined, normal serum, and a drop of salt solution in the place of the preventive serum. This second control will indicate whether the organism in question is very attenuated and so liable to transformation by the normal serum alone without any cholera serum. If the second control also gives Pfeiffer's phenomenon, which is unlikely, no definite conclusion may be drawn as to the pathogenic nature of the organism examined.

Since the preparations remain only two hours at body temperature, perfect asepsis to prevent growth of other contaminating bacteria is unnecessary. Examination may be made either in the fresh condition or by means of stained preparations. There is frequently some difficulty in staining the granules, particularly when the preparation has been left too long in the incubator and the 
reaction has gone too far. Carbolated thionin is a good stain to use. The slide is fixed by heat and stained for one minute with thionin and then differentiated in water until there is no longer any noticeable diffusion of the stain (about ten minutes when there are many blood corpuscles present or four or five minutes when clear serum has been used). The granules stain a purplish blue; the red blood corpuscles greenish. If the preparations are left too long in water, the granulations will be completely decolorized. The formation of thionin crystals may be avoided by drying the preparation with filter paper. The preparations may also be stained with a concentrated aqueous solution of methylene blue or by a very dilute solution of Ziehl's fuchsin. When the methylene-blue stain is used, fixation by a saturated solution of picric acid is recommended, with a counterstain of alcoholic eosin.

This method is evidently only a modification or rather a simplification of the method that Pfeiffer has described. It depends on the same principle and its value is equally open to criticism. Are we indeed sure of the diagnostic value of these results? Owing to the specificity of bactericidal sera, it seems to us that if an organism agrees culturally and gives this phenomenon with a true anticholera serum we may identify it as a true cholera vibrio. But if, on the other hand, we are dealing with an organism obtained from a case that clinically resembles cholera, and this organism possesses the morphological and cultural peculiarities of Koch's vibrio but fails to give this reaction with cholera serum, we should not be authorized in saying, with our present knowledge, that the vibrio in question is not identical with the cholera organism and that the case is not one of true cholera. Different cholera vibrios from various sources show variations in their resistance to serum. More serum is necessary, for example, to transform the Saint-Cloud vibrio than the vibrio from Eastern Prussia. Although the sera that one employs are active, they have nevertheless their limitations. If the Saint-Cloud organism had been slightly more resistant or the serum we used slightly weaker, we should not have obtained the phenomenon. It is far from being proved that there may not be organisms, which, although essentially true cholera organisms and of the same speicies as the Koch organism, may not have been more highly differentiated as regards resistance to destructive 
organic substances than usual. And, what is more, it has by no means been proved that organisms that differ slightly from Koch's organisms are incapable of producing cholera. For example, the vibrio of Massaouah, which is unlike the cholera organisms usually met with, is unquestionably a cause of cholera. (Fermi's experiment.) This organism, however, does not fulfill Pfeiffer's condition.

Nevertheless, since the majority of cholera vibrios recovered from the stools of cholera cases give the phenomenon, the method may be recommended, with a certain reserve as to negative results.

\section{Pfeiffer's Phenomenon with the vibrio METChNiKovi.}

Will all vibrios, even the most virulent of them, undergo transformation outside the protoplasm of leucocytes when injected into the peritoneal cavity of specifically immunized guinea-pigs? Is the vibrio Metchnikovi, the virulence of which is recognized, easily altered by the peritoneal fluid?

We injected $\frac{1}{10}$ of a 24 -hour agar culture of vibrio Metchnikovi into a guinea-pig immunized by repeated injections of killecl or living cultures. The serum of this animal was highly preventive. The organisms were rapidly clumped, but very few of them were morphologically changed. After an hour, for example, in addition to the majority of intact vibrios, a few individuals were found in these clumps broken up into fine granulations and others that stained poorly, but were morphologically intact. The typical transformation into large granulations was only to be found in the protoplasm of polynuclear leucocytes which contained intact vibrios as well. One or two hours after injection the leucocytes were found clumped together. Later they increased in number and many were seen scattered throughout the fluid; 6 hours after injection the number of leucocytes was relatively large and the vibrios were few. In a preparation from the exudate 6 hours and 20 minutes after injection an unphagocyted and morphologically intact clump of vibrios was, however, noted. It is evident, then, that not all vibrios undergo granular transformation equally well. 


\section{Factors Concerned in the Production of Pfeiffer's Phenomenon in vitro.}

Let us consider the experiment for producing the granular transformation of the cholera vibrio in vitro by the combination of normal serum and serum of an immunized goat. We repeat that it is not necessary for cells to be present either in the preventive serum or in the normal serum to produce a complete Pfeiffer's phenomenon.

There are three factors necessary in the experiment; each of these factors may be studied in turn and we may determine whether there are conditions that render these factors unfit to produce the phenomenon and also whether any one of them may be omitted or replaced by any other factor. The first factor is the cholera vibrio. We have already seen that the cholera vibrio from Eastern Prussia which was used in producing the anticholera serum may be replaced by any other of the true vibrios. There are two other factors: the normal serum and the preventive serum. Is the presence of both of these substances necessary? Or may one of them be omitted and the phenomenon still occur? These are the first questions that arise. We already know that the normal serum employed has little or no power in itself to change the form of the vibrios.

The preventive serum used in these studies was from a goat that had been well vaccinated against the Eastern Prussia vibrio. It produces no transformation of vibrios in any dose when not associated with normal serum. When used alone the only effect that this serum has on vibrios is to immobilize them. A very small amount of serum rapidly paralyzes their motility. It is also to be noted that the vibrios collect in small clumps in the fluid.*

The serum we first used had been drawn from the goat three weeks previous to our experiments. It might reasonably be supposed that during this period the serum had lost some of its properties, since it is known that the bactericidal substance is easily affected.

* We have not been able to decide whether this clumping is an active phenomenon due to the vibrio itself or a purely physical affair. If the red blood cells of a normal guinea-pig are placed in their proper serum, they diffuse uniformly and soon collect at the bottom of the hanging drop. But when our protected goat serum is added to such normal serum the corpuscles collect in small separate clumps and give the drop a granular appearance. 
And this idea proved to be correct. We then tried recently obtained serum from the same animal. This fresh serum brings about transformation of the vibrio without the addition of normal serum. The phenomenon, to be sure, was not complete; there were a few vibrios that remained unaltered and others that seem to have been killed without any modification, since they failed to take the stain. The immunized goat serum is not as suitable a medium in which to demonstrate transformation as is the serum or blood of a vaccinated guinea-pig. Fresh immune serum from this latter animal also brings about complete transformation without the slightest necessity for the addition of normal serum.

Let us consider, first, a preventive serum which does not alone cause the granular transformation. Since the aid of normal serum is necessary, we may better study the influences that affect its activity.

All the following experiments have been modeled after a fundamental experiment and, in order to be comparable with it, they have all been performed in an identical manner; a single factor, the normal serum, has been modified. The hanging drops examined were always made in the same way:

The hanging drops were made from two equal-sized drops placed side by side on the slide and then mixed. These two drops were each from the same platinum loop. One of the drops consists of the fluid whose bactericidal property is tested (in this case the normal serum) and the other consists of an emulsion of vibrios (24-hour agar culture suspended in 6 c.c. of normal salt solution) mixed or not, as the case may be, with the preventive serum. This latter mixture was made as follows: A drop of the emulsion of vibrios was dropped from a capillary tube into a watch glass and to it a large platinum loop of preventive serum was added. The preventive serum, then, in the final hanging drop is very small in amount, much less than the normal serum. Controls were also made in each instance by using an emulsion to which no preventive serum had been added. An experiment may be represented by this abbreviation:

Cholera + preventive goat serum + normal serum,

or

Cholera + normal serum. 
In the tables that follow the + sign indicates that the granular transformation took place. Now that we have considered the technique certain points may be consideréd.

First. Does the method of observation indicated show delicate differences in bactericidal power between two fluids?

It is already known that normal serum alone will sometimes produce a partial transformation of the vibrio, but this effect is relatively slight. We also know that the injection of normal serum or even bouillon into the peritoneal cavity of a normal guinea-pig increases within certain limits the non-specific bactericidal property of this guinea-pig's serum. This increase in bactericidal property may be detected by our method. A small amount of blood is taken from a guinea-pig; the animal is then given 4 c.c. of normal guinea-pig serum and 24 hours later a new specimen of blood is taken. The effect of these two sera on the cholera vibrio without the addition of cholera serum is then determined. A relatively weak culture is used. Since no preventive serum is used the transformation of the vibrios with either of these sera is only partial. It may, however, be noted that the serum obtained from the animal after injection of normal serum causes metamorphosis in many more vibrios than does the serum obtained before injection; there are also many more non-motile vibrios. The difference between the two preparations is very distinct and demonstrates the delicacy of this method. We repeat that the phenomena, although relatively different in degree, are only partial in both instances and incomplete unless a drop of preventive serum is added.

Second. Is the bactericidal substance of normal serum still present in the blood of animals that have succumbed to certain infections or intoxications? Is it also present in the animals that have been immunized against organisms other than the cholera vibrio?

The blood of a guinea-pig that had died of anthrax, the blood of a rabbit that had just succumbed to a pneumococcus infection, the pleural exudate of a guinea-pig that had died of diphtheria toxin, and the blood of a guinea-pig that had been vaccinated against cholera and had died of cholera toxin, all gave Pfeiffer's phenomenon in the presence of cholera serum. The blood of the last animal, in spite of the fatal intoxication, brought about Pfeiffer's transformation without the addition of cholera serum, that is, the blood 
remained both bactericidal and preventive. The persistence of the preventive power under these conditions has already been noted by Metchnikoff. The blood of guinea-pigs immunized against the vibrio Metchnikovi or the Massaouah vibrio and incapable of producing the phenomenon alone, produces it very well when added to the preventive cholera serum.

The bactericidal substance, then, is to be found generally in experimental animals and persists even after fatal infections. It occurs both in the blood of vaccinated animals and in normal animals.

Third. The effect of heat on the fresh defibrinated blood (or serum) of a normal guinea-pig.

Fresh blood serum from a normal guinea-pig was placed in several tubes. One of these tubes was left unheated; the others were heated for 5 minutes to temperatures of 50 degrees, 55 degrees, 60 degrees, and 64 degrees respectively.

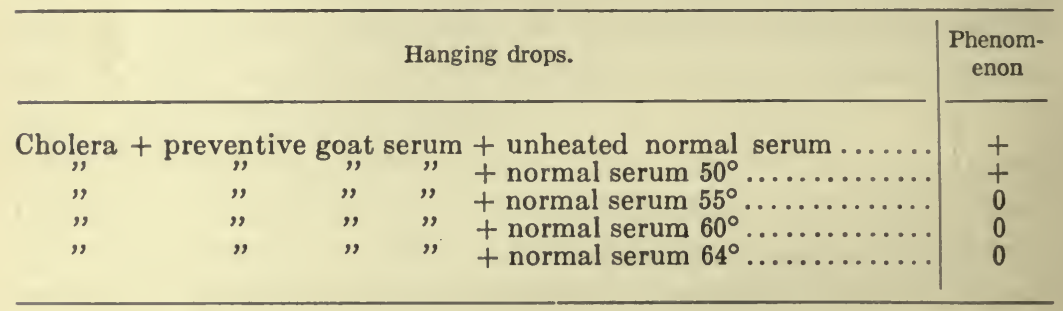

From this table we may conclude that the bactericidal substance of normal serum necessary to produce Pfeiffer's phenomenon is destroyed if the serum is heated for 5 minutes to from 50 degrees to 55 degrees. It may be noted that, even in those tubes where there was no transformation, the vibrios were motionless and clumped owing to the continued presence of the preventive serum.

Fourth. The effect of heat on the preventive serum. Pfeiffer has recently shown that preventive serum heated to 65 degrees still produces metamorphosis of the vibrio when placed in the peritoneal cavity with the cholera organism. The same fact is true in vitro. It is interesting to consider the effect of heat on quite fresh serum from an immunized guinea-pig; as we have already seen, this serum 
alone produces Pfeiffer's phenomenon without the aid of normal serum. Tubes of fresh serum from well-immunized guinea-pigs were heated to 50 degrees, 55 degrees, 60 degrees, and 64 degrees for 5 minutes. Each one of these sera was then mixed with an emulsion of cholera.

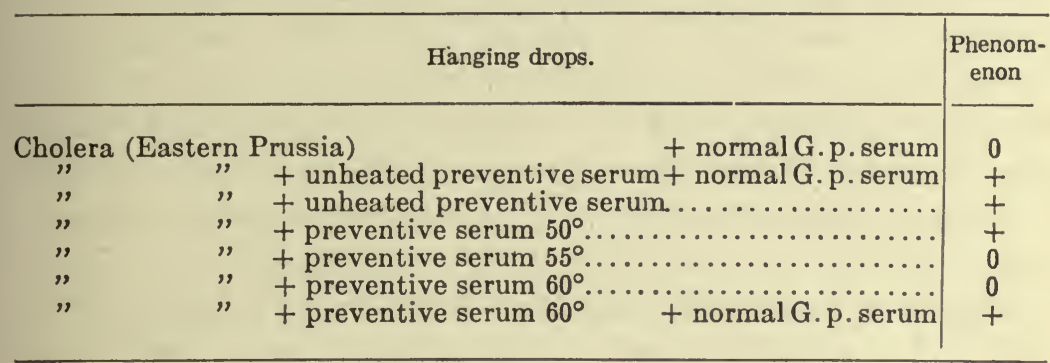

As may be seen from this table, serum heated to 50 degrees transforms the vibrio; heated above this temperature it has no effect. The bactericidal substance in the serum of immunized animals, then, is destroyed at the same temperature as in normal animals. But this heated serum, which alone cannot produce Pfeiffer's phenomenon, brings it about perfectly well, and apparently with undiminished vigor, when normal serum is added. In other words, normal serum restores to the immune serum the property lost by heat. A mixture of two fluids, 'each of which alone has no bactericidal property, forms a fluid that has high bactericidal property for a specific organism. We have already described this experiment and in the previous description we demonstrated the regeneration of bactericidal power, not by means of Pfeiffer's phenomenon, but by gelatin plate cultures.

What conclusions may be drawn from these facts? We have already considered them partially and these experiments only confirm them. It would seem as if the serum of vaccinated animals had no particular bactericidal substance, but that a similar bactericidal substance is present in the blood of normal as well as of immunized animals. This bactericidal substance is not specific unless mixed with the preventive substance, and under its normal conditions will affect only attentuated vibrios. Its energetic action depends on the combined presence of a preventive substance that is present only in 
the serum of immunized animals. It may be that this specific preventive substance has of itself some harmful effect on the vibrios that predisposes them to feel the power of the bactericidal substance. At least the preventive substance alone has no real antiseptic properties, for it cannot kill a culture or even prevent its growth. It lends to the bactericidal substance present with it, however, a character of specificity. When fresh, the serum of vaccinated animals has bothsubstances, but when heated to 55 degrees for a few moments or kept for a long time, the preventive substance alone remains; the addition of fresh serum is necessary to restore to it its bactericidal property. It is indifferent whether this fresh serum containing the bactericidal substance comes from a normal animal or an immunized animal, or even from an animal that has just died of such an infection as that caused by anthrax or the pneumococcus.

Fifth. Is the bactericidal substance present in equal amounts in normal and in immunized animals? While the immunized animal is forming the specific preventive substance, does it increase to any extent the bactericidal substance present before immunization? One may determine at least in an approximate manner the amount of bactericidal substance present in normal and immune serum. Let us mix a loop of well-immunized guinea-pig serum with a drop of culture. We have established, let us suppose, by a preliminary experiment, that a drop of this mixture placed with a drop of normal serum gives a complete Pfeiffer's phenomenon; that is to say, in a drop of this emulsion there is enough preventive substance present. It must be noted that the fresh preventive substance alone may give metamorphosis of the vibrio. But under the conditions noted, that is, one loop of the serum to one drop of cholera emulsion, no distinct phenomenon takes place. But, as we have already shown, there is enough preventive substance there; it is the bactericidal substance that is lacking. If we add another loop of the same serum to the mixture, the phenomenon occurs very distinctly and becomes still more complete if a third loop is added. At the same time, if we add normal serum to a similar mixture of cholera emulsion and active preventive goat serum, we find that only three loops are necessary to bring about the usual degree of the phenomenon. The amount of fresh serum, whether from a normal or 
from an immunized animal, necessary to add to the preventive substance is in either case little and, within broad limits, apparently the same. It seems evident, then, that the bactericidal substance in immune serum is not sensibly greater in amount than in normal serum.

\section{Leucocytic Secretions and Pfeiffer's Phenomenon.}

Experiments that have been given in a previous article* showed us that during life the bactericidal substance is within the leucocytes. When blood is taken out of the vessels this substance is liberated in the surrounding medium and endows the serum with bactericidal properties. Both serum deprived of some of its leucocytes, and edema fluid, are less bactericidal than serum obtained under normal conditions. They are also less preventive. It is easy to determine whether edema will produce Pfeiffer's phenomenon when added to preventive serum. Hanging drops are prepared containing on the one hand, cholera vibrio, preventive serum and serum of a normal guinea-pig; and, on the other hand, cholera preventive serum and edema fluid from the same normal guinea-pig. These mixtures are prepared in the usual manner, so that the doses are equal in each preparation.

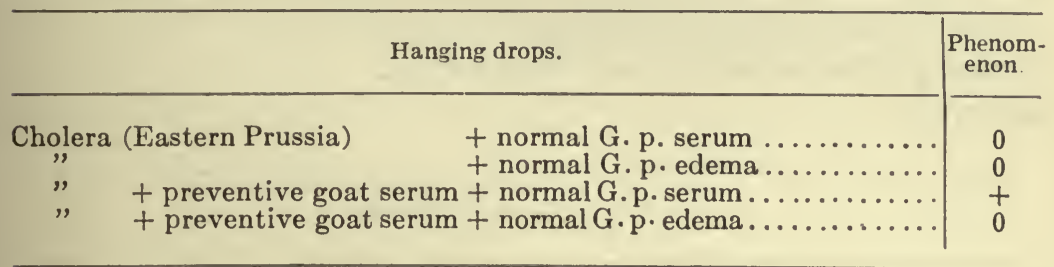

It is to be noted that edema fluid causes no Pfeiffer's phenomenon. It is evident that the bactericidal substance in this fluid is too small in amount, since there is complete transformation in the preparation containing serum. In the same manner it may be determined that goat milk, aqueous humor from the guinea-pig, and urine, tears, or saliva (human) when added to preventive serum produce no metamorphosis of vibrios. Edema fluid from a guineapig infected with anthrax, and pleural exudate from a guinea-pig

* See page 24 et seq. 
killed by diphtheria toxin, both produce the phenomenon; in these latter fluids numbers of leucocytes are present.

\begin{tabular}{|c|c|c|}
\hline & Hanging drops. & $\begin{array}{l}\text { Phenom- } \\
\text { enon. }\end{array}$ \\
\hline \multicolumn{2}{|c|}{ Cholera (Eastern Prussia) } & 0 \\
\hline$"$ & + preventive goat serum + normal G. p. serum......... & + \\
\hline$"$ & + preventive goat serum + normal goat milk......... & 0 \\
\hline$"$ & 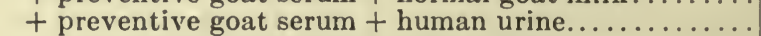 & 0 \\
\hline " & + preventive goat serum + human tears. . . . . . & 0 \\
\hline$"$ & 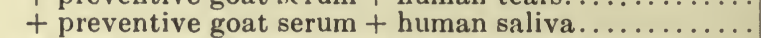 & 0 \\
\hline ", & 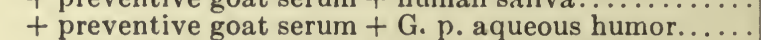 & 0 \\
\hline$"$ & + preventive goat serum + edema anthrax G. p....... & + \\
\hline$"$ & + preventive goat serum + pleural ex. dipt. G. p..... & + \\
\hline
\end{tabular}

What is the action of edema fluid from a guinea-pig that has been highly immunized against cholera? It is known that fresh serum from this animal will alone produce the phenomenon, since it contains both the bactericidal and the preventive substances. It is therefore of interest to determine whether the edema fluid from such a guinea-pig will lack one or both of these substances. Edema fluid alone, without either normal serum or preventive serum, does not cause the phenomenon. But are both substances or is only one of them lacking? To determine whether the bactericidal substance is lacking, a hanging drop is prepared containing the vibrio, preventive serum and edema fluid. Controls are made as indicated.

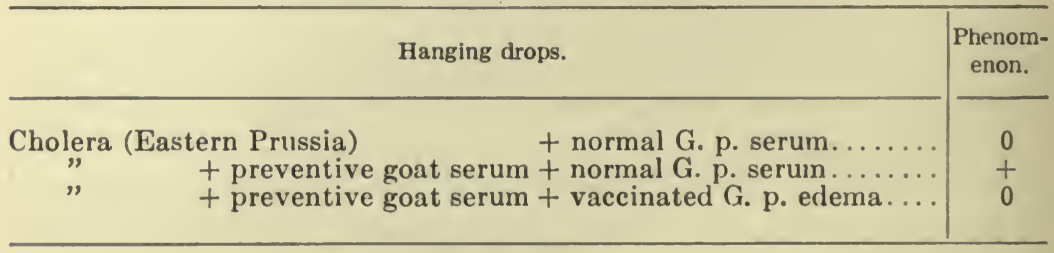

From this table it is evident that the edema fluid from immunized animals as well as that from normal animals contains no demonstrable bactericidal substance. But is the preventive substance entirely lacking? It will be recalled that in previous experiments the edema fluid was shown to be distinctly preventive for animals although inferior in this respect to serum. In the following table 
preparations were made with vibrio, edema fluid and normal serum, and transformation takes place:

\begin{tabular}{|c|c|c|}
\hline \multicolumn{2}{|c|}{ Hanging drops. } & $\begin{array}{c}\text { Phenom- } \\
\text { enon. }\end{array}$ \\
\hline $\begin{array}{c}\text { Cholera (Eastern Prussia) } \\
\text { " } \quad+\text { preventive goat serum } \\
\text { + vaccinated G. p. edema }\end{array}$ & $\begin{array}{l}+ \text { normal G.p. serum } \ldots \ldots \\
+ \text { normal G.p. serum } \ldots \ldots \\
+ \text { normal G.p. serum } \ldots \ldots\end{array}$ & $\begin{array}{l}0 \\
+ \\
+\end{array}$ \\
\hline
\end{tabular}

It is evident, then, that the edema fluid contains enough of the preventive substance, of which a very small amount is necessary to produce the phenomenon, and it may be added that it is difficult to obtain an edema fluid without leucocytes. Even if there were no leucocytes present and it were distinctly proved that leucocytes are necessary for the presence of the preventive substance, it might well be that a certain amount of it would be liberated during life into the fluids of the body and eventually be excreted; it is well known, indeed, that the preventive power falls rapidly when injections are stopped. But there is a body fluid that under normal conditions is almost entirely deprived of cells, that is, the aqueous humor. It may be shown that the aqueous humor in vaccinated guinea-pigs has neither bactericidal nor preventive power. Whether mixed with normal serum or with immunized goat serum, aqueous humor produces no change in the vibrio.

Hanging drops.

Cholera (Eastern Prussia)

+ normal G. p. serum..... 0

+ preventive goat serum

+ normal G. p. serum..... +

+ vaccinated G. p. aq. humor + normal G. p. serum.....

+ preventive goat serum + vaccinated G.p. aq. humor

0

0

It would seem, therefore, that the function of leucocytes in the genesis of the specific properties of serum is well determined.

In a consideration of bactericidal and preventive power we have covered several of the more important body fluids. We may now consider intestinal transudate from the same point of view. In human cholera the vibrio is not a parasite in the tissues; it invades 
the organism only after death and during life usually remains in the intestine; that is to say its culture medium is the intestinal secretion. It is important, therefore, to consider the properties of this secretion. Metchnikoff, as we know, succeeded in producing true intestinal cholera in young rodents. His method consists in introducing the cholera vibrio accompanied by certain bacteria that facilitate their development, into the stomach of these animals. Under these conditions cholera is regularly produced. When one autopsies young rabbits treated in this way the intestine is found filled with a rather clear fluid swarming with vibrios. Metchnikoff has further determined that this intestinal cholera may be produced in animals that have received large injections of a strong preventive serum quite as well as in animals which have had no previous treatment. In such animals the vibrio increases in the intestine, and remains motile and unmodified. It may be easily shown that intestinal secretion plus our preventive serum causes no metamorphosis of the vibrio. The same negative result is obtained if normal serum be added to the intestinal fluid from a young rabbit killed by cholera. This animal, however, had received a previous dose of cholera serum and its blood was strongly bactericidal for the vibrio. An intestinal secretion of this sort contains no leucocytes; it does contain, however, epithelial cells which apparently give the fluid no distinct activity.

Does the cholera vibrio become accustomed to bactericidal sera? Can we by repeated passage through bactericidal fluids adapt it so that it no longer feels the bactericidal effect with the same intensity and fails to give granular transformation?

If we inoculate a rather large quantity of the cholera vibrio (Eastern Prussia) in a mixture of fresh guinea-pig serum and immunized goat serum the metamorphosis takes place rapidly. But if the amount inoculated is sufficient the vibrio will eventually grow and after 24 hours a considerable number of normal organisms will be found.

A few drops of this growth may then be inoculated in a mixture similar to the first and when this culture has grown out a new transfer made. If this transfer is repeated twenty times we find that after each inoculation a rapid metamorphosis of the vibrio occurs, and even after two days, when the culture has finally grown 
out, a great number of granules will be found with the normal vibrios.

After twenty passages through bactericidal serum, the vibrio is quite as apt to be transformed as in the beginning. It is quite evident, then, that the vibrio acquires no tolerance and becomes no less susceptible to the bactericidal effect of the fluid. It continues to show a granular change which is not strictly a disintegration, but an active contraction of the organism which would appear to serve some purpose. In this new form it presents in a given volume a smaller surface of contact to the surrounding fluid, and, therefore, better avoids its harmful effect.

The fact that the vibrio does not become accustomed to contact with the bactericidal substances from leucocytes explains the difficulty in increasing its virulence. After a few passages through the animal body a definite virulence is reached which is not easily increased. The cholera vibrio does not seem to possess the characters necessary for a dangerous general parasite and does not give generalized infections. It is not necessary for the organism to acquire a great resistance to the bactericidal properties of the animal body in order to live in the intestinal contents and form toxins there, for, as we have already shown, no bactericidal substance is present in the intestinal secretion. It is, therefore, by no means proved that a vibrio that is only slightly pathogenic in the peritoneal cavity may not be very pathogenic when present in the digestive tract.

\section{REMARKS ON THE MECHANISM OF IMMUNITY.}

I. Active Immunity. Bactericidal Properties of Leucocytes. The Conception of a "Unity of the Bactericidal Substance."

Active immunity is the immunity shown by animals vaccinated by repeated injections of living or sterilized cultures of bacteria or of bacterial products. It is established slowly, but it is much more enduring than immunity brought about by preventive sera. This form of immunity is accompanied by cellular changes that are sufficiently lasting to create temporarily in the immunized animals properties that are not to be found in normal animals.

The study of immunity has convinced us that the resistance of animals to pathogenic organisms is regularly due to the activity of phagocytes. In a previous article we have considered the more 
important proofs of the accuracy of this conception and the facts brought out in this article do not change our opinion. It has been established that in vaccinated animals the phagocytes have their protecting properties distinctly increased. Not only is the tactile sensitivity of their phagocytes more perfect as Massart has shown,* so that they can take up the struggle with their adversaries more preparedly, but we believe that it may be granted that the bactericidal properties of their body fluids are increased. In certain cases, as with the vibrios, the antiseptic property of leucocytes is increased to such an extent that it is liberated in the surrouncling fluid, either on removing the blood from the body or by some harmful effect on the leucocytes. The substances diffused from these leucocytes in the blood fluid may then bring about destructive changes in bacteria. When serum is endowed with bactericidal properties it owes them to the leucocytes; the bactericidal power of serum is only a reflection of the power within leucocytes, and is present only in a relatively feeble degree.

A bactericidal property is not always present in the serum of immunized animals. The sera from animals vaccinated against tetanus, diphtheria, hog-cholera, etc., do not destroy their respective organisms. May we conclude in these instances that the bactericidal substance is lacking in the serum? Must we make a distinction between those instances in which the serum of immunized animals is bactericidal and those in which it is not? Since the bactericidal power of serum indicates the destructive power of phagocytes, are we authorized in these instances of diphtheria or hog-cholera in considering the absence of bactericidal properties in serum as indicative of little bactericidal activity in the phagocytes?

Such a conclusion would not be legitimate. It would seem rather that there is a regular increase of bactericidal property in phagocytes in immunity and that we know now only of certain general examples of this fact. And if the increase of this property is not evident in certain cases by a notable increase in the bactericidal property of the serum, it is apparently due to the fact that certain microorganisms possess a relatively higher resistance to such properties. Not all bacteria are equally easily destroyed by those diluted bac-

* J. Massart, Le chemiotaxisme des leucocytes et l'immunité. Annales de l'Institut Pasteur, May, 1892. 
tericidal substances, which, although strong within the leucocytes, are necessarily weakened when liberated into the serum. When phagocytosis of the cholera vibrio by the leucocytes of a wellimmunized animal is studied in vitro the granular transformation of the organism and its changes in color reaction are found, not only in the leucocytes, but also in the surrounding fluid. When such an experiment is made with the diphtheria bacillus and the leucocytes of a guinea-pig immunized the day before by a large dose of antidiphtheria serum (3 c.c.), it is found that the organisms within the leucocytes lose their power to stain blue and take eosin, whereas outside the leucocytes the bacteria show no tinctorial change. In other words, the activity has remained within the protoplasm of the phagocyte. It is well known, moreover, as Behring has shown us, that the body fluids of animals immunized against diphtheria have no effect on Löffler's bacillus, which differs from the findings in the case of cholera. And, although there is apparently a different set of facts to consider in each of these cases, it is to be noted that energetic bactericidal activity on the part of the phagocytes occurs in both instances.

If there is a distinction, then, to be drawn between bactericidal sera and those that are not, it is due to a difference in resistance of the specific organisms and not to the absence or presence of a bactericidal substance in the serum.

The best evidence of this fact is that the more strongly animals are immunized the more frequently do we find examples of the humoral bactericidal activity and extracellular destruction of bacteria. In other words, the destructive function has been increased to such a point that it is manifest even outside the phagocytes. The use of highly immunized animals was necessary to discover Pfeiffer's phenomenon. Later Pfeiffer* and Dunbar $†$ found that the typhoid bacillus also shows granular transformation when injected into the peritoneal cavity of an animal together with a certain amount of preventive serum. Dunbar has noted similar facts with phosphorescent vibrios and Bacillus pyocyaneus. These observations agree entirely with those originally made on the

* Pfeiffer, Deutsche medicinische Wochenschrift, No. 48, 1894.

$\dagger$ Dunbar, Zum Stande des bakteriologischen Cholera Diagnose. Deutsche medicinische Wochenschrift, February, 1895. 
cholera vibrio by Pfeiffer. The analogy is so complete that it seems justifiable to assert that the substance that gives rise to the morphological change in the colon bacillus, the typhoid bacillus, and the phosphorescent vibrios is of leucocytic origin as well as in the case of the cholera vibrio. Indeed, when B. typhosus or B. coli is mixed with leucocytes from a normal guinea-pig the transformation occurs only inside cells. Oval granulations are formed there similar to the cholera granulations. It is evident, then, that Pfeiffer's phenomenon, whether with the colon bacillus or the typhoid bacillus or Koch's vibrio, follows the same rule. The colon bacillus and the typhoid bacillus give further examples of the fact that phagocytes may become so active in well-immunized animals as to bring about a diffusion of their substances into the surrounding fluid.

We have already seen that the bactericidal substance that affects vibrios is not in itself specific. It is the preventive substance that, while adding to its activity, endows it with specificity. We have shown, accordingly, that bactericidal sera from animals immunized against various organisms are to be distinguished only by the specificity of their preventive substance. The bactericidal substance, properly speaking, in immunized animals is, with the possible exception of certain quantitative changes, quite the same as in normal animals. And since Pfeiffer's phenomenon occurs with bacteria other than the cholera vibrio and under identical conditions, it seems evident that the bactericidal substance that phagocytes use in their struggle against bacteria is the same, whatever may be the organism attacked. In an animal immunized against a given infection the bactericidal substance is directed against the specific organism on account of the presence of the preventive substance, the specificity of which depends on the organism used for immunization. It is due to the presence of this peculiar preventive substance that the animal directs its destructive power against a given organism. What is the nature of this active and distinctive body? By what mechanisn have the body cells been enabled to create this specific substance that is so valuable to the animal? This is one of the most important problems of cellular biology, and, at the same time, at present the greatest enigma with which immunity confronts us. 
II. The Mechanism of the Immunity Conferred By Preventive Serum and the Evidence for Considering this Immunity as a Purely Chemical Phenomenon.

There are certain conclusions to be drawn from the facts that we have offered as to the nature of the immunity conferred by serum.

Cholera serum, as we know, is not antitoxic; animals vaccinated against cholera have no real immunity against cholera toxin. The serum gives an immunity only against the organism itself. It contains, when fresh, two substances: a bactericidal substance and a preventive substance. Serum that has been kept for some time or heated to 55 degrees, no longer contains the bactericidal substance. As Fraenkel and Sobernheim were the first to show, such a serum, although deprived of its bactericidal substance, still retains its immunizing properties.

It is easy to understand why the presence of this bactericidal substance is not indispensable for the preventive action of the serum. It is due to the fact that the bactericidal substance is not a peculiar property of this serum but exists in any normal serum, so that a normal animal given an injection of cholera serum is protected because it already possesses the bactericidal substance.

The substance that the normal animal does not have is the preventive substance, and it is, therefore, this latter substance that it is important to furnish. One may ask how this substance when introduced into the tissues produces immunity. It is certain that leucocytes, in common with other sensitive living cells, react to the presence of the preventive serum. Stimulated by this serum they give evidence of positive chemiotaxis. We know, moreover, that in animals immunized by serum, phagocytosis occurs with remarkable intensity. It is difficult, then, in view of these facts, not to believe in the idea advanced by several observers and notably by Metchnikoff, Roux, and Sanarelli that serum acts on cells as a "stimulin" that excites phagocytosis.

But there is another phenomenon in passive immunity which is perhaps even more important. The injection of preventive cholera serum causes the appearance of a very distinct bactericidal property against the cholera vibrio, for which the serum injected is specific. We know already that the preventive substance, incapable in itself 
of destroying the vibrio, when mixed with normal serum, which in itself is only faintly bactericidal, acquires new and energetic destructive properties. It is not necessary to imagine any reaction on the part of cells, due to preventive serum, to form this normal substance contributed by the normal serum, since it has been shown to be regularly present in the normal animal. This substance is not uniformly dissolved in the plasma during life, but is confined rather within the leucocytes.

What happens, then, when preventive serum is inoculated? The active substances penetrate into the leucocytes as we already know by the fact that leucocytes show evidence of chemiotaxis in presence of this serum. The preventive substance finds in the leucocytes the bactericidal substance there present. From this moment the leucocyte has acquired a powerful and specific bactericidal property. If it takes up vibrios it can destroy them; but if, for any reason, it should suffer injury, it will liberate the bactericidal substance into the surrounding fluid and so bring about an extracellular destruction of the vibrio. If we consider the facts from this point of view, as seems justifiable, we must admit that in addition to cellular stimulation there is present a purely chemical phenomenon that may be repeated in vitro and which is of great importance in passive immunity. This phenomenon is the union of the two substances that produce a bactericidal power either in the animal or in the test tube. An immunity of this sort brought about in this manner need not be very powerful, and it is not necessary for its formation that a profound cellular change should be caused as would be essential for its persistence. Passive immunity is essentially ephemeral and evidence of it is not long shown by the cell. As soon as the preventive substance is eliminated the leucocytes will have no more than a limited bactericidal property, and the refractory condition so rapidly obtained will be quite as rapidly lost. 


\title{
IV. ON THE MODE OF ACTION OF PREVENTIVE SERA.*
}

\author{
BY DR. JULES BORDET.
}

\section{Preparateur at the Pasteur Institut.}

\section{(From the Laboratory of Professor Metchnikoff.)}

The properties of the blood serum of immunized animals are being attentively studied. While it is still rather difficult to explain the antitoxic properties in certain sera, our knowledge of the preventive power of serum, that is to say the property of immune serum to protect animals against the invasion of bacteria, has recently been greatly increased. In the present article we shall deal, not only with certain new facts, but shall also review the conceptions we have held and refer to the various opinions of other writers on these subjects. We must repeat, then, not only those facts which we personally have demonstrated, but also the researches of other observers, notably of Pfeiffer and of Gruber. We shall frequently refer to the work of Metchnikoff $\dagger$ whose experience and advice is of such signal value to those who have the opportunity of working with him.

The attention of many observers has naturally been directed to those substances that are harmful for bacteria which the animal body uses in its struggle against infections, and the presence of which may be easily demonstrated, particularly in animals immunized against the cholera vibrio. In such animals the serum is endowed, not only with a preventive power but also with a distinct bactericidal power for Koch's vibrio. One of the chief points of disagreement is whether this bactericidal substance is uniformly dissolved in the body fluids during life or is confined to cells. The action of preventive sera on bacteria in vitro has been carefully

* Sur le mode d'action des serums prévéntifs. Annales de l'Institut Pasteur, 1896, X, 193.

$\dagger$ Particularly the article: Réchérches sur la destruction extracellulaire des bactéries, Annales de l'Institut Pasteur, June, 1895. 
studied. Furthermore, attempts have been made to gain an insight into the mechanism of the immunity conferred by serum, and to determine the relation between this kind of immunity (passive immunity) and the immunity caused by repeated injections of bacteria (active immunity). In these discussions the question has repeatedly recurred as to the part which is played in animal protection by the body fluids on the one hand and by the living cells on the other.

\section{The Localization of the Bactericidal Substance During Life.}

If cholera vibrios are placed in the serum of an animal immunized against these bacteria they are destroyed at least partially. After a certain time cultures from this serum on artificial media are either negative or show very few colonies. We have already given the reasons that have led us to conclude that the bactericidal substance present in serum comes from leucocytes. We were forced to the conclusion that during life the bactericidal substance is present in leucocytes and that when the white blood cells are removed from the blood vessels they liberate into the surrounding serum those bactericidal substances which they normally retain.* We based our conclusions on the comparative study of the bactericidal property of the serum of a vaccinated animal with the edema fluid from the same animal, and by a comparison between the destructive property of the serum from whole blood and the serum from blood previously deprived within the body of part of its leucocytes.

The means of determining the existence of bactericidal power and of estimating its intensity was simply to inoculate culture media (gelatin) with organisms that had been allowed to remain in contact with the body fluids mentioned for variable lengths of time. We have also considered another reaction that has been given to investigators through the observations of Pfeiffer.

Vibrios and other bacteria may show the harmful influences of a bactericidal substance, not only by losing more or less completely their power to develop on such artificial media as gelatin, but also by the morphological change they show when affected by this 
harmful substance. The existence of bactericidal power may thus be recognized, and its nature studied in detail by means of a mörphological change evident on microscopic examination.

As is well known, Pfeiffer found that when cholera vibrios are injected into the peritoneal cavity of a well-immunized animal they lose their motility and undergo rapid transformation. The same phenomenon occurs in a normal animal provided that the emulsion of vibrios is mixed with a certain amount of active preventive serum. Pfeiffer thought that this phenomenon of granular transformation could occur only within the animal body, and that the substance which caused this metamorphosis of vibrios came from an active secretion of the endothelial cells; he thought that the leucocytes had no function in the elaboration or in the storing of these destructive substances. Metchnikoff was then able to show that Pfeiffer's phenomenon could be produced in vitro, if the vibrios are mixed with a small amount of preventive serum and a little peritoneal lymph containing leucocytes. On the other hand, the injection into a normal animal of vibrios plus preventive serum in a region where there are no leucocytes (for example, a leg in which an edema has been formed) is found to produce no transformation in spite of the presence of the preventive serum. Some time after injection, when leucocytes appear, transformation does take place, but only within the cells. We found, moreover, that the metamorphosis of the vibrio could be very well brought about in vitro by the action of the fresh serum of an immunized guinea-pig,* even when no cells are present. We found, too, that this transformation did not take place if, instead of serum (that is, a fluid formed outside the body that has been in contact with leucocytes and therefore liable to contain products of leucocytic disintegration), we used edema fluid from the same animal; in other words, a fluid separated within the animal body and almost entirely free from leucocytes.

Aqueous humor and various secretions from an animal immunized against the cholera vibrio also fail to produce a transformation of the organism.

It was also shown that other bacteria (for example, B. typhosus, B. coli and B. pyocyaneus) "that have been found to be suscep-

* And also in a mixture of fresh normal serum with a small amount of preventive serum. 
tible to granular transformation in vaccinated animals even outside the cells may show the same change in normal animals. But in the latter case the transformation occurs only in those places where the bactericidal substance is most concentrated, that is to say, within the phagocyte." We may add that if we inject B. coli into a guinea-pig with preventive serum we find that granular transformation takes place within the leucocytes even when the bacteria in the surrounding fluid are unaffected.

These facts indicate very clearly the superiority in bactericidal power of the white corpuscles over the body fluids, as is shown by a production of granules. They show, moreover, that the bactericidal power in an exudate owes its origin entirely to the leucocytes in the fluid. We must consider why this essential function of leucocytes in the elaboration of bactericidal substance has been so much doubted and is still denied by many observers, and why the activity of the body fluids in destroying bacteria has seemed in certain cases superior to the cell activity. It is due simply to the following fact: in well-immunized animals a very small dose of bactericidal substance is sufficient to cause a granular change in a large number of vibrios.* A very few leucocytes endow the exudate with a bactericidal power which can produce the metamorphosis of a surprising number of bacteria.

On the other hand, a large supply of leucocytes is necessary to effect the phagocytosis of a moderate number of bacteria. When vibrios are injected into the peritoneal cavity the few leucocytes present suffice to give the peritoneal fluid a very distinct bactericidal activity, but as they are few in number they cannot take up many organisms. For this reason the attention of the observer is immediately monopolized by the extracellular bactericidal activity, so that he overlooks the essential importance of the cells present in the fluid. Later on the leucocytes come up in large numbers and phagocyte the intact or altered vibrios completely and then it is quite impossible even on the most superficial examination to overlook the importance of their protective function. In short, extracellular transformation of vibrios in the peritoneal cavity is due to

* Two or three platinum loops of the serum of one of our vaccinated guineapigs, when mixed with a drop of emulsion of vibrios, cause a generalized metamorphosis, and yet our serum is not, as a rule, as active as Pfeiffer's. 
the chance of there being sufficient leucocytes in the cavity when the injection is made to cause the surrounding fluid to become bactericidal, but too few of them to cause phagocytosis on a large scale. This is the essential condition for the production of Pfeiffer's phenomenon. We should expect, then, that if we increase the number of phagocytes at the point of inoculation we should change the appearance of the conflict between the animal body and the infection; and this is indeed what happens. Metchnikoff injected a mixture of preventive serum and vibrios into the peritoneal cavity of a guinea-pig that had received an injection of bouillon the day before; this previous injection caused an increase of leucocytes, so that the vibrios subsequently inoculated encountered large numbers of cells. Under these conditions the vibrios were immediately taken up without any evidence of extracellular morphological change. A morphological change goes on, to be sure, but within the phagocyctes where all the bacteria are to be found.* We have already shown that vibrios are immediately phagocyted when injected into the circulation of vaccinated animals. On the other hand, if the vibrio is injected into a region deprived of leucocytes, for example, subcutaneously, or into an edema area, no extracellular change is found. Phagocytes come up gradually and, as soon as they appear in the infected region, they take up the bacteria which have remained normal. Phagocytosis under these conditions occurs as the initial phenomenon, beginning with the arrival of the cells, and dealing with intact bacteria.

The experiments which we have just reviewed as well as the conclusions that we have drawn from them have given rise to objections which we must consider. Pfeiffer admits that vibrios are changed in vitro by a mixture of fresh guinea-pig serum and preventive serum, but he thinks that this effect on the vibrios is only passing and not equal in bactericidal force to that which occurs in the animal body. We cannot share this opinion. If vibrios are injected into the peritoneal cavity of an immunized guinea-pig, transformation allowed to take place, and some of the exudate is then withdrawn, it is found on placing it in the incubator that the

* We do not understand how Gruber could use this experiment to contest the origin of the bactericidal substance from the polynuclear leucocytes. (Wiener klinische Wochenschrift, 1896, No. 12, p. 207.) 
organisms grow out again quite as well as when the phenomenon has been produced in a serum mixture in vitro.*

Pfeiffer's objection, then, is untenable. As a matter of fact, the power of transforming vibrios is very marked in immune serum. There is nothing astonishing in the fact that after a certain time the bactericidal properties are exhausted and, with in-vitro experiments when the substance is used up, there is no means of renewing it. In the animal body, however, the exudate keeps on increasing after injection. Moreover, it would not be surprising to find that the exudate is more bactericidal than serum, since even at the moment of injection it of ten contains more leucocytes than does the blood. And finally, there is every reason to believe that the extent of the humoral bactericidal activity which occurs in the peritoneal cavity of immunized animals is exaggerated. Certain vibrios resist, and either retain or recover their motility; and, under the usual conditions of Pfeiffer's experiment, it is not reasonable to speak of an extracellular destruction that occurs 3 or 4 hours after injection for the simple reason that by this time all the organisms, whether altered or not, are within leucocytes. After this time any further destruction of the infective agent must be attributed, not to the fluid, but to the cells. Pfeiffer has repeated Metchnikoff's experiment of injecting into a normal guinea-pig a mixture of vibrios and preventive serum in an area where edema has been caused by venous compression. Under these conditions Metchnikoff found that the vibrios outside the cells were not changed into granules. In this experiment naturally a preventive serum that has not been freshly obtained must be used, since a fresh serum might of its own accord produce a metamorphosis of the vibrio. Pfeiffer obtained a different result from the one noted by Metchnikoff, that is to say, he did find an extensive extracellular change in the organisms. Metchnikoff's results, however, were constant. We have found, moreover, that in vitro the cholera vibrio is unchanged by a mixture of preventive serum and edema fluid, whereas it is transformed by a mixture of preventive serum and fresh normal serum. The unexpected result of Pfeiffer's experiment may be due to the fact that this edema was not pure plasma, but contained a certain amount of blood. The punctures that have to be made to obtain the exudate may very

* This was very clearly shown in Metchnikoff's experiments. 
well produce slight hemorrhages, and we know that even a small amount of blood serum will affect vibrios in presence of the preventive substance.

Pfeiffer, moreover, was unable to find the almost instantaneous occurrence of phagocytosis in animals prepared by a previous injection of bouillon, under which conditions it will be recalled the vibrios immediately encounter a large number of phagocytes. It may be that if one injects a large amount of bacterial emulsion at a low temperature a momentary paralysis of the phacocytes may be caused and an extracellular transformation may therefore occur. It does not seem evident that Pfeiffer's conditions were essentially different, which fact renders his results still more inexplicable, as we regularly obtain rapid phagocytosis under these conditions.

Pfeiffer mentions the case of a goat that had been well immunized and that died of intoxication following a subcutaneous injection of vibrios; in this instance the destruction of the culture was brought about, according to Pfeiffer, entirely without the aid of phagocytes; no white cells were found at the point of inoculation, although the cultures were negative. When dealing with well-immunized animals a few scattered leucocytes may be sufficient to give the body fluid a distinct bactericidal power. What is more, it is quite probable that in a dead animal the diffusion of bactericidal substances does not follow the laws that obtain in a living animal. We know, indeed, that when leucocytes are taken out of the animal body they liberate certain substances more readily than they do under normal conditions, and the leucocytes in a dead or dying animal can scarcely be considered to be under normal conditions.

\section{The Effect of Sera on Bacteria in Vitro. The}

Diagnosis of Bacteria by Serum.

Before endeavoring to determine the mechanism of passive immunity more exactly we must consider the exact effect of serum on vibrios in vitro. To be perfectly clear, we must consider this subject from the beginning, which will necessitate the repetition of certain details that have already been published.

In the first place we may recall that the serum of an immunized animal causes the granular transformation of the vibrio used for 
immunization, which phenomenon serves as an index of the bactericidal power which this fluid possesses. If an emulsion of vibrios is mixed with preventive serum, they lose their motility, collect into compact clumps and finally undergo transformation.* This effect by serum is rather markedly specific, as we shall consider in detail later on.

Serum that has been kept for some time or has been heated to 60 degrees remains preventive and may still immunize animals, as Fraenkel and Sobernheim $\dagger$ were the first to note, but loses its bactericidal power. Under these conditions it also loses, as might be imagined and as we have pointed out, the property of producing granular transformation of the vibrio. But, as we have particularly emphasized, the bactericidal power may be restored to such serum and it may again become able to transform vibrios. Although

* It had not been distinctly recognized before our experiments that preventive serum, when added in very small amount to an emulsion of the bacteria against which the serum is active, eauses their clumping in little masses that float in the fluid. Observations on the clumping action of the serum of an immunized animal had, however, been made. Charrin and Roger (Développement des microbes pathogènes dans le serum des animaux vaccinés, Société de Biologie, 1889, p. 667) may be credited with this observation. These authors noted that "a culture of B. pyocyaneus becomes clear and transparent when placed in the serum of an immunized animal. The organisms are collected in tiny clumps which may be separated by shaking the tube, but which fall again to the bottom when left standing; bacilli placed in normal serum show no such peculiarity. In the serum of refractory animals, however, the appearance is very distinctive; the organisms are united in chains, etc. Finally, these elements tend to collect together and, instead of floating freely, as do normal bacilli, they pile up in little clumps, which explains the clotted appearance of the culture."

Later on Metchnikoff (Annales Pasteur, 1891, p. 473 et 474) noted the same phenomenon with the vibrio Metchnikovi; he found that this organism develops on the blood and serum of non-vaccinated guinea-pigs as it does in any ordinary fluid medium. In the serum of immunized animals, on the contrary, the culture shows definite clots that float about in a clear fluid on agitation and then fall to the bottom of the tube. He noted the same fact with the organism of pneumonia, and his observation was confirmed two years later (Annales Pasteur, 1893) by Issaef, who, later still, in collaboration with Ivanhoff, found that the same phenomenon occurred with a vibrio discovered by the latter.

Although Metchnikoff was inclined to regard these phenomena of clumping as of general significance, he made no definite statement to this effect, because the phenomenon did not occur in cultures of the organism of pneumo-enteritis on adding the serum of animals immunized against it (Annales de l'Institut Pasteur, 1892).

† Hygienische Rundschau, 1894. 
heated immune serum contains only the preventive substance, its strong antiseptic power against the vibrio may be restored by mixing with it fresh normal serum, which in itself is only faintly bactericidal. This simple experiment led us to the rather paradoxical conclusion that "two sera, neither of which is distinctly bactericidal, form a mixture which has marked antiseptic properties against vibrios."

Only a very small amount of preventive substance is necessary to endow the preformed substance of normal serum with great activity and with specificity. Normal serum heated to 55 degrees loses its power to form a bactericidal mixture with preventive serum. Edema fluid, aqueous humor, etc., from a normal guinea-pig differ from normal serum, since, mixed with preventive serum and an emulsion of vibrios, they cause no metamorphosis. The preventive substance in immunized animals passes more rapidly into the edema fluid than does the bactericidal substance as is shown by the fact that the edema fluid of a vaccinated guinea-pig, which does not cause granular transformation, will produce it on the addition of a small amount of fresh serum.

We were led to the conclusion that the intense bactericidal property of immune serum is due to the combination of two substances; one, the specific preventive substance which resists heating to 60 degrees or even more; the other, the bactericidal substance properly speaking, which is not specific and in itself is only slightly active and is present in normal as well as in immunized animals. This latter substance is sharply differentiated by the fact that it is destroyed by keeping for any length of time, or by heating to 55 degrees. The serum of vaccinated animals when fresh contains both substances; it may easily be deprived of one of them by heat, but the original properties of the serum may be restored synthetically by adding to it the part that has been lost, that is to say, the bactericidal substance (or alexin) of normal serum.

Since experiment has shown us that the simultaneous presence of both substances is necessary to constitute bactericidal power against the vibrio we must now consider what the respective action of each substance in the mixture is. If we add to an emulsion of vibrios preventive serum alone, that is to say, serum previously heated for half an hour to 60 degrees, we find that the organisms remain 
morphologically intact, but that many of them become motionless and clumped.*

If a mixture is made of vibrios and normal serum without the specific preventive substance, certain bacteria lose their motility and form round granules; this transformation, however, is only slight.

It is evident from these facts that the preventive substance immobilizes and clumps, and that it becomes still more active when accompanied by the bactericidal substance. Of the two substances the normal bactericidal substance is the only one which alone can cause even a partial morphological change in the organism. It appears probable then "that the preventive substance exerts a certain unfavorable effect on the vibrios, although it is not a real antiseptic; and that this effect causes them to react more distinctly to the power of the bactericidal substance."

The origin of this important bactericidal substance which occurs in normal as well as in immunized animals, and also in sick animals or animals that have died of various infections, is known. It is the substance found within the leucocytes, and which even in normal animals is sufficiently concentrated in the cells to bring about a granular modification of organisms ingested by the phagocytic protoplasm. As a result of the coagulation of the blood it is partially liberated in the serum, and gives evidence there of its activity when combined with the preventive substance, although this activity is necessarily weakened by dilution.

This represented the status of our knowledge a year ago. In a series of recent articles Gruber has carefully repeated the majority

* It must be noted that this phenomenon of immobilizing and clumping is not nearly so striking with heated serum as with fresh scrum. In heated serum, moreover, the vibrios grow rapidly, and after they cease to feel the clumping influence of the serum spread diffusely throughout the fluid. Some time ago Metchnikoff, in his work on the vibrio Metchnikovi, noted that the vibrios grew unclumped in the inflammatory exudate of the immunized guinea-pig, whereas they were agglutinated when inoculated in the serum of the same animal. This offers yet further analogy between the properties of the exudate and the heated serum, which we have already found to be very similar. Both fluids contain (provided they have been taken from immunized animals) the preventive substance; but their bactericidal activity is very much less than that of intact serum. It may also be added that bacteria cultivated in heated preventive serum undergo clumping and granular transformation when a certain amount of normal serum is added to the culture. This addition restores to the serum its original qualities, even after it has served as a culture medium. 
of the experiments which we have mentioned and added others, and as we shall see presently he has drawn certain conclusions as to the mechanism of passive immunity which are in part similar to our own. This confirmation is gratifying, but we shall not consider further such experiments and conclusions as have already been mentioned. We shall consider more particularly the new facts and interpretations which he has offered. First of all Gruber finds that B. coli and B. typhosus react under similar conditions in the same way as do the vibrios, that is to say, they lose their motility and become clumped.

Gruber thinks that a swelling and viscosity of bacteria will explain their clumping by preventive sera. He thinks, moreover, that this viscosity actually produces the collection and adhesion of separated organisms into clumps. On this account Gruber calls those substances that produce clumping "agglutinins." However fitting this term may be, we must consider first whether the swelling and viscosity of bacteria can explain a collection and clumping of disseminated micro-organisms. It is quite conceivable that viscosity may prevent the separation of bacteria already clumped, but it is not quite so clear how this change can bring them together, particularly when we are dealing with non-motile organisms. Tetanus bacilli and streptococci are clumped by sera; and motile organisms (such as the cholera vibrio) are clumped, even when they have been killed with chloroform and so lost their motility.

When cholera vibrios are mixed with heated cholera serum clumping occurs, but no granular transformation is to be noted. The clumping, however, occurs very well, even when serum that has been kept for some time is used; clumping, moreover, occurs very rapidly, whereas the metamorphosis takes some time. Vibrios that have been rapidly brought together into definite masses when colored by the usual stains show no distinct alterations.*

It is evident then that the viscosity of clumped bacteria cannot be directly demonstrated and consequently must be regarded as purely hypothetical. This idea of a surface modification of bacteria which Gruber regards as certain becomes less admissible when we

* When about to send these pages to the press we found that Pfeiffer has noted that same fact in the last number of the Deutsche medicinische Wochenschrift, April 9, 1896. 
study the clumping produced by specific sera in organisms other than the vibrios. If, for example, we examine the clumps of tetanus bacilli which are well formed by the action of an antitoxic horse serum, we find that the bacteria, although collected in small masses, do not adhere well. A similar fact is suggested on examination of stained preparations of masses of vibrios. The preparations made from a clumped culture of tetanus suggest in appearance a handful of pins that has been carelessly thrown on a table. The clearly defined stiff rods cross each other in all directions without giving evidence of an intimate contact, and with rather large spaces between them. The appearance of the bacilli is absolutely normal, even when they have remained for a long time in the serum. An examination of clumped streptococci gives similar results. Clumping may be noted also in cells other than bacteria. Red blood corpuscles, for example, may show it very distinctly. If a small amount of rabbit serum is added to guinea-pig serum containing a few blood corpuscles the latter very rapidly undergo a curious modification; instead of remaining scattered uniformly throughout the fluid they collect in perfectly definite clumps which stand out as little red points in a clear liquid.

But if instead of using rabbit serum, we add the serum of one guinea-pig to the corpuscles of another, no change takes place in the uniform turbidity of the fluid. Horse serum when added to red blood corpuscles of the guinea-pig or of the rabbit clumps them energetically. In a similar manner rat serum clumps rabbit corpuscles and vice versa; goat serum produces the same effect on guinea-pig corpuscles. These facts would indicate that as a general rule red blood corpuscles are clumped by serum from a different animal species.

It may be added that the cholera vibrio, when cultivated in guinea-pig serum containing corpuscles, clumps them. It would be interesting to ascertain whether red blood corpuscles subjected to the effect of foreign serum, or taken from an infected animal, still show normal osmotic properties and act in the usual manner with salt solutions.

There is a striking analogy between the clumping of red blood cells by a foreign serum and the effect of preventive serum on bacteria. If we imagine that bacteria swell up and become viscous 
because we find them clumped, we should draw the same conclusion as to the clumping of corpuscles. If, however, we examine the clumps of red blood corpuscles produced by horse serum, it is found that they show no distinct difference from normal corpuscles whether examined fresh or in stained preparations. If two or three drops of such clumped cells are heated on a slide to 60 degrees, the corpuscles separate quickly as the temperature rises; on cooling again the clumping reappears and disappears again when reheated; and this procedure may be repeated two or three times. A rise in temperature is found to produce the same effect on clumped bacteria, although in this case the phenomenon is not so readily observed.* Corpuscles or bacteria that have been separated from the clumps by means of heat are morphologically and tinctorially normal. The hypothesis that cells such as bacteria or red blood cells undergo some modification when subjected to specific serum has little probability. This hypothesis, moreover, we may repeat, would account for the persistence of the clumps, but not for their formation; it does not explain the action of heat just noted nor is it in harmony with the microscopic appearance of the organisms, which, although clumped, show no visible alterations. These clumping phenomena seem due rather to some phenomenon of molecular physics. The slightest effects, as we know, may cause chemical precipitates which have remained uniformly suspended in a fluid to fall to the bottom.

It is probable that serum acts on bacteria by changing the relations of molecular attraction between the bacteria and the surrounding fluid. As far as the effect of heat is concerned it may be explained by the well-known effect of temperature changes on molecular phenomena, as, for example, on the superficial tension of fluids.

This point of view would imply that bacteria in suspension act as inert particles when clumped by a serum. There can be no question of active participation, as we know that cholera vibrios killed by chloroform may still be clumped.

The second point which has been considered by Gruber and Durham, $\dagger$ and which they have explained in a rather peculiar man-

* Heat has little effect on clumps of streptococci, but this is due to the fact that the chains of organisms are so interlaced as to hold together.

† Eine neue Methode zur raschen Erkennung der Choleravibrio und des Typhusbacillus. Münchener med. Wochenschrift, March 31, 1896. 
ner, is the question of the specificity of sera and the value of this specificity as a means of certain diagnosis of bacterial species. The diagnostic method founded on the instance of specificity offered by Pfeiffer is well known. The serum of an animal vaccinated against the cholera vibrio, for example, when injected together with a culture of the true vibrio into the peritoneal cavity of a normal guinea-pig causes loss of motility and then rapid transformation of the vibrios into roundish granules. We are safe in concluding that all vibrios which give this reaction in presence of an anticholera serum are true cholera vibrios. According to Pfeiffer, all the true cholera vibrios do act in the same way, which fact gives us a means of distinguishing Koch's vibrio from similar vibrios and from other species of bacteria.

This method, however, has not appeared to all to be absolutely certain. When we say, indeed, that sera are specific and that one may utilize this specificity as a means of diagnosis two distinctly independent facts are implied, each one of which demands a separate demonstration. It means, in the first place, that the animal body during vaccination acquires certain new properties which are in a direct and intimate relation to the kind of bacteria that have been used in immunization. It would seem that this fact has already been justified by experiment; we have found, for example, that the serum of a guinea-pig vaccinated against cholera is specifically bactericidal for the true cholera vibrios and has little or no effect on vibrios that are known to differ from the cholera vibrios, for example, the vibrio Metchnikovi.* But it is at the same time asserted that bacteria which have once reacted in a positive or negative manner to a given preventive serum will thereafter always act in the same way whatever may be their subsequent condition of existence. This statement implies that there are no transitional forms between organisms which react positively on the one hand and those which act negatively on the other; such a lack of transitional forms, indeed, is necessary to render the method precise. This conception, which tends to establish distinct, insuperable limitations

* These facts were demonstrated first by Pfeiffer. We had previously noted a similar phenomenon, namely, that the serum of animals vaccinated against the cholera vibrio when injected into a normal guinea-pig endows the serum of this animal with intense bactericidal power against the cholera vibrio and against this vibrio only. 
between bacterial species that are so difficult to define and separate, would scarcely appear to be in harmony with our present.knowledge of the origin of species. Moreover, we know that the vibrio of Massaouah, which is certainly a cholera vibrio, does not show the granular change with cholera serum. The method, however, in many instances may be of real value and aid considerably in confirming the diagnosis.

Instead of injecting vibrios and serum into the peritoneal cavity of an animal, there is a simpler method, which we have described as a modification of the Pfeiffer diagnostic procedure, and which consists in making a mixture of the vibrio and fresh preventive serum in vitro (in case the preventive serum is not fresh, fresh normal serum may be added). If the organism is susceptible to the action of the serum, in other words, if it belongs to the strain against which the animal that has furnished the serum has been vaccinated, it will lose its motility by clumping and show granular transformation as already described. We described this procedure in vibrios only, whereas Pfeiffer has applied his method not only to vibrios, but to the colon bacillus and the typhoid bacillus as well.

Gruber and Durham make a diagnosis, not only between vibrios, but even between B. coli and B. typhosus, using as the single criterion the immobilizing and clumping effect which each specific serum shows for its respective micro-organism. For example, it is assumed that a suspension contains typhoid bacilli if the organisms in it are clumped by antityphoid serum.

As far as vibrios are concerned Gruber and Durham's method is only a mutilation of our own in-vitro method. Our method, as we have just described it, gives three distinct indications of the reaction of the bacteria in question to the serum that is used. These indications are loss of motility, clumping, and granular transformation. Gruber and Durham pay no attention to this latter important indication and consider only the first two. A priori it would seem impossible to have too many distinctive indications in dealing with a task so delicate as a diagnosis between two closely related species of bacteria, and it would seem that ignoring one of these indicating signs would scarcely constitute progress. In other words, we should consider all the indications of specificity that our data may afford us. 
If those substances in sera which clearly and actively cause the immobilization and clumping of a given bacterial species are to be found in the serum of a vaccinated animal; and if the clumping by a specific serum occurs only with bacteria which are similar to those used to vaccinate the animal from which the serum is derived, and if, thirdly, bacteria of the same species always act in a similar manner with a given serum; we may then regard as exact diagnoses based on clumping alone; without considering such other characters as granular transformation. Not all these conditions, however, are realized.

First:-Clumping of bacteria may be produced by sera other than specific preventive sera; for example, normal horse serum clumps cholera vibrios energetically. A similar, though slightly feebler effect, is produced on the vibrio Metchnikovi; it also happens frequently with B. tetani, B. coli and B. typhosus and less distinctly with the streptococcus (the culture used was very virulent).* This property persists in horse serum that has been kept or heated for half an hour to from $60^{\circ}$ to $62^{\circ} \mathrm{C}$.; under this latter condition, however, the property is somewhat diminished.

If two drops of horse serum are added to 1 c.c. of an emulsion of vibrios (a 24-hour agar culture suspended in 20 c.c. of normal salt solution) the bacteria fall rapidly to the bottom of the tube and the supernatant fluid becomes absolutely clear within two hours. The clumping property, then, is well developed in horse serum, and much better developed relatively than is the immobilizing property. For it is found on examining hanging drop preparations from the emulsion of vibrios and serum, that there are vibrios which, although brought together in definite masses, have not entirely lost their motility; these motile vibrios, moreover, frequently cause a movement or turning of the entire mass. These facts may be noted even when a large amount of serum is employed. But if such mixtures have been made according to Gruber's method in test tubes, the deposition of bacteria is complete, as clumps fall to the bottom in spite of their oscillating movements. Although the specific preventive serum of the immunized guinea-pig gives complete immobilization more easily, horse serum produces the same effect on the

* Normal guinea-pig serum has practically no effect on these different bacteria. 
majority of the vibrios and causes very energetic clumping. If we note the clarification of tubes containing an emulsion of cholera and a very small dose of specific serum we find that it takes considerable time for completion. It is partial at first, owing to a rapid deposition of clumps, but proceeds gradually by the falling of isolated motionless vibrios that are not clumped, and therefore sink to the bottom slowly.

We have then two sera which, although essentially different, have similar properties. We might look on the horse as having naturally the preventive substance against the cholera vibrio and on this supposition we might postulate that the injection of horse serum would give guinea-pigs a certain immunity against this organism.* But the clumping substance of normal horse serum differs very distinctly from the similar clumping substance of the immunized guinea-pig. The first causes an agglutination of the red blood corpuscles of the normal guinea-pig, but the second does not. We may conclude, then, that the property of clumping bacteria does not belong exclusively to the specific preventive substance, and we cannot refer to "clumping substance" and "preventive substance" as synonymous. All that we can say is that in animals whose serum normally is neither clumping nor preventive, vaccination may give rise to both properties.

Second:- If the objection which we have just outlined were the only one, we might use the clumping effect of preventive sera as a means of diagnosis if we took care to use only an immune serum from animals in whose serum no such property is present before immunization (the guinea-pig).

But the effect which we are considering is never entirely specific, even in serum from such animals. Gruber himself mentions certain examples which invalidate the law of specificity. We must consider this point rather in detail. We find that serum from guineapigs vaccinated against the cholera vibrio (Eastern Prussia) agglutinates energetically the vibrios from Eastern Prussia, of Massaouah and less completely, but very distinctly, the colon bacillus. And

* This fact has been noted by Pfeiffer and we can confirm it. The preventive power, however, is never very strong. We may add that the subcutaneous injection of 3 c.c. of horse serum in the guinea-pig markedly increases the bactericidal property of the animal. The increase, however, is not nearly so great as that caused by specific serum. 
again, the serum of a rabbit vaccinated against Massaouah (which, however, was not very powerful) clumped the Massaouah organism, and had only the slightest effect on the vibrio of Eastern Prussia as we have already noted in conjunction with Mesnil. It is, therefore, not certain that in using different sera we shall always obtain the same result.

Third:-A micro-organism which ordinarily is clumped by a given serum may undergo such changes as to be no longer distinctly affected by this serum. In 1891 Metchnikoff found that the vibrio Metchnikovi grown in the exudate of an immunized animal might either form clumps or else grow as individual organisms, according to the previous conditions under which the culture had lived. Metchnikoff and I have recently convinced ourselves that a very virulent vibrio from Eastern Prussia, after living for some time within the protoplasm of phagocytes, was affected only very slightly by serum from a horse vaccinated against cholera. This vibrio had been injected subcutaneously into a horse and the exudate withdrawn after phagocytosis was complete. Vibrios from the original culture transplanted on agar were very strongly clumped by the serum of this animal, whereas the organisms drawn from the exudate of the animal remained scattered and caused a permanent cloudiness in the fluid. This modification of the vibrio, moreover, lasts for several generations.

When dealing with an organism that has been recently isolated we have very indefinite knowledge as to its previous conditions of existence. It may well be that in nature certain influences change organisms in such a way that they become insusceptible to the clumping action of serum. Since the property of collecting bacteria into clumps does not belong exclusively to specific preventive substances; and since the clumping effect of a preventive serum is not absolutely specific; since bacteria are susceptible to changes in respect to clumping, and their reaction to a given serum is inconstant and varying, it must be admitted that suppressing such a diagnostic sign as the granular transformation does not add to the accuracy of this type of investigation. 
III. The Function of Cells and of Body Fluids in Immunity. The Mechanism of Passive Immunity and its Relations to Active Immunity.

In the first section of this article we recalled to the reader experiments that demonstrated the respective activities of the cells and the body fluids on vibrios that had got into the tissues. It was mentioned that cells may act either by liberating bactericidal properties in the surrounding fluid or by taking up intact or modified bacteria. It was evident also that of these two modes of action the second is the more important and the only essential one. Phagocytosis takes place in all cases and neither previous granular change nor loss of motility are necessary for its occurrence. The body fluids in transforming or changing vibrios act simply because they have received active principles from the leucocytes. Although it is true that the fluids may in certain cases destroy large numbers of vibrios, phagocytosis nevertheless occurs markedly and is the ultimate means of destroying the infection.

Neither the clumping nor immobilizing of bacteria is to be considered as indicative of their destruction or as an indication of their elimination without phagocytosis, even to the extent that granular transformation is. We have already shown that clumping of bacteria is no indication of their alteration, that their surface has become viscous, nor that they are swollen or incapable of reproduction. Moreover, this phenomenon is never complete in the animal body and, when the transformation of vibrios into rounded bodies follows in the peritoneal cavity, many granules are seen that remain separate. Moreover, the clumping power of a given serum bears no relation to the resistance of the animal that furnished the serum to the bacterium in question. Horse serum clumps tetanus bacilli energetically, but the horse is very susceptible to tetanus; much more so, indeed, than is the rabbit, and yet the serum of this latter animal has no effect on the tetanus bacillus. The rat is refractory to cholera, but its serum has no effect on the cholera vibrio.

An organism may show most evident clumping and yet retain its virulence completely. The pneumococcus when grown on the serum of immunized animals forms clots; such cultures, how- 
ever, as Issaeff (Annales Pasteur, 1893) has shown are still very virulent.

As a general thing cultures of pathogenic organisms grown in the serum of immunized animals remain virulent whether the organisms are clumped or not (the pneumococcus on the one hand and the bacillus of hog-cholera described by Metchnikoff in 1892 on the other).

Horse serum and even horse edema fluid has a remarkable clumping effect on the cholera vibrio. The struggle against the vibrios, however, in the body of this animal takes place just as it does in other animals, namely, by means of phagocytosis. If we inject an agar culture of a virulent cholera vibrio subcutaneously in a horse, ${ }^{*}$ an influx of leucocytes occurs. A purulent fluid may be removed the next day from the area of inoculation and is found to contain leucocytes, in the protoplasm of which are found the organisms in various stages of degeneration; there are no free bacteria present. The inoculation of this pus on agar even 30 hours after injection gives an abundant culture. In other words, the bacteria have been taken up alive and eventually die within the phagocytes, as is shown by the fact that the animal rapidly recovers.

Does this mean that the extracellular changes in bacteria, the most important of which is granular transformation, is without importance in immunity? In no manner; by this means the number of invaders may be decreased, their development hindered and time given the animal to combat them advantageously by means of the leucocytes, that have eventually come up, instead of being faced by an invincible number of adversaries and their elaborated toxic substances.

In order to explain the mechanism of passive immunity we must account for one very important property acquired by animals treated with preventive serum. To what is the bactericidal power that is given these animals due? We have nothing essentially new to add to observations already made on this subject. The mixture in vitro of fresh normal serum and a small amount of preventive serum, neither of which sera is in itself bactericidal, produces a fluid that is strongly bactericidal. We have repeatedly emphasized this experiment because it appears to us to be the explanation of the appearance of a bactericidal property in the blood of passively

* The culture was kindly furnished us by Dr. Salimbeni. 
immunized animals. The study of an infection in such passively immunized animals shows that their resistance is due to a mechanism similar to that which we described in June, 1895 as occurring in vitro. Bactericidal power arises in the animal body, just as it docs in a test tube, by the union of two substances: a specific preventive substance, and the normal bactericidal substance present in the blood, not only of immunized, but also of normal animals. Separately, each one of these substances has only the slightest effect; together, they change the micro-organism most evidently.

The normal animal has the normal bactericidal substance. By treating this animal with immune serum we give to it the preventive substance which is lacking. How do these two substances unite? If the bactericidal substance under normal conditions is uniformly dissolved in the body fluids, the union would take place in these fluids. But we know that this bactericidal substance is concentrated in the leucocytes. The antiseptic mixture, then, must be formed primarily by a union within the leucocytes and can take place outside the cells only when a certain amount of the alexin has been liberated by the cells into the surrounding fluid.

Passive immunity is due then, at least partially, to the chemical effect of two preëxisting substances on the vibrios. One of these substances is present in the animal before injection and the other comes from the serum injected. The phenomenon is chemical in the sense that it can occur without the aid of any vital reaction or any new cellular secretion, as we know from the fact that it occurs in fluids that contain no cells. With the aid of this new means of defense, which has been acquired without reaction, the leucocytes are more than ever able to utilize their protecting power.* Passive immunity then may be explained, at least in part, as a passive increase of the phagocytic bactericidal power. Gruber tacitly agrees to this explanation, at least so far as the production of a bactericidal property by means of the union of two substances is concerned. It is, moreover, evident that he does accept this explanation, since he has drawn similar conclusions in his recent articles.

* We do not wish to imply that the leucocyte does not react at all to this injected serum, although a reaction by the leucocyte is not indispensable for the genesis of bactericidal power. There is no reason to suppose that the leucocyte may not react by an acceleration of its phagocytic activities. 
To be sure Gruber does not believe that union takes place within the protoplasm of the phagocyte, simply because he does not believe that the phagocyte is the source of the normal bactericidal substance. He does not bring any new facts to bear on this discussion, but simply accepts Pfeiffer's opinion which we have already considered.

The task of explaining the intimate changes which take place in bacteria as a result of the activity of the serum (immobilization, clumping, granular transformation) is far from finished. Gruber, to be sure, has offered an explanation of clumping, but, as we have already seen, it is far from satisfactory.

Pfeiffer has a different point of view on the action of sera. He thinks that the immunizing substances in immune serum are present in these sera in an inactive form, and become active by undergoing certain changes within the animal body.

In the first place it is certain that sera contain active substances, since they can produce the same modifications on bacteria in vitro as in vivo. These modifications, moreover, are exactly alike, and equal whether in vivo or in vitro. And, further, it is easy to show that the preventive substance injected is simply diluted in the blood of the animal and does not become more active.

The serum of a guinea-pig vaccinated against the cholera vibrio acquires a property that it did not previously have, namely, the property of clumping the cholera vibrio. We know, moreover, that the better a serum clumps the more actively preventive it is. The preventive value, then, may be measured by the clumping property. If we place in separate tubes a given amount of an emulsion of vibrios and add to each tube a varying number of drops of preventive serum we can determine exactly what this clumping power is. Controls, of course, are made with emulsion without preventive serum. After a certain time the deposition of bacteria and the clarification of the supernatant fluid reaches its maximum (about 24 hours when the doses of serum are small). In this way the smallest amount of serum which will cause either a beginning of clarification or complete limpidity may be determined.

A guinea-pig weighing 360 grams was bled and then injected with 1 c.c. of active preventive serum; the next day the animal was again bled. The serum obtained before injection had only a 
faint clumping power for the cholera vibrio, even in a large dose. The serum taken the day after injection, however, clumped them distinctly, and it was possible to compare its power with that of the preventive serum used for injection. By means of a series of tubes it was found that the serum obtained after injection of cholera serum is only one thirtieth as active as the original cholera serum. For example, in a tube containing 1 c.c. of bacterial emulsion and eight drops of this serum the opacity is the same as that in a tube containing 4 c.c. of bacterial emulsion and one drop of anticholera serum.* In this experiment the same results were obtained as if the 1 c.c. of cholera serum had been diluted in 30 c.c. of liquid. This volume corresponds fairly well to the amount of blood in a guinea-pig of this weight, 360 grams.

This experiment, moreover, shows why passive immunity cannot be transmitted indefinitely and indicates that the active principles of the serum are simply diluted in the body without acquiring new and more energetic properties. A word might be added about active immunity. The preventive substances, which are so valuable for animals injected with them, are also evidently equally valuable for those that have elaborated them. It must be admitted that active immunity is evidenced by a perfection of the bactericidal power of phagocytes. $\dagger$ But active immunity has also other characteristics, such as an augmentation of the number of phagocytes and an increase of chemiotactic sensitivity on the part of leucocytes, as has frequently been recognized by Metchnikoff and clearly evidenced by Massart in his experiments. We cannot, therefore, consider the two kinds of immunity as equally dependent on the presence of a preventive substance.

* To be sure the clarification took place more rapidly, although no more completely, in the tube containing serum from the passively immunized animal. This is due to the fact that the serum in this tube was in large amount and therefore acted not only as a diluted preventive substance, but also as a normal serum, and as is well known, normal serum increases the clumping action of preventive serum. The eventual amount of clarification in the two tubes, however, was equal.

$\dagger$ See article, page 8 . 


\section{A CONTRIBUTION TO THE STUDY OF ANTISTREPTOCOCCUS SERUM.*}

By DR. JULES BORDET, PREPARATEUR AT THE PASTEUR INSTITUT.

I.

\section{STREPTOCOCCUS INFECTION.}

\section{A. Streptococcus Infection in the Guinea-pig.}

Before beginning the study of antistreptococcus serum and its properties a consideration of the principal characteristics of streptococcus infection and the methods of reproduction of the streptococcus in the animal body is indispensable. We must therefore consider the characters of the micro-organism that we have used in our experiments and describe briefly the general aspects of the infection which it causes.

The streptococcus we have used in the majority of instances is one whose virulence was increased by Dr. Marmorek by methods described in the Pasteur Annals of July, 1895. Marmorek has been so kind as to place his organism and his serum at our disposal for these researches and we wish herewith to express our thanks for his kindness.

The readers of the Pasteur Annals are already aware that the streptococcus in question is extremely virulent; it kills rabbits in a dose of a fraction of a millionth of a cubic centimeter. Its minimal lethal dose may be as low as a thousand millionth of a cubic centimeter.

We have regularly used, as a culture medium suitable to maintain the virulence of this organism, a mixture of peptonized bouillon and human ascitic fluid, as already described by Marmorek. On this medium the organism grows rapidly and keeps its pathogenic qualities through many successive transplantations.

* Contribution à l'étude du sérum anti-streptococcique. Annales de l'Institut Pasteur, 1897, XI, 177. 
The infection caused by this streptococcus, although not yarying in general appearance, differs somewhat in details according to the animal species employed.

Much larger doses are necessary to kill a guinea-pig than are fatal for a rabbit. The minimal intraperitoneal dose for a mediumsized guinea-pig sufficient to cause a generalized infection and death of the animal is generally 0.2 of a cubic centimeter. The results following such an intraperitoneal dose in a normal guinea-pig are as follows: By puncturing the abdominal wall of the animal and withdrawing a little exudate at intervals with a small pointed tube it is easy to follow the course of the infection.

A fatal dose. - Following the inoculation of the culture the peritoneal fluid is more or less limpid and contains only a small number of cells. This is the period of phagolysis which, as Metchnikoff has shown, regularly follows the introduction of cultures into the peritoneal cavity.* The leucocytes in this exudate, consisting generally of the mononuclear type, with admixtures of a few true eosinophiles and infrequent amphophiles, do not long remain scattered either because they are destroyed, as Metchnikoff is inclined to believe, or because they adhere to the peritoneal walls, as Durham thinks.

This period of phagolysis is usually short, particularly when the amount of fluid introduced is inconsiderable, and is followed by the appearance of mononuclear and polynuclear phagocytes. These latter cells appear particularly during the first hours of the phenomenon and increase rapidly in number, so as to constitute the majority of cells present.

The first polynuclears arriving on the scene (say an hour after injection) take up a few streptococci; and a considerable number of the organisms introduced are soon found to be phagocyted. The mononuclears also frequently take up a good many of them. There are, however, among the micro-organisms injected, certain ones which are not engulfed. These latter, to be sure, are very few if the minimal lethal dose is employed and they remain scattered in the fluid in the midst of an increasing number of cells.

* This phenomenon of phagolysis is very slight if as small an amount as 0.2 of a cubic centimeter of culture is injected; but it becomes more marked if the amount of fluid injected reaches 0.5 to 1 c.c. 
The number of phagocytes gradually becomes increased; the cells become infinitely more numerous than is necessary to take up all the streptococci present. There always remain, however, certain free bacteria and these bacteria soon multiply. These organisms are differentiated by the fact that they are surrounded by an areola never present under normal conditions, which takes a pale pinkish-violet color with Kühne's blue. These organisms after 2 or 3 hours give rise to new individuals also surrounded by an areola, similarly able to avoid engulfing, and usually occurring in the form of diplococci or in short chains. The number of these new bacteria, as a rule, becomes considerable in 6 or 7 hours. In an exudate taken at this time we find both a large number of cells and a large number of bacteria. The majority of the phagocytes, however, are empty and no longer capable of capturing bacteria. We have already shown in a previous article* that such leucocytes not only are not paralyzed, but are, on the contrary, even more motile than usual and are still able to engulf other phagocytable micro-organisms, such as the B. diphtherix or the B. proteus vulgaris. If a culture of Proteus or Diphtheria is added to such an exudate, the leucocytes immediately take up the new bacteria, although they still refuse streptococci; in other words, they choose between the species of bacteria. Streptococci, then, secrete a substance which, although it does not inhibit the influx of leucocytes into the peritoneal cavity, affects adjacent phagocytes unfavorably and prevents them from accomplishing their protective engulfing function. We may say that they exercise a negative chemiotactic influence on the phagocytes. The number of these cells increases until it becomes enormous and, as a rule, the guinea-pig dies in from 15 to 20 hours with a purulent peritonitis accompanied by an invasion of the heart's blood by the bacteria. The leucocytes retain their faculty of engulfing other micro-organisms for a long time. So far as the extracellular streptococci are concerned they stain well, are morphologically normal, are surrounded by an areola and show at no stage the slightest evidence of degeneration.

A non-fatal dose.-If a smaller amount of culture, for example, 0.1 of a cubic centimeter, is injected intraperitoneally in a guineapig instead of the minimal lethal dose rapid and complete phagocytosis 
occurs. The streptococci being in too small numbers are engulfed before they have time to adapt themselves to the medium, and acquire their intense repelling property for leucocytes. Certain ones among them, to be sure, resist and remain free relatively longer than the others. But as their number is inconsiderable in proportion to the cells present they finally encounter leucocytes which are so vigorous that they yield to them. The totality of the streptococci injected are eventually contained within the cells. The guinea-pig recovers without any further trouble.

We have already mentioned that the streptococci that are able to protect themselves against the attack of the guinea-pig leucocytes are surrounded by an areola which takès a peculiar stain. We may further note that in those experiments in which a sublethal dose of streptococci is employed the more resistant micro-organisms, that is, those last to be engulfed, are also usually those with the most marked areola.

The condition necessary for a fatal infection.-In order that streptococci injected into the peritoneum of the guinea-pig may produce a fatal infection the following condition must be satisfied; when phagocytosis by the rare leucocytes begins, the streptococci should be in sufficient quantity to permit the more virulent of them to remain outside the cells long enough to become accustomed to the chemical composition of the exudate so that they and their descendants may acquire to the highest degree the faculty of remaining free amid the increasing leucocytes. It would follow, then, that if we inject a fatal dose of streptococci, not into a normal intraperitoneal cavity where the cells at first are few and little adapted to phagocytosis, but into a peritoneal cavity rich in vigorous phagocytic cells capable of bringing about a rapid engulfing, the bacteria will not have the necessary time for adaptation and for increasing their resistance. This, indeed, is what happens. If the number of active phagocytes in the peritoneal cavity of a guineapig is increased by a previous injection of bouillon, a dose of streptococci equal to at least twice the ordinary minimal lethal dose may be injected without fatal effect.* We shall not consider such an experiment for the moment, but shall take it up again when we come to

* The amount that can safely be injected naturally has its limits. If as much as 1.5 or 2 c.c. is given, phagocytosis is incomplete, even in a prepared guinea-pig. 
consider the relative sensitivity of the rabbit and the guinea-pig to the streptococcus.

If something near the minimal lethal dose of a virulent culture has been injected into the peritoneal cavity of a guinea-pig a prognosis may generally be given by the presence or absence of streptococci with an areola outside the cells. If, for example, four hours after infection the exudate contains a small number of extracellular streptococci with areola and a great many leucocytes, the fate of the animal is doubtful. If the number of such bacteria is very small, so that they may be found on a slide only with difficulty, they frequently become the prey of exceptionally active phagocytes. But if their number is at all considerable we may feel sure that they will soon multiply unrestrictedly.

The issue of streptococcus peritonitis is thus soon indicated and the outcome of the conflict between cells and bacteria is clear very early.

Mechanism of cure. - We may review the evolution of streptococcus peritonitis in the guinea-pig by saying that the cure of an animal is due to phagocytosis. The existence in the bacteria of a negative chemiotactic influence preventing the accomplishment of phagocytosis, excludes a cure. Since the streptococci that in the beginning have been able to protect themselves from phagocytes multiply outside the cells without changes of morphology or color reaction or any diminution in their virulence, there is nothing that leads us to suppose that when animals withstand an infection there are any factors in their cure other than phagocytosis. It may be simply mentioned here that virulent streptococci taken up by the phagocytes of the guinea-pig are absorbed without losing their activity.

The injection of a small amount of guinea-pig peritoneal exudate containing no free streptococci, but with leucocytes containing engulfed streptococci, into a rabbit within four hours after removal, kills as a rule in 24 hours.

We have already said that a guinea-pig that has received a dose of streptococci small enough to allow the bacteria to be entirely engulfed by phagocytes recovers without any further trouble. As a general rule, indeed, when complete phagocytosis takes place the animal is at once out of danger and soon recovers. Within two or three days the exudate ceases to contain living bacteria. In a few rare instances an animal that has lived several days without showing 
any free bacteria in its peritoneal exudate and that has apparently returned to normal condition becomes sick again and undergoes a new streptococcus infection. Under these conditions the streptococci found in the peritoneal exudate are of a peculiar form.

They often occur in short chains with beadings of unequal diameter; chains are also found here and there of extraordinary length and surrounded by a distinct and well-marked areola differing considerably from the one that ordinarily encloses the streptococcus. The chromogenic substance in certain of these chains is a line of nearly regular granulations as is usual in the streptococci, but in other larger chains, the chromogenic substance appears to be more indefinite and at intervals shows indefinitely walled off and poorly stained sac-like dilatations. We repeat that these cases of fatal relapse in apparently cured guinea-pigs occur only rarely and at certain definite stages of the infection. It is probable that the culture that brings about these delayed reinfections is possessed of peculiar qualities of resistance not ordinarily present.

Larger doses ( 0.5 to 2 c.c.) are necessary when injected subcutaneously to cause a fatal infection in a guinea-pig.

\section{B. Streptococcus Infection in the Rabbit.}

As we have just seen, a dose of about 0.2 of a c.c. is the minimal amount of bacteria necessary to produce a fatal peritonitis in the guinea-pig. This is an infinitely larger amount than is necessary to cause death in the peritoneal cavity of a normal rabbit. The streptococcus finds a very favorable culture medium in the clear peritoneal fluid of this animal which contains only few leucocytes. The streptococci injected into the rabbit are usually scattered and within half an hour become surrounded by an areola not ordinarily present. It is evident that the production of this areola is due to the activity of the bacterial secretions, as its color changes as the infection proceeds.

Shortly after inoculation the areola is seen as a distinctly outlined zone which does not color with Kühne's blue and consequently appears whitish against a bluish background. In one and a half to two hours it begins to take a pale violet or pink color that subsequently becomes darker.

The original injected streptococci may take on this areola, but 
it is usually more distinct and more deeply colored in those cocci generated in the exudate.

Leucocytes soon appear in the peritoneum following injection of the streptococci. After an hour or two there are present some mononuclears and many polynuclears. But these leucocytes take up only a relatively small number of the bacteria injected, which develop unrestrictedly and soon become very numerous. Although the influx of the leucocytes is considerable they never become sufficient to give a purulent appearance to the exudate even several hours after inoculation. As soon as bacteria become very numerous the leucocytes do not sensibly increase in number. At this stage death is not very far distant. A rabbit that has received 0.1 of a cubic centimeter of a culture into the peritoneal cavity usually dies in 8 or 10 hours, rarely as late as 12 hours. The greatest number of leucocytes, as a rule, are present 4 to 6 hours after injection.

A short time before death the exudate changes remarkably in appearance. Two hours before death it is only slightly cloudy. At death the exudate is reddish in color and composed of serum which contains a rather large number of scattered red blood cells. In this fluid leucocytes are still present and they are frequently intact in appearance, but often degenerated and with no distant protoplasmic outlines. The leucocytes, which were previously abundant, are usually found collected in masses on the walls of the peritoneum, particularly about the mesentery. The exudate changes, then, in composition toward the end and becomes a harmful medium for leucocytes and red blood cells.

Two hours before death, when the exudate is no longer turbid, the leucocytes present in the fluid which refuse to take up the streptococcus will still take up other organisms (for example, the diphtheria bacillus). Phagocytosis, however, under these conditions is never so energetic as under similar conditions in the guinea-pig. In general, it may be said that the exudate in streptococcus peritonitis in the guinea-pig is always richer in leucocytes than it is in the rabbit.

A generalized infection, that is, an invasion of the blood by the streptococci, occurs soon after intraperitoneal inoculations in the rabbit. As soon as the bacteria in the exudate become very 
numerous, that is, in 4 or 5 hours or even less after the inoculation of 0.1 of a cubic centimeter, the blood is invaded. A culture taken at this time from the heart's blood grows as luxuriantly as from the peritoneum. Three hours before death the bacteria in the heart's blood are still rather difficult to find in stained preparations, but an hour later they are very numerous there and their numbers increase rapidly from this time on. As a general rule a normal rabbit injected with streptococci, whether intraperitoneally or elsewhere, gives an abundant culture from the heart's blood at death. An examination of the blood shows evident changes in the red blood cells. They are found to have almost entirely disappeared. When a rabbit is autopsied immediately after death, the heart contains large red clots and serum filled with diffused hemoglobin. In trying to express red blood cells from this clot into the serum only débris are obtained, which, when examined after staining with eosin, have no distinct cellular outlines. A few leucocytes, more or less altered, and also endothelial cells are found.

These grave alterations, which are so incompatible with life, appear late in the disease and only in the agonal period. They are not to be found when, for one reason or another, the animal dies with few streptococei in the blood.

When these alterations do occur a reddish exudate similar to that present in the peritoneum at the time of death is also found in the pericardium and the pleura. These late changes in the peritoneal exudate (the appearance of scattered red blood cells and diffused hemoglobin) are correlative with changes in the blood and appear only when such changes are present.

Animals given subcutaneous injections of streptococci die with the same lesions and always rapidly.

If streptococci are injected into the ear vein of the rabbit, they grow in the blood without any resistance. For example, if a drop of blood is taken immediately after injecting 0.1 of a cubic centimeter and inoculated on agar, only a few colonies are found; 3 hours after injection numerous colonies are found on inoculating the same amount of blood. After 7 hours the blood gives confluent colonies of streptococci on agar. It is evident that in the blood, as in the peritoneal exudate, there is no hindrance to the growth of the inoculated micro-organisms. 
From these observations, then, it is easy to realize that Marmorek's streptococcus owes its extreme virulence for the rabbit, not only to the great rapidity of its development in the body fluids, but particularly to its power to prevent its own engulfment by leucocytes. It exercises a negative chemiotactic influence on rabbit leucocytes, and this repelling action is characteristic not only of streptococci adapted to the body fluids by origin in them, but also of organisms grown on culture media.

Indeed, if 0.5 of a cubic centimeter of a culture is injected into the peritoneum of a rabbit that has received 6 c.c. of bouillon the night before and contains, therefore, numerous leucocytes, the majority of these bacteria remain free and soon become surrounded with an areola. And yet under such conditions the number of bacteria injected is relatively small when compared with the large number of phagocytes. We know, moreover, that the phagocytes present in an exudate caused by injecting bouillon, are very active and show remarkable phagocytic power for various bacteria.

In this experiment, however, phagocytosis is not entirely absent; there are always a few cocci that become the prey of cells. If the number of bacteria inoculated is markedly diminished, if, for example, 0.1 of a cubic centimeter is injected into the prepared peritoneum of such a rabbit, the number of engulfed bacteria increases; this fact indicates, it would seem, that there are certain cells in this exudate that are particularly active and absorb a certain number of bacteria. But however small may be the dose inoculated, there always remain certain free bacteria whose multiplication rapidly takes place. A previous inoculation of bouillon into the peritoneal cavity increases the number of active leucocytes in the exudate, but will not protect the rabbit against a subsequent inoculation of streptococci even when these organisms are very few in number in proportion to the phagocytes.

This chemiotactic influence is present, particularly, in young streptococci and is present, most markedly, in organisms undergoing active multiplication. It is easy to prove that the streptococci from a three- or four-day-old culture are much less repelling. If even a large dose of such a culture (several cubic centimeters) is injected into the prepared peritoneum of a guinea-pig, active phagocytosis occurs, and usually of the majority of the organisms 
present; the same culture, when 24 hours old, entirely escapes such phagocytosis. Even with the old culture, however, new.organisms are formed in the exudate after a certain time and kill the animal.

The fluid in which cultures have been grown for several days apparently does not contain any perceptible amount of the substance that prevents phagocytosis. Streptococci injecteci into the peritoneal cavity of a guinea-pig prepared by bouillon are rapidly and more or less completely taken up, depending on the dose employed. This engulfing occurs to just as marked a degree if 3 c.c. of a filtrate from a 24-hour culture is injected with the bacteria. The negative chemiotactic influence, then, is a property which belongs exclusively to living streptococci. We should like to insist on a correlation between a negative chemiotactic influence coming from the bacteria and the presence of an areola about them; this areola may develop, not only about bacteria in the peritoneal cavity, but also under the skin, in the blood and in the aqueous humor.

The resistant qualities of streptococci. - The property of repelling leucocytes, which is of such value to the streptococcus, is not the only property which this organism utilizes in growing in animal tissues. We have already seen that a fatal relapse may occur in guinea-pigs even several days after an apparent cure.

The momentary arresting of the infection and the apparent cure are due to the intervention of complete phagocytosis and the consequent disappearance of free bacteria. These cases, which in guinea-pigs are very rare, indicate that certain streptococci may be very resistant and may remain alive within phagocytes, regain their activity, and after several days give rise to a new culture that kills the animal.

This delayed outgrowth of streptococci, after an apparent cure of the animal lasting some days, happens in rabbits that have been partially immunized by the injection of a certain amount of preventive serum. The following experiment shows that the streptococci in such a rabbit may remain latent for a long time in the phagocytes and after a long interval grow again.

A rabbit was given 6 c.c. of pepton bouillon into the peritoneal cavity and 3 c.c. of antistreptococcus serum subcutaneously. On the following day 6 c.c. of a 3 -day-old culture of streptococcus was 
inoculated into the peritoneum, which contained many leucocytes. As we have already shown, these old cultures are much more easily phagocyted than are young cultures. The exudate examined 3 or 4 hours after injection showed no free bacteria; all the cocci had been taken up by the cells. Animals similarly inoculated, but without serum, usually die in 24 hours. The animal that has received the serum resists infection and, on the following day, the exudate contains many leucocytes, some of them containing more or less altered streptococci; there are no free bacteria. Nor are there any bacteria present the following day, and it would seem as if they had been entirely taken up. The animal, however, although remaining well for several days, at the end of the week succumbs to a generalized streptococcus infection. These instances of a late outgrowth of streptococci are frequently met with in experiments; they occur, irrespective of the point of inoculation, in animals that have received either too small a dose of serum or too large a dose of bacteria. In such cases death may not occur for two weeks and, exceptionally, not even until the third week. To sum up, the virulent streptococcus has two qualities that render it dangerous, and particularly so for the rabbit: it repels leucocytes, and it can remain living for a long time in an animal that is apparently cured.

\section{A Comparison of Streptococcus Infection in the Guinea- PIG AND IN THE RABBIT.}

We must now compare the appearance of the streptococcus infection in the guinea-pig and in the rabbit in order to understand why the first of these animals resists the infection so much better than the latter.

We have already shown in a previous article that the normal serum of rabbits or of guinea-pigs is a favorable cuiture medium for the streptococcus and has no bactericidal power against it.

We have just shown, moreover, that streptococci grow very well and rapidly in the peritoneal exudate of infected guinea-pigs; the only condition necessary for a uniform development is that they remain free in the fluid and protect themselves from the phagocytes by their negative chemiotactic power. The guinea-pig, then, has no more antiseptic properties in its fluids than has the rabbit. But there is a very striking difference between the phagocytes of these 
two animals: guinea-pig leucocytes are much less sensitive than rabbit leucocytes to the repelling action of the streptococcus. As much as 0.5 of a cubic centimeter of a young culture of streptococcus or even more is rapidly taken up in the peritoneal cavity of a guineapig previously injected with bouillon. The same amount of streptococci injected into a rabbit similarly prepared suffers very little engulfing and extracellular development occurs rapidly.

This difference in reaction of the leucocytes explains clearly the difference in the evolution of the disease in the two animals.

The exudate in a guinea-pig given a fatal dose is always richer in leucocytes; the purulent peritonitis which usually occurs in an infected guinea-pig is not found in an infected rabbit at autopsy. When a guinea-pig dies as the result of an intraperitoneal injection the exudate contains, in addition to a large number of leucocytes, numerous streptococci. The blood of such an animal, however, contains fewer organisms than does that of a rabbit under the same conditions. It need scarcely be insisted upon that the more active the phagocytic apparatus against a given bacterium, the more difficult does the invasion of the blood by this organism become. The extreme alterations in the blood (destruction of red blood cells) found in the blood of rabbits and resulting from an abundant growth of the streptococcus is not found in the guinea-pig at autopsy.

Cases of streptococcus reinfection occurring after a period of apparent cure, although rare in guinea-pigs, are, on the contrary, very frequent in rabbits that have received a small dose of protective serum. What is the cause of this difference?

Among the leucocytes that take up bacteria there are always a certain number that die after having destroyed the parasites that they contain; and so it is easy to understand how such bacteria, which may be almost intact, are liberated. These liberated bacteria are much more likely to be taken up again in a guinea-pig than in a rabbit, since the guinea-pig leucocytes are more actively phagocytic for the streptococcus. It may be, too, that guinea-pig leucocytes have a greater destructive power for the streptococcus than rabbit leucocytes. This leads us to a consideration of the alterations in streptococci taken up by cells in the two animals we are studying. In both of these animals the leucocytes cause destructive alterations in at least some of the phagocyted bacteria. 
When streptococci have remained for several hours in the protoplasm of the phagocyte some of them take acid dyes in preference to basic dyes; in a counter stain with eosin and methylene blue such bacteria take a more or less red stain. This phenomenon occurs both in the rabbit and in the guinea-pig, but in the latter is more evident on account of the greater extent of the phagocytosis.

\section{II.}

\section{ANTISTREPTOCOCCUS SERUM.}

Marmorek, in an article published in July, 1895, in the Pasteur Annals, has described his method of obtaining this serum by immunizing animals against the streptococcus.

We shall not reiterate his methods. It may be noted simply that the serum we have used was from animals that had been under immunization for about a year. One of these, the serum of which was remarkably active, had received twenty-three injections of a very virulent 24 -hour culture. The total amount of culture injected during this time was 3800 c.c.

The preventive activity of these sera is most evident. When injected into a rabbit before inoculation of bacteria, they permit the animal to withstand many times the minimal fatal dose of streptococcus.

The amount of bacteria that can be safely injected in animals immunized by serum varies a great deal according to the region in which the injection is given. Animals that have received serum do not tolerate intraperitoneal injections of streptococci nearly as well as they do subcutaneous injections. Intravenous or intraocular injections are also much more dangerous, even in animals that have received a very highly active serum. In determining the preventive value of a given serum the portal of entry chosen for inoculation of the culture should therefore be indicated. For this reason we shall frequently mention the doses of serum and culture used in our experiments.

A few figures from our notebook may be given at once as showing the result of injecting a culture subcutaneously in passively immunized animals.

An animal that has received 10 c.c. of serum subcutaneously can 
be given 0.5 of a cubic centimeter of culture subcutaneously the next day without any effect. A control given 0.001 of a cubic centimeter of culture dies. The rabbit that receives serum remains perfectly well, although it has received at least 500 times the fatal dose of streptococci.

A smaller dose of serum (5 c.c.) protected a rabbit against 0.001 of a cubic centimeter of a virulent culture, while the control that had received one-tenth of this amount (that is 0.0001) died. We offer these figures, not as a systematic tabulation of the preventive power of horse serum but as results that occurred regularly during our experiments.*

A suitable dose of preventive serum, as in the case just mentioned, protects rabbits against the inoculation of streptococci. If too small doses in proportion to the number of organisms injected are given, the animals may simply resist longer than the controls or show an apparent cure lasting long after any original manifestation of the disease, but eventually succeeded by a rapid reinfection with the streptococcus.

These conditions will be considered later. For the moment we shall consider briefly the effect of the sera on bacteria in vitro.

\section{Action of the Serum in Vitro.}

Marmorek's preventive serum is from a horse immunized against the streptococcus. The serum has no bactericidal power for the streptococcus. Bacteria inoculated in it do not, to be sure, grow very rapidly or abundantly, but they grow quite as well in the immune serum as they do in normal horse serum.

Preventive serum mixed with fresh normal rabbit serum has also no inhibiting power on the growth of the organism. Streptococci grow equally well in a mixture composed of 1.5 c.c. of normal rabbit serum and 5 c.c. of preventive serum, or in a mixture in like pro-

* Petruschky, in two recently published articles (Zeitschrift für Hygiene), as a result of his experiments concludes that Marmorek's serum does not in the least protect rabbits against streptococcus infection. Treated rabbits showed no appreciable difference over the controls according to this author. It is evident that Petruschky has experimented with a serum that was spoiled in some manner. The efficacy of Marmorek's serum may be shown by the most elementary experimentation, and consequently the experiments and conclusions of Petruschky need not concern us as they do not deal with antistreptococcus serum as obtained at the Pasteur Institute. 
portions with normal horse serum instead of preventive serum. Streptococci grown in these media, however, show certain peculiarities that may be noted.

In both these mixtures of normal rabbit serum and either normal or immune horse serum the growth begins rapidly if the cultures inoculated are young. An equal and considerable growth is evident three hours after inoculation. In the tube containing the normal horse serum the streptococci are in numerous short chains; in the other, containing preventive serum, they form much fewer and longer chains and these chains are frequently coiled. There is a difference then in the arrangement of the cocci, although the total number is about the same in either medium.

After $5 \frac{1}{2}$ hours these same characteristics are still present. Later on (19 hours after inoculation) the chains become longer in the tube containing normal serum, so that the difference in length of chains between the two tubes is less manifest. There is still no difference to be noted in the richness of the culture. We have found no retarding property on growing streptococci in antistreptococcus horse serum any more than did Denys and Marchand.*

If in such an experiment a less virulent streptococcus is used (one that kills a rabbit in a dose of 0.25 of a cubic centimeter intravenously), similar results are obtained; with such an organism, however, the difference in the length of the chains is much less.

The injection of 10 c.c. of preventive serum in a normal rabbit does not endow this animal's serum with any bactericidal power. The serum 25 hours after injection is just as good as a culture medium for streptococcus as before the injection of antistreptococcus serum. In both of them it grows rapidly and well.

Antistreptococcus serum has a slight but distinct agglutinating property. We demonstrated in 1895 that a trace of a preventive cholera serum, when introduced in a fluid containing cholera vibrios in suspension, produces in a very short time their immobilization, and clumps them into distinct masses that stand out as white points in a clarified fluid. This is the invariable effect of this preventive serum. The following year Gruber and Durham noted a similar fact, not only with vibrios, but also with $B$. typhosus and

* Denys and Marchand, Immunité conferée au lapin par l'injection de sérum anti-streptococcique de cheval. Bull. Académie royale de Belgique, 1896. 
B. coli, all of which are found to clump when placed in contact with a specific serum.

Although the agglutination of the streptococcus by its preventive serum is generally distinct, it is never very marked; to cause it, large quantities of serum are necessary, at least one-third the volume of the culture fluid. Not all of the chains of the streptococci are affected; some of them remain separate. Cultures of streptococci grown in preventive serum or in a fluid containing a certain amount of it (for example, equal parts of bouillon and immune serum) show only a slight grouping of the organisms at best. In short, preventive serum causes no profound alteration in the streptococcus. The growth of the organism is not sensibly diminished and its morphology remains the same, with the exception of certain variations in the length of the chains. And even the agglutinating property that has been found by recent studies to be present in numerous immune sera is only slightly developed in antistreptococcus serum.

We must consider whether a preventive serum with so slight an effect on the morphology and development of the streptococcus does not have some weakening influence on its virulence. Cultures of streptococci grown in a mixture of equal parts of preventive serum and pepton bouillon retain a very great virulence, as may be determined by comparing them with cultures made on bouillon plus normal horse serum. After 24 hours' growth in an incubator the turbid culture fluids are filtered through filter paper. There remain enough bacteria on the filter paper and they may be washed and freed of serum. A small amount of these bacteria is taken with sterile forceps and suspended in a few cubic centimeters of salt solution. Two emulsions are thus obtained, both slightly and as near as possible equally turbid: one containing bacteria cultivated in normal serum and the other bacteria cultivated in preventive serum. Both these fluids are extremely virulent, although they contain few bacteria; 0.0005 of a cubic centimeter of either one kills a rabbit in 24 hours. In other words, there has been no detectable attentuation by growing in preventive serum. And, what is more, the whole culture containing preventive serum and bacteria is also very dangerous for rabbits, killing them as rapidly as controls, that is, in 24 hours. 
The latter result may seem astonishing. One might expect rabbits that have received the whole culture to receive some benefit from the serum in the fluid and to be enabled to resist the invasion of the bacteria. But it must be noted that this culture contains a considerable number of bacteria, and experiment shows us that 1 c.c. of pure serum fails to protect animals against a much smaller dose of bacteria than is present in a cubic centimeter of such a culture. It is not surprising, then, that the culture is virulent, for it contains too many bacteria in proportion to the serum.

This experiment that we have just mentioned* simply shows that streptococci grown in preventive serum show no deterioration that decreases their virulence in subsequent generations. Does it show, however, that preventive serum when injected into an animal before infection is quite incapable of any enfeebling effect on the subsequently injected steptococci? By no means, for we may imagine that a serum incapable of modifying bacteria in vitro may, when in the tissues, affect them, owing to the additional or combined effect of certain adjuvant factors furnished by the animal body. Have we not seen, for example, that cholera serum which, when kept for some time or heated, is incapable of causing granular transformation in vitro can bring about this modification when injected into an animal? We make these reservations on a priori grounds without considering for the moment whether or not they are justified by experimental facts.

The fluids hitherto examined, that is, normal rabbit serum, and preventive serum either pure or mixed with bouillon or rabbit serum, have no bactericidal effect on the streptococcus. There is a body fluid, however, which shows an evident destructive effect on this micro-organism. It is the clear fluid separated by centrifugalizing an exudate rich in leucocytes. The streptococcus grows with great difficulty in this fluid and, as Denys and Leclef have already noted, a destruction of bacteria ensues even when a large amount of culture is inoculated. $\dagger$ If a drop of a young culture is

* This experiment is similar to those of Metchnioff (1892), Issaeff, and Sanarelli (1893) with the bacteria of hog-cholera, pneumonia and the vibrio Metchnikovi:

† Denys et Leclef, Sur l'Immunité du lapin vacciné contre le streptocoque. La Cellule, 1895. 
inoculated in such a fluid, no development takes place and the next day the fluid is found to be sterile.

This serous fluid loses its bactericidal power when heated for a few moments to 60 degrees, and in such heated fluid the streptococcus grows in long chains of rather small organisms. As the fluid part of rich leucocytic exudate is bactericidal in vitro, the question arises as to whether it is as much so in the animal body. Experiment gives a negative reply to this question. When streptococci are injected into the rabbit peritoneal cavity containing many leucocytes the organisms are not engulfed, but develop well. If a little of this exudate is removed when the streptococci are not yet very numerous, and placed in the incubator, the growth stops; at times the fluid becomes quite sterile after 2 or 3 days and at other times the organism finally grows out again after a delay of 24 hours or so.

Experiments performed in vitro with leucocytic exudates cannot be accepted offhand without many reservations as necessarily corresponding to the conditions of similar experiments in the animal body.

\section{The Action of the Serum in the Animal Body. INTRAPERITONEAL INJECTION.}

Let us now consider the phenomenon which takes place in rabbits that have received a preventive injection of serum and are subsequently inoculated with the steptococcus. We shall consider first an instance in which the serum is injected subcutaneously 24 hours before the culture is injected intraperitoneally.

The study of the struggle between the cells and the bacterial culture in the peritoneal cavity is extremely instructive as, by extracting at intervals a little peritoneal exudate with a capillary tube, the course of the infection may be followed step by step.

The exudate is homogeneous, that is to say, throughout the peritoneal cavity it has an identical constitution in so far as the number, quality, and appearance of cells and bacteria is concerned. The subcutaneous exudate, on the contrary, frequently differs even in adjacent regions, either in the amount of fluid or in the number and condition of cells and bacteria.

It is possible to produce artificial variations in the number of 
cells present in the peritoneal exudate before injecting bacteria. By means of a simple injection of bouillon a great many active phagocytes may be made to participate at the very beginning of the infection. This method is very useful in studying the course of an infection and we have frequently employed it.

In order to understand the phenomena of a peritoneal infection in passively immunized animals we must consider a fact evidenced on simultaneous injection of streptococci into a normal and into an immunized rabbit. A virulent streptococcus inoculated into the circulation of a normal rabbit in a dose of 0.1 to 0.25 of a cubic centimeter of culture develops so well as to give an abundant culture from the blood in seven or eight hours. The same dose of culture injected intravenously into a rabbit that has received serum subcutaneously is markedly inhibited in growth. A drop of blood taken from any part of the body immediately after injection and inoculated on agar gives rise to a few colonies. Streptococci may still be found in the blood five to seven hours later or even on the following day, but their number is not increased. Such a rabbit, however, succumbs in two, three, or four days, but at autopsy many less bacteria are found in the blood than are present in the rabbit that has received no serum. More bacteria, it is true, are found in the liver, spleen, bone marrow, and particularly in the lung; some of them free and others within leucocytes.

In this connection we may emphasize the fact that, as a general rule, in rabbits that have received an injection of serum and an excessive dose of baeteria and have survived the controls only two or three days, there are never so many bacteria present in the heart's blood as there are in control animals. The contrast is very marked from whatever region the cultures are made.

The only exceptions to this rule are those instances where rabbits that have apparently been cured, after a certain time have a fatal relapse, under which conditions the blood may contain nearly as many bacteria as a rabbit infected without serum.

The peritoneal infection in normal rabbits is accompanied by a rapid invasion of the blood by the micro-organism; the increase of streptococci in the blood is so marked that it, and not the peritonitis, must be regarded as the immediate cause of death. Therefore, since there is less growth of bacteria in the blood of 
the vaccinated animal, a peritoneal infection in such an animal may last much longer than in a control, without leading to a septicemia. This is the point of importance to us for the moment.

A. Young cultures. - What happens to virulent streptococci in a young, 24-hour culture when injected into the peritoneum of a rabbit containing many leucocytes owing to a previous injection of 10 c.c. of serum subcutaneously and 6 c.c. of bouillon intraperitoneally? There are two distinct conditions to be considered.

First:- The number of bacteria injected may be very few, less than 0.5 of a cubic centimeter of culture; let us take, for example, 0.1 of a cubic centimeter. Under these conditions the number of bacteria in the peritoneal cavity is relatively small in proportion to the number of cells, so that they are frequently difficult to find in stained preparations. Under these conditions the culture is rapidly and, as far as may be estimated, completely engulfed. For example, after an hour or two no free cocci are found; when stained with boracic carmin followed by Gram they are seen to have become the prey of cells. As a result, no extracellular development of bacteria takes place under these conditions and the animal gets well. In a control animal that has received an intraperitoneal injection of bouillon but no protective serum a very distinct phagocytosis is generally found if small doses of bacteria are injected; free bacteria are only exceptionally found in such preparations. A multiplication, nevertheless, takes place, and within three or four hours numerous cocci with an intense negative chemiotactic influence are found. Such an animal usually dies within twelve hours with the ordinary symptoms occurring in normal animals as already described.

Second:- The results are quite different if instead of a very small amount of culture, that is 0.1 of a cubic centimeter, a dose of 0.5 of a cubic centimeter or slightly more is given. Under these conditions*

* We may mention that when 0.5 of a cubic centimeter of a culture is injected into a peritoneum prepared by bouillon the number of streptococci at first in the exudate is very small in proportion to the number of cells. Notwithstanding this fact reproduction occurs and as we shall see presently the animal is saved only by a delayed phagocytosis.

Denys and Leclef (La Cellule, 1895) made a mixture of normal rabbit leucocytes and preventive serum in vitro. When they injected this mixture with the streptococcus they found there was a distinct inhibition in the growth of their culture owing to an abundant initial phagocytosis, although the organism grows rapidly 
the engulfing that takes place in the first few hours is only partial. As in the previous experiment streptococci are found within apparently vigorous cells, but since the number of streptococci injected is too great, many of them remain free and develop. In these doses there is not so much difference as regards phagocytes between a rabbit treated by serum and a normal rabbit. The streptococci increase without any notable retarding effect on their development. Eight or ten hours after injection the organisms in the vaccinated animals are surrounded by an areola and are growing in the midst of leucocytes while the control is nearing death. The animal recovers, however, although the bacteria are always extremely numerous and phagocytosis is either insignificant or absent.

The appearance of the exudate changes, however. It becomes thicker and thicker and more concentrated until it is almost white and its proportion of leucocytes great. This condition lasts for a longer or shorter period. When the thickened exudate comes to resemble a homogeneous white pus, say 20 hours after injection, phagocytosis suddenly appears. Within a few hours later, 3 or 4 at the most, all the streptococci that were swarming outside the cells are capiured by the phagocytes. A great majority of the cells contain cocci and often in numbers. This complete engulfing is followed by either a final or a temporary cure; if the number of bacteria was too great a relapse may occur 2 or 3 days later although the phagocytosis sēemed complete.

This delayed phagocytosis may be only partial if the culture has developed too extensively. Under such conditions the animal simply lives longer than the controls. Complete phagocytosis is the essential condition for a cure.

Conditions necessary for the occurrence of a delayed phagocytosis:

Delayed phagocytosis may occur in animals that have received sufficient serum whether subcutaneously or intraperitoneally. It is most conveniently studied in a rabbit prepared by an injection of bouillon and thereby rendered more resistant to peritoneal infection. Under these conditions it may occur even with an amount in a mixture of serum and normal rabbit leucocytes without the preventive serum. We have obtained different results under such conditions. We found at the beginning a certain degree of phagocytosis whether the preventive serum was added or not. Subsequent development took place in both mixtures. 
of bacteria relatively large in proportion to the amount of serum employed (for example, a rabbit may be given 6 c.c. of bouillon intraperitoneally and the next day 1 c.c. of culture plus 5 c.c. of serum; a delayed and complete phagocytosis occurs about 25 hours later).

Delayed phagocytosis may also occur in animals that have simply received serum subcutaneously without any preparation of the peritoneum. But under these conditions the dose of bacteria injected must be considerably less. In rabbits protected by serum without previous intraperitoneal preparation by bouillon, an intraperitoneal injection is much more dangerous than a subcutaneous one and more harmful still if the culture used is young and rapidly growing. Even in a vaccinated animal the inoculated streptococci find a very suitable culture medium in the limpid exudate;* when leucocytes finally come up in considerable numbers they meet with a large number of bacteria.

In rabbits immunized by serum, in whose peritoneal cavity a delayed phagocytosis occurs, the number of phagocytes gradually becomes considerable. In the first hours, however, this influx of leucocytes is no more marked than in normal rabbits.

Intraperitoneal injection of filtered culture fluid causes an abundant influx of leucocytes both in normal and in treated rabbits. When week-old cultures are used or dead or attenuated cocci, similar results occur in a normal rabbit.

B. Old cultures.-Part of the danger from injection of young cultures lies in the extreme rapidity of their increase before the phagocytes arrive. If streptococci that do not show active reproduction for several hours are used, a delayed phagocytosis occurs much more readily even if the serum is injected after the bacteria. A brief résumé of such an experiment follows: Two normal rabbits received each an intraperitoneal injection of 8 c.c. of a fourday-old culture on ascites bouillon. Six hours later the phagocytes had become very numerous and the bacteria injected were engulfed (old streptococci, as we have already seen, are easily phagocyted).

* We have never found a disappearance of bacteria, as noted by Denys and Leclef following inoculation of streptococcus into the pleura of vaccinated rabbits, after injecting a small dose of streptococcus intraperitoneally in a treated rabbit. 
No growth had as yet occurred and no free bacteria were seen. At this stage one of the rabbits was given 3 c.c. of preventive serum intraperitoneally and 5 c.c. subcutaneously.

The other rabbit that received no serum died of a generalized infection in 18 hours, the delay being due to the slow growth of the culture. Twenty-four hours after beginning the experiment the peritoneal cavity of the rabbit that had received serum contained numerous extracellular bacteria scattered in the midst of abundant leucocytes. Growth, then, had taken place. But a few hours later the phagocytic crisis occurred and all the free bacteria were engulfed.

In this experiment an extracellular growth occurred and was followed by a delayed phagocytosis. And yet the serum had been given some time after the injection of the culture. When the serum is injected the day before, and old cultures are used it is generally found that a generalized phagocytosis occurs and is not followed by the appearance of extracellular bacteria.

Engulfing may also take place in a normal rabbit and often no invasion of the bacteria is noted for a number of hours; the streptococci have remained in a latent condition. But the growth soon starts up again and new cocci are developed and invade the exudate. Do these new organisms come from the few streptococci that have remained free in spite of the generalized phagocytosis? Or are they derived from bacteria that were taken up, but have resisted the phagocytes? Either explanation is possible. We have already seen that the streptococci may remain alive for a long time in the animal body even after they have been taken up by cells.

Rabbits treated with preventive serum may resist an infection with very large amounts of an old culture.* In a general way these are the phenomena in rabbits vaccinated with serum and subsequently inoculated intraperitoneally with the streptococcus. We must now consider delayed phagocytosis in some detail.

* The activity of several-day-old cultures naturally depends much on the nature of the culture medium. When this medium is better than usual the streptococci remain "younger" for a longer time, if this expression may be permitted; in other words, they retain better their property of growing rapidly on a new soil. 


\section{Delayed Phagocytosis or Phagocytic Crisis.}

The phagocytic crisis may be readily studied in the peritoneum of rabbits treated with serum. It occurs suddenly on top of an extracellular and usually abundant development of bacteria; it occurs only in an exudate that has become thick and purulent in appearance; other things being equal, it takes place more rapidly and certainly in rabbits prepared by bouillon than in those with a normal peritoneum at the time of injection. The preparation of the peritoneum allows this crisis to occur more readily owing to the extreme abundance of leucocytes in the exudate.

The phagocytic crisis may be complete or incomplete and its time of occurrence varies according to the gravity of the case. If the crisis is incomplete or too much delayed the animal simply survives longer than the controls. It frequently occurs after 20 hours and even later when incomplete.

If the number of bacteria developed is too great or the dose of serum too small the animal may die before phagocytosis is complete; under such conditions the free bacteria at the time of death are restricted in number.

Death may also occur following a partial phagocytosis succeeded by a new growth of new streptococci that, owing to their adaptation, are again able to invade the exudate.

Such an instance of delayed and partial phagocytosis we may now consider in detail; a rabbit received 6 c.c. of bouillon intraperitoneally and was given an injection the next day, also intraperitoneally, of 1 c.c. of a streptococcus culture to which was added 5 c.c. of preventive serum.* In accordance with the appearance of the bacteria and cells the process of infection may be divided into four stages.

First stage or stage of free development. - Following the injection a very restricted number of bacteria are engulfed by the relatively numerous cells. The growth of the organisms takes place actively. A preparation made $10 \frac{1}{2}$ hours after injection shows a complete absence of phagocytosis; the leucocytes are very numerous however.

* The control received 0.1 of a cubic centimeter of culture and 5 c.c. of normal horse serum and died eleven hours later with the usual findings at autopsy. 
The bacteria are extremely numerous, normal as regards size and color reaction, and are present in short chains or as diplococci.

Second stage or the stage of incomplete phagocytosis. - Twenty-two hours after injection the number of bacteria is not very much greater than at ten hours and phagocytosis is still incomplete although leucocytes are numerous and the exudate less fluid. The appearance of the bacteria in the fluid, however, remains the same. They are, as a general thing, distinctly smaller and stain more faintly with methylene blue. A large number of very small diplococci are also found. There are to be noted also short chains composed of very small cocci which are frequently compressed or unequally spaced and often irregularly colored. More rarely still indistinct chains, containing only a few stained cocci, appear.

There are found scattered throughout the exudate well stained normal chains with individual organisms as large or larger than usual.

There is a very distinct contrast between these normal or nearly normal forms and the bacteria with the peculiar characteristics just mentioned. In other words the streptococci, instead of being uniform in appearance, show distinct variations.

Third stage or phagocytic stage. - Six hours later (that is 28 hours after injection) there is generalized phagocytosis.

The exudate has gradually become thicker, and contains a large number of leucocytes of the polynuclear type and some mononuclear cells of variable sizes. It is to be noted that the mononuclears, particularly the large macrophages, have distinctly outstripped the microphages in phagocytic activity. Many of the microphages are empty, whereas the mononuclear cells have taken up considerable numbers of bacteria. On staining with Kühne's blue it is found that the phagocyted bacteria are generally the small ones taking the faint stain and showing the peculiarities just mentioned. On account of their smallness and their poor staining reaction it is frequently difficult to detect them inside the phagocytes when staining with methylene blue. This latter remark is applicable to all instances of partial or total delayed phagocytosis; some cells, to be sure, contain cocci of normal appearance that are easily distinguishable in the cells, but the great majority of them occur as small bluish indistinct points inside the cells. To demonstrate 
phagocytosis well boracic carmine followed by Gram's stain should be used.*

Fourth stage. Post phagocytic stage or stage of reinfection.Preparations from the exudate 34 hours after injection still show a few small unphagocyted bacteria, but whereas these organisms are in relatively small numbers, the number of well stained, plump and normal appearing chains is considerably increased. That is to say this latter form of the streptococcus has increased proportionately over the other. New streptococci have been formed by the multiplication of the normal appearing organisms that have resisted engulfing. The animal dies shortly after, say 40 hours after inoculation.

A few scattered streptococci in short chains and normal in appearance are found in the blood, as is usual at the time of death in rabbits injected with serum.

This description of a peritoneal infection characterized by a partial phagocytosis naturally varies considerably. The duration of the different stages may vary and the relative proportion of the normal and abnormal bacteria before phagacytosis may also change. $\dagger$

Sometimes the animal, being exhausted, dies at the beginning of a phagocytosis that concerns only a small proportion of the bacteria present in the exudate. But one observation is constant, namely, a peculiar appearance of the bacteria at the moment when phagocytosis begins; this peculiarity of faint staining and smallness which is found in the majority of bacteria that are being taken up by phagocytes is shown best by staining with methylene blue.

* Staining bacteria with gentian violet followed by Gram's stain and decolorization with alcohol and clove oil is not always satisfactory. It stains bacteria rather violently and does not bring out the differences between individual streptococci. To bring out clearly details in the appearance of bacteria a more delicate stain like Kühne's blue is necessary.

The normal appearing chains already mentioned are rarely to be found within cells. They remain extracellular and are somewhat increased in number, whereas the number of altered bacteria has greatly decreased outside the cells owing to phagocytosis.

† Partial phagocytosis can appear only relatively late. For example, we have seen a partial phagocytosis appear after nearly 48 hours. But even in this instance the exudate was full of leucocytes at 12 hours and later became purulent. Such cases prove the extreme persistence of the negative chemiotaxis of the micro-organism. 
Another point which constantly recurs is the more or less distinctly superior phagocytic activity of the mononuclears over the microphages particularly to be noted in beginning phagocytosis. Another regular finding is a relation between the consistency of the exudate and the occurrence of phagocytosis.

In exudates containing large numbers of leucocytes and bacteria there are often to be found, before partial phagocytosis begins, localities where cells are collected in more or less compact masses; these leucocytes frequently show changes; their protoplasm is not distinctly outlined and they are frequently confluent; these clumps are surrounded by a slimy layer that does not stain well; this layer is apparently mucilaginous and has some distinct relation to cellular disintegration. Within this layer there are large numbers of streptococci to be found that are small and poorly colored, in other words that show distinct evidence of abnormality, having apparently developed there and been retained by the more fluid nature of the exudate at this point.

When taken from the body the exudate coagulates, the leucocytes are killed and the fluid therefore becomes distinctly bactericidal.

B. Delayed complete phagocytosis. - In order for a complete phagocytosis to take place it is necessary for the bacteria to be in not too great numbers and for the animal not to be too exhausted. The pre-phagocytic period is similar to the corresponding period in incomplete phagocytosis, but is not so long. When the animal survives, a progressive diminution in the number of living bacteria within the phagocytes is to be noted day by day; the cocci inside the phagocytes are separated.

The phagocytic activity in complete phagocytosis is very great, not only on the part of the mononuclears, but also by the polynuclears. The macrophages do not content themselves with taking up streptococci, but also take up more or less degenerated polynuclears; which may already themselves have taken up bacteria. This point has already been brought out by Metchnikoff in studying the phagocytosis of the streptococcus in erysipelas.

There are all transitions between delayed phagocytosis and immediate phagocytosis. The latter, as we have seen, occurs in rabbits immunized by serum and prepared by bouillon on the intraperitoneal injection of a small amount of streptococcus. Without 
being absolutely instantaneous, phagocytosis in these cases is so rapid as to prevent any extracellular reproduction of the streptococcus. In addition, cases are met with in which the phagocytic crisis, although somewhat delayed, is yet so rapid that no exuberant development of the bacterium takes place; we have found such phagocytosis 10 hours after inoculation. Cases like this show a connection between the two forms of phagocytosis and it seems logical to admit that if the leucocytes in prepared rabbits, with small doses of culture, can rapidly take up all the bacteria inoculated, that it is owing to a mechanism identical with that which brings about delayed phagocytosis.

As we have already seen, phagocytosis may be brought about by injecting into the peritoneal cavity of a rabbit previously prepared with bouillon, a mixture of preventive serum and young streptococcus culture. The question arises whether the accomplishment of this delayed phagocytosis is favored or accelerated when the mixture of bacteria and serum injected has been in contact for several hours; or whether the phagocytic phenomena are as distinct and as rapid when the two factors are injected separately into the peritoneal cavity without having been previously mixed.

Let us take two rabbits of the same weight and prepare them by injecting 6 c.c. of pepton bouillon into the peritoneal cavity of each. On the following day rabbit $\mathrm{A}$ is given 4 c.c. of preventive serum intraperitoneally; at this time of course the peritoneum contains many leucocytes. Rabbit B is given 4 c.c. of normal horse serum at the same time. There have been prepared a few hours before two mixtures composed as follows: No. 1, 4 c.c. of preventive serum and 0.5 of a cubic centimeter of young streptococcus culture; No. 2, 4 c.c. of normal horse serum and 0.5 of a cubic centimeter of the same streptococcus culture.* These mixtures have been kept at room temperature. At times varying from onehalf or three-quarters of an hour to seven or eight hours after the first injection of rabbits $\mathrm{A}$ and $\mathrm{B}$, these two mixtures may be injected into the respective peritoneal cavities as follows: rabbit $\mathrm{A}$, that has received preventive serum, is given the mixture containing normal serum. Rabbit B that has received theinjection of normal horse serum, subsequently receives the mixture containing preven-

* We have varied these doses in different experiments. 
tive serum. Both these rabbits have received, then, in the same region the same amounts of bacteria and of serum; they differ, in that one has received both substances separately and the other received them at the same time.

Rabbit B, that received preventive serum plus bacteria subjected to it, not only survives longer, but shows the most complete and accelerated phagocytosis. Neither rabbit recovers, as the amount of bacteria given was purposely too large for the dose of serum, but both live longer than the control. In correspondence with the greater rapidity of phagocytosis in rabbit B the total development of streptococci has remained distinctly more restricted; before phagocytosis occurs the peculiarities already noted in the bacteria are shown more clearly.

This experiment, which we have repeated a number of times, gives the same results uniformly. The same results are also observed in experimenting on rabbits that have not been previously prepared by means of bouillon.

It is to be noted, however, that the differences between the two rabbits in the experiment cited are only relative differences. In both animals phagocytosis is delayed, but it appears more readily in the one than in the other. At the beginning of the experiment, during the first hours, however, the phenomena are similar and the bacterial growth goes on actively in both. A previous contact between the bacterium and the serum in vitro may favor a cure, but even prolonged contact with the serum apparently causes no modification in the micro-organism evident after its injection into the animal.

IV. Intravenous, Intraocular and Subcutaneous InocuLation of the Streptococcus in Rabbits Treated by Serum.

We have already seen that a dose of 0.1 to 0.25 of a cubic centimeter of streptococcus culture injected intravenously in animals vaccinated by serum does not increase to any great extent in the body. It is evident, however, that the injected bacteria live, as the animal dies in 2 to 4 days. At autopsy few bacterla are found in the blood, but more are present in the liver, spleen, bone marrow and particularly the lung.

If a small number of bacteria are injected in the first place, it is 
difficult to find them microscopically. It is quite probable that these organisms have been taken up by leucocytes in the blood, since the serum of animals that have received preventive serum shows no bactericilal property that apparently would offer resistance to the growth of the micro-organism in the circulating blood. We know, to be sure, that when small amounts of streptococci are inoculated into a region containing many leucocytes (a prepared peritoneum) they are rapidly taken up and their growth inhibited. Moreover, at autopsy of such rabbits after death there are frequently to be found in the internal organs (lungs or spleen) leucocytes, particularly mononuclears, containing numerous streptococci.

A subcutaneous inoculation is the one most easily tolerated by animals that have received serum. No edema occurs at the point of inoculation (except with inoculations in the ear), which renders the study of this condition rather difficult. The bacteria do not pass into the blood and it is probable that the cells of the lymph spaces in the glands have an important function in defending the body. We have many things still to consider on this point. Denys and Leclef, particularly by studying the effects of subcutaneous inoculations in the ear, have established the fact that the immunity is due to phagocytosis. Inoculation into the aqueous humor is dangerous. The very smallest amount of streptococci when introduced here increases rapidly. The influx of leucocytes is slow, not occurring to any extent under 24 hours, and is not sufficient to protect the animal for any length of time. Later on well stained chains of streptococci surrounded by an areola are found free amid leucocytes that contain few bacteria; a generalized infection finally takes place. The serum injected into such a rabbit, however, was very active; as it protected another rabbit in the same dose against the subcutaneous inoculation of 0.25 of a cubic centimeter of a young and very virulent culture. 


\title{
VI. ON THE AGGLUTINATION AND DISSOLUTION OF RED BLOOD CELLS BY THE SERUM OF ANIMALS
} INJECTED WITH DEFIBRINATED BLOOD.*

\author{
By DR. JULES BORDET.
}

In an article published in $1895 \dagger$ we called attention to the following facts:

First:- The serum of animals which have been vaccinated against the cholera vibrio causes a remarkable phenomenon when mixed with a culture of vibrios suspended in salt solution or bouillon. A very small dose of this serum will cause a loss of motility in the bacteria and will collect them in masses or clumps. If the serum has been freshly obtained and added in a sufficient dose to the emulsion its action on the bacteria is still more extensive. The vibrios that have been clumped are soon transformed into granules identical with those observed by Pfeiffer in the peritoneal cavity of immunized guinea-pigs on injecting a culture, and the same as those produced by Metchnikoff in vitro by mixing an emulsion of vibrios, preventive serum, and peritoneal exudate containing leucocytes. This granular transformation is the visible indication of an extensive destruction of the bacteria.

Second:- Serum kept for some time or heated to 55 degrees loses its power to produce a granular transformation in vibrios but still clumps them. This clumping always occurs in the presence of preventive serum and may, therefore, be very marked with a serum deprived of its bactericidal activity.

We may note that Fraenkel and Sobernheim (Hygienische Rundschau, January, 1894) had already noted that cholera serum heated to 55 degrees or even as high as 60 degrees or 70 degrees $\mathrm{C}$. loses its bactericidal power but retains its preventive power.

* Sur l'agglutination et dissolution des globules rouges par le serum d'aninaux injectés de sang defibriné. Annales de l'Institut Pasteur, 1898, XII, 688.

$\dagger$ See p. 8 . 
Third: - If we add the fresh serum of a normal animal to cholera serum previously heated to 55 degrees, and which consequently no longer effects a granular transformation but still clumps the vibrios, we find that the bactericidal power is restored to the preventive serum, that is, it again produces granules; and yet heated preventive serum alone is an excellent culture medium, and normal serum alone has only slight bactericidal power. In other words, the two constituents of the mixture, separately, are slightly, if at all, bactericidal; when united, however, they act energetically on the vibrio. Normal serum restores to the preventive serum that substance which heat has destroyed, but it is incapable of restoring this property when itself heated to 55 degrees. It is rather striking to note how very small an amount of preventive serum, whether fresh or heated to 55 degrees, will endow normal serum with strong bactericidal activity. From these facts we concluded that the intense destructive power against bacteria present in immune serum is due to the action of two distinct substances, one of which is characteristic of immunized animals, is endowed with specificity, is capable of acting in a very small dose, and resists heat; and the other of which is present in normal as well as immunized animals, is destroyed by heating to 55 degrees, is not in itself specific, and has only a slight activity when not associated with the first substance. Without indulging in hypotheses as to the intimate mechanism of the action of the two substances, we offered as a probable explanation that the specific substance by immobilizing and clumping bacteria renders them more susceptible to the bactericidal substance (alexin) present in the serum of normal as well as of immunized animals.

It may easily be understood then why the injection in normal animals, of either fresh or heated cholera serum, gives rise to a specific bactericidal power in their serum;* the specific substance unites in the injected animal with the alexin already present. The serum obtained from an animal after such an injection contains, therefore, the two substances, the presence of both of which is necessary to affect the vibrio seriously as indicated by granular metamor-

* We noted in 1895 that if a normal guinea-pig is injected with preventive serum active against the vibrio Metchnikovi, the serum of this animal becomes bactericidal for the vibrio Metchnikovi, but not for the cholera vibrio. 
phosis. This theory of "two substances" as explanatory of the origin of the bactericidal power in the serum of passively immunized animals was accepted in the following year by Gruber and Durham; we shall return presently to this subject and particularly to certain objections that Pfeiffer has made to our theory.

Other facts were soon added to those just mentioned. In the early part of 1896 both Gruber and we ourselves recognized that the property of immobilizing and clumping bacteria was not found exclusively in the serum of immunized animals. We noted, for example, that normal horse serum clumps the cholera vibrio, $\mathrm{B}$. coli, B. typhosus and B. tetani very distinctly. The serum of other animals also agglutinates them, but usually to a less extent. This property of agglutination which is so marked in immunized animals occurs, as it were, in a primitive condition in the serum of normal animals. We also called attention in 1895 and subsequently* to the fact that, as a general rule, the serum of one animal will clump the red blood cells of an animal of a different species. This power also is frequently present to a very marked extent; thus it is found that the serum of the hen clumps rat corpuscles and especially rabbit corpuscles with surprising energy. Thanks to the researches of Prof. Buchner we have known for some time that a given serum is frequently able to destroy the red blood cells of an animal of another species by diffusing their hemoglobin and so making them transparent; a.good example of this phenomenon is the action of rabbit serum on guinea-pig red blood corpuscles. Buchner also showed that a temperature of 55 degrees will destroy this destructive power for red blood cells in serum as well as the analogous power for bacteria.

It is easy to determine that these two phenomena of clumping and of destruction of corpuscles by serum from a different animal species are due to two separate substances. The destructive substance which causes corpuscles to lose their hemoglobin is destroyed at 55 degrees, as Buchner showed, but the clumping substance resists heating to this temperature. In experiments of this kind I have usually heated the sera to 55 degrees for half an hour. For example, we find that fresh hen serum agglutinates and then destroys rabbit corpuscles; when heated to 55 degrees it still clumps them as

* See page 92. 
well as before, but does not destroy them, as is shown by the fact that they retain their color and normal appearance.

It is evident that there is a striking parallel between those changes shown by vibrios subjected to cholera serum and those in red blood cells affected by serum from an alien species. We have noted that the clumping effect, which is more or less evident in both instances, is due to substances which resist heating to 55 degrees or even more; it has also been noted that the destructive properties necessitate the presence of a more susceptible substance, which is destroyed by heating to 55 degrees. In a general way certain analogies are present in the sera of normal animals, as a weak clumping power both for bacteria and red blood cells is frequently found in them; normal sera, moreover, have usually some altering or destructive effect both for alien red blood cells and susceptible micro-organisms. As has already been noted, the cholera vibrio if attenuated and only slightly resistant may show at least partial transformation with a normal serum.

If a normal animal is vaccinated with the cholera vibrio, the original clumping and destructive properties of its serum are considerably increased.* On account of the parallelism that we have just indicated and on account of analogies in the action of sera on cells and bacteria, a question immediately arises. Would it be possible by injecting a normal animal with the defibrinated blood of an animal of a different species to inerease the clumping and destructive property of its serum for the corpuscles injected? Experiment gives a positive answer to this question. Guinea-pigs were injected intraperitoneally five or six times with 10 c.c. of defibrinated rabbit blood. $\dagger$ The animals stand this treatment very well. After a time their blood is withdrawn and the serum is found to have the following characteristics:

First:- When added to defibrinated rabbit blood it clumps the red corpuscles energetically. For example, one part of serum will

* We use the cholera vibrio as an example, although it is well known that vaccination with other bacteria will also cause the appearance of agglutinating power. The cholera vibrio, however, on account of its susceptibility is the best organism to demonstrate the bactericidal substance.

$\dagger$ These doses, first used by Dr. Bordet to produce a hemolytic serum, have since been found in his hands, as well as those of other investigators, to be unnecessarily large. Quite as good results can be obtained by injecting much smaller amounts, for example, 1 c.c. (Ed.) 
clump the red blood cells in fifteen parts of defibrinated rabbit blood.

Second:- The corpuscles that have been clumped by this serum subsequently undergo rapid and complete destruction; for example, in a mixture of one part of defibrinated rabbit blood and two or three parts of active serum the mixture becomes red and perfectly transparent in 2 or 3 minutes. Microscopically, nothing but the stromata of the corpuscles are found in the fluid; they appear more or less distorted, very transparent, without their usual sheen, and rather difficult to discern.

Third:- If this active guinea-pig serum is heated to 55 degrees for half an hour it loses the property of destroying rabbit corpuscles, but still agglutinates them.

Fourth:- if a certain quantity of fresh normal guinea-pig serum is added to a mixture of defibrinated rabbit blood and specific serum heated to 55 degrees, the phenomena of destruction reappear in their entirety. The mixture becomes limpid and red in a few minutes. It is rather surprising to find, moreover, that the experiment succeeds perfectly if fresh serum from the very rabbit whose corpuscles are affected is added to the mixture of corpuscles and heated specific serum. That is to say, the corpuscles of this rabbit have become susceptible to their own alexin under the influence of the foreign clumping substance from a guinea-pig treated with defibrinated rabbit blood.

Fifth:- Although it is true that active guinea-pig serum loses its destructive property by heating to 55 degrees it is not quite exact to say that defibrinated rabbit blood when mixed with such a serum remains wholly intact. There is sufficient destruction of red blood cells to give the fluid a more or less reddish color, although the destruction is only partial and very slow. This destruction is due to the fact that the defibrinated blood contains, not only corpuscles, but also serums containing a certain amount of alexin, and, as we have just seen, normal rabbit alexin will act on rabbit corpuscles when the latter have been affected by the clumping substance of active serum. The amount of alexin in the defibrinated rabbit blood, however, is not sufficient to destroy a large number of corpuscles, which explains why the destruction of red blood cells is very slow and only partial in such a mixture. 
Sixth:-The phenomena mentioned do not occur if normal guineapig serum is used instead of the serum from a guinea-pig that has been treated with frequent injections of defibrinated rabbit blood. Normal guinea-pig serum has only a very slight clumping effect on rabbit corpuscles and its destructive power against them is practically nil.

Seventh:-The specific serum of a treated guinea-pig has no effect on the defibrinated blood of a normal guinea-pig. It has, moreover, no effect on the red blood cells of the pigeon. It agglutinates energetically rat and mouse corpuscles, but no more so than normal guinea-pig serum. This guinea-pig serum, which affects rabbit blood, has slightly more destructive properties for rat and mouse corpuscles than does normal guinea-pig serum; but the destruction of corpuscles in a mixture of this active serum and rat or mouse blood is very much less complete and rapid than in a mixture of the serum with rabbit corpuscles. We intend trying the effect of this serum on the corpuscles of a great number of species in order to determine just how far the phenomenon is specific; the specificity, however, from the data 'that we have already given would seem to be very distinct, if not absolute.

Eighth:- If a small amount (2 c.c. for example) of defibrinated rabbit blood is injected into the peritoneal cavity of a treated guineapig (that is a guinea-pig that has received several injections of rabbit blood) the corpuscles are rapidly destroyed. The fluid withdrawn from the peritoneal cavity ten minutes later is red and limpid. The corpuscles remain intact much longer if injected subcutaneously. If such an injection is made into the peritoneal cavity of a normal guinea-pig the corpuscles remain unchanged and are finally taken up by the macrophages.

Ninth:- If rabbit blood plus a small amount of active serum previously heated to 55 degrees, is injected into the peritoneal cavity of a normal guinea-pig a similar destruction of corpuscles occurs.

Tenth:-As might be expected an active serum with so marked an effect on rabbit corpuscles is toxic for this animal. Two cubic centimeters injected into the ear vein is fatal. We shall later return to a discussion of the symptoms and lesions which such injections cause. 
It must be quite evident to the reader how close an analogy there is between the action of cholera serum and of this anticorpuscular serum, the properties of which we have merely outlined. In the preceding pages the description so much resembles the description of a specific cholera serum that it would hold for the latter if the words "defibrinated blood" were replaced by the words "culture of vibrios" and the expression "destruction of rabbit blood cells" by the expression "granular transformation of the vibrio." The analogy is still more striking when we consider that the alexin that affects rabbit blood cells is probably identical with that which causes a granular transformation of the vibrios. At least the intense destructive power which is evident in both instances is destroyed at 55 degrees. In both instances this destructive property would seem to be widely distributed, not only in the serum but in the peritoneal exudate, and it would seem to be absent from subcutaneous edema fluid obtained by venous compression. If mixtures of defibrinated guinea-pig blood and rabbit serum are made on the one hand and defibrinated blood and edema fluid from the same rabbit on the other hand, it will be found that there is destruction of the guinea-pig corpuscles in the serum tube, but none in the edema tube. As we already know edema fluid also fails to produce a metamorphosis of vibrios treated with heated cholera serum.

What conclusions may be drawn from these analogies? We may conclude that the properties with which cholera serum is endowed have not been manufactured by the animal body for the simple purpose of combating an infection, if we may so express it, but are due simply to the starting up of certain preëxistent functions that may be directed according to chance conditions either against such harmful substances as vibrios or else against such wholly innocuous elements as red blood cells. As we have already shown, on injecting animals with harmless substances such as red blood cells we obtain a serum which affects these cells just as a cholera serum affects the cholera vibrio. In the case of the cholera vibrio these properties do not arise spontaneously, expressly to defend the animal against this organism, any more than does phagocytosis, the very keystone of immunity, owe its existence to a need of combating bacterial infections. One of the most important conclusions to be drawn from Metchnikoff's work is that immunity 
is simply an instance of intracellular digestion and entirely a chance and efficient application to animal defense of a primitive function which would exist even if there were no pathogenic organisms in existence. Since this form of intracellular digestion is so admirably fitted to aid in the survival of the individual it has been made use of for this purpose. 


\title{
VII. THE MECHANISM OF AGGLUTINATION.*
}

\author{
BY DR. JULES BORDET.
}

The expression "phenomenon of agglutination" is usually employed to indicate that bacteria, in a homogeneous suspension in a fluid like bouillon or isotonic salt solution collect in clumps and fall to the bottom of the tube when acted on by a specific serum. We demonstrated the first instance of this phenomenon in 1895 by showing that cholera vibrios suspended in salt solution lose their motility when subjected to the action of a small dose of fresh or heated anticholera serum and that they then rapidly collect in small masses that float in the fluid. $\dagger$

This agglutination must be considered from several different standpoints. In the first place it must be studied as an entity without any reference to its physiological significance. When looked at from this viewpoint agglutination has evident relation both with physics and with chemistry. If we attempt to define the importance of this phenomenon in immunity, when we wish, for instance, to know whether it is functional in defense of the animal body or what cells secrete the substances that cause it and liberate them in the serum and so endow it with its particular activity, we must consider agglutination from the physiological standpoint.

In the present study we shall consider the mechanism of agglutination and shall begin by reviewing the principal theories that have been offered to explain the phenomenon. We may note at once that, to be satisfactory, a theory on a subject like this should have a general bearing, and should not deal simply with the agglutination of bacteria. Bacteria are not the only cells clumped by serum; the agglutination of red blood cells by the serum of an animal of another species, must also be taken into consideration. We have

* Le méchanisme de l'agglutination. Annales de l'Institut Pasteur, 1899, XIII, 225.

† See article, p. 8. 
already shown that a specific serum with very marked agglutinating properties for red blood cells may be obtained by injecting an animal of another species with a given blood.* Any acceptable explanation then should be applicable, not only to the agglutination of bacteria but also to the agglutination of blood corpuscles and, as we shall see further on, to the agglutination of particles of casein suspended in milk.

With this remark we may consider the different hypotheses that have been proposed.

1. Gruber's hypothesis. - Gruber thinks that the agglutinin changes the bacterial substance essentially. According to his interpretation it renders the membrane of the micro-organisms more viscous and this viscous condition of the superficial part of the bacteria causes them to stick together and explains their collection in definite clumps.

This conception explains well enough why bacteria that have once been united remain together, but it does not in any way explain how the organisms approach each other to form the clumps. In explaining the fact it emphasizes exclusively the structure of the cells affected by the agglutinin, without admitting that the phenomenon may be explained even in part by simple physical laws. Since this hypothesis depends entirely on the supposed existence of a change in a cell membrane, namely, a swelling accompanied by the production of an adhesive substance, it in no way explains the agglutination of inorganic chemical particles. It rules out any relation between the agglutination of bacteria and the possible clumping of chemical precipitates in a fluid.

2. Bordet's hypothesis. - The conception which we gathered from a study of this phenomenon in 1896 is essentially different. It appeared to us that in the case of the agglutination of the cholera vibrio by its specific serum we have to deal with a phenomenon in which the bacteria play only a passive rôle and in which their vitality is not concerned. It is evident that motility is not necessary, since agglutination occurs not only in bacteria that have lost their motility, but also in red blood cells which are inert. The passive rôle of bacteria is still further evident when the agglutination of dead micro-organisms is considered. Gruber's hypothesis, more-

* See article p. 134. 
over, arouses certain other objections, to which we shall later refer, and it appeared to us that agglutination "is due to some phenomenon of molecular physics. The slightest effects may cause chemical precipitates which have remained uniformly suspended in a fluid to fall to the bottom of the tube. It is probable that serum acts on bacteria by changing the relations of molecular attraction between the bacteria and the surrounding fluid."

It is evident that this interpretation does not explain the intimate nature of the phenomenon any more than does Gruber's; it simply compares particles of such different natures as bacteria, red blood cells and chemical precipitates, each of which when suspended in a fluid may be brought together in masses by certain influences. Contrary to Gruber's conceptions, this explanation implies the existence of analogies between the various forms of agglutination whatever may be the substances agglutinated; it presupposes the predominant intervention of physical laws in the phenomenon.

We must be quite clear on this point. Does this hypothesis mean that bacteria when affected by the agglutinin act simply as inert particles in all phases of the phenomenon? Certainly not. Agglutinins are specific; and there is, moreover, not the slightest doubt that they act directly on the bacteria since these cells rapidly lose their motility in the very first stages of the phenomenon. In the first phase of the phenomenon the action of the agglutinin evidently takes into account the particular biological nature of the element that it affects: this is evident since it affects certain organisms and not others. But the subsequent changes which bring the affected organisms together may be brought about by the slightest modifications in the active substance provided they are sufficient to change the relation of molecular adhesion between bacteria and fluid. From that point on, according to the hypothesis, the biological nature of the substances affected would no longer count. The bacteria are thenceforth agglutinated according to physical laws which are applicable also to certain inorganic particles, and it is unnecessary to suppose the presence of an adhesive substance or of sticky or viscous membranes to explain the clumping and the adhesion of the micro-organisms. Gruber's hypothesis excludes physical laws; our hypothesis would attribute considerable impor- 
tance to them, particularly during the phase of the phenomenon in which the bacteria which have been affected by the agglutinin are still scattered and are beginning to collect to form the typical agglutination.

These two hypotheses, which were formulated in the beginning of studies on agglutination, could not rest on a very firm basis from lack of sufficient data. An experimental fact of importance was furnished by Kraus.* Kraus showed that if the serum of animals vaccinated against the cholera vibrio is mixed with a limpid filtered culture of this organism a precipitate is formed in the fluid. This reaction is specific and does not occur when any other serum is used in place of cholera serum. When this precipitate has been formed it soon collects in small clumps that recall in appearance masses of agglutinated bacteria. Kraus also showed that the same result could be obtained with other bacteria and their corresponding antisera.

These experiments would seem to corroborate, a priori, the second of the two hypotheses that we have mentioned. It would seem, moreover, to invalidate Gruber's hypothesis, which recognizes as the sole cause of agglutination a structural modification of the affected cells. These experiments indeed show that a flaky precipitation resembling true agglutination can be formed by mixing with serum a fluid containing no definite bacteria but simply the materials of bacterial disintegration. This fact of Kraus' would seem, then, to rule out Gruber's theory entirely.

3. Nicolle's hypothesis. $†$ - Nicolle has a somewhat different idea. He has confirmed Kraus' results and agrees that the agglutinin precipitates the agglutinable (or agglutinated) substance of bacteria.f He thinks, moreover, that this agglutinable substance, which in old cultures may become diffused into the surrounding fluid, is present in large amounts in the membrane of the outer

* Kraus, K. K. Gesellschaft der Aertze in Wien, April 30, 1897, and Wiener klinische Wochenschrift, August 12, 1897, No. 32.

† Annales de l'Institut Pasteur, March, 1898.

$\ddagger$ This is more than a simple adaptation of Kraus' experiment; the expression indicates, perhaps rather hastily, that we must consider the substance precipitated as the one which is of importance in the agglutination of bacteria, the substance which, in other words, represents in bacteria that part which is susceptible to the agglutinin. 
surface of young and healthy bacteria. This superficial layer includes the substance that is susceptible to attack and precipitation by the agglutinin; when the agglutinin acts, this external layer of the bacteria "swells up, becomes apparent and sticks to the external layer of adjacent bacteria. Our opinion as to the intimate nature of the phenomenon of agglutination is quite similar, then, to that offered by Gruber and later defended by Roger. We believe that agglutination consists in the coagulation and coalescence of the external layers of the agglutinable bacteria by the agglutinating serum." *

As may be seen, Nicolle attaches or adds on Kraus' experiment to Gruber's theory, but it is precisely this addition, on which the whole value of the idea depends, that appears to us incomprehensible and the weak point in the reasoning. Why should a precipitation of the agglutinable substance within the external layer of the bacterium, which we will not deny off hand, lead to a swelling and viscosity which may bring about the coalescence and junction of the external layers of neighboring organisms?

However this may be, this interpretation, as well as Gruber's, takes no account of the intervention of the physical laws of molecular adhesion in explaining the fact. Nor does it presuppose any relation between the collection of certain chemical precipitates and bacterial agglutination, since its explanation of the phenomenon depends essentially on the presence of a membrane and external layer or of a ciliated covering susceptible to swelling and stickiness.

Nicolle describes a rather curious experiment in his article. He found that the precipitate caused by the interaction of an active serum with a culture filtrate has the property of carrying down with it, in its clumping, inert particles like talcum powder in the form of definite masses. Although this experiment is interesting we do not think it is of any direct importance in explaining the phenomenon of agglutination, its resemblance to which is only apparent. These particles of talcum that collect into masses are drawn together mechanically and collected by a precipitate which is forming. To admit that this non-specific phenomenon, which resembles superficially true agglutination, is of importance, would be to admit 
that agglutination proper is also due to the formation of a precipitate outside of the bacteria which retracts and becomes agglutinated and thus presses together the bacteria and forces them to unite and become adherent. As a matter of fact this idea was already offered by Paltauf * before Nicolle's work. Dineur in a recent article has discussed this theory and offered objections to it. $\dagger$ We shall consider this hypothesis in its proper place.

4. Paltauf's hypothesis. - According to this author the agglutination of bacteria is due to their being mechanically drawn together in the interstices of a coagulum formed outside of the bacteria in the surrounding fluid, as a result of the reaction between the agglutinin and the agglutinable substances from the bacteria.

5. Dineur's hypothesis. - According to Dineur the clumping is due to the formation of an adhesive substance which keeps the bacteria together. This adhesive substance is formed particularly on the cilia. As may be seen, Dineur attributes essential importance in agglutination to the presence of cilia. Agglutination would be caused, then, by an adhesion and interlacing of these cilia.

$$
*^{*} *
$$

We have thus reviewed in the preceding pages the various interpretations of agglutination that have been proposed, with emphasis on their exact significance. We have, however, only touched on the experimental facts that corroborate or eliminate them. There are already enough of these facts to allow of a discussion founded on adequate data, and it is this consideration that we propose to take up.

Among the interpretations that have been offered there are some which evidently are so little in relation to fact that they may be dismissed preëmptorially. For example, Dineur's hypothesis which attributes a maximum importance to the existence of cilia. Such an opinion is evidently unsatisfactory, as agglutination may occur with bacteria that have no cilia or even with such elements as red blood cells, or particles of casein which obviously do not possess these appendages. Dineur, to be sure, also emphasizes the production of an adhesive substance which collects the bacteria subjected

* Wiener klinische Wochenschrift, 1897.

$\dagger$ Dineur, Recherches sur le mécanisme de l'agglutination du bacille typhique. Bulletin de l'Academie de Medecine de Belgique, 1898, p. 652. 
to the agglutinin. In this respect he agrees with Gruber's interpretation which we shall consider later.

Paltauf's interpretation, which would explain the agglutination of bacteria by a retraction of a precipitate (Kraus) forming in the fluid and collecting bacteria in its own clumping, meets with grave objections. To begin with Kraus' phenomenon of precipitation does not occur constantly; even when it does occur the precipitate is never abundant, and is formed so slowly that it scems incredible that it occurs before the rapid and energetic bacterial agglutination and is, therefore, the cause of it. Moreover no one has been able to demonstrate a coagulum about agglutinated bacteria. Dineur, indeed, has repeatedly attempted to find one unsuccessfully, and he very correctly states that if there were such a coagulum enclosing the bacteria we might expect to demonstrate it since coagula take basic colors as shown by Nicolle.

Certain other observations may be noted at this point. Rabbits which have received several intraperitoneal injections of defibrinated hen blood give a scrum which has an agglutinating and dissolving power for hen corpuscles. This active serum has still another property. When mixed with hen serum it caused a precipitate to form in the fluid which gradually increases and finally flocks out. This property of forming a precipitate with the causative serum present in the serum of animals injected with this serum was noted recently for the first time by Tchistovitch at the Pasteur Institut. Tehistovitch found that the serum of rabbits which had received several injections of eel serum caused this latter serum to become cloudy; he noted the same occurrence with the serum of rabbits immunized against horse serum on mixing the two sera. As Tchistovitch noted, these precipitates are soluble in small amounts of alkali (potassium, sodium or ammonia), as we also found to be the case with our rabbit serum specific for hen blood.

It would seem reasonable that these phenomena are similar to those which Kraus noted. The specific serum from animals injected with blood serum from another animal causes an opacity in the serum of the species used for inoculation. Serum from animals injected with bacterial cultures causes an opacity in the culture fluid in which the organism used for vaccination has grown. The precipitate that we have mentioned bears the same relation to Kraus' 
precipitate that the agglutinin for red blood cells does to the agglutinin for bacteria. It may be shown experimentally that in the case of blood these precipitates not only are not indispensable for the occurrence of strong agglutination but have no definite relation to it. As we have already mentioned, the serum of a rabbit that has been repeatedly treated with hen blood has the property of agglutinating and dissolving hen corpuscles and of precipitating hen serum. It also forms a precipitate with pigeon serum. We therefore might expect that this serum would also agglutinate pigeon corpuscles; as a matter of fact it has no more effect on these corpuscles than does normal rabbit serum, which when mixed with hen serum remains quite limpid. A slight agglutination, to be sure, does occur either with the normal or specific rabbit serum, but it is not so much as, for example, the agglutination of rabbit corpuscles by normal hen serum, in which latter instance also no precipitate occurs. Guinea-pigs which have been given several injections of defibrinated rabbit blood furnish a serum that has a very intense clumping power for rabbit corpuscles, but which produces no clouding with rabbit serum. In other words there is no necessary parallel between precipitate formation and an intense clumping power, and any opinion that regards the formation of such precipitates as the sine qua non of agglutination would seem to be no longer tenable.

Let us now consider Gruber's hypothesis which from the very beginning has been extremely open to criticism. It is easy enough to conceive that a sticky substance coming from the covering of the bacteria should hold the micro-organisms together, but it is not so easy to understand why it should bring these organisms together. No evidence of the morphological modification which this hypothesis implies has been found by Pfeiffer or ourselves on microscopical examination of either living or stained preparations of bacteria or red blood cells. Trumpp * thought that he did find an alteration in agglutinated vibrios, but the bacteria in which he noted this swelling had been in fluids containing not only agglutinin, but alexin (or lysin), that is, the bactericidal substance which is destroyed by a temperature of 55 degrees. This alexin is very destructive both for vibrios and for red blood cells; it dissolves the latter and 
causes swelling, granular transformation and often destruction of the former. If he wished to study the effect of the agglutinin alone, Trumpp evidently should have used fluids that had been previously deprived of the alexin normally present.* Red blood corpuscles clumped by the serum of an animal of a different species seem to keep their normal appearance; they also remain normal when subjected to an active anticorpuscular serum that has been previously heated to 55 degrees and thus deprived of its dissolving alexin without losing its agglutinin. It seems, moreover, hardly reasonable that such different cells as bacteria and red blood cells undergo the same modifications when affected by an active serum. When we deal with chemical particles such as milk casein instead of cells like bacteria and red blood cells, the existence of a viscous change is still less probable. And yet a serum may be produced that "agglutinates" milk, that is to say, which clumps particles of casein.

Is it reasonable to suppose that these particles become sticky or viscous when affected by the active serum? Is it to be supposed that their sticking together is due to such a viscosity? If we agree to this we must suppose that particles of clay in a homogeneous aqueous suspension are also covered with a special viscous and sticky coating when we add a little sodium chloride to the fluid in which they are suspended. As is already known, the addition of salt to such a fine clay suspension causes flecks to form which settle to the bottom of the tube; this is a fact which interests geologists extremely as a means of explaining sedimentations.

The existence of an adhesive substance, which is the foundation of both Gruber's and Dineur's hypotheses, seems to the latter observer to be corroborated by a very significant experiment. Dineur has noted that if an emulsion of bacteria to which a specific serum has been added is gently shaken, the clumping of the bacteria is very much increased. Dineur supposes that this mechanical rolling of bacteria tends to bring them together, to interlace their cilia, and to allow the sticky substance with which their cilia are supposedly covered to bring about final adhesion.

* The same criticism may be made of the statements of Roger (Revue générale des Sciences, 1896) concerning the modification in the oidium albicans subjected to an active serum. Kraus and Seng in a recent article (Wiener klin. Wochenschrift, 1899, No. 1) have offered the same objections to the experiments of Trumpp and Roger. 
The fact that Dineur has reported is exact, but its interpretation does not seem to be so. As a matter of fact, the favorable effect of motion may also be noted in the formation of flecks in inert inorganic precipitates. If a drop of serum is added to 5 or 6 c.c. of a 0.7 per cent salt solution and nitric acid then added, an albuminous opacity is formed which, if left alone, clumps very slowly. But if, when the precipitate has been formed, a small amount of the fluid is poured into another tube and this tube held almost horizontally and gently agitated, the precipitate is agglutinated in a few moments in small white masses which float in a clear fluid. The fluid which has been left standing, however, remains homogeneous for a long time and the contrast between the two is very striking. The same experiment may be performed with other albuminous precipitates, as, for example, with a precipitate formed by nitric acid in whey. The phenomenon to which Dineur attaches so much importance is also distinctly visible when milk is agglutinated by its specific serum. Dineur's observation, then, instead of pleading for Gruber's theory offers a still further analogy between the agglutination of bacteria and of inorganic particles. But there is another still more significant analogy.

We know that the collection of precipitates is frequently controlled by such apparently insignificant causes as the presence of salts in solution in the fluid. A clear example of this is offered by clay, which forms a very fine and homogeneous emulsion in distilled water, but clumps and falls rapidly in the tube if placed in water containing sodium chloride. If we believe, then, that the agglutination of bacteria depends on laws of molecular adhesion, we might suppose that salts would have some effect on this phenonenon as well; and such indeed proves to be the case. Several 24-hour cultures of the cholera vibrio are suspended in salt solution (10 c.c. to a culture) and to the homogeneous emulsion obtained in this manner is added a powerful agglutinating dose of cholera serum. The bacteria soon form flecks which fall to the bottom of the tube. The tube is centrifugalized, the supernatant fluid removed, and a compact mass of agglutinated bacteria left in the bottom of the tube. These bacteria are then suspended in water so as to form a rather thick emulsion, which is divided in two equal parts and placed in two separate tubes. To the first 
tube is added distilled water and to the second normal salt solution. After agitation these tubes are again centrifugalized. It is found that the bacteria in the tube containing salt solution go to the bottom much more rapidly than in the one containing distilled water. When they are finally deposited the supernatant fluid is removed from each tube and replaced by a second amount of the same fluid, that is to say, salt solution in one and distilled water in the other. The bacteria are then shaken up in the fluid. It is found that clumps form rapidly in the tube containing salt solution, but that the bacteria remain indefinitely in suspension in the tube containing distilled water. If a small amount of the cloudy fluid, say 10 c.c., is taken from the second tube and placed in a fresh tube and to it is added 0.07 grams of $\mathrm{NaCl}$, agglutination reappears and the deposition of bacteria takes place.*

The same phenomenon occurs with the cholera vibrio and a normal agglutinating serum in place of a specific cholera serum. We noted three years ago that normal horse serum agglutinates the cholera vibrio and other bacteria such as B. typhosus, B. coli and B. tetani very markedly. If this last experiment is repeated with normal horse serum instead of the specific serum the same results are obtained.

In this latter case it is not necessary to remove all the traces of $\mathrm{NaCl}$ by repeated washing. The salt solution containing the clumped bacteria is simply centrifugalized and the supernatant fluid decanted; the deposition is then divided into two parts and placed in two separate tubes; one tube is filled with distilled water and the other with salt solution. On agitation the agglutination recurs only in the presence of salt. $\dagger$ A similar experiment with normal horse serum and B. typhosus gave the same result.

* It must be noted that this. "reagglutination" of bacteria on the addition of salt to distilled water does not occur quite so rapidly as in the tube in which the bacteria have remained in contact with salt solution, especially when the contact with distilled water has been prolonged. It is probable that the micro-organisms must retain a certain amount of salt in order to agglutinate well.

$\dagger$ The presence of an agglutinating power in serum has doubtless vitiated many researches on the bacterial power of body fluids. Many observers, indeed, have used the method of inoculating at intervals small amounts of a mixture of serum and bacteria in gelatin in order to determine the destructive power of the serum. It is quite possible that the serum in question, when the amount of bacteria is small, may so clump them that each clump of bacteria will give rise to a single colony 
The same results are also obtained if, instead of bacteria, Kraus' precipitate, obtained by mixing cholera serum with an old filtered culture of the vibrios, is used. This precipitate is treated exactly as described for bacteria, and it is found that it clumps very much more markedly in fluids containing salt than in distilled water.

It may be worth while to give the results obtained by the same technique on the agglutination of a fine emulsion of potter's clay in distilled water after filtering through paper. Tubes which do not contain salt remain opaque for days, whereas in tubes containing 0.7 per cent salt solution there is a very distinct agglutination and a rapid deposition. The resemblance between floating clumps of agglutinated bacteria and the whitish flecks of clay suspended in salt solution and falling slowly to the bottom, is very striking.

These experiments on the absence of agglutination in distilled water are very strongly confirmatory of the idea that the agglutinin acts by producing on isolated elements such changes in their properties of molecular adhesion as are shown by particles of clay. In each case the adding of salt suffices to produce the physical phenomenon of agglutination which, without it, is impossible. This, in our opinion, is a confirmation of an hypothesis that we formerly offered and have just recalled.

$$
*^{*} *
$$

If we conceive of the phenomenon of agglutination in this way, interesting generalizations may be drawn, particularly in the light of the explanation that Duclaux has proposed for coagulation. What, indeed, is agglutination? It is the union into masses of organized scattered particles, by some peculiar influence that changes only. This technical error is naturally of great importance in researches on easily agglutinable bacteria like $\mathrm{B}$. typhosus.

It is certain that real bactericidal properties have been presumed to exist when agglutinins alone were present. It is also probable that at times certain properties of the agglutinins have been attributed to the alexins. For example, Buchner states that alexin loses its activity, to a large extent at least, when mixed with distilled water. The presence of distilled water might apparently diminish the bactericidal property of a serum by weakening its agglutinating power and consequently by increasing the number of colonies that grow on gelatin. We have found that alexin acts very well in a medium with very little salt; vibrios treated with preventive serum and then washed and suspended in fifteen parts of distilled water may show granular transformation when one part of normal serum is added to these fifteen parts of emulsion, without any agglutination. 
the properties of molecular adhesion. What is coagulation according to Duclaux? It is the uniting in groups of particles which may have been so finely divided as to appear in solution, by some peculiar influence which modifies the molecular relations between the particles and the fluid. Before the intervention of this influence the liquid remains homogeneous, but as a result of it "the state of equilibrium between gravity and molecular forces is disturbed, either because the adhesion between the fluid and the solid has diminished, or, more probably, because the attraction between the particles of the solid is increased so that they unite into more or less voluminous collections which become visible to the naked eye and are precipitated." *

On account of these changes of molecular adhesion, particles, the chemical nature of which may differ greatly in the various instances and which are often so small as not to be microscopically visible, collect into masses which are still invisible to the naked eye, but which, by progressive clumping, gradually increase in size and render the fluid opaque, until by molecular condensation they form more and more voluminous masses.

It is not necessary to follow the systematic way in which Duclaux has elaborated this idea, nor to indicate how simplifying this conception is, combining as it does facts that were so separated as to appear unrelated. Such a conception connects agglutination with the phenomenon of coagulation, as he conceived of it. The agglutination of bacteria is due to a change in molecular adhesion between the bodies of the bacilli and the surrounding fluid. As Duclaux expresses it this phenomenon "as a whole and in detail recalls our observations and description given in the chapter on the phenomenon of coagulation." $\dagger$

Therefore, if, as Duclaux affirms, we have the right to regard agglutination as a phenomenon of coagulation and if we are authorized in giving henceforth the active substance in serum the more suggestive name of "coagulin" instead of agglutinin, which latter term simply indicates its activity without any reference to its relations or cause, we may suppose that the animal body, owing to its functional plasticity and the multiplicity of its resources, would be able to elaborate, when necessary, active clumping principles,

* Duclaux, Traité de microbiologie, vol. 2, p. 263 † Duclaux, Ibid, p. 706. 
not only against organized cells but against such chemical substances as have been recognized as coagulable.

Experiment, indeed, justifies this supposition. If rabbits are given several successive intraperitoneal injections of milk, previously heated to 65 degrees to sterilize it partially, after a proper interval they give a serum that has specific properties against milk.

A certain amount of this serum (for example 3 c.c.), is placed in a tube and the same amount of normal rabbit serum is placed in another tube. To each of these tubes milk is added (10 to 15 drops for example). The tube containing normal serum remains opalescent and homogeneous. In the tube containing active serum small particles rapidly appear that soon increase in size and form thick flecks. The fluid then becomes separated into two parts, one of which is quite limpid and the other of which contains clumped masses which generally fall to the bottom of the tube, leaving the clear fluid above. This sedimentation goes on better if milk that contains little fat or, better still, milk that has been passed two or three times through filter paper and so deprived of part of its fat globules, is used.* If milk containing a good deal of fat is used, the clumps may float to the top carried up by the fat corpuscles that they have enclosed.

If these mixtures of milk with normal serum or with "lactoserum" are passed through a filter paper, the latter mixture filters quite clear without any of the whitish opacity which milk produces; the mixture containing normal serum remains cloudy after filtration.

Microscopical examinations of these mixtures of milk with sera show that the lactoserum causes the formation of abundant granular masses that do not occur in mixtures with normal serum. This granular precipitate resembles the clots of casein formed by rennin. $\dagger$

* The experiment is more striking if filtered milk of this sort is used. Such milk does not stick to glass that it touches, and renders the fluid less opaque, so that the agglutination may be more readily estimated.

$\dagger$ We do not wish at all to assert that the agglutinin of lactoserum is identical in action with rennin. There are distinct differences between these two substances. The action of the agglutinin is much less dependent on a suitable temperature than is rennin; as a matter of fact, it acts at a temperature so low that rennin is almost inactive. Nor does our serum have the property of clotting relatively enormous quantities of casein, as does rennin. Moreover, in a mixture containing a large amount of normal serum and a small amount of milk, rennin has slightly, any effect, whereas under these conditions agglutination appears on addition of lactoserum. 
Certain of these clumps are composed entirely of a fine granular precipitate; and others enclose a large number of fat globules in their midst. If a little more milk than can be agglutinated is added to lactoserum, there is nevertheless an abundant deposit of the agglutinated substance formed. After the deposition of all the clumps, even to the smallest ones, the supernatant fluid is found to be quite limpid. When taken off and mixed with normal serum this supernatant fluid gives no opacity. If added to lactoserum, however, it causes a slight cloud which soon increases and is followed by flecks which form a considerable deposit. In other words, the lactoserum has clumped casein that was in so fine a state of division that it did not render the liquid turbid, and that had escaped the agglutinating action of the first insufficient dose of active serum.

A similar experiment may be performed in the following manner: as we have already stated, normal serum containing milk (for example, 4 c.c. of serum to ten drops of milk) passes through filter paper as an opaque fluid. If the filtration is repeated a number of times through the same paper, a much clearer fluid, which is only almost imperceptibly opalescent, is finally obtained. On microscopic examination this fluid contains very few fat globules and nothing else. If a small amount of this is mixed with equal parts of normal serum, nothing happens. When mixed with lactoserum in equal parts the fluid that was at first transparent immediately becomes turbid, and rather voluminous white masses of casein form, which, microscopically, are granular clumps, identical with those formed by rennin.

Similar precipitates may also be formed with whey produced by adding rennin to milk. This whey when filtered is faintly opalescent and causes no turbidity with normal serum; when added to lactoserum it causes a very distinct turbidity which soon settles down in the form of flecks. It is well known that whey still contains casein which has escaped the effect of the rennin, but which gives a voluminous precipitate on addition of an acid.

$$
*^{*} *
$$

Are there no analogies to be drawn between the appearance of flaky precipitates in limpid fluids containing sufficient casein to render them faintly opalescent when lactoserum is added, and the 
precipitates produced on mixing two sera under the conditions already described? Our specific serum from a rabbit injected with hen blood causes an abundant precipitate with hen serum. May we not assume in this case, too, that the active serum collects molecular groups which have previously remained scattered and dissociated to such an extent that they did not cloud the limpid fluid? The precipitate produced in such mixtures of sera would seem to be caused by a phenomenon of agglutination,* or, if preferred, of coagulation, for we are at a loss to know which of the two terms to use.

May we not also draw an analogy between the appearance of these precipitates and the agglutination of bacteria or of red blood cells which is nothing more than the collecting into voluminous masses of separate defined cells? The only point of difference between the phenomena in question is that in certain of them the agglutinable particles are so small and separate that before being collected they fail to affect the limpidity of the liquid; in certain other cases, as with bacteria or corpuscles, they are sufficiently large to produce a visible cloud before being clumped.

But this variation in size of the particles concerned is only an accessory fact which, in our opinion, certainly does not affect the essentials of the phenomena themselves. Such a distinction is only secondary and cannot in the least affect the conclusion that there is no fundamental difference between the phenomena of agglutination and of coagulation. For example, the coagulation of clay is closely allied to the coagulation of milk, according to Duclaux's ideas. It is, moreover, closely related to the agglutination of bacteria, as is shown by the effect of sodium chloride; and further, the agglutination of bacteria resembles the coagulation of milk, as we learn from the experiment with agglutinating lactoserum, which produces coagulation similar to that caused by a mixture of normal serum and

* The following fact corroborates this point of view. The specific property of the active rabbit serum that produces a precipitate with hen serum is weakened on heating to 65 degrees for half an hour; it is destroyed on heating for the same length of time to 70 degrees. On heating to 65 degrees or, still more so, by heating to 70 degrees the serum also loses to a great extent its agglutinating power for hen corpuscles. In other words, the precipitating substance is affected by heat in the same way as is the agglutinin. The precipitable substance of hen serum, on the contrary, resists heating to 75 degrees for half an hour, as is shown by its forming a precipitate when added to active rabbit serum. 
antiserum. This specific precipitating serum, moreover, is produced by treating animals much in the same way as to produce agglutinating sera for bacteria. There are numerous evidences of similarity between these different phenomena, so that one is forced to accept a single general explanation for them all, and to attribute the occurrence of agglutination to changes in molecular adhesion.

If we were to choose the specific agglutination of bacteria as an example of these phenomena we might say that the agglutinin which unites with the bacteria acts by modifying the relations of molecular attraction both between the individual bacterial particles and between these particles and the surrounding fluid. The agglutinin affects only a certain definite bacterial species. During the first period of agglutination the individual constitution of the bacteria in question is much in evidence. It is, perhaps, essential that bacteria, in order to be affected by an agglutinin, should be sufficiently intact, as would seem indicated by certain of Malvoz's* experiments.

But as soon as the change in molecular adhesion has been produced the bacteria collect as do inorganic particles. It is not necessary to imagine that their structure has anything to do with it; nor to think that the bacteria must stick to one another as a label sticks to a bottle, by means of some peculiar adhesive substance which covers the cilia, or owing to a swollen outer membrane. This phenomenon of the collection of particles by means of some influence which changes their molecular attraction should by definition be placed among the phenomena of coagulation as Duclaux has described them.

From this standpoint then the phenomenon of agglutination is divided into two distinct phases. In the first phase the scattered bacteria are affected by the agglutinin and absorb it. This causes modifications in their properties of molecular adhesion. The existence of these modifications during the second phase brings about agglutination properly speaking.

This division into two periods is neither artificial nor imaginary. These two phases may indeed be separated and the first be brought about without the second. The experiment already mentioned that shows

* Recherches sur l'agglutination du bacille typhique. Annales de l'Institut Pasteur, July, 1897. 
the function of sodium chloride does this very thing. The bacteria that we washed and suspended in distilled water had been affected by the agglutinin. They are immobilized and are ready to be agglutinated energetically.* But to bring about the second phase of the phenomenon or agglutination, properly speaking, a little salt must be added to the emulsion.

As far as Kraus' phenomenon is concerned we are as yet in no position to interpret it. It is by no means demonstrated that Kraus' precipitates have anything to do with the real agglutination of bacteria; it may be that this precipitate is similar to that obtained in a mixture of defibrinated hen blood and specific rabbit serum (that is, serum from a rabbit injected with hen blood), which apparently has no relation to the agglutination of the corpuscles themselves. But if Kraus' precipitate is formed from the agglutinable substance of bacteria it seems to us that we must compare it, from the standpoint of its formation, with the casein precipitates which lactoserum produces with whey, in which instance the substance is so finely divided as not to disturb the limpidity of the fluid. In other words this precipitation would resemble the agglutination of bacterial substances in a finely divided state.

\section{***}

The idea that the agglutination of cells, corpuscles, bacteria or non-differentiated particles like casein has the characteristics of a phenomenon of coagulation might suggest certain observations on the significance of the active properties of serum. Let us enumerate briefly, without repeating the observations that we have formerly made, the essential properties which we have found in specific sera by experiments performed in vitro. To be more exact we shall consider the two sera which we used as typical, that is cholera serum, and a serum active against rabbit corpuscles obtained by injecting guinea-pigs with rabbit blood. These sera, as we have recently pointed out, have similar properties which are as follows:

1. Both sera agglutinate cellular elements and suppress their motility if they have any.

2. When either the vibrios or the corpuscles have been in contact with their respective serum they are more susceptible to the

* We may also add that they have become very susceptible to the effect of alexin. 
destructive effect of alexin. This alexin or lysin is the bactericiclal or globulicidal substance that is destroyed at 55 degrees, and affects certain delicate cells as a sort of digesting diastase. The specific properties of these sera resist heating to 55 degrees or 60 degrees.

3. The sera when fresh contain alexin; and it is due to the presence of this substance that they are able to alter profoundly or to cause partial dissolution of those cells which they sensitize.

If we were to enumerate the really essential properties of these sera we might eliminate the third, namely the possession of alexin, as this substance is also present in the serum of normal animals. After destroying this alexin in the specific serum by heating it to 55 degrees and so removing its bactericidal or globulicidal power, the power may be restored by the addition of a small amount of normal serum which contains alexin.*

There remain then two other properties, which, to be sure, occur in normal sera to a slight extent, but may be considered as characteristic of the serum of vaccinated animals.

We shall not consider here the question as to whether these two properties are due to two distinct substances or are to be attributed to the action of one and the same substance, reserving such discussion for another time. In all events the most remarkable of these two properties is the sensitizing of cells to the action of the alexin. When we say that there exists in specific sera a sensitizing substance (substance sensibilisatrice) it implies that these sera act directly on the cells. Indeed this sensitizing substance has a particular predilection for fixing itself on those cells which it affects.

When cholera vibrios are placed in a suitable amount of fluid containing cholera serum they absorb its active principles. After centrifugalizing and decanting the clear supernatant fluid is found to have lost both its agglutinating power and its power of sensitizing new bacteria to the action of the alexin. In other words, new vibrios, when placed in contact with this fluid, are not immobilized or agglutinated and may be injected into the peritoneal cavity of a guinea-pig or mixed with normal guinea-pig serum in vitro without showing granular transformation. The same phenomenon of absorption or fixation occurs if the vibrios are cultivated in bouillon

* The details of these experiments for the cholera vibrio will be found in the article beginning page 56, and for the red blood cells in the article on page 134 . 
to which a moderate amount of cholera serum has been added. The bacteria in their development remove all the particular properties from the fluid.* The same facts hold for sera active against red blood cells. When red blood cells are placed in contact with their specific serum they absorb both the agglutinating substance and the sensitizing substancet. The serum separated by centrifugalizing such a mixture is inactive for fresh corpuscles. It may also be added that the agglutinins of normal sera are likewise absorbed by corpuscles or bacteria. $\ddagger$ This all indicates that animals during vaccination are to be distinguished, not by the elaboration of large amounts of the "dissolving diastase" or alexin, but by formation of those substances which favor the action of these diastases, that is, of principles which unite with the cells and sensitize them to the effect of this alexin.

* This result completely contradicts the facts observed by Pfeiffer, who performed this last experiment. (Centralblatt für Bakt., 1896.) Pfeiffer found that the agglutinin was absorbed under these conditions, but stated that the fluid in which bacteria had grown still retained the property of causing a granular transformation when injected with new vibrios into the peritoneal cavity of a normal guinea-pig. Pfeiffer's conclusion that active sera do not act on bacteria in the same manner in vitro as they do in the peritoneal cavity is erroneous. The effect in vivo and in vitro is precisely the same. Experiment shows us that the smallest amount of cholera serum necessary to cause granular transformation of a given dose of vibrios is the same whether the transformation takes place by means of the alexin in the peritoneal cavity, or in vitro on the addition of the alexin of normal serum. This minimal destructive dose for vibrios is very similar to the minimal agglutinating dose, as we shall consider presently.

$\dagger$ It must be noted that Ehrlich (Collected Studies on Immunity, EhrlichBolduan, Wiley \& Co., page 1) has recently noted that hemolytic serum exhausted by contact with its specific corpuscles no longer forms a dissolving mixture for new corpuscles on the addition of normal serum.

¥ We should like to mention briefly a curious experiment which, strictly speaking, is rather irrelevant. If a given dose of normal serum with strong agglutinating property for cholera vibrios (normal horse serum) is placed with these vibrios, agglutination takes place. If the mixture is then centrifugalized and the clear supernatant fluid taken off, it is found that it no longer agglutinates cholera vibrios, but still does agglutinate the typhoid bacillus. Conversely, normal horse serum mixed with typhoid bacilli leaves a supernatant fluid after agglutination and centrifugalization that no longer agglutinates the typhoid bacillus, but still agglutinates the cholera vibrio. It seems certain, then, that there are two distinct agglutinins, one for each of these micro-organisms, in the same serum. Such experiments may one day throw light on the obscure question of the origin of the specificity of active properties in serum. It is quite conceivable that vaccination with a given bacterium may cause the production of a large amount of an agglutinin which has already existed in small amounts. 
It is owing to the presence of these facilitating substances that the sera of vaccinated animals can produce marked evidence of digestion if the cells affected are not too resistant, as, unfortunately, is the case with many bacteria.* We may consider such sera, then, as analogous to digestive secretions.

This analogy between the properties of active sera and digestive secretions is still more evident when we consider that the active substances in serum arise in the digestive cells that Metchnikoff demonstrated; the function in immunity of such cells is very important and, in the course of evolution, they become identified with those ameboid cells which, in simple organisms, are able to assure the nutrition of the individual, owing to their intracellular digestive functions. It is such cells that, as Metchnikoff has found, take up the function of digestion in slightly differentiated species. What is more, such ameboid cells represent the origin of our digestive apparatus, for, as Metchnikoff has shown, digestion, which at first is only intracellular, becomes, in the course of evolution, extracellular, as these cells acquire the property of excreting their dissolving juices. The source of the agglutinins and the sensitizing substances is not, to be sure, well defined, but, as far as the alexin or dissolving principle is concerned, we know from numerous observations that it is of leucocytic origin.

In the present state of our knowledge of this as yet obscure subject we do not wish to state too dogmatically such facts as occur to us as correlated. There are at least important characteristics which suggest the relation of active sera to digestive juices, and the analogy would be explicable if, as numerous facts and suppositions render it probable, we were to attribute the elaboration of these active substances to the phagocytes, in other words, to that group of cells endowed with digestive properties which are retained during evolution.

If future facts point to the same conclusions, we shall finally regard immunity, not only from the biological standpoint, as we do at the present moment, but also from the chemical standpoint, as an instance of the physiology of digestion.

* As we know, the typhoid bacillus and the colon bacillus show only slight granular transformation in the presence of an active serum. Many other bacteria are still less susceptible to bactericidal properties. 


\section{Conclusions.}

I. Theories that explain bacterial agglutination by a swelling and viscosity either of the membranes or cilia of the bacteria meet with numerous objections and do not serve to explain all the phenomena of agglutination. The theory that regards the formation of a precipitate in the fluid as the cause of agglutination is open to the same objections.

II. Agglutination may affect such different elements as red blood corpuscles, bacteria, and casein. In all these instances of agglutination by serum the same explanation must be accepted.

III. We may conclude that the agglutinins by uniting with the agglutinable substances lead to changes in molecular attraction between the elements affected, either as among themselves or as between them and the surrounding fluid. The entire phenomenon of agglutination should be divided into two phases, of which the first may be experimentally produced without the second. The first is a period during which the isolated elements are affected by the agglutinin, and the second a period of agglutination, properly speaking. The individuality of the elements affected counts only in the first phase. During the second phase, cells in obedience to molecular attraction show, in their agglutination, only such peculiarities as occur in the clumping of mineral particles.

IV. The phenomena of agglutination resemble the phenomena of coagulation very closely.

V. The phenomena of true agglutination may be brought about in limpid fluids in which the particles are extremely finely divided.

VI. Active sera may be compared with digestive juices in respect to their coagulating and dissolving properties. It would seem as if immunity would come to be regarded more and more from a chemical standpoint as an instance of the physiology of digestion.

VII. As was previously stated in an article on sera active against blood corpuscles, the production of bacteriolytic substances in animals during the course of vaccination cannot be regarded teleologically. The animal body does not form these harmful sub- 
stances for the purpose of defending itself, but simply puts into action preëxisting functional capacities that, under proper conditions, might act on such innocuous substances as red blood cells or milk casein, as well as bacteria. The special properties that are found in the sera of vaccinated animals are present in a primitive form in normal sera. This fact probably has a distinct bearing on the specific nature of these substances in immune sera. 


\title{
VIII. THE AGGLUTINATION AND DISSOLUTION OF RED BLOOD CELLS BY SERUM.* (SECOND MEMOIR.)
}

\author{
BY DR. JULES BORDET.
}

In the present article we shall offer certain facts in addition to those recently published. $\dagger$ In our preceding memoir we enumerated the properties found in serum of guinea-pigs that have received several injections of defibrinated rabbit blood and we insisted on the close analogy between the properties of such a serum and those of an antimicrobial serum like cholera serum.

We shall now consider, first, whether the cellulicidal property is the sole characteristic of antihematic sera or whether there are, in addition, certain antitoxic properties. We shall then endeavor to draw a still further comparison between antihematic sera and antimicrobial sera. For example, we shall consider whether an antihematic serum injected into a normal animal endows its fluids with a cellulicidal property, for we know that the injection of cholera serum into an animal gives rise in the serum of the recipient to an intense bactericidal power.

A comparison must be drawn between the important properties of these different sera. It does not suffice simply to compare antihematic sera with antimicrobial sera. We must still further consider whether normal sera and specific sera have any characters in common, and whether the active properties which develop and are specialized as a result of immunizing injections are present in a primitive form in normal animals. It is already known that the majority of normal sera have a faculty of agglutinating and destroying alien red blood cells and also certain bacteria in some degree. These evident analogies must be carefully outlined.

* Agglutination et dissolution des globules rouges par le sérum. Annales de l'Institut Pasteur, 1899, XIII, p. 273.

$\dagger$ See article, page 134. 


\section{The Properties of Antihematic Sera.}

The active serum used in our previous work was furnished by guinea-pigs that had received several injections of defibrinated rabbit blood. This serum has a harmful effect on rabbit corpuscles in that it agglutinates them and brings about their dissolution. In other words, it attacks the identical cells which have been used to inject the animals that have furnished the serum.

But it might well be imagined that such a serum also possesses "defensive properties" in addition to "attacking properties."

Let us consider, for example, the serum of a rabbit that has been injected with normal hen blood. Normal hen serum has the property of agglutinating and dissolving rabbit corpuscles. It may well be, then, that the rabbit that has been "vaccinated" against hen blood should furnish a serum that is able, not only to attack hen red blood corpuscles energetically, but also to defend rabbit corpuscles against the harmful effect of hen serum. In other words, our active serum might be endowed to a certain extent with antitoxic properties in addition to the antihematic property, which is comparable to the antibactericidal property of cholera serum; in such a case the toxin would be hen serum. We shall consider these sera, then, from two standpoints, first as regards their antihematic property and secondly as regards their antitoxic property.

A. Antihematic property. - The antihematic property is present in the serum of animals treated with several iniections of defibrinated blood from a different animal species.* The serum of rabbits that have received intraperitoneally several injections of 10 c.c. each of defibrinated hen blood shows properties similar to those found in the serum of guinea-pigs treated with rabbit blood. Although normal rabbit serum has only the faintest agglutinating and dissolving effect on hen red blood corpuscles this active serum agglutinates and dissolves them energetically. This action, however, is

* Rabbits that have received six intraperitoneal injections of 10 c.c. of defibrinated rabbit blood show no particular property in their serum. This is reasonable enough. The production of the active substances found in serum is evidently due to a stimulation of the cells in the animal body on the introduction of foreign substances not normally present, which may have some effect on the cell, or, in other words, which may lead to a change in the chemical or physical constitution of the cell. 
only on the protoplasm of the corpuscle and does not affect the nucleus.

A microscopical examination of a mixture of hen blood and active serum shows that the red blood corpuscles are clumped in more or less compact masses and reduced to their nuclei. Nothing remains of the protoplasm but a very delicate stroma. On staining with eosin followed by methylene blue no protoplasmic outline is visible; the affinity of protoplasm for eosin has disappeared; the nuclei, however, stain blue as usual. Such lesions of the red blood corpuscles, however, are not characteristic of an active specific serum alone, but may occur when hen corpuscles are placed in contact with a sufficiently active normal serum, as for example dog serum. The active serum is distinguished only by the remarkable intensity of its properties against the corpuscles in question.

The destructive property is destroyed on heating the serum to 55 degrees, but may be restored by the addition of fresh normal rabbit or guinea-pig serum to the heated serum. This fresh normal serum contains, as we know, alexin.* The heated active serum retains both its agglutinating property and the property of forming with alexin a mixture that has intense hemolytic power. We shall not insist further on the significance of these facts nor on the analogies between antimicrobial sera and antihematic sera in this respect, since they have already been pointed out in our preceding article.

If defibrinated hen blood, to which heated active serum has been added, is introduced into the peritoneal cavity of a normal rabbit, a rapid destruction of the protoplasm of the corpuscles may be noted under the influence of the alexin from the peritoneal exudate. The nuclei of the corpuscles, deprived of their protoplasm and resisting the action of the body fluid in vivo as well as in vitro, are found in such an exudate. These nuclei are later taken up by the macrophages of the peritoneal cavity.

The question arises as to whether an antihematic serum from a treated animal will endow a normal animal of the same species with a "passive immunity" analogous in its characteristics to that

* The normal serum may be replaced by the peritoneal exudate from a normal rabbit which also contains alexin. 
afforded by injecting antibacterial sera. We know that cholera serum when injected into a normal animal causes a remarkable phenomenon: the serum of the treated animal becomes bactericidal for the cholera vibrio.* It is important to determine whether the serum from a rabbit injected with a serum active against hen corpuscles becomes destructive for these corpuscles.

A small amount of blood is taken from a normal rabbit and gives serum A. After bleeding, the rabbit is given 10 c.c. of active serum subcutaneously. On the following day the animal is bled again and serum $B$ is obtained from this bleeding. The hemolytic properties of serum A may be compared with those of serum B. It is found that serum $B$ has a distinct hemolytic property for hen corpuscles, whereas serum $\mathrm{A}$, from the normal rabbit, has only the faintest activity. Serum B also has an agglutinating property, which, however, is slight. This property has been transmitted, although much weakened by dilution in the fluids of the normal rabbit. It is found, indeed, that although the active serum employed agglutinates three or four volumes of hen blood if diluted with 20 to 25 parts of normal salt solution it causes no rapid agglutination in any dose. $\dagger$ We have already noted the fact that agglutinins when injected subcutaneously into a normal animal go into the blood rapidly. $\neq$

The most natural conclusion to be drawn from this experiment is that the active substances in serum, when injected subcutaneously, are simply diluted in the fluids of the recipient. It follows, therefore, that the serum of this latter animal should acquire to a less degree all the properties which characterize the active serum injected. And this, in fact, is what happens. When heated to 55 degrees, serum B loses its destructive property, but recovers it on the addition of a little of serum A (normal serum). It is scarcely necessary to note that experiments such as these are controlled

* This important fact was noted for the first time by Fraenkel and Sobernheim, Hygienische Rundschau, 1894, No. 1.

$\dagger$ Under these conditions the agglutination occurs only after a considerable period. The same is true of serum $B$. When agglutination is very slow heated sera must be used.

I See article, page 56. Agglutinins injected under the skin are also found in the peritoneal exudate, but in smaller amounts than in the blood. (The experiment to demonstrate this fact was done with cholera serum.) 
with accuracy as to dosage. For example, in such an experiment the following mixtures would be made:

Mixture (a): one part of defibrinated hen blood; four parts of serum A (heated for one-half hour to 55 degrees); three parts of unheated serum A. This is the control.

Mixture (b): one part of defibrinated hen blood; four parts of serum B (55 degrees); three parts of unheated serum A.

A drop from each mixture is suspended on a hollow ground slide. In mixture (a) the corpuscles remain intact. On the following day a few free nuclei are found, but the destroyed corpuscles are in the great minority. As we already know, normal rabbit serum has a slight hemolytic activity. In mixture $(b)$ the destruction of red blood cells occurs rapidly, so that at the end of an hour there are nothing but free nuclei left.

In comparing the dissolving activity of different fluids in such an experiment, care must be taken to keep them at the same temperature. The dissolution of red blood cells is very much accelerated by heat, as is the granular transformation of the cholera vibrio.

As this experiment clearly shows, there is no reason to suppose that the injection of active serum into normal animals brings about any secretion of a particular dissolving alexin which is not present in normal serum. To all intents the substances injected have been simply diluted in the body fluids of the recipient. Any experiments that can be performed with active serum may also be done with the serum of a passively immunized animal.

Consequently, if we regard the proper substances of the active serum as simply diluted in the body fluids without any further change on injection into normal animals, we should also expect to obtain a fluid which is exactly as active as serum B, by mixing serum A with a certain amount of the active serum employed. Such a mixture of active serum and serum A, should be made, to be sure, in proportions that are comparable to the relation between the amount of active serum injected and the amount of fluid in the animal body.

This is found to be true. In the preceding experiment serum B (which was obtained from a rabbit that weighed about 2000 grams and had received 10 c.c. of active serum) shows activity similar to that obtained by diluting 1 part of active serum with 20 parts of serum A. 
This experiment may be done more exactly as follows: A normal guinea-pig of 600 grams is given 3 c.c. of guinea-pig serum active against rabbit corpuscles. A normal serum has been previously obtained from this guinea-pig before injection (serum A). Serum B is obtained by bleeding the guinea-pig 24 hours after injection. A mixture is made of one part of active serum (the same as used for injection) and nineteen parts of serum A (equals serum C). This mixture and also serum $\mathrm{B}$ are then heated for half an hour to 55 degrees to destroy the alexin, since the amount of alexin in these two fluids might differ. A comparison of the power of sera $\mathrm{B}$ and $\mathrm{C}$ to form a dissolving mixture for rabbit corpuscles with normal serum A containing alexin, may then be made. This is done by determining the smallest amount of $\mathrm{B}$ and of $\mathrm{C}$ respectively, which, in the presence of a given amount of serum A, will completely dissolve a given quantity of red blood cells. B is found to be slightly less active than C. From the result we should conclude that the active serum when injected subcutaneously in a guinea-pig of 600 grams is simply diluted in slightly more than 60 c.c. of body fluid. This dilution corresponds pretty well to what actually takes place.

The different facts considered up to this point, namely, concerning the identity in action of the sera on corpuscles whether in vivo or in vitro, lead us to the conclusion previously offered for antimicrobial sera. Antihematic sera have no particular cellulicidal substance; the cellulicidal substance (alexin) destroyed at 55 degrees is present, not only in immunized animals, but also in normal ánimals. Although it is only slightly active in normal serum it acts energetically in the serum of treated animals because it occurs there in conjunction with the proper substance of this active serum which resists heating to 55 degrees or 60 degrees and favors the action of the alexin. The intense cellulicidal power which appears in the serum of a normal animal following the injection of active serum is not due to a reaction on the part of the animal nor to any transformation of the substance injected, but simply to the encounter within the animal body of the two substances that are necessary to form intense cellulicidal power. The animal already contained one of the substances, the alexin, and the other, a specific substance characteristic of active serum, is furnished by the injection. The union of these two substances, which occurs in the animal body, may also be produced in vitro by simply mixing normal serum with either intact or previously heated active serum.*

* We shall not consider at this point in all its details the conclusions that we offered in 1895 concerning antimicrobial sera (see p. 79) applicable in this instance to antihematic sera. 
The specificity of rabbit serum active against hen blood.-We have not done many experiments on this subject, but it seems certain that this serum is quite as specific from all appearances as are antimicrobial sera. This active rabbit serum naturally enough has no effect on rabbit corpuscles and it also has no more effect on guinea-pig corpuscles than has normal rabbit serum; nor does it have a distinctive action on pigeon, human or mouse corpuscles. With none of these corpuscles is the agglutination and energetic dissolution that occurs with hen corpuscles to be noted.

The fixation by corpuscles of the specific substances of antihematic serum. - Corpuscles that are susceptible to a given antihematic serum fix the active substances of this serum. We have already considered this fact in a recent article;* and have also noted that bacteria absorb all the active substances from antimicrobial sera in a similar manner. Corpuscles that are not affected by a serum do not absorb its active properties and this remark applies to normal sera as well as to specific sera. For example, if normal hen serum is added to rabbit corpuscles the corpuscles are energetically agglutinated; and the supernatant fluid is found to be deprived of agglutinating properties for fresh rabbit corpuscles. But if hen serum is placed in contact with corpuscles that it affects only slightly, for example, with guinea-pig corpuscles, the supernatant fluid still retains its agglutinating property for susceptible corpuscles.

The technique employed in absorbing the active principles of an antihematic serum follows: the active serum employed is heated to 55 degrees for half an hour and thereby deprived of its dissolving effect. The following mixtures are then made in test tubes:

$A, 3$ c.c. of normal rabbit serum plus 0.5 of a cubic centimeter of active guineapig serum.

$B, 3$ c.c. of defibrinated rabbit blood plus 0.5 of a cubic centimeter of active guinea-pig serum.

The first tube is a control. Both tubes contain equal amounts of active serum diluted in a similar manner. The second tube, however, contains corpuscles, whereas the first does not. After contact the tubes are centrifugalized and the supernatant fluids decanted. The clear fluids thus obtained are tested for agglutinating property. Fluid A, which has not been subjected to corpuscles, agglutinates rabbit corpuscles energetically whereas fluid B does not agglutinate- them. A determination is then made of the power of the two fluids to form hemolyzing mixtures with alexin, as follows:

* Mechanism of agglutination, p. 142. 
$a$, one part of normal defibrinated rabbit blood plus one part of fluid A plus five parts of fresh normal guinea-pig serum.

$b$, one part of normal defibrinated rabbit blood, one part of fluid B, five parts of fresh normal guinea-pig serum.

Dissolution occurs rapidly in tube $a$, but the corpuscles remain intact in tube $b$.

It is evident that red blood corpuscles fix the active substances in antihematic sera with avidity. It is also found that the effect of these substances on the corpuscles that leads to their agglutination and sensitization to alexin is very profound, since it is not eliminated by several washings. These washings are easily accomplished. Several drops of salt solution containing numerous corpuscles are placed in a test tube* and a small amount of active serum, heated to 55 degrees and so deprived of dissolving effect, is added. The tube is filled with salt solution, shaken and centrifugalized; the supernatant fluid is decanted; fresh salt solution is added to the deposition of corpuscles and the washing repeated several times until a deposit of well-washed corpuscles is obtained.

These washed sensitized corpuscles are as easily dissolved by a dose of fresh normal serum as are unwashed sensitized corpuscles.

These facts seem to prove that the particular substance present in the serum of vaccinated animals which resists heat and permits the energetic action of the alexin acts upon the corpuscles themselves in such a way as to sensitize them to the action of the alexin. We might have supposed, indeed, that this particular substance acted, not on the corpuscles, but directly on the alexin in a way to render it more energetic. This latter hypothesis, however, is not in accordance with facts and, moreover, would not explain so well the evident specificity. It would seem to be demonstrated by the preceding experiment that in a mixture of corpuscles, active heated serum, and normal serum there is no reaction in the fluid part of the mixture between the alexin and the particular substance of the active serum. This is shown by the fact that the specific substance rapidly separates from the fluid and fixes itself on the corpuscles, which then become susceptible to the subsequently added alexin; this is shown by adding it only after the sensitizing substance has been combined

* In such an experiment the corpuscles employed have been previously washed to remove the serum present in defibrinated blood; in such an experiment no trace of alexin should be left. 
with the corpuscles. Similar experiments may be made with bacteria and their corresponding antisera.* We may consider, then, the conception that we previously arrived at, namely, that specific sera contain a sensitizing substance that renders the corpuscles or bacteria susceptible to attack on the part of the alexin, as sufficiently proved.

We have not yet considered the close analogies between the properties in the serum of a guinea-pig active against rabbit blood and those present in the serum of a rabbit active against hen blood. There is, however, a difference between these two sera that should be noted.

As we have already noted in a previous article, active guinea-pig serum heated to 55 degrees when added to defibrinated rabbit blood still produces a slight effect on these corpuscles, as is shown by a distinct reddish coloration of the fluid. This is due, as we have already noted, to the fact that these corpuscles are slightly attacked by the small amount of rabbit alexin present in the defibrinated blood. If, indeed, corpuscles previously washed in salt solution are used instead of ordinary defibrinated blood, no dissolution takes place; that is to say, the alexin has been eliminated by washing. On the other hand, if to such washed corpuscles, subsequently sensitized, a sufficient dose of normal rabbit serum is added, complete and rapid dissolution occurs. Rabbit corpuscles, then, under the influence of the sensitizing substance, become susceptible to the action of their proper alexin. We should expect that hen corpuscles when treated with active heated serum (from a rabbit injected with hen blood) would dissolve in normal hen serum. Such dissolution, however, does not-occur. In spite of the effect of the sensitizing substance hen corpuscles do not become susceptible to hen alexin.

This shows clearly enough that the alexins are not quite identical in different animal species. Their more important characteristics are common; that is, they act more or less in the same way on a given bacterium, but they show differences in their action upon red blood cells. The idea, moreover, was a priori evident from a study of the effect of normal sera on corpuscles, from which we

* Cholera vibrios treated with cholera serum and then carefully washed show granular transformation upon the addition of normal serum or when introduced in the peritoneal cavity of a normal guinea-pig. 
learned that the red blood cells of an animal are not attacked by the alexin from the same animal, whereas they are susceptible to alexins from other animals.

We might compare in a very gross way the modification which the sensitizing substance causes in the corpuscles by likening it to a change in the structure of a lock by means of which one or several keys that previously could not open it are enabled to do so. Any two keys that are sufficiently alike would enter the lock indifferently. In the same way the alexins, both of the guinea-pig and of the rabbit, "enter" rabbit corpuscles or hen corpuscles once these corpuscles have been sensitized. On the other hand, hen alexin, that apparently differs too greatly from guinea-pig or rabbit alexin, does not "enter" hen corpuscles even when they are previously sensitized. It may be that the entrance or the efficient working of the alexin depends, not only on the nature of the alexin, but also on the nature or the particular origin of the sensitizing substance. There are evidently many experiments yet to be made. In the absence of sufficient data we shall content ourselves for the moment with pointing out a method of obtaining some insight into the mode of action of these substances.

The effect of heat on the active substances of serum. - On heating rabbit serum, active against hen corpuscles, to 55 degrees the agglutinating and sensitizing properties persist, whereas, as we have already seen, the dissolving property is suppressed.

On heating for half an hour to 65 degrees, both the agglutinating and the sensitizing power are still intact; heating to 65 degrees diminishes the agglutinating property slightly, and it becomes very weak on heating to 70 degrees for the same length of time. It should be remarked at this point that unheated serum when previously diluted with, say, 20 parts of salt solution has no visible agglutinating effect in any dose, which shows that the agglutinin must be present in a certain state of concentration in order to affect corpuscles, and, moreover, when the phenomenon is absent no conclusion can be drawn as to complete absence of the active substance. The sensitizing substance, however, is still distinctly manifest in serum heated to 70 degrees and also in serum that has been diluted with 20 parts of salt solution. One part of defibrinated blood mixed with two parts of serum heated to 70 degrees plus four parts of 
normal rabbit serum, is rapidly hemolyzed. But, with the same amount of defibrinated blood and normal serum, the addition of relatively small doses of active serum heated either to 60 degrees or to 70 degrees shows that dissolution takes place more slowly with the serum heated to 70 degrees; that is, the sensitizing property of the serum has been diminished by heating to 70 degrees. It is very attenuated, but is not completely destroyed by heating for half an hour to 75 degrees.

It may be noted that a mixture of defibrinated hen blood, normal serum, and active serum heated to 70 degrees is hemolyzed without any preliminary agglutination; the corpuscles are soon reduced to separate nuclei. If the agglutinin is not destroyed it has at least become too feeble or too small in amount to produce clumping; sensitization, however, still goes on.

Precipitating property. - In addition to the antihematic property in active rabbit serum we should note its property of forming a precipitate with normal hen serum. We have already considered this question in a preceding article* and in this connection we have mentioned the researches of Tchistovitch, to whom we owe the first observations of a similar phenomenon. When the two sera are mixed a turbidity appears that at first is slight but soon increases and finally condenses into flecks. The most abundant precipitate is formed by a mixture of one or two parts of hen serum with eight or nine parts of active serum.

If, instead of treating rabbits with hen blood, hens are treated with rabbit blood, their serum is found to precipitate normal rabbit serum. The phenomenon of precipitation, however, does not occur regularly in all instances examined; the serum of guinea-pigs treated with rabbit blood, for example, causes no precipitate with rabbit serum. A mixture of sera from normal animals of different species never causes a precipitate.

B. Antitoxic property. - It seemed probable that we should be able to produce sera endowed with an antitoxic property and capable of opposing the destructive action of certain given sera on

* See p. 142. We shall not here consider the significance of this phenomenon. We have already mentioned the effect of heat on the precipitating property and determined to what extent the precipitins are specific. We may recall that the serum in question (serum rabbit $>$ hen) also precipitates pigeon serum. 
red blood cells. As a matter of fact, Camus and Gley and Kossel have shown that in the serum of animals immunized against cel serum there are substances that protect their corpuscles against the dissolving effect of this toxic serum.

In order to make such results evident the "toxin" employed should be powerful. Animals of species A should be injected with serum or defibrinated blood of species B, and the serum of species B should be able to agglutinate and dissolve the red blood corpuscles of species A energetically. The rabbit and the hen fulfill these conditions: the agglutinating and dissolving effect of normal hen serum on rabbit corpuscles being very energetic. The antihematic property of rabbit serum for guinea-pig corpuscles, on the contrary, is only slight; and therefore the serum of a guinea-pig vaccinated against rabbit blood is not suited to the study of this antitoxic or antihemotoxic property.

Normal hen serum agglutinates an equal amount. of defibrinated rabbit blood energetically. Two or three parts of this serum will dissolve one part of rabbit blood corpuscles. Rabbits that have received intraperitoneally three injections of 10 c.c. each of hen blood have an antitoxic property in their serum, although it is not very intense. It may be demonstrated in a mixture containing normal rabbit blood, hen serum (fresh) and active rabbit serum; and, as a control, normal rabbit blood, fresh hen serum and normal rabbit serum. The exact proportions in these tubes are as follows:

Tube A, one part of normal rabbit blood; three parts of hen serum; ten parts of active rabbit serum.

Tube B, one part of normal rabbit blood; three parts of hen serum; ten parts of normal rabbit serum. In the second of these tubes the rabbit blood corpuscles rapidly collect into thick compact masses that after two or three hours hemolyze. In tube "A" the corpuscles remain indefinitely intact and only a slight agglutination in very small clumps is evident. In addition to a property of preventing the dissolution of corpuscles the active serum has a distinct anti-agglutinating property.

The control mixture "B" shows that normal rabbit serum has no antitoxic properties.

If the amount of active serum is diminished to any extent or the amount of dissolving hen serum is increased, the antitoxic effect 
is no longer detectable. So, for example, in a mixture of one part of rabbit blood, three parts of active serum and six parts of hen serum the corpuscles are dissolved without any appreciable delay.

In order to determine more clearly the effect of the "anti-agglutinin" in the serum, hen serum heated to 55 degrees and therefore without dissolving property may be used; such a serum still agglutinates very energetically an equal volume of rabbit blood. For example, the following mixtures are prepared:

Tube A, defibrinated blood, one part; active serum, ten parts; hen serum, 55 degrees, one part.

Tube B, defibrinated blood, one part; normal rabbit serum, ten parts; hen serum, 55 degrees, one part.

Agglutination is rapid and very strong in mixture " $\mathrm{B}$ " and is negative or extremely feeble in mixture "A."

When the active serum is mixed with hen serum a precipitate is formed. We might imagine that the active serum does not contain, strictly speaking, an antitoxic substance, but that the precipitate in its formation removes the harmful substances of the hen serum and so renders it inactive. This objection, however, is not well founded, as is shown by the fact that active rabbit serum heated to 70 degrees loses the property of producing a precipitate with serum, but still manifests its anti-agglutinating properties. It retains, moreover, the property of preventing the dissolution of corpuscles by hen alexin.

This shows that the substance that opposes destruction by alexin is quite different from alexins themselves.

The serum of a guinea-pig vaccinated against rabbit blood also shows antitoxic properties, but only to a slight extent.

\section{Analogies Between Specific and Normal Sera. Anal- ogies Between Substances Active Against Bacteria and Those Affecting Blood Corpuscles.}

It is well known that the various properties in specfic sera are also present to a slight degree in normal sera. They are manifest by an agglutinating action or dissolving action either on bacteria or on corpuscles. It is quite natural to believe that the properties acquired by the animal body as a result of immunization are only 
a perfectioning of, or an increase in, preëxisting-properties, and it is therefore of interest to ascertain whether the active substances in the sera of vaccinated animals have characters in common with similar substances in normal sera and whether, indeed, the two are identical. For example, we may compare the antihematic properties which are so strongly marked in the serum of animals treated with defibrinated blood with similar properties present in normal sera.

The property of agglutinating and dissolving alien red blood cells is found very commonly in normal sera. We know that these properties affect the corpuscles from different animal species only. Following is a recapitulation of the agglutinating power of certain normal sera on certain varieties of red blood cells. In these experiments we usually mixed one part of defibrinated blood with three or four parts of serum:

Guinea-pig serum agglutinates the corpuscles of the rabbit and the hen slightly and rat corpuscles more energetically.

Rabbit serum has slight agglutinating properties for the red blood corpuscles of the guinea-pig, man, the hen and the rat.

Hen serum has an energetic agglutinating property for the corpuscles of the dog and rat and especially for the rabbit. Its agglutinating action on guinea-pig corpuscles is slight. It agglutinates pigeon corpuscles rather strongly.

Pigeon serum has a very weak agglutinating property for the corpuscles of the hen, the rabbit, man, and also for bacteria.

Dog serum agglutinates the corpuscles of the rabbit and the rat strongly, and the corpuscles of the guinea-pig and the hen faintly.

Rat serum agglutinates the corpuscles of the rabbit and the guinea-pig slightly.

Goat serum agglutinates the corpuscles of the rabbit and the guinea-pig distinctly.

Horse serum agglutinates the corpuscles of the guinea-pig, the rabbit and the hen distinctly and the corpuscles of the rat energetically.

The dissolving properties of these animal sera for red blood corpuscles are always due to the alexin, or thermolabile substance. It is a general rule, applicable to all sera, that the 
power to destroy red blood corpuscles disappears on heating to 55 degrees.*

The most destructive and powerful action that we have noted in studying sera is the effect of hen serum on rabbit corpuscles. One part of defibrinated rabbit blood is rapidly dissolved by two or three parts of hen serum. The destructive effect of this serum for rat corpuscles is also very distinct; it is very slight for guinea-pig corpuscles.

Dog serum dissolves hen corpuscles energetically, although it leaves their nuclei intact; it also attacks rabbit and guinea-pig red blood corpuscles.

Guinea-pig serum and rabbit serum have both a very slight destructive effect on hen and human corpuscles. Guinea-pig serum has only a very slight and delayed effect on rabbit corpuscles. Rabbit serum, however, is much more active against guinea-pig corpuscles, but is almost entirely without effect on rat corpuscles.

It should be noted in these tests that the agglutinating and the dissolving property differ in different specimens of the same variety of normal serum; that is, there are individual differences which may be rather marked.

No general rules can be drawn from these facts. There is no means, for example, of classing corpuscles in groups aceording to their sensitivity to agglutinating or dissolving action.

The corpuscles of a given species may be very easily agglutinated by one normal serum and resist another. In the same way it cannot be shown that a serum that shows itself very active for one species of corpuscles will be equally active for other varieties of corpuscles. For example, hen serum affects rabbit, dog and rat corpuscles, but has almost no effect on guinea-pig corpuscles. Nor can we assert, as a general rule, that a serum that is capable of dissolving a given species of corpuscles will necessarily agglutinate them energetically. For example, although hen serum dissolves rabbit corpuscles easily and also agglutinates them strongly, we find, on the other hand, that dog serum agglutinates hen corpuscles only faintly, although it destroys them actively. It is evident, then, that

* There is, however, an apparent exception. Dog red blood corpuscles are still dissolved by sera heated to 55 degrees. These corpuscles, however, are also dissolved by their proper serum. It is, therefore, not a question of alexic activity, but of a particular fragility on the part of dog corpuscles. 
there are two distinct factors concerned in the effect of sera on corpuscles: First, the greater or lesser amount of active substances in the serum and, secondly, the particular sensitivity of the corpuscles in question to the substances in the serum employed. Corpuscles may be very susceptible to the effect of the active substances of one serum and much more refractory to those in another normal serum. Although the agglutinins in different normal sera are similar in their method of action they are not identical; there must be certain slight differences of chemical constitution between them to explain the diversity of their effect on a given kind of corpuscles. The same remarks apply also to the alexins from different animal species. The corpuscles, too, of different species differ in constitution and each variety has its particular way of reacting with a given alexin or a given agglutinin.

We have, moreover, offered in our memoir on the mechanism of agglutination an experiment which indicates that a given normal serum contains several different agglutinins.

$$
*^{*} *
$$

It is well to consider how great a resemblance there is between substances with similar properties in different sera. For example, we may compare the agglutinins of normal sera with those of immune sera and the agglutinins and sensitizing substances for blood corpuscles with the corresponding substances for bacteria.

We have already frequently repeated that, in the matter of alexins, normal sera do not differ from immune sera, whether antimicrobial or antihematic.

And, what is more, the alexins that affect corpuscles seem to be quite identical with those that affect bacteria. Both the property of changing red blood corpuscles and the power of producing a granular transformation of vibrios, are frequently met with among sera. Vibrios, especially when sensitized by cholera serum, are readily changed into granules when placed in contact with normal serum from the rabbit, guinea-pig, goat, hen, dog, rat or pigeon; only a slight difference in intensity of action is shown between these different sera.* These sera lose their activity against bacteria when

* We are dealing here, it may be stated, with those bactericidal effects which depend on alexins and which do not oceur with sera heated to 55 degrees. The typical example of this activity is the granular transformation of vibrios. There 
heated to 55 degrees; and at the same time are deprived of their activity against corpuscles. The differences in alexins from different sources, so far as their action on corpuscles is concerned, are not to be reckoned with when dealing with vibrios.

Although the presence of alexins is not distinctive of the sera of vaccinated animals, since they occur in the same form in normal serum, the agglutinating properties of the serum of vaccinated animals are clearly to be distinguished from those of normal sera as more intense and specific. It is worth considering, however, whether the agglutinins in immunized animals, in spite of these differences, are affected in the same way by heat as are the agglutinins of normal sera.

We may begin by establishing to what extent heat weakens the agglutinating power of a given normal serum for corpuscles and for bacteria respectively. For such an experiment bacilli easily clumped by various normal sera are chosen. We have particularly employed the bacillus typhosus. Without going into the details of these experiments we may say in general that a given high temperature causes a normal serum to lose its property of agglutinating both bacteria and cells to an equal extent. In the sera that we have tried the agglutinating properties remain unaffected after heating to 55 degrees for half an hour. Heating to 61 to 62 degrees for the same period diminishes but does not entirely destroy the agglutinating properties of guinea-pig, goat, dog, horse and hen serum. With poorly agglutinating sera there is practically no clumping action after heating to this degree; when dealing with strongly agglutinating sera it is still very distinct after such treatment. Hen serum, for example, even after heating to 67 degrees still agglutinates rabbit corpuscles distinctly. As the temperature is raised the agglutinating power becomes weaker and weaker, so that normal sera heated for a half hour to 70 degrees no longer agglutinate.

are, however, in certain sera bactericidal substances quite distinct from alexins. Rat serum heated to 55 degrees is quite incapable of producing a transformation in cholera vibrios, sensitized with cholera serum, although this property is present in intact rat serum. But such heated rat serum destroys the anthrax bacillus with apparently undiminished activity. Sawtchenko has already noted that this particular bactericidal property of rat serum for the anthrax bacillus resists heating to from 55 to 60 degrees. 
Normal rabbit serum is the one in which agglutinins best resist heating, so far as we have studied them. Rabbit serum must be heated to approximately 65 degrees to cause any distinct diminution in agglutinating power either for corpuscles or bacteria.

When we come to compare the effect of heat on the normal and on the specific agglutinins we find very distinct correspondence. In such experiments it should be remembered that specific agglutinins are very much more energetic and, consequently, any diminution in their activity is less evident than in normal sera, the energy of which is slight to begin with. In experiments on the sera of the goat, rabbit, or guinea-pig vaccinated against the cholera vibrio, and the serum of the dog vaccinated against B: typhosus, the specific agglutinating property is distinctly diminished by any temperature that weakens a normal agglutinating power; in the case of the guinea-pig and the goat this diminution is evident after heating to 61 to 62 degrees; and the sera heated to 70 degrees are almost inactive. The agglutinins in immune rabbit serum are much more resistant; and, as we have just seen, the same fact holds true for the normal agglutinins in normal rabbit serum. Two cholera sera coming from a rabbit and a guinea-pig respectively resist heat differently; on heating them to 70 degrees there is marked diminution of activity in the specific guinea-pig serum, but only a very faint diminution in the specific rabbit serum. It may be noted that rabbit serum also resists coagulation by heat better than does guinea-pig serum. Guinea-pig serum mixed with equal parts of normal salt solution becomes opalescent, but is not coagulated, on heating to 65 degrees for a half hour; rabbit serum treated in the same way does not even become opalescent; it becomes faintly opalescent only on heating to 70 degrees. We need scarcely emphasize the fact that there is apparently some close relation between the appearance of a coagulation or opalescence and the deterioration of active substances.

In short, it may be said that agglutinins, whether affecting corpuscles or bacteria, are similarly affected by heat whether they occur in normal sera or in specific sera. There is a progressive diminution in activity as the temperature is raised, but it is impossible to define a critical temperature below which they remain intact and above which they are destroyed. Agglutinins appear to be more resistant 
in the serum of certain animals than they are in the serum of other animal species.

Since heat weakens the agglutinating property of sera for corpuscles or bacteria, the question arises as to whether the property of sensitizing cells to the action of alexin present in specific sera is diminished in a similar way. The question is not without interest, as it may throw some light on the problem of whether the phenomenon of sensitization is due to the same or different substances than the phenomenon of agglutination. We shall not undertake to consider this subject here, but reserve for a future time a discussion of the significance of facts bearing on this question.*

We shall content ourselves with noting that the exposure of specific sera to temperatures ranging from 65 to 75 degrees, which distinctly enfeebles their agglutinating and immobilizing power, also distinctly diminishes their sensitizing power. When cholera serum from the rabbit has been heated to 70 degrees it is less energetically agglutinating for the vibrio and is also slightly less sensitizing. Such a diminution in strength may be demonstrated by placing vibrios in contact with small doses of the serum heated to 55 degrees and to 70 degrees respectively. These emulsions are not equally well agglutinated. On adding normal serum to each emulsion it is found that the granular transformation is much less extensive in the emulsion containing serum heated to 70 degrees than in the one with serum heated to 55 degrees. A similar difference in transformation may be noted in vivo on intraperitoneal injection of the emulsion. Guinea-pigs receiving the emulsion with serum heated to 55 degrees have an exudate showing a granular transformation of the vibrios, and the animals recover; in the other animals motile vibrios remain and a fatal infection ensues. If more than minimal doses of serum are employed, the difference between the two emulsions naturally is not so distinct; we may repeat that the weakening effect of heat on the properties of the serum is only partial. A

* Agglutination is a complex phenomenon. For example, when motile bacteria are concerned agglutination includes and necessitates an immobilization of these bacteria. It has not been demonstrated, so far, whether the two phenomena of immobilization and agglutination are due to the same substance. It would seem probable when we consider the experiments that we have already performed and particularly those dealing with the effect of salt solution on agglutination that agglutination depends on the coöperation of several different factors. 
similar diminution, as already stated, may be noted on heating rabbit antihen serum to a temperature of 70 degrees.

The sensitizing property of cholera serum from the guinea-pig is weakened by heating to a lower temperature than 70 degrees, as is true of its immobilizing and agglutinating property; the weakening, indeed, is very distinct even on heating to 65 degrees.

Are there sensitizing properties in normal sera? Is not the action of the alexin in these sera aided by some other substances? It is difficult to answer these questions because, so far, we have not succeeded in separating alexins from the other substances present in serum. On combining two normal sera, however, the existence of a sensitizing property may sometimes be shown. For example, cholera vibrios, immobilized and agglutinated by horse serum, are easily transformed to granules on the addition of normal guineapig serum. No generalized statement, however, should be made on the basis of this observation; it is not safe to conclude that it suffices to agglutinate bacteria or corpuscles in order to sensitize them to the action of alexin. For example, rabbit red blood corpuscles, although strongly agglutinated by heated hen serum, are no more susceptible to the slight dissolving effect of normal guinea-pig serum than are normal rabbit corpuscles. The presence of agglutination, then, does not indicate any particular sensitivity to alexin. An answer to these questions will necessitate further study.

\section{Conclusions.}

I. The serum of animals treated with defibrinated blood from a different animal species shows active properties, consisting in the agglutination and energetic dissolution of corpuscles similar to those used for injection. There is also in certain instances a power to produce a precipitate with serum (or defibrinated blood) similar to that used in immunization.

II. The dissolving action of the active serum on corpuscles is due to the presence of two substances: one that belongs properly to the active serum; the other (alexin) which occurs not only in active sera but also in normal sera. The first substance acts by sensitizing the corpuscles to the action of the second substance. These facts are strictly comparable with those already established by us for cholera serum and the cholera vibrio. 
III. The injection of an antihematic serum in a normal animal of the same species that furnished the serum causes the appearance of a similar property in the serum of the treated animal. The occurrence of this hemolytic property should be explained as we explained in 1895 the genesis of a bactericidal property in the serum of animals treated with cholera serum. In other words, this antihematic property occurs as the result of a meeting in the animal body of the characteristic substance of active serum with the alexin which the recipient possessed before injection.

IV. The specific antihematic substances characteristic of active serum resist heating to 55 degrees and combine energetically with the corpuscles they act on. Washing does not remove from corpuscles their agglutination or their sensitization to alexin acquired by contact with specific serum. In this respect the analogy to bacteria and their specific sera also holds.

V. Alexins from different animal species although very similar in their action on a given bacterium show distinct differences in their action on corpuscles. The sensitization of given corpuscles to the action of the alexins of certain animal species does not necessarily imply that these corpuscles are equally destroyed by all alexins.

VI. Antihematic sera have also a distinct antitoxic property; they protect their own corpuscles against agglutination and dissolution by the normal serum used in injecting the animals that have produced the active serum.

VII. There is a close analogy between the active substances of specific sera and similar properties that occur in normal sera in respect to the effect of heat; there is also an analogy between the substances affecting bacteria and those affecting red blood corpuscles in this respect. Heating to from 60 to 70 degrees weakens both the agglutinating and the sensitizing property.

VIII. Destruction by alexin may take place in non-agglutinated corpuscles. The fact that corpuscles are clumped by serum does not necessarily imply that they are sensitized to the action of alexins. 


\section{HEMOLYTIC SERA AND THEIR ANTITOXINS, AND THEORIES CONCERNING CYTOLYTIC SERA IN GENERAL**}

\section{BY JULES BORDET.}

\section{Additional Ideas Concerning Hemolytic Sera.}

In the present article we propose to consider first of all certain novel facts concerning antihematic sera; $\dagger$ these sera, as we know, are obtained from animals that have been treated with the blood of other species. We shall then consider the principal properties of an antitoxin capable of opposing the destructive effect of a hemolytic serum on red blood corpuscles. And finally, we shall take up the theories that have been offered to explain the cytolytic properties of various immune sera and shall consider which of these theories are best corroborated by experimental facts.

In the following pages we shall deal with a single hemolytic serum and for this purpose have chosen the serum of guinea-pigs treated with rabbit blood. This is the serum that we first used in describing antihematic sera. It is easy to obtain, as guinea-pigs furnish a very active serum after receiving two or three injections subcutaneously of 3 to 5 c.c. of defibrinated rabbit blood. The serum agglutinates and destroys rabbit blood corpuscles, but has no effect on the corpuscles of other animals.

We described the properties of this serum fully in a previous article (1898); it seems, therefore, scarcely necessary to consider again in detail the effect of the two substances present in an antihematic

* Les sérums hémolytiques, leurs antitoxines et les théories des sérums cytolytiques. Annales de l'Institut Pasteur, XIV, 1900, 257.

† We shall frequently use the expression "hemolytic sera," or, preferably, "hemotoxins" to designate antihematic sera. If the word hemotoxin is used, the antitoxin to a hemolytic serum should be called antihemotoxin. These terms are convenient, They were suggested by Metehnikoff, who called a serum that was active against spermatozoa a spermotoxin and one active against leucocytes a leucotoxin. The expression "cytolytic" sera or "cytotoxins" may be used to designate various immune sera that are able to destroy bacteria or cells like red blood cells. 
serum, namely, the alexin* or cellulicidal substance, properly speaking, destroyed at 55 degrees; and the sensitizing substance or specific antibody (preventive substance) that resists heat much better. We must later consider these facts in considering the theories concerning bactericidal and hemolytic sera. We may mention simply that one of these substances, the alexin, is found, not only in immume serum, but also in normal serum. The function of the sensitizing substance is to render the corpuscles susceptible to the cytolytic influence of the alexin. It is scarcely necessary to repeat in the following experiments that heating to 55 degrees for half an hour destroys the cytolytic activity of the sera employed.

1. The dissolving properties of different alexins in the presence of the sensitizing substance.

We know that our hemotoxin loses its hemolytic activity when heated to 55 degrees and that the addition of normal guinea-pig serum restores its primitive energy; this added serum, however, is only slightly cellulicidal in itself. The same result may be obtained by using normal rabbit serum instead of normal guinea-pig serum: we have already mentioned this remarkable fact and shall frequently return to it, that the alexin in normal rabbit serum which is wholly inoffensive for its proper corpuscles becomes strongly hemolytic for them when associated with a sensitizing substance. $\dagger$ We have then two different alexins, normal rabbit serum and normal guineapig serum, either of which can destroy sensitized rabbit corpuscles. Do the alexins from other animals act in the same way? In other words, does the sensitizing substance increase the destructive property of various normal sera considerably?

An experiment to answer this question shows that sensitized rabbit corpuscles are rapidly dissolved by the normal serum of the rat, the goat and the dog, none of which, however, without the addition of the sensitizing substance is more than faintly destructive.

* We think it quite unnecessary to replace the word alexin by any other; this word was introduced some time ago by Buchner and has been frequently employed by numerous observers and become a customary term. It matters little what word is used provided its significance is understood.

$\dagger$ We may mention once for all that by the word sensitizing substance we mean a hemolytic serum heated to 55 degrees and so deprived of alexin and containing only the sensitizing substance or specific antibody. 
The presence of the sensitizing substance then increases the hemolytic power of these sera distinctly. It also increases to a less extent the activity of normal pigeon serum. The normal sera of the hen and the goose are in themselves very hemolytic for rabbit corpuscles and the addition of sensitizing substance does not appear to increase their properties to any great extent.

It is to be noted that guinea-pig serum is the one that affects corpuscles subjected to sensitizing substance most actively; this serum is much more effective than rabbit serum. Very large doses of rabbit serum are necessary to destroy sensitized rabbit corpuscles when a small dose of sensitizing substance is used.* An analogous fact has recently been observed by Buchner. $\dagger$ According to this observer bovine red blood corpuscles treated with an appropriate sensitizing substance from the rabbit are easily destroyed by normal dog serum. In a general way these facts have been known for some time. Five years ago we mentioned an analogous series of facts in showing that cholera vibrios treated with a little anticholera sensitizing substance (that is to say, immune serum from a goat heated to 55 degrees) were changed in vitro into roundish granules when subjected to normal serum from the guinea-pig, rabbit, rat, man or goat. More recently we added to this list sera of the dog, hen and pigeon. $\neq$ In these instances the sensitized vibrios are affected by the alexins present in normal sera.

The same facts hold for corpuscles.

For completeness we must recall an already published observation. A year ago§ we first mentioned that sensitized corpuscles are not indiscriminately dissolved by any normal serum, or, in other words, by every alexin. For example, sensitized hen corpuscles (hemolytic serum from the rabbit heated to 55 degrees) are readily dissolved by several normal sera, but remain quite intact in normal hen serum. This seems then to be a certain contradiction to the statement that sensitized rabbit corpuscles are destroyed by rabbit serum. When we mentioned this apparent contradiction a

* If the energy of the sensitizing substance is slightly diminished, small doses of rabbit serum will frequently fail to dissolve the corpuscles, although under the same conditions normal guinea-pig serum is still very effective.

$\dagger$ Münchener medicinische Wochenschrift, 1900.

$\ddagger$ See p. 180 .

$\S$ See p. 173. 
year ago we said that the modification of the corpuscle by the sensitizing substance might be likened, as Fischer has done for diastases, to a change in a lock so that keys that could not previously open it were enabled to do so. In such a way certain alexins would "enter" a sensitized corpuscle and destroy it; and other slightly different alexins could not attack it. There must exist, then, certain suitable relations between the sensitizing substance and the alexin employed in order to bring about a destruction of the corpuscles. We shall not insist further on these conceptions, which have, indeed, been made use of by Wassermann in a recent article.*

2. The identity in a given serum of the bacteriolytic and the hemolytic alexin.

A normal serum, say from the guinea-pig, in presence of an anticholera sensitizing substance attacks vibrios and causes their granular transformation. The same normal serum destroys red blood corpuscles in presence of a hemolytic sensitizing substance. When the normal serum is heated to 55 degrees it has no longer either bacteriolytic or hemolytic effect even in the presence of appropriate sensitizing substances. This fact is explained by saying that heating to 55 degrees destroys the alexin, which causes cytolysis. $\dagger$ The question arises as to whether normal guinea-pig serum contains a single alexin that is both hemolytic and bacteriolytic, or several alexins, one or several of which can destroy bacteria and the other, or several of which, preferably attack red blood corpuscles. This question is open to experimental proof.

When normal cholera vibrios are mixed with normal guinea-pig serum they are not destroyed, but are only slightly affected, since, not being sensitized, the alexin cannot react with them. $\neq$ If to such a mixture sensitized corpuscles are added (that is to say, rabbit corpuscles plus hemotoxin heated to 55 degrees) we find that they are at once destroyed, which proves that the normal cholera vibrios have neither transformed nor fixed the alexin necessary for destruction of corpuscles.

We may repeat this experiment with a modification. We may

* Wassermann, Deutsche medicinische Wochenschrift, 1900.

$\dagger$ Heating to 55 degrees destroys the cytolytic property, not only of guinea-pig serum, but also of sera from the rabbit, rat, goat, hen, etc.

$\ddagger$ We already mentioned some time ago (see p. 61,1895 ) that normal serum destroys few vibrios unless they be very attenuated. 
add to the same dose of normal guinea-pig serum cholera vibrios sensitized with cholera serum instead of normal vibrios. Under such conditions the sensitized vibrios show rapid granular transformation. If we then add to the mixture, as we did in the first experiment, sensitized corpuscles we find that they remain indefinitely intact. From this experiment we conclude that the alexin necessary to destroy the corpuscles was used up before they were introduced. In the presence of the cholera sensitizing substance the alexin reacted with the vibrios and, in transforming them into granules, was fixed by them. Consequently the alexin that unites with sensitized vibrios and alters them is identical with the one that produces hemolysis.

Experiment. An emulsion of vibrios is prepared by suspending a 24-hour agar culture in 5 c.c. of salt solution. The cholera sensitizing substance employed is from a vaccinated rabbit and has been heated to 55 degrees. As a control for this active serum normal rabbit serum, also heated to 55 degrees, is used.

Mixture A. 0.5 c.c. of normal guinea-pig serum plus 0.3 c.c. of cholera sensitizing substance plus 0.5 c.c. of vibrio emulsion.

Mixture B. 0.5 c.c. of normal guinea-pig serum, 0.3 c.c. of normal rabbit serum ( 55 degrees) plus 0.5 c.c. of vibrio emulsion.

Mixture C. Identical with A but containing no vibrios.

An hour's contact is allowed and there is then added to each mixture 0.2 of a cubic centimeter of hemosensitizing substance ( 55 degrees) plus 2 drops of washed rabbit blood.*

The results of the experiments are as follows: the corpuscles are rapidly destroyed in $\mathrm{B}$ and $\mathrm{C}$, but remain intact in $\mathrm{A}$.

A similar experiment with the cells (bacteria and corpuscles) used in the inverse order gives a similar result. We know from the work of Ehrlich and Morgenroth as well as from our own that an alexin that does not destroy given red blood cells is not fixed by them, but is, on the contrary, fixed by cells that it can dissolve.

An alexin that under normal circumstances has no effect on a given species of corpuscles and therefore is not fixed by them is fixed by these same corpuscles when they have been treated with a suitable sensitizing substance. The fixation of the alexin by

* In the majority of experiments we use previously washed blood instead of simple defibrinated blood. This washing gives us rabbit blood corpuscles without the addition of the serum present in defibrinated blood. The corpuscles are washed by adding a relatively large amount of salt solution to a small amount of defibrinated blood, centrifugalizing, and decanting the supernatant fluid. 
sensitized corpuscles is the important fact that Ehrlich and Morgenroth were the first to show experimentally.* This fact would seem to be a remarkable confirmation of the idea that we established several years ago, namely, that under the influence of a suitable sensitizing substance (preventive substance) the animal body directs this bactericidal (or cellulicidal) power particularly against a given cell.

Normal rabbit corpuscles remain intact when mixed with fresh normal guinea-pig serum and the alexin is not absorbed. If we subsequently add sensitized cholera vibrios to this mixture, they soon show a granular transformation, which proves that the fluid contains free alexin. But if in another tube we mix normal guineapig serum with rabbit corpuscles treated with a hemosensitizing substance, they are destroyed, absorb the alexin, and sensitized vibrios subsequently added remain normal and show no transformation.

EXPERIMENT. An emulsion of cholera vibrios is made by suspending an agar culture in $10 \mathrm{cec}$. of salt solution. About a third of this volume of cholera serum from the rabbit, heated to 55 degrees for half an hour, is then added. This forms an emulsion of sensitized vibrios. The blood used is washed rabbit blood.

Mixture A. 0.3 of a cubic centimeter of rabbit blood plus 0.6 of a cubic centimeter of hemolytic serum ( 55 degrees) plus 0.3 c.c. normal guinea-pig serum. Complete and rapid hemolysis occurs.

Mixture B. Identical with A with the exception that 0.6 of a cubic centimeter of normal guinea-pig serum ( 55 degrees) is used instead of a specific serum. No hemolysis.

Mixture C. Identical with A but with guinea-pig blood, 0.3 of a cubic centimeter, replacing the rabbit blood. In this mixture there is no hemolysis, as the guinea-pig corpuscles are not affected by fresh guinea-pig serum and heated hemolytic serum.

In 1 to 2 hours 0.1 of a cubic centimeter of the emulsion of sensitized vibrios is added to each mixture. The mixtures are then placed in the incubator for an hour with the following results: in mixture A, where hemolysis took place, the vibrios undergo no transformation. There is complete granular transformation in mixtures $\mathrm{B}$ and $\mathrm{C}$, in which the corpuscles were intact.

3. The fixation by corpuscles of the active substances of hemolytic serum. The action of the stromata.

Rabbit corpuscles when mixed with our hemolytic serum previously heated to 55 degrees remain intact, with the exception of being agglutinated. Under these conditions they fix the sensitizing

* See "Collected Studies on Immunity," Ehrlich-Bolduan, Wiley \& Co., p. 1. 
substance.* The same corpuscles, when mixed with either fresh hemolytic serum or a mixture of fresh normal serum plus heated hemolytic serum, are destroyed. Under these conditions it is easy to demonstrate that they have fixed both the sensitizing substance and the alexin.

We may now consider what part of the corpuscles absorbs the active substances. If corpuscles are placed in distilled water they lose their hemoglobin, as we know, and are reduced to transparent stromata floating in a red fluid. We may determine whether the hemotoxic substances are fixed by the substances dissolved in the laky fluid or by the stromata suspended in it. For such an experiment the stromata are separated from the fluid in which they are suspended.

We take 3 c.c. of defibrinated rabbit blood, and preferably of blood that has been washed in salt solution. To this is added 15 c.c. of distilled water and a very red and nearly transparent fluid is obtained. Microscopically the stromata are scarcely visible, are very translucent, with a full round outline, and apparently are filled with water. To this laked red fluid is added 1 c.c. of distilled water containing 0.0975 of a gram of sodium chloride. The addition of this salt solution restores the laked fluid to a tonicity corresponding to normal salt solution. This addition of salt solution produces an immediate clouding of the nearly transparent fluid. This cloudiness settles out in whitish flecks which gradually fall to the bottom of the tube, leaving a clear supernatant fluid. Microscopical examination shows these flecks to be composed of agglutinated stromata. These stromata have lost their very transparent and regularly rounded form, have become more visible, and are flattened out into thin concave disks. When seen in cross section they seem to have undergone a veritable plasmolysis and it would seem as if the stromata were simply inclosing membranes or veritable closed sacs that swell in hypotonic fluids, and contract and flatten out in more concentrated solutions.

However that may be, the fluid is easily separable into two parts, either by deposition or, better, by centrifugalization: an upper part, which is absolutely limpid, with no microscopically visible elements; and the other reddish, opaque, and including large numbers of sus-

* As we have already previously noted, they also fix the agglutinin. 
pended stromata.* To equal amounts of these two fluids equal amounts of sensitizing substance and of alexin (from a normal guinea-pig) are added. The alexin should not be in too large amount. The mixtures are then shaken and, after a certain time, well-sensitized rabbit corpuscles are added to each fluid. It is found that these corpuscles are not hemolyzed or are only slightly hemolyzed in the fluid containing the stromata, but, on the contrary, are rapidly dissolved in the limpid red fluid. From this experiment it may be concluded that the property possessed by the red blood corpuscles of absorbing alexin in presence of the sensitizing substance is due to their stromata.

For another experiment a suitable dose of sensitizing substance (that is, hemolytic serum heated to 55 degrees) may be added to the two fluids. After shaking and allowing to stand for a time the second mixture is centrifugalized to separate out the stromata, and the supernatant fluid is decanted. It is then demonstrable that this fluid contains no sensitizing substance, since the subsequent addition of red blood cells and normal guinea-pig serum gives no hemolysis. The property of absorbing the sensitizing substance also belongs, then, to the stromata.

In yet another experiment it may be shown that the stromata absorb alexin when the sensitizing substance is present, whereas they do not fix it if the latter substance is absent. For this experiment a thick emulsion of stromata is prepared to which a large amount of salt solution is added. The mixture is centrifugalized and the limpid pinkish supernatant fluid decanted. The washing with salt solution is repeated until the emulsion of stromata is quite white and free from hemoglobin. The emulsion is then divided into two equal parts, to each of which the same dose of alexin (serum of a normal guinea-pig) is added. To the first mixture a small amount of sensitizer is then added and to the second the same amount of normal guinea-pig serum also heated to 55 degrees. It may be demonstrated in the usual manner that in the first mixture the alexin has disappeared from the fluid by fixation on the stromata; there is no absorption of the alexin in the second fluid containing no sensitizing substance.

* It is well to determine, of course, that there are no intact corpuscles remaining among these stromata. 
In brief, then, red blood corpuscles owe their characteristic absorbing properties to their stromata. Is the property in rabbit corpuscles of producing a hemolytic power when injected in guineapigs also due to their stromata? To answer this question stromata that have been freed from all soluble substances by carefully washing in salt solution are obtained and injected into guinea-pigs. Each guinea-pig receives the stromata from 4 to 5 c.c. of defibrinated blood. Corresponding guinea-pigs are injected with a limpid reddish fluid containing the soluble substances obtained from corpuscles by treating them with distilled water and subsequently adding salt. This fluid is then centrifugalized to deprive it of suspended stromata. After injection lasting for three weeks it is found that guinea-pigs injected with stromata furnish an active hemolytic serum; those that receive the limpid fluid containing hemoglobin have a serum like that of normal guinea-pigs.

Corpuscles fix the active substances of hemolytic serum. Can the nature of this phenomenon of fixation be more exactly determined? Is it a question of true chemical combination by the union of certain elements of the red blood cells with the active principles, and does it take place according to fixed proportions? Or should we consider the fixation by corpuscles as more like a dyeing phenomenon? A substance to be dyed, as we know, absorbs extremely varying amounts of the dye, so as to take shades of varying intensity. The amount of dye that is fixed is subject to wide variations; chemical reactions, properly speaking, on the other hand, are characterized by a reaction in definite proportions.

We shall not consider this difficult problem fully, as we have not as yet sufficient data, but a single experiment may be given which may prove important in future studies of this subject.

A small amount of defibrinated rabbit blood (for example, 0.1 of a cubic centimeter) is added to a certain amount of fresh hemolytic serum (for example, 0.4 of a cubic centimeter). The corpuscles are rapidly destroyed. The hemolysis although still rapid is somewhat slower if a large dose of blood is added (say 0.3 or 0.4 of a cubic centimeter). The destruction of corpuscles naturally enough varies in rapidity with the amount of blood added to the hemotoxin. And yet the serum that is used can destroy completely and relatively 
rapidly an equal or even a slightly superior volume of rabbit blood. For example 0.5 of a cubic centimeter of blood added to 0.4 of a cubic centimeter of serum gives a complete destruction of all the corpuscles in about an hour.

In this latter experiment we have supposed an instance in which the amount of blood ( 0.5 of a cubic centimeter) was added all at once to the 0.4 of a cubic centimeter of serum. But the blood may also be added in fractions; we may add to the 0.4 of a cubic centimeter of hemotoxin, first 0.2 of a cubic centimeter of blood, and later 0.1 of a cubic centimeter and, after an hour or two, a third 0.2 of a cubic centimeter. In other words, we determine whether a given amount of serum is uniformly able to destroy the same number of corpuscles whether they be added all at once or in divided doses. As a matter of fact it is found that the dissolving property of serum is rapidly exhausted if the blood is introduced gradually in small amounts, particularly when the time intervals between the doses are somewhat prolonged. We find that 0.4 of a cubic centimeter of fresh hemolytic serum - an amount capable of destroying completely at least 0.5 of a cubic centimeter of blood when added at once - dissolves no more than 0.2 of a cubic centimeter of blood if the corpuscles are added to the serum in divided doses. The first doses introduced are well dissolved until the total amount of blood added is about 0.2 of a cubic centimeter, but subsequently added corpuscles remain intact. At least twice as much blood then may be dissolved when added to serum all at once as when added in divided doses. It is evident that in the latter instance the first corpuscles added become in some manner supersaturated with the active substance and exhaust the fluid so that it becomes inactive for other corpuscles. In other words, the first corpuscles absorb more active substances than is necessary for their dissolution.

This seems to us to favor the conception that the absorption of active principles by the corpuscles should be compared to a dyeing phenomenon. In such phenomena, as we know, the substances to be dyed absorb varying amounts of the dye under conditions that are quite similar to those just outlined for corpuscles.

If we pour 10 c.c. of a diluted rather pale solution of methy! violet in a crystallizing dish, a piece of filter paper placed in it becomes colored. It soon takes on a uniform pale tint, while the fluid, as it 
is exhausted, becomes decolorized. Let us now take the same amount of coloring fluid and a piece of filter paper of exactly the same size as before. Instead of plunging this entire piece of paper into the fluid we cut it up into pieces. When we place the first piece of paper in the fluid it takes on a very dark shade and deprives the coloring fluid of a good deal of its color. We then add a second fragment of paper, and, after another interval, a third. These latter pieces take only a very faint tinge owing to the more or less complete decolorization of the fluid. The fluid soon becomes entirely decolorized and the last fragments added remain quite white. By analogy we may consider that, in the first instance, when red blood corpuscles are added in a single dose they become susceptible to a loss of hemoglobin, although they only "stain faintly" with the active principles, but that in the latter conditions, when added in divided doses, they absorb a much larger dose of these substances and so exhaust the serum and prevent the destruction of subsequently added corpuscles.

It is difficult to understand just how alexin destroys sensitized corpuscles and causes their hemoglobin to pass out. We may mention a fact that may have some bearing on researches along this line. We add defibrinated rabbit blood to a rather small dose of hemotoxin and wait until hemolysis is complete. At the same time we add a corresponding amount of normal guinea-pig serum to the same amount of blood, in which case the corpuscles remain intact. We then add to each mixture a large amount of distilled water, which will destroy the corpuscles in the second mixture as well. We have then two fluids; in the first the corpuscles have been destroyed by active serum and in the second by distilled water. To each of these fluids we then add sufficient salt to render them isotonic. In the mixture in which the corpuscles were dissolved by distilled water the stromata show energetic plasmolysis and retract. In the other fluid containing hemotoxin the stromata keep their primitive rounded form and apparently do not show any effect from the increased concentration. It would seem as if the alexin from the active serum had destroyed or digested something in the corpuscles that has to do with the action of plasmolysis and phenomena of osmosis. The diffusion of soluble hemoglobin would perhaps be the result of such destruction. 


\section{The Antitoxin to Hemolytic Serum.}

A serum with antitoxic properties for the hemotoxin that we have been considering in the preceding pages is easily obtained. It is, in other words, a substance capable of protecting rabbit corpuscles from the destructive power of our hemolytic serum.* This hemolytic serum, as already shown, is toxic for the rabbit. When injected in doses of 5 c.c. or thereabouts intravenously it kills almost instantaneously. At autopsy clots bathed with reddish serum are found in the heart and large vessels, which indicates that hemolysis has taken place in vivo. Disseminated hemorrhagic effusions, are also frequently found in the kidney and muscles, particularly in the psoas muscle. The hemotoxin when injected subcutaneously into rabbits in a small dose produces no severe effect and animals treated in this manner acquire an antitoxic power in their serum.

The hemotoxin is injected two or three times, at intervals of 15 days, in a dose of from 2 to 3 c.c. The rabbits are then bled 12 to 15 days after the last injection.

The antitoxic (antihemolytic or antihemotoxic) serum obtained by this relatively short treatment is not, to be sure, very powerful; it is sufficiently so, however, to permit a study of its properties.

The simplest experiment to demonstrate its antitoxic property consists in adding a relatively large dose of freshly obtained antitoxic serum to a small amount of fresh hemolytic serum. To this mixture is then added a small amount of rabbit blood. As a control a mixture of hemolytic serum, normal rabbit serum and rabbit blood is made at the same time and in the same proportions.

If the dose of antitoxin is sufficient, no dissolution of corpuscles takes place in the first mixture. In the second mixture, on the contrary, containing the same dose of normal rabbit serum instead of antitoxic serum, the corpuscles are destroyed.

In such an experiment a considerably greater dose of antitoxin than of hemolytic serum (10 to 20 times) must be used to protect

* We have already shown (see p. 175) that an antihemolytic antitoxin similar to that described by Camus and Gley and Kossel for eel serum may be obtained. The serum that we described opposed the hemolytic effect of hen serum.

Metchnikoff has recently described an antispermatoxin (Annales de l'Institut Pasteur, 1900, No. 1). 
the corpuscles properly. It will be recalled that in these experiments we employed an active hemotoxin from guinea-pigs that have received three injections of 4 to 5 c.c. of rabbit blood.

We may note at once that the antitoxic serum is in reality more active than would seem from this preliminary experiment; by a very simple procedure it may be made to neutralize much larger quantities of hemolytic serum perfectly. If the serum is heated to 55 degrees it is found that three or even two volumes of antitoxic serum will completely neutralize one volume of fresh hemolytic serum.

As we shall presently see, the explanation of this fact is simple. When the antitoxic serum is fresh it contains an excess of rabbit alexin, which, in the presence of the sensitizing substance, is dangerous for the rabbit corpuscles. In order, therefore, to demonstrate its maximum protective power one should eliminate this harmful substance by heating the serum to 55 degrees.

That the antitoxic serum does possess an alexin capable of destroying rabbit corpuscles when the sensitizing substance is present as well as normal rabbit serum, is easily demonstrable. On mixing hemolytic serum heated to 55 degrees with equal parts of fresh antitoxic serum and adding a small amount of rabbit blood we find that the corpuscles are dissolved. This destruction is due to the alexin in the antitoxin since there is none in the hemolytic serum. If we should add too great a dose of antitoxin to the sensitizing substance, there would be no hemolysis. For, as we shall see, the sensitizing substance will have been neutralized and under these conditions the alexin in the antitoxin can have no harmful effect on the corpuscles. In the presence of the sensitizing substance, then, a small dose of antitoxin destroys the corpuscles but a large close protects them.

In order; then, to study the properties of the antitoxin to best advantage it is better to heat the serum to 55 degrees as a general procedure.

Antisensitizing substance and anti-alexin. - We know that hemolytic serum destroys rabbit corpuscles owing to the action of two distinct substances: the specific sensitizing substance (antibody or preventive substance) and the alexin. This latter substance, which 
occurs in normal rabbit serum, attacks rabbit corpuscles treated with the sensitizing substance.

Since hemolytic serum has two active substances, both of which must be present to produce hemolysis, it is at once evident that the antitoxin may protect corpuscles by neutralizing either one of these substances. Which substance, then, does the antitoxin neutralize? Or does it affect them both simultaneously?

The following experiments that we are about to describe answer this question; but we may state the conclusion at once, namely, that antitoxic serum neutralizes both the sensitizing substance and the alexin of hemolytic serum.

In order to demonstrate the neutralizing effect of the antitoxin for the sensitizing substance we make use of the fact that rabbit corpuscles affected by the sensitizing substance may be destroyed, not only by normal guinea-pig serum, but also by normal rabbit serum. Normal rabbit serum is, of course, under ordinary conditions quite harmless for rabbit corpuscles and it therefore serves as an excellent indicator of the presence of the sensitizing substance in a fluid. It may be shown, moreover, by a suitable experiment that our antitoxin from the rabbit has no neutralizing effect on rabbit alexin. Consequently, if, in a suitably chosen mixture of heated hemolytic serum, heated antitoxic serum, and fresh normal rabbit serum, subsequently introduced corpuscles remain intact, we may conclude that the sensitizing substance of the heated hemolytic serum has been neutralized by the antitoxin. The control naturally is composed of identical doses of each substance with normal rabbit serum heated to 55 degrees replacing the antitoxic serum. In such a control mixture corpuscles treated with sensitizing substance are easily destroyed by the alexin present in the normal rabbit serum.

From such experiments we learn that the antisensitizing function of our antitoxin is only slightly developed, and at least 15 parts of antitoxin are necessary to neutralize the sensitizing substance in 1 part of heated hemolytic serum. The intensity of the antialexic property in the antitoxin is much greater.

To demonstrate the anti-alexic property a mixture of antitoxin (55 degrees) and a rather large dose of fresh hemolytic serum is used. For example, two parts of antitoxin and one part of hemotoxin are mixed together and rabbit corpuscles added. These 
corpuscles remain intact even after a long time. But if a small amount of fresh rabbit serum is added to the mixture, a rapid dissolution takes place, although this added serum is in itself harmless for the corpuscles. This shows that the original mixture contained an excess of sensitizing substance, as demonstrated by the destruction of the corpuscles on the addition of normal rabbit serum. Consequently, although the original mixture did not affect the corpuscles, there was no lack of sensitizing substance and the protection is due to the neutralization of the guinea-pig alexin also present in the fresh hemolytic serum by the antitoxin.

To show the anti-alexic activity in another way we prepare a mixture of 1 c.c. of fresh normal guinea-pig serum and 2 or 3 c.c. of antitoxin ( 55 degrees). As a control a mixture 1 c.c. of fresh guineapig serum and 2 or 3 c.c. of normal rabbit serum ( 55 degrees) is also made. To each mixture is added a strong enough dose of sensitizing substance (heated hemolytic serum) to resist neutralization by the antitoxin; rabbit corpuscles subsequently added to each mixture remain intact in the first and are destroyed in the second. To make this experiment more conclusive a second control may be prepared containing, like the first, antitoxin and sensitizing substance, but with the normal guinea-pig serum replaced by an equal amount of normal rabbit serum. This mixture destroys corpuscles, which proves that the dose of sensitizing substance used was larger than could be destroyed by the antitoxin; and it proves also that our antitoxin although effective against guinea-pig alexin has no effect on rabbit alexin.

The idea that antitoxin has only a weak "antisensitizing" power whereas it has a very much more distinct "anti-alexic" power explains very clearly the fact that we have already noted, namely, that heated antitoxin combats the effect of fresh hemolytic sera better than non-heated antitoxin. A moderate dose of antitoxin added to fresh hemolytic serum may neutralize the alexin of this hemolytic serum, but leave a certain amount of sensitizing substance still active.

If the antitoxin has not been heated, it contains an additional dose of alexin, which aids in the destruction of corpuscles the sensitization of which it has not been able to prevent. When heated to 55 degrees it does not have this disadvantage; it has then only 
one of the active substances of the hemolytic serum to neutralize in order to protect the corpuscles, namely, the alexin. Before heating it must neutralize completely not only the alexin but also the sensitizing substance.

It is very probable that the antisensitizing substance and the antialexin are two distinct substances. An antitoxin supersaturated with sensitizing substance still retains intact its property of neutralizing normal guinea-pig alexin.

Antihemolytic and antibactericidal properties of the antitoxin:In bleeding three guinea-pigs, the first normal without any treatment, the second treated by injection of rabbit blood, the third immunized against cholera vibrio, we know that we shall obtain three different sera each possessing the same alexin. The identity of the alexin of normal serum with that of immune serum is one of the important points in the theory we offered five years ago to explain the mode of action of preventive sera on vibrios, a theory to which we shall later return. Two of these three sera are immune sera, and, although identical as regards alexin, differ profoundly in their antibodies or sensitizing substances. The identity of the alexin in normal and immune sera explains why normal serum can restore the original destructive properties of various heated immune sera deprived of their alexin indifferently. If, therefore, we add to normal guinea-pig serum containing alexin an anti-alexin neutralizing this latter substance, the normal serum should become incapable, not only of dissolving sensitized red blood cells, but also of producing a granular transformation in cholera vibrios treated with cholera-sensitizing substance. As our antitoxin neutralizes guinea-pig alexin we should expect it to protect various sensitized cells against the effect of this alexin. Such an antitoxin, therefore, would be both antihemolytic and antibactericidal.

Experiment confirms these suppositions. On mixing antitoxin (55 degrees) with a certain amount of fresh normal guinea-pig serum we remove both its property of restoring cellulicidal activity to hemolytic serum and also the power of restoring the bactericidal property to anticholera serum (55 degrees). But if we use normal rabbit serum instead of normal guinea-pig serum the antitoxin no longer shows any protective property either for vibrios or corpuscles. Although 
active against guinea-pig alexin it has no effect on rabbit alexin. Experimental details follow:

An emulsion of sensitized cholera vibrios, prepared by suspending an agar culture in 10 c.c. of salt solution, and adding 2 c.c. of cholera serum from a rabbit heated to 56 degrees, is used.

\section{Mixtures Containing Sensitized Vibrios.}

a. Fresh normal guinea-pig serum, 0.2 c.c.; antitoxin ( 55 degrees), 0.4 c.c.

b. Fresh normal guinea-pig serum, 0.2 c.c.; normal rabbit serum (55 degrees), 0.4 c.c.

c. Fresh normal rabbit serum, 0.2 c.c.; antitoxin (55 degrees), 0.4 c.c.

d. Fresh normal rabbit serum, 0.2 c.c.; normal rabbit serum ( 55 degrees), 0.4 c.c.

To each of these four mixtures 0.2 c.c. of an emulsion of sensitized vibrios is then added. The mixtures are left for an hour at 37 degrees. Granular transformation is complete in mixtures $b, c$, and $d$. The vibrios in mixture $a$, containing guinea-pig alexin neutralized by antitoxin, on the contrary, have kept their normal appearance.

\section{Mixtures Containing Sensitized Blood.}

This blood consists of 0.2 of a cubic centimeter of rabbit blood (previously washed in salt solution) plus 1 c.c. of hemolytic serum heated to 55 degrees.

e. Identical with $a$.

$f$. Identical with $b$.

g. Identical with $c$.

To each of these mixtures 0.1 c.c. of the sensitized blood is added. The corpuscles are rapidly dissolved in $f$, less rapidly in $g$ and remain intact in $e$.

For the sake of completeness we may mention that the bactericidal effect is evident, not only by granular transformation of the vibrios, but by their complete destruction, as shown by inoculating agar tubes with the mixtures. In this way we find that a small amount of sensitized vibrios mixed with normal guinea-pig serum plus normal rabbit serum (55 degrees) becomes completely sterile. The same dose of - vibrios mixed with guinea-pig serum plus antitoxin from the rabbit (55 degrees), on the contrary, remains alive; cultures from this mixture on gelatin at intervals show numerous colonies.

The specificity of the anti-alexic action of the antitoxin. - Our antitoxin neutralizes the alexin of guinea-pig serum. We have already noted that it has no effect on the alexin of rabbit serum. Does it affect the alexins in sera from other animal species? This may be answered experimentally by adding doses of fresh serum from different animals to a mixture composed as follows: 
Antitoxin, 55 degrees; fresh alexic serum to be tested; sensitizer in sufficient dose (that is, hemolytic serum heated to 55 degrees). The effect of this mixture on subsequently added rabbit corpuscles will indicate whether or not the alexin in the serum added is neutralized by the antitoxin.

A control series is made with similar mixtures containing normal rabbit serum ( 55 degrees) in place of antitoxin. This series shows the intensity of corpuscle destruction by the combined action of the sensitizer and the normal sera when antitoxin is absent. A third series containing normal rabbit serum, 55 degrees, plus the fresh normal sera under consideration but without sensitizer is also useful; this series indicates what dissolving properties are present in the normal sera alone without any sensitizer. The rabbit blood added to these mixtures should previously have been washed in salt solution in order to free the corpuscles from rabbit serum.

Without going into minute details of the results of such experiments we may state that the antitoxin neutralizes guinea-pig alexin efficiently, but has no effect on alexin from the rat, dog, rabbit, goat, goose or hen.

It does, however, have a distinct neutralizing effect on pigeon alexin. We may therefore conclude that this anti-alexic activity is relatively but not absolutely specific. With a certain exception (pigeon alexin) our anti-alexin has no neutralizing effect on any animal species tested except the guinea-pig. These results confirm distinctly the idea that the alexin in the sera of different animal species is not uniformly identical; this idea was already probable from the fact that the corpuscles of a given species are attacked by foreign normal sera with an intensity that varies according to the species from which this serum has been obtained.

The direct action of the antitoxin on the toxin. - Our anti-alexin counteracts the harmful effect of guinea-pig alexin on rabbit corpuscles. There are reasons, moreover, for believing that anti-alexin combines with alexin or acts directly on this toxic substance by destroying or modifying it. This conclusion, however, cannot be accepted offhand, as proven. We might imagine, perhaps, although it seems scarcely reasonable, that antitoxin has no effect on the 
alexin, but that in some way it prevents the corpuscles from yielding to its harmful effect. As is well known, various observers and in particular Cherry and Martin, in their study of other toxins and antitoxins, have offered certain facts that favor the hypothesis that antitoxins act directly on toxins.

It is worth while considering, however, whether this hypothesis is exact so far as our toxin and antitoxin are concerned. Two questions immediately arise on consideration of this subject: first, if to the first of two tubes containing the same amount of antitoxin there is added a little fresh alexin and to the second the same amount of serum heated to 55 degrees, and if both mixtures are then heated to 55 degrees, shall we find that their antitoxic effect on a new dose of fresh active serum is the same? Or this question may be expressed in another way: when alexin has been heated to 55 degrees, and has thereby lost its toxicity for corpuscles, will it still neutralize as much antitoxin as it could before being heated? Second: does a neutral mixture of alexin and antitoxin heated to 55 degrees become antitoxic (by a neutral mixture we mean a mixture that has little or no effect on sensitized corpuscles)?

If antitoxin and alexin are not combined, but continue to exist side by side in a free condition, heating which destroys the toxin (alexin), but has no effect on antitoxin, might leave this latter substance intact. And further, if the antitoxin does not act directly on the alexin in the two mixtures under question, we should have the same antitoxic value after heating to 55 degrees. As a matter of fact the activity or non-activity of the alexin added to antitoxin should not affect the result. For the sake of clearness we shall answer these questions at once and then consider the experiments by means of which we arrived at these answers: first, alexin heated to 55 degrees and deprived of its cellulicidal activity has lost wholly or to a great extent its power of saturating antitoxin; second, the antitoxin is not recovered from a neutral mixture of antitoxin and fresh alexin by heating it to 55 degrees, although this temperature destroys the activity of the toxin. The antitoxin must then have been definitely neutralized.

Both these results lead to the conclusion that antitoxin acts directly on the toxin by destroying its toxic activity. The experiment to prove these facts follows: 
It was first determined that two parts of antitoxin (56 degrees) will neutralize one part of alexin (fresh normal guinea-pig serum). Two mixtures were prepared as follows:

a. Antitoxin, 2 c.c.; fresh normal guinea-pig serum, 1 c.c.

b. Antitoxin, 2 c.c.; normal guinea-pig serum heated to 55 degrees, 1 c.c.

As a control the following mixtures were prepared:

c. Antitoxin, 2 c.c.; salt solution, 1 c.c. In this mixture the antitoxin is simply diluted.

d. Normal rabbit serum ( 55 degrees), 2 c.c.; salt solution, 1 c.c.

These four mixtures are then heated for half an hour to from 55 to 56 degrees. The mixtures are subjected to exactly similar conditions, but mixture $a$, containing fresh alexin, is naturally the one most affected, as the alexin is destroyed at this temperature.

After heating, the antitoxic value of the four mixtures is determined. For this purpose a suitable dose of fresh normal guinea-pig serum is added to each tube.*

Later well-sensitized rabbit blood is added to each tube. The amount of sensitizer used is sufficiently large to prevent the antisensitizing effect of the antitoxin from being noticeable.

It is evident that the blood will hemolyze in mixtures in which the toxin (alexin) has not been neutralized; in other words, in mixtures that do not contain active anti-alexin. Hemolysis takes place quickly in $d$, which contains no antitoxin. Corpuscles remain intact in $c$, containing a mixture of antitoxin and salt solution. Mixtures $a$ and $b$ do not act exactly alike. In $b$ the corpuscles remain intact, as in $c$, for a long time, but finally become partially hemolyzed; in mixture $a$ hemolysis is very energetic. The contrast between $a$ and $b$ is very striking. In $a$ there is only a slight antitoxic effect: unheated normal serum neutralizes antitoxin much more distinctly than does heated serum.

We shall not consider farther for the present the mechanism by which the antitoxic serum protects corpuscles from the hemotoxin, but shall hope to return later to a consideration of this question. We may mention in conclusion that the antitoxin has also antiagglutinating properties against the hemolytic serum. It also causes a precipitate both with hemolytic serum and also with normal guinea-pig serum. This precipitating property exists, not only in animals that furnish antitoxin (i.e., rabbits treated with hemolytic serum), but also in rabbits given injections of defibrinated blood or normal guinea-pig serum. We refer to our previous article for more information on this property of serum. $\dagger$

* The amount of normal serum that is best to add is determined by using a series of tubes corresponding to each mixture $a, b$, etc., to each one of which a different amount of alexin is added.

$\dagger$ We may recall simply that the "precipitating property" of such a serum is quite distinct from its antitoxic property. Nor has it any relation to the agglu. tinating property of the serum for corpuscles. 


\section{Observations on the Theories of Chemical IMMUNITY.}

Immunity is due primarily to phagocytic activity, that indispensable function of the body in the taking up and destruction of bacteria or alien cells. Substances having a digestive effect on bacteria may also be found in the blood serum, particularly during the condition of artificial immunity. As our knowledge of these active substances of serum has increased it has become evident that they, too, owe their origin to those phagocytic cells the function of which is to protect the animal body.

The cytolytic properties of serum have come to be regarded more and more as simply a new manifestation of the activity of the protective cells. The tendency in the study of immunity has been to harmonize these humoral manifestations with the functions of the phagocytes, which, as Metchnikoff has shown, are both in origin and function the digestive cells, fitted to form substances that digest and destroy alien cells.

But these substances in serum are themselves worthy of study apart from any consideration of their origin. The study of this "immunity of a chemical nature" has consisted hitherto in a comparison between the sera of normal animals and the sera of animals that are in a condition of artificial immunity. In the sera of these latter animals, particularly, the existence of specific cellulicidal properties, frequently of great intensity, has been noted.* But this cytolytic power is not the only characteristic of immune sera; they have also the curious property of creating on injection an intense cellulicidal property in the serum of a normal animal, as Fraenkel and Sobernheim first pointed out in 1894. It is notable that immune sera still retain this transferring property, even after they have lost their own power to destroy cells by being heated to 55 degrees. In the example given by Fraenkel and Sobernheim, cholera serum, whether intact or heated to from 55 degrees to 60 degrees, when injected into a normal animal endows the latter's serum with an intense bactericidal power. As another example it may be noted that hemolytic serum from a rabbit "vaccinated"

* We owe our conception of the specificity of bactericidal phenomena to Pfeiffer's researches particularly. 
with hen blood, on injection into a normal rabbit, confers on the serum of the latter an intense hemolytic power for hen corpuscles.* For the sake of completeness we may add that the serum of the normal animal that has received the immune serum has a cellulicidal property quite as specific as that of the immune serum employed. We demonstrated in 1895 that, when a guinea-pig is injected with an immume serum specific for the vibrio Metchnikovi, its serum acquires a bactericidal property only against the vibrio Metchnikovi and not against such vibrios as the V. choleræ.

How may the destructive effect of a cytolytic serum be detected in the affected cell? What lesion is produced when this cell is acted on by the serum which indicates the destructive effect? It varies naturally with the nature of the cell in question. In vibrios it consists in a granular transformation; in red blood cells, in hemolysis. As is well known, the granular metamorphosis of the cholera vibrio was first noted by Pfeiffer in the peritoneal cavity of actively or passively immunized animals. This investigator thought that this modification of the vibrio could be brought about only in the animal body and never in vitro; according to him this transformation is indicative of an essentially vital action, and is distinct from the bactericidal effect of cholera serum in vitro. According to this author there would be two distinct varieties of bactericidal action, the one occurring exclusively in vivo, and indicated by a granular transformation, and the other produced in vitro, similar in effect but less marked and of less significance. If this conception had proved correct the subject would have been excessively complicated. We know now, however, that it does not agree with the facts: Metchnikoff, in a classical experiment that inaugurated the more comprehensive study of these phenomena, was able to obtain the granular transformation of vibrios in vitro by mixing the organisms with' cholera serum plus the peritoneal exudate from a normal guinea-pig. We subsequently demonstrated that fresh cholera serum alone is able to produce this metamorphosis, even when quite limpid and free from cells.

The important point to be emphasized is that the bactericidal action of a fluid or serum on the cholera vibrio is evidenced both in vivo and in vitro by a granular transformation of the organism. The

$\because$ See p. 170 . 
analogous action of hemolytic sera is detectable in vitro as well as intraperitoneally by a destruction of the corpuscles with a diffusion of hemoglobin.

To summarize: First, the sera under consideration obtained from immunized animals are cytolytic, the cytolysis consisting in such changes as the granular transformation of vibrios, and the hemolysis of red blood cells. Certain fluids from the immunized animals, as the peritoneal fluid, show similar cytolytic effects in vivo or in vitro. And second, a given immune serum on injection into normal animals confers on their serum and body fluids similar cytolytic properties likewise demonstrable in vivo and in vitro. Immune serum transmits this property even when itself deprived of its destructive property by heating to 55 to 56 degrees.

These are the facts to be coördinated and explained by a theory. The first theory proposed was our own, offered first in 1895, and confirmed without any essential addition by our subsequent researches. It might seem unnecessary to rehearse our theory, as we have already outlined and repeated it several times.* This theory, however, has not been universally accepted and certain other observers have preferred a different one: it would therefore seem advisable to reconsider it and to compare it with other theories.

Bordet's theory (1895). - Our conception as expressed five years ago is based on the possibility (first demonstrated by Metchnikoff) of producing a granular transformation of vibrios in vitro, and depends particularly on the following facts:

1. Fresh cholera serum produces a granular transformation of cholera vibrios; the phenomenon produced in vitro is identical with the one first observed in the peritoneal eavity by Pfeiffer; it is just as highly specific and may be similarly employed for the diagnosis of vibrios. Cholera serum has also the power of clumping a culture of vibrios. $\dagger$

2. When heated to 55 degrees cholera serum loses its bactericidal property and the power of producing granular transformation, but still retains its agglutinating power.

* See articles on pp. 8, 56, 81, and 134 .

$\dagger$ This is the first instance deseribed of the rapid agglutination of a microorganism by a speeific serum in high dilution. 
3. Heated cholera serum recovers its original bactericidal energy on the addition of a normal serum that is itself only faintly bactericidal. A small amount of intact or of heated cholera serum suffices to confer an intense specific bactericidal power on a considerable amount of normal serum. This power is evidenced by a granular transformation in the added vibrios.*

4. Normal serum alone frequently causes a granular transformation of vibrios to a less degree, particularly if the vibrios are attenuated.

5. Normal serum heated to 55 degrees loses the property of restoring the bactericidal activity to heated cholera serum.

Our theory founded on these facts is comprised in the two following propositions:

A. The bactericidal property of cholera serum, or of analogous sera, is due to the presence of two distinct substances: the first, which may be called preventive substance or antibody (later called sensitizer), is characteristic of immune serum, is specific and resists a temperature of 55 to 60 degrees and even more. The other, or proper bactericidal substance, the alexin, occurs in the serum of normal as well as of vaccinated animals, and is destroyed on heating to 55 degrees. Heating cholera serum to 55 degrees does not destroy the preventive substance, but simply eliminates the alexin. As this alexin is present in normal serum the addition of such serum restores to heated cholera serum its original activity. $\dagger$

In such a mixture cholera serum again contains both substances originally present, the collaboration of which is essential in producing intense specific bacteriolysis.

The preventive substance, in other words, heated cholera serum, is not at all bactericidal. Normal serum on account of its alexin

* We advocated such a mixture in vitro as a practical diagnostic method for the cholera vibrio.

$\dagger$ We have been surprised to find that certain authors, particularly in Germany, give a very inexact historical account of these ideas, frequently attributing the first consideration of these facts to authors who have only recently considered the question. Consequently we may be permitted to refer to certain passages in our memoir of 1895 . See, for example, on p. 58, and on p. 59.

We shall later quote other references in the text, particularly when we come to consider the unity of the bactericidal substance in different immune sera, and the occurrence of bactericidal power in the fluids of passively immunized animals. 
has a slight and non-specific bactericidal activity affecting, generally, only attenuated bacteria. But in presence of the preventive substance of an immune serum the alexin energetically attacks that particular race of bacteria for which the immune serum is specific. The activity of the alexin against other bacteria is not, however, increased.

In short, the preventive substance, in itself not bactericidal, acts specifically on a cell by increasing the destructive effect of the alexin on it. We now call the preventive substance the "sensitizer" as it sensitizes the cell to the alexin.

It may be stated at this point that the specific hemolytic sera, first demonstrated by us in 1898, agree in general characteristics with the bacteriolytic sera, and any conception of one generally applies to the other. The majority of observers and particularly Ehrlich and Morgenroth agree with us on this point. The only difference lies in the cell affected, and hemolysis corresponds to bacteriolysis.

B. When an immune serum, as, for example, cholera serum, is given to a normal animal, the introduction of the preventive substance (sensitizer) transmits the specific bactericidal power to this animal's body fluids. The introduction of an alexin, present, of course, in fresh immune serum, is not necessary and therefore a heated immune serum will serve equally well. As we stated in 1895 , "It is quite evident why the presence of the bactericidal substance (alexin) is not indispensable for the preventive activity of serum. It is not characteristic of this serum alone, but is present in normal serum as well, and the animal injected with cholera serum already possesses this substance, so that its addition is unnecessary. What the normal animal does not possess is the preventive substance, and it is this substance, then, that must be given." As soon as the animal obtains the preventive substance it has the two factors necessary for intense specific bacteriolysis. On bleeding such an animal we find that its serum contains the preventive substance as well as the normally present alexin and will, therefore, destroy the vibrio. "The encounter of the two substances produces as energetic a bactericidal power in the animal body as in a test tube."

As we have already stated, this theory is also applicable to hemo- 
lytic sera.* The important fundamental idea is that the bactericidal (or cellulicidal) substance in various immune sera that endows them with their properties is similar to the one found in normal serum. This is the principle of the unity of the cytolytic substance which we expressed in 1895 by saying that, in normal and in vaccinated animals, "the bactericidal substance is in general respects the same, whatever may be the invading organism. In animals vaccinated against a given infective agent the bactericidal energy affects that bacterium particularly, owing to the presence of the specific preventive substance, which varies according to the micro-organism used in immunizing. It is owing to the intervention of this peculiar preventive substance that the animal directs its destructive energy against a given infective agent."

In this description the word "bacterium" may be replaced by the word "cell," referring to bacterium or blood corpuscles, as the case may be. And further, a reservation should be made, based on the subsequent study of hemolysis, that, although the alexin is the same in normal and vaccinated animals of the same species, there are certain differences in the alexins from different animal species. The alexins of most animals, however, have the same essential characteristics. We have already made another reservation, namely, that certain normal sera contain, beside the alexin, other bactericidal substances of less general import. The bactericidal substance for B. anthracis in rat serum, for example, is certainly not an alexin. $\dagger$

As far as the absolute identity of the hemolytic with the bacteriolytic alexin in a given serum is concerned, it would seem to have been firmly established in the present article.

The fact, moreover, that "it is owing to the intervention of the sensitizer that the animal directs its particular cellulicidal activity against a given cell" is fully confirmed by the experiments detailed in the first part of this article, experiments that prove, not only that the same normal serum, but that the same alexin destroys at one time a vibrio and at another a red blood cell depending on whether a hemosensitizer or a cholera sensitizer is used.

* It is probably also applicable to the immune sera active against other cells, such as the spermotoxic serum discovered by Landsteiner, the leucotoxic serum of Metchnikoff, and the epitheliotoxic serum of V. Dungern.

$\dagger$ Rat serum has in addition to this substance an alexin similar to that of other sera. See note on p. 180. 
Our theory makes two assumptions: First, that cytolytic phenomena in vitro, produced by adding the sensitive cell to the two substances of active serum, are quite similar to those which occur in vivo with the same substances. This may indeed be experimentally demonstrated provided that relatively similar amounts of each substance are present in both cases. For example, we find that the smallest amount of cholera sensitizer that will cause a given amount of vibrios to be transformed by alexin in vitro is similar to the amount necessary to destroy the same number of vibrios in a normal peritoneal cavity. This fact has been already mentioned.

Second, that a normal animal injected with an immune serum acts simply as a passive container for the sensitizer. The animal receives the substance, but does not modify it, and it becomes diluted in the fluids of the body, particularly in the blood. The serum from this injected animal should act, then, like a dilution of the sensitizer in normal serum. This we believe we have proved experimentally.* This conclusion holds as well for the agglutinating property. $\dagger$

$$
\text { *** }
$$

Pfeiffer's theory, (1896). - The preceding theory has not been the only one to awaken interest in the study of bactericidal sera. A year later Pfeiffer offered an explanation of the granular transformation of vibrios that differs from our own in several important particulars. Pfeiffer, who has carefully studied the bacteriolytic changes in vibrios in the peritoneal cavity, draws a sharp distinction between such phenomena and those that take place in vitro; the latter, according to this investigator, are much less energetic. As Pfeiffer understands it, vaccinated animals have a specific antibody that has two forms: a stable and inactive form that may be kept for a long time, and an active bactericidal form which is less stable. The antibody can easily change from one form to another. The change from inactive to active form is brought about by means of a ferment-like substance of cellular origin; this change takes place easily in the animal body, so that the antibody can attack the vibrios in its most destructive form. Serum, on the contrary, contains the antibody in its stable, inac-

† See, "The mode of action of preventive sera," p. 81 . 
tive form, as it is stored up against possible necessity in the body. In this latter form the antibody is incapable of destroying vibrios. To be sure, Pfeiffer does not deny that a granular transformation may occur in vitro with fresh cholera serum, but he thinks this effect is due to a small amount of the ferment substance in the serum that renders the antibody bactericidal. It is evident that Pfeiffer has been obliged to agree essentially with our point of view in order to explain bacteriolysis in vitro, at least to the extent of admitting that two substances are necessary. The resemblance between the two theories, however, is only superficial.

According to Pfeiffer and his pupils the substance present in fresh normal serum, that has been named alexin, would not be in the strict sense a bactericidal substance, but rather the ferment which transforms the inactive antibody into its active form. The transformation of vibrios in vitro by fresh cholera serum would be due to the effect of the active antibody and not to a direct action of the alexin, the one function of which is to produce the active antibody. Consequently the real cellulicidal substance would always be specific; instead of being the same in various immune sera, as we think of it, it would vary in each one, since the antibodies in immune sera are essentially different.

But if the alexin bears no direct causal relation to alterations shown by vibrios and corpuscles, why do normal sera, which have no specific antibodies, show a distinct if inferior bactericidal effect on vibrios? How, as Buchner has emphasized in his valuable researches,* can these normal sera produce a certain amount of hemolysis?

This hemolysis, by normal serum, although less, is nevertheless quite comparable to that produced by hemolytic sera. Artificial immunization, as indicated by the production of specific antibodies, does not produce cytolytic power, but renders it specifically more intense by forming a sensitizer or specific antibody. All this tends to invalidate Pfeiffer's theory.

This theory, to be sure, would seem more reasonable if cytolytic phenomena in vitro were much less intense than those in the animal body (peritoneal cavity). There is, however, no difference in inten-

* Buchner, as we know, brought out the fundamental fact that normal serum heated to 55 degrees loses both its bactericidal and globulicidal properties. 
sity, as we have already stated. To be sure, if a mixture is made in vitro containing too little cholera serum in proportion to the vibrios to give complete bacteriolysis, the injection of this mixture into the peritoneal cavity of a normal animal will frequently result in a granular transformation. It is evident, however, that by this procedure we increase the amount of alexin (peritoneal exudate), and such an increase of one of the two substances required for bacteriolysis naturally produces a greater effect.* To estimate bactericidal power correctly either in vitro or in vivo the vibrios used must be mixed with comparable amounts of the two active substances; if this precaution is observed it will be found that vibrios or corpuscles are altered as well in vitro as in vivo.

The properties of the antitoxin studied in the preceding pages suggests another objection to Pfeiffer's theory.

Let us mix a certain amount (e.g., 0.4 of a cubic centimeter) of hemolytic serum, 55 degrees (sensitizer), with a little fresh, normal guinea-pig serum ( 0.2 of a cubic centimeter). Similar tubes are prepared containing, in the place of guinea-pig alexim, rabbit and rat alexin respectively. Any one of these mixtures is strongly hemolytic for rabbit blood, although no one of the constituents alone is so. What has happened according to Pfeiffer's theory? The alexin employed has transformed the inactive thermostable antibody into the active and specifically globulicidal antibody. And consequently, in such a mixture, it is this active antibody and. not the alexin that destroys the corpuscles. It may be added that of the three alexins mentioned the one from the guinea-pig forms the best hemolytic mixture with the sensitizer.

Since each mixture contains the same hemolytic substance, we should expect, according to Pfeiffer's theory, that an antitoxin capable of neutralizing the destructive effect of one would also

* We may recall the point demonstrated by us in 1895 , that a fresh immune serum does not contain appreciably more alexin than normal serum. The sensitizing property of an immune serum, however, is so great that it can sensitize many more vibrios than its alexin is able to destroy. Conversely, the amount of alexin in a given dose of immune serum is too small to destroy all the vibrios that the serum can sensitize. For this reason fresh immune serum is able to destroy many more vibrios when fresh normal serum is added on account of the additional amount of the alexin. On summing up the facts on which our theory is based we noted that a small dose of immune sarum suffices to endow a relatively large dose of normal serum with intense bactericidal energy. 
neutralize either of the other mixtures. Let us add, then, to the most strongly hemolytic mixture, namely sensitizer plus guinea-pig alexin, some of our heated antitoxin ( 0.8 of a cubic centimeter). The mixture becomes harmless for rabbit corpuscles. The addition of the same amount of antitoxin to either of the other mixtures containing rabbit or rat alexin does not produce any neutralizing effect. This result, then, is contrary to a logical deduction from Pfeiffer's theory.* On the contrary, the results are quite explicable: the different alexins act directly on the sensitized corpuscles to destroy them. And the antitoxin, being specific for guinea-pig alexin, naturally affects the activity of this alexin only.

With this we conclude our remarks on the theories of the cytolytic sera. To sum up, our theory, offered in 1895, explains all the facts observed and is in disagreement with none of them. From a general standpoint this theory and facts on which it is founded contains three general ideas:

1. Artificial immunization simply renders the cytolytic manifestations of normal serum specifically more intense. This conception we demonstrated by showing that both normal and immune serum (cholera serum) produce a similar effect on the cholera vibrio, which effect, however, is of unequal intensity in the two instances. This conception has been confirmed by our being able to exalt the hemolytic activity of a given normal animal's serum by vaccinating the animal against red blood cells.

2. The immunized animal does not have its cytolytic substance increased over normal, but simply produces a large amount of the specific substance which favors the activity of the cytolytic substance. $\dagger$

3. A reaction of immunity, consisting in the production of an antibody, noted in an animal injected with harmless cells, such as red blood corpuscles, is identical with the reaction shown by an animal to an infective agent, for purposes of protection.

* Such an experiment naturally comprises suitable controls containing heated normal rabbit serum instead of the antitoxin. Hemolysis occurs in all the control mixtures and most markedly in the one containing guinea-pig alexin.

$\dagger$ The mechanism by means of which the sensitizing substance favors activity has recently given rise to interesting studies. Among them may be mentioned, in particular, the researches of Ehrlich and Morgenroth, which have been discussed in the present article. 


\section{Conclusions.}

Under this heading we shall note only the facts subjected to experimental proof in the first two sections of this article:

1. Corpuscles sensitized by a given sensitizer are destructible by the different alexins (normal sera) of many if not of all animal species.

2. In a given serum the bacteriolytic is identical with the hemolytic alexin.

3. The fixing properties of corpuscles for the active substances of hemolytic sera lie in their stromata. This fixation is similar to a process of dyeing.

4. An antitoxin to a hemolytic serum may be produced. This antitoxin has both an antisensitizing and an anti-alexic property.

5. Owing to its anti-alexic property the antitoxin is both antihemolytic and antibactericidal.

6. The anti-alexin would seem to neutralize the alexin by acting directly upon it.

7. The anti-alexin in question is specific, although not absolutely so. It neutralizes guinea-pig alexin, but has no effect on alexins from most other animals. 


\section{ON THE EXISTENCE OF SENSITIZING SUB- STANCES IN THE MAJORITY OF ANTIMICROBIAL SERA**}

BY DRS. JULES BORDET AND OCTAVE GENGOU.

(From Professor Metchnikoff's Laboratory.)

The serum of many animals contains alexin, that ill-defined, chemically unknown substance to which is due the property of sera in general of producing a harmful effect on various cells and on certain bacteria. The activity of the alexin is destroyed on heating serum to 55 degrees. Alexin is found, in relatively similar amounts, in the serum of normal and of vaccinated animals: artificial immunization changes neither its amount nor its properties.

When an animal is vaccinated against the cholera vibrio there is formed in the body a particular substance, the preventive or sensitizing substance, that resists heating to relatively high temperatures. This substance is not in itself in any way destructive for the vibrio. It does, however, faciliate in a specific manner the destructive action of the alexin on this micro-organism. It may further be said that the specific bactericidal property of cholera serum, although primarily due to the alexin, properly speaking, depends on the collaboration of two substances, the alexin and the favoring or sensitizing substance. This conception, offered by one of us in 1895, explains the various properties of cholera serum. It is closely applicable also to the specific hemolytic sera between which and cholera serum most evident analogies exist. In brief, the intense destructive power in bacteriolytic or cytolytic serum is due to the presence of a specific antibody, the sensitizer, in addition to the ordinary alexin.

In the preceding description, in referring to sera specific for bacteria, we have mentioned cholera serum only. As a matter of fact

* Sur l'existence de substances sensibilisatrices dans la plupart des sérums antimicrobiens. Annales de l'Institut Pasteur, XV, 1901, 290. 
it would have been hasty to assert that specific sensitizers are present in antimicrobial sera in general. Hitherto these bodies have been demonstrated with certainty only in the specific antisera for the true cholera or other similar vibrios.

This is quite understandable when we come to consider the known method of demonstrating the sensitizing substance. This method described by one of us in the case of cholera serum, and later applied to hemolytic sera, is as follows:

Mixtures are made in suitable proportions of cholera vibrios with normal serum and specific serum respectively. In the second mixture an intense destruction of the bacteria occurs, as is evidenced by their complete granular transformation. In the first mixture, on the contrary, a morphologically similar, but relatively insignificant, change takes place. Both cholera serum and normal serum lose their bactericidal power completely on being heated to 55 degrees. But the addition of a trace of heated cholera serum to unheated normal serum forms a mixture that is as strongly bactericidal as fresh cholera serum; it enables it to produce granules in the vibrios. This proves that heated cholera serum, although harmless alone, still favors the bactericidal energy of the alexin of normal serum.

This method, then, of demonstrating a sensitizer depends on the presence of some microscopically detectable lesion of the bacterium affected; bacteriolysis must occur. With hemolytic sera the criterion is the occurrence of hemolysis.

Not all bacteria, however, fulfill this condition. Many of them not only are undestroyed, but remain apparently unchanged in the presence of serum from highly immunized animals. In such cases the method described is of no avail and should be replaced by another.

We have, indeed, another method to offer for the demonstration of sensitizers in the sera of animals immunized against such bacteria as B. pestis, first anthrax vaccine, B. typhosus, the bacillus of swine plague, and $B$. proteus vulgaris.

But first we must recall an experiment described a year ago in the Pasteur Annals * the essentials of which follow:

If well-sensitized rabbit blood corpuscles (that is, corpuscles * See p. 186. 
treated with a hemolytic serum heated to 55 degrees) are added to fresh guinea-pig serum containing alexin, their dissolution follows. If after a certain time sensitized cholera vibrios (i.e., vibrios treated with heated cholera serum) are added and the mixture placed in the incubator, no transformation or change takes place in the vibrios. From this result we know that there was no free alexin in the mixture, for, if present, it would have transformed the vibrios. From a control tube we learn that a transformation occurs in the vibrios if the red blood corpuscles added in the first place are not sensitized.

The converse of this experiment also holds. If sensitized cholera vibrios are added to normal alexic serum, subsequently added sensitized corpuscles are not hemolyzed.

From such experiments we drew, it will be recalled, two distinct conclusions: First, corpuscles or bacteria when sensitized are able to absorb alexin with avidity and to remove it from the surrounding fluid; second, in a given serum the same alexin may produce either hemolysis or bacteriolysis.*

These conclusions find still further confirmation in the following article. In the present article a fact suggested by the experiment just outlined is of preëminent interest: To demonstrate the existence of a sensitizer in an antimicrobial serum we may make use of its property of causing the bacterium it affects to absorb alexin.

As an experiment to demonstrate this fact is practically the same with any one of the antimicrobial sera studied, it may be described in detail once for all. We shall take as an example antiplague serum.

Serum of a horse vaccinated against B. pestis. - This strongly preventive serum was kindly furnished us by Dr. Dujardin-Beaumetz, who has charge of preparing it and testing its potency at the Pasteur Institute.

This serum and normal horse serum were heated to $56^{\circ} \mathrm{C}$ for one half hour to destroy the alexin. A 24-hour agar culture of B. pestis was suspended in a small amount of salt solution so as to form a thick emulsion of bacteria. Fresh guinea-pig serum obtained by bleeding the guinea-pig the day before, and freed from corpuscles by centrifugalization, was also at hand and was used for alexin.

* The unity of the alexin in a given serum is also admitted by Buchner. 
The following mixtures were then prepared in test tubes:

(a) Alexic serum, 0.2 c.c.; emulsion of plague bacilli, 0.4 c.c.; antiplague serum, 56 degrees, 1.2 c.c.

(b) Same as " $a$," with normal horse serum, 56 degrees, replacing the antiplague serum.

(c) Same as "a," but containing no emulsion of plague bacilli. (d) Same as "b," but containing no emulsion of plague bacilli. Each of these four mixtures contains the same amount of alexin (normal guinea-pig serum).

(e) Emulsion of plague bacilli, 0.4 c.c.; antiplague serum, 56 degrees, 1.2 c.c.

(f) Emulsion of plague bacilli, 0.4 c.c.; normal horse serum, 56 degrees, 1.2 c.c.

These two tubes are the same as " $a$ " and " $b$ "respectively, without alexin.

The mixtures are left at room temperature (15 to 20 degrees) for about 5 hours. To each tube in turn is added 0.2 of a cubic centimeter of a mixture composed of 2 cubic centimeters of serum from a guinea-pig immunized against rabbit blood and previously heated to 55 degrees, and 20 drops of defibrinated rabbit blood.* In other words, each tube receives 2 drops of well-sensitized blood.

The result of the experiment is as follows:

Hemolysis takes place rapidly and nearly simultaneously in tubes "b," "c," and "d." In ten minutes no intact corpuscles remain. In tube " $a$," containing alexin, bacilli and antiplague serum, no hemolysis occurs. The corpuscles also remain intact in tubes " $\mathrm{e}$ " and "f," to which no alexin was added. We find then, first, that the plague bacillus mixed with normal horse serum does not absorb alexin; and second, that the bacillus with antiplague serum from the horse does absorb alexin with avidity; and third, that antiplague serum alone has no effect on the alexin.

Consequently we must conclude that the serum of a horse vac-

* As already mentioned in previous articles, blood that has been washed in salt solution is generally used in experiments of this nature. 1 to 2 c.e. of defibrinated blood is placed in a centrifuge tube and the level marked on the glass. After centrifugalization the supernatant fluid is drawn up with a bulb pipette, leaving the deposited corpuscles. Enough salt solution is added to restore the blood to its original level. This constitutes defibrinated blood with the serum replaced by salt solution, in other words, containing no alexin. 
cinated against B. pestis contains a sensitizer that endows this bacterium with the power of absorbing alexin. This sensitizer then acts as do the corresponding substances present in cholera serum and in hemolytic sera.

It may be added that plague bacilli added to a mixture of alexin and antiplague serum show no morphological alteration after 3 hours at 37 degrees. Consequently in this instance the presence of a sensitizer can be demonstrated only by the fixation of the alexin.

If in such an experiment much smaller doses of bacilli and antiplague serum with the same dose of alexin are employed, a complete fixation does not take place. The subsequently introduced corpuscles are finally hemolyzed, but only after a more or less considerable delay.

The serum of guinea-pigs vaccinated with the first anthrax vaccine. - Guinea-pigs were given four successive intraperitoneal injections of the first anthrax vaccine. Five- to six-day pepton-bouillon cultures were used for the first two injections. Three- or four-day agar cultures suspended in salt solution were used for the last two injections.

An experiment similar to the one with antiplague serum was then performed. As a control, in the place of the serum of the vaccinated guinea-pigs, normal guinea-pig serum was used. As a bacterial emulsion a 24-hour agar culture of the first vaccine suspended in salt solution was used. Fresh normal guinea-pig serum furnished the alexin.

The results were identical with those obtained with antiplague serum. The first vaccine plus normal serum absorbs little or no alexin. There is complete fixation with the specific serum. In this instance, also, the fixation of alexin causes no visible lesion of the bacterium.

Serum of a horse immunized against swine plague.-Dr. Frasey of the Pasteur Institute was so kind as to furnish us with strongly preventive serum for the swine-plague bacillus. In this serum we were also able by the same method to demonstrate a sensitizer which allows the swine-plague bacillus to fix alexin.

Serum of guinea-pigs vaccinated against B. typhosus. - These guinea-pigs were given three injections of B. typhosus suspended from agar cultures in salt solution. An experiment modeled after 
the others showed that the active serum of these guinea-pigs causes an energetic fixation of alexin by B. typhosus.

It seemed worth while in this instance to determine whether this sensitizer, that causes a fixation of alexin, is strictly specific. For this purpose the experimental tubes were doubled in the following manner: One series of tubes contained mixtures of normal and specific sera with or without the emulsion of specific bacilli, as has already been described. In a second series of tubes the amounts of serum were identical with the first series, but an emulsion of $\mathrm{B}$. coli instead of B. typhosus was used. Twenty-four-hour agar cultures of each organism suspended each in 4 c.c. of salt solution were used. The agar surface covered was approximately the same with each organism; and yet the colon suspension, judging from its growth, contained more organisms. We mention this point so that a failure of B. coli to fix the alexin in presence of antityphoid serum may not be attributed to an insufficient dose of bacteria.

The result of such an experiment is very clear. The antityphoid serum shows very marked and yet not absolute specificity. In fact the colon bacillus when mixed with antityphoid serum acquires the property of absorbing alexin to a certain degree. Whereas relatively small doses of the typhoid bacillus and antityphoid serum absorb alexin completely, much larger doses of $\mathrm{B}$. coli with the same serum are required to produce even a partial fixation. For example, if sensitized blood corpuscles are added to a mixture of 0.2 c.c. of guinea-pig alexin, 0.2 c.c. typhoid emulsion and 0.6 c.c. antityphoid serum ( 56 degrees) that has been standing for a few hours, they remain indefinitely intact; in a mixture containing the same amount of alexin and twice the dose of antityphoid serum and colon emulsion, the same dose of corpuscles is hemolyzed after an hour's delay. There is a very distinct delay in this tube, as the controls containing bacteria are completely hemolyzed in 15 minutes, and those without bacteria in 2 minutes.

It is evident, then, that the colon bacillus reacts distinctly, though much less powerfully, than the typhoid bacillus to the sensitizing effect of antityphoid serum.*

Serum from convalescent typhoid patients. - Dr. Widal has been

* It may be noted that this serum agglutinates B. typhosus well, but has no more effect on $B$. coli than normal serum. 
so kind as to allow us to take 3 to 4 c. c. of blood from two of the convalescent cases of typhoid in his hospital service. Both these women had shown the classical symptoms of typhoid fever. At the time the blood was taken the temperature had been normal for from 20 to 30 days.

The sera obtained from the two patients were heated, together with control sera from each of us, who have never had typhoid, to 56 degrees for a half hour. A smail amount of one of the control sera was kept unheated for alexin.

The result of the experiment was very convincing. In tubes containing alexin 0.2 c.c. (unheated human serum), emulsion of B. typhosus $(0.5$ c.c.)* neither of our heated sera caused any fixation of the alexin. Sensitized rabbit corpuscles added a few hours later were hemolyzed as rapidly as in control tubes containing the sera without bacterial emulsion. In the tubes containing human alexin, typhoid bacillus, and either of the heated sera from typhoid convalescents, subsequently added sensitized corpuscles remained intact for days. In similar mixtures of sera without bacilli, hemolysis occurred with customary rapidity.

Consequently, the power of causing the typhoid bacillus $\dagger$ to absorb human alexin is very marked in the serum of patients convalescent from typhoid.‡

It would be of interest to determine just how highly specific such a serum is, particularly by comparing the reaction as between B. typhosus and B. coli. The time in the course of the disease at which this power appears in the serum is also of interest, but would necessitate an examination of a large number of cases. Hitherto we have had neither the time nor the material to consider these problems.

Serum of guinea-pigs vaccinated against B. proteus vulgaris. - The method described for demonstrating the existence of a sensitizer by

* An emulsion prepared by suspending a 24-hour agar culture of B. typhosus in 5 c.c. salt solution of 0.7 per cent.

$\dagger$ The culture of B. typhosus used was one that had been carefully controlled by Dr. Binot of the Pasteur Institute, who was so kind as to give it to us.

‡ These particular sera were only faintly agglutinative for B. typhosus. In this connection the observations of Pfeiffer and Kolle may be recalled; they showed that the agglutinating power in such sera does not run parallel to the bactericidal power. 
fixation of alexin is superfluous in the case of antiproteus serum. The proteus bacillus we have used gives a granular degeneration similar to the one shown by the cholera vibrio in cholera serum when placed in contact with fresh normal serum.

We know that the vibrio, too, is slightly affected by normal guineapig serum. Proteus vulgaris gives a complete granular transformation with a sizable dose of normal serum, but a much smaller amount suffices if a little fresh or heated antiproteus serum is added. This, of course, demonstrates the presence of a sensitizer in the immune serum.

But we have also done the alexin fixation experiment with Proteus vulgaris. The expected result was obtained: the organism in the presence of normal guinea-pig serum absorbs little or no alexin, but, when affected by the specific serum, takes it up. In the latter instance, of course, sensitized corpuscles are not destroyed.

In the experiments up to this point we have uniformly employed sensitized rabbit corpuscles (i.e., those treated with specific serum from the guinea-pig heated to 55 degrees) to prove the presence or absence of free alexin in the fluid. In view of the conception of the unity of the hemolytic and bacteriolytic alexin as proved a year ago we might have used as an indicator either other corpuscles or sensitized bacteria, such as cholera vibrios, to show the presence of free alexin by a morphological change. Such an experiment we have performed with $\mathrm{B}$. proteus vulgaris as an indicator:

A 24-hour agar culture of Proteus vulgaris was suspended in 6 c.c. of salt solution. An agar culture of the cholera vibrio was treated in the same manner. Fresh normal guinea-pig serum, as alexin, and heated proteus serum and cholera serum were also at hand. The following mixtures were prepared:

(a) Alexic serum, 0.2 c.c.; proteus emulsion, 0.3 c.c.; proteus serum, 56 degrees, 0.6 c.c.

(b) Alexic serum, 0.2 c.c.; proteus emulsion, 0.6 c.c.; normal guinea-pig serum, 56 degrees, 0.6 c.c.

(c) Same as "a," without bacteria.

(d) Same as "b," without bacteria.

Five hours later 0.2 of a cubic centimeter of the following mixture was added to each tube: Emulsion of cholera vibrios 0.5 cubic centimeter, cholera serum, 1 cubic centimeter 56 degrees (from a 
vaccinated guinea-pig). After $1 \frac{1}{2}$ hours in the thermostat stained preparations were made from each tube.

In tube " $a$ " we found that the cholera vibrios kept their normal appearance, but all the Proteus rods showed granular degeneration. In tube "b," on the contrary, numerous Proteus rods were found but the vibrios had all lost their normal appearance and become completely metamorphosed. In tubes " $\mathrm{c}$ " and " $\mathrm{d}$," containing no Proteus, as might be expected, the vibrios were completely transformed.* It is obvious, then, that in tube "a" the sensitized proteus bacilli have absorbed the alexin and so protected the subsequently added vibrios.

The mixtures were left at room temperature overnight and the next day placed in the incubator for 6 hours. When stained preparations were then made the results were striking.

In tube " $a$, " in which the proteus bacilli were destroyed, the cholera vibrio has undergone marked multiplication. In tube "b," containing non-sensitized Proteus, the reverse is true; the Proteus has grown out, but no vibrios are found. Therefore in these two tubes containing the same amount of alexin the bactericidal power has been directed, in one, against one organism, in the other, against another. Although the vibrios in each mixture were equally sensitized, they have grown in tube "a," because the harmful influence of the alexin was diverted by the properly sensitized Proteus vulgaris; this bacillus has acted, in a way, as a shield for the vibrio. $\dagger$

The data considered in the present article, when added to those we already have, give the conception, that under immunization an animal forms an appropriate sensitizer capable of causing the bacterium it affects to absorb alexin, the appearance of a general law.

We shall not here consider how far the presence of a sensitizer in

* In controls of both the vibrio and Proteus made each with its heated specific serum alone there is agglutination, but no metamorphosis.

$\dagger$ This is a correct demonstration of the principle established by one of us in 1895: "That the bactericidal substance is the same in normal as in vaccinated animals. In the case of animals vaccinated against certain diseases the energy of the bactericidal substance acts particularly against a given microbe owing to the specific preventive substance (sensitizer), which varies according to the bacterium used for immunization. It is by means of this peculiar preventive substance that the animal body directs its destructive power against a particular infection." 
any one of these sera endows it with curative or preventive properties for normal animals. We may say simply that the function of sensitizers in protecting animals must vary with the bacteria under consideration. Certain of these bacteria are easily destroyed in fixing alexin; others under the same conditions resist better (leaving aside any purely physiological alteration that may occur); and others doubtless absorb alexin with impunity.* Several therapeutic sera owe their activity in great part to the presence of antitoxins. The seat of infection must enter into account, as alexin is not uniformly distributed throughout the body. It is certain that the part played by each substance of active serum in a cure by serum therapy must vary with the infection under consideration.

There is one fact frequently mentioned in this article that we may consider for a moment. The bacteria studied had little or no alexin-fixing property unless sensitized, and even when sensitized the bacteria had to be relatively numerous tc absorb alexin. Consequently, in animals invaded by a pathogenic micro-organism, we should scarcely imagine death to be due to an insufficiency of alexin in the body. An assertion that this is the cause of death is, moreover, to lose sight of the fundamental and well-proved idea that yielding to an infection is primarily due to an inability of the phagocytes to take up the infective agent. And then - even supposing protection by alexin to be the essential factor in immunity - death would not be due to a lack of alexin, but rather to a lack of utilizing or absorbing it.

We should scarcely expect in treating human bacterial infections that - as Wassermann has expressed the hope - the administration of certain normal sera or alexins in addition to the specific serum could be recommended. It is unreasonable, since the alexins from foreign species affect not only bacteria, but body cells. And, moreover, the animal would soon protect itself from such injections by forming anti-alexins.

* We intend to determine whether the serum of tuberculous individuals contains a sensitizer capable of causing Koch's bacillus to absorb alexin. If such a sensitizer is present, it would be a good example of one that serves no distinctly useful purpose. 


\section{Conclusioin:}

1. Specific sensitizers are formed in vaccinated animals as a general rule. The sensitizers active against various bacteria have the common property of causing the organisms they affect to absorb alexin.

2. The extent of the damage that alexin absorption causes in bacteria varies with the organism concerned. 


\section{ON THE MODE OF ACTION OF CYTOLYTIC SERA; AND ON THE UNITY OF THE ALEXIN IN A GIVEN SERUM.*}

BY DR. JULES BORDET.

(From Professor Metchnikoff's Laboratory.)

The conception that bacteriolysis by cholera serum and that hemolysis by hemolytic sera are due to the combined action of two distinct substances, as proved by us in 1895 and 1898, is now generally accepted. These two substances are, first, the alexin, the cellulicidal and bactericidal substance, properly speaking, occurring in the serum both of normal and of immunized animals; and, second, the specific sensitizing substance that endows an immune serum with the particular property of favoring alexic activity. In collaboration with Dr. Gengou we have described in the preceding article a method of demonstrating the existence of sensitizing substances. This method depends essentially on the fact that a specific bacteriolytic or hemolytic serum heated to 55 degrees, and so deprived of its own destructive power, will confer a bactericidal or hemolytic power on normal alexic serum to which it is added. We further demonstrated by a new method that these sensitizing substances are present, certainly, in many, and probably in all, antimicrobial sera resulting from artificial immunization.

But the elementary fact that the destructive action of a serum is due to the collaboration of the alexin and the sensitizer is not sufficient. An attempt should be made to discover the inner mechanism of the phenomenon and to determine the exact nature of the reaction between the active substances and the affected cells. It is only since 1899 that this new problem has been attentively considered; the data obtained are not as yet very numerous and yet three important facts have been firmly established. These facts we shall enumerate and shall then consider the interpretations to which they have given rise.

* Sur le mode d'action des sérums cytolytique et sur l'unité de l'alexine dans un même sérum. Annales de l'Institut Pasteur, XV, 1901, 303. 


\section{Does the Alexin Unite with the Sensitizing Substance?}

The first of the three facts mentioned concerns normal sera particularly. In many instances when a certain amount of normal serum from one species is added to the red blood corpuscles of another species only slight hemolysis occurs. It is particularly true that a large number of corpuscles remain intact if the contact is brief. If we then separate these corpuscles by centrifugalization, and wash them so as to remove the normal serum, we find on adding a sensitizer (that is, a heated serum active against the corpuscles in question) that no hemolysis occurs. This experiment was first performed by Ehrlich and Morgenroth.* It proves that non-sensitized corpuscles mixed with normal serum do not absorb the alexin, for we know that if the alexin is present the addition of an appropriate sensitizer will hemolyze the corpuscles. This fact, that unsensitized corpuscles do not fix the alexin of normal serum, is applicable only to those corpuscles that have remained intact, but bears no relation to the few corpuscles that have been destroyed. There are certain normal sera that have great hemolytic activity for certain species of corpuscles. For example, hen serum destroys rabbit corpuscles energetically. $\dagger$ When these corpuscles are added to hen serum they absorb alexin very distinctly, as is shown by the fact that fresh rabbit corpuscles added to such a hemolyzed mixture remain intact. $\ddagger$

We may conclude from these two experiments that, when mixed with normal serum, corpuscles that remain intact do not affect the alexin, whereas corpuscles that are destroyed absorb a certain amount.

The two other facts about the specific hemolytic sera, obtained by injecting animals with defibrinated blood, are the following:

When a hemolytic serum, previously heated to 55 degrees, is mixed with the corpuscles affected by that serum, these corpuscles

* See Collected Studies on Immunity. Ehrlich-Bolduan, John Wiley and Sons, p. 1.

$\dagger$ See article, p. 134.

$\ddagger$ They remain intact even if heated hen serum is also added to the mixture, which proves that it is indeed the alexin that has been absorbed. The objection indeed might be raised that the first corpuscles destroyed have not absorbed much alexin, but have fixed the normal sensitizer of hen serum necessary for a subsequent hemolysis; the addition of heated hen serum answers this objection. 
absorb the sensitizer energetically and deprive the fluid of it. This fact was first irrefutably proved by Ehrlich and Morgenroth.*

The third fact established by us a year ago $\dagger$ is that, in a mixture of fresh normal serum (alexin) and sensitized corpuscles or bacteria (that is, treated with an appropriate heated hemolytic or bacteriolytic serum) the alexin that destroys the sensitized cells is absorbed by them and disappears from the fluid. This fixation may be so complete that the fluid completely loses its power to destroy subsequently added sensitized cells.

These are the principal facts derived from experiments, apart from any theory. We may now attempt to explain the reactions between sensitive cells and active substances in the light of these facts.

According to Ehrlich and Morgenroth the specific antibody (sensitizer) acts as a real intermediary body (Zwischenkörper, Amboceptor), a joining link united on the one hand to the corpuscles and on the other to the alexin. In other words, the absorption of the alexin by the sensitized corpuscles is not due to any distinct affinity of the corpuscles for the alexin. The absorption of the alexin is indirect only: the corpuscle is joined to the intermediary substance which unites chemically by its other pole with the alexin.

Our conception of the phenomenon is quite different. We regard the sensitizer as uniting with the corpuscle and so modifying it as to allow a direct absorption of the alexin. The action of the sensitizer on cells would be similar to that of fixing or mordanting agents that give certain substances or, in histological technic, certain cells the power of absorbing dyes that were previously not taken. We know that slight modifications suffice to make cells take stains that normally do not affect them. To be sure, in speaking of mordanting we do not mean that all the phenomena of dyeing must agree with the phenomena under consideration; we have simply offered a comparison in order to simplify our explanation. The hypothesis we wish to emphasize is that in presence of hemolytic serum the corpuscle itself becomes capable of absorbing alexin directly, owing to its modification by the sensitizer. In other words, we see no reason for considering that the sensitizer itself combines

* Loc. cit., p. 6.

$\dagger$ See p. 186. 
with the alexin, as Ehrlich and Morgenroth believe, or that this union is necessary in order that the alexin may reach the corpuscle.

It must be admitted that both of these interpretations are purely hypothetical and have been sanctioned by no well-proved fact. We should then find out how far either of these hypotheses conforms with reality by determining how far deductions from them agree with experimental fact.

Let us consider a fresh hemolytic serum containing both alexin and sensitizer. According to Ehrlich and Morgenroth, this sensitizer is combined with alexin-if not with all of it, owing to its possible excess, at least with a more or less considerable amount of it. When corpuscles are added the sensitizer unites with them, dragging after it the alexin fixed by its other pole. The amount of alexin that can affect the corpuscles must be only that portion previously fixed by the sensitizer. If there is more alexin in the serum than is necessary to saturate the sensitizer, the excess could have no effect on the corpuscles and would consequently not be utilized. We may therefore conclude that the added corpuscles are unable to modify in the slightest degree the established relations between alexin and sensitizer; they simply fix the sensitizer and are destroyed by the alexin already united with this intermediary body.

What will happen if we add to such an active hemolytic serum, specific, let us say, for rabbit corpuscles, another sensitizer, say a heated serum affecting hen corpuscles? The result is, as might be anticipated, that the mixture obtained destroys hen or rabbit corpuscles indifferently. In accordance with the theory of Ehrlich and Morgenroth one must consider the two sensitizers as struggling to share the alexin; part of it unites with sensitizer $\mathrm{A}$ and the rest with sensitizer B. If we then add the corpuscles affected by sensitizer A, obviously they will absorb it and be injured by the alexin already united to this intermediary body $\mathrm{A}$. But there is no logical reason to suppose that these corpuscles will be attacked by the rest of the alexin united to the other sensitizer that has no combining influence with them. Consequently, on adding subsequently the other species of corpuscles, we should expect them to be destroyed by the remainder of the alexin attached to sensitizer $B$ which is specific for these corpuscles. 
Such an experiment planned to corroborate these theoretical deductions from the Ehrlich and Morgenroth's hypothesis fails completely to do so. Two exactly similar mixtures A and B are prepared, containing each 0.2 of a cubic centimeter of fresh hemolytic serum from a guinea-pig that had been given four injections (from 4 to 5 c.c.) of rabbit blood, and 1 c.c. of the heated (55 degrees) serum of a rabbit treated with hen blood. To mixture $\mathrm{A}$ is added 0.6 of a cubic centimeter of defibrinated washed hen blood; the corpuscles are soon destroyed. Nothing is added to mixture $\mathrm{B}$ for the moment.

A few hours later 2 drops of defibrinated rabbit blood are added to each mixture. In mixture $\mathrm{B}$ destruction of the corpuscles is complete in about 45 minutes. There is then enough alexin present to destroy these corpuscles; we have, moreover, admitted that the portion of the alexin that produces this effect was already combined with the sensitizer for the corpuscles in question. The other mixture, however, proves that this is not so.

Mixture A is identical with B except it contains in addition hemolyzed hen corpuscles. The rabbit corpuscles remain intact in this mixture; even on the following day they may be discerned among the nuclei of the hen corpuscles.* The hen corpuscles then have used up all the alexin, and if we were to follow the hypothesis of Ehrlich and Morgenroth, we should be forced to conclude that the sensitizer acting on hen corpuscles had combined with all the alexin, preventing the other sensitizer (active against rabbit corpuscles) from sharing it. Such a conclusion must also hold for mixture B containing the same doses of the same sera. And yet in this mixture B the sensitizer that has not combined with the alexin has been able to destroy its appropriate corpuscles. There is no reason, then, in explaining hemolysis, to assume that the sensitizer combines with the alexin. On the contrary, it seems clear to us from this experiment that the corpuscles modified by their union with the sensitizer absorb the alexin directly and prevent it from acting on other cells. When the corpuscle is not present the sensitizer in no way fixes the alexin or prevents it from acting on the first properly

* The destruction of the rabbit corpuscles takes place on addition of alexin (normal guinea-pig serum); the corpuscles are then rapidly destroyed, showing that they have absorbed their appropriate sensitizer. 
sensitized cell with which it comes in contact. It would be well, then, to give up the terms Zwischenkörper, Amboceptor, and Complement, words chosen under the domination of theories which, although ingenious, and capable of having advanced science by the experiments they have suggested, are not justified by experiment.*

This experiment we have just quoted is similar to those we have already discussed in previous articles. We wish here simply to emphasize more fully the conclusions that may be drawn from them; it also seems wise to repeat the experiment with certain variations to show that it invariably gives the same results.

For example, we can make a mixture of 0.5 of a cubic centimeter of fresh unheated, normal guinea-pig serum, 2.5 c.c. of heated serum from a guinea-pig immunized against rabbit blood and 2.5 c.c. of heated serum from a guinea-pig immunized against hen blood. One-tenth of a cubic centimeter of this mixture, containing alexin and the two sensitizers, destroys 0.1 of a cubic centimeter of defibrinated washed hen blood. The hen blood, however, remains intact in twice the amount of mixture, 0.2 of a cubic centimeter, if there has previously been added to it 0.3 of a cubic centimeter of washed rabbit blood. The rabbit corpuscles are destroyed and absorb all the alexin present without leaving any for the hen-corpuscle sensitizer.

In a similar mixture of fresh normal guinea-pig serum, heated guinea-pig serum affecting rabbit blood, and heated rabbit serum active against hen corpuscles, the reverse experiment may be performed. . This mixture is divided in equal parts. To one is added corpuscles A and a few hours later corpuscles B; to the other, first $\mathrm{B}$, then $\mathrm{A}$. As in the preceding experiments, the introduction of

* In the experiment just considered there is a third mixture $\mathrm{C}$ containing, as the others, 0.2 of a cubic centimeter of fresh hemolytic serum specific for rabbit corpuscles. Instead of heated rabbit serum active against hen corpuscles it contains the same amount (1 c.c.) of heated normal rabbit serum. When 0.6 of a cubic centimeter of hen blood is added there is no hemolysis. On adding 2 drops of rabbit blood hemolysis is as rapid as in B. Since B and C act alike we conclude that neither the sensitizer for hen corpuscles, nor hen corpuscles themselves, are able alone to absorb alexin; the two must be united to produce this result. As we have already shown in a similar experiment (see p. 186), corpuscles from species other than the one affected by the sensitizer that is present leave the alexin unchanged. 
one kind of corpuscle prevents destruction of the second kind. Controls show that the amount of either species of corpuscles added is rapidly destroyed with even half the amount of each mixture provided that the other kind of corpuscles has not previously been added.

The experiments reported in collaboration with Dr. Gengou in the previous article are also of significance in this connection. They prove that the addition to alexic serum of a sensitizer active against bacteria does not deprive the serum of its power to destroy subsequently added sensitized corpuscles or sensitized bacteria of another variety. But if the sensitizer added in the first place is accompanied by the bacteria for which it is specific, the serum is entirely deprived of its alexic power. Here again it is the sensitizer plus bacterium and not the sensitizer alone that takes up the alexin.

We may now consider an objection that Ehrlich and Morgenroth have raised to our hypothesis. These invėstigators imagine that to agree with our conception a sensitized corpuscle should be equally affected by any alexin coming from any animal species. They mention the fact that rabbit corpuscles must be much more powerfully sensitized to be hemolyzed by rabbit alexin than when guineapig alexin is employed.

The fact is exact; we are all the more ready to admit it as we ourselves mentioned it in the article that gave rise to Ehrlich and Morgenroth's objection.* We are at a loss, however, to understand how these investigators should imagine that in our opinion sensitized corpuscles should be equally susceptible to various alexins. Our idea is quite the opposite. Since the alexins in the different animal species are not identical, it is quite obvious that they should not all have an equal tendency to destroy a given corpuscle. And consequently a weak sensitization may at times render a corpuscle susceptible to certain alexins, while a strong sensitization would be necessary to cause weaker alexins to produce as great an effect. In the instance under consideration, it is quite conceivable that rabbit corpuscles must be strongly sensitized to yield to rabbit alexin, which, under normal conditions, is quite harmless for its proper corpuscles. It is evident, then, that a given dose of a given sensi- 
tizer would seem to affect a corpuscle differently, according to the alexin used. In different instances the sensitizer may be the same, but the dose would vary with the alexin employed.

From this easily explicable fact, that the amount of sensitizer necessary varies with the alexin employed, Ehrlich and Morgenroth feel justified in concluding that the serum contains several sensitizers, all of which affect rabbit corpuscles, but which differ from one another. One of these sensitizers renders the corpuscle susceptible to guinea-pig alexin, and another to rabbit alexin. This complicates unnecessarily the already sufficiently complicated question of hemolytic sera.*

\section{On the Unity of the Alexin in a Given Serum.}

We know that the alexins from different animal species are not identical. But does a given alexic serum, say from the guinea-pig, contain a single alexin or several of them that differ in chemical constitution? It is rather difficult to reply to the question put in this way, as our knowledge of the chemical nature of the alexin is practically nil. We may question, however, whether the alexin is functionally simple, that is to say, whether the alexin (or alexins, if there are several) can attack various cells such as corpuscles and bacteria indifferently, particularly when these cells are sensitized. In this form the question is of lively interest in studies on immunity and may be more easily answered.

We know that Buchner in his first studies on the alexins of normal sera, $\uparrow$ that have by now become classical, considered the substance that destroys bacteria as identical with the one that produces hemolysis. We offered facts a year ago that seemed to corroborate this opinion.

We have just recalled these facts in the preceding article. When a serum is deprived of its alexin by the addition of a sensitized bacterium or corpuscle, the fluid becomes incapable of destroying another sensitized cell, even when it is different from the one that has taken up the alexin in the first place. It must then be always

* By the same process of reasoning Ehrlich and Morgenroth think that in a given normal serum there are several intermediary bodies (sensitizers), all active against the same corpuscles, but each requiring a different alexin.

$\dagger$ See, for example, Verhandlungen der Congresses für innere Medicin, 1892. 
the same alexin that unites with and destroys various cells. In the preceding article with Dr. Gengou we found that various bacteria absorb the alexin which is essential for the destruction of red blood cells or of other bacteria.

We may mention two other similar experiments. The red spirillum, a non-pathogenic organism, is readily destroyed by the serum of a normal guinea-pig, even without the addition of a sensitizer. In each of two tubes is placed 0.2 of a cubic centimeter of fresh hemolytic serum obtained from guinea-pigs immunized against rabbit blood. To tube "a" 0.3 of a cubic centimeter of guinea-pig blood is added; to tube " $b$ " 0.3 of a cubic centimeter of washed rabbit blood. After a few hours 2 drops of an emulsion of the spirillum are added to each tube and they are placed in the incubator. The spirilla are destroyed in tube " $a$," in which the guineapig corpuscles have remained intact; they remain normal in tube "b" containing hemolyzed rabbit corpuscles. Sensitized hen corpuscles subsequently added to both tubes are hemolyzed in " $a$," but remain intact in "b." Rabbit corpuscles, then, by absorbing the alexin protect two very different cells - the spirillum and hen corpuscles.

In the other experiment we have to deal with a rather peculiar instance. We may ask whether the rabbit alexin that destroys sensitized rabbit corpuscles differs from the alexin in the same serum which attacks other species of corpuscles. Experimentally we find that it does not. In each of two tubes " $a$ " and " $b$ " is placed 0.2 of a cubic centimeter of washed hen blood; 0.2 of a cubic centimeter of fresh normal rabbit serum is then added to each. To tube "a" is then added 0.6 of a cubic centimeter of serum from a rabbit treated with hen blood and heated to 56 degrees. To tube "b" 0.6 of a cubic centimeter of normal rabbit serum ( 56 degrees) is added. The hen corpuscles are hemolyzed in " $a$," but remain intact in "b." After a few hours 0.2 of a cubic centimeter of the following mixture is added to each tube: Washed rabbit blood, 20 drops; serum of a guinea-pig treated with rabbit blood and heated to 56 degrees, 2 c.c.

These sensitized corpuscles are destroyed in " $b$ " and remain intact in "a."

The fact that a given alexin may attack different cells seems to 
us sufficiently proved by all these experiments.* We must now take up certain objections that have been offered to this opinion, some of which seem rather serious.

Ehrlich and Morgenroth found that the serum of a goat immunized against sheep blood, when heated to 56 degrees or even higher, loses its power of destroying various species of corpuscles, such as the guinea-pig and the rabbit, which it previously attacked. This loss affects the non-specific hemolytic power present in normal goat serum and acting on corpuscles not used in the immunization of this animal. But this goat serum still shows a distinct ability to destroy sheep blood, for which it is specific. Ehrlich and Morgenroth conclude that this serum contains several alexins, some of which are destroyed at 56 degrees and others of which resist this temperature. It is just as easy to imagine that we have to deal with a single alexin modified or attentuated by heating. The alteration is sufficient to inhibit the hemolysis of non-sensitized corpuscles, which is never very strong, but does not prevent the destruction of corpuscles that are sensitized and so rendered more susceptible by the same serum.

In another experiment Ehrlich and Morgenroth pass the serum of a normal goat, that destroys guinea-pig and rabbit corpuscles, through a Pukal filter. After filtration the serum still destroys guinea-pig corpuscles, but does not affect rabbit corpuscles. According to these authors one of the alexins is retained by the filter and the other passes through; this would imply that the two alexins in question must have well-marked chemical differences to act so differently in respect to the filter. It is to be noted that here again we are dealing with hemolysis by a normal serum which is never intense and may be affected by slight variations. It is quite evident that the serum after filtration does not have as much alexin as before, but there is no reason for supposing that there are two distinct alexins. It may well be imagined that filtration, by removing from serum some of its elements, has so modified its physical properties as to render it less favorable for preserving corpuscles; we may well imagine, then, that certain species of corpuscles,

* It is also in harmony with the fact we previously established, that an antialexic serum, neutralizing the alexin of the serum of a given species, protects the various cells that are affected by this alexin, even when they are sensitized. 
which are more susceptible than others to the physical changes in the surrounding fluid, should yield more readily to traces of alexin. However this may be, this experiment does not deal with sensitization by specific serum and therefore does not affect our thesis that the same alexin can destroy various sensitized cells energetically.*

Neisser, $\uparrow$ a partisan of the plurality of alexins in a given serum, emphasizes experiments both by Bail $\ddagger$ and by himself, the principle of which is as follows: if a normal serum is mixed with cell $\mathrm{A}$ (bacterium or blood cell), and, after a sufficient contact, centrifugalized, it is found that the supernatant serum has lost its property of destroying cell A, but will still destroy cells B or C. Neisser concludes from this that the alexin affecting $\mathrm{A}$ is not identical with the one attacking $\mathrm{B}$ or $\mathrm{C}$.

The experiment contains a serious experimental error and consequently any conclusion from it is erroneous. In the majority of instances, when even large amounts of non-sensitized cells are mixed with normal serum, they absorb only a small amount of alexin. Our experiments just related as well as those with Gengou prove this fact conclusively. Under these conditions the fluid still retains its property of destroying subsequently added sensitized cells. It is reasonable to expect, therefore, that if an unsensitized or feebly sensitized cell is added to normal serum, for example corpuscles of species A, there will soon be a state of equilibrium established and a division of the alexin between the cell and the fluid; the fluid, however, will retain the greater part. Hemolysis, moreover, never goes beyond a certain point. It is quite possible that destroyed corpuscles liberate a substance that prevents the fluid from acting further on corpuscles of the same variety, without inhibiting its effect on other varieties of corpuscles. In other words, a state of equilibrium for corpuscle A may be far from being so for another cell, which, when subsequently added, is destroyed by the fluid.

In view of these facts we should not feel justified in saying that

* The experiment, indeed, does not inform us as to whether the filtered serum would have a varying hemolytic power for two different species of corpuscles, each sensitized with specific heated serum.

†Ueber die Vielheit der in normalen Serum vorkommenden Antikörper. Deutsehe med. Wochen., 1900, No. 49.

$\ddagger$ Archiv. für Hygiene, 1899, Bd. XXV. 
even after a prolonged contact (16 hours) normal guinea-pig serum (3 c.c.) mixed with defibrinated, carefully washed rabbit blood (6 c.c.) has lost all of its alexin for rabbit corpuscles. The supernatant fluid from such a mixture after centrifugalization is very red* and has no effect on freshly added rabbit corpuscles; in other words; it has become inactive for these corpuscles; but it will still produce a distinct hemolysis of hen corpuscles.

We may be sure that this fluid still contains a definite amount of alexin, to the presence of which is due the destruction of hen corpuscles. As far as rabbit corpuscles are concerned, a state of equilibrium in the division of the alexin has been established (and in producing this state the products from destroyed rabbit corpuscles have perhaps contributed), and further attack on the same corpuscles thereby prevented.

This state of equilibrium is easily broken: it suffices to increase by means of a sensitizer the avidity of the rabbit corpuscles for alexin. The reddish fluid under consideration will still destroy fresh rabbit corpuscles energetically if they are sensitized. In a mixture of 0.2 of a cubic centimeter of red fluid, the same amount of washed rabbit blood, and 0.6 of a cubic centimeter of heated specific guinea-pig serum, hemolysis occurs rapidly and completely. To render the experiment more complete another mixture is prepared, containing, in the place of the sensitizing serum, 0.6 of a cubic centimeter of heated normal guinea-pig serum (56 degrees). In this mixture there is, of course, no destruction of the rabbit corpuscles. A few hours later 0.3 of a cubic centimeter of specific antihen serum (56 degrees) plus 0.1 of a cubic centimeter of defibrinated washed hen blood is added to each tube. The hen corpuscles are rapidly destroyed in the second tube, but remain quite intact in the first.

It is evident, then, that the normal alexic serum has by no means been exhausted by the long contact with an excess of rabbit corpuscles, but has retained a considerable amount of the alexin fitted to destroy these same corpuscles. If the rabbit corpuscles are sensitized, the absorption of alexin takes place much more energetically, and new corpuscles of another species subsequently introduced remain unchanged.

* There are many intact rabbit corpuscles present in the sediment. 
This criticism of Neisser's experiments is significant: it indicates that much prudence should be exercised in drawing conclusions from similar experiments dealing with normal sera; the multiplicity of active substances in such sera have been too hastily presumed. We feel that it is the more permissible for us to venture this assertion, as it is perhaps applicable also to a certain experiment of our own on the multiplicity of agglutinins in normal horse serum. At any rate the subject is still far too obscure to allow of any exact opinion on substances other than the alexins.

In this chapter we have considered only the question of the unity or plurality of the alexin (Ehrlich and Morgenroth's complement) in a given serum. We have not sought to invalidate the idea, drawn from certain experiments of Ehrlich and Morgenroth* in particular, that normal sera may contain in addition to the alexin (complement) one or several normal sensitizers which, although weaker than those in specific sera, facilitate the activity of the alexin.

* These experiments indeed agree with our own (p. 97), that indicate the existence of a substance in normal horse serum that sensitizes the cholera vibrio to a certain extent to the alexin of another normal serum. 


\section{ON THE SENSITIZERS OF SERA ACTIVE AGAINST ALBUMINOUS SUBSTANCES.*}

BY DR. OCTAVE GENGOU.

The experimental study of anticholera immunity in vivo led Pfeiffert to discover the phenomenon that now bears his name, which consists in an extracellular granular transformation of Koch's vibrios in the peritoneal cavity of guinea-pigs vaccinated against cholera. The same phenomenon also occurs in the peritoneum of normal guinea-pigs, as Pfeiffer himself showed, on injecting cholera vibrios mixed with serum from well-immunized animals.

This destructive transformation of vibrios indicates an energetic bactericidal action. Pfeiffer attributed to the fixed endothelial cells of the peritoneum the property of rapidly secreting substances harmful to the vibrios whenever these organisms were injected into the peritoneal cavity. Metchnikoff $\ddagger$ soon showed that this interpretation is incorrect. He was able to produce the granular change of vibrios in vitro by mixing them with preventive serum plus the peritoneal exudate of a normal guinea-pig; this exudate contains leucocytes, but no endothelial cells.

Pfeiffer in his experiments failed to obtain a transformation on mixing the vibrios in vitro with the serum of vaccinated animals. From our present knowledge it is evident that the serum he used must have been too old to have retained its bacteriolytic properties.

Bordet $\S$ indeed produced Pfeiffer's phenomenon in vitro by simply mixing the vibrios with fresh preventive serum. He further

* Sur les sensibilisatrices des sérum actifs contre les substances albuminoïdes. Annales de l'Institut Pasteur, XVI, 1902, 734.

$\dagger$ Pfeiffer, Zeit. für Hyg. XVIII, 1894.

$\ddagger$ Metchnikoff, Annales de l'Institut Pasteur, June, 1895.

$\$$ Bordet, see p. 66 . 
showed that the bacteriolytic property of anticholera serum necessitates the collaboration of two substances: one, the specific preventive substance, or sensitizer as he called it, formed during immunization in the animal, and able to resist rather high temperatures (65-70 degrees); the second, Buchner's alexin, occurring in the serum of normal as well as of immunized animals, disappearing rapidly on standing, and easily destroyed by heat ( 55 degrees). The alexin alone has little effect on normal vibrios, but becomes infinitely more active when the vibrios have been acted on by a specific sensitizer.

This idea of the duality of the bacteriolytic substances was established by Bordet in 1895 by means of a series of experiments, of which the following is the most important: Heating cholera * serum to 55 degrees deprives it of its bacteriolytic properties, but, on adding fresh serum from a normal animal to this heated serum, the latter entirely recovers the energetic property it possessed before being heated. In other words, fresh normal serum "reactivates" heated cholera serum. Indeed, the two substances, the collaboration of which is necessary, are present in such a mixture.

The alexin, as Metchnikoff and his school have shown, is leucocytic in origin; even the destruction of vibrios within the leucocytes of normal animals is due to this alexin, as is evident from Metchnikoff's studies on phagocytosis.

Bordet* later injected animals with red blood cells of other species in the place of vibrios. The serum of such animals acquires the property of hemolyzing in vitro the corpuscles used for injection, and this hemolysis is also due to the collaboration of two substances: the normal alexin, destroyed by heating to 55 degrees, and an acquired sensitizer. What, then, are the intimate reactions between the active substances of the serum and the sensitive cells?

We know that if a suitable immune serum, previously heated to 55 degrees, is added to its specific red blood corpuscles, there is no hemolysis because the alexin has been destroyed. Ehrlich and Morgenroth $\dagger$ showed that under such conditions the corpuscles

* See p. 134.

$\dagger$ See Collected Studies on Immunity. Ehrlich-Bolduan. John Wiley \& Sons, p. 1. 
fix the sensitizer of the immune serum, and the decanted serum is deprived of its specific properties. But blood cells fix substances other than sensitizers. If we add corpuscles to fresh immune serum instead of to heated serum, they deprive the fluid, as Bordet* proved, of both sensitizer and alexin. This same phenomenon of alexin absorption also occurs if a mixture of heated hemolytic serum and fresh normal serum is employed instead of fresh hemolytic serum; we know from the experiment of Bordet, to which reference has been made, that an immune serum is completely regenerated on the addition of fresh normal serum and becomes quite as active as it was before being heated. But how may the absorption of alexin in this mixture of corpuscles, alexin and suitable sensitizer be detected? The matter is quite simple: fresh sensitized cells, say cholera vibrios, treated with heated cholera serum, are added to the mixture. If there is still alexin present, the sensitized vibrios subsequently introduced will certainly undergo granular transformation, but if they remain intact it shows that the alexin has disappeared from the fluid. This experiment may be reversed by mixing the alexin with the vibrios plus heated cholera serum and then adding sensitized corpuscles: in this instance it is the vibrios that absorb the alexin and are destroyed, and the subsequently added corpuscles remain intact.

From these experiments Bordet drew the conclusion that the alexin that is fixed by and that destroys sensitized blood cells is identical with the alexin that acts on sensitized bacteria. In a given serum, then, there would be only one alexin attacking bacteria and corpuscles indifferently. Whether we agree with this observer as to the unity, or at least the functional unity, of this substance, or with Ehrlich and Morgenroth in believing that a normal serum contains a large number of different alexins, each particularly adapted for a given cell or bacterium, the fact remains that, in presence of a suitable sensitizer, a bacterium or a cell deprives fresh serum of its alexin and renders it inactive either for the same cell or for other sensitized cells.

It is hardly necessary to state that in these experiments the alexin, obtained as a rule from fresh serum of the rabbit or guineapig, is not absorbed by the cells unless a sensitizer is present. In 
other words, if, instead of the mixture of alexin, corpuscles and hemolytic serum ( 55 degrees), a mixture is prepared in which the specific hemolytic serum is replaced by normal serum from the same species, the fluid remains full of alexin and retains its destructive power for sensitized cells. Normal sera, then, as a rule are unable to produce alexin absorption. If normal serum contains sensitizers, they are either too little in amount or too inactive to cause a fixation of alexin as determined by the method described.

There are, however, certain exceptions to this general rule. There are normal sera the alexin of which may be fixed by certain cells without the presence of an immune serum. Malvoz* has recently shown that this is the case with dog serum mixed with B. anthracis; this serum, moreover, in presence of this organism will cause the fixation of the alexins of other sera (rabbit, guinea-pig, rat); in other words, it acts as if it contained a true sensitizer.

In a similar way Bordet and I have recently found that, in a mixture of fresh normal dog serum and washed rabbit corpuscles, hemolysis occurs rapidly, and a fixation of alexin takes place to such an extent that subsequently added sensitized vibrios undergo no transformation.

Such cases, however, are the exception. The normal serum of the majority of laboratory animals does not affect cells enough to cause them to fix alexin to an appreciable extent; immune sera, on the contrary, render cells very avid of this substance. The reaction of alexin fixation in general shows a distinction between an immunized and a normal animal of the same species. In other words, it demonstrates the presence of the specific sensitizers produced by vaccination.

Previous to Bordet's experiments on alexin absorption a sensitizer could be defined simply as a substance that renders a given cell destructible, or at least morphologically alterable, by a dose of alexin that normally has no effect on it. But since the sensitizer has not only the property of increasing the harmful effect of the alexin, but also the property of causing the cell it affects to absorb

* Annales de l'Institut Pasteur, August, 1902. 
alexin, no morphological change in the specific cell is necessary to prove the existence of a sensitizer in a given serum. A disappearance of the alexin from the fluid is the only essential criterion. By this means Bordet and Gengou* were able to find sensitizers in the majority of antimicrobial sera, as, for example, in the sera of animals injected with $B$. pestis, the bacillus of swine plague, the first anthrax vaccine, B. typhosus, B. proteus vulgaris, and in the serum of convalescents from typhoid fever. If, for example, we put the same amounts of plague bacilli suspended in salt solution, and of alexin, in two tubes, and then add to the first a given dose of normal horse serum heated to 55 degrees, and to the second the same amount of heated serum from a horse vaccinated against $B$. pestis, it will be found that sensitized rabbit corpuscles, subsequently added, undergo complete hemolysis in the first tube, but remain intact in the second. In other words, the alexin has remained free in the first and disappeared in the second. Suitable controls show that it is indeed the plague bacilli influenced by the preventive serum that fix the alexin.

The facts have not been questioned so far as we are aware; Aschoff, $\uparrow$ however, has recently criticised the method employed by Bordet and Gengou on the ground that the production of hemolysis does not necessarily indicate the absence of a sensitizer (probably in a normal serum); for, as he says, "besides the alexins suitable for bacteriolytic amboceptors there may be alexins fitted for hemolytic amboceptors." This is not the point at issue, for B. \& G. have not sought to establish that there is no sensitizer in normal serum, but that there is one in immune serum. And, what is more, if Aschoff believes in the existence of an antiplague sensitizer in normal horse serum as well as in antiplague serum, how does he explain that the latter fixes these "hemolytic alexins," whereas the former does not?

From this summary it is evident that hitherto sensitizers have been demonstrated and studied only in such sera as act on definite cells. We may go further and consider whether the substance

* See p. 217.

$\dagger$ Aschoff: Ehrlich's Seitkettentheorie und ihre Anwendung auf die künstlichen Immunitätsprozesse. Zeitsch. f. allgem. Physiol., 1902, 3 Hft 1 ter Bd. p. 159. 
injected must be of definite morphology to give rise to distinct sensitizers. Substances without any cellular structure have already been used for injection. Bordet,* for example, obtained a serum that precipitates cow's milk by injecting this milk into rabbits. Wassermann $\dagger$ obtained similar results with several varieties of milk. Tchistovitch $\ddagger$ and Bordet $\S$ injected various foreign sera into rabbits and obtained corresponding precipitating sera. Mijers $\|$ produced sera that precipitate egg albumin, sheep-serum globulin, ox-serum globulin, and pepton. These experiments have been repeated and extended by other authors whose work need not be mentioned.

But hitherto only a precipitating property has been described in the serum of animals injected with foreign non-cellular organic substances. It seemed to us desirable to ascertain whether an animal immunized in this way does not produce substances similar to antimicrobial and hemolytic sensitizers as well. For the demonstration of these sensitizers we have used the method of Bordet and Gengou, which, as already stated, is based on the fixation of the alexin by a sensitized cell. We have endeavored to determine whether this fixation may be produced by the serum of rabbits injected with such fluids as cow milk, hen-egg albumin, pure horse fibrinogen, and dog serum heated to 55 degrees, and, finally, with the serum of guinea-pigs immunized against rabbit serum ( 55 degrees); in other words, we have endeavored to determine whether the sera of these treated animals contain a sensitizer as well as a precipitin for the substances injected.

The serum of rabbits vaccinated against cow milk.-We injected rabbits with relatively large amounts of cow milk previously heated to 70 degrees for one-half hour. They were given, in successive doses, 10, 10, 12 and 12 c.c. at intervals of 7 days. They were bled 14 days after the last injection and the separated serum heated for a half hour to 56 degrees; this serum we refer to as Serum rabbit $>$ milk 56 degrees. Fresh normal rabbit serum freed from corpuscles, after standing overnight at room temperature $\left(16^{\circ} \mathrm{C}\right)$ was uniformly employed as alexin.

* Mechanism of agglutination, p. 142.

† Deutsche med. Wochensehr., 1899, No. 80.

¥ Tehistovitch, Annales de l'Institut Pasteur, March, 1899.

$\S$ Bordet, see pp. 142, 175.

\| Centralblatt für Bakt., 1900, XXVII. 
With these materials the following tubes were prepared:

$\begin{array}{lll}\text { Tube } 1 & \text { Cow milk } & 0.2 \text { c.c. } \\ & \text { S. rabbit }>\text { milk, } 56 \text { degrees } & 0.6 \text { c.c. } \\ \text { Tube } 2 & \text { Rabbit alexin } & 0.1 \text { c.c. } \\ & \text { Cow milk } & 0.2 \text { c.c. } \\ \text { Tube } 3 & \text { S. normal rabbit, } 56 \text { degrees } & 0.6 \text { c.c. } \\ & \text { Rabbit alexin } & 0.1 \text { c.c. } \\ \text { Tube } 4 & \text { Salt solution of } 0.75 \text { per cent } & 0.2 \text { c.c. } \\ & \text { S. rabbit }>\text { milk, } 56 \text { degrees } & 0.6 \text { c.c. } \\ \text { Tube } 5 & \text { Sabbit alexin } & 0.1 \text { c.c. } \\ & \text { S. normal rabbit, } 56 \text { degrees } & 0.2 \text { c.c. } \\ & \text { Rabbit alexin } & 0.6 \text { c.c. } \\ \text { Tube } 6 & \text { Cow milk } & 0.1 \text { c.c. } \\ & \text { S. rabbit }>\text { milk, } 56 \text { degrees } & 0.2 \text { c.c. } \\ & \text { Salt solution } & 0.6 \text { c.c. } \\ & \text { Cow milk } & 0.1 \text { c.c. } \\ & \text { S. normal rabbit, } 56 \text { degrees } & 0.2 \text { c.c. } \\ & \text { Salt solution } & 0.6 \text { c.c. }\end{array}$

Tubes 1 and 2. In these tubes the action of the rabbit $>$ milk serum on cow milk is compared with that of normal rabbit serum.

Tubes 3 and 4. Show the effect of the sera alone on the alexin.

Tubes 5 and 6. Contain no alexin and show that heated serum will not produce hemolysis without alexin, and act as controls to those tubes in which the alexin is not affected.

These tubes are left 5 hours at room temperature, with agitation from time to time. To each tube is then added one-thirtieth of a cubic centimeter of sensitized hen corpuscles.* Following is the resultant hemolysis after 2 hours at room temperature, which result is found to remain constant the next day:

In tubes 2, 3 and 4 hemolysis is complete and uniformly rapid; it is evident in 30 minutes and has become complete in 1 hour and 30 minutes. Tubes 1,5 and 6 show no hemolysis even on the following day. What conclusion is to be drawn? In tubes 5 and 6 there is no hemolysis because no alexin is present; in tubes 3 and 4 the alexin has remained free when mixed with the heated rabbit $>$ milk serum or with heated normal rabbit serum; and in tube 2 the milk plus normal serum has caused no fixation. Tube 1 has acted as tubes 5 and 6 that had no alexin. The alexin added could not have been fixed by the heated rabbit $>$ milk serum (tube 3) nor by

* Defibrinated hen blood is washed in an excess of normal saline, centrifugalized, the supernatant fluid pipetted off, and the original level of the blood reestablished. This washed blood is mixed with two volumes of heated (56 degrees) serum from a rabbit that has received several injections of hen blood. 
the milk (tube 2), unless the latter had undergone some combination with the specific serum similar to that described by Bordet and by Bordet and Gengou for bacteria and corpuscles treated with their appropriate immune sera. We may admit, then, that the serum of rabbits that have been given injections of cow milk contains, in addition to the precipitin, a sensitizer similar to those of antimicrobial and hemolytic sera, which gives cow milk the power to fix the alexin of normal serum.

The serum of rabbits injected with egg white.-These animals were given large doses of egg white; at intervals of 7 days they received $10,10,12$ and 10 c.c. of egg white and 14 days after the last injection they were bled. The serum - designated serum rabbit $>$ egg was heated to $56^{\circ} \mathrm{C}$. for 30 minutes; clear normal rabbit serum was used as alexin.

An experiment wholly analogous to the one with rabbit $>$ milk serum, 56 degrees, was made with this rabbit $>$ egg serum. In place of milk, egg white was used.

The results of such an experiment are identical with those described for heated rabbit $>$ milk serum. It is found that alexin is fixed by hen-egg white in the presence of rabbit>egg serum (56 degrees), whereas no such fixation occurs with heated normal rabbit serum (tube 2) or with egg white alone (tubes 3 and 4 ).

The rabbit > egg serum also gives a precipitate with egg white which normal rabbit serum fails to do.

The sera of rabbits injected with heated dog serum (56 degrees). This serum, rabbit > dog serum, 56 degrees, was obtained in a manner similar to the other sera described. The rabbits were given at week intervals 8,10 and 12 c.c. of dog serum that had been heated to 56 degrees. The treated rabbits were bled 2 weeks after the last injection and the separated serum was heated to 56 degrees for one-half hour.

Experiments similar to those described for the other active sera were performed with this serum active against dog serum; similar controls were also made and need not again be specified. The important tubes are as follows:

(a) Rabbit alexin, 0.1 of a cubic centimeter, serum rabbit $>$ dog serum, 56 degrees, 0.6 of a cubic centimeter, dog serum, 56 degrees, 0.2 of a cubic centimeter. 
(b) Same as last tube, with heated normal rabbit serum replacing the specific serum.

These mixtures are left, as usual, for 5 hours at room temperature and then one-thirtieth of a cubic centimeter of sensitized hen corpuscles is added to each tube.

The results are wholly in harmony with those already detailed. The corpuscles are destroyed by the alexin in " $b$ "; in " $a$ ", on the contrary, there is no hemolysis. The rabbit serum specific for dog blood has fixed the alexin when dog serum is present; in addition to Tchistovitch's precipitin there is also a sensitizer, as in the case of rabbit $>$ milk serum and rabbit $>$ egg serum.

The serum of guinea-pigs treated with heated rabbit serum.-We decided to study this combination on account of certain peculiarities that it was known to present. We know from Bordet's researches that, contrary to the general rule, guinea-pigs injected with rabbit serum form no precipitin for this serum. On account of this unusual occurrence the question might well arise as to whether multiple injections instead of the usual two injections might not give a different result. We therefore gave our guinea-pigs six successive injections of from 4 to 5 c.c. of rabbit serum. By this means we obtained a serum which, although it produced no real precipitate, did give rise to a distinct opalescence when rabbit serum was added.

As we presupposed from the work of others, the guinea-pig produces only very weak precipitins to rabbit serum. We have also tested for the presence of a sensitizer in this "guinea-pig > rabbit" serum. We found that guinea-pig alexin is indeed fixed by a mixture of rabbit serum and heated guinea-pig > rabbit serum; this fixation, however, is distinctly less than in the other instances considered and is indeed a mere delay in hemolysis rather than a total inhibition. We consider, however, that a sensitizing property may be claimed for this serum, although it is as slight as is the precipitating property.

The serum of rabbits treated with pure horse fibrinogen. - We wished to determine whether injecting pure fibrinogen into rabbits would lead to the formation of sensitizers for this chemically pure substance, as we already know that coagulins for the globulins, 
casein, etc., may be produced in such a way. We have used Hammarsten's method of obtaining horse fibrinogen.*

The rabbits were given at intervals of 7 days 10,12, 12 and 15 c.c. of this solution of fibrinogen and were bled 2 weeks after the last injection. The serum, designated rabbit $>$ fibrinogen serum, 56 degrees, was tested with the pure fibrinogen. An abundant precipitum is formed at once on mixing; no such precipitate is formed with normal rabbit serum. We were further able to determine by the method described that the rabbit $>$ fibrinogen serum fixes rabbit alexin in the presence of fibrinogen. In other words, this immune serum contains a sensitizer for fibrinogen.

From these facts we may conclude that rabbits injected with such substances as milk, egg white, fibrinogen, and serum, form both precipitins and sensitizers for the respective substances. The production of sensitizers, then, is not dependent on the morphology of the substance injected and the animal body will react as well against amorphous material as against substances of definite histological structure.

Just as the sensitizers of antimicrobial and hemolytic sera fix the alexin of normal serum in presence of the corresponding antigen, so do the sensitizers of sera active against amorphous organic products fix the alexin in presence of the substance in question.

The majority of the immune sera we have worked with act on complex mixtures like serum, milk and egg albumin. It would be of interest to determine whether the sensitizer acts on the complex as a whole or more particularly on one or several components of it.

Several writers have considered this question in respect to coagulins and with somewhat divergent results. It may be considered proved, it seems to us, that the coagulin affects the globulins

* Oxalated horse plasma ( 1 to 1000 ) is centrifugalized and mixed with an equal volume of 30 per cent solution of sodium chloride. The precipitate is redissolved in 8 per cent salt solution and again precipitated with the 30 per cent solution; after three or four successive precipitations the fibrinogen is redissolved in sterile distilled water, as is possible owing to the sodium ehloride carried down by the precipitate. To test its purity the fibrinogen is mixed on the one hand with ealcium chloride and on the other with fresh blood serum. In the second mixture coagulation occurs, proving there is fibrinogen present in solution. No coagulation takes place in the first tube, showing that the proferment is absent. We further determined by heat that no other albumins or globulins were present. 
(Nolf, ${ }^{*}$ Mijers, $\uparrow$ F. Hamburger, $\ddagger$ Van Steenberghe, $\S$ Leblano $\|$ ). It is difficult to determine the effect on the albumins; F. Hamburger claims to have obtained a coagulin for lactalbumin, Mijers one for egg albumin, and Leblanc one for serum albumin; but Nolf, on the other hand, could obtain no coagulin for serum albumin.

We have made a few observations along this line, restricting ourselves to the action of rabbit $>$ dog serum and rabbit $>$ milk serum on the separable substances of their respective antigens.

The effect of rabbit $>$ dog serum on the globulins and the albumins of dog serum. - We separated the globulins from dog serum by saturation with magnesium sulphate. After redissolving in distilled water the globulins were again precipitated by sodium chloride; this precipitation was repeated twice and the final globulins were dissolved in a volume of distilled water to equal their original serum volume. Solution is made possible by the $\mathrm{NaCl}$ taken down by the globulins.

The albumin is obtained from the fluid of the original precipitation with magnesium sulphate by adding acetic acid, and is redissolved in normal salt solution; the fluid is then neutralized to litmus by the addition of a few drops of 2 per cent $\mathrm{NaOH}$.

With these two products, two series of tubes are prepared containing mixtures of normal rabbit serum or specific rabbit serum with and without rabbit alexin. In the mixtures without alexin the sensitized hen corpuscles added 5 hours later remain intact; in other words, the solutions of globulins and albumins employed were not in themselves hemolytic. In the mixture of normal rabbit serum and alexin hemolysis occurred; there is no hemolysis, however, in the mixtures containing rabbit $>$ dog serum.

It would seem, then, that rabbit $>$ dog serum will fix rabbit alexin with either the globulin or the albumin of dog serum. We do not wish, however, to attach too much significance to this experiment, which we were unfortunately unable to repeat owing to lack of material.

The effect of rabbit $>$ milk serum on casein, lactoglobulin and

* Nolf, Annales de l'Institut Pasteur, 1900.

$\dagger$ Mijers, loc. cit.

¥ F. Hamburger, Wien. klin. Wochens., 1901, p. 1202.

$\S$ Van'Steenberghe, Annales de l'Institut Pasteur, 1901.

\| Leblanc, La Cellule, t. XVIII, 2nd fascic. 
lactalbumin from cow milk. - These experiments with the various milk derivatives have been frequently repeated and always with the same result.

We obtained milk casein by the well-known method of diluting with three volumes of water and precipitating with 0.1 to 0.2 per cent acetic acid. The resultant precipitate is redissolved with ammonia, 1 to 200, and reprecipitated twice with acetic acid.

The casein purified in this manner is dissolved in water, rendered alkaline with ammonia and made equal in amount to the original volume of the milk; neutralization is then made with phosphoric acid.

The lactoglobulin and the lactalbumin were obtained from whey by the same method employed for dog serum. They were all finally redissolved in a much smaller amount of fluid than the original whey volume. We know from Bordet's studies that in order to produce complete fixation of alexin by bacteria and specific serum that a considerable number of bacteria are necessary. Since lactoglobulin and lactalbumin are present in such small amounts in milk we should not expect them, in absence of casein, which forms the bulk of organic substances, to fix all the alexin in the presence of rabbit $>$ milk serum. •

With these substances prepared in this way three identical series of tubes are prepared corresponding to those in the experiment with rabbit $>$ milk serum and whole milk. In addition to a fourth control series of milk and rabbit $>$ milk serum there are the three other series containing casein, lactoglobulin, and lactalbumin respectively in place of whole milk.

In the tubes of each series that contain normal rabbit serum, 56 degrees plus each of the substances in turn, hemolysis is complete; the alexin is not fixed by any of these substances without rabbit $>$ milk serum. But in the mixtures of milk or milk derivatives and specific serum, hemolysis occurs only in the mixture containing lactalbumin, and is lacking in the tubes containing casein and lactoglobulin as well as in the one with whole milk. In other words, alexin is fixed by rabbit $>$ milk serum with both easein and lactoglobulin; it is not fixed, however, with lactalbumin. We may add that the precipitating property of the serum parallels the sensitizing property; we obtain a distinct precipitate by adding casein or lactoglobulin to the specific serum, whereas the addition of lactalbumin 
produces no such effect. We do not agree, therefore, with F. Harnburger on this point.

One of the principal properties of antimicrobial and of hemolytic sensitizers is their specificity. As a general rule immune sera are active only against those cells which by injection have given rise to them. There is, we believe, no exception to this rule, although Ehrlich and Morgenroth have noted that the serum of rabbits treated with ox blood sensitizes not only ox corpuscles, but also goat corpuscles.

On the other hand there are numerous exceptions to this rule among the coagulins. Wassermann and Schütze,* Stern, $\dagger$ and Nuttall $\ddagger$ in particular have shown that the serum of animals injected with human serum precipitates not only this serum, but also the serum of anthropoid apes. Grünbaum, $\S$ by injecting rabbits with the sera from three different species of monkeys, obtained sera affecting the serum of these three species and also of man. Linossier and Lemoine $\|$ found that the serum of animals given ox serum would precipitate sera of other animal species. And Moro I has recently shown that a serum that precipitates cow milk will also precipitate goat milk.

It seemed to us well to determine to what extent our sera active against albuminous substances were endowed with specificity, particularly as regards their sensitizing property.

Rabbit serum active against milk. - This serum from rabbits that had been given injections of cow milk was heated to 56 degrees and added to different milks: cow milk, ewe milk, goat milk, mare milk and human milk.

With each milk, mixtures were made with normal rabbit serum, 56 degrees, and with specific serum, 56 degrees.

Tube 1

Milk (e.g., cow)

0.2 c.c.

Serum rabbit $>$ milk, 56 degrees

0.6 c.c.

Alexin (rabbit) 0.1 c.c.

Tube 2

Milk

0.2 c.c.

Serum normal rabbit, 56 degrees

0.6 c.c.

Alexin

0.1 c.c.

* Wassermann and Schütze. Berlin klin. Wochen., XXXVIII, 1901, 187.

† Stern, Deutsche med. Wochens., 1901, p. 135.

$\ddagger$ Nuttall, The Journal of Hyg., July, 1901, 3.

$\S$ Grünbaum, The Lancet, January 18, 1902.

\|I Linossier and Lemoine, Comptes rend. Soc. de Biol., 1902, 85.

I Moro, Wiener klin. Wochsch., 1902, 121. 
In addition to this series, comprising two tubes for each milk tested, other tubes with decreasing doses of active and normal serum, e.g., $0.4,0.2$ and 0.1 of a cubic centimeter, but all containing the same amount of alexin and milk, were prepared.

In none of the tubes containing normal rabbit serum, 56 degrees, do the various milks fix alexin; in all tubes containing rabbit $>$ milk serum, on the contrary, an alexin absorption occurs. There is no evidence of specificity with this serum in a dose of 0.6 of a cubic centimeter of serum, 0.1 of a cubic centimeter of alexin, and 0.2 of a cubic centimeter of each milk in turn. Nor does decreasing the active serum to 0.1 of a cubic centimeter show any difference between the cow, ewe and goat milk. Human milk, however, was less perfectly sensitized by the serum, even in a dose of 0.6 of a cubic centimeter of serum to 0.2 of a cubic centimeter of milk; fixation was not quite complete, and is almost nil with 0.2 of a cubic centimeter of serum.

Mare milk seems to lie between the first group of milks and human milk; a total fixation occurs with 0.6 of a cubic centimeter of serum, 0.4 of a cubic centimeter or even with 0.2 of a cubic centimeter, but only an imperfect fixation with 0.1 of a cubic centimeter.

To sum up; the sensitizer in our rabbit > milk serum is not specific. It acts energetically on milks from various allied animal species (cow, ewe, goat) and somewhat less distinctly on other milks (human and mare).

Rabbit > egg serum, 56 degrees. - Similar experiments were performed with the sensitizer of rabbit $>$ egg serum. The serum was obtained by injecting hen-egg white and was tested with egg white from the hen, the pigeon, the turkey and the duck. On the first experiments we used uniformly 0.2 of a cubic centimeter of serum; an intense precipitate was found with all the albumins, and the alexin was fixed in all cases so that no hemolysis of sensitized hen corpuscles occurred. Hemolysis was complete in controls with normal rabbit serum.

We have not tried to establish a difference between the various egg whites by using varying amounts of active serum, as in the rabbit > milk experiments; such an experiment was tried, however, between hen and pigeon albumin. With both species fixation is complete with 0.6 or even 0.5 of a cubic centimeter of active serum. 
With 0.4 of a cubic centimeter it is nearly complete and with 0.2 of a cubic centimeter very slight. There is no differentiation, then, between pigeon-egg white and hen-egg white by sensitization with rabbit > hen-egg white serum.

In brief, we have found no specificity either as regards precipitin with or sensitizer with rabbit > egg serum.

Rabbit > fibrinogen serum, 56 degrees. - We have found no more evidence of specificity as regards precipitation and sensitization with this serum than with the two preceding sera. The serum was obtained by injecting large amounts of pure fibrinogen from the horse and was tested with fibrinogens from the horse, the ox and the dog, all prepared in exactly the same way. An abundant and immediate precipitate occurred with each fibrinogen; there was apparently no difference in amount even when the active serum was decreased to 0.1 of a cubic centimeter, with 0.2 of a cubic centimeter of fibrinogen and 0.1 of a cubic centimeter of rabbit alexin.

The same results were obtained as regards sensitization of the three fibrinogens. Whatever the dose of active serum employed $(3,2,1$ or one-half volume to 1 volume of fibrinogen solution, and one-half volume of rabbit alexin), a complete fixation of alexin occurred with each fibrinogen with the largest dose of serum, and progressively less as the serum was decreased.

Rabbit serum active for dog serum, 56 degrees. - On account of the practical value of the serum precipitation, first demonstrated by Tchistovitch, we have studied the specificity of our rabbit $>$ dog serum.

Varying amounts of serum $(0.6,0.4,0.2$ and 0.1 of a cubic centimeter) were mixed with 0.2 of a cubic centimeter of various sera heated to 56 degrees plus 0.1 of a cubic centimeter of rabbit alexin.

We tested in this way dog, horse, ox and guinea-pig serum.

With dog serum the precipitate formed with 0.6 and 0.4 of a cubic centimeter of specific serum is very distinct; it is less with 0.2 of a cubic centimeter and no longer visible with 0.1 of a cubic centimeter. With the other sera no precipitate occurred. Similarly, the alexin was fixed only with dog serum and more or less completely according to the amount of specific serum employed.

The active serum, however, produced no alexin fixation with any of the other sera in any dose. 
The specificity of the sensitizer in this instance was as distinct as that of the precipitin, and is analogous to the specificity of antimicrobial and hemolytic sensitizers.

There remains to describe certain experiments that we have made, suggested by the lack of specificity in many of our sera. We have endeavored to determine whether these sera were active only against the particular substances used in immunization and were without effect on other substances from the same animal species. We can simply add a few examples to those described by other authors. Leclainche and Vallée,* Mertens, $†$ Dieudonné, $\ddagger$ Zuelzer $\S$ and Schütze $\|$ found that antihuman sera produced by injecting human blood would give precipitates with urine and pleural exudates from human beings; conversely, the injection of exudates and transudates gives rise to a serum that precipitates human blood.

Schütze $\int$ by injecting powdered human muscle, obtained a serum that sensitized human red blood cells. F. Hamburger** has shown that sera that precipitate cow lactoglobulin and lactalbumin will also precipitate bovine blood serum.

We have considered whether hen-egg white might not be precipitated and sensitized by a serum other than the rabbit $>$ egg serum. In the following experiment we determined the effect of the serum of a rabbit treated with defibrinated hen blood, and very active against the corpuscles in question, on hen-egg white:

Tube 1

Hen-egg white

0.2 c.c.

S. rabbit $>$ hen blood, 56 degrees

0.6 c.c.

Rabbit alexin

0.1 c.c.

Tube 2

Hen-egg white

0.2 c.c.

S. rabbit > egg, 56 degrees

0.6 c.c.

Rabbit alexin

0.1 c.c.

Tube 3

Hen-egg white

0.2 c.c.

S. normal rabbit, 56 degrees

0.6 c.c.

Rabbit alexin

0.1 c.c.

After 5 hours contact, one-thirtieth of a cubic centimeter of sensitized hen blood was added to each tube.

* Leclainche and Vallée, La Semaine médicale, 1901, No. 4.

$\dagger$ Mertens, Deutsche med. Woch., 1901, No. 1.

$\ddagger$ Dieudonné, Münch med. Woch., 1901, No. 4.

§ Zuelzer, Deutsche med. Woch., 1901, p. 219.

॥ Schutze, Zeit. für Hyg., XXXVI, 1901, 459.

T Schütze, Deutsche med. Woch., XXXVIII, 1901, 487.

** F. Hamburger, Wiener klin. Woch., 1901, 1202. 
In the first two tubes a marked cloudiness appeared, which was lacking in tube 3. In these two first tubes the fixation of alexin also was complete, but there was none in tube 3. The serum of rabbits injected with defibrinated hen blood is capable of precipitating and of sensitizing hen-egg white as well as rabbit > egg serum.

Is the converse true? That is to say, will rabbit $>$ egg serum agglutinate and sensitize hen blood corpuscles? The experiment to answer this question contains three tubes:

$\begin{array}{lll}\text { Tube } 1 & \text { Washed hen corpuscles } & 0.1 \text { c.c. } \\ & \text { S. normal rabbit, } 56 \text { degrees } & 0.3 \text { c.c. } \\ \text { Tube } 2 & \text { Rabbit alexin } & 0.2 \text { c.c. } \\ & \text { Washed hen corpuscles } & 0.1 \text { c.c. } \\ \text { Sube } 3 & \text { S. rabbit }>\text { egg, } 56 \text { degrees } & 0.3 \text { c.c. } \\ & \text { Wabbit alexin } & 0.2 \text { c.c. } \\ & \text { Washed hen corpuscles } & 0.1 \text { c.c. } \\ & \text { S. rabbit }>\text { hen blood, } 56 \text { degrees } & 0.3 \text { c.c. } \\ & \text { Rabbit alexin } & 0.2 \text { c.c. }\end{array}$

There is no hemolysis in tube 1 and rapid hemolysis in tube 3 ; in 2, hemolysis occurs, but slowly, and apparently is not accompanied by agglutination.

We then determined whether this rabbit > egg serum would act on normal hen serum; there was no effect. In a similar manner rabbit $>$ milk serum has no effect on cow serum. In short, it is evident that if an immune serum is tested on elements other than those used for immunization, but from the same animal species, the results vary and cannot be prognosticated in an untried combination. Although rabbit $>$ milk serum has no effect on cow serum and rabbit $>$ egg serum. none on hen serum, we find that rabbit $>$ egg serum will sensitize hen corpuscles to a certain extent, and that rabbit $>$ hen blood serum is very active against hen-egg white.

\section{Conclusions}

These experiments would, we believe, lead to the conclusions:

1. That in the sera obtained by injecting rabbits with large doses of cow milk, egg white, pure horse fibrinogen, or heated dog serum there are, in addition to the precipitins of Bordet and Tchistovitch, substances analogous to the sensitizers described by Bordet in bacteriolytic and hemolytic sera, and later found in the majority of antimicrobial sera. The same is true in respect to the 
sera of guinea-pigs injected with rabbit blood, although in this case the sensitizer appears less powerful.

2. The sensitizers studied by Bordet cause blood cells or bacteria to fix alexin. The sensitizers we have just described produce the same phenomenon and differ only in being directed against nondifferentiated substances. We have dealt with amorphous chemical substances and not with morphologically defined elements.

3. The sensitizer in the serum of rabbits injected with dog blood would seem to act both on the globulin and the albumin of dog serum; the sensitizer in the serum of rabbits injected with cow milk acts on the casein and the lactoglobulin, but not on the lactalbumin.

4. It is known that the sensitizers of antimicrobial and of hemolytic sera generally have the character of marked specificity. We have found this to be true also of the serum of rabbits active against dog serum. On the contrary, the sensitizers of most of the sera we have studied have slight specificity or none at all. Such sera, notably, are those from animals injected with milk, egg white and fibrinogen. It is true that these substances-the albuminoids of milk, fibrinogen and egg white - show an almost identical constitution in various animal species, at least sufficient to render them all susceptible to a given active serum irrespective of their origin.

5. The sensitizers of immune sera may often act on substances other than those used for immunization, but from the same animal species. For example, the serum of an animal injected with hen blood acts on hen-egg white. There is no general rule in this respect, however, as individual cases vary. 


\title{
XIII. ON THE MODE OF ACTION OF ANTITOXINS ON TOXINS.*
}

\author{
BY DR. JULES BORDET.
}

The majority of scientists who have studied antitoxins believe that they modify the poison for which they are the antidote. In other words, antitoxin protects the animal, not by rendering it more resistant, but by reacting with the toxin and destroying its harmful properties.

This conclusion has been drawn from numerous important researches, among which may be mentioned those of Martin and Cherry. Its accuracy became very evident as soon as we were able, thanks to Ehrlich, to eliminate experiments on the living animal, with the accompanying uncertainty of individual variation, and replace them by cells that are susceptible to the poison. Thereafter we were able to study the effect of antitoxin on toxin in vitro, as the sensitive cell is changed when toxin is present, but remains intact if this poison has been neutralized by a suitable dose of antitoxin.

The conception that antitoxin not only modifies toxin, but that it also unites with it, is in perfect harmony with the data we have concerning the other active substances of sera, and may therefore be accepted with relative certainty. The action resembles closely the effect produced by agglutinins, sensitizers and alexin on susceptible cells; in a similar way the precipitins, which are strikingly analogous to the agglutinins, agree to a certain extent with true antitoxins in that they unite with non-differentiated chemical substances.

Since we may regard it as proven that antitoxin destroys the harmful properties of toxin by direct combination, an attempt may be made to determine the intimate reaction between these two

* Sur le mode d'action des antitoxines sur les toxines. Annales de l'Institut Pasteur, XVII, 1903, 161. 
substances. Many investigators have endeavored to solve this problem, but, of necessity, with only partial success. As the molecular constitution of these substances is a mystery and their chemical nature unknown, their interaction naturally could not be so exactly and clearly demonstrated as is possible in dealing with the well-known substances with which chemists work.

We must be content, then, for the present with outlining the general characters of the reaction, in describing its appearance and in endeavoring to determine the laws to which it is subject. The first question to answer obviously is: does antitoxin unite with toxin in definite proportions, as does a monobasic acid with an alkali, in which case the neutralized product has a fixed and invariable composition; or does one of the substances unite with the other in varying amounts? If this second supposition is correct, it is evident that the combination formed by union of the two substances will not always be the same or endowed with the same characteristics, but will vary in composition according to the relative proportions of the two reacting substances. For instance, a mixture of equal volumes of toxin and antitoxin would produce a different substance than a mixture of one part of toxin with two of antitoxin. The resulting substance would vary with the respective doses of the two components employed: all would contain the same elements, toxin and antitoxin, but would differ in that one substance would be more or less saturated by the other. According to this hypothesis, the reaction of toxin and antitoxin would resemble, at least in regard to the variation of proportions, the reaction of iodine on starch. Starch, as we know, absorbs varying amounts of iodine and correspondingly varies in the intensity of its blue color; for this reason chemists regard this reaction as belonging to the phenomena of dyeing. Dyed substances take widely varying amounts of the dye.

Let us consider for a moment this comparison with dyeing phenomena, a comparison that we have previously made use of, and it would seem inadvisedly, since it has led many who have read our preceding articles to a thorough misconception of them.

As we have determined,* the maximal amount of red blood cells that a given dose of hemolytic serum can destroy varies in accord- 
ance with the manner in which the blood is added to the serum. If, for example, we add a given amount of blood in a single dose to, say, 1 c.c. of serum, this amount, A, may be relatively considerable and yet be entirely hemolyzed. But if this amount $\mathrm{A}$ is divided into several fractions added one after another at sufficiently spaced intervals to the same amount of the active serum, only a few of the corpuscles, say $\frac{A}{2}$, will be destroyed. It would seem as if the first doses of corpuscles had taken up all the hemolytic substances, and so deprived the subsequently added corpuscles of their share.

A blood corpuscle, then, absorbs varying amounts of active substances, the maximal dose that can be fixed being distinctly greater than the amount necessary to produce complete solution.* With this explanation we may offer, as a preliminary hypothesis, that the absorption of the active principles of serum by the fixing portion of blood cells does not follow the law of fixed proportions, but resembles, rather, the absorption of dyes by substances that take them, which absorption varies considerably in amount. Consequently we should expect the absorbing energy to vary to a large extent, depending on the conditions of the experiment (concentration of the substance considered, length of contact, establish-

* As we know, hemolysis depends on the collaboration of the alexin and the sensitizer. To which of these two substances is this result due? In other words, which one is absorbed in variable doses by the red blood cell? Both substances have this property, but the alexin shows it the more distinctly; and to the alexin, then, is due the greater part in the phenomenon. To a tube containing 0.5 of a cubic centimeter of alexin (fresh guinea-pig serum) is added 0.3 of a cubic centimeter of well-washed rabbit blood, and immediately afterward 0.9 of a cubic centimeter of sensitizer (guinea-pig $>$ rabbit serum, 55 degrees), hemolysis follows rapidly. Three hours later 0.1 of a cubic centimeter more of blood and 0.3 of a cubic centimeter of sensitizer is added, and 1 hour later 0.1 of a cubic centimeter more of blood and 0.3 of a cubic centimeter of sensitizer. The last corpuscles remain intact. We may then prepare a mixture containing the same total amount of each substance ( 0.5 c.c. of alexin, 0.5 c.c. of blood and 1.5 c.c. of sensitizer), but mix them together at once. Complete hemolysis occurs. The intact corpuscles in the first mixture are well sensitized, but lack alexin. This is shown by the fact that the subsequent addition of alexin produces complete hemolysis. We may conclude, then, that the stromata of the first hemolyzed corpuscles are loaded with alexin that they refuse to yield to other sensitized corpuscles. The stroma-alexin complex is stable and does not break up. We shall later return to this fact in considering one of Morgenroth's experiments. 
ing of equilibrium between the dose of active substance absorbed and that which remains free, and so forth), and this idea we have endeavored to prove experimentally.

We may add that this interpretation has appealed to various observers who have performed similar experiments; similar and even more demonstrative results have been obtained, as certain of these other experiments are less open to criticism than our own. The researches of Eisenberg and Volk,* giving definite information on the relations between agglutinins and bacteria and leading to many new and important experiments on this subject, are particularly instructive in this connection. These authors found that the law of definite proportions does not apply to the union of the agglutinin with'the agglutinable substance of bacteria. In the course of their work they noted that if a dose A of bacteria is added to a given dose of agglutinating serum the results are the same as those just described for hemolytic serum, and depend on whether $\mathrm{A}$ is added all at once or in divided doses: less bacteria are agglutinated when added little by little. Bacteria can absorb much more agglutinin than is necessary to clump them. If the amount of agglutinin is considerable, an equilibrium is established between the fraction that remains free and the one absorbed by the bacteria, the degree of saturation depending on the concentration of the agglutinin. We cannot reproduce here in detail the interesting conclusions of these observers (variations in the coefficient of absorption in proportion to the dose of agglutinin, the function of the relative concentrations of lagglutinin and agglutinable substances, etc.); the most important fact is that the agglutinable substance absorbs amounts of agglutinin that vary according to the relative proportions of the two reacting substances.

It would seem legitimate, then, to assume that the law of fixed proportions is not applicable to the absorption phenomena by cells or bacteria for the active principles of specific sera. The proportions would seem to vary as markedly as do the conditions of experiment. The conditions are very unlike those met with in straight chemistry, which depend on equations and equivalents. It is simply for the purpose of expressing this idea more emphatically that we have compared these phenomena with those of dyeing.

* Zeit. für Hygiene, Vol. XL, 1902. 
Certain observers in discussing this comparison have supposed that we overlooked the "chemical nature" of the combination of active serum with the fixing substance of the corpuscle. In other words, they have imagined that we regard this fixation as depending entirely on mechanical causes (surface adhesion, etc.), to the exclusion of any elective or specific affinity.*

To read these authors it would almost appear as if, in our opinion, corpuscles absorb the active substances of suitable immune sera indifferently and without any special affinity, as charcoal collects various gases indiscriminately! We have never committed ourselves on the intimate nature of the reaction, but simply as to its general appearance. The expressions "purely mechanical causes" and "surface adhesion" do not occur in our descriptions. We shall not go any further into the discussions as to whether dyeing phenomena should be qualified as "physical" or as "chemical." The point of importance is that these reactions differ from those of ordinary chemistry in that they are not expressed by equations; the proportions in which the substances unite vary according to the conditions of the experiment. It is simply this idea of a variability in proportions which led to our comparison and which to our thinking would justify it. The results of Eisenberg and Volk, which agree with our own, render this comparison still more legitimate. That in the case of specific sera and susceptible cells we have to do with real affinities would seem evident from the principle of specificity on which we have so much insisted and which no one questions. It is certain that these cells show a truly specific and exclusive avidity for their appropriate antibodies. That is no reason, however, for their absorption in fixed proportions nor that the resulting compound should be of fixed and invariable composition.

Having finished this digression we may return to toxins and antitoxins and reconsider the question already stated. Does the combination of these elements occur in fixed and constant proportions, and is the resulting product always the same, or may the proportions vary within wide limits and the resulting compounds

* We may remark in passing that it seems unwise to assert, as some authors do, that dyeing phenomena should never be considered from a chemical standpoint, and that dyed substances absorb dyes in a purely mechanical manner, owing to physical properties (texture, porousness), and never owing to certain chemical affinities that depend on their composition. 
differ according to the experimental conditions, and contain with a given dose of one substance variable amounts of the other?

Before seeking an answer we must recall certain experimental data. In the first place it has been shown that if an amount $\mathrm{A}$ of antitoxin is necessary completely to neutralize an amount $\mathrm{T}$ of toxin, that $2 \mathrm{~A}, 3 \mathrm{~A}$ or $\mathrm{nA}$ is necessary to neutralize $2 \mathrm{~T}, 3 \mathrm{~T}$ or $\mathrm{nT}$ respectively. If we regard the antitoxin as acting directly on the toxin and combining with it, there is nothing surprising in this fact. It would, moreover, seem, a priori, evident. This fact, however, affords no definite information as to whether antitoxin and toxin unite in definite and constant proportions.*

There is a second very important fact mentioned by Ehrlich. Having determined very exactly the minimal lethal dose of toxin, let us suppose that it is necessary to add 100 fatal doses of this toxin to a quantity $\mathrm{A}$ of antitoxin to produce a neutral mixture. We admit that the dose $\mathrm{A}$ of antitoxin is just sufficient to neutralize the toxin or, in other words, to produce a harmless mixture containing no excess of antitoxin. Let us now prepare a mixture containing also a dose $\mathrm{A}$ of antitoxin, but 101 minimal lethal doses of toxin. We might suppose that this mixture would kill a test animal, since it contains an excess of toxin equal to one fatal dose. This is not what happens, however; the animal shows only slight symptoms.

Mixtures may be prepared containing a considerably increased amount of toxin, even as much as 200 lethal doses, to the same dose A of antitoxin, without proving fatal for animals within the usual time limit. The injection of such mixtures produces slight edemas if the excess of toxin is slight, and more serious ones if the excess is considerable.

This is "Ehrlich's phenomenon." It evidently offers a notable objection to explanation by the law of fixed proportions. The simplest explanation of the phenomenon is evidently the one we have already mentioned, namely, that the antagonistic substances

* It would seem unnecessary to insist on this fact, if it were not that a bacteriologist has recently asserted, in an analogous manner, that the agglutinin unites in definite proportions with the agglutinable substance of bacteria, basing the assertion on the obvious fact that, if a dose $\mathbf{A}$ of serum is necessary to agglutinate a dose $\mathrm{B}$ of emulsion, that $2 \mathrm{~A}$ is necessary to produce the same effect on $2 \mathrm{~B}$. If we were to reason in this way, we should assert that a paint unites in definite proportions with the surface of a wall, since, if a quantity A of paint is needed to paint 10 square meters, $2 \mathrm{~A}$ is necessary to paint 20 square meters. 
(toxin and antitoxin) combine in variable proportions. We may imagine that each molecule of toxin is able to unite with or fix a variable number of molecules of the antitoxin. Let us suppose, for example, that a molecule $\mathrm{T}$ of toxin can unite either with a single molecule or with $2,3,4$ or 5 molecules $\mathrm{A}$ of antitoxin. Five compounds then are possible that may be designated $\mathrm{TA}^{1}, \mathrm{TA}^{2}$, $\mathrm{TA}^{3}, \mathrm{TA}^{4}$ and $\mathrm{TA}^{5}$. These mixtures will be more or less toxic, according to the amount of antitoxin present. The first, $\mathrm{TA}^{1}$ would be rather poisonous, although less so than pure toxin; the following $\mathrm{TA}^{2}$ and $\mathrm{TA}^{3}$ would be successively less toxic; $\mathrm{TA}^{4}$ and $\mathrm{TA}^{5}$ may be supposed to have no toxic effect.

If we were to mix with one volume of toxin containing 100 molecules (T) a volume of antitoxin containing 200 molecules (A), we should have a compound $\mathrm{TA}^{2}$. The antitoxin would be equally distributed over all the molecules present to form a compound of distinct though slight toxicity. This compound represents the toxic molecule partially saturated with antitoxic molecules. It is an attenuated but not a neutralized toxin.

If to the same number of toxin molecules $(100 \mathrm{~T})$ we were to add 300 molecules of antitoxin, the compound $\mathrm{TA}^{3}$ would be formed, and so on. In each instance, and notwithstanding the dosage, the antitoxin will be equally distributed among all the toxic molecules. The resultant compounds would differ according to the amount of antitoxin. As a result, we would never find in such mixtures quite free and intact toxin in conjunction with a toxin completely saturated with antitoxin.

On the other hand, if toxin and antitoxin united regularly in a fixed, constant and uniform proportion, it would be easy to obtain a mixture containing both intact toxin and saturated toxin by adding to a certain volume of toxin a relatively small amount of antitoxin.

In brief, we must regard a mixture of toxin with an incomplete dose of antitoxin* as one or the other of two very different compounds, in accordance with whether we accept the hypothesis of a

* We may note at once that it is precisely such non-fatal mixtures that give rise to Ehrlich's phenomenon, that is, mixtures containing a dose of antitoxin capable of neutralizing 100 lethal doses of toxin plus a slight excess of toxin, say 120 doses. 
combination in fixed proportions, or the hypothesis of union in variable proportions. In the first instance we conceive of the fluid as containing two substances - free active toxin and saturated and inactive toxin. On the second supposition we conceive of the mixture as containing a single substance - an incompletely saturated, non-neutralized, or simply an attenuated, toxin.

It is evident that non-identity in the composition of the mixtures would lead to a marked difference in action on the animal body. And, in accordance with the view adopted as to the union of the antagonistic substance, one would presuppose very different harmful properties in a given fluid. It is quite understandable, however, that a fluid containing attenuated toxin should be less dangerous than one containing a certain dose of intact toxin along with completely neutralized toxin. Ehrlich's phenomenon would appear easily explicable if we accept the idea of a combination in variable proportions.

The preceding statement is evidently schematic, and it is the fault of such schemes to be, in general, too dogmatic. Nor is our own working hypothesis free from this criticism, and should therefore not be taken too literally. For example, it would imply that the union of toxin and antitoxin is a simple molecular union; it also of necessity implies that our two substances obey the law of multiple proportions strictly (as that the dose of antitoxin in $\mathrm{TA}^{3}$ for example, is an exact multiple of that in TA). As a result, therefore, in order to simplify expression we may look on each substance in question as one elemental particle, as one molecule, which is by definition indivisible. It is evident that our purpose is not to determine whether toxin and antitoxin unite by a union of molecules or by an exchange of atoms, nor to find out whether we are dealing with combinations in exactly multiple, or simply in variable, proportions. Such problems are beyond our present range, and experiment is unable to solve them.

We are dealing with a single idea, and our schema has been used simply to express this idea. If the hypothesis of a union in variable proportions is exact, the essential characters of the reaction would be as follows:

1. When, to a given amount of toxin, antitoxin is added in an amount that does not suffice completely to neutralize, the anti- 
toxin molecules are not monopolized by certain of the toxin molecules, whose affinities become thereby satisfied, leaving the remaining toxic units intact. On the contrary, the antitoxin molecules are shared by and divided equally among all the toxic molecules present, which thenceforth are partially saturated and lose to a certain extent their original toxicity.

2. The phenomena of intoxication caused by injecting this compound into animals may not be the same as those produced by a mixture of neutralized toxin plus intact toxin.

3. Between the extremes of free toxin and entirely saturated or innocuous toxin, all transitions or stages of progressive attenuation may exist. Each time that a mixture of toxin and antitoxin is made in a given relation the same degree of attenuation will be produced.

Ehrlich, as we know, interprets his phenomenon in quite a different way. He thinks that toxin and antitoxin unite in fixed proportions. To harmonize his phenomenon with the law of fixed proportions, Ehrlich makes the supposition that the composition of toxic bouillon is very complex; that it contains, indeed, several poisons: one, an active poison, is the toxin properly speaking; another, less dangerous poison, is the toxon.

A molecule of toxin absorbs as much antitoxin as does a molecule of toxon. In this respect the two substances are equivalent. But the toxin exceeds the toxon in the energy of its affinity for, or in other words is more avid of, antitoxin. In order to neutralize completely a toxic bouillon enough antitoxin must be added to neutralize both toxin and toxon. But if an additional amount of toxic bouillon is added, the additional toxin that it contains will seize antitoxin that has already combined with the original toxon, and so liberate this latter substance. In other words, if to antitoxin a few doses of toxin in excess of the proper amount for neutrality are added to obtain a fluid containing no free toxin, there is, to be sure, uncombined toxon; but since this is relatively harmless the animal withstands the injection of such a mixture.

As a matter of fact we have simplified this explanation considerably, for Ehrlich has attributed an extraordinarily complicated composition to the toxic bouillon in order to make his theory agree 
fully with experiment. The explanation is unquestionably ingenious but the existence of certain of the substances, particularly of toxons, is purely hypothetical. The question, then, is still an open one.

\section{Mode of Action of Anti-alexin on Alexin.}

We might have expressed the preceding elementary ideas some time ago. In fact, they were suggested to us by some experiments we did on the neutralization of alexin by anti-alexin in 1900 at the Pasteur Institute (Professor Metchnikoff's service). But it seemed well to render the study more complete by considering the effect of certain other toxins on their antitoxins. We have not as yet been able to carry out this work. We shall therefore limit ourselves here to a consideration of the facts we obtained some time ago so as to obviate their future consideration.

We may consider, then, the effect of a suitable anti-alexin; that is to say, the serum (heated to 55 to 56 degrees) of an animal of species $B$ that has received two or three injections of fresh serum from species $\mathrm{A}$, on the alexin in the fresh serum of A.*

Having mixed these two antagonistic sera we need a reagent capable of detecting whether or not free alexin is present. For this purpose we use red blood bells well sensitized by a specific hemolytic serum (55 degrees). We know that alexin is absent when these cells remain intact.

Such an experiment naturally comprises a control mixture containing the same factors as the preceding mixture, but with heated serum from a normal animal of the same species as the one that furnished the anti-alexic serum.

As may be imagined, our first experiments are to determine whether the reactions of our antagonistic sera will give the "Ehrlich phenomenon," which is so difficult to reconcile with the hypothesis of neutralization according to fixed proportions. In the following experiments certain precautions must be taken to avoid experimental error. In the first place only a single antitoxic effect, namely, the one against the alexin under consideration, must be allowed to

* An anti-alexic serum must be heated to 55 degrees to destroy its proper alexin, in a study of this sort. The only alexin in the experiment should be in the form of the fresh serum of the animal against which the antiserum is active. (See "Hemolytic sera, their antitoxins, etc," p. 186.) 
intervene. As sensitized corpuscles are employed the anti-alexic serum should be without effect on the sensitizer used for these corpuscles. The heated normal serum used as control to the antialexin or the sensitizer should have no particular properties and should be simply an inert fluid. And of course the absence of hemolytic activity in the heated sera should be controlled. The reagent employed (sensitized red blood corpuscles) should be added only in very small doses in order that the smallest amounts of free alexin may be detected.

To fulfill these conditions we use the following materials: As alexin, fresh guinea-pig serum is employed. As anti-alexin, the heated serum of a rabbit that has been given injections two or three times of fresh normal guinea-pig serum. For sensitized corpuscles hen red cells washed in salt solution and treated with rabbit $>$ hen serum, 56 degrees, are employed. As controls of the antialexin and the sensitizer, normal rabbit serum, 55 to 56 degrees.

In short, the toxin (alexin) is from the guinea-pig. The other sera (antitoxin, sensitizer and control) are all from the rabbit and have no effect on one another. No accessory reactions, therefore, should destroy the accuracy of the experiment.

The strength of the anti-alexin is first defined by the following experiment:

Tube $a$. Alexin, 0.2 of a cubic centimeter; anti-alexin ( 55 degrees), 0.3 of a cubic centimeter.

Tube $b$. Alexin, 0.2 of a cubic centimeter; normal rabbit serum ( 55 degrees), 0.3 of a cubic centimeter.

An hour or two later 0.2 of a cubic centimeter of sensitizer (serum rabbit $>$ hen, 56 degrees) is added to each tube and then one drop of washed hen blood. The corpuscles are hemolyzed in 20 minutes in " $b$." There is no hemolysis in " $a$," even on the following day.

This lack of hemolysis in " $a$ " may be shown to be due solely to a neutralization of the alexin. The sensitizer, present in relatively large amount in respect to the corpuscles, is unaffected.*

Tube $a$. Sensitizer, 0.1 c.c; anti-alexin, 2 c.c; fresh rabbit serum (alexin), 2 c.c.

Tube $b$. Same as " $a$," with 2 c.c. normal rabbit serum, 55 degrees, replacing the anti-alexin.

* This is shown by replacing the guinea-pig alexin, which is specifically affected by theanti-alexin, by another alexin, say from the rabbit, on which the anti-alexin has no effect. 
Tube $c$. Rabbit alexin 2 c.c; normal rabbit serum, 55 degrees, 2 c.c. (Same as " $b$ " without sensitizer.)

To each tube is added one drop of hen blood. Hemolysis occurs rapidly in " $a$ " and " $b$." It is only partial in " $c$ " after 3 hours. It is evident, then, that the anti-alexin can have no effect on the sensitizer, which, in the experiment, is only one-twentieth of the volume of the antiserum. It is evident, too, that the sensitizer acts energetically although present in small amount and diluted in 4 c.c. of fluid. In the rest of the article, for the sake of simplicity, these control experiments need not be insisted on in each instance.

We then determine the potency of the anti-alexin. For this purpose varying amounts of alexin (say from 0.05 to 1.2 c.c.) are added to a constant volume ( 0.3 of a cubic centimeter) of antialexic or of normal serum ( 55 degrees). The following tubes are prepared:

A. Tubes containing 0.3 of a cubic centimeter of anti-alexin:

a. Alexin, 0.05 c.c.; b. Alexin, 0.1 c.c.; c. 0.2 c.c.; d. 0.3 c.c.; e. 0.4 c.c.; f. 0.5 c.c.; g. 0.6 c.c.: h. 0.7 c.c.; $i .0 .8$ c.c.; j. 0.9 c.c.; k. 1.2 c.c.

B. Same as preceding tubes, with heated normal rabbit serum (non-anti-alexic) replacing anti-alexin.

To each tube is then added 0.2 of a cubic centimeter of sensitizer and an hour later one drop of a suspension of hen corpuscles in $\mathrm{NaCl}$. The mixtures are kept at room temperature $\left(18^{\circ} \mathrm{C}\right.$.).

In the tubes that do not contain anti-alexin, hemolysis takes place rapidly in, say, 15 minutes in those tubes containing most alexin, and in 30 minutes in the one with the smallest amount. Consequently 0.05 of a cubic centimeter of alexin represents the minimal dose for hemolysis in a half hour, which may be taken as a unit. We may add that the dilution of this small amount of alexin in heated serum, one volume of alexin in ten of heated serum, for the sake of mensuration has no effect on its activity.

We may now consider the tubes containing anti-alexin. Let us consider first the final reading, say on the following day, in order to allow for the maximal action of the smallest amounts of free alexin. On the following day hemolysis is complete in the tubes containing 0.5 of a cubic centimeter or more of alexin; it is partial in the 0.4 c.c. tube and slight in the 0.3 c.c. tube. Two-tenths of a cubic centimeter or less shows no hemolysis.

It may be noted that such results argue somewhat against the hypothesis of a combination in fixed proportion. If there is slight 
hemolysis in tube " $d$," it is due to an almost negligible" trace of free alexin; the next mixture, " $e$," which contains two additional minimal doses of alexin, should show complete hemolysis but actually does show only partial hemolysis. Ehrlich's phenomenon, then, is present, but is not well marked. It is also to be noted that a volume of anti-alexin equal to that of the alexin must be used in order to protect corpuscles effectively.

The most interesting results are evident soon after the mixtures are made, and, if the moment when hemolysis is complete is noted, Ehrlich's phenomenon is then very striking. The mixture of 1.2 c.c. of alexin and 0.3 of a cubic centimeter of antialexin should, according to the hypothesis of neutralization in fixed proportions, contain 0.3 of a cubic centimeter of neutralized alexin and 0.9 of a cubic centimeter of intact alexin. In such a mixture hemolysis is complete only after 70 minutes, which is at least twice as long as is required in a mixture containing a single fatal dose of alexin without antitoxin. Hemolysis is complete in this latter mixture before it begins in the former.

In mixtures of anti-alexin with doses of alexin from 0.4 to 0.9 of a cubic centimeter, corpuscles are eventually hemolyzed, the rapidity in beginning and completion of the process varying directly with the amount employed. For example, although hemolysis is complete in 70 minutes in a tube with 1.2 c.c. of alexin, there is none at this time with a dose of 0.9 of a cubic centimeter (" $j$, ,), $2 \frac{1}{2}$ hours being necessary for complete hemolysis, and at $2 \frac{1}{2}$ hours hemolysis is only partial in tube " $i$," has barely begun in tube " $h$," and so on. In short, the time necessary for the liberation of hemoglobin varies indirectly with the amount of alexin employed.

The experiment shows that a dose of anti-alexin that can completely neutralize 6 fatal doses of alexin (one-half hour) will check 24 fatal doses to such an extent that they produce less rapid hemolysis than a single unaffected fatal dose. It is impossible, therefore, to imagine that anti-alexin added to a large dose of alexin completely neutralizes part of it, leaving the excess free: such mixtures do not act at all as do simple dilutions of alexin.

Anti-alexin affects all the alexin present equally, in accordance with a law of varying proportions, as has just been fully explained. Ehrlich's explanation for diphtheria toxin, presupposing the exist- 
ence of toxons, will not explain the results just considered. This is evident in considering the proportions of sera employed, the fact that the minimal dose of alexin ( 0.05 c.c.) is strongly hemolytic, and the fact that the time necessary for the appearance of hemolysis decreases regularly and gradually with the amount of alexin employed.

The conclusion, then, is that each of the alexin-antialexin mixtures forms a new substance or complex containing neither of the antagonistic substances in pure state, but depending for composition on the relative proportion of each substance employed. The complex is different in each successive mixture, being more or less toxic in accordance with the degree of saturation of the toxin by the antitoxin. The anti-alexin attenuates the alexin until it completely neutralizes it if the dose is sufficient.

It follows from this experiment that it is impossible to prepare an exactly neutral mixture of alexin and anti-alexin, that is to say, a mixture that is absolutely non-toxic and non-antitoxic. This conception, to be sure, is not novel, but is simply a restatement of Ehrlich's phenomenon. Take, for example, a mixture containing a moderate dose of alexin (" $f$ " or " $g$ ") in addition to the anti-alexin. Such a mixture is toxic, since the corpuscles finally hemolyze. It is also antitoxic, since similar mixtures containing the same amount of anti-alexin, but more alexin, are simply hemolyzed more slowly. Such a result is a corollary to the idea of a combination in variable proportions, according to which a whole series of degrees of progressive attenuation between an active toxin and a neutralized toxin may be formed.

The proof that such a mixture contains a distinct anti-alexic power in addition to a real toxicity lies in comparing it with a similar mixture containing a little more alexin, in which case hemolysis is very slow. A more direct proof might be preferable. We may prepare such a mixture, supposed to be at once toxic and antitoxic, by mixing 0.3 of a cubic centimeter of anti-alexin with 0.5 of a cubic centimeter of alexin.* As a control a mixture of the same amounts of non-antialexic serum (normal rabbit serum, 56 degrees) and inactive alexic serum (56 degrees) is prepared. Two or three hours later a little active alexin (0.1 c.c.) is added to each

* In such an instance hemolysis takes place after several hours. 
tube. It may be presupposed that this substance will be distinctly attenuated in the first mixture, and such proves to be the case although the result is not very striking. If sensitized hen corpuscles are subsequently added to these tubes, hemolysis is more rapid in the control than in the first tube, although the difference is not extremely marked. And why?

The first mixture contains in addition to the anti-alexin a total of 0.6 of a cubic centimeter of alexin added in two successive fractions. A comparison of the hemolytic power of this mixture with that of a mixture containing 0.6 of a cubic centimeter of alexin added in a single dose is interesting.

We prepare, then, liquid $\mathrm{A}$, containing 0.3 of a cubic centimeter of anti-alexin plus 0.5 of a cubic centimeter of alexin. Three hours later we add 0.1 of a cubic centimeter of alexin to it and at the same time prepare mixture $B$, which contains 0.3 of a cubic centimeter of anti-alexin plus 0.6 of a cubic centimeter of alexin. We then add to each mixture 0.2 of a cubic centimeter of rabbit $>$ hen sensitizer and an hour later two drops of hen blood to each tube. Hemolysis requires an hour in tube $\mathrm{A}$, and an hour and three quarters in B. An anti-alexic serum then neutralizes a given dose of alexin better when it is added all at once than when it is added in successive fractions. This experiment recalls the one in which red blood corpuscles are added to a hemolytic serum either all at once or in divided doses.

In a mixture of alexin with anti-alexin the latter substance is uniformly distributed over all the alexin, and all the toxic molecules are equally modified; the composition of the mixtures is homogeneous. But if we subsequently add more alexin to such a mixture it tends to remove the antitoxin from the combination into which it has entered, tends, in other words, to break up its established distribution. For example, if the original complex is $\mathrm{TA}^{2}$, the addition of another $\mathrm{T}$ would tend to form $2 \mathrm{TA}$. But since the combination $\mathrm{TA}^{2}$ is already formed, a new reaction (i.e., removal of the anti-alexin from the complex) is somewhat difficult to bring about, and the additional dose of alexin, in consequence, is not readily attenuated.* In other words, $\mathrm{TA}^{2}$ does not give a part of its

* If all the alexin had been added at once, the complex $T A$ would of course have been formed. 
antitoxin unresistingly. The difficulty in such a reaction naturally depends on the toxins and antitoxins under consideration,* and it is probable that the stability of the complex will vary in different instances. If the complex is very stable, an additional $\mathrm{T}$ will remain intact; on the other hand, it is readily attenuated when the complex is unstable.

It would seem quite evident from a recent experiment of Morgenroth's $\uparrow$ that these various possibilities may well exist.

This investigator sensitized well-washed red blood cells with their specific serum (55 degrees), centrifugalized, removed the supernatant fluid and washed the corpuscles several times in salt solution. The resulting sensitized corpuscles were suspended in a medium that contained no free sensitizer. To such a suspension he then added normal unsensitized corpuscles of the same sort. If alexin in moderate amount is immediately added to such a mixture, only part of the corpuscles are destroyed, namely, only those that were sensitized. But if some time elapses before the alexin is added, all the corpuscles are hemolyzed indiscriminately. Morgenroth very properly draws the conclusion that the normal corpuscles are able after a certain interval to remove a certain amount of sensitizer from the sensitized corpuscles. $\ddagger$ In other words, a change in distribution of the sensitizer analogous to the change in distribution of the anti-alexin in the preceding experiment has taken place.

It is evident that the complex formed by the union of the sensitizer with the fixing substance of the cell (which may be designated $\mathrm{CS}^{2}$ ) gives up a part of its sensitizer to other cells rather easily and becomes, say, CS. In this instance the complex is rather unstable. We may compare this result with the one noted at the beginning of this article, which dealt with the effect of adding alexin in a single or in divided doses to sensitized cells, in which instance the complex "cell-sensitizer-alexin" (or, better, stroma-alexin) is remarkably stable. Destroyed blood cells laden with alexin do not yield

* And even with a given toxin and antitoxin the more or less complete saturation of the complex must be considered. A complex $T A^{4}$ would probably give up its antitoxin more readily than does $T A^{2}$.

$\dagger$ Münch. med. Wochenschrift, 1903, No. 2.

$\ddagger$ It is also quite probable. as Morgenroth states, that the fluid serves as a medium of passage for the sensitizer from one cell to the other. 
it to other corpuscles even when they are strongly sensitized. This justifies the preceding remarks as to the varying stability of complexes obtained.*

It is evident that for these reasons and from these experiments we believe in the hypothesis of a combination in variable proportions. It is well, however, to verify this hypothesis in its more important bearings.

Two different standpoints may be assumed in determining the toxicity of any poisonous substance. In the first place an estimation of the strength of a poison may be made by determining how many cells or what weight of animal it will destroy. On the other hand, the toxicity in relation to the time necessary to accomplish a given result may be determined.

In considering a mixture of alexin and anti-alexin, let us mix 0.3 of a cubic centimeter of anti-alexin with 1.2 c.c. of alexin (guinea-pig). This 1.5 c.c. of fluid (A) contains, according to our hypothesis, only attenuated alexin, and only slightly attenuated at that, as the dose of anti-alexin is small. In other words, the antialexin has not neutralized part of the alexin and left the rest unaltered. We have then to deal, not with a simple quantitative diminution of the alexin, but with a complex that, as a whole, is less toxic, in that it hemolyzes even a small dose of corpuscles remarkably slowly.

Since all the alexin has been transformed, but none of it, properly speaking, destroyed, and since we are dealing, not with a quantitative diminution, but with a modification of the totality of alexin, it is conceivable how the mixture can destroy a relatively considerable amount of red blood cells, although the hemolysis may be slow.

* An idea of Morgenroth's phenomenon may be gained from a certain staining phenomenon that would appear in a rough way to be suggestively analogous. We place about half of a strip of filter paper at the bottom of a crystallizing dish and pour over it a little solution of methylene blue. In a short time the paper removes all the color from the fluid. We then take two more bits of filter paper and place one of them (A) at the bottom of the dish and the other (B) very near the original strip that has been lying in the solution and that has absorbed the blue. Care is taken that B does not actually touch the original paper. B soon becomes more colored than A. The first strip is decolorized at the point nearest to B. The distribution of color between this original strip and B tends to homogeneity, as does the sensitizer in Morgenroth's experiment tend to be equally shared by all the cells present. 
We may now compare mixture $\mathrm{A}$, that contains much attenuated alexin, with a second mixture B having the same volume, but a different constitution. This second mixture has been obtained by adding a very small amount of normal alexin to an inactive, certainly not anti-alexic serum (normal guinea-pig or rabbit serum, 55 degrees). The mixture, then, is a simple dilution of active alexin in an inert fluid. For example, B may be a mixture of 0.3 of a cubic centimeter of heated normal rabbit serum plus 0.1 of a cubic centimeter of alexin diluted in 1.1 c.c. of the same serum heated to 55 degrees.

It is evident that small amounts of sensitized hen corpuscles added to mixture $\mathrm{B}$ will be rapidly destroyed owing to the presence of the active alexin. But since the amount of this alexin is relatively inconsiderable, it is evident that if much sensitized blood is added hemolysis even on long standing will be only partial.

As will be suspected, the two mixtures $A$ and $B$, containing each the same volume and both capable of producing hemolysis, are in reality endowed with very different properties. If the hemolytic property of the mixtures is estimated by means of a small dose of sensitized corpuscles, e.g., one drop, B will appear more active than A; that is to say, it will hemolyze a few corpuscles more rapidly. But the opposite result will be obtained if the hemolytic power is measured by the total amount of corpuscles that each will destroy. For example, if a large dose of sensitized blood is added (say, 1.5 c.c.) on the following day it will be found that most of the corpuscles in $\mathrm{B}$ are intact, whereas all the corpuscles in $\mathrm{A}$ are hemolyzed. In other words, the alexin is quantitatively greater in A, and qualitatively more active (rapidity of action) in B.

These experimental results agree with our hypothesis. It is no longer possible to assume that an insufficiently neutralizing dose of anti-alexin changes alexic serum to a mixture of perfectly neutralized and of intact alexin. If it were, the resultant fluid would be simply a dilution of normal alexin in a certain amount of inert fluid, in other words, a mixture identical with B.

It would not be legitimate to draw too generalized conclusions from these experiments on alexin and anti-alexin; the further study of various toxins and antitoxins, from the point of view of method 
of combination, is necessary. We may, however, note that certain well-known hitherto enigmatic facts become easily explicable on the hypothesis of union in variable proportions. One or two examples may be given:

It has been noted (particularly with tetanus) that a toxin-antitoxin mixture that is harmless for an animal of species $\mathrm{A}$ shows evident toxicity for an animal of species B. Instances of this sort have been mentioned, particularly by Buchner and by Roux and Vaillard. Such apparently peculiar results are an almost necessary corollary to our hypothesis. A mixture of toxin with even a weak dose of antitoxin contains no primitive toxin, but in its place an attenuated toxin, a new complex endowed with individual characteristics, and it will not necessarily act in the same manner on different animals. It is reasonable to anticipate that its toxic power will be so attenuated as to produce no trouble with certain animals, but distinct effect on others; why, indeed, should they all react alike to this new compound? It is even theoretically conceivable that completely saturated toxin should poison certain species or certain individuals. According to our conception there is no sharp, radical difference between attenuation and neutralization of a toxin, or, in other words, there is no absolute neutralization. There is simply a greater or less degree of attenuation in accordance with the more or less complete saturation of the toxin by antitoxin. This saturation is often practically equivalent to a neutralization. The expression "neutralization" implies that the toxin has become irrevocably inactive for very sensitive animals owing to a radical abolition of the substance that renders it toxic. With such complete neutralization the degree of a given animal's susceptibility is not a significant factor in the result. When, however, an apparent neutralization is, in reality, only a very marked attenuation, the degree of susceptibility must be taken into account, as the attenuation may be very marked as far as animal $\mathrm{A}$ is concerned, but very little for animal B. The conception of variation in attenuation, then, must constantly take into account the relation between the sensitivity of the animal body and the harmful nature of the substance under consideration.

It is quite comprehensible, then, how a mixture that is rich in antitoxin (i.e., with the toxin well saturated) and inoffensive for 
healthy animals may yet be dangerous for animals that are weakened and so rendered more sensitive. It may be possible to explain in this manner the intoxication by means of diphtheria toxin of animals that are actually producing active diphtheria antitoxin. Although the modification of toxin is still going on, the animal may have become very sensitive to even a saturated toxin, which, of course, is without effect on normal animals. The susceptibility of guinea-pigs immunized against the cholera vibrio to a tetanus toxin-antitoxin mixture (Roux and Vaillard) is apparently another example of this fact.*

A relatively small amount of anti-alexin suffices to protect normal unsensitized corpuscles from alexin, but a large amount is necessary to protect sensitized blood cells. As Morgenroth and Sachs $\dagger$ have already shown, more anti-alexin is necessary to protect heavily sensitized corpuscles than slightly sensitized ones; in other words, the toxicity of the alexin-antialexin complex depends on the strength of the sensitization.

This. leads us to a consideration of results obtained by investigators on the injection of a toxin that is partially neutralized by antitoxin. As we know, such a substance is simply attenuated toxin, inoffensive for certain animals, but distinctly harmful for others. Dryer and Madsen have shown that such an incompletely saturated mixture of diphtheria toxin, which is quite innocuous for the guinea-pig, produces slight symptoms, (edema, etc.) in the rabbit. The toxicity of such a mixture might be increased by varying the respective amounts of the antagonistic substances and so preparing a slightly less attenuated mixture. Under such conditions an animal that had only shown edema would show distinct poisoning. Such results, indeed, were obtained by Dryer and Madsen.

These investigators consider that such incompletely saturated mixtures contain only toxon, that is to say, a different and less active substance than the true toxin. This toxon seems to us to be simply our complex - toxin incompletely saturated with antitoxin. Such a complex has all the characteristics attributed to toxons: it is by definition less toxic than free toxin and also less avid of antitoxin,

* Such mixtures, of course, are inactive for normal guinea-pigs.

$\dagger$ Sce Studies on Immunity, Ehrlich-Bolduan, John Wiley and Sons, p. 250. 
since its affinity for it is already partially satisfied. It is evidently related to toxin as far as origin and composition are concerned, but still is a new substance and therefore gives rise to different symptoms than a small amount of diluted toxin. Since it contains the toxic radical its injection, of course, leads to formation of antitoxin. For Dryer and Madsen have shown that antitoxin can be produced by injections of the substance they call toxon.*

We may add further, since we consider the existence of toxons as distinct substances as improbable, that we do not wish in any way to cast doubt on Ehrlich's results as to the spontaneous weakening of toxins by conservation and the corresponding formation of toxoids from them. We regard the effect of antitoxins simply as one factor in the attenuating of toxins, but there may well be others, such as oxygen and light, the exact effect of which is not well determined, although their weakening action on toxin is well defined.

* Zeitschrift f. Hygiene., XXXVII, 1901. 


\title{
XIV. THE PROPERTIES OF ANTISENSITIZERS AND
} THE CHEMICAL THEORIES OF IMMUNITY.*

\author{
By DR. JULES BORDET.
}

One of the most important problems in the study of immunity, and it must be confessed one of the most difficult of solution, is the specificity of serum. The problem is, of necessity, complex. We have known for some time that the antibodies of immune sera are specific in the sense that they affect certain substances and do not affect others. But in addition to this specificity of action we apparently must recognize a specificity of origin. For example, a given sensitizer (amboceptor or fixateur) should be designated, not only in respect to the blood corpuscles or bacterium that it attacks, but also in respect to the animal species that has formed it. Two sensitizers, both active against the cholera vibrio, but derived in one case from the rabbit and in the other from the guinea-pig, do not act exactly alike under all circumstances; the same statement holds for antitoxic sera. We know, for example, that the duration of a passive immunity given by a preventive serum varies, depending on whether the serum is derived from the same species as the recipient or from an alien species.

A study of antisensitizers is particularly useful as throwing light on the specificity of sera, and particularly as to whether this specificity is as absolute as would appear. It would seem, a priori, reasonable to suppose that the law of specificity should be most evident in dealing with these antisensitizers or, more generally speaking, in dealing with anti-antibodies. In this instance we deal with substances that are not only antibodies, but are specific both as regards action and origin. It would seem reasonable, then, that they should afford the most notable and instructive instances of specificity.

* Les propriétés des antisensibilisatrices et les théories chimiques de l'immunité. Annales de l'Institut Pasteur, XVIII, 1904, 593. 
Antisensitizers may be demonstrated in the blood of the animals that have been vaccinated against a hemolytic serum. Following the work of Camus and Gley and Kossel on the antitoxin to eel serum, we demonstrated in $1899 *$ that the injection of an animal of species A (rabbit) with the serum of species B (hen) gave rise to a property in the serum of $\mathrm{A}$ that neutralizes the hemolytic effect of serum B on the corpuscles of A, and we later studied in detail the properties of such antihemolytic sera. On injecting rabbits with specific hemolytic serum from guinea-pigs immunized against rabbit blood we found that these rabbits formed an antiserum that would inhibit the hemolytic effect of the specific guinea-pig serum, and that, strangely enough, acted against both substances necessary in hemolysis. In other words, it destroyed the characteristic sensitizer, and was also antitoxic for guinea-pig alexin (anti-alexic property). On account of its anti-alexic property this antiserum protected, not only rabbit corpuscles, but also other sensitized cells, against guinea-pig alexin. For example, it was found that sensitized cholera vibrios (treated with heated cholera serum) could be subjected to fresh guinea-pig serum without showing granular disintegration, provided a suitable dose of antiserum was added. $\dagger$ In short, the addition of antiserum to guinea-pig alexin prevented both its hemolytic and its bacteriolytic action. The anti-alexin was found, moreover, to be strictly specific, having no effect on the hemolytic or bactericidal action of sera from animals other than the guinea-pig.

The anti-alexic property of such antisera was later studied by several observers. Wassermann in particular confirmed our experiment on the antibactericidal effect of anti-alexin and in addition noted an interesting modification. He neutralized the alexin of normal serum by anti-alexin in vivo instead of in vitro; he found that he could annul the bactericidal power of the peritoneal exudate by injecting antiserum into the peritoneal cavity.

The antisensitizing property has recently interested other experimenters. In the present article we shall consider the facts that have been brought out concerning it.

* Agglutination and dissolution of red blood cells by serum, p. 165 .

Hemolytic sera, their antitoxins, etc., p. 186.

$\dagger$ This antiserum, of course, had been deprived of its own alexin by heating to 55 degrees. This temperature does not effect the anti-alexin that protects the vibrios. 


\section{Properties of Antisensitzers.}

The antisensitizer should be chosen with some care in order to render its study most fruitful. The antisensitizing power is frequently only slightly developed in antisera and is often detectable only by delicate methods; in which cases very large doses of antiserum must be used to neutralize the sensitizer effectively, and such doses are often inconvenient for various reasons.

For this reason one must use an antiserum with marked antisensitizing properties and also an active sensitizer. It is necessary, too, that the normal sera of the animals furnishing the sensitizer and the antisensitizer respectively should be as inactive as possible, as they are used as controls to contrast with the peculiar properties of the immune sera. These conditions are very satisfactorily realized in the following example:

The sensitizer we have generally used is the serum of rabbits that have been given three or four injections of from 5 to 7 c.c. each of defibrinated bovine blood. In certain other experiments we have used as well the serum of rabbits immunized against other blood corpuscles, for example, human and hen corpuscles. The antisensitizer employed is the serum of guinea-pigs that have received two or three injections of 3 to 5 c.c. each, at intervals of from 12 to 15 days, of normal rabbit serum. These animals are bled 2 weeks after the last injection.

Before being used for experiments, both sera are deprived of alexin by heating for a half hour to 55 to $56^{\circ} \mathrm{C}$. As we already know, this temperature has no effect on the sensitizer or antisensitizer. For simplicity, we refer to our sensitizers as "rabbit $>0 x$, 56 degrees," "rabbit $>$ hen, 56 degrees," "rabbit $>$ human, 56 degrees," and the antisensitizer as guinea-pig $>$ rabbit antiserum, 56 degrees. The control sera are normal rabbit serum, 56 degrees, normal guinea-pig serum, 56 degrees.

What is the best method of demonstrating antisensitizing power? Two widely divergent methods may be considered.

First, sensitizer and antiserum may be mixed, the corpuscles used as a reagent subsequently added, and a determination made as to whether they have become sensitized by subsequently adding alexin. This method is one frequently employed. It makes use 
of the antiserum as a preventive, the sensitizer being neutralized before it has affected the corpuscles.

We may also attempt to "cure" corpuscles already treated with the sensitizer by means of the antiserum. Attempts of this sort have been made by Pfeiffer and Friedberger,* who worked with bacteria instead of blood corpuscles. Such experiments are also similar to those of Madsen $\uparrow$ and Kraus and Lipschütz, $\ddagger$ who cured blood cells intoxicated with bacterial poisons by means of antitoxins.

This second method of experimentation has seemed to us preferable. It allows one to treat a single sensitizer with the antiserum to the exclusion of other sensitizers. In addition to the specific sensitizer in rabbit $>$ ox serum there are one or, according to certain authors, a large number of similar normal sensitizers already present in the animal before immunization. If we begin by mixing the antiserum with rabbit $>$ ox serum, it is probable that the normal sensitizers will take part in the reaction. But if, instead, we first mix the sensitizing serum with the corpuscles and then wash them to remove the excess of serum, and add them, laden as they are with the specific substance, to antiserum, the latter should affect only the specific sensitizer united with the blood cells.

It remains to choose a suitable alexin, which should be one that is not destroyed or weakened by the antiserum. The simplest thing is to employ fresh guinea-pig serum, as this is the species of animal that furnishes the antiserum. Not only is this alexin not affected by the antiserum, but it acts very well in conjunction with the sensitizers employed.§

Following is the method of demonstrating antisensitizing action: Sensitized blood, and also control non-sensitized blood, are first prepared. In each of two large tubes is placed 1 c.c. of washed bovine blood.\| To tube $\mathrm{A}$ is then added 2 c.c. of rabbit $>$ ox

* Centralblatt für Bakt. Orig., XXXIV, 72.

$\dagger$ Zeitschrift für Hygiene, XXXII.

$\ddagger$ Idem, XLV, 49 .

§ Human alexin may also be used.

\| This washed blood has been restored to its original volume, that is to say, contains as many red blood cells to a given volume as did the original blood. A small amount of defibrinated blood is poured into a tube and the level marked on the glass. After filling with salt solution and centrifugalization, the supernatant fluid is removed and sufficient salt solution added to restore the sediment to its original volume. 
serum, 56 degrees, and to tube B, 2 c.c. of normal rabbit serum, 56 degrees. Half an hour later the two tubes are filled with salt solution, shaken and centrifugalized. The supernatant fluids are pipetted off and the sedimented corpuscles in each tube are suspended in 3 c.c. of salt solution. These two corpuscle suspensions differ only in that the first corpuscles are laden with specific sensitizer. We may then prepare the following mixtures:

Tube $a$. Sensitized blood, 0.1 c.c.; Guinea-pig $>$ rabbit antiserum, 56 degrees, 0.3 c.c.

Tube $b$. Same as last, but containing 0.3 c.c. of normal guinea-pig serum, 56 degrees, instead of antiserum.

Tubes $c$ and $d$. Same as " $a$ " and " $b$ " respectively, but containing nonsensitized blood in place of sensitized blood.

One hour later 0.1 of a cubic centimeter of guinea-pig alexin (fresh serum) is added to each tube.

It is found that hemolysis takes place rapidly in tube " $b . "$ In tubes " $a$," " $c$," and " $d$ " there is no hemolysis even after 24 hours. The lack of hemolysis in " $a$ " is due to the neutralization of the sensitizer by the antiserum, as may be shown by adding a small amount of rabbit $>$ ox serum, 56 degrees, which at once produces hemolysis. The same results are obtained if we use any other alexin (for example, human serum) that is not affected by the antiserum.

This method of experimentation allows us to measure the antisensitizing power of the antiserum. We may add in place of 0.3 of a cubic centimeter of pure antiserum to 0.1 of a cubic centimeter of sensitized blood, 0.3 of a cubic centimeter of a dilution of antiserum in a greater or less amount of heated normal guinea-pig serum. On trial we find that the antisera from different guineapigs vary much in potency. The antisensitizing property of antiserum is frequently so strong that 0.1 of a cubic centimeter will suffice to protect 0.1 of a cubic centimeter of sensitized blood from the alexin.

Having settled on our technic, we may now consider certain questions that naturally arise concerning antisensitizers: Certain of the questions ( $\mathrm{B}, \mathrm{C}, \mathrm{D}$ and $\mathrm{E}$ ) relate particularly to the effect of the antiserum on the sensitizer; others ( $\mathrm{A}$ and $\mathrm{F}$ ) relate rather to the origin of and the variations in antibodies.

(A) Is an antiserum obtained by immunizing an animal of 
species A with the serum of normal untreated animals of species B capable of neutralizing the specific sensitizers formed by species $\mathrm{B}$ against such cells as red blood corpuscles? Experimentally, we learn that it is; for our antiserum, which, as already shown, is actively antisensitizing, was obtained from guinea-pigs that had received only normal rabbit serum. The same results are true if we replace the sensitized ox blood by hen or human blood, each sensitized by its respective hemolytic serum from the rabbit (rabbit $>$ hen, 56 degrees, or rabbit $>$ human, 56 degrees). This agrees very well with the findings of various writers and particularly with those of Ford.* This writer, having found that hen corpuscles are agglutinated, not only by the serum of a rabbit immunized with hen blood, but also to a certain extent by normal rabbit serum, injected hens, on the one hand, with normal rabbit serum, and on the other with the specific serum of rabbits that had been immunized against hen blood (rabbit $>$ hen serum). He found that either antiserum from the hen neutralized both the specific agglutinin of rabbit $>$ hen serum and the normal agglutinin of normal rabbit serum. Pfeiffer and Friedberger† obtained analogous results with antisera for bacteria.

We may conclude, then, from these results that as a general rule antiserum obtained by injecting the normal serum of species A, and acting, therefore, on the normal antibodies, will also neutralize the various specific antibodies formed by $\mathrm{A}$ in response to immunization.

(B) Is the antisensitizer used up in producing its effect as are the other antibodies already studied? In other words, is not the amount of sensitizer that an antiserum can neutralize, limited? It is almost superfluous to add that this turns out to be true; there is a minimal dose of antiserum, a less amount than which fails to protect sensitized corpuscles. And, moreover, it may be shown that the addition of a sufficient amount of washed sensitized bovine corpuscles to antiserum deprives it of its antisensitizing property, as is shown on adding additional sensitized corpuscles to such treated antiserum. $\ddagger$ Of course antiserum treated with the same

* Zeit. für Hygiene XL, 1902, 363.

$\dagger$ Berliner klin. Woch., 1902, No. 1, and Centralblatt für Bakt., XXXIV, 1903, 74 .

$\ddagger$ We shall consider this experiment in detail in another connection. 
amount of non-sensitized corpuscles (i.e., treated with normal rabbit serum, 56 degrees, and then washed) loses no antisensitizing power.

(C) Does the antiserum neutralize the sensitizer directly, like a true antitoxin, or does it simply neutralize the effect of this substance in some antagonistic manner? To answer this question we must determine whether corpuscles sensitized and then cured by antiserum remain refractory to alexin even after being washed in salt solution.

Experimentally, we find that they do. We add to 0.1 of a cubic centimeter of sensitized blood 0.3 of a cubic centimeter of antiserum; in another tube we add to a similar amount of sensitized blood 0.3 of a cubic centimeter of heated normal guinea-pig serum. After a certain time we fill both tubes with salt solution, centrifugalize, decant the supernatant fluid,* and suspend the sedimented corpuscles in 0.3 of a cubic centimeter of heated normal guinea-pig serum. We then add 0.1 of a cubic centimeter of guinea-pig alexin to each tube. There is no hemolysis in the tube that contained antiserum, but rapid hemolysis in the other. The curing of sensitized corpuscles, therefore, does not depend on permanent contact with the antiserum.

(D) Does the antiserum inhibit the effect of the sensitizer in respect to its various manifestations? The most important property of the sensitizer is to render the suitable cells susceptible to destruction by alexin. But, as we have already shown, sensitizers also have the property (a property, moreover, correlative with the other) of conferring on the specific cells the power of absorbing alexin and of so removing it from the surrounding fluid. Consequently we must ascertain whether corpuscles that are sensitized and then treated with antiserum will still fix alexin. Experimentally, we find that under these conditions the alexin is not absorbed.

Defibrinated washed ox blood is mixed with either two volumes of rabbit $>$ ox serum, 56 degrees, or of normal rabbit serum, 56 degrees. After sufficient contact the tubes are filled with salt solution, centrifugalized, and the supernatant fluids decanted. One volume of salt solution is added to each blood sediment. As a result we have two similar emulsions of red blood corpuscles, one of which is sensitized. There are then prepared the following tubes:

* This washing may be repeated several times. 
Tube $a$. Sensitized blood, 0.3 c.c.; Normal guinea-pig serum, 56 degrees, 1.2 c.c.

Tube $b$. Same as last, with guinea-pig $>$ rabbit antiserum, 56 degrees, replscing the normal serum.

Tubes $c$ and $d$. Same as " $a$ " and " $b$ " respectively, with non-sensitized blood in place of sensitized blood.

One hour later 0.1 of a cubic centimeter of alexin (fresh guineapig serum) is added to each tube. Hemolysis takes place in tube " $a$ " in a few minutes; no hemolysis in the other tubes. The tubes are shaken from time to time and after about five hours are centrifugalized. The clear supernatant fluids are placed in separate tubes, and 0.4 of a cubic centimeter of a mixture composed of one part of washed ox blood to two parts of rabbit $>$ ox serum, 56 degrees, is added to each tube.*

These new sensitized corpuscles are rapidly hemolyzed in all tubes except the one containing supernatant fluid " $a$," where no hemolysis occurs. $\dagger$ We conclude from this experiment that the antiserum removes from sensitized corpuscles their power of fixing alexin; such corpuscles act as do normal corpuscles.

(E) Does the antisensitizer drive out the sensitizer from the corpuscles by a process of washing, or does it unite with the sensitizer joined to the corpuscle?

We have just seen that if a sufficient amount of sensitized corpuscles is added to the antiserum the latter becomes inactive. We might suppose that the antisensitizer drives out the sensitizer from the corpuscles, and the fact that it becomes inert might be due to a mutual saturation of the two antagonistic substances.

Let us take 0.1 of a cubic centimeter of sensitized ox blood and add to it 0.3 of a cubic centimeter of antiserum. After a little contact we wash the corpuscles carefully in salt solution. After centrifugalization and decanting we suspend the sedimented corpuscles in 0.3 of a cubic centimeter of an active normal guinea-pig serum, and add 0.1 of a cubic centimeter of alexin. No hemolysis occurs. Has the sensitizer with which the corpuscles were laden been driven out by the antiserum and then removed from the fluid

* The sensitizing serum in this mixture is relatively so large in amount that no neutralization of it could take place in tube " $b$," even if the antisensitizer had not been used up.

$\dagger$ Hemolysis will take place in this tube on addition of a little more alexin. 
by washing? To settle this question we add 0.1 of a cubic centimeter of normal rabbit serum, 56 degrees.* On this addition hemolysis soon appears. In control tubes we find that this normal rabbit serum has in itself no power to sensitize ox corpuscles. We must therefore conclude that the sensitizer has remained with the corpuscles, that the antisensitizer has joined with it, but has not expelled it, and that normal rabbit serum contains a substance (normal sensitizer?) that is able to break up the combination of specific sensitizer and antisensitizer by replacing the first in its union with the second substance. And if normal rabbit serum under these conditions seems to sensitize, it does so indirectly by liberating the specific sensitizer that has been neutralized.

We shall later return to this experiment in other connections, particularly in considering the multiplicity of active substances in a given immune serum. We may note simply, for the moment, that this phenomenon, which may be designated as a suppression by normal serum of a cure effected by antiserum, varies in rapidity with the conditions of the experiment. We have already remarked that the reappearance of sensitization on adding normal rabbit serum becomes slower and more difficult in proportion to the time of contact between the corpuscle and the antiserum before the addition of the normal serum. In other words, it would seem, so far as our experiments go, that the combination between specific sensitizer and antisensitizer becomes more perfect in some way with time and less apt to be affected by the sensitizers of normal serum. The combination would also seem to be more stable when the antiserum employed is very powerful.

It is not surprising that normal rabbit serum should contain substances with an affinity for the antisensitizer. We showed in 1899 that substances similar to sensitizers $\uparrow$ may be detected in normal sera, and Ehrlich and Morgenroth have furnished numerous analogous examples. In consideration of the experiment we have

* It may be remembered that our antiserum was obtained by injecting guineapigs with normal rabbit serum.

$\dagger$ Although these substances would scem to belong to the same eategory as sensitizers, they are, with few exceptions, very inferior to them as regards their affinity for the sensitive cells. They are, moreover, very little known, and we call them "normal sensitizers," only with a certain reservation. 
just discussed, it would seem superfluous to add that if normal rabbit serum, 56 degrees, is added in the first place to the antiserum the latter loses its power to protect sensitive corpuscles from alexin. It is indeed obvious, since even when the corpuscles have been cured by antiserum the subsequent addition of normal rabbit serum will neutralize the protection already afforded.

Another fact resulting from the evidence of the preceding experiment is that sensitized ox corpuscles cured with antiserum and washed, and resistant to guinea-pig or human alexin, will be hemolyzed by rabbit alexin, since this serum restores the sensitization.

Is the power of annulling the cure of corpuscles by antiserum present in normal rabbit serum that has been heated to temperatures considerably above 56 degrees? Heating to 70 degrees for a half hour will not destroy it. On the other hand, the fluid expressed from a clot of serum coagulated at 100 degrees no longer retains this power. The power is only feebly, if at all, present in rabbit aqueous humor heated to 56 degrees. It is evident, then, that if it be due to normal sensitizers, as is likely, they are not present in the aqueous humor. This fact is in harmony with our experimental results published in $\mathbf{1 8 9 5}$, in which we demonstrated that the bactericidal power of cholera serum is due to the collaboration of two distinct substances, one a specific one which is thermostable and occurs only in the serum of vaccinated animals (preventive substance or sensitizer); and the other, the alexin, or proper bactericidal substance, destroyed at 55 degrees, and present in the serum of both normal and vaccinated animals in approximately equal amounts. The familiar experiment of mixing the aqueous humor ( 56 degrees) of an immunized animal with fresh serum of a normal animal and vibrios gave no bacteriolysis; bacteriolysis was energetic, however, if heated serum from the same animal replaced the aqueous humor. In other words, aqueous humor contains no sensitizer.

(F) If we find that a given antiserum neutralizes, as does the one we are studying, several different specific sensitizers, each active against different cells, but all derived by immunizing animals of the same species, are we to conclude that this antiserum contains several different antisensitizers, each one of which combines with a separate sensitizer? Or are we to conclude that the antiserum 
contains a single antisensitizer that neutralizes various sensitizers indifferently?

Although this question has not been put to experimental proof, it would seem to be answered by certain results on another problem considered by Wassermann* and by Ford. $\dagger$ These investigators have iried to elucidate the following point: In many instances, as we know, the serum of a normal animal has a distinct agglutinating power, without any immunization, for blood corpuscles A, let us say.f If this animal is immunized against corpuscle $\mathrm{A}$, is the specific agglutinin that is formed to be considered as identical with the one present in the normal serum before treatment? In brief, does immunization simply increase a preëxisting principle already present in small amount, without giving rise to new and particular substances, properly speaking? If this is so, immunization would be equivalent simply to a purely quantitative modification.

Wassermann and Ford think that the fact brought out by Ford, to which reference has been made, $\S$ makes clear this obscure point and proves conclusively that antibodies active against a given cell in normal or in immune serum are identical. The fact, to repeat, is that the antiserum from hens immunized against normal rabbit serum neutralizes the agglutinating effect of either normal rabbit serum or of the serum of rabbits immunized against hen corpuscles for the corpuscles in question. Their conclusion is as follows: if the antiserum obtained by injection of normal agglutinin neutralizes both normal and immune agglutinin, it proves that these agglutinins are identical.

We do not see why Wassermann and Ford consider this conclusion logical. It would be true only if it had been proved that a given anti-agglutinin (or antisensitizer) could under no circumstances neutralize several different agglutinins (or sensitizers). And this is precisely what Wassermann and Ford have not proved. Why, therefore, if we were to formulate in the beginning the opposite

* Wassermann, Zeitschrift für Hygiene, XLII, 1903, 267.

$\dagger$ Ford, Zeitschrift für Hygiene, XL, 1902, 363.

$\ddagger$ Wassermann and Ford deal, it is true, with agglutinins and not with sensitizers. It is not, however, unreasonable to apply the results obtained with agglutinins to sensitizers and consequently conclusions concerning anti-agglutinins to antisensitizers.

$\S$ See p. 285. 
hypothesis - namely, that one and the same anti-agglutinin (or antisensitizer) will neutralize indifferently the various agglutinins formed by a given animal species-should we not be justified in asserting that the two agglutinins in question, both affecting hen blood corpuscles, are not identical, but show distinct differences as marked, let us assume, as those between immune agglutinins from the same animal species affecting different cells? Why, in fact, should we accept without experimental proof the thesis that Wassermann and Ford consider axiomatic, that there is a specific antibody exclusively fitted for each active substance and that, conversely, when two substances are neutralized by a given antibody that they are identical? Why, in short, must we conclude from Ford's experiment that immunization simply increases the amount of the active substance without changing it qualitatively? It is evidently indispensable to consider this question before we can admit Wassermann and Ford's conclusion, the importance of which in increasing our comprehension of the genesis of active substances in immune sera is evident. We shall see from the following experiments, as a matter of fact, that a given antisensitizer can neutralize distinctly different sensitizers affecting different cells.

When we mix our antiserum with sensitized and washed ox corpuscles the antisensitizer is, as we know, used up in curing the corpuscles, so that the supernatant fluid after centrifugalization no longer protects sensitized blood corpuscles. But will this fluid still protect other corpuscles sensitized with a different rabbit sensitizer, for instance, hen corpuscles treated with rabbit $>$ hen serum? The experiment, the details of which follow, proves that it will not. The same antisensitizer neutralizes, then, two different sensitizers (rabbit $>$ ox and rabbit $>$ hen). In a control it is shown that ox corpuscles treated with rabbit $>$ hen serum (which they do not absorb) will not remove from the antiserum the power of protecting sensitized hen or ox corpuscles. In the same way it is shown that rabbit $>$ ox serum does not sensitize hen corpuscles.

Washed ox blood, the volume of which equals the primitive blood, is placed in equal amounts in two large tubes (1.5 c.c.) and two volumes (3 c.c.) of rabbit $>$ ox serum, 56 degrees added to one, and the same amount of rabbit $>$ hen serum, 56 degrees, to the 
other. After contact the corpuscles are carefully washed, centrifugalized, and the supernatant fluids decanted; 1.5 c.c. of salt solution is then added to each tube. In one tube, then, the blood is sensitized and in the other it is not.

In the same way hen blood, sensitized and not sensitized, is prepared by treating it with each specific serum.

Antiserum is then diluted with an equal volume of normal guineapig serum, 56 degrees, to facilitate mensuration. We refer to this as diluted antiserum.* We then prepare the following mixtures of antiserum and the two ox bloods treated as described:

Tube A. Sensitized ox blood, 1 c.c.; diluted antiserum, 2.5 c.c.

Tube B. Same as A, with non-sensitized ox blood in place of sensitized blood.

One hour later the tubes are centrifugalized and the supernatant fluids $\mathrm{A}$ and B decanted. Fluid A is deprived of antisensitizer, as may be imagined. These fluids are used in making the following tubes:

Tubes $\mathrm{C}$ and D. Each contains 1 c.c. of fluid A; to $\mathrm{C}$ is added 0.1 of a cubic centimeter of sensitized ox blood. To $\mathrm{D}$ is added 0.1 of a cubic centimeter of sensitized hen blood.

Tubes $\mathrm{E}$ and $\mathrm{F}$. The same as tubes $\mathrm{C}$ and $\mathrm{D}$ respectively, with fluid $\mathrm{B}$ replacing fluid $\mathbf{A}$.

In addition there are controls containing each 1 c.c. of normal guinea-pig serum, 56 degrees, and 0.1 of a cubic centimeter of hen or ox blood sensitized and not sensitized respectively. These control the tubes containing antiserum.

Three-quarters of an hour later 0.2 of a cubic centimeter of alexin (fresh guinea-pig serum) is added to tubes C, D, E, F, and to the controls.

There is no hemolysis in tubes $\mathrm{E}$ and $\mathrm{F}$ containing liquid $\mathrm{B}$, in which the antiserum has not been deprived of its antisensitizing power by sensitized corpuscles. There is, of course, no hemolysis in the controls with non-sensitized corpuscles and without antiserum. In tubes $\mathrm{C}$ and $\mathrm{D}$ that contain fluid $\mathrm{A}$, in which the antisensitizer has been previously used up by the sensitized ox corpuscles, the hemolysis of both the ox and the hen corpuscles takes place as rapidly as in the controls without antiserum. The same antisensitizer, then, neutralizes the two sensitizers under consideration, which differ markedly, as one is specifically suitable for ox corpuscles and combines with them, whereas the other does not.

* It may be noted that four parts of this antiserum completely protect one part of the sensitized blood used against the subsequent action of alexin. In a control tube in which the antiscrum is replaced by normal guinea-pig serum, 56 degrees, hemolysis is complete in 5 minutes. 
This experiment is not indispensable, as a similar conclusion might be drawn with assurance from an observation that has been made concerning normal rabbit serum. We have already noted that on adding a little normal rabbit serum, 56 degrees, to a mixture of sensitized corpuscles, antiserum and alexin, in which no hemolysis is present, hemolysis appears; we must conclude, then, that the normal serum contains a substance that can replace the specific sensitizer in its union with the antitoxin, at least in part. That this substance is not to be regarded as a rabbit $>$ ox sensitizer preëxistent in small amounts in normal rabbit serum is evident from the fact that the normal serum does not sensitize ox corpuscles to alexin. But in order to be quite certain of this point and to forestall any possible objection, we may ascertain whether normal rabbit serum that has been treated with an excess of ox corpuscles acts in a similar manner.

Let us place 1 c.c. of defibrinated ox blood in a large tube, fill with salt solution, centrifugalize, and then remove the supernatant fluid, leaving a sediment of blood corpuscles to which 1 c.c. of heated rabbit serum is added. On the following day we centrifugalize, remove the supernatant serum and add to it the fresh sediment of another cubic centimeter of washed ox blood. We may hope that two successive contacts with ox corpuscles will have absorbed all the combinable substances in the normal serum. We then prepare sensitized and washed ox blood as in the former experiment.

Two tubes, $\mathrm{A}$ and $\mathrm{B}$, are prepared, containing, each, 0.1 of a cubic centimeter of sensitized washed blood, 0.3 of a cubic centimeter of antiserum and 0.1 of a cubic centimeter of guinea-pig alexin; to tube $\mathrm{A}$ is then added 0.2 of a cubic centimeter of the normal rabbit serum treated with ox blood as described. The corpuscles are hemolyzed in 10 minutes in tube $\mathrm{A}$, but remain intact in tube $\mathrm{B}$. A control shows that the treated normal serum in the same doses does not hemolyze a mixture of antiserum, alexin and non-sensitized corpuscles.

The same results are obtained by using in place of normal rabbit serum, rabbit $>$ ox serum that has previously been deprived of its specific activity by two successive contacts with ox corpuscles. Such treated rabbit $>$ ox serum still combines with the anti- 
sensitizer, although deprived of all its specific sensitizing property for ox blood; or, in other words, when it contains only normal sensitizers with no particular affinity for ox corpuscles.*

We are not justified in concluding, therefore, as Wassermann and Ford do, that two sensitizers (or agglutinins) are identical simply because they are neutralized by the same antibody. The conclusion of these observers concerning the identity of two agglutinins affecting the same blood cells, and of which one is present in normal sera and the other is produced by immunization, may not reasonably be drawn from their experiments. It may be exact, but has not yet been proved.

The answer to question $\mathrm{F}$ is that a single given antisensitizer can neutralize several different sensitizers from the same animal species, but active for different cells. When we come to consider the fact noted by other observers (Ehrlich and Morgenroth, Pfeiffer and Friedberger), namely, that an antiserum obtained by injecting an animal of species A with the serum of species B has little or no effect on sensitizers from either species $\mathrm{C}$ or $\mathrm{D}$, we must conclude that, as far as sensitizer action is concerned, there is a closer relation between sensitizers from a common source active against different cells than there is between sensitizers active against the same cell and obtained from different animal species.

\section{Observations on the Chemical Theories of Immunity.}

The study of antisensitizers suggests various remarks of which note should be made; these remarks, indeed, would seem to facilitate the comprehension of certain insufficiently explained or inaccurately interpreted already known facts. We shall consider, therefore, first of all, Ehrlich's theory and later the mechanism of passive immunity.

There is'yet another subject which may be considered more attentively, and that is the mode of action of toxins on antitoxins, toward the elucidation of which the study of antisensitizers would seem to offer interesting information. Indeed, owing to the

* We may repeat that, in designating these substances as normal sensitizers, we simply mean that they have the power, as do specific sensitizers, of combining with antisensitizer. We know, however, relatively little of their nature and properties. We give them a definite name simply to facilitate expression. 
property that blood cells have of extracting their specific sensitizer from heated hemolytic serum, we are able to simplify the toxic solution, or, in other words, to isolate the toxin to be tested with the antitoxin. And the fact that the normal sensitizers of normal rabbit serum (which are not toxic for the ox corpuscles, but have as great an affinity for the antitoxin as the specific sensitizer), when added to neutralized toxin (i.e., sensitized corpuscles + antiserum), liberate it by uniting with a certain amount of antitoxin, may allow us to determine the law that governs the equilibrium between antagonistic substances. But we shall not discuss in the present article the theories of the interaction of toxins and antitoxins, as our researches on this subject are not yet finished. We may simply mention a fact that would seem to indicate that the destruction of toxin by antitoxin is rarely absolute-whether because the reaction is incomplete, so that the mixture always contains traces of free toxin (Arrhenius and Madsen), or because, as we believe, the neutralization of a toxin by an antitoxin is, in reality, simply a greater or less attenuation depending on whether the toxin fixes more or less antitoxin. According to our hypothesis the two substances unite in variable properties and may therefore give rise to a series of combinations ranging from free toxin to completely saturated toxin, being less and less toxic (without becoming, of necessity, quite non-toxic) in proportion to the increase in antitoxin. As we know, Ehrlich's toxons, which occur in mixing a toxin with an incompletely neutralizing dose of antitoxin, represent in Arrhenius and Madsen's conception small amounts of free toxin; according to our conception they are toxic molecules insufficiently saturated with antitoxin in which the toxicity is simply decreased without being eliminated. It would seem to us, then, that in mixing antiserum and sensitizer we form toxons from the toxin, in that the sensitizer, when affected by antiserum, is simply weakened, but not deprived of its original power. The sensitization is, as we know, very slight, so that under the ordinary conditions of experimentation, that is to say, in a mixture containing serum, the red blood cells remain intact. The sensitizing power has not, however, been entirely destroyed, for we find evidence of it if the corpuscles are made less vulnerable by being placed in a less favorable medium (salt solution); in other words, under conditions that facilitate sen- 
sitization. Under these conditions the antiserum is unable to prevent hemolysis.

Let us add 0.6 of a cubic centimeter of antiserum, which is a large dose, to 0.2 of a cubic centimeter of sensitized ox blood. We fill the tube with salt solution, centrifugalize, and remove the supernatant fluid. To the sediment we add 0.6 of a cubic centimeter of normal guinea-pig serum, 56 degrees, and 0.2 of a cubic centimeter of guinea-pig alexin. Under these conditions no hemolysis occurs. The experiment is repeated, with the variation of adding 0.6 of a cubic centimeter of normal salt solution in place of the heated guinea-pig serum after washing and then adding the alexin $(0.2$ c.c.). Hemolysis occurs completely in about an hour.* The cure of the corpuscles, then, was only partial and the sensitization, although weakened, is evident when the corpuscles are placed in a medium that lowers their resistance. $\dagger$

Are we not justified in comparing this fact with the one noted some time ago by Roux and Vaillard, namely, that a mixture of tetanus toxin and antitoxin that is harmless for normal guinea-pigs is dangerous for guinea-pigs that have been weakened by vaccination with the cholera vibrio?

Without considering these questions further for the moment, we may return to the subject properly under consideration.

Ehrlich's theory.-Relates to the origin of antibodies and is as follows: on injecting an animal with a substance that gives rise to an antibody, the substance injected unites with certain chemical elements (receptors) in certain definite cells. These cells are disturbed and react. In rëestablishing their previous condition they form new receptors which, being produced in excess, are forced out of the cell into the surrounding fluids and constitute the antibodies.

The theory offers, then, an easy means for determining the nature of antibodies. Let us apply it to the antisensitizer we have been studying. When guinea-pigs are immunized against rabbit serum we must conceive of them as having, according to the theory,

* A control shows that the same corpuscles without sensitization are unaffected. We know, furthermore, that heated normal guinea-pig serum has no antisensitizing effect.

$\dagger$ It is a well-known fact that salt solution renders corpuscles less resistant to traces of hemolytic serum. 
receptors capable of combination with certain active principles (normal sensitizers) of normal rabbit serum. The receptors affected by the injection are reproduced in excess by a cellular reaction and poured into the serum, which fluid becomes endowed with the property of neutralizing the specific rabbit $>$ ox sensitizer. Consequently, the antisensitizer is composed of receptors identical with, or similar to, the receptors in the ox corpuscles that unite with the sensitizer.* It should then act as these corpuscle receptors do, for it is, so to speak, only a solution of such receptors in serum. This conception of the antisensitizer is indeed the one recently accepted by Morgenroth. $\dagger$

The inaccuracy of such a conception founded on Ehrlich's theory is evident from the facts we have just offered. In the first place, if the antisensitizer were identical with the corpuscle receptors used, it would not combine with the sensitizer already saturated with these receptors, in other words, bound to the corpuscles. And the cure of sensitized cells by antiserum would be impossible.

Moreover, if the antisensitizer were identical with the receptors in question, it is evident that any substance which would combine with one would combine with the other. Normal rabbit serum contains, as we know, no substances that combine with the receptors of ox corpuscles (that is, these corpuscles remove nothing from the serum), and yet this serum is so avid of antisensitizer that it can compete successfully with rabbit $>$ ox sensitizer in combining with it. Rabbit $>$ ox serum, moreover, even when entirely deprived of its specific sensitizer for ox corpuscles, will still saturate antisensitizer. And, what is more, since the same antisensitizer unites with various sensitizers indifferently, whether or not they combine with ox corpuscles the result is that antiserum treated with sensitized ox corpuscles no longer protects other varieties of sensitized corpuscles.

The theory under discussion is also in direct opposition to the fact that antiserum removes from sensitized corpuscles their power of absorbing alexin. It is evident that if the antisensitizer were made from corpuscle receptors, its combination with the sensitizer

* According to Ehrlich's terminology, these receptors all have the same haptophore group.

$\dagger$ Studies on Immunity, Ehrlich-Bolduan, John Wiley and Sons, p. 241. 
should take up alexin energetically in the same way as sensitizer plus corpuscle does. This is not found to be true.*

To sum up: first, the antisensitizer unites with the sensitizer already fixed on the specific corpuscles, which latter it cures; second, the antiserum owes its activity to a single unique substance that endows it with the property of protecting various sensitized cells; in other words, of neutralizing various sensitizers that combine each with a different type of receptor; for example, the same antisensitizer combines not only with rabbit $>$ ox sensitizer, but also with other normal or specific sensitizers that do not unite with ox corpuscles; third, the antisensitizer removes from sensitized corpuscles their power of absorbing alexin. All the facts appear to be irreconcilable with Ehrlich's theory, and tend to prove the general thesis that antitoxins (or other antibodies) are not assimilated by the receptors that fix their respective toxins. We consider them all, whether acting against bacterial, vegetable or animal toxins, as substances of the same nature and of a common cellular origin, in the same general category and with marked similarity. If the receptor theory were true, the various antitoxins would be united by no analogy, since it would seem reasonable that the receptors attacked by various toxins must be widely different, according to the nature and property of the poison in question.

Ehrlich and Morgenroth have given over the larger part of one memoir to a consideration of the antisensitizers, $\uparrow$ the importance of which is essential to partisans of the lateral-chain theory. This article, indeed, is one of those that have most contributed to make this conception acceptable and to offer the most convincing arguments of its accuracy. The facts brought forth in this article are in direct opposition to our own and must, therefore, be considered. We shall first of all, however, deal with an article recently published by Morgenroth. $\ddagger$

* If we were to aecept the ideas of the Ehrlich school, and particularly the ideas that the sensitizer combines with the alexin and that each property manifested by a substance is evidenced by a particular grouping in the molecule, one might say that the antisensitizer should unite only with the cytophilic group of the sensitizer and not with its complementophilic group. There is no experimental evidence for this.

$\dagger$ Studies on Immunity, Ehrlieh-Bolduan, John Wiley and Sons, p. 88.

‡ Morgenroth, Komplementablenkung durch hämolytische Ambozeptoren. Centralblatt für Bakt., XXXV, 1904, 501. 
The hypothesis of "complement deviation" (Komplementablenkung) formulated to explain the observations of Neisser and Wechsberg on the inhibiting influence of too large an amount of sensitizer on bacteriolysis when the amount of alexin is relatively small, is well known.* It has been claimed, without direct demonstration that, since the bacteria in such an experiment cannot absorb the large amount of sensitizer, the excess rernaining in the fluid unites with the alexin and monopolizes a larger or smaller part of it, so that it does not attack the bacteria. It is rather strange that the inhibiting effect of an excess of sensitizer has never been noted in hemolytic experiments, and Morgenroth has attempted to fill this rather important gap in the theory. He admits in the first place that hemotoxic sensitizers must first be combined with the receptors of the appropriate corpuscles in order to show any marked affinity for the alexin. $\dagger$ Consequently, in a mixture of corpuscles, alexin, and too large a dose of sensitizer, the excess of the latter substance remaining free in the fluid cannot take up the alexin because it needs corpuscle receptors in order to become avid of the complement. The introduction of these receptors is necessary to produce complement deviation. On the supposition that the antisensitizer is identical with corpuscle receptors as far as affinities are concerned (since they both possess the same haptophore group, according to the lateral-chain theory), Morgenroth conceived the idea that a mixture of sensitizer and antisensitizer should be able to fix a certain amount of alexin. Such a mixture should act, in other words, precisely as we have shown $\ddagger$ that sensitizer and corpuscles do, that is, should absorb alexin energetically. It is evident that if these two substances - corpuscle receptors and antisensitizer - are considered as identical the addition of either one of them to a mixture of sensitizer and alexin should bring about the fixation of a certain amount of the active substance and conse-

* Studies on Immunity, Ehrlich-Bolduan, John Wiley and Sons, p. 120. See also in this connection this volume, p. 357 .

† It may well be questioned why antimicrobial sensitizers should not be subject to the same necessity. It is certain that if the experiment had turned out the other way, that is to say, if the phenomenon of complement deviation had been found, not in bacteriolysis, but in hemolysis, that the same explanation would have been forthcoming; it would simply have been necessary to apply it to the other instance.

$\ddagger$ See p. 191. 
quently diminish the hemolytic power of the fluid. Sensitized corpuscles, therefore, used as a reagent for the destructive power, would be less likely to be hemolzyed when added to a mixture of alexin, antisensitizer and sensitizer, than when added to a mixture of antisensitizer and alexin without sensitizer. This difference would be due to the fact that in the first instance part of the alexin would be consumed by the complex "sensitizer-antisensitizer," which acts precisely as does "sensitizer-corpuscle," since, according to Morgenroth, the terms antisensitizer and corpuscle receptor are synonymous.

Experiment confirms Morgenroth's expectations. By mixing, in carefully chosen proportions, guinea-pig alexin, rabbit $>$ ox sensitizer (56 degrees) and goat $>$ rabbit serum ( 56 degrees) it may be shown that the alexin does not remain free. If sensitized corpuscles are subsequently added to such a mixture and at the same time to another similar mixture without the sensitizer, hemolysis appears in the second, but not in the first.

Morgenroth, however, does not prove that the results are really due to the substance which he holds responsible for them. There is no proof that the disappearance of alexin is due to its combination with the rabbit $>$ ox sensitizer united to the receptors of antisensitizer. To prove that it is this sensitizer that uses up the alexin, a control should have been made to show that the same result is not obtained in a mixture of alexin, antisensitizer, and an immune serum that has been already deprived of its specific sensitizer.*

It seems to us that Morgenroth's phenomenon should be explained differently. In addition to the alexin there are two sera in the experiment to be considered. The first (antisensitizer) has been obtained by injecting goats with the second serum, namely, of rabbits immunized with ox corpuscles. The first is certainly antisensitizing for the second in the sense that it neutralizes its specific sensitizer. But from another standpoint it may be regarded as sensitizing for the same serum, and this fact Morgenroth does not take into account. The antiserum (serum I) is from animals injected with alien serum (serum II), and in this respect should sensitize serum II if we consider this serum simply

* Or in a mixture of alexin, antisensitizer and normal rabbit serum. 
as a solution of albuminous substances, precisely as the serum of animals vaccinated with milk sensitizes milk; in other words, confers on certain of the constituents of milk a power to absorb alexin.* Gengou has demonstrated the interesting fact that "anti-albuminous sensitizers," similar to antimicrobial or antihematic sensitizers, may be obtained, which have a similar power of fixing alexin when united with the antigen. Gengou proved the existence of such sensitizers, not only in the serum of animals immunized against milk, but also in the serum of animals treated with an alien serum. Such an antiserum plays an obvious rôle in Morgenroth's experiments. We are justified in supposing, then, that the alexin absorption is brought about, not by a union of antisensitizer with sensitizer, but simply by certain sensitized albuminoids of the rabbit $>$ ox serum.

Morgenroth's conclusion, therefore, is not acceptable in the present state of our knowledge. As far as we are concerned, the theory of complement deviation by amboceptor (sensitizer) is a myth. We have already stated that an identity of receptors with antibodies cannot be admitted. It is not only incompatible with our own results, but receives no experimental confirmation from the work of Pfeiffer and Friedberger, who are zealous upholders of Ehrlich's theory.

We may note in passing that Morgenroth has not noticed in his experiments that his antisensitizer can cure already sensitized corpuscles. In this his result differs from our own; but we used a different antiserum. The question, however, properly arises as to whether the lavish use of normal salt solution (which, as we have seen, tends to annul corpuscle protection to a great extent and should therefore be, as much as possible, eliminated from hemolytic experiments $\dagger$ ) has not affected the accuracy of this author's observations.

Let us now consider as briefly as possible Ehrlich and Morgenroth's ideas on antisensitizers as stated in their sixth memoir on hemolysis.

These authors employ an immune serum from rabbits immunized against ox blood. They find that this serum gives hemolysis with various alexins and particularly with those of the guinea-pig

* See Gengou, p. 241. $\dagger$ See also Gay, p. 333. 
and the goat. They find, however, that when they use goat alexin the corpuscles must be more heavily sensitized (that is, more heated immune serum added) than when they use guinea-pig alexin. The fact, however, is not surprising. It is well known that the alexins from different animals are not strictly identical; and, since they differ somewhat, it would be strange indeed if they had equal hemolytic power for a given blood cell; and it is not astonishing that the corpuscles have to be made more vulnerable by a heavier sensitization in order for some alexins to destroy them, whereas a weak sensitization suffices with other alexins. Ehrlich and Morgenroth, however, reject so simple an explanation. They think, rather, that the immune serum contains two (or more) distinct sensitizers, " $\mathrm{x}$ " and " $y$ "; the one in greatest abundance ( $\mathrm{x}$ ) is very efficient with the guinea-pig alexin, but finds no fit complement in the goat serum; this first sensitizer, then, has nothing to do with an hemolysis caused by goat alexin. This alexin, however, suits the second sensitizer (y) of the serum very well, but, since this " $y$ " is present in relatively small amounts, a large amount of immune serum must be used when goat alexin is employed. Let us accept, for the sake of argument, this idea of the multiplicity of sensitizers in a given immune serum in spite of its improbability, and go on to the next point.

Ehrlich and Morgenroth have a second serum, an antiserum from goats obtained by immunizing them with rabbit $>$ ox immune serum, which latter serum it neutralizes. First of all, they add to a large dose of this antiserum (Dose A, let us say) a small amount of rabbit $>$ ox serum and then add ox corpuscles. These corpuscles are then washed and guinea-pig alexin added to them. There is no hemolysis, showing that the sensitizing power has been abolished by the antiserum. Their conclusion is as follows: The antiserum is antitoxic for sensitizer " $\mathrm{x}$ " suitable for guinea-pig alexin.

Ehrlich and Morgenroth then perform a second similar experiment, using in this instance goat alexin. Under these conditions the antiserum apparently does not protect the corpuscles and hemolysis occurs. This is the conclusion: The antisensitizer is so specific that, although it can neutralize sensitizer " $x$," it has no effect on sensitizer " $y$," which is particularly suited to goat alexin. This fact goes to show that there are indeed two distinct sensitizers.

Their second experiment, however, differs from the first in an 
important detail. Imbued with the idea that the rabibit $>$ ox immune serum contains only a small amount of " $y$ " suitable for goat alexin, Ehrlich and Morgenroth think it indispensable to mix with the given dose $\mathrm{A}$ of antiserum, as, in the first experiment, a much larger amount of rabbit $>$ ox immune serum than was used in the first instance. They think this technic is justified because even a large amount of the serum would contain only a small amount of the " $y$ " which is to be tested against the antiserum and is alone of importance, since it is the only sensitizer that can hemolyze the corpuscles in conjunction with goat alexin. They do not consider that, in adding this excess of immune serum, they introduce, not only a large amount of specific sensitizer, which is the only substance they have in mind, but also a large amount of normal sensitizers, which, as we know, monopolize a greater part of the antisensitizing power and so prevent the specific sensitization from being neutralized by the antiserum. Of course under these conditions the corpuscles are hemolyzed. A small amount of normal serum would have been just as effective in hiding the antisensitizing effect as was the excess of immune serum. Under such conditions no corpuscle protection would have been evident even if guineapig alexin were used instead of goat alexin. Ehrlich and Morgenroth's laborious considerations on the nature of antisensitizers, on the multiplicity of antibodies, and particularly on the multiplicity of sensitizers in a given immune serum lead to an incorrect conception of the experimental results. It is evident from this example that the logic employed in defending the lateral-chain theory is far from unassailable; indeed, in certain instances it is open to severe criticism and cannot be accepted without argument.

Theories of passive immunity. - The immunity conferred by injecting preventive serum presents certain peculiarities that have recently attracted considerable attention. It was formerly thought that the animal that received antitoxin and benefited from it acted simply as a passive recipient without any reaction, or the elaboration of any antagonistic substance even when the serum injected was from an alien species. The fact that the duration of passive immunity is brief was always supposed to be due to a gradual elimination of the antibodies. This was the general opinion, which we ourselves shared when we demonstrated in 1895 
that the collaboration of two substances was necessary for the bacteriolysis of the cholera vibrio. We were able to satisfy ourselves that an animal immunized with cholera serum, and thereby acquiring, as Fraenkel and Sobernheim showed, a bacteriolytic power in their body fluids, owes this newly obtained power to, first, the alexin already present in the normal animal, and, secondly, the specific sensitizer in the injected cholera serum. The bactericidal power, then, is generated in the fluids of the treated animal as it is in a test tube containing fresh normal serum on the addition of a little anticholera sensitizer. In either instance a combination of the two substances is required.* The fact that the immunity soon disappears is due simply to disappearance of the sensitizer from the fluids.

This explanation of the transitory nature of passive immunity is doubtless correct in those instances in which the injected serum comes from animals of the same species. But it has been noted by several observers that the duration of the immunity is remarkably short when the serum is obtained from an alien species. $\dagger$ Pfeiffer and Friedberger offered the plausible hypothesis in the case of cholera serum that, when passive immunity disappears very rapidly, it is owing to the fact that the treated animal reacts to the substances injected by elaborating antagonistic substances, like antisensitizers, that neutralize them.

The question may well arise as to whether animals of species A inoculated with any immune serum from species B (antitoxins, agglutinins, lactoserum and so forth) do not form antisubstances to them, comparable to those that neutralize hemolytic sensitizers. As we have noted, Pfeiffer and Friedberger obtained an anticholera serum and Schütze $\ddagger$ has obtained an antilactoserum. Kraus and Eisenberg§ have made systematic studies of these substances and failed to find in the serum of treated rabbits substances capable of neutralizing the diphtheria antitoxin or the typhoid agglutinins present in specific sera from the horse. Such results appear irrecon-

* See p. 80.

† See particularly among recent articles, Schütze, Ueber das Verschwinden verschiedener Immunsera aus dem tierischen Organismus. Festsch.v 60 Geburtstag. v. R. Koch, p. 657.

$\ddagger$ Sehütze, Berlin. klin. Wochen., 1901, No. 50, 1263.

$\S$ Kraus and Eisenberg, Cent. f. Bakt. Orig. XXXI, 1902, 208. 
cilable. Why is not the law that controls these phenomena more general?

To explain the contradictory facts in harmony with Ehrlich's theory the following explanation has been offered: Diphtheria antitoxin and typhoid agglutinins have affinity for only such receptors as occur in their respective micro-organisms. It is natural, then, that these substances should remain free in the injected animal, since they find no appropriate receptors; as these receptors are lacking, they are not reproduced and consequently no active antiserum is formed. It might be objected that if this explanation were true that Pfeiffer and Friedberger would have obtained no antisensitizer to cholera serum. But this objection could, in turn, be answered by saying that although the injected animal has no receptors identical with those of typhoid or diphtheria bacilli and consequently fitted to fix the active substances that affect these organisms, they do possess receptors similar to those of the cholera vibrio. If the experiment had turned out the other way, that is to say, if an antityphoid serum had been obtained more easily than an anticholera serum, the converse explanation of the respective absence or presence of receptors could have been offered. In short, whether an antiserum is obtained or not, the receptor theory is upheld; in unfavorable cases receptors are lacking; in favorable cases they are present. Whatever happens, the use of the term "receptors" makes the facts easily explicable.

Another explanation of these divergent results may, however, be offered. As we have already seen, the serum of a guinea-pig immunized against rabbit serum neutralizes the various specific sensitizers from the rabbit indifferently, and also the normal sensitizers (or substances of this nature) in normal rabbit serum. And, what is more, a single given antisensitizer confers all these antagonistic powers on the antiserum or is able, in other words, to show these various affinities by uniting with the various sensitizers.

It follows, therefore, that to neutralize a given specific sensitizer (e.g., rabbit $>$ ox sensitizer) economically by means of an antiserum it is well to have the sensitizer in a relatively pure condition, without admixture of other sensitizers, before treating it with.the antiserum. Under these conditions the neutralizing effect of the antiserum is directed only against the sensitizer in question. 
This purification of the sensitizer is accomplished in our experiments in which we have used corpuscles treated with their specific serum.* If this precaution is neglected by mixing the whole immune serum, which contains other analogous substances in addition to the specific sensitizer, with the antiserum in the first place, the neutralizing effect is wasted. When we add to our sensitized corpuscles, either before or after mixing them with antiserum, normal sensitizers in the form of normal rabbit serum, 56 degrees (or indeed rabbit $>$ ox serum deprived of its specific sensitizer by contact with ox corpuscles) their cure is gravely compromised, since the neutralizing power of the antiserum ceases to be concentrated on the sensitizer affecting the corpuscles. If, therefore, the mixture is made in the usual manner, antiserum and whole immune serum containing the specific sensitizer (or antitoxin or agglutinin, for these remarks are apparently applicable to any of the antibodies) to be neutralized, the chance of obtaining a neutralization depends on the relation of specific and normal sensitizer content in the immune serum. The greater the relative amount of normal sensitizers the less the specific sensitizer will be affected by the antiserum, so that its neutralization may be practically nil. And if the antisensitizers-or, one may say, the anti-antitoxins-. are found to be of relatively little potency or practically negative, it is due at least in part to the fact that not all their activity is evident. When we expect to neutralize a given immune body in an immune serum we do not consider that energy may be exhausted in saturating other substances that are not taken into account in the experiment and of the presence which we may actually be ignorant. It would be strange, indeed, that the relative proportion of normal and specific sensitizers should be the same in all immune sera from no matter what animal species. It must be expected, then, that

* It would be desirable, to be sure, to obtain pure sensitizer in some other way. - It would then be possible to treat it with antiserum before it was united with the blood cells. Hitherto such a separation has not been possible, although it would be theoretically better than the method we have employed. It is indeed possible that the antisensitizer should show no preventive action on pure sensitizer, although it can cure sensitized corpuscles. It would seem, indeed, from our experiments that there is some struggle between the affinity of the sensitizer for the corpuscle on the one hand, and for the antisensitizer on the other. And it may be that in certain cases the combination between sensitizer and corpuscle may be so stable as to prevent any curative action by an antiserum. 
anti-antibodies are not always detectable experimentally, which may account in part for the variation in results. There are different affinities to be considered in these experiments. There is the affinity of the anti-antibody for the specific antibody and for the normal antibody, and then there is the affinity of the antibody for the sensitive cell used as reagent. The susceptibility of this reacting cell must also be taken into account. The net result from these factors varies, and yet is the only result that we take into consideration. The laws that govern these phenomena may in reality be general, although the apparent results differ. The study of immunity is full of such instances. It is a general rule that the injection of bacteria gives rise to sensitizers, and these sensitizers increase the destructive power of the alexin. It is exceptional, nevertheless, that the immune serum obtained in this way is strongly bactericidal for the specific bacterium; and why? It is due to the fact that, although there are certain bacteria that are so delicate as to be destroyed by immune sera, the majority of them are resistant and suffer no injury from the serum in a suitable medium, as Metchnikoff has shown by numerous examples.

When we inject animals with immune serum in the hope of obtaining an anti-antibody we naturally think of the animal as reacting particularly to the antibody that we are studying. If the experiment succeeds, as is the case with cholera serum, and if we believe in Ehrlich's theory, we conclude that the animal has formed an anticholera serum owing to the reproduction of receptors analogous to those of the cholera vibrio. But this conception does not represent the true state of affairs. If the cholera sensitizer is neutralized, it is not on account of its specific peculiar qualities and simply because it affects the cholera vibrio. If it is neutralized it is not owing to any "personal equation," so to speak, but simply because it belongs in common with all sensitizers (and probably also all antitoxins) to a group of substances belonging to species $A$, which, on injection into species $B$, cause a reaction. To speak colloquially, we might say that the injected animal is not concerned as to whether it is given antibodies that affect tetanus or diphtheria toxin, or those acting on cholera or typhoid bacilli ; haptophore groups of toxins and receptors of bacilli do not particularly appeal to it. 
Antibodies belong simply to a group of active substances in sera. The only affair of the treated animal is to react against certain or all members of this group, representing, as they do, an alien substance. Nor must it be lost sight of that in injecting immune serum we inject, not only specific antibody, but normal serum as well. It is simply the unaccustomed presence of all these foreign substances that leads the animal to form an antagonistic substance, and as a result this substance is not strictly specific. Such an antiserum may be used to protect bacteria or various kinds of blood cells against the respective antibodies obtained from the same animal species. Since the antibodies obtained by immunizing with normal serum will neutralize any antibody from the animal species corresponding to the normal serum employed, it is obvious that it acts on the entire category of substances in this serum.

Theoretically, at least, we might expect that an animal would react more violently on injection of toxic immune serum specific for its own blood cells.* This condition, however, is not indispensable in order to produce a reaction, as is reasonable when we consider that normal serum frequently has a harmful effect on animals of a different species.

When, however, an animal is injected with a specific immune serum acting on bacteria or cells that have nothing in common with the vaccinated animal, and as a result an antiserum is formed that neutralizes the injected antibody, there is no legitimate reason for us to conclude that there is any necessary identity or even relation in the composition of this animal's cells and the substances (bacteria, cells, or bacterial products) against which the serum injected is specific. There is no reason for introducing the conception of common receptors. As a matter of fact the antiserum obtained under these conditions does not differ from that obtained on injecting normal serum; we repeat that it acts indiscriminatingly on any of the active substances present in the foreign serum.

We believe, then, that the rapid disappearance of passive immunity

* It may be noted, however, that Kraus and Eisenberg did not obtain any detectable active antiserum on injecting dogs with an immune serum active for dog corpuscles. 
afforded by the injection of an alien serum should be attributed, as a rule, to the secretion of antagonistic substances. The fact that this substance is not easily detectable experimentally is due to its inhibition by certain of the conditions to which reference has been made. Such conditions depend on the doses used and the relations of affinity between the substances that take part in the reaction. In so far as dosage is concerned, it should be noted that the amount of immune serum administered for passive immunity is very small in proportion to the volume of blood in the recipient; under such conditions the antagonistic power that soon develops has greater chance to manifest itself than in test-tube experiments, where the tendency is to minimize the amount of antiserum.

\section{Conclusions.}

The study of an antiserum obtained by injecting animals of species A with the normal serum of species B gives rise to the following remarks:

I. Various red blood cells, sensitized each by its appropriate heated hemolytic serum obtained from an animal of species B, lose their sensitization to alexin when treated with the antiserum. The sensitization is generally diminished rather than completely abolished; it may, indeed, frequently be demonstrated when the corpuscles are placed in a medium which tends to diminish their resistance, for example in salt solution.

II. It is not necessary in obtaining an antiserum that neutralizes various specific sensitizers, obtained in each instance from an animal of species $B$, to inject animals with the respective specific sensitizers, but simply with normal serum from species B.

III. The power of this antiserum to neutralize various specific sensitizers as well as the normal antibodies (or sensitizers) in B serum may be attributed to the presence in this antiserum of a single antisensitizer. There is no need of assuming the existence of a multiplicity of antisensitizers. As far as the action of antisensitizer is concerned, there is a closer relation between sensitizers from the same source acting on different cells than between sensitizers from different sources acting on the same cell.

IV. The antisensitizer is used up in acting. The addition of sen- 
sitized corpuscles to antiserum removes from it the power of protecting additional sensitized corpuscles either of the same or of a different variety. The normal sensitizers in serum B also neutralize the antiserum.

V. The antiserum cures sensitized corpuscles by uniting with the sensitizer that is fixed on them. Corpuscles protected in this way resist alexic activity even when the excess of protective serum has been washed away.

VI. It would seem as if the complex antisensitizer-sensitizerred blood cell were broken up on adding normal sensitizers of serum B (i.e., normal serum or immune serum deprived of specific sensitizers by contact with blood cells), because these sensitizers take away at least a part of the antisensitizer that has already been combined with the specific sensitizer.

VII. The power in normal serum B of inhibiting the curative effect of the antiserum for sensitized corpuscles resists heating to 70 degrees, but not to $100^{\circ} \mathrm{C}$. It is not present in aqueous humor from species B. It has previously been ascertained that the aqueous humor of vaccinated animals contains no specific sensitizer.

VIII. When antiserum affects the sensitizer attached to red blood cells it removes from the complex the property of fixing alexin.

IX. The identity of the antibodies of normal and of immune serum affecting the same cell, as assumed by certain writers, must be considered as not proved.

X. Ehrlich's theory, which supposes that specific antibodies are identical with the cell receptors that combine with those substances against which the animal becomes immunized, is erroneous. The arguments to support this theory obtained from the study of antisensitizers are not sound. The thesis that a given hemolytic immune serum contains several separate specific sensitizers has no experimental justification. The conception of complement deviation due to an excess of sensitizer is purely hypothetical.

XI. The short duration of the passive immunity afforded by injecting an immune serum from an alien species would seem to be due to the fact that the recipient generally elaborates an antagonistic substance. The effect of this substance is not directed especially against the antibody that gives immunity, but in a 
general way against any and all sensitizers, normal or specific, present in the alien serum. The production of this antagonistic substance is not in any way dependent on the identity or even on a close relation in composition between the cells of the animal body and the substances injected. There is no reason, in other words, to assume the existence of common receptors. 


\section{RESEARCHES ON THE AGGLUTINATION OF RED BLOOD CELLS BY CHEMICAL PRECIPITATES AND ON THE SUSPENSION OF SUCH PRECIPI- TATES IN COLLOIDAL MEDIA.*}

Bx Dr. OCTAVE GENGou.

Colloidal substances play so important a rôle in vital phenomena that biologists have been following with great interest the advances made by chemistry and physics in our knowledge of these substances. Any analysis of the properties of organic colloids has been extremely complicated by our ignorance of their composition; for this reason we have resorted to such information as may be gained from a study of simpler colloids. We have, in general, drawn our conclusions as regards organic colloids from the rules that have been drawn from the better-known reactions with inorganic colloids. One of the best-known phenomena afforded by these two groups of substances is agglutination. Works dealing with this phenomenon are numerous, and the recent publications of Perrin on colloidal substances have stimulated biologists to a most active study of it.

Although the agglutination of bacteria and of red blood cells by sera has been investigated with much diligence, the essential principles of the reaction are still a mystery. The apparent analogy between agglutination and the precipitation of colloids has given rise to the hope that a careful analysis of this latter phenomenon may lead to an explanation of agglutination. The works dealing either with the precipitation of colloids or the agglutination of red blood cells by suspensions (that is, by colloids or chemical precipitates) have been very numerous since the publications of Perrin.

Landsteiner and Jagic $\uparrow$ were, we believe, the first to draw atten-

* Recherches sur l'agglutination des globules rouges par les precipités chimiques et sur la suspension de ces precipités dans les milieux colloïdaux. Annales de l'Institut Pasteur, XVIII, 1904, 678.

† Landsteiner and Jagic, Wien. klin. Wochenschr., 1904, No. 3. 
tion to the fact that red blood cells may be agglutinated by a welldefined colloid, namely, by colloidal silicic acid. Shortly afterward we ourselves* reported examples of agglutination and hemolysis of red blood cells by means of such chemical precipitates as $\mathrm{CaFl}_{2}$ and $\mathrm{BaSO}_{4}$. Girard Mangin and Henri† have since studied this question in a very exhaustive and careful manner with different colloids.

As we have already demonstrated, certain chemical precipitates as well as colloids agglutinate red blood cells, provided the latter are washed free of serum; this agglutination is followed by hemolysis, which latter phenomenon we have referred to in our first article. This agglutination and hemolysis is inhibited by the presence of even very small amounts of serum.

In the present article we shall consider simply the agglutination of corpuscles and at some later period take up their laking. Mme. Girard-Mangin and V. Henri found that the agglutination of red blood cells is brought about by negative as well as by positive colloids. Red blood cells, however, have a negative electric charge, as is shown by the fact that they are deposited at the anode. The fact that a negative emulsion (corpuscles) may be agglutinated by equally negative colloids evidently does not coincide with the general ideas that we have gained concerning the action of colloids having effect on one another or other colloids with the same electric charge. A mixture of colloids having the same electrical charge indeed causes no flocculation; both colloids remain in suspension, $\ddagger$ and so Mme. Girard-Mangin and V. Henri did not conclude that the agglutination of red blood cells by colloidal substances is due to a direct interaction of these substances. They believe that this agglutination is only an indirect and secondary affair; they think that the corpuscle is passive in the phenomenon and that the active functions are exerted by the colloidal substances on the one hand and by endocorpuscular salts liberated by the corpuscles on the other.

It is true, indeed, that certain colloidal substances, as, for example, those studied by Mangin and Henri, are flocculated by electrolytes. When red blood cells are left in normal salt solution they liberate

* Gengou, Comptes rendus de l'Acad. des Sciences, April 11, 1904.

$\dagger$ Mme. Girard-Mangin and V. Henri, Soc. de Biol., 1904, Nos. 19, 20, 21, 24, 25.

$\ddagger$ Henri, Lalou, Mayer and Stodel, Soc. de Biol., 1903, Dec. 19. 
a certain part of their salts and it is perfectly evident that these salts in the course of their diffusion from within the blood cells will be more abundant in the pericorpuscular zone near the corpuscle than in the fluid between the corpuscles at a relatively considerable distance from the corpuscles. And so Mangin and Henri think that colloidal substances mixed with a suspension of corpuscles will by preference be precipitated in the more concentrated pericorpuscular zone.

Following this first stage, a second would occur, during which the particles of colloidal substances that have been flocculated about the corpuscles by means of the endocorpuscular salts would collect into masses and drag the corpuscles with them; hence would result the formation of masses composed of colloids and corpuscles.

According to this theory the agglutination of red blood cells by colloidal substances depends on a preliminary precipitation of these colloids about the corpuscles by means of the diffused electrolytes from within the corpuscles. The corpuscles remain quite passive during the entire phenomenon and are simply brought together by means of the collection of particles of colloidal substances that have been precipitated about them.

We do not believe that the phenomenon of agglutination of corpuscles by colloids should be interpreted in this way; we think rather that the agglutination is due to a direct action of one of these elements on the other.* We think, indeed, that we are justified in applying the conclusions drawn from the facts we have observed with chemical precipitates to the agglutination of corpuscles by colloids. According to the opinion of Bredig $t$ these two substances are of the same order and differ from one another simply in a variation in size of their particles. This opinion indeed is shared in so far as the present phenomena are concerned by Landsteiner and Jagic $\ddagger$ and by Mangin and Henri. $ई$

It is to be noted in the first place that the colloids studied by these latter authors are very susceptible to flocking by electrolytes;

* It is evident that there is no dispute about the well-known action of salts in the phenomenon of agglutination in general; we are dealing here simply with the function attributed by Mangin and Henri to the salts that have diffused from the red blood cells on their agglutination by colloids.

$\dagger$ Bredig, Anorganische Fermente.

$\ddagger$ Landsteiner et Jagic, Münch. med. Woch., No. 27, 1904.

$\$$ Mme. Girard-Mangin et V. Henri, Soc de Bıol., No. 19, 1904. 
they may, therefore, be easily flocculated by the electrolytes diffused from the corpuscles, but it does not follow that this explanation serves to explain their agglutinating power over corpuscles.

It is not essential for a substance to be precipitated by salts coming from corpuscles in order that the agglutination by this substance be possible. We have already noted that barium sulphate agglutinates corpuscles readily and barium sulphate, as we know, is much more susceptible to gravity than it is to electrolytes. It is no more flocculated by a strong concentration of salt solution than it is by distilled water, in which latter solution it sediments rather rapidly.

We have undertaken to determine whether a fine suspension that is flocculable by electrolytes can subsequently agglutinate corpuscles after having been flocculated.

For this purpose we have used calcium fluoride, which is a precipitate of rather colloidal appearance flocculable by 0.8 per cent $\mathrm{NaCl}$. We find that calcium fluoride that has been previously flocculated by 0.8 per cent or by 2 per cent sodium chloride will still agglutinate corpuscles in 0.6 per cent salt solution quite as well as if it were in suspension in distilled water. If the saline concentration is increased to 3 per cent the calcium fluoride so treated no longer agglutinates as well. This would prove simply, according to our opinion, that the very dense accumulated masses of colloidal precipitates are less effective on corpuscles, owing to the fact that with such masses an intimate mixture of the corpuscles and the calcium fluoride is no longer possible. When the masses, on the contrary, are not so dense (for example, $\mathrm{CaFl}_{2}$ in sodium chloride, 0.8 per cent or 2 per cent) and consequently more easily broken up, the effect of the suspension on the corpuscles is only slightly attenuated. We should not conclude from these facts, however, that the agglutination of corpuscles by calcium fluoride is possible only when the state of dissociation of this substance is such that it may be flocculated by the salts about corpuscles as they pass out, but simply that the greater the intimacy of mixture between the corpuscles and the calcium fluoride, the more intense the agglutination.*

* It is probable that the same holds true for the colloidal substances, which become distinctly less active, as they are less easily flocculated by electrolytes. Mme. Girard-Mangin and V. Henri asserted that flocculated colloids no longer aggluti- 
If colloids are flocculated about corpuscles before agglutinating them, as Mme. Girard-Mangin and V. Henri think, such a concentration depends on the difference of saline concentration between the rich pericorpuscular zone and the relatively weak fluid between the corpuscles. If this difference in concentration were eliminated, there would be no reason for the colloids to flocculate about the corpuscles, and consequently the agglutination of the latter by the colloids would be nil or, at least, very much diminished. We have endeavored to determine whether such a result may be verified experimentally. We introduce in a large volume of normal saline a certain amount of well-washed red blood corpuscles and allow the mixture to stand for 3 or 4 hours, taking care to shake it from time to time. It is evident that the longer a diffusion of intracorpuscular salts goes on the more will the fluid between the corpuscles increase in tonicity and the more perfect will the equilibrium between this fluid and the pericorpuscular zone become. And, what is more, in proportion as the corpuscles lose their electrolytes, the concentration of the fluid surrounding them becomes more nearly equal to their tonicity. Consequently, after a certain period there will be an equilibrium between the corpuscles and the surrounding fluid. If we centrifugalize at this point and decant the supernatant fluid, we shall have a fluid A (salt solution plus diffused salts from the corpuscles) and the red blood cells $\mathrm{D}$, which have lost their salts.

Let us place in tube 1 a given amount of fluid $\mathrm{A}$ and in tube 2 an equal amount of salt solution; we then add to both the same amount of $\mathrm{CaFl}_{2}$ and allow the mixtures to stand for a half hour in order to allow the intracorpuscular salts in fluid A to act upon the suspension. We then add to each tube the same amount of corpuscles D. Agglutination of the corpuscles immediately takes place and there is no difference in its intensity in the two tubes, although the experimental conditions differ. It is indeed to be

nate corpuscles. Landsteiner and Jagic, however, find that the previous flocculation of colloids does not diminish their agglutinating property for corpuscles. It is possible that both experimenters are correct, and that one may flock out colloids at various stages and so obtain colloids that are either agglutinating or not so, in accordance with whether the electrolytes have formed loose or dense clumps, that is to say, depending on whether a mixture of the colloids with the corpuscles is or is not possible. 
noted that in this experiment we used D corpuscles and not fresh corpuscles. The reason that we have employed D corpuscles is because they have been for a long time in contact with fluid $\mathrm{A}$ and supposedly are very similar in tonicity to this fluid, so that they have nothing more to give out into it. Under these conditions we should not expect the pericorpuscular zone to be richer in salts than is the fluid between the corpuscles. In tube 2, on the contrary, D corpuscles are placed in contact with saline solution that does not contain the corpuscular salts, and their tonicity is therefore higher than that of the salt solution. We should expect them, therefore, to diffuse their own salts and to create a pericorpuscular zone that is more concentrated than the fluid between the corpuscles. To sum up, in tube 1 we have a pericorpuscular zone and an intercorpuscular fluid of the same concentration; in tube 2 we have a pericorpuscular zone that is more concentrated than between the corpuscles. The agglutination of the corpuscles by calcium fluoride should therefore be more intense in tube 2 than in tube 1 if the salts in the pericorpuscular zone play any rôle in the phenomenon.

And there would be still another reason why the intensity of the agglutination should differ in the tubes in our experiment if this hypothesis were true. As we introduced $\mathrm{CaFl}_{2}$ some time before the corpuscles, this suspension was subjected to the flocculating effect of salt solution in tube 2 and to the effect of fluid A in tube 1 . As fluid A contains the intracorpuscular salts it should have more flocculating action than salt solution, and consequently $\mathrm{CaFl}_{2}$, being more markedly flocculated in tube 1, should in tube 2 have less effect on the corpuscles. As we have already seen, the results do not justify this conception, and, what is more, $\mathrm{CaFl}_{2}$ is not flocculated in tube 1 any more than it is in tube 2 . In other words, the supposed increase of salt in tube 1 from the corpuscles does not give any more marked flocculation than does salt solution.

This experiment demonstrates that the agglutination of corpuscles by $\mathrm{CaFl}_{2}$ does not lose in intensity when the flocculating effect that the pericorpuscular zone might have on the suspension is reduced to the minimum.

The objection may be raised that the diffusion of salts from the corpuscles is never pushed sufficiently in such an experiment and 
that the saline concentration of the pericorpuscular zone is under any condition sufficient to bring about a precipitate of the colloid or of the suspension around the corpuscles. We have noted a fact in our first article that answers this objection: we caused a diffusion of all the substances that the corpuscles contain. For this purpose we laked corpuscles with distilled water and then washed them several times in 0.7 per cent salt solution. As a result of several such washings we presumed that the saline tonicity of the stromata and consequently of the pericorpuscular zone should not exceed that of the fluid between the corpuscles, and that, consequently, with such stromata we have to deal simply with the sodium chlorid in salt solution. Such stromata as they contain no intracorpuscular salts should not be agglutinable by a colloid or by a chemical suspension. We find, however, that on adding $\mathrm{CaFl}_{2}$ to such stromata an agglutination takes place which is quite comparable with that of red blood cells; the $\mathrm{CaFl}_{2}$, moreover, may be replaced by barium sulphate or by colloidal ferric hydroxide.

We see no reason for supposing that the agglutination of stromata by colloids and suspensions is to be explained in any different way than that of red blood cells. In fact the agglutination of stromata by such a suspension as $\mathrm{CaFl}_{2}$ follows in all its details the phenomena present in the agglutination of red blood cells. In both instances the intensity of the agglutination depends on the amount of $\mathrm{CaFl}_{2}$ employed; the maximum agglutination occurring with an optimal dose, a smaller or larger amount giving rise to less agglutination. In each instance, moreover, the addition of a small amount of serum prevents agglutination. The phenomena are absolutely interchangeable, which shows, according to our opinion, that in agglutination of corpuscles by a suspension the salts from the corpuscles do not first flocculate with the suspensions in order to bring about agglutination. The fundamental action takes place between the albuminoid substances (stromata) of the corpuscles and the suspensions in question.*

* We find, indeed, that agglutination of stromata may be produced by barium sulphate whether suspended in distilled water or in 2 per cent salt solution, and it seems difficult to imagine that a suspension that is so little affected by electrolytes should be influenced by the traces of salts that might come from the stromata. Mangin and Henri have noted similar phenomena in the agglutination 
There is, to be sure, one conclusion in the theory of Mangin and Henri which seems experimentally verified, but as we shall see the verification is only apparent and their conclusion incorrect. This is the subject in point. If it is true that the salts within corpuscles have something to do with flocculating colloids and suspensions about the corpuscles, on extracting the salts from these corpuscles by laking, we should obtain a fluid L, which, when the stromata are removed, will prevent the agglutination of fresh corpuscles by a suspended precipitate. This in particular would be true if the suspension were placed in fluid $\mathrm{L}$ before the fresh corpuscles were, thus allowing it to be flocculated by the corpuscular salts present in the fluid. We proceed, then, by laking blood corpuscles with distilled water and subsequently adding enough sodium chlorid to restore its tonicity to that of physiological salt solution, and we then separate the stromata by centrifugalization and decant the supernatant fluid, which contains all the substances that the corpuscles liberated. To a given amount of this fluid we add a known quantity of $\mathrm{CaFl}_{2}$, of barium sulphate or of colloidal ferric hydroxide and, after a short period, we add to each tube a small amount of fresh washed corpuscles. No agglutination occurs. The laked fluid, then, deprived of stromata completely prevents the agglutination of other corpuscles either by suspensions or by ferric hydrate.

At first sight this fact agrees very well with Mangin and Henri's ideas, but it is not, however, explicable as we see it by their theory. If the inhibiting effect of this fluid is in reality due to diffused intracorpuscular salts, it must be due to the precipitation of the colloids or suspensions by these salts, which precipitation in this case would occur without the subsequent addition of corpuscles. We find, however, no precipitation of colloids or suspensions by this laked fluid; the reverse indeed is found. Salt solution rapidly flocculates $\mathrm{CaFl}_{2}$ and ferric hydrate and allows barium sulphate to sediment rather quickly, but fluid L, deprived of stromata, keeps these substances in suspension; even the ferric hydrate, which is so susceptible to the presence of salts. Since the electrolytes in

of corpuscles by colloids; they find that stromata are also agglutinable by these substances. Under such conditions we see no reason for attributing an active function to the corpuscular salts, as do these authors. 
this fluid can only have a flocculating action they are evidently not responsible for producing this suspension.*

We believe (although our opinion is based on no irrefutable experimental proof) that if ferric hydrate and $\mathrm{CaFl}_{2}$ fail to be precipitated by the electrolytes of fluid L, and if barium sulphate remains in a state of fine suspension in this fluid, it is due to the fact that they are held in suspension by the colloidal substances of an albuminous nature that have come from the corpuscles through laking. The phenomenon, indeed, is identical with the dissociating effect of serum on barium sulphate, to which we have already referred and which we shall presently again consider. This dissociating power in the laked fluid can be exhausted, as can the same power in serum, and if we presume that the precipitate is due to some substance we may suppose that this substance can be removed from the laking fluid. As a proof let us add a rather large amount of $\mathrm{CaFl}_{2}$ to fluid $\mathrm{L}$ containing no stromata; after 15 minutes' contact we will remove the $\mathrm{CaFl}_{2}$ by centrifugalization and obtain, on decanting, a fluid $\mathrm{A}$ which differs from fluid $\mathrm{L}$ only in having been subjected to a large amount of fluoride. We find that the sedimentation and flocculation of the various substances to which we have referred, although prevented by fluid $\mathrm{L}$, occur very readily in fluid A. And, what is more, an agglutination of red blood cells by suspensions and by ferric hydrate takes place perfectly in A, although it does not occur in L.

The large amount of $\mathrm{CaFl}_{2}$, then, has removed something from fluid $\mathrm{L}$ that prevents the flocculation of $\mathrm{CaFl}_{2}$ and of ferric hydrate by electrolytes as well as the sedimentation of barium sulphate. The property of preventing the agglutinating of corpuscles by means of these three substances has also been removed from fluid $\mathrm{L}$ by this treatment. We believe that this substance or substances are albuminous colloids that come from the corpuscles as a result of the laking, and we base this hypothesis on the results obtained

* Neisser and Friedemann* have noted, it is true, that colloids flocculated by a given dose of salts no longer give such a result if the amount of salt is increased. In their experiments, however, they deal with the salts of heavy metals and they have compared this fact to the formation of colloidal hydrate by hydrolysis of its electrolytes. This evidently is not the case with the salts obtained from red blood cells.

- Neisser and Friedemann, Münch. med. Woch., 1904, No. 11. 
by studying the inhibiting effect of serum on the agglutination of corpuscles by suspensions.*

Once these colloids are removed we have a fluid (A) that allows the flocculation of $\mathrm{CaFl}_{2}$ and of ferric hydrate by the intracorpuscular salts present in this laked fluid. This flocculation, as we know, takes place. This fact, however, does not prevent it from agglutinating subsequently added corpuscles. These experiments show, as we believe, that the intracorpuscular salts extracted by laking do not antagonize the agglutination of fresh corpuscles by suspensions or colloids; we have just recently seen, moreover, that the stromata, deprived of intracorpuscular salts, are agglutinable by colloids and suspensions as well as are corpuscles.

As a result of this we conclude that in order to explain the agglutinating effect of these suspensions with which we have been dealing, on corpuscles, it is not necessary to suppose that a flocculation of them by means of the intracorpuscular salts takes place. We have indeed seen, first, that this agglutination is likewise possible by suspensions that have previously been flocculated by electrolytes; and second, that there is no change when the excess of salts in the pericorpuscular zone of the corpuscles is reduced to the minimum so as to annihilate the hypothetical flocculating effect of this zone on the suspension; and third, the phenomenon of the agglutination of stromata is identical with that of corpuscles; fourth, the sensitivity of these suspensions to the flocculating action of salts is not a necessary preliminary to their agglutinating property toward corpuscles. Inasmuch as all the phenomena of the agglutination of corpuscles by a suspension take place just as well with colloids, we think, whether or not these latter are flocculated by diffused intracorpuscular salts, that this has no effect on their agglutinating property over red blood cells. We have endeavored to prove this fact more directly. We have attempted to produce phenomena of agglutination analogous to those already described by allowing our suspensions to act upon an emulsion, the particles of which contain no salts that might be diffused, in place of red blood cells. For this purpose we have used emulsions of oil prepared by

* We have already noted in our first article that although serum prevents the agglutination of suspensions with corpuscles this inhibiting property may be removed by treating it previously with a large dose of $\mathrm{CaFl}_{2}$ or of barium sulphate. 
adding 5 drops of olive oil to 10 c.c. of distilled water plus 0.001 of a cubic centimeter of sodium carbonate. Such feebly alkaline emulsions may be neutralized or even acidified without showing any change. In our experiments we have used such emulsions neutralized as carefully as possible. Working with them we find that the tiny drops of oil agglutinate on the addition of suspensions of $\mathrm{CaFl}_{2}$ and barium sulphate and also of colloidal ferric hydrate. The flocks obtained in this manner are found microscopically to consist of a suspension of the hydrate with drops of oil scattered among them. It is found, moreover, that the addition of a small amount of serum prevents the agglutination of oil corpuscles by suspensions and by ferric hydrate in the same way as it does the agglutination of red blood cells by chemical precipitates.

Inasmuch as these two forms of agglutination apparently agree, we believe that their respective causes are due to the same factors and that the agglutination of red blood cells by suspensions and colloids is not an indirect result of the effect of electrolytes on these suspensions, but due to a direct effect of one element on the other.

We have just seen that certain suspensions agglutinate blood cells. If in place of the corpuscles we add serum to either of these two suspensions we find that $\mathrm{CaFl}_{2}$ becomes clumped, while barium sulphate is unaffected. Suspended in salt solution barium sulphate sediments rapidly to the bottom of the tube. In serum, on the contrary, it does not fall or falls only very slowly, and remains in the fluid in the form of a fine suspension. This dissociating effect of serum on barium sulphate is very marked and evident even on the addition of 1 drop of serum diluted 1-20 to 1 c.c. of 0.8 per cent salt solution containing 4 drops of an emulsion of barium sulphate.

We have then, on the one hand, an agglutination (barium sulphate plus corpuscles) and on the other hand quite a different phenomenon, namely, a dissociation of the suspension (barium sulphate plus serum). It is generally supposed that the albuminous substances of serum are in a state of colloidal solution. An emulsion of corpuscles, then, is the same as serum in that both are emul- 
sions of albuminous substances, but in the case of the corpuscles the particles are larger than in the case of serum. Both serum and corpuscles have the same negative electric charge.*

How is it, then, that the addition of a given substance-barium sulphate-produces such divergent effects in these two similar emulsions? Agglutination and dissociation would indeed seem to be the direct opposites of one another, but are they indeed as different as at first seems? Are they not rather two different results of a single fundamental phenomenon appearing either in the form of an agglutination or of a dissociation, as the case may be?

The agglutination of corpuscles with barium sulphate leads us to think of some adhesion between the suspension and the corpuscles. In the dissociation of barium sulphate by serum, is there not also an adhesion between the suspension and the albuminous particles of the serum? According to this explanation the fundamental phenomenon would be adhesion between the suspension and the corpuscles or the albuminous substances of serum; this adhesion, however, would be followed in the one case by agglutination and in the other by dissociation.

The following experiments seem to prove the accuracy of this hypothesis:

EXPERIMENT 1. If there is some adhesion between the albuminous particles of serum and barium sulphate, the dissociating property of the serum for the powder should have a definite limit. In order to demonstrate this we add a large amount of barium sulphate to a small amount of serum. We begin by centrifugalizing five tubes, each of which contains 2 c.c. of our emulsion of sulphate, and then throw off the supernatant fluids. One of these sediments is then suspended in the serum employed, namely 0.5 of a cubic centimeter of fresh horse serum diluted with 1.5 c.c. of salt solution. After allowing short contact the tube is then centrifugalized and the second original sediment of sulphate is suspended in the supernatant fluid of the first tube; this procedure is repeated with the third and fourth sediment and so on. We then add to the serum that has been treated in this manner a small amount of barium sulphate and find that this serum has little or no dissociating property for the suspension. Nor does it agglutinate $\mathrm{CaFl}_{2}$ or inhibit the agglutination of corpuscles by either $\mathrm{CaFl}_{2}$ or by barium sulphate. The colloidal solution, which constitutes the serum, loses its albuminous substance, then, as a result of contact with larger amounts of barium sulphate, just as an emulsion of red blood cells in agglutinating the sulphate solution loses its corpuscles.

* It is to be noted that although Mangin and Henri consider serum as a negative colloid, other writers, notably Neisser and Friedemann, are doubtful of this fact on account of Hardy's work.

Mme. Girard-Mangin et V. Henri., Soc. de Biol., 1904, No. 24.

Neisser et Friedemann, 1. c. 
EXPERIMENT 2 If the comparison is exact it must be true that dissociation of barium sulphate by serum is due to an adhesion of the colloidal substances of the serum similar to that which takes place between the corpuscles and sulphate in their agglutination. Repeated washings in salt solution of these masses formed by the adhesion of corpriscles and suspension lead to no change in the clumps. Shall we find in a similar way that barium sulphate that has been dissociated by serum will remain dissociated after washing, in other words, that the adhesion of the suspension to the dissociating colloids of the serum will persist? Twelve drops of our emulsion of barium sulphate are mixed with a large dose of horse serum, that is, with 3 c.c. of fresh serum diluted 1-4, and after 5 minutes the mixture is centrifugalized. The sediment is subsequently washed in salt solution until the wash water contains no trace of free serum, as evidenced by the fact that on boiling it does not become white and that it fails to dissociate fresh sulphate. We then suspend this sulphate treated by serum in salt solution and find that it remains in the form of an emulsion. This emulsion differs absolutely from fresh barium sulphate in salt solution; it is very colloidal in appearance and resembles milk; it finally clarifies, but without any evidence of an agglutination of particles, since a slight jar suffices to restore it to the form of an emulsion.

\section{This experiment is the complement of the preceding; serum} owes its dissociating property to colloidal substances that adhere to the barium sulphate, just as red blood cells adhere to it.

EXPERIMENT 3. We have endeavored to demonstrate more directly the union that occurs between the colloids of serum and the dissociated suspensions. As we shall see, we have succeeded in liberating or separating these colloids from the suspension to which they were attached. In order to do this it is necessary to eliminate the original suspension by dissolving it. For this purpose we have employed tricalcium phosphate as an emulsion in distilled water instead of barium sulphate, which is difficult to dissolve. This tricalcium phosphate is similar to barium sulphate in that it sediments rather rapidly in salt solution and distilled water, but remains for a long time as an emulsion in the presence of horse serum.

Three drops of our emulsion, for example, remain dissociated for a long time in 1 c.c. of serum diluted 1-10. We saturate a rather large dose of tricalcium phosphate, for example, 20 drops of our emulsion, with 4 c.c. of serum diluted 1-4 in salt solution, and after 15 minutes' contact centrifugalize the precipitate and wash repeatedly with salt solution until the wash water contains no free serum. The phosphate is then suspended in as much salt solution as the amount of serum employed in the first place and gives a homogeneous emulsion impregnated with serum as in the case of barium sulphate.

We place 1 c.c. of this emulsion in tube 1, and an equal amount of fresh phosphate in tube 2, a corresponding amount of fresh phosphate plus 1 c.c. of salt solution and a very small amount ( 0.025 c.c.) of serum in 1 c.c. of sall solution in tube 3 . We then add to each tube one drop of acetic acid. There is no change in tube 3 ; a certain amount of the phosphate in tubes 1 and 2 is dissolved and the rest remains suspended. Fiftcen minutes later these two tubes are centrifugalized and the supernatant fluid decanted. These fluids differ in this respect: The one is obtained by dissolving the fresh phosphate (tube 2), and the other by dissolving phosphate that has previously bcen impregnated with the dissociating substance of serum (tube 1). If we have succeeded in liberating the dissociating substances in tube 1 by dissolving the phosphate 
to which they were fixed, we should be able to demonstrate it by a dissociation of barium sulphate added to this fluid. This indeed proves to be true: barium sulphate added to these two fluids and also to tube 3 is found to sediment rapidly in tube 2 and to remain dissociated in tube 1 and in tube 3 . Control tubes show that neither the wash water nor the acetic acid have the slightest dissociating effect on barium sulphate. On choosing, therefore, a suspension that is dissociated by serum and easy to dissolve, the combination between the suspension and the colloidal substances that dissociated it may be broken up.*

These experiments show, it seems to us, that the phenomena of agglutination and dissociation, at least in the instances we have studied, although of different appearance, are nevertheless due to the same fundamental fact, namely, the affinity of the suspension for the corpuscles or the colloids of serum. Why are so widely different phenomena produced under such apparently closely related conditions? We cannot attempt to give the ultimate reason for this difference, but will simply offer an hypothesis that we have supported by a few facts. Since we know that an adhesion occurs in both instances between a suspension and the particles either of the corpuscle suspension or of the serum, the question arises if, when these adhesions are produced, the respective properties of the substances employed do not have some effect on the result of the reaction? As we know, barium sulphate will sediment by its own weight. If we add corpuscles which are particles that also tend to sediment, to barium sulphate, may we not consider the fact that the combination falls to the bottom of the tube as due to the tendency of barium sulphate to sediment, which tendency is only slightly modified by a contrary tendency on the part of the corpuscles? In a mixture of barium sulphate and serum, however, the sulphate meets with colloids which are very difficult to precipitate and the tendency of barium sulphate to sediment would be counterbalanced by a marked opposing tendency. If this opposing tendency prevails, a combination with barium sulphate would not sediment at all, but remain dissociated in the fluid, as indeed happens. According to this hypothesis the dissociation of barium sulphate by serum would be due to the result of a struggle

* It is to be noted that if hydrochloric acid is used in place of acetic acid and the phosphates thus totally dissolved, barium sulphate is not dissociated by the resultant fluid; it is also to be noted that barium sulphate sediments in serum to which the same amount of hydrochloric acid has been added. In other words, hydrochloric acid inhibits the dissociating effect of the serum. 
between opposing influences. And if this is true we should be able to modify the outcome of the struggle by enfeebling one or the other of the influences present; and this we have done in the following manner: It is well known that heating serum to 60 to 65 degrees brings it through a series of transitory stages to a dense coagulum. This coagulum may be avoided by a preliminary dilution of the serum, under which conditions the serum is still coagulable more or less completely, according to the degree of dilution, but never in a single mass. For example, if we leave horse serum, that has been diluted with three times its volume of salt solution, for a quarter of an hour in boiling water, we obtain a whitish fluid containing albuminoids coagulated to a greater or less extent, but not in a single mass; such a fluid is a true colloidal solution, but in comparison with unheated serum it represents a coagulated solution, the particles of which have a greater tendency to clump and sediment.

We now prepare two tubes which contain the same quantity of barium sulphate in the same volume of salt solution and add to one a given dose of non-heated serum diluted 1-4 in salt solution, and to the other the same amount of the same diluted serum that has been heated for 15 minutes in boiling water. The barium sulphate becomes dissociated in the first tube, but in the second forms clumps that are quite different from the spontaneous sedimentation in salt solution. After this agglutination is finished and the clumps have fallen to the bottom of the tube, the supernatant fluid has lost much of its milky appearance and the subsequent addition of a fresh amount of barium sulphate leads to only a slightly increased clarification in the fluid, the agglutinating property of which has become very much diminished. The agglutination of barium sulphate by heated serum is due, then, to a combination between the suspension and the colloidal substances which give the serum its milky appearance. Since this is true the agglutinating property may be removed from heated serum by adding sufficiently large amounts of barium sulphate. We collect at the bottom of the tube by centrifugalization the sulphate contained in 4 c.c. of our emulsion and remove the supernatant fluid and then suspend the sediment in a mixture containing 0.6 of a cubic centimeter of horse serum plus 1.8 c.c. of salt solution (the mixture 
having been heated for 15 minutes in boiling water). After a few minutes we again centrifugalize.

The supernatant fluid is absolutely limpid and has no effect on fresh barium sulphate and does not turn white on boiling.

To sum up, we have seen that the dissociation of barium sulphate by serum and its agglutination by red blood corpuscles is due fundamentally to the same phenomenon, namely, the adhesion of the sulphate with the particles of the serum or of the corpuscles, as the case may be. We have offered the hypothesis that the result of such a combination depends on the tendency of the particles united with barium sulphate to remain in suspension, and we have shown that by diminishing this tendency the property of the sulphate to sediment becomes preponderant and leads to an agglutination instead of a dissociation. This hypothesis is supported by the experiment to which reference has just been made.*

* Two objections may be raised which it may be well to meet before going farther. It might be possible that the substances causing agglutination or dissociation with barium sulphate, in accordance with whether the serum has or has not been heated, are not the same; and it might be supposed that heat has simply caused the agglutinating property to appear by destroying the dissociating property. Such, however, does not seem to be the case. We find that on treating a certain amount of fresh serum ( 0.5 c.c. plus 1.5 c.c. of salt solution) with large amounts of barium sulphate, we remove, as we have already mentioned, all the dissociating properties of the serum. If the diluted serum treated in this manner is then heated for one-quarter hour to 100 degrees with a tube of serum that has not been treated with barium sulphate, the latter becomes milky through coagulation of its albuminous substances, whereas the first tube shows no appreciable whitening. The addition of this latter treated serum to a small amount of barium sulphate produces little or no agglutination. By removing the dissociating substance from fresh serum by adding large doses of sulphate, we thus remove at the same time its property of agglutinating the sulphate after heating. This renders it very probable that the different actions on barium sulphate are due to the same substances.

The objection may also be raised that the dissociating property of serum is retained even after heating, but is masked by the agglutinating properties which this heating develops. This, however, does not seem to us to be correct. We have just seen that the agglutinating properties may be removed from heated serum by treating it with large doses of barium sulphate, and it may also be noted from the doses that we have given that this removal is much easier than the removal of the dissociating property from unheated serum. Thus we find that whereas 4 c.c. of our emulsion of sulphate suffices to remove the agglutinating property from 2.4 c.c. of serum diluted $1-4$ and heated to 100 degrees, 10 c.c. of the same emulsion suffices only to weaken the dissociating properties of 2 c.c. of the same serum diluted 1-4 and unheated. If the agglutinating and dissocia- 
If it be true that the agglutination of barium sulphate and heated serum depends on the diminution of the colloidal condition of the latter, we should expect to obtain different phenomena, in accordance with whether we add à large or small amount of heated serum to a given amount of suspension. We put the same amount of barium sulphate ( 4 drops) in two tubes and add to one 0.95 c.c. of salt solution plus 0.05 c.c. of heated serum and to the other 0.6 c.c. of salt solution plus 0.4 c.c. of the same serum. In the first tube, containing a small amount of serum, a fine clumping occurs, but the sulphate remains dissociated in the second tube. The loss of dissociating property of the serum by heat may be made up for, then, by adding a larger amount of the heated serum.

Not only will a large dose of heated serum dissociate fresh barium sulphate, but it will also restore to a fine suspension sulphate that has been previously agglutinated by a small dose of serum. We prepare two tubes containing each 0.95 of a cubic centimeter of salt solution and 0.05 c.c. of heated serum and 4 drops of our emulsion of sulphate; when agglutination has become complete, to one of the tubes we add 0.35 of a cubic centimeter of the same heated serum and to the other the same amount of salt solution, and shake. The agglutination persists in the second tube, but the suspension becomes dissociated in the first tube; the first tube then looks exactly as if 0.4 of a cubic centimeter of heated serum had been added in a single dose to 4 drops of sulphate.*

A large dose of heated diluted serum is necessary to dissociate barium sulphate. The serum, however, may be heated to 100 degrees in such a way as to preserve its dissociating property almost

ting properties of serum were due, then, to different substances, we should be able to remove the first by a small dose of barium sulphate and thus leave the second intact. We may then treat a given amount of heated serum with an amount of sulphate which is just sufficient to remove its cloudiness; in this way we remove all the agglutinating properties, but we find that no trace of dissociating property reappears.

We conclude, then, that the two different actions of serum on barium sulphate are due to the same substance which becomes changed by heat.

* There is an evident resemblance between this fact and the observation of Henri and Mayer, who found that the clumps resulting from an agglutination of two colloids of different charges become dissolved in an excess of one or the other of these colloids.

* Henri and Mayer, Soc. de Biol., 1904, No. 19. 
intact; if we dilute the serum, not with 3 volumes of salt solution, but with 3 volumes of distilled water and heat for 15 minutes to 100 degrees, the dissociating property of the serum for suspensions is almost intact. It should be noted, however, that whereas serum diluted in salt solution and heated, becomes white, serum diluted in distilled water scarcely changes in color, that is to say, the albuminous substances of the serum are very incompletely coagulated. This modification of the albuminous substances is indispensable, then, in order to bring about a preponderating influence of the barium sulphate over the tendency to remain in solution and so allow it to form clumps. If we heat serum diluted in salt solution for onequarter hour to different temperatures (55 degrees, 70 degrees, 85 degrees and 100 degrees), we find that an agglutination of barium sulphate is produced by serum heated to 85 or to 100 degrees, but that dissociation is produced by serum heated to 55 or to 70 degrees. It is to be noted that serum heated to 70 degrees has not whitened perceptibly, whereas serum heated to 85 degrees has become milky.

These facts all go to show that in a mixture of barium sulphate and serum either an agglutination ordissociation is produced, according to the more or less marked colloidal condition of the serum employed. These two very different phenomena, however, depend on the same fact. The adhesion of the colloids to the suspensions is just the same as the union between the suspension and blood corpuscles. The agglutination of corpuscles by chemical precipitates and the suspension of these precipitates in serum have one common explanation, although their appearance is quite different.

It would seem to us that the existence of an adhesion between serum and a suspension like barium sulphate explains the inhibiting effect that the serum plays in the agglutination of corpuscles by means of suspensions. This adhesion is the result, evidently, of an affinity between the suspension and serum, and we need only imagine that this affinity is stronger than the one between the corpuscles and the suspension. The suspension, then, would choose to unite with the serum rather than with the corpuscles.

Up to the present point we have simply referred to the experiments with suspensions, corpuscles and serum and suggested the conclusions drawn from the data obtained. We should like now to 
glance for a moment at the phenomena observed in the study of colloidal substances in the light of what we have learned from our study of the suspensions, even though such a consideration must be largely hypothetical. Henri, Lalou, Mayer, and Stodel found that, on mixing a stable negative colloid, that is to say, one that was little affected by electrolytes, with a colloid of the same charge that is unstable, that is to say, very susceptible to salts, there is no precipitation and the mixture becomes even more insusceptible to electrolytes than is the unstable colloid. In a precipitate caused by a flocculation from electrolytes both colloids are present. Unfortunately this fact gives no indication of the relation between the two colloids while in solution. One is tempted to suppose that the stable colloid protects the unstable colloid from the electrolytes owing to an adhesion between their particles. Such protection of an unstable colloid by a stable colloid evidently resembles the protection of barium sulphate against gravity by serum. We have already shown that this protection, which leads to a suspension of the sulphate, is due to an adhesion between this substance and the colloids of the serum. It would therefore seem to us not unreasonable to suppose that, in mixtures with the same electric charge, the protection of the unstable by the stable colloid is due to an adhesion between the particles of the two substances. There would be, then, an adhesion, as in mixtures of two colloids of opposite charges; but whereas, in this latter case, a flocculation occurs, no such result happens in mixtures of colloids with the same charge. An adhesion thus would seem to take place between colloids without any relation to their electric charge. Flocculation subsequently does or does not occur, according to the case under consideration. This flocculation, however, is not indispensable as a proof of adhesion between the two colloids and its absence cannot be interpreted as a certain indication of an absence of adhesion. The precipitation of these colloids would seem due to factors some of which are already known (electrolytes and contrary electric charges).

The existence of such an adhesion would readily explain a phenomenon that has been observed by Mangin and Henri which deals with the opposition to agglutination of corpuscles by means of colloids, both negative and positive, produced by small amounts 
of serum.* If we consider the serum as a negative colloid, it is easily understandable how it opposes the action of positive colloids on corpuscles; it flocks these colloids as any negative colloid flocks a positive one. This explanation, however, according to Mangin and Henri, is not applicable in the case of negative colloids, since the latter are not precipitated by serum. These authors have explained the inhibiting effect of serum on the agglutinating property of negative colloids as due to the opposition that the stable colloid has on the flocculation of unstable colloids of the same charge by means of electrolytes, which, in the case in point, are the intracorpuscular salts diffused in the fluid. As we have already seen, this interpretation is based on the explanation that they have given of the agglutination of red blood cells by means of colloids, with which we have already dealt.

If the hypothesis of adhesion between stable colloids (serum) and unstable colloids of the same electric charge is exact, it would suffice, in explaining the inhibition of agglutination of corpuscles with negative unstable colloids by serum, to consider that the affinity of the colloids for serum agrees with their affinity for corpuscles. The inhibition or agglutination of red blood cells by serum, and the flocculation of positive colloids by serum, and the non-flocculation of negative colloids and the dissociation of barium sulphate would all be due to the same cause.

\section{Conclusions.}

1. Certain chemical precipitates agglutinate and then hemolyze washed red blood cells.

2. This agglutination is due to the direct action of these precipitates and corpuscles upon one another.

3. It is probable that the agglutinating property of colloids for corpuscles is due fundamentally to a direct interaction of these two substances.

4. Serum even in small doses prevents the agglutination and hemolysis of corpuscles by precipitates.

* This is evidently analogous with the fact that we have referred to in our first article, namely, that serum inhibits the agglutination and hemolysis of red blood cells by suspensions. 
5. Fresh serum retains in suspension certain fine precipitates, such as the sulphate of barium.

6. This dissociation of barium by serum is due to an adhesion between this substance and the albuminous colloids of the serum, and is similar, therefore, to the agglutination of barium sulphate by corpuscles, in that they are both due to the adhesion of particles in suspension to the precipitate. The adhesion of the albuminous substance of serum with suspensions they have dissociated is easily demonstrable by the fact that suspensions dissociated by serum remain so when the excess of serum is removed and also by the fact that with certain precipitates the colloidal substances that have been attached to them and have kept them in suspension may be liberated by dissolving the precipitates (tricalcium phosphate).

7. The intensity of the tendency with which the particles attached to the suspension tend to remain in suspension plays a distinct rôle in the appearance of an agglutination or of a dissociation of the precipitate.

8. It is possible that, in a mixture of two colloids of the same electric sign, one stable and the other unstable, the protection of the first for the second against the flocculating action of electrolytes is due to a reciprocal adhesion of the particles of the colloids. 


\title{
XVI. OBSERVATIONS ON THE SINGLE NATURE OF HEMOLYTIC IMMUNE BODIES, AND ON THE EXIS- TENCE OF SO-CALLED "COMPLEMENTOIDS." *
}

\author{
BY FREDERICK P. GAY, M. D.
}

This article deals primarily with two hemolytic phenomena, by means of which two hypotheses have apparently been proved experimentally. Although I have dealt in each instance with the classical example only, my results are doubtless applicable to hemolysis in general.

\section{The Nature of a Hemolytic Immune Body as Regards its Reactivating Sera.}

As Bordet $\dagger$ was first to show, hemolysis of rabbit red blood corpuscles may be accomplished, in the presence of a suitable heated immune body (sensibilisatrice), by any one of several alexins, even by rabbit alexin; in this latter case, however, the amount of immune body necessary to produce hemolysis is sensibly increased over the amount necessary when guinea-pig alexin is used. Ehrlich and Morgenroth $\ddagger$ were able to corroborate this observation and also to add several analogous instances which show clearly that the relation of such immune body dosage is a variable one. The explanation which they offer, in harmony with their "Lateralchain theory," is briefly as follows: In a given case the amount of heated serum of a guinea-pig immunized against rabbit corpuscles necessary to hemolyze a given dose of rabbit washed corpuscles is ten times as great if rabbit "complement" (fresh serum) is used to reactivate, as when guinea-pig "complement" is used. This is due to the fact that a given immune serum contains a series of "partial immune bodies," all active against the specific corpuscles, but

* Centralbl. f. Bakt., etc., I. Abt. Originale. Bd. XXXIX, 1905, p. 172.

$\dagger$ Bordet, see p. 134.

¥ Ehrlich and Morgenroth, See Collected Studies on Immunity, EhrlichBolduan, John Wiley \& Sons, p. 67. 
having each an affinity for one certain "complement." By natural law, only the smallest number of such bodies in serum would have "complementophilic" arms suitable to join with the proper "complement" of the corpuscles to be destroyed. In the given case a hemolytic dose of the guinea-pig > rabbit immune serum with guinea-pig "complement" may be represented by the formula $1 \mathrm{~A}$ $+\frac{1}{10} \mathrm{~B}$, "A" being partial immune bodies joining only with the guinea-pig complement, " $\mathrm{B}$ " being partial immune bodies joining only with the rabbit complement. In order to obtain hemolysis with rabbit complement it is necessary, logically, to have ten such doses (i.e., $10 \mathrm{~A}+1 \mathrm{~B}$ ) in order to have one whole dose of "B." Ehrlich and Morgenroth* have sought further to establish this hypothesis of the multiplicity of immune bodies in a given immune serum by means of certain experiments with anti-immune bodies - a subject which we need not here consider.

After my first results on the subject I found that this interesting question had already been considered in part by Muir and Browning, $\dagger$ who have come to practically the same conclusions as myself, although they were unable wholly to refute the hypothesis of the multiplicity of immune bodies on account of certain technical difficulties; these difficulties I have been able to obviate by a specially devised method.

Although nowhere expressly stated by Bordet or by Ehrlich and Morgenroth, I have found, in common with Muir and Browning, that, when we reactivate sensitized rabbit corpuscles (heated guineapig > rabbit immune serum) with rabbit alexin, not only is the requisite dose of immune body large, but also the dose of rabbit alexin relatively high. The relative amounts of the immune serum necessary in the presence of sufficient alexin of either animal, and the relative amounts of each alexin when sufficient immune body is present are so well shown in the tables of Muir and Browning that I omit similar examples. As regards the relatively high dose of rabbit alexin against its own corpuscles the following (Table I) shows that it takes nearly three times as much alexin to destroy numerically a smaller number of its own sensitized corpuscles than of sensitized ox corpuscles.

* Ehrlich and Morgenroth, See Collected Studies on Immunity, EhrlichBolduan, John Wiley \& Sons, p. 101.

$\dagger$ Muir and Browning, Proceed. Royal. Soc., Vol. LXXIV, 1904, p. 298. 


\section{TABLE I.}

Red blood corpuscles of ox per c.mm., 5,880,000. Red blood corpuscles of rabbit per c.mm., $4,240,000$.

\begin{tabular}{|c|c|c|c|c|}
\hline No. & Washed rabbit cor- & S. guinea-pig > rabbit, & Rabbit alexin. & Hemolysis. \\
\hline \multirow[t]{3}{*}{$\begin{array}{l}1 \\
2 \\
3 \\
4 \\
5\end{array}$} & $\begin{array}{l}0.025 \text { c.c. } \\
0.025 \text { c.c. } \\
0.025 \text { c.c. } \\
0.025 \text { c.c. } \\
0.025 \text { c.c. }\end{array}$ & $\begin{array}{l}0.25 \text { c.c. } \\
0.25 \text { c.c. } \\
0.25 \text { c.c. } \\
0.25 \text { c.c. } \\
0.25 \text { c.c. }\end{array}$ & $\begin{array}{l}0.2 \text { c.c. } \\
0.15 \text { c.c. } \\
0.1 \text { c.c. } \\
0.075 \text { c.c. } \\
0.05 \text { c.c. }\end{array}$ & $\begin{array}{l}\text { complete } \\
\text { partial } \\
\text { slight } \\
\text { slight } \\
\text { slight }\end{array}$ \\
\hline & Washed ox corpuscles. & S. rabbit $>$ ox, $55^{\circ}$. & & \\
\hline & $\begin{array}{l}0.025 \text { c.c. } \\
0.025 \text { c.c. }\end{array}$ & $\begin{array}{l}0.15 \text { с с } \\
0.15 \text { c.c. }\end{array}$ & $\begin{array}{l}0.075 \text { c.c. } \\
0.05 \text { c.c. }\end{array}$ & $\begin{array}{l}\text { complete } \\
\text { slight }\end{array}$ \\
\hline
\end{tabular}

As regards the technic of hemolytic experiments, it may be well to note the rôle played by an excessive amount of isotonic salt solution in the suspensions of test corpuscles employed by many observers. It would seem, a priori, desirable to deal with corpuscles as nearly as possible in their normal condition of suspension in the blood, but the advantages of a 5 per cent suspension are manifest, since smaller amounts can be accurately measured and a great waste of often precious materials obviated. But I have noted repeatedly that the dilution of a small amount of alexic serum by so great an excess of isotonic solution unquestionably affects its activity and often completely inhibits it. This is well shown in the case of sensitized rabbit corpuscles when acted on by rabbit alexin.

TABLE II.

\begin{tabular}{|c|c|c|c|c|}
\hline \multicolumn{2}{|c|}{ Washed rabbit corpuscles. } & \multirow{2}{*}{$\begin{array}{l}\text { S. guinea-pig > } \\
\text { rabbit, } 55^{\circ} \text {. }\end{array}$} & \multirow{2}{*}{ Rabbit alexin. } & \multirow{2}{*}{ Hemolysis. } \\
\hline $\begin{array}{l}5 \% \text { suspension in } \\
\mathrm{NaCl}(0.85 \%)\end{array}$ & $\begin{array}{c}\text { Centrifugalized } \\
\text { deposit of } 5 \% \text { sus- } \\
\text { pension. }\end{array}$ & & & \\
\hline $\begin{array}{l}2 \text { c.c. } \\
2 \text { c.c. } \\
\ldots \ldots\end{array}$ & $\begin{array}{l}\cdots \cdots \\
=10 \mathrm{c} . \mathrm{c} . \\
\cdots \cdots \\
=\frac{1}{10} \mathrm{c} \cdot \mathrm{c} .\end{array}$ & $\begin{array}{l}0.4 \text { c.c. } \\
0.4 \text { c.c. } \\
0.4 \text { c.c. } \\
0.4 \text { c. c. }\end{array}$ & $\begin{array}{l}0.4 \text { c.c. } \\
0.4 \text { c.c. } \\
1.2 \text { c.c. } \\
1.2 \text { c.c. }\end{array}$ & $\begin{array}{l}\text { none } \\
\text { partial } \\
\text { slight } \\
\text { complete }\end{array}$ \\
\hline
\end{tabular}


A similar and yet more striking example of the effect of a great excess of isotonic solution will be given later. To retain the usefulness of the 5 per cent suspension and to obviate its errors, I have employed this method of centrifugalizing the suspension in the actual experimental tube, removing the supernatant fluid, and subsequently adding the small amount of physiological solution necessary to bring the blood suspension to its primitive volume.

Another source of possible error in experiments of this nature is the presence of anti-alexic action in the excess of heated immune serum, which, in rare instances, inhibits the activity of the proper alexin of the corpuscles. The occurrence of such an action is not surprising when we consider that the usual procedure for the production of an immune serum is to immunize animals with the whole defibrinated blood, that is, with corpuscles plus serum. The remedy, of course, consists in removing the excess of immune serum after it has been allowed to come in contact with the corpuscles.

Although the amount of rabbit alexin necessary to hemolyze sensitized rabbit corpuscles is relatively large, it is easy to show that smaller doses combine perfectly with the corpuscle-immune body complex. As Muir expresses it, the combining power is perfect although the toxic power is slight. Such variations in toxicity were previously noted by Bordet* in consonance with his quantitative idea of the difference in alexins.

If we take Table I, for example, and add to each of the supernatant fluids of tubes 1 to 5 , in which more or less hemolysis has occurred, sensitized ox corpuscles (ox corpuscles, 0.025 of a cubic centimeter + S. rabbit $>$ ox, 55 degrees, 0.05 of a cubic centimeter), we obtain no trace of hemolysis in any tube, which shows that the rabbit alexin has already combined perfectly with the sensitized rabbit corpuscles.

Before proceeding to the proof of the simple nature of the guineapig-rabbit immune body, it will be necessary to describe an experiment which offers a valuable technical method for the solution of this problem, and which may also prove of service in other hemolytic experiments. It is already known that when corpuscles have been hemolyzed by means of a heated specific serum, and a dose of alexin, which is not in excess, added, the resulting stromata have

* Bordet, see p. 234. 
still the ability to absorb additional portions of alexin. I have found also that stromata produced by heating corpuscles to 55 degrees for half an hour retain unimpaired their power of absorbing immune body, or, if previously sensitized, of absorbing alexin. Rabbit corpuscles are completely hemolyzed by this temperature, but ox corpuscles, although showing a reduction of hemoglobin, remain, for the most part, microscopically intact. The affinities of ox corpuscles after heating to 55 degrees are shown by the following experiment:

\section{THREE TUBES ARE PREPARED AS FOLLOWS:}

Tube A. Ox corpuscles previously heated to 55 degrees (not hemolyzed)

0.2 c.c.

Serum rabbit $>$ ox, 55 degrees $\quad 0.8$ c.c.

S. guinea-pig (alexin) 0.5 c.c.

Tube B. Ox corpuscles, 55 degrees

0.2 c.c.

S. normal rabbit, 55 degrees

S. guinea-pig

0.8 c.c.

0.5 c.c.

Tube C. Ox corpuscles, $0.2+$ Serum rabbit $>$ ox, 0.8 , heated to 55 degrees

S. guinea-pig

0.5 c.c.

In tubes $\mathrm{A}$ and $\mathrm{C}$ hemolysis is complete; in $\mathrm{B}$ nul.

To the centrifugalized supernatant fluids of $\mathrm{A}, \mathrm{B}$ and $\mathrm{C}$ is added subsequently

Ox corpuscles $\quad 0.1$ c.c.

S. rabbit $>$ ox, 55 degrees 0.4 c.c.

Tube D (control). Ox corpuscles 0.1 c.c.

S. rabbit $>$ ox, 55 degrees $\quad 0.4$ c.c.

S. guinea-pig 0.2 c.c.

In tubes $\mathrm{B}$ and $\mathrm{D}$ hemolysis is complete.

In tubes $\mathrm{A}$ and $\mathrm{C}$ hemolysis nul.

Similar results may be obtained with rabbit corpuscles with the exception that they are hemolyzed by heating to 55 degrees.

In considering the nature of the guinea-pig $>$ rabbit immune body I shall give an example in which the relative doses of the immune body and the alexin of rabbit or of guinea-pig chanced to be the same as in the classical example of Ehrlich. Of course it is understood that this relation is not a fixed one, as. Ehrlich has shown. In this case, in order to produce hemolysis of rabbit corpuscles in the presence of sufficient rabbit alexin, it took ten times the amount of heated immune serum as when using guinea-pig alexin. In the language of the Ehrlich hypothesis, the necessary dose of immune serum with rabbit alexin may be represented by 10 " $\mathrm{A}$ " +1 "B," " $\mathrm{A}$ " being the partial immune body suitable fơr guinea-pig alexin 
and " $\mathrm{B}$ " the partial immune body for rabbit alexin. Rabbit alexin cannot join "A" or hemolysis would be produced with the same dose of immune body as when employing guinea-pig alexin. Now, if we produce hemolysis with rabbit alexin in the presence of the necessary amount of immune serum, the 1 " $\mathrm{B}$ " will be satisfied, that is, one-eleventh only of the amboceptors will have their "complementophilic" arms plugged, while 10 "A," or the remaining tenelevenths, will be attached to the stromata and be still ready to fix many hemolytic doses of guinea-pig alexin. This is the logic of the Ehrlich hypothesis. Experimentally we obtain the following results:

Rabbit corpuscles are hemolyzed with rabbit alexin and guineapig > rabbit heated immune serum; the stromata are then heated to 55 degrees, which will destroy any rabbit alexin that may be free, or prevent any further toxic action from it, but will leave the corpuscle-immune-body complex still unaffected as regards its further absorption of the guinea-pig alexin by its supposedly unsatisfied 10 " $\mathrm{A}$ " amboceptors. In fact, we find that the alexin of the guinea-pig subsequently added remains entirely free; not one slight portion of it has been attached, as is shown by suitable controls. In other words, the fixation of rabbit alexin will completely prevent the subsequent fixation of guinea-pig alexin. The details of the experiment follow.

Minimal hemolytic dose of guinea-pig > rabbit immune serum, 55 degrees, for 0.025 of a cubic centimeter of washed rabbit corpuseles.

with rabbit alexin.

0.25 c.c.

with guinea-pig alexin

0.025 c.c.

Relation $10: 1$.

\section{THREE LARGE TUBES ARE PREPARED:}

Tube A. Washed rabbit corpuscles

S. guinea-pig $>$ rabbit, 55 degrees

S. rabbit (alexin)

Tube B. Washed rabbit corpuscles

S. guinea-pig $>$ rabbit, 55 degrees

S. rabbit, 55 degrees

0.125 c.c.

1.25 c.c.

1.25 c.c.

0.125 c.c.

1.25 c.c.

1.25 c.c.

Tube C. Washed rabbit corpuscles

0.125 c.c.

S. normal guinea-pig, 55 degrees

S. rabbit, 55 degrees

Contact at $37^{\circ} \mathrm{C}$ for 1 hour.

1.25 c.c.

Tube A is perfectly hemolyzed.

Tube B, agglutination; no hemolysis.

Tube $\mathrm{C}$, no change. 
The contents of the three tubes are then heated to 55 degrees for one-half an hour. Hemolysis complete in all tubes. From each of the tubes $\mathrm{A}, \mathrm{B}$ and $\mathrm{C}$ are prepared three smaller tubes, each containing 0.5 of a cubic centimeter of the stroma mixture, that is, the stromata of 0.025 of a cubic centimeter of rabbit corpuscles. To the corresponding tubes in each series is added the same amount of fresh guinea-pig alexin, as follows:

\begin{tabular}{|c|c|c|c|}
\hline \multirow[t]{4}{*}{ "A" series. } & Tube 1. & "A" mixture & 0.5 c.c. \\
\hline & & S. guinea-pig & 0.025 c.c. \\
\hline & & S. guinea-pig & 0.05 c.c. \\
\hline & Tube 3. & "A" mixture & $\begin{array}{l}0.5 \text { c.c. } \\
0.1 \text { c.c. }\end{array}$ \\
\hline \multirow[t]{4}{*}{ "B" series. } & Tube 4. & " $\mathrm{B}$ " mixture & 0.5 c.c. \\
\hline & & $\begin{array}{l}\text { S. guinea-pig } \\
\text { "B" mixture }\end{array}$ & $\begin{array}{l}0.025 \text { c.c. } \\
0.5 \text { c.c. }\end{array}$ \\
\hline & & S. guinea-pig & 0.05 c.c. \\
\hline & Tube 6. & "B" mixture & 0.5 c.c. \\
\hline \multirow[t]{4}{*}{ “C" series. } & Tube 7. & "C" mixture & 0.5 c.c. \\
\hline & & S. guinea-pig & 0.025 c.c. \\
\hline & Tube 8 & $\begin{array}{l}\text { "C" mixture } \\
\text { S. guinea-pig }\end{array}$ & $\begin{array}{l}0.5 \text { c.c. } \\
0.05 \text { c.c. }\end{array}$ \\
\hline & Tub & $\begin{array}{l}\text { "C" mixture } \\
\text { S. guinea-pig }\end{array}$ & $\begin{array}{l}0.5 \text { c.c. } \\
0.1 \text { c.c. }\end{array}$ \\
\hline
\end{tabular}

Contact at $37^{\circ} \mathrm{C}$ for 1 hour. Tubes centrifugalized. The supernatant fluid of each tube is placed in a new tube containing:
Washed rabbit corpuscles
0.025 c.c.
S. guinea-pig $>$ rabbit, 55 degrees
0.05 c.c.

Resultant hemolysis is as follows:
"A" series
"B" series
"C" series
1: Marked
4. None
7. Marked
2. Complete
5. None
8. Complete
3. Complete
6. None
9. Complete

In series " $A$ " the guinea-pig alexin is left entirely free because the avidity of the corpuscle-immune-body complex for alexin has been entirely satisfied by the rabbit alexin. In series " $B$ " the sensitized corpuscles, subjected to the same conditions of heat and serum dilution as series "A," absorb perfectly the guinea-pig alexin. In series " $\mathrm{C}$ " we have a control, with no immune serum, which leaves just as much but no more guinea-pig alexin free than does series "A." The certain conclusion from this experiment is that the immune body against rabbit corpuscles is of a simple nature as regards combination with one or another alexin. 


\section{The Nature of a So-Called "Complementoid."}

In his analogy between antitoxic and hemolytic sera Ehrlich presupposed the existence of substances called "complementoids" -derived from "complements," as "toxoids" are supposed to be derived from toxins. These "complementoids" are depicted as possessing a "haptophore group" but no "zymotoxic" group, in distinction from complements, which have both. That is, they have none of the toxic or hemolytic activity of complements, but may give rise, when injected, to "anticomplements," and may, under certain conditions, combine with amboceptors. After the hypothesis followed apparent experimental proof of the existence of such bodies. Ehrlich and Sachs* described a phenomenon which occurs in dog serum which they call "Verstopfung der Ambozeptoren durch Komplementoïde," - the plugging of amboceptors by means of complementoids. The details are briefly as follows: Fresh dog serum hemolyzes guinea-pig corpuscles. Dog serum heated to $51^{\circ} \mathrm{C}$ for one-half hour loses its power to produce this hemolysis owing to the destruction at this temperature of the very labile complement. This heated serum (Ambozeptor) may be reactivated by guinea-pig "complement." If, however, the corpuscles are left in contact with the heated $\operatorname{dog}$ serum at $37^{\circ} \mathrm{C}$ for 2 hours the subsequent addition of fresh guinea-pig serum produces no hemolysis. $\dagger$ This fact is ascribed to a plugging of the "complementophilic arm" of the dog amboceptor by means of the "complementoids" present in the serum heated to 51 degrees. The amboceptor is said to be destroyed by a temperature of 60 degrees; the somewhat unusual nature of this amboceptor is fully discussed in another place by Sachs, $\ddagger$ but need not concern us here.

* Ehrlich and Sachs, See Colleeted Studies on Immunity, Ehrlich-Bolduan, John Wiley \& Sons, p. 209.

$\dagger$ Although experimental proof that the subsequently added alexin has not been combined is not offered by the authors, such a matter is easy to determine. If we centrifugalize such a tube and to the supernatant fluid add heated dog serum, and then corpuscles; hemolysis is produced, showing that the alexin has really been kept out of combination by the heated dog serum united to the corpuscles. The control is a tube in which hemolysis has occurred by the addition of dog serum and alexin at the same time, in which case no subsequent alexic activity is detectable.

$\ddagger$ Sachs, H., See Colleeted Studies on Immunity, Ehrlich-Bolduan, John Wiley \& Sons, idem, p. 186. 
In dealing with this phenomenon, I have found the results as given do not express the entire truth as I have found it. The doses given in the "plugging" experiment described, are as follows:

Guinea-pig washed corpuscles, 5 per cent suspension

Dog serum, 50 to 51 degrees

Guinea-pig serum
1 c.c.

$0.5 \mathrm{c} \mathrm{c}$.

0.5 c.c.

which doses I shall use in the experiments to be described. I have found, as a matter of fact, that dog serum heated to the degree mentioned does always give a slight hemolysis of guinea-pig corpuscles, a fact which is masked by the excess of salt solution used in the 5 per cent suspension. If we deal with the centrifugalized deposit of 1 c.c. of a 5 per cent suspension, or simply with 0.05 of a cubic centimeter of washed corpuscles, this hemolysis is clearly shown. That dog serum heated for one-half hour to 50 degrees, 51 degrees or 52 degrees dóes possess distinct hemolytic power for guinea-pig corpuscles may be shown more distinctly in the following way: If we prepare a series of tubes each containing 1 c.c. of suspensions of corpuscles of 5 per cent, 3 per cent, 1 per cent and one-half per cent respectively, and to each tube add the given dose of heated dog serum ( 0.5 of a cubic centimeter, 50 to 51 degrees), we find after 2 hours at $37^{\circ} \mathrm{C}$ that in the tubes at 1 per cent and one-half per cent hemolysis is complete; or, better still, if we use the centrifugalized deposits of such a series of suspensions without the excess of salt solution, we find that hemolysis is complete in the tubes at 3 per cent, 1 per cent and one-half per cent and marked in the 5 per cent tube. It is evident from such an experiment that the so-called "complementoid" is nothing more than a "complement" the hemolytic activity of which has been impaired, but not destroyed, by the heating. That the subsequent addition of guinea-pig serum does not sensibly increase the hemolysis in such a series of tubes is perfectly true, which means that there is a "plugging" (Verstopfung) by means of the partially destroyed "complement." The combining power of the "complement" has been no more destroyed than the toxic power. If, instead of dog serum heated to 51 degrees, we use dog serum heated to 56 degrees, we find another more striking example of this modified "complement." To a similar series of tubes at 5 per cent, 3 per cent, 1 per cent and one-half per cent respectively add 0.5 of a cubic centimeter of dog serum heated to 56 degrees for 
one-half hour. After 3 hours at $37^{\circ} \mathrm{C}$ slight hemolysis is present only in the tube at one-half per cent (complete hemolysis if the deposit of such a suspension is used), which shows that not even at this temperature is the toxic action of the "complement" entirely destroyed. If we add 0.5 of a cubic centimeter of fresh guinea-pig serum to an analogous series of tubes, it is found that hemolysis is all but complete throughout the series, that is, only the slightest "plugging" has occurred. The combining ability of the dog complement is impaired equally with and proportionately to the toxic ability.

The tables follow:

TABLE I.

\begin{tabular}{|c|c|c|c|c|c|}
\hline $\begin{array}{l}\text { Washed } \\
\text { guinea-pig } \\
\text { corpuscles in } \\
\text { suspension of }\end{array}$ & $\begin{array}{l}\text { Dog serum, } \\
50-51^{\circ} \mathrm{C} \text {. }\end{array}$ & Contact, $37^{\circ}$. & $\mathrm{NaCl}$ sol. & Contact, $37^{\circ}$. & Hemolysis. \\
\hline $\begin{array}{r}5 \% 1 \text { c.c. } \\
3 \% 11 \text { c.c. } \\
1 \% 1 \text { c.c. } \\
0.5 \% 1 \text { c.c. }\end{array}$ & $\begin{array}{l}0.5 \text { c.c. } \\
0.5 \text { c.c. } \\
0.5 \text { c.c. } \\
0.5 \text { c.c. }\end{array}$ & $\begin{array}{l}2 \text { hours } \\
2 \text { hours } \\
2 \text { hours } \\
2 \text { hours }\end{array}$ & $\begin{array}{l}0.5 \text { c.c. } \\
0.5 \text { c.c. } \\
0.5 \text { c.c. } \\
0.5 \text { c.c. }\end{array}$ & $\begin{array}{l}1 \text { hour } \\
1 \text { hour } \\
1 \text { hour } \\
1 \text { hour }\end{array}$ & $\begin{array}{l}\text { trace } \\
\text { marked } \\
\text { complete } \\
\text { complete }\end{array}$ \\
\hline
\end{tabular}

TABLE II.

\begin{tabular}{|c|c|c|c|c|c|}
\hline $\begin{array}{l}\text { Deposit of } 1 \text { c.c. } \\
\text { G. p. corpuscles; } \\
\text { suspension of }\end{array}$ & $\begin{array}{l}\text { Dog serum, } \\
50-51^{\circ} \mathrm{C} .\end{array}$ & Contact, $37^{\circ}$. & $\mathrm{NaCl}$ sol. & Contact, $37^{\circ}$. & Hemolysis. \\
\hline $5 \%(.05$ c.c. $*)$ & 0.5 c.c. & 2 hours & 0.5 c.c. & 1 hour & $\begin{array}{l}\text { nearly com- } \\
\text { plete }\end{array}$ \\
\hline $\begin{array}{c}3 \% \text { (.05 c.c. }) \\
1 \%(.05 \text { c.c. }) \\
0.5 \% \text { (.05 cc. })\end{array}$ & $\begin{array}{l}0.5 \text { c.c. } \\
0.5 \text { c.c. } \\
0.5 \text { c.c. }\end{array}$ & $\begin{array}{l}2 \text { hours } \\
2 \text { hours } \\
2 \text { hours }\end{array}$ & $\begin{array}{l}0.5 \text { c.c. } \\
0.5 \text { c.c. } \\
0.5 \text { c.c. }\end{array}$ & $\begin{array}{l}1 \text { hour } \\
1 \text { hour } \\
1 \text { hour }\end{array}$ & $\begin{array}{l}\text { complete } \\
\text { complete } \\
\text { complete }\end{array}$ \\
\hline
\end{tabular}

* Represents the actual volume present.

TABLE III.

\begin{tabular}{|c|c|c|c|c|c|}
\hline $\begin{array}{l}\text { Washed } \\
\text { guinea-pig } \\
\text { corpuscles in } \\
\text { suspension of }\end{array}$ & $\begin{array}{l}\text { Dog serum, } \\
50-51^{\circ} \mathrm{C}\end{array}$ & Contact, $37^{\circ}$. & $\begin{array}{l}\text { Alexin guinea- } \\
\text { pig. }\end{array}$ & Contact, $37^{\circ}$. & Hemolysis. \\
\hline $\begin{array}{c}5 \% \\
3 \% \\
3 \% \text { c c c } \\
1 \% 1 \text { c c. } \\
0.5 \% 1 \text { c c. }\end{array}$ & $\begin{array}{l}0.5 \text { c.c } \\
0.5 \text { c.c. } \\
0.5 \text { c.c } \\
0.5 \text { c.c }\end{array}$ & $\begin{array}{l}2 \text { hours } \\
2 \text { hours } \\
2 \text { hours } \\
2 \text { hours }\end{array}$ & $\begin{array}{l}0.5 \text { c c. } \\
0.5 \text { c c. } \\
0.5 \text { c c } \\
0.5 \text { c c }\end{array}$ & $\begin{array}{l}1 \text { hour } \\
1 \text { hour } \\
1 \text { hour } \\
1 \text { hour }\end{array}$ & $\begin{array}{l}\text { trace } \\
\text { marked } \\
\text { complete } \\
\text { complete }\end{array}$ \\
\hline
\end{tabular}


TABLE IV.

\begin{tabular}{|c|c|c|c|c|c|}
\hline $\begin{array}{l}\text { Deposit of } 1 \\
\text { c.c. G. p. } \\
\text { corpuscles; } \\
\text { suspension of }\end{array}$ & $\begin{array}{l}\text { Dog serum, } \\
50-51^{\circ} \mathrm{C} .\end{array}$ & Contact, $37^{\circ}$. & $\begin{array}{l}\text { Alexin guinea- } \\
\text { pig. }\end{array}$ & Contact, $37^{\circ}$. & Hemolysis. \\
\hline $5 \%(.05$ c.c. $)$ & 0.5 c.c & 2 hours & $0.5 \mathrm{c} \mathrm{c}$. & 1 hour & $\begin{array}{l}\text { nearly com- } \\
\text { plete }\end{array}$ \\
\hline $\begin{array}{r}3 \% \text { (.05 c.c. }) \\
1 \%(.05 \text { c.c }) \\
0.5 \%(.05 \text { c.c. })\end{array}$ & $\begin{array}{l}0.5 \text { с с. } \\
0.5 \text { с с } \\
0.5 \text { с с } .\end{array}$ & $\begin{array}{l}2 \text { hours } \\
2 \text { hours } \\
2 \text { hours }\end{array}$ & $\begin{array}{l}0.5 \text { c.c. } \\
0.5 \text { c.c } \\
0.5 \text { c.c. }\end{array}$ & $\begin{array}{l}1 \text { hour } \\
1 \text { hour } \\
1 \text { hour }\end{array}$ & $\begin{array}{l}\text { complete } \\
\text { complete } \\
\text { coinplete }\end{array}$ \\
\hline
\end{tabular}

TABLE $Y$.

\begin{tabular}{|c|c|c|c|c|c|}
\hline $\begin{array}{l}\text { Washed } \\
\text { guinea-pig } \\
\text { corpuscles in } \\
\text { suspension of }\end{array}$ & $\begin{array}{c}\text { Dog serum, } \\
56^{\circ} \mathrm{C} \cdot\end{array}$ & Contact, $37^{\circ}$. & $\mathrm{NaCl}$ sol. & Contact, $37^{\circ}$. & Hemolysis. \\
\hline $\begin{array}{r}5 \% 11 \text { c.c. } \\
3 \% 11 \text { c.c. } \\
1 \% 11 \text { c.c. } \\
0.5 \% 11 \text { c.c. }\end{array}$ & $\begin{array}{l}0.5 \text { c.c. } \\
0.5 \text { c.c. } \\
0.5 \text { c c. } \\
0.5 \text { c.c. }\end{array}$ & $\begin{array}{l}2 \text { hours } \\
2 \text { hours } \\
2 \text { hours } \\
2 \text { hours }\end{array}$ & $\begin{array}{l}0.5 \text { c.c. } \\
0.5 \text { c.c. } \\
0.5 \text { c.c. } \\
0.5 \text { c.c. }\end{array}$ & $\begin{array}{l}1 \text { hour } \\
1 \text { hour } \\
1 \text { hour } \\
1 \text { hour }\end{array}$ & $\begin{array}{l}\text { none } \\
\text { none } \\
\text { none } \\
\text { marked }\end{array}$ \\
\hline
\end{tabular}

TABLE VI.

\begin{tabular}{|c|c|c|c|c|c|}
\hline $\begin{array}{l}\text { Deposit of } 1 \\
\text { c.c. G. p. cor- } \\
\text { puscles : sus- } \\
\text { pension of }\end{array}$ & $\begin{array}{l}\text { Dog serum, } \\
56^{\circ} \mathrm{C} .\end{array}$ & Contact, $37^{\circ}$. & NaCl sol. & Contact, $37^{\circ}$. & Hemolysis. \\
\hline $\begin{array}{r}5 \% \text { (.05 c.c. }) \\
3 \% \text { (.05 c.c.) } \\
1 \% \text { (.05 c.c.) } \\
0.5 \% \text { (.05 c.c. })\end{array}$ & $\begin{array}{l}0.5 \text { c.c. } \\
0.5 \text { c.c. } \\
0.5 \text { c.c. } \\
0.5 \text { c.c. }\end{array}$ & $\begin{array}{l}2 \text { hours } \\
2 \text { hours } \\
2 \text { hours } \\
2 \text { hours }\end{array}$ & $\begin{array}{l}0.5 \text { c.c. } \\
0.5 \text { c.c. } \\
0.5 \text { c.c } \\
0.5 \text { c.c. }\end{array}$ & $\begin{array}{l}1 \text { hour } \\
1 \text { hour } \\
1 \text { hour } \\
1 \text { hour }\end{array}$ & $\begin{array}{l}\text { none } \\
\text { none } \\
\text { slight } \\
\text { complete }\end{array}$ \\
\hline
\end{tabular}

TABLE VII

\begin{tabular}{|c|c|c|c|c|c|}
\hline $\begin{array}{l}\text { Washed } \\
\text { guinea-pig } \\
\text { corpuscles in } \\
\text { suspension of }\end{array}$ & $\begin{array}{c}\text { Dog serum, } \\
56^{\circ} \mathrm{C}\end{array}$ & Contact, $37^{\circ}$. & $\begin{array}{c}\text { Alexin guinea- } \\
\text { pig. }\end{array}$ & Contact, $37^{\circ}$. & Hemolysis. \\
\hline $5 \% 1$ c.c. & $0.5 \mathrm{c} \mathrm{c}$ & 2 hours & $0.5 \mathrm{c} \mathrm{c}$ & 1 hour & $\begin{array}{l}\text { nearly com- } \\
\text { plete }\end{array}$ \\
\hline $\begin{array}{r}3 \% 11 \text { c.c. } \\
1 \% 1 \text { c.c. } \\
0.5 \% 1 \text { c.c. }\end{array}$ & $\begin{array}{l}0.5 \text { c.c. } \\
0.5 \text { c.c } \\
0.5 \text { c.c. }\end{array}$ & $\begin{array}{l}2 \text { hours } \\
2 \text { hours } \\
2 \text { hours }\end{array}$ & $\begin{array}{l}0.5 \text { c c. } \\
0.5 \text { c.c. } \\
0.5 \text { c.c. }\end{array}$ & $\begin{array}{l}1 \text { hour } \\
1 \text { hour } \\
1 \text { hour }\end{array}$ & $\begin{array}{l}\text { complete } \\
\text { complete } \\
\text { complete }\end{array}$ \\
\hline
\end{tabular}


TABLE VIII

\begin{tabular}{|c|c|c|c|c|c|}
\hline $\begin{array}{l}\text { Deposit of } 1 \\
\text { c.c. corpus- } \\
\text { cles; suspen- } \\
\text { sion of }\end{array}$ & $\begin{array}{l}\text { Dog serum, } \\
56^{\circ} \mathrm{C} \text {. }\end{array}$ & Contact, $37^{\circ}$. & $\begin{array}{c}\text { Alexin guinea- } \\
\text { pig. }\end{array}$ & Contact, $37^{\circ}$. & Hemolysis. \\
\hline $5 \%(05 \mathrm{c} \mathrm{c})$ & $0.5 \mathrm{ccc}$ & 2 hcurs & $0.5 \mathrm{c} \mathrm{c}$. & 1 hour & $\begin{array}{l}\text { nearly com. } \\
\text { plete }\end{array}$ \\
\hline $\begin{array}{r}3 \%(05 \text { c c }) \\
1 \%(05 \text { c.c }) \\
0.5 \%(05 \text { c c })\end{array}$ & $\begin{array}{l}0.5 \mathrm{c} . \mathrm{c} \\
0.5 \mathrm{c} \mathrm{c} \\
0.5 \mathrm{c} . \mathrm{c}\end{array}$ & $\begin{array}{l}2 \text { hours } \\
2 \text { hours } \\
2 \text { hours }\end{array}$ & $\begin{array}{l}0.5 \text { c c } \\
0.5 \text { c c. } \\
0.5 \text { c c. }\end{array}$ & $\begin{array}{l}1 \text { hour } \\
1 \text { hour } \\
1 \text { hour }\end{array}$ & $\begin{array}{l}\text { complete } \\
\text { complete } \\
\text { complete }\end{array}$ \\
\hline
\end{tabular}

Controls:

G. p. corpus-

cles, $5 \%$ sus-

pension in

$0.85 \% \mathrm{NaCl}$.

Hemolysis.

1 c c. S. $\operatorname{dog} 50-51^{\circ} .0 .5$ c.c. Alex. guinea-pig 0.5 c.c. complete 10 mins.

1 c.c. $S$ dog $56^{\circ} .0 .5$ c.c. Alex. guinea-pig 0.5 c.c. complete $10 \mathrm{mins}$.

In these experiments we again see the disadvantage of employing a 5 per cent suspension owing to the inhibiting action of the great. excess of salt solution on a weak alexin (series I and II, III and IV, etc.). Heating dog serum to 51 degrees merely weakens the toxic and combining activity of the dog alexin; $56^{\circ} \mathrm{C}$ does not wholly destroy either the toxic or the combining activities of the alexin, but destroys one as much as the other. The normal immune body apparently suffers no impairment by heating to 56 degrees (Controls).

\section{Conclusions.}

1. Suspensions of blood corpuscles for hemolytic experiments of 5 per cent are disadvantageous unless the excess of normal saline solution be removed, as alexic power is often entirely inhibited by the great excess of fluid.

2. Heating to 55 degrees for one-half hour does not affect the power of ox or rabbit corpuscles, even when hemolysis is produced, to absorb suitable immune bodies or alexins.

3. A given immune serum, active against rabbit corpuscles, contains a simple immune body as regards affinity for various alexins.

4. So-called "complementoids" (to judge from the test example) are simply "complements" (alexins) in which both the combining power and the hemolytic power are weakened. 
(Sachs (Central. für Bakt., etc., Abt. I, Orig. Bd. XXXIX, 1905) has questioned the experimental accuracy of the part of this article bearing on complementoids. In a reply Gay (Central. für Bakt., $\mathrm{XL}, 1906,695)$ has repeated the experiments and proved that dog serum heated to $51^{\circ} \mathrm{C}$ is not only slightly hemolytic for guinea-pig corpuscles, but will hemolyze more markedly either rabbit or guineapig corpuscles that have been treated with a heated immune serum. The conclusion, then, is that in classical examples of "complementoids" we have to deal with a "complement" the toxicity of which has simply been attenuated, and that no satisfactory experimental proof of the existence of "complementoids" exists. F. P. G.) 


\section{THE FIXATION OF ALEXINS BY SPECIFIC SERUM PRECIPITATES.*}

BY FREDERICK P. GAY, M.D.

We possess already a wealth of experimental detail relative to the specific immune bodies formed in the sera of animals injected either with simple cells or with such complex fluids as defibrinated blood or blood serum. Among the best known of these immune bodies are the specific hemolysins and bacteriolysins, the activities of which have been most fruitfully studied. We know, for example, that a given hemolytic immune body (substance sensibilisatrice, amboceptor) formed after the injection of foreign red blood cells has two important properties, namely, the property of sensitizing the causative cell in such a manner as to allow it to be destroyed by the alexin (complement) of various fresh normal sera; and secondly, the power, when it has formed a complex with the causative cell, of fixing an alexin. This second property is perhaps the more important, since the alexin can of ten be shown to have been absorbed by the cell-immune-body complex even when the cell itself is not destroyed. Indeed, this absorption of alexin has given the means of determining the existence of sensitizing substances where they might not otherwise have been shown to exist. Bordet and Gengou $\dagger$ have shown that the majority of antimicrobial sera contain "substances sensibilisatrices" that absorb alexin in instances where destruction of the specific bacteria is not produced, and Gengou $\ddagger$ has further shown that the injection of certain albuminoids may likewise give rise to specific sensitizing sera which may form with the causative substances mixtures that absorb alexin.

\footnotetext{
* Centralblatt für Bakt., I Abt., Orig. XXIX, 1905, 603.

$\dagger$ Bordet and Gengou, see p. 217.

$\ddagger$ Gengou, see p. 241 .
} 
I.

I was led recently to consider the effect that hemolytic immune serum heated to $55^{\circ} \mathrm{C}$, and then left in contact for a time with the specific red blood corpuscles, might have on the activity of an alexin. It surprised me to find that such a treated serum, centrifugalized long enough to free it of the corpuscles, had the power of neutralizing the hemolytic activity of an added alexin, as could be shown by subsequently introducing sensitized corpuscles. In controlling more carefully such an experiment I found that the treated immune serum, although freed of all corpuscles by the first short centrifugalization, would, when centrifugalized a second time, show a slight cloudiness at the bottom of the tube, which microscopically exhibited the amorphous character of specific precipitates. If this precipitate was removed from the treated serum, no fixation of the alexin took place. It seems, then, quite evident that a specific serum precipitate has the power to fix alexin, and the causative factors of such a precipitate must now concern us.

Of the precipitate-forming factors in the experiments to which I have referred the precipitin was of course furnished by the immune serum, but the source of the precipitinogen is not so evident, since washed corpuscles and not native blood were used. Further observations showed that although the corpuscles for these experiments had been washed once or twice with a relatively large amount of physiological solution, such washing was not sufficient to remove all the serum which bathed the corpuscles, and which, although markedly diluted, contained the very small amount of precipitinogen necessary to form a precipitate with the large amount of immune serum present. As a matter of fact it is extremely difficult to free blood corpuscles of all traces of serum, as is clearly shown by some recent experiments of Gengou, to whom I am indebted for the following unpublished observations: A four per cent alcoholic solution of mastic, forms, on the addition of distilled water in the proportions of nine parts of water to one of mastic, an emulsion, which affords a delicate reagent for the albuminoids of blood serum. One drop of normal salt solution containing a trace of serum gives rise to the rapid agglutination and precipitation of 1 c.c. of mastic emulsion, whereas such an amount of the fresh physiological solution pro- 
duces no effect. If 2 c.c. of fresh blood of the rabbit is washed in 20 c.c. of salt solution, the water of washing, removed after centrifugalizing, gives an immediate serum reaction with the mastic. If the corpuscles are washed a second time with a fresh amount of physiological solution and centrifugalized, the supernatant fluid gives a like reaction. Even the third water of washing gives a positive result with the mastic emulsion. The fourth wash water usually shows no evidence of the presence of serum.

It is evident from these preliminary observations that enough precipitinogen is present in the diluted serum which surrounds blood corpuscles washed in the ordinary manner, to give a precipitate in the presence of immune serum. Let us now consider more closely the power of this specific serum precipitate to fix alexin. The work of Gengou*demonstrated that a serum active against a foreign serum will, when mixed with this causative albuminoid, absorb alexin, as shown by the absence of hemolysis in sensitized corpuscles subsequently added. And the author notes particularly that this absorption of alexin is in proportion to the presence of specific serum precipitates; but he did not determine whether it is the precipitate itself or some other albuminoid in solution that exercises this alexin-fixing property. In the light of the present communication it is evident that it is the precipitate itself that fixes the alexin.

The following experiment shows that a specific serum precipitate will fix alexin. To furnish the precipitinogen necessary for the formation of this precipitate I have used, instead of dilute separated serum, the supernatant salt solution that had been employed to wash native blood. Such a serum dilution furnishes the same conditions as are obtained when insufficiently washed corpuscles are mixed with the immune serum.

\section{EXPERIMENT I.}

To 2 c.c. of fresh ox blood is added 38 e.c. of salt solution of 0.85 per cent; the suspension is then centrifugalized and the supernatant washing solution removed. The blood is washed with another 38 c.e. of the physiological solution and both the washing fluids are used for the following experiment:

$$
\text { * Gengou, l. c. }
$$


Tube 1. First $\mathrm{NaCl}$ washing solution

0.2 c.c.

Serum rabbit $>$ ox, 55 degrees*

0.6 c.c.

Tube 2. Second $\mathrm{NaCl}$ washing solution

0.2 c.c.

Serum rabbit $>$ ox, 55 degrees

0.6 c.c.

0.2 c.c.

Tube 3. Fresh $\mathrm{NaCl}$ solution

0.6 c.c.

Serum rabbit $>$ ox, 55 degrees

Tube 4. First $\mathrm{NaCl}$ washing solution 0.2 c.c.

Serum normal rabbit, 55 degrees

0.6 c.c.

Tubes are left at room temperature 2 hours.

In tube 1. Abundant precipitate.

In tube 2. Trace of precipitate.

In tubes 3 and 4 . No precipitate.

Tube 1 is then centrifugalized and the supernatant fluid forms tube 1a, while the precipitate is brought to the original volume ( 0.8 of a cubic centimeter) with salt solution and forms Tube 1 . To each of the tubes 1, 1a, 2, 3 and 4 is then added fresh rabbit serum ( 24 hours), 0.075 of a cubic centimeter, and contact allowed for 2 hours at room temperature. To each tube is then added 0.025 of a cubic centimeter of sensitized rabbit corpuscles ( $\mathrm{S}$. rabbit $>$ ox, 55 degrees), and the resultant hemolysis is as follows:

Tube 1 (precipitate). No hemolysis.

Tubes 1a, 2, 3 and 4. Hemolysis complete.

This experiment shows clearly that it is the specific precipitate that fixes the alexin. In tube 2 the hemolysis, although finally complete, is distinctly delayed owing to partial absorption of the alexin by the very slight precipitate.

The marked difference in dosage between the precipitinogen and the precipitin is indicated by the dilutions of ox serum represented by the washing solutions of the last experiment. In fact, very small traces of the precipitinogen suffice to give a maximum precipitate, provided sufficient immune serum (precipitin) is used. A more accurate idea of the relation of dosage and dilution between the two precipitate-forming sera than is given incidentally in the following experiments need not concern us here, since we are to deal rather with the properties of precipitates than with their formation.

The question may properly arise as to whether the sensitizing activity of the immune body for the corpuscles has been diminished by the formation of a specific precipitate, and is directly answered by the following experiment:

* Which abbreviation is used to indicate the serum of a rabbit immunized against ox blood. Such serum, as indicated, has been heated to $55^{\circ} \mathrm{C}$ for one-half hour to deprive it of alexin. 


\section{EXPERIMENT II.}

Two large tubes are prepared.

Tube A. Serum of ox, 55 degrees

0.05 c.c.

Serum rabbit $>$ ox, 55 degrees $\quad 2.00$ c.c.

Tube B. $\mathrm{NaCl}$ solution, 0.85 per cent

0.05 c.c.

Serum rabbit $>$ ox, 55 degrees

2.00 c.c.

Contact, 2 hours. Tube A gives a dense precipitate; B, none. Tube A is centrifugalized and the supernatant fluid used for the following small tubes:

$\begin{array}{lllll}\text { Series A. } & \text { Tube 1. } & \text { Treated serum A } & 0.2 & \text { c.c. } \\ & \text { Tube 2. } & \text { Treated serum A } & 0.1 & \text { c.c. } \\ & \text { Tube 3. } & \text { Treated serum A } & 0.05 \text { c.c. } \\ \text { Series B. } & \text { Tube 4. } & \text { Treated serum A } & 0.025 \text { c.c. } \\ & \text { Tube 5. } & \text { Treated serum B } & 0.2 \text { c.c. } \\ & \text { Tube 6. } & \text { Treated serum B } & 0.1 & \text { c.c. } \\ & \text { Tube 7. } & \text { Treated serum B } & 0.05 \text { c.c. } \\ & \text { Tube 8. } & \text { Treated serum B } & 0.025 \text { c.c. }\end{array}$

Each tube is brought to the same volume ( 0.2 of a cubic centimeter) with salt solution, and then to each tube is added

Washed ox corpuscles $\quad 0.05$ c.c. (washed four times) Alexin of rabbit 0.05 c.c.

Resultant hemolysis is as follows:

Series A.

Tube 1. Complete

Tube 2. Nearly complete

Tube 3. Marked

Tube 4. Marked
Series B.

Tube 5. Complete

Tube 6. Nearly complete

Tube 7. Marked

Tube S. Marked.

As is evident from this experiment, the hemolytic immune body is not affected by the formation of the specific precipitate.

The alexin-fixing power of the precipitate is not specific as regards alexin - that is, it is aible to absorb alexins other than those of the species furnishing the precipitin. The fixation of guineapig alexin, for example, is shown by:

\section{EXPERIMENT III.}

Two tubes are prepared:

Tube 1 . Serum $>$ ox, 55 degrees 0.025 c.c. ( 0.1 c.c. of a dilution of 1 to 4 )

Serum rabbit $>$ ox, 55 degrees 2.00 c.c.

Tube 2. $\mathrm{NaCl}$ solution

0.1 c.c.

Serum rabbit $>$ ox, 55 degrees 2.00 c.c. 
To each tube is added alexin of the guinea-pig one-thirtieth of a cubic centimeter, and contact allowed for one-half hour. In tube 1 a considerable precipitate is formed; in tube 2 , none. Then to each tube is added 0.05 of a cubic centimeter of ox corpuscles sensitized with S. rabbit $>$ ox, 55 degrees ( $1 \frac{1}{2}$ hemolytic doses).

\section{Resultant Hemolysis.}

Tube 1. No hemolysis.

Tube 2. Hemolysis complete.

Which shows that the precipitate has fixed the guinea-pig alexin.

\section{II.}

That a disregard of this fixation of alexin by specific precipitates has led to many erroneous impressions of the mechanism of hemolysis will undoubtedly prove true, but I wish to commit myself only on such phases of the question as I have been able to submit to experimental study.

Recently Pfeiffer and Friedberger* have given the résumé of a study of the antibacteriolytic, or "antagonistic," substances which are said to occur in normal sera. These authors have found that certain normal sera, which in themselves possess no antilytic properties, acquire distinct antibacteriolytic power when previously put in contact with the bacteria on which they are destined subsequently to act. For example, normal rabbit serum treated with typhoid bacilli has the power to prevent in vivo the destruction of sensitized typhoid organisms; untreated serum has no such power, nor does the serum treated with typhoid bacilli show any antilytic effect for cholera vibrios sensitized with anticholera serum. A further consideration of these most interesting observations concerning bacteria need not concern us here, but an analogous series of facts in hemolysis, which was soon published by Sachs, $\dagger$ and the conclusions of this author must be regarded more in detail. Normal rabbit serum heated to 55 degrees when treated with equal parts of sedimented red blood corpuscles of the sheep or of the pig inhibits the action of guinea-pig alexin on the properly sensitized corpuscles of the blood in question. Normal untreated serum has no such

* Pfeiffer und Friedberger, Deutsche med. Wochenschr., 1905, No. 1, p. 6.

$\dagger$ Sachs, Deutsche, med. Wochenschr., 1905, No. 18, p. 705. 
antihemolytic property, and the treated serum itself acts only to protect the species of corpuscles with which this serum has been digested. The explanation which Sachs offers for the facts up to the present point is as follows. Normal rabbit serum contains a series of normal hemolytic amboceptors, of which some are specific for sheep corpuscles. When treated with sheep corpuscles rabbit serum loses its sheep amboceptors, but the remaining amboceptors (active against other corpuscles), although unattached to their specific cells, have a greater affinity for complements than do the sheep corpuscles sensitized with serum rabbit $>$ ox, added as a test for alexin; and hemolysis of these test corpuscles does not take place.*

If we repeat in detail Sachs' first experiment, together with a control suggested by the facts I have already adduced, it is evident that his explanation of this interesting phenomenon is certainly incorrect. I have worked with sheep blood only and have employed the specific serum used by Sachs, that is, the serum of a rabbit immunized against ox blood (which, of course, readily destroys ox red blood corpuscles, but also works satisfactorily against the red cells of the sheep).

\section{EXPERIMENT IV.}

Three tubes are prepared as follows:

Tube A. Sheep corpuscles (the sediment of blood washed once in 15 volumes of $\mathrm{NaCl}$ solution of 0.85 per cent 1.5 c.c. Normal rabbit serum, 55 degrees $\quad 1.5$ c.c.

Tube B. Normal rabbit serum, 55 degrees $\quad 1.5$ c.c.

Tube C. Sheep corpuscles (the sediment of blood washed five successive times with fresh volumes of $\mathrm{NaCl}$ )

Normal rabbit serum, 55 degrees

1.5 c.c. $\bullet$

1.5 c.c.

Of these tubes, $\mathrm{A}$ and $\mathrm{B}$ correspond exactly in dosage to those given by the German author. Just how completely he washed

* As is usual with the Ehrlich school, an hypothesis was invented in harmony with the lateral-chain theory, to explain the Neisser-Wechsberg phenomenon; and it is this hypothesis and not fundamental expcrimental facts which is used as a foundation for further hypotheses. It has never been proved that an alexin can unite with an immune body unless the latter has formed a complex with the cell or substance, the injection of which has given rise to the specific serum. The Neisser-Wechsberg phenomenon, which has been accepted by the Ehrlich school as proving this union, is, in reality, unquestionably due to another cause, as I shall consider later. 
the sheep corpuscles he does not state, but we may presume not far differently from the manner employed in tube $\mathrm{A}$, since the result is the same. Tube $\mathrm{C}$ differs from tube $\mathrm{A}$ only in the fact that every trace of sheep serum has been removed by the repeated washings. The succeeding steps follow exactly the conditions and dosage of Sachs.

Tubes $\mathrm{A}, \mathrm{B}$ and $\mathrm{C}$ are left at $37^{\circ} \mathrm{C}$ for 1 hour. Tubes $\mathrm{A}$ and $\mathrm{C}$ are then centrifugalized and the supernatant treated sera as well as the contents of tube B serve to make the following tubes:

$\begin{array}{cll}\text { Tube } 1 . & \text { Treated serum A } & 1.0 \text { c.c. } \\ & \text { Serum of guinea-pig (alexin) } & 0.1 \text { c.c. } \\ \text { Tube 2. } & \text { Treated serum A } & 0.2 \text { c.c. } \\ & \text { Alexin, guinea-pig } & 0.1 \text { c.c. } \\ \text { Tube 3. Serum B } & 1.0 \text { c.c. } \\ & \text { Alexin of guinea-pig } & 0.1 \text { c.c. } \\ \text { Tube } 4 . & \text { Treated serum C } & 1.0 \text { c.c. } \\ & \text { Alexin, guinea-pig } & 0.1 \text { c.c. } \\ \text { Tube 5. Treated serum C } & 0.2 \text { c.c. } \\ & \text { Alexin, guinea-pig } & 0.1 \text { c.c. }\end{array}$

Contact at 37 degrees for one-half hour. Then to each tube is added 1 c.c. of a 5 per cent suspension of washed sheep corpuscles ( 5 times) plus 0.4 of a cubic centimeter of serum rabbit $>0 x$, 55 degrees (about two hemolytic doses). Resultant hemolysis is as follows:

$\left.\begin{array}{l}\text { Tube } 1 \\ \text { Tube } 2\end{array}\right\}$ No hemolysis $\left.\begin{array}{l}\text { Tube } 3 \\ \text { Tube } 4 \\ \text { Tube } 5\end{array}\right\}$ Hemolysis complete

That is, in rabbit serum treated with imperfectly washed sheep corpuscles, there is a substance that prevents the hemolysis of test corpuscles added at the end; this is the Sachs experiment. If the corpuscles are washed so as to remove all sheep serum, there is no antagonistic substance found. That there is an alexin-fixing substance present in tube " $\mathrm{A}$ " is true, but it is the precipitate formed at the end by the interaction of the immune serum and the sheep precipitinogen carried by the treated rabbit serum, and not the treated serum itself. That no true "anticomplement action" exists in the digested normal rabbit serum itself in the experiment of Sachs is easy of proof and would have been evident in the tubes of this experimenter had he only subjected the tubes which he compares, to the same experimental conditions. The details of 
his last experiments, which show a grave experimental error, are the following: The normal rabbit serum treated with insufficiently washed sheep corpuscles brought about the inhibition of hemolysis already noted, when, for the sensitizing of the test corpuscles, he used S. rabbit $>$ ox, 55 degrees. In this case the excess of sensitizing serum was left with the test corpuscles, and of course a precipitate was formed in the last stage of the experiment, and hemolysis thereby inhibited. No such "anticomplement action" took place if he sensitized the corpuscles both with serum rabbit $>0 x, 55$ degrees, and with heated normal rabbit serum. In this instance he removed the excess of both sensitizing sera, and no precipitate was formed. And again he notes that no inhibition of hemolysis occurred if, for sensitizing the test corpuscles, he used normal rabbit serum alone. Incidentally, the serum was removed in this case, but of course no inhibition would have taken place anyway, as no precipitate was formed.* Manifestly, the fixation of alexin (inhibition of hemolysis) occurs only in the presence of a precipitate formed by the interaction of an excess of immune serum and the precipitinogen of the sheep serum carried from the first incompletely washed corpuscles. The following experiment comprises a complete refutation of Sachs' hypothesis and puts in evidence the alexin-fixing precipitate:

\section{EXPERIMENT V.}

The tubes are prepared as follows:

Tube A. Sheep corpuscles (washed once)

Tube B. Sheep corpuscles (washed five tim

3 c.c.

3 c.c.

Serum normal rabbit, 55 degrees

3 c.c.

Contact, 1 hour at $37^{\circ} \mathrm{C}$

3 c.c.

Centrifugalization, and, from the supernatant treated sera $\mathrm{A}$ and $\mathrm{B}$, are formed two tubes:

$\begin{array}{lll}\text { Tube } \mathrm{A}^{1} . & \text { Treated serum "A" } & 2.5 \text { c.c. } \\ & \text { Alexin guinea-pig } & 0.25 \text { c.c. } \\ \text { Tube } \mathrm{B}^{1} . & \text { Treated serum "B" } & 2.5 \text { c.c. } \\ & \text { Alexin guinea-pig } & 0.25 \text { c.c. }\end{array}$

* Sachs, l.c.; compare Tabelle 2, Kol. I and II; Tabelle 3, Kol. B, and Tabelle 2, Kol. I. 
Contact, three-quarters of an hour at room temperature, and then the following tubes are made:

$\begin{array}{lll}\text { Tube } 1 . & \mathrm{A}^{1} \text { mixture } & 1.1 \text { c.c. } \\ & \text { Serum rabbit }>\text { ox, } 55 \text { degrees } & 0.4 \text { c.c. } \\ \text { Tube 2. } & \mathrm{A}^{1} \text { mixture } & 1.1 \text { c.c. } \\ & \text { Serum normal rabbit, } 55 \text { degrees } & 0.4 \text { c.c. } \\ \text { Tube } 3 . & \mathrm{B}^{1} \text { mixture } & 1.1 \text { c.c. } \\ & \text { Serum rabbit }>\text { ox, } 55 \text { degrees } & 0.4 \text { c.c. } \\ \text { Tube } 4 . & \mathrm{B}^{1} \text { mixture } & 1.1 \text { c.c. } \\ & \text { Serum normal rabbit, } 55 \text { degrees } & 0.4 \text { c.c. }\end{array}$

After contact, a precipitate is seen in tube 1 but none in tubes 2,3 and 4 . Then to each tube is added 0.05 of a cubic centimeter of washed sheep corpuscles sensitized with S.rabbit $>$ ox, 55 degrees, and with the excess of the immune serum removed by centrifugalizing.

The resultant hemolysis is as follows:

Tube 1. No hemolysis.

Tubes 2, 3 and 4. Hemolysis complete.

This experiment clearly demonstrates that the so-called "amboceptor anticomplement" action of Sachs is simply due to specific precipitates.

As will suggest itself, the alexin-fixing action of serum precipitates may readily be brought forward to explain the Neisser-Wechsberg phenomenon of "complement deviation." This, it will be remembered, was demonstrated in the case of bacteria, where it was found that an excess of immune serum prevented the complete destruction of a given dose of bacteria by an amount of alexin that destroyed perfectly the same amount of organisms if the optimal dose of immune serum were used. The authors reconciled these experiments with the Ehrlich hypothesis by supposing that the mass action of the excess of free amboceptor deviated the complement. But no one has been able to demonstrate the hemolytic analogue of this phenomenon. Morgenroth,* it is true, by making certain suppositions as regards the union of "complements" with free amboceptors, and by introducing certain other bodies ("antiamboceptors"), has obtained somewhat similar results, but his analogy is far from exact. The discussion of this subject, together with * Morgenroth, Centralbl. f. Bakt., etc., Bd. XXXV, 1904, p. 504 
definite experimental demonstration that deviation of the alexin may exist in hemolysis under conditions absolutely identical to those described for bacteriolysis by Neisser and Wechsberg, will appear presently in the Pasteur Annals.* I may note simply that it is indeed the conjectured rôle of precipitates that does cause this alexin deviation in hemolysis.

\section{Conclusions.}

1. As was noted by Gengou, the serum of an animal of species $A$, injected with the blood serum of species $B$, contains specific "substances sensibilisatrices" which, when the immune serum A is mixed with serum B, forms a complex which fixes alexin. This alexin-fixing substance is the specific serum precipitate formed by the interaction of the two sera.

2. Repeated washings of blood with relatively large amounts of physiological solution are necessary to remove all traces of serum. A very small amount of this serum contains enough precipitinogen to form a large precipitate if enough precipitin (immune serum) be present.

3. The formation of a serum precipitate does not affect the sensitizing strength of the hemolytic immune body.

4. The so-called "anticomplements of normal sera" of Sachs and probably also the "antagonistic substances" of Pfeiffer and Friedberger are simply specific serum precipitates capable of fixing alexin.

5. A disregard of the presence and the alexin-fixing properties of serum precipitates has doubtless given rise to many erroneous impressions of the mechanism of hemolysis.

* See p. 357. 


\section{DEVIATION OF THE ALEXIN IN HEMOLYSIS.*}

By FREDERICK P. GAY.

Since the observations of Neisser and Wechsberg $†$ on the inhibiting influence on bacteriolysis of too large a dose of specific immune serum (previously heated to 55 degrees) when the amount of alexin is relatively small, many attempts have been made to determine the mechanism of this interesting phenomenon. The interpretation that Neisser and Wechsberg gave is well known; it is wholly in harmony with Ehrlich's theory. According to these authors, in a mixture of bacteria, a relatively small dose of alexin, and a relatively large dose of heated immune serum, or, in other words, of sensitizer (amboceptor), not all the alexin is utilized in destroying bacteria. Not all the large amount of sensitizer present can be absorbed by the bacteria; the excess remains in the surrounding fluid and, owing to its affinity for alexin, takes up a greater or less amount of this substance that consequently is of no service in producing bacteriolysis. The inhibiting effect of too much immune serum would be explained as a real deviation of the complement (Komplementablenkung) by the excess of sensitizer not combined with the bacteria. This hypothesis, to be sure, has neither been experimentally proved nor refuted. It is by no means necessary to accept it, as it is based on a supposition that has never been proved experimentally, namely, that the sensitizing substance can fix alexin even when uncombined with bacteria. It must be admitted that certain defenders of the hypothesis, notably Lipstein $₫$ have been able to reply to certain objections that have been raised to it, without, however, bringing forward any really definite proofs of its correctness. The inhibiting effect of an excess of immune serum

* La déviation de l'alexine dans l'hemolyse. Annales de l'Institut Pasteur, XIX, 1905, 593.

$\dagger$ See Studies on Immunity, Ehrlich-Bolduan, John Wiley \& Sons, p. 120.

$\ddagger$ Lipstein, Ehrlich-Bolduan, Wiley \& Sons, p. 132. 
evident in bacteriolysis has never been shown to exist in hemolysis. The experiments by Morgenroth* demonstrating such a phenomenon in hemolysis are evidently incorrectly conceived, as Bordet $\uparrow$ has shown, and should not,be admitted as evidence.

The interpretation we wish to offer of this Neisser and Wechsberg phenomenon has an evident relation to certain experimental results that have already been described. $\ddagger$ These experiments dealt with the alexin-fixing action of the albuminous precipitates obtained by mixing a precipitin serum with the proper antigen. The results may be summarized as follows:

1. Gengou § showed that the serum of animals of species A immunized against an albuminous substance of species B has not only a precipitating property for these substances, but also endows them with the property of absorbing alexin. These albuminous substances treated with the specific immune serum act, in other words, as do blood corpuscles or bacteria treated with suitable sensitizers.

2. We were able to carry Gengou's observations farther by showing that the fixation of alexin in this instance is brought about exclusively by the precipitated albuminous substances and not by those in solution.

3. It is worth noting that a very weak dilution of precipitinogen (precipitable serum) in salt solution will give a relatively abundant precipitate with a suitable dose of precipitin. To avoid the occurrence of a serum precipitate in hemolytic experiments, it is indispensable to wash the corpuscles employed several times with salt solution to remove the accompanying serum entirely. This serum if present will form a precipitate with the immune serum used to sensitize the blood corpuscles in such an experiment.

4. This precaution in washing has apparently not been taken by many investigators; as a result, in their mixtures of incompletely washed blood (that is, blood containing traces of serum) and hemolytic substances (sensitizer and alexin) a precipitate was formed that could independently fix a greater or less amount of the alexin employed. This fixed alexin would, of course, not affect the corpuscles, and consequently a true deviation of alexin would have occurred in a hemolytic experiment.

* Centralblatt f. Bakt., XXV, 1904, 501. 
5. This last paragraph is applicable to certain recent researches of Sachs * on the so-called "anticomplement" power of normal sera. For the discussion of these experiments we may refer to the previous article. Pfeiffer and Friedberger have also observed certain analogous facts with bacteria, and it would seem as if their work might be explained in a similar manner.

We shall now consider more attentively the experimental results to which reference has been made in paragraphs 2 and 3 above. We have seen, first, that the albuminous substances precipitated by a specific serum are in reality sensitized, or, in other words, able to absorb alexin, and, secondly, that small amounts of precipitinogen suffice to form an abundant precipitate with the precipitin serum. We must further emphasize that a relatively large amount of this precipitin serum is necessary to cause a precipitum sufficient to fix alexin well. The following experiment indicates the relation between the two sera, the precipitum obtained, and the degree of alexin absorption. As precipitinogen, ox serum is used, and, as precipitin, the serum of a rabbit immunized against ox blood and heated to 55 degrees. $†$

$\begin{array}{lll}\text { Tube 1. } & \text { Ox serum, } 55 \text { degrees } & 0.025 \text { c.c. } \\ \text { Serum rabbit }>\text { ox, } 55 \text { degrees } & 0.1 \text { c.c. } \\ \text { NaCl solution }(0.85 \text { per cent }) & 1.9 \text { c.c. } \\ \text { Tube 2. } & \text { Ox serum } & 0.025 \text { c.c. } \\ & \text { Serum rabbit }>\text { ox, } 55 \text { degrees } & 0.6 \text { c.c. } \\ \text { Tube 3. } & \text { Ox serum } & 1.4 \text { c.c. } \\ & \text { Serum rabbit }>\text { ox, } 55 \text { degrees } & 0.025 \text { c.c. } \\ & 2.0 \quad \text { c.c. }\end{array}$

These three tubes contain the same total volume and the same amount of ox serum. They differ in their respective amounts of precipitin serum, which in the small doses is replaced by salt solution, so that the total volumes are the same. Three control tubes (4, 5 and 6) are also prepared corresponding to tubes 1, 2 and 3 respectively, but containing salt solution in place of ox serum. After one-half hour at 37 degrees there is no evident precipitate in tubes $1,4,5$ and 6 ; there is a distinct though slight precipitate in tube 2 ; and in tube 3 there is a voluminous precipitate.

* Deutsche med. Woch., 1905, No. 18, 705.

$\dagger$ This serum, for the sake of abbreviation, is referred to as "Sorum rabbit >ox." 
To each tube there is then added one-thirtieth of a cubic centimeter of normal rabbit alexin (serum from blood obtained the day before). Fifteen minutes later 0.3 of a cubic centimeter of sensitized ox blood is added to each tube. This sensitized blood is a mixture of one part of carefully washed ox blood $*$ to five parts of heated rabbit $>$ ox serum.

There is complete hemolysis in tubes $1,4,5$ and 6 ; partial hemolysis in tube 2 ; and no hemolysis in tube 3 . The alexin, therefore, has remained free in tubes $1,4,5$ and 6 containing no precipitate, has been partially absorbed in tube 2 containing a moderate precipitate, and has been completely absorbed in tube 3 that contains the largest precipitate. In other words, the greater the amount of precipitate the greater the alexin absorption and corresponding absence of hemolysis.

As may be imagined, this experiment may be performed somewhat differently. A mixture containing the albuminous precipitum and the sensitized corpuscles may be made and the alexin subsequently added. Under these conditions we should expect that both sensitized elements that are avid of alexin, namely, the precipitate and the corpuscles, would struggle to obtain the alexin, and hemolysis would be more or less inhibited or even entirely prevented if the dose of alexin were not too large. To produce these conditions we may place in tube A small amounts of well-washed ox blood ( 0.05 of a cubic centimeter) and of ox serum ( 0.025 of a cubic centimeter), and then add a very large dose of heated rabbit $>$ ox serum (2.5 c.c.). A few seconds later let us add rabbit alexin (one-thirtieth of a cubic centimeter). There is no hemolysis, as the precipitum has absorbed all the alexin. In a control tube B prepared at the same time, and containing the same components except the ox serum, hemolysis is complete, as no precipitate forms. All that is necessary to produce hemolysis in tube A is to add a small additional dose of alexin.

We have already noted that considerable immune serum is necessary to obtain an abundant precipitate with ox serum. Consequently, if a dose of rabbit > ox serum barely sufficient to sensitize

* This blood had been carefully washed in normal salt solution and then restored to its original volume; it eorresponds, then, to blood in which the original serum is replaced by salt solution. 
the corpuscles, but too little to produce an abundant precipitate, had been put in tube A we should expect that hemolysis would have taken place. This may be experimentally verified. In a tube containing 0.025 of a cubic centimeter of ox serum, 0.05 of a cubic centimeter of corpuscles and only 0.2 of a cubic centimeter of immune serum, hemolysis oceurs as well as in a control that contains no ox serum.

In such experiments, however, it is evident that if the amount of rabbit $>$ ox serum is too much diminished no hemolysis will occur owing to the fact that the corpuscles are not sufficiently sensitized.

In the following tables are given the results of experiments in which the amounts of rabbit $>$ ox serum vary. In table $\mathrm{A}$ the mixtures contain ox serum; in B there is no ox serum, but salt solution in its place; the doses of ox corpuscles and rabbit alexin are constant.

TABLE A.

\begin{tabular}{|c|c|c|c|c|c|c|}
\hline Tube. & Ox serum, $55^{\circ}$ & Ox corpuscles. & $\begin{array}{r}\text { Rabbit }>\text { ox } \\
\text { serum, } 55^{\circ} .\end{array}$ & $\begin{array}{l}\mathrm{NaCl} \\
\text { sol. }\end{array}$ & Rabbit alexin. & Hemolysis. \\
\hline $\begin{array}{l}1 \\
2 \\
3 \\
4 \\
5 \\
5 \\
6 \\
7 \\
8\end{array}$ & $\begin{array}{l}0.025 \text { c.c. } \\
0.025 \text { c.c. } \\
0.025 \text { c.c. } \\
0.025 \text { c.c. } \\
0.025 \text { c.c. } \\
0.025 \text { c.c. } \\
0.025 \text { c.c. } \\
0.025 \text { c.c. }\end{array}$ & $\begin{array}{l}0.05 \text { c.c. } \\
0.05 \text { c c. } \\
0.05 \text { c.c. } \\
0.05 \text { c.c. } \\
0.05 \text { c.c. } \\
0.05 \text { c c. } \\
0.05 \text { c.c. } \\
0.05 \text { c.c }\end{array}$ & 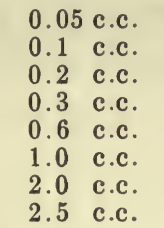 & $\left|\begin{array}{ll}2.45 & \mathrm{c} . \\
2.4 & \mathrm{c} . \\
2.3 & \mathrm{c} . \\
2.2 & \mathrm{c} . \\
1.9 & \mathrm{c} . \\
1.5 & \mathrm{c} . \\
0.5 & \mathrm{c} . \\
0.0 & \mathrm{c} .\end{array}\right|$ & $\begin{array}{l}0.033+c . c . \\
0.033+\text { c.c. } \\
0.033+\text { c.c. } \\
0.033+\text { c.c. } \\
0.033+\text { c.c. } \\
0.033+\text { c.c. } \\
0.033+\text { c.c. } \\
0.033+\text { c.c. }\end{array}$ & $\begin{array}{l}\text { incomplete } \\
\text { complete } \\
\text { complete } \\
\text { complete } \\
\text { incomplete } \\
\text { slight } \\
\text { none } \\
\text { none }\end{array}$ \\
\hline
\end{tabular}

TABLE B.

\begin{tabular}{|c|c|c|c|c|c|c|}
\hline Tube. & $\mathrm{NaCl}$. & $\begin{array}{l}\text { Ox cor- } \\
\text { puscles. }\end{array}$ & $\begin{array}{l}\text { Rabbit }>\text { ox } \\
\text { serum, } 55^{\circ} \text {. }\end{array}$ & NaCl sol. & Rabbit alexin. & Hemolysis. \\
\hline $\begin{array}{l}1 \\
2 \\
3 \\
4 \\
5 \\
6 \\
7 \\
8\end{array}$ & $\begin{array}{l}0.025 \text { c.c. } \\
0.025 \text { c.c. } \\
0.025 \text { c.c. } \\
0.025 \text { c.c. } \\
0.025 \text { c.c. } \\
0.025 \text { c c. } \\
0.025 \text { c.c. } \\
0.025 \text { c.c. }\end{array}$ & $\begin{array}{l}0.05 \text { c.c. } \\
0.05 \text { c.c. } \\
0.05 \text { c.c. } \\
0.05 \text { c.c. } \\
0.05 \text { c.c. } \\
0.05 \text { c.c. } \\
0.05 \text { c.c. } \\
0.05 \text { c.c. }\end{array}$ & 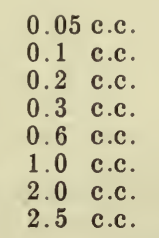 & $\begin{array}{l}2.45 \text { c c } \\
2.4 \text { c c } \\
2.3 \text { c.c } \\
2.2 \text { c c } \\
1.9 \text { c c } \\
1.5 \text { c c } \\
0.5 \text { c c } \\
0.0 \text { c c }\end{array}$ & $\begin{array}{l}0.033+c . c . \\
0.033+c c \\
0.033+c c . \\
0.033+c c . \\
0.033+c . c . \\
0.033+c c . \\
0.033+c \text { c. } \\
0.033+c . c .\end{array}$ & $\begin{array}{l}\text { incomplete } \\
\text { complete } \\
\text { complete } \\
\text { complete } \\
\text { complete } \\
\text { complete } \\
\text { complete } \\
\text { complete }\end{array}$ \\
\hline
\end{tabular}


These experiments show a Neisser-IVechsberg phenomenon in hemolysis. The result may be described by saying that blood (i.e., serum plus corpuscles) is not hemolyzed by a small dose of alexin unless the amount of sensitizer is suitably small. Too much sensitizer protects the corpuscles by deviating the alexin. This deviation (Komplemenablenkung), however, is not to be explained as Neisser and Wechsberg have done. It is not due to alexin absorption by means of a certain amount of uncombined sensitizer remaining free in the fluid. The fixation of alexin is brought about by a sensitized precipitum that competes successfully with the corpuscles in absorbing this active substance.

It is particularly to be noted that no Neisser and Wechsberg phenomenon occurs if the corpuscles employed have been washed free of the serum present in the primitive blood.

Will this interpretation of alexin fixation in hemolysis account for the Neisser-Wechsberg phenomenon in bacteriolysis? We expect to take up this question at a later time. It may be noted here that a culture or emulsion of bacteria contains elements that correspond rather closely to those present in blood. The bacteria correspond, of course, to the corpuscles, the bacterial precipitinogens, that, as Kraus has shown, torm precipitates with the specific antiserum, moreover correspond to the albuminous substances in serum. 


\section{ON THE RELATIONS OF SENSITIZERS TO ALEXIN.*}

By DRS. J. BORDET AND F. P. GAY.

Those who have made a study of hemolysis hold very divergent opinions as to the relations between the susceptible corpuscle and the substances that affect it, namely, the sensitizer (amboceptor) and the alexin (complement). It is well known that the blood corpuscles fix the sensitizer (Ehrlich and Morgenroth), and that corpuscles so modified have the new property of energetically absorbing all the alexin from the surrounding fluid. (Bordet.) It is evident, then, from these facts that the sensitizer acts as an intermediary agent in bringing about the union between the sensitive cell and the toxic substance or alexin.

It is perfectly evident that there is some not well understood substance in the red blood cell which unites and forms a complex with the sensitizer. This much and no more has been experimentally demonstrated; the intimate nature of the reaction is unknown.

It is scarcely profitable to explain this simple fact in any elaborate fashion. To say that the corpuscle receives and holds the sensitizer by means of a "receptor," or that the sensitizer combines with such a receptor because it has a combining cytophilic group, is to pretend to a knowledge not yet obtained. We may content ourselves by saying that a complex is formed.

But how does this complex (sensitizer-corpuscle) fix alexin? To which constituent of the complex is the affinity for this substance due? It is certainly not the corpuscle itself, for we find that normal unsensitized corpuscles do not take up alexin. Is it, then, the sensitizer, or is it the combination of the two, that shows an avidity for alexin that neither one of its constituents alone possesses?

Both hypotheses have been suggested. The first one, namely,

* Sur les relations des sensibilisatrices avec l'alexine. Annales de l'Institut Pasteur, XX, 1906, 467. 
that the sensitizer combines with the alexin, has been suggested by Ehrlich and Morgenroth. According to these authors the sensitizer molecule has, in addition to the atom group that binds it to the cell receptor (cytophilic group), a second distinct group (complementophilic) that unites with the alexin. This explanation, which regards the sensitizer as a bond of union between corpuscles and alexin, suggests two corollaries.*

In the first place the corpuscle has no direct participation in alexin absorption, but simply takes hold of the sensitizer and then ceases to function. It is, then, the sensitizer that comes into play in alexin absorption by means of its own affinities. And further, on Ehrlich and Morgenroth's thesis, alexin absorption is a purely chemical reaction due to affinities between atoms or groups of atoms. The complex receptor-sensitizer-alexin may be regarded as a single large molecule, the nucleus of which is the sensitizer and the side chains of which are the receptor and the alexin.

The alexin, then, would unite with a new and definite chemical complex, and its absorption is not comparable with phenomena of adhesion (the fixation of a toxin by a precipitate, for example) nor with the various dyeing phenomena, in which cases the molecules of the substance to be stained attract the molecules of the dye without intervention of atomic affinities. Nor is it comparable to the common facts observed in the precipitation, agglutination and coagulation of colloidal substances, nor, in short, to the many phenomena due to molecular adhesion.

According to the second explanation, proposed several years ago by one of the authors of the present article, the sensitizer does not of itself combine with the alexin. It unites with the corpuscles and forms a complex which has the new property of uniting with the alexin and of removing it from the surrounding fluid; in other terms, neither the proper substance of the corpuscle nor the sensitizer by itself has any perceptible affinity for the alexin. Such an affinity becomes evident only when the proper substance in the corpuscle has become modified (sensitized) as a result of union with the sensitizer and so changed into an alexin-attracting complex.

According to this hypothesis, there is no question of a comple-

* In accordance with this conception Ehrlich and Morgenroth have given the name of amboceptor to the sensitizer. 
mentophilic group in the sensitizer fitted to take hold of alexin without intervention of the corpuscle; the corpuscle itself takes part in the fixation inasmuch as it is one of the constituent members of the absorbing complex.*

It is to be noted that this second point of view, in accordance with which the sensitizer has no complementophilic group, is much less compatible with the idea that alexin fixation is a true chemical reaction, implying the formation of a new well-defined compound.

Unless we suppose that the union of the sensitizer with the corpuscle receptor is more than a simple attachment, and unless we admit that this union modifies the affected molecules very profoundly by causing a new distribution of their atoms, it is difficult to see how this combination can give rise to atom groups avid of alexin when no trace of them is present in either of the two bodies that take part in the reaction.

This latter conception, however, harmonizes with the idea that the alexin is taken out and absorbed by a process of molecular adhesion rather than by a true chemical reaction.

We have simply to consider the substance of the corpuscle uniting with the sensitizer as so modified in its properties of adhesion as to form a complex capable of sticking to alexin, as calcium fluoride and other inert chemical precipitates in fine colloidal suspension remove the fibrinogen from plasma.

Do we not find analogous if not identical instances in the change

* Ehrlich and Morgenroth, to be sure, have subsequently modified their original theory, at least as regards certain sensitizers or alexins and certain corpuscles. They admit that in certain instances the sensitizer shows no affinity for alexin until it is combined with the cell. It is naturally difficult to discuss or appraise the value of a theory that changes so markedly from year to year.

To state that the sensitizer combines with the alexin only aftcr union with the corpuscles is practically to adopt Bordet's conception, according to which neither one of the constituents alone is able to fix alexin. Such a statement, moreover, renounces the theory that was so definitely stated at first, namely, that the alexin unites with the sensitizer even when no corpuscles are present.

If the intervention of the corpuscles is admittedly necessary, the two theories really differ only in logic subtleties.

It is only fair to add that although Ehrlich and Morgenroth admit that the sensitizer in certain instances can combine only after cell union, they state that in other cases the opposite condition occurs, namely, that the union of the alexin with the sensitizer increases the affinity of the latter for the corpuscle. 
of molecular adhesion that is the essential cause of the agglutination of bacteria? Is it not a change in molecular adhesion that makes bacteria treated by an agglutinin flock out when sodium chloride is added, although normal bacteria do not?

From this viewpoint alexin fixation assumes a more real and general interest than was at first apparent. In endeavoring to understand the reactions that take place in the body by comparison with simpler and better-known facts, is it necessary to look for analogies in pure chemistry alone, as do Ehrlich and Morgenroth? Such phenomena, as we know, follow laws of definite proportions, and give rise to compounds of unvarying constitutions described by a formula. But may we not also cite analogies among the phenomena of molecular adhesion, flocculation, coagulation, emulsion, dyeing, stickiness and the like?

When we try to prove the principal proposition of Ehrlich and Morgenroth experimentally, namely, that the sensitizer can combine with the alexin without the presence of corpuscles, negative results are obtained; the alexin remains quite free, as was demonstrated in earlier experiments, to which the reader is referred.*

In endeavoring to prove their assertion Ehrlich and Sachs $\dagger$ have laid much stress on the supposed complement deviation evidenced by the well-known experiments of Neisser and Wechsberg on the antibacteriolytic effect of an excess of sensitizer in presence of a relatively small dose of alexin.

This complement deviation is due, according to the authors in question, to the fact that the excess of sensitizer which is refused by the saturated bacteria remains free in the fluid and takes up on its own account part of the alexin and so prevents it from destroying bacteria; this supposition was never proved experimentally. Several authors have recently questioned Neisser and Wechsberg's explanation of their phenomenon and have offered new explanations which have no part with the thesis defended by the Ehrlich school. (Gay, $\ddagger$ Moreschi, $§$ Buxton.\|)

* Bordet, The cytolytic sera, etc., p. 228.

$\dagger$ Ehrlich and Sachs, Studies on Immunity, Ehrlich-Bolduan, John Wiley \& Sons, p. 217.

$\ddagger$ Gay, see p. 357 .

\$ Moreschi, Berliner klinische Wochenschrift, 1906, p. 100.

|| Buxton, Journal Med. Research, XII, 1905, 431. 
In their studies on snake venoms Kyes and Sachs * found an apparent confirmation of this explanation of complement deviation, but Hideyo Noguchi $\uparrow$ later showed that their phenomena were to be explained quite differently.

There is, then, no valid reason for conceiving, as Ehrlich and Morgenroth do, that, when an alexin is not able to destroy a given corpuscle sensitized with a certain sensitizer, the failure is due to the inability of the alexin to combine with the sensitizer in question. The fact that an alexin does or does not destroy in a given case is not dependent on its adaptability or non-adaptability to a suppositious complementophilic group of the sensitizer employed. The designations "passende" or "nicht passende Komplemente" do not indicate any real condition.

And, indeed, with what alexin should a sensitizer in horse serum most readily unite logically? It is evident that it should combine with an alexin from the same animal species - the horse. But as a matter of fact, although horse serum contains a sensitizer that hemolyzes guinea-pig corpuscles in conjunction with guinea-pig alexin, no such result occurs with horse alexin.

Are we to suppose that this sensitizer unites with the first alexin better than with the second? Or is it not more reasonable to conclude that both alexins are absorbed by the sensitized corpuscles, but that the horse alexin is simply less toxic and less liable to cause hemolysis? $\ddagger$

As another example we may note that normal rabbit serum contains a sensitizer for goat corpuscles. This sensitizer, however, is much more effective with guinea-pig alexin (which alone does not hemolyze, as the serum of the guinea-pig contains no sensitizer) than with rabbit alexin.\$

* Kyes and Sachs, Studies on Immunity, Ehrlich-Bolduan, John Wiley \& Sons, p. 443.

$\dagger$ Noguchi, Jour. of Exp. Medicine, VII, 1905.

$\ddagger$ In the same way we shall later show that although horse alexin is well absorbed by sensitized ox corpuscles it fails to hemolyze them. Analogous instances have already been noted by Muir (Proceedings of the Royal Society, Vol. 74, 1904, 305) and by Gay (this volume, p. 336).

\$ Experiment. One cubic centimeter of a 10 per cent suspension of goat blood in salt solution is placed in each of four tubes. To tube " $a$ " is added 0.4 of a cubic centimeter of fresh rabbit serum, to tube " $b$ " 0.2 of a cubic centimeter of fresh guinea-pig serum, to tube " $\mathrm{c}$ " 0.2 of a cubic centimeter of rabbit serum 
The important factor that Ehrlich and Morgenroth neglect is that each alexin has a definite toxic property of its own for a given corpuscle. It is quite evident that the hemolytic or bacteriolytic power of serum will vary in different animal species as their alexins differ in intensity.

There is, however, one experiment reported by Ehrlich and Sachs* which, if the interpretation they give to it were correct, would prove indisputably that the alexin really unites with the sensitizer. It would actually seem in the experiment in question as if the sensitizer does unite with the corpuscles subsequent to its union with the alexin.

This alexin-sensitizer union would seem to be so indispensable that, if the alexin were destroyed, a real paralysis of the sensitizer would result, so that its affinity for the corpuscle would be destroyed or at least inhibited; in other words, the cytophilic group would seem to react with the corpuscle only after the complementophilic group, uniting with the alexin, is satisfied.

It remains to be seen, however, whether Ehrlich and Sachs have not entirely misinterpreted their own experiment. The sensitizer in question is present in inactivated (56 degrees) normal bovine serum, the corpuscles affected are from the guinea-pig, and the alexin employed is in the form of fresh horse serum.

We may first of all summarize the facts that Ehrlich and Sachs have noted. Bovine serum, inactivated at 56 degrees, naturally does not hemolyze guinea-pig corpuscles, as its alexin has been destroyed. Fresh (alexic) horse serum also has only the slightest hemolytic effect on these cells. The heated ox serum, however, apparently sensitizes the corpuscles so that they are hemolyzed when fresh horse serum is added. Strong hemolysis (Experiment I) is evident when guinea-pig corpuscles, heated bovine serum and fresh horse serum are mixed together in suitable doses. $\dagger$ So far there is plus 0.1 of a cubic centimeter of guinea-pig serum, and to tube "d" 0.4 of a cubic centimeter of rabbit serum plus 0.1 of a cubic centimeter of guinea-pig serum. Resultant hemolysis. Complete in one-half hour in " $\mathrm{d}$ "; complete in one hour in "c." There is only a trace of hemolysis in " $a$ " and none in " $b$ " in an hour.

* Ehrlich and Sachs, Studies on Immunity, Ehrlich-Bolduan, John Wiley \& Sons, p. 209.

$\dagger$ For example, 1 c.c. of a 5 per cent suspension of guinea-pig blood plus 0.3 of a cubic centimeter of bovine serum ( 56 degrees) plus 0.5 of a cubic centimeter of fresh horse serum. 
nothing remarkable in the experiment. But in a second experiment the corpuscles are placed in contact with the ox serum, and after a certain period the mixture is centrifugalized and the supernatant fluid decanted. On the addition of horse serum to the sedimented corpuscles no hemolysis takes place. It would seem, then (and such is the conclusion of Ehrlich and Sachs), that the corpuscles have failed to absorb the sensitizer from the ox serum in spite of contact with it. This conclusion seems further corroborated by the fact that the supernatant fluid that has been used in treating the corpuscles is just as active as before contact. Fresh guinea-pig corpuscles when added to this supernatant fluid plus fresh horse serum are readily hemolyzed. And, what is more, the treated corpuscles, which are not hemolyzed by fresh horse serum alone, are destroyed as readily as fresh corpuscles by a mixture of heated ox serum and horse alexin.

Ehrlich and Sachs' interpretation as already stated is as follows: The bovine sensitizer unites readily with the corpuscles as soon as its affinity for alexin (horse) is satisfied, in other words, when its complementophilic group is saturated. This is the reason that hemolysis takes place in a mixture of the two sera. But the sensitizer shows little or no affinity for the corpuscles unless previously combined with the alexin. This explains why the corpuscles remain intact when treated successively with ox serum and then with horse serum. If these interpretations are correct, as Ehrlich and Sachs affirm, we are forced to admit that a saturation of the complementophilic group by means of alexin increases the chemical affinity of the cytophilic group, in other words, the affinity for the corpuscles.

Theoretically, it is rather difficult to conceive of such a repercussion as this, since, according to Ehrlich's theory, the two atom groups are distinct and independent. It is better at all events to remain within the bounds of experimentation. The experiments which we have just mentioned are the only ones that Ehrlich and Sachs have referred to. As we shall see, it would have been preferable for them to have investigated somewhat further and to have adduced other experiments before offering their interpretation.

There is one fact in particular which, although remarkable and certainly of significance in any correct interpretation, seems to have 
escaped the attention of these writers. Heated bovine scrum has only a slight agglutinating property for guinea-pig corpuscles. Fresh horse serum does agglutinate them, but only slowly and when present in relatively large amounts; several hours may be required to clump the corpuscles in any considerable masses. A mixture of the two sera, however, agglutinates in a very few minutes, and the corpuscles soon form veritable chunks that stick to the glass. We may mention in this connection an experiment with fresh horse serum (alexin), bovine serum heated to 56 degrees, and a 5 per cent suspension of washed guinea-pig corpuscles; mixtures are prepared as follows:

1. Corpuscle suspension, 1 c.c.; bovine serum, 56 degrees, 0.5 of a cubic centimeter.

2. Corpuscles, 1 c.c.; horse serum, 0.5 of a cubic centimeter.

3. Corpuscles, 1 c.c.; 0.5 of a cubic centimeter of a mixture in equal parts of the two sera.

There is intense agglutination in 3 in a few minutes; hemolysis begins shortly after and later becomes complete; mixtures 1 and 2 not only are not hemolyzed, but show only a very delayed agglutination which is never comparable with the one in 3.

A slight variation in the experiment may be made by making mixtures 1 and 2 and then mixing them. The corpuscles rapidly agglutinate in a mixture of the two sera and are soon laked.* It should be noted at once that if horse serum heated to 56 degrees is added in place of fresh horse serum to the heated bovine serum there is not only no hemolysis, but also none of this intense agglutination. The agglutination, then, would seem in some way dependent on the presence of active alexin.

This is a rather curious condition. If an explanation were sought according to Ehrlich and Sachs' explanation, we should have to conclude that the agglutinin as well as the sensitizer must combine with the alexin before uniting with the corpuscle. Such a conclusion is unusual, for there are no facts that would lead us to assume that the agglutinins need alexins to be efficient.

Ehrlich and Sachs' theory of the mode of action of these two sera seems already open to criticism. We must therefore consider in

* This agglutination takes place at room temperature, but is more rapid at 37 degrees. 
detail the logic they have adopted in arriving at their conclusion. Let us follow, then, their argument step by step: First: In order to prove that the sensitizer of heated bovine serum does not combine with guinea-pig corpuscles when horse serum is absent, these authors rely on the fact that corpuscles treated with bovine serum remain intact when subjected to horse alexin. Such an argument is valid only on the supposition that horse alexin is capable of hemolyzing sensitized corpuscles. Such, however, is not the case. Horse alexin, indeed, differs from the alexins of most sera in this very respect. Ehrlich and Morgenroth have themselves noted* that bovine corpuscles, sensitized by an inactivated hemolytic serum from the rabbit, are not hemolyzed by horse alexin, although very small doses of rabbit or guinea-pig alexin suffice to destroy them. They have explained the fact by saying that the horse alexin does not "fit" the rabbit sensitizer, that is to say, fails to combine with the complementophilic group of this sensitizer; in other words, the alexin is not absorbed by the sensitized cells. It may be noted, however, that this latter fact is not true, for, as we shall later see, the alexin does become fixed by such corpuscles, although it fails to destroy them. $\dagger$

There is no proof, then, that because guinea-pig corpuscles treated by bovine serum remain intact in horse alexin, it is due to their not being sensitized, since, when they are undoubtedly sensitized, they give no hemolysis with this alexin. If, indeed, we take guineapig corpuscles, sensitized by a specific inactivated serum from the rabbit, we find that, although they are hemolyzed by traces of fresh rabbit serum or even by guinea-pig serum, they remain intact in moderate doses of horse alexin and are destroyed by large doses only after a long period.

It is, moreover, easy to prove that there is a moderately powerful sensitizer in bovine serum acting on guinea-pig corpuscles in the ordinary way, that is, by uniting with them when no alexin is present. A few tenths of a cubic centimeter of bovine serum ( 56 degrees) will hemolyze 1 c.c. of a 5 per cent suspension of guinea-pig cor-

* Ehrlich and Morgenroth, Studies on Immunity, Ehrlich-Bolduan, John Wiley \& Sons, p. 88.

$\dagger$ This fact might be stated in the Ehrlich parlance by saying that horse alexin has no toxophore group or that this group is too weak. 
puscles on the addition of guinea-pig alexin ( 0.3 of a cubic centimeter). No hemolysis, however, occurs if, instead of ordinary heated bovine serum, the same serum previously treated with guineapig corpuscles and separated from them by centrifugalization is used. Such corpuscles have absorbed the sensitizer.

Second: Ehrlich and Sachs think that the bovine serum (56 degrees) is the only sensitizing serum in their experiment. No proof of this is given, and, as a matter of fact, the horse serum also contains a sensitizer for guinea-pig corpuscles which is usually stronger than the one in bovine serum. A mixture of horse serum ( 0.3 of a cubic centimeter) and guinea-pig corpuscles ( 1 c.c. of a 5 per cent suspension) gives hemolysis on the addition of guineapig alexin ( 0.3 of a cubic centimeter). It is not surprising that fresh horse serum alone fails to hemolyze the blood, although it contains both alexin and sensitizer, when we take into consideration, as just shown, that the horse alexin fails to hemolyze these corpuscles even when they are well sensitized. It is to be noted in passing that the horse sensitizer is very thermolabile, being almost entirely deprived of its power by heating to 56 degrees.

Third: Ehrlich and Sachs think that ox serum acts only as a sensitizer in their experiment.

Is it true that the bovine serum owes its entire or even the greater part of its efficacy to its sensitizing property? May there not also be, in addition to the sensitizer, some particular substance in bovine serum that has not as yet been described in this or in other sera? No such possibility has occurred to Ehrlich and Sachs, who regard the bovine serum simply as containing a sensitizer. It is evident that to decide such a question the sensitizing property of the serum must first be removed. In other words, we must have conditions in which the sensitizer not only need not but actually does not enter into consideration. If, under such conditions, heated bovine serum, although without a sensitizer, hemolyzes corpuscles with horse alexin, we must conclude that the serum contains some other as yet unsuspected substance that is of capital importance in the experiment.

These conditions are easy to fulfill by adding well-sensitized bovine blood corpuscles* instead of guinea-pig corpuscles to a

* Previously treated with immune serum from the rabbit and then washed. 
mixture of heated bovine serum and horse alexin. It is evident in such an experiment that not only is a sensitizer in the bovine serum unnecessary, but that no such an iso- or auto-sensitizer can exist.*

We take, then, bovine corpuscles that have been well sensitized by immune serum from the rabbit. On the addition of alexic horse serum neither hemolysis nor agglutination occurs. $\dagger$ But the addition of the horse serum plus heated bovine serum produces an extreme agglutination followed by slow but distinct hemolysis.

The details of this experiment are as follows:

Well-washed bovine corpuscles are treated with three volumes of rabbit antibovine serum. Two or three hours later the excess of serum is removed by washing in salt solution and the supernatant fluid rejected. Salt solution is then added to the corpuscle sediment so as to make a 20 per cent suspension. Three tubes are made as follows:

Tube 1. Fresh horse serum, 0.3 c.c.

Tube 2. Bovine serum (56 degrees), 0.3 e.c.

Tube 3. Fresh horse serum, 0.3 c.c. + bovine serum, 56 degrees, 0.3 c.c.

To each tube is then added 0.3 of a cubic centimeter of sensitized bovine corpuscles. As a result, no agglutination or hemolysis in tubes 1 and 2; rapid agglutination followed by hemolysis in tube 3.

Such a result is unexpected and paradoxical in view of current ideas on sera. It is quite conceivable that sensitized ox corpuscles should remain intact in horse alexin, for we know that the alexins of certain species are too weak, or are unsuitable for hemolysis. But the fact that the addition of the very serum that should be most inactive, namely, the proper serum of the corpuscles employed, should destroy the corpuscles is at least peculiar. This serum, moreover, has lost its alexin through heating. It is also a surprising fact that this form of agglutination requires the coöperation of two sera, neither of which alone affects the corpuscles in question.

The analogy between this experiment and the one described by

* In most of these experiments bovine serum and corpuscles from the same individual were employed.

$\dagger$ It is also to be noted that the rabbit antibovine serum, although highly sensitizing, has little or no agglutinating property. 
Ehrlich and Sachs is evident. In their experiments guinea-pig corpuscles which remain intact, either in heated bovine serum or in fresh horse serum, are destroyed by a mixture of the two. In our experiment sensitized ox corpuscles act in the same manner. As far as agglutination is concerned the analogy also is perfect. It is probable, then, that both experiments are subject to a common explanation. And, what is more, the explanation of Ehrlich and Sachs would already seem untenable.

It is not to be supposed that bovine serum contains an amboceptor that unites with its own corpuscle and, on the other hand, with the alexin of horse serum, or that this combination with the alexin is necessary to produce a hypothetical union with the corpuscles. A careful study of the facts, moreover, renders such a supposition quite impossible. We may consider, then, in detail the data offered by the experiment that we have reported.

a. Is it necessary, in order to produce agglutination and hemolysis of bovine corpuscles by means of horse serum and heated bovine serum, that these corpuscles should be sensitized? We add to 0.5 of a cubic centimeter of a 20 per cent emulsion of unsensitized corpuscles 0.3 of a cubic centimeter of each serum. Nothing occurs and the corpuscles remain intact. Experiment shows, then, that the corpuscles must be sensitized in order to be agglutinated and destroyed, and that the necessary amboceptoracting upon ox corpuscles is not present in ox serum. Experiment further shows that horse serum has no distinct sensitizing effect for bovine blood corpuscles.

$b$. The presence of alexin in the mixture is obviously necessary for hemolysis; but is it equally indispensable for agglutination? We add 0.3 of a cubic centimeter of heated bovine serum and 0.3 of a cubic centimeter of horse serum heated to 56 degrees to 0.5 of a cubic centimeter of a suspension of sensitized bovine corpuscles. There is no hemolysis or agglutination.

c. Does the horse serum simply furnish the alexin or does it also furnish the principle that causes agglutination? If it only furnishes the alexin it may evidently be replaced by any other fresh serum, as, for example, fresh rabbit serum. This proves to be the case, as the following experiment shows: We add to each of two tubes 0.5 of a cubic centimeter of a suspension of sensitized bovine corpuscles and to the first tube add 0.2 of a cubic centimeter 
of rabbit alexin; to the second 0.2 of a cubic centimeter of rabbit alexin plus 0.3 of a cubic centimeter of heated bovine serum. The corpuscles in both tubes hemolyze, as the rabbit alexin is strongly hemolytic for the sensitized corpuscles. But the hemolysis is preceded in the second tube by an extraordinary agglutination which does not occur in the first. A control tube shows that neither agglutination nor hemolysis would have taken place if an emulsion of normal bovine corpuscles, instead of sensitized bovine corpuscles, had been employed. It is, then, the bovine serum and not the horse serum that furnishes the agglutinating principle, and, as we have already determined, agglutination takes place only when the corpuscles are treated both with a sensitizer and with an alexin. The origin of this alexin, however, is indifferent and may be either from horse serum, rabbit serum or even, as we shall see, from bovine serum itself.

We add to each of two tubes 0.2 of a cubic centimeter of fresh non-heated bovine serum. To the first tube we then add 0.5 of a cubic centimeter of normal bovine corpuscles and to the second tube an equal amount of bovine corpuscles that have already been treated with rabbit antibovine serum. There is a rapid agglutination of the corpuscles in the second tube, but none in the first.

To sum up, we find that, in presence of any alexin, bovine serum will agglutinate corpuscles of the same species (and even of the same animal) provided they be sensitized. When the alexin is only weakly hemolytic, as is the case with horse alexin, its task is greatly facilitated by the addition of bovine serum. In other words, this serum not only agglutinates sensitized corpuscles that have been subjected to alexin, but changes these corpuscles in such a way that alexin that might otherwise be impotent becomes strong enough to produce hemolysis. This explains why sensitized bovine corpuscles which show no effect when subjected to horse alexin are agglutinated and hemolyzed when heated bovine serum is added.*

Bovine serum causes bovine corpuscles to be more easily destroyed

* It is to be noted that we do not say that it is on account of its agglutination that ox serum renders corpuscles more accessible to the action of a weak alexin. What we do say is, that bovine serum renders corpuscles more accessible to alexic activity and in addition produces a very marked agglutination. It is not owing to the fact that the corpuscles are clumped that they become more susceptible to the alexin, as we shall later see. 
by horse alexin; one might almost say that it increases their sensitivity. We purposely avoid using this expression, since it might lead to a supposition that bovine serum contains a real sensitizer for its own corpuscles. If the term sensitization were loosely used, it might, perhaps, be employed in this case, that is to say, if it were used in the sense of rendering the corpuscles more susceptible to destruction, as is the case when the amount of sodium chloride present is diminished; the word sensitization, however, has been used in a more exact sense by one of us for some time, which suffices to prohibit its use in the present instance as applied to bovine serum. We know, indeed, that the true sensitizers do not affect their proper corpuscles and, on the other hand, that corpuscles that have once been properly sensitized do not need the aid of another sensitizer in order to be hemolyzed by alexin. In a similar way it is evident that this substance in bovine serum should not be confused with the ordinary agglutinins, although it does lead to an agglutination.

We find, in fact, only one logical explanation for this peculiar activity of bovine serum; and in order to elucidate the following discussion we may announce this explanation at once, although it is experimentally proved only in subsequent pages. We believe that there exists in bovine serum a peculiar substance that resists heating to 56 degrees (and, it may be added, is retained in heated serum for months), of an apparently albuminous and colloidal nature, which shows no effect on red blood cells so long as they are under normal conditions, but which unites with them as soon as they are laden with sensitizer and alexin. We have to deal, we believe, with a pure phenomenon of molecular adhesion. From the point of view of properties of molecular adhesion it is evident that normal corpuscles differ from the complex that results in a mixture of corpuscles, sensitizer and alexin, inasmuch as this complex has the property of attracting and binding to it this substance in ox serum that the normal corpuscle does not possess. The adhesion between this substance and sensitized and alexinized corpuscles produces their agglutination in large clumps and also leads to a modification in them which renders them more easily hemolyzed by alexins of moderate potency.

We shall refer briefly from this time on to this substance in bovine 
serum that attaches itself to sensitized and alexinized corpuscles as "bovine colloid."

Before we endeavor to explain the accuracy of the interpretation that we have just offered, we may apply it to the hemolysis of guineapig corpuscles, since these are the corpuscles that are used in Ehrlich and Sachs' experiment. To render the transition between the experiments we have just recounted and the experiment of Ehrlich and Sachs more simple, we may deal first with guinea-pig corpuscles that have been sensitized in the same manner as were our bovine corpuscles, that is to say, with a specific antiguineapig serum (a serum, heated to 56 degrees, from a rabbit that had been immunized against guinea-pig blood).

These sensitized guinea-pig corpuscles are hemolyzed by fresh guinea-pig serum, although the hemolysis is rather slow, particularly when the amount of alexin employed is small, owing to the fact that the alexin comes from the same animal species. Under such conditions, by analogy with the experiments already considered, the addition of heated bovine serum should have a distinct accelerating action on the hemolysis. The addition of this serum, moreover, to these sensitized corpuscles that have been mixed with alexin should bring about very marked agglutination.

These expectations are experimentally confirmable. Sensitized guinea-pig corpuscles are, to be sure, already distinctly agglutinated by rabbit antiguinea-pig serum, but as soon as the alexin and heated bovine serum are added the agglutination becomes much more marked. The corpuscles are immediately collected into large glistening clumps which are soon hemolyzed. The bovine serum produces no such agglutination with the sensitized corpuscles when no alexin is present; the bovine colloid, we repeat, affects corpuscles only when they have been both sensitized and alexinized. The analogy, then, between this experiment and the one with sensitized ox corpuscles is complete. The details of the experiment follow:*

* It is to be noted that the particular rabbit antiguinea-pig serum that we have used for this experiment was obtained by injecting rabbits with carefully washed guinea-pig red blood cells. This antiguinea-pig serum was neither precipitating nor anti-alexic for guinea-pig serum; if it had been, the experiment might have been vitiated by a neutralization of the guinea-pig alexin in the tubes 3 and 4 following. 
The following mixtures are made:

Tube 1. Guinea-pig alexin, 0.1 c.c.; bovine serum ( 56 degrees), 0.3 c.c.

Tube 2. Rabbit antiguinea-pig serum ( 56 degrees), 0.15 c.c.; bovine serum (56 degrees), 0.3 c.c.

Tube 3. Guinea-pig alexin, 0.1 c.c.; rabbit antiguinea-pig serum (56 degrees), 0.15 c.c.; bovine serum ( 56 degrees), 0.3 c.c.

Tube 4. Guinea-pig alexin, 0.1 e.c.; rabbit antiguinea-pig serum (56 degrees), 0.15 c.c.

Tube 5. Rabbit antiguinea-pig serum, 0.15 c.c.

To each tube is then added 0.5 of a cubic centimeter of a 5 per cent suspension of washed guinea-pig blood. At room temperature the following results occur:

In tube 3 , in which the bovine serum affects sensitized and alexinized corpuscles, a very powerful agglutination occurs in a few moments, and hemolysis is complete in 10 minutes. In mixture 4, which is identical with 3 except in not containing bovine serum, there is only slight agglutination, and hemolysis is incomplete in half an hour. Tubes 2 and 5 that contain no alexin give a slight agglutination, but no hemolysis. In tube 1 that contains alexin, but no sensitizer, the corpuscles are not agglutinated and show only. a very slow partial hemolysis which is incomplete on the following day. This hemolysis is due, as we have already seen, to the fact that heated bovine serum contains a weak sensitizer for guinea-pig corpuscles.*

Now that we have determined that the heated bovine serum acts by adhesion of the colloidal substance on sensitized and alexinized corpuscles both of the ox and of the guinea-pig, Ehrlich and Sachs' experiment is readily understood. It may be explained just as the preceding experiments, namely, by supposing that in the mixture of fresh horse serum and heated bovine serum the

* This mixture containing alexin and corpuscles sensitized simply with heated bovine serum (and not with the specific antiserum) is not sufficiently sensitized or fixed with alexin to bring about an energetic adhesion of the colloid; this latter substance, as we shall later see, under these conditions is somewhat absorbed, so that hemolysis although slight is increased.

It is to be noted that the weakness of the bovine sensitizer is due perhaps to heat. This substance indeed appears to be more powerful in unheated bovine serum. We find that guinea-pig corpuscles are energetically clumped by fresh serum, which shows that the colloid has adhered; this means, of course, that they have been well sensitized and also alexinized by the fresh bovine serum; hemolysis subsequently occurs. 
guinea-pig corpuscles become laden with sensitizer and alexin and are therefore susceptible to absorb the colloidal substance which brings about agglutination and facilitates hemolysis.

Which of the two sera is it that produces the sensitization? We have already seen that heated bovine serum contains a sensitizer inasmuch as its addition to a mixture of corpuscles and guinea-pig alexin produces a distinct hemolysis. This sensitizer may produce some effect in the experiment, but it is by no means necessary, because we have already found that heated bovine serum treated with guinea-pig corpuscles and thereby deprived of its sensitizer for these corpuscles retains intact its property of endowing fresh horse serum with a hemolytic property. It is, moreover, easy to understand why the bovine sensitizer is not necessary inasmuch as another sensitizer of superior potency is present in Ehrlich and Sachs' experiment. This is the sensitizer contained in fresh horse serum in addition to the alexin.*

Our interpretation of Ehrlich and Sachs' experiment is therefore the following: When guinea-pig corpuscles are added to a mixture of the two sera they are affected by the sensitizer of the horse serum and to a certain extent by the sensitizer in heated bovine serum. This second sensitizer is, however, superfluous. Its presence is by no means necessary for the experiment. When this sensitization is effected the corpuscles are then in a condition to fix the horse alexin. This alexin, however, has only slight hemolytic power. But once the corpuscles have become sensitized and laden with alexin, they are modified in their properties of molecular adhesion to such an extent that they become able to attract the colloidal substance of bovine serum, which unites with them. The adhesion of this new substance produces two results: It causes the blood corpuscles to be more easily destroyed by alexin and also agglutinates them energetically. Consequently, a powerful clumping followed by hemolysis is observed.

This interpretation explains all the facts that have been noted by Ehrlich and Sachs. It is evident, for example, that: $(a)$ bovine serum (56 degrees) that has been treated with guinea-pig corpuscles will retain its property of forming a hemolytic mixture with horse

* It is to be recalled that the demonstration of this sensitizer is easy. Guineapig corpuscles are hemolyzed by a mixture of guinea-pig alexin and horse serum. 
serum. The guinea-pig corpuscles, in other words, may remove all the sensitizer from the bovine serum, since this substance is not necessary in the hemolysis, but they cannot remove the colloidal substance. This colloidal substance is absorbed only by corpuscles that have been treated both with sensitizer and alexin.

(b) These treated guinea-pig corpuscles that have fixed the sensitizer but not the colloidal substance of bovine serum are not hemolyzed if the excess of serum is removed and horse serum added. Such corpuscles, although they are sensitized and fix the alexin in the horse serum, are not subjected to the effect of the colloidal substance, which has been removed in removing the excess of bovine serum.

Inasmuch as the preceding experiments, and in particular those dealing with bovine corpuscles, have shown that Ehrlich and Sachs' interpretation formulated on purely theoretical and preconceived grounds is inacceptable, we feel scarcely called upon to consider it further. It is well, however, to verify the accuracy of our own explanation.

First: We think that the fixation of the colloidal substance occurs only when the corpuscles have been treated with sensitizer and alexin. If this be true we should expect that corpuseles so sensitized and alexinized and subsequently carefully washed in salt solution would absorb the colloidal substance of heated bovine serum added to it. We should not expect the presence of free alexin to be necessary for this absorption.

Inasmuch as clumping is a visible symptom of the absorption of the colloid, we should expect sensitized, alexinized and subsequently washed corpuscles to show this phenomenon on the addition of heated bovine serum. In such an experiment the sensitized corpuscles must of necessity not be destroyed by the alexin, and for this purpose horse serum which has only slight hemolytic properties is best.

We prepare, then, a 10 per cent suspension of ox blood that has been sensitized with a specific serum from the rabbit and subsequently washed, and also a 10 per cent suspension of nonsensitized ox blood. The following mixtures are made in three large tubes: 
(a) Suspension of sensitized corpuscles, 1 c.c.; fresh horse serum, 0.3 c.c.

(b) Sensitized corpuscles, 1 c.c.; horse serum, 56 degrees, 0.3 c.c.

(c) Non-sensitized corpuscles, 1 c.c.; fresh horse serum, 0.3 c.c. Half an hour later the tubes are filled with salt solution, centrifugalized and the supernatant fluids decanted; this washing is repeated. After the second decanting 1 c.c. of salt solution plus 0.4 c.c. of bovine serum ( 56 degrees) is added to each sediment of corpuscles. The result is, no agglutination in tubes " $b$ " and " $c$," but a strong and almost instantaneous agglutination in tube " $a . "$

Second: We have just said that the colloidal substance is absorbed by sensitized and alexinized corpuscles. If this is true, heated bovine serum that has been treated with such corpuscles should not be able to agglutinate fresh corpuscles. Experiment verifies this expectation. Well-sensitized bovine corpuscles are washed, restored to primitive volume and mixed with three parts of fresh horse scrum. Two hours later the corpuscles are washed again, restored to primitive volume and an equal amount of heated bovine serum is added. The bovine serum is found, subsequent to this contact, to retain little if any of its colloidal substance.*

Third: Bovine serum that has lost the greater part of its colloidal substance in this manner should show very much less activity than intact serum when mixed with fresh horse serum and guinea-pig corpuscles. We find, indeed, that agglutination and hemolysis of these corpuscles are very much less in the presence of this exhausted serum than with a corresponding dose of untreated bovine serum or with bovine serum that has been treated simply with guinea-pig corpuscles and has thereby lost its sensitizer only.

Fourth: The colloidal substance is absorbed and produces agglutination only when the corpuscles are alexinized as well as sensitized. In the Ehrlich and Sachs' experiment it is evidently horse serum that furnishes the alexin. We should expect, then, that if, to a mixture of guinea-pig corpuscles and heated bovine serum, horse serum that has been treated with any sensitized corpuscles

* It is to be noted that very small amounts of colloidal substance suffice to agglutinate sensitized and alexinized corpuscles. A complete removal of the colloidal substance would therefore be necessary to bring about an entire loss of agglutinating property. 
is added, the corpuscles would show neither hemolysis nor agglutination. Experimentally we find this to be true. Bovine blood is sensitized with three volumes of serum from an immunized rabbit. The corpuscles are then washed, the original volume of the blood reëstablished and an equal amount of fresh horse serum added. After a time this mixture is centrifugalized and the supernatant fluid is employed as horse serum deprived of alexin. As a control we use horse serum that has been treated in exactly the same manner with unsensitized bovine corpuscles. The following mixtures are then prepared:

(a) 5 per cent suspension of guinea-pig corpuscles, 1 c.c.; bovine serum ( 56 degrees), 0.3 c.c.; treated horse serum, 0.6 c.c.

(b) Corpuscle suspension, 1 c.c.; bovine serum (56 degrees), 0.3 c.c.; control horse serum, 0.6 c.c.

(c) Corpuscle suspension, 1 c.c.; bovine serum (56 degrees), 0.3 c.c.; a mixture of equal parts of salt solution and fresh horse serum, 0.6 e.c.

As a result, agglutination appears rapidly in " $b$ " and " $c$," followed by hemolysis. There is no agglutination or hemolysis in " $a$."

The same experiment with sensitized ox corpuscles in place of guinea-pig corpuscles gives a similar result.

It is to be noted in passing that this experiment also furnishes a proof of the functional unity of the alexin. Horse serum that has been deprived of alexin for bovine corpuscles has likewise lost its alexic activity for guinea-pig corpuscles. Both species of corpuscles, therefore, are sensitive to the same alexin.

Fifth: According to our idea, in Ehrlich and Sachs' experiment the fresh horse serum not only furnishes the alexin for the guinea-pig corpuscles, but is also of prime importance in sensitizing them. Guinea-pig corpuscles that have been placed in contact with fresh horse serum alone, and then washed and so deprived of the excess of serum, should be both sensitized and alexinized, in other words, able to fix the colloidal substance; they should therefore be agglutinated on the addition of heated bovine serum, whereas normal guinea-pig corpuscles will show no such effect. We find this, experimentally, to be true. As a control, it is shown that corpuscles treated in the reverse manner, that is to say, by heated bovine serum first, washed, and then horse serum, show no agglutination. 
Tube $a$. Ten per cent suspension of guinea-pig corpuscles, 1 c.c.

Tube $b$. Ten per cent suspension of corpuscles, 1 c.c.; fresh horse serum, 0.3 c.c.

Tube $c$. Suspension of corpuscles, 1 c.c.; bovine serum (56 degrees), 0.3 c.c.

An hour later the tubes are filled with salt solution, the mixtures well shaken and centrifugalized. The supernatant fluids are then decanted and 1 c.c. of salt solution is added to each sediment. To tubes " $a$ " and " $b$ " is then added 0.3 of a cubic centimeter of bovine serum ( 56 degrees), and to tube " $c$ " 0.3 of a cubic centimeter of fresh horse serum. As a result, there is a powerful and almost immediate agglutination in " $b$," but nothing of the kind in " $a$ " or " $c$. "

Sixth: According to the preceding results we should expect that horse serum that has been treated with guinea-pig corpuscles would be deprived both of its sensitizer and of its alexin for these cells, and that consequently it would have no power to form an agglutinating and hemolytic mixture for fresh guinea-pig corpuscles with bovine serum. This exhaustion of horse serum by contact with guineapig corpuscles is easily verified experimentally, as Klein* has already shown. This fact, moreover, is quite irreconcilable with Ehrlich and Sachs' explanation. There is one fact, however, that must be noted in performing this experiment. The absorption of the active substances from horse serum takes place perfectly if the serum is mixed with a suspension of corpuscles, that is to say, corpuscles sufficiently diluted in salt solution. But if the salt solution is removed, that is, if a sediment of corpuscles is employed, the absorption is incomplete and the serum retains the larger part of its original properties. This fact has also been noted by Klein. This would show that the fixation of active principles in a serum is influenced by very slight variations, the importance of which well may be overlooked, and, consequently, the usual method of specific absorption as employed by Ehrlich and his followers in the study of hemolysis may lead to erroneous conclusions. In the present example, for instance, one might be led to conclude that the cor-

* Ueber die Beeinflussung des hämolytischen Komplements durch Agglutination und Präzipitation. Wiener klinische Wochenschrift, 1905, No. 48. 
puscles did or did not possess receptors for the active substances of horse serum in accordance with whether salt solution was or was not present. One of us and also Landsteiner have already noted the causes of error in experiments in specific absorption of this nature. The following experiment will illustrate this point:

Tube $a$. Fresh horse serum, 0.4 c.c.; washed guinea-pig blood, 40 per cent suspension in salt solution, 1 c.c.

Tube $b$. Fresh horse serum, 0.4 c.c.; the sediment of corpuscles derived from 1 c.c. of 40 per cent suspension.

The tubes are centrifugalized after an hour's contact and the supernatant fluids " $a$ " and " $b$ " are poured into tubes " $a a$," which contain 0.05 c.c. of pure guinea-pig blood, and " $b b$," which contain this amount of blood plus 1 c.c. of salt solution. To each tube " $a a$ " and " $b b$ " is then added 0.4 c.c. of bovine serum (56 degrees). As a result, there is no agglutination or hemolysis in tube " $a a$," but in tube " $b b, "$ on the contrary, agglutination and hemolysis are almost as rapid and powerful as in a control mixture containing 0.05 c.c. of blood, 1 c.c. of salt solution, 0.04 c.c. of horse serum and 0.4 c.c. of bovine serum ( 56 degrees).

We then take the sediments in tubes " $a$ " and " $b$ " respectively, and add to each 1 c.c. of salt solution plus 1 c.c. of heated bovine serum. The corpuscles in " $b$ " show only slight and very gradual agglutination; the corpuscles in " $a$ " are much more rapidly agglutinated, which means that they have absorbed the colloidal substance better, and are better sensitized and alexinized.

In the same way, as we find that the active substances of horse serum are more easily absorbed in the presence of salt solution, we also find that agglutination and hemolysis of guinea-pig corpuscles in a mixture of bovine serum plus horse serum occur better when the mixture contains a certain amount of physiological solution than when it does not.

Seventh: In Ehrlich and Sachs' experiment the sensitization of the corpuscles is essentially brought about, as we have already seen, by horse serum. This serum would naturally lose its alexin when added to sensitized bovine corpuscles, and would keep its sensitizer for guinea-pig blood.

If we should add, then, to such a serum alexin in the form of fresh guinea-pig serum, it should recover its property to hemolyze guinea- 
pig corpuscles, and the property would become more energetic if one added in addition heated bovine serum.

To make the experiment more demonstrative, the bovine serum may first be deprived of its sensitizer for guinea-pig corpuscles by contact with them; in this case its action is due entirely to its colloidal substance, the sensitizer being furnished by the horse serum.

Under these conditions a mixture of guinea-pig corpuscles, bovine serum ( 56 degrees) that has been treated with an equal volume of guinea-pig corpuscles, fresh horse serum that has been treated with an equal volume of sensitized bovine corpuscles, and guinea-pig alexin, gives a marked hemolysis.

Proper controls show that, in this experiment, if bovine serum is not present the hemolysis by the treated horse serum plus guineapig alexin is much slower, and also, in another control, that, if horse serum is not present, treated bovine serum plus alexin from the guinea-pig produces no hemolysis.

Eighth: One final remark on the hemolysis of guinea-pig corpuscles by heated bovine serum plus guinea-pig alexin occurs to us. It is evident that in such a mixture the colloidal substance may take a certain additional part, inasmuch as the corpuscles are somewhat sensitized by the bovine serum, and therefore capable of fixing the alexin and subsequently of absorbing a certain amount of colloid. This latter substance, as we know, tends to increase hemolysis.

Under these conditions the corpuscles should be more actively destroyed than when they have been first sensitized by addition of bovine serum and then washed and guinea-pig alexin subsequently added. If we proceed in this manner we suppress the colloid absorption by sensitized and alexinized corpuscles. In other words, we find experimentally that hemolysis is better when the bovine serum is left with the corpuscles and the alexin than when added to the corpuscles, and the excess eliminated before the fresh guineapig serum is added.

Of course no such peculiarity occurs in the hemolysis of corpuscles in presence of alexin and horse serum. This latter serum, as we know, acts only as a sensitizer and shows none of the properties of the bovine colloid.

The interpretation of Ehrlich and Sachs' experiment that we have offered is therefore experimentally confirmable. There is, 
however, one fact that at first sight might seem not to agree with our explanation.

Wher guinea-pig corpuscles are treated with a sufficient amount of fresh horse serum, washed, and then added to heated bovine serum, an energetic agglutination occurs, due to the absorption of the colloidal substance by sensitized and alexinized corpuscles. It would also seem as if under these conditions an active hemolysis should take place, since we know that hemolysis is also favored by the addition of colloid. The hemolysis under these conditions, however, is very slow and slight, although the agglutination is rapid and strong.

It is to be noted that in such an experiment the alexin and colloid are fixed successfully and at relatively long intervals apart. It would seem as if the fact that the alexin had been acting for a long time had increased the properties of molecular adhesion on the part of the corpuscles to its maximum, and as if the exaggerated absorption of the colloid had brought about an unusually intense agglutination. In other words, it would seem as if there were some antagonism between hemolysis and agglutination. It would seem as if, when agglutination is too powerful, the corpuscles would, so to speak, coagulate, and so become more resistant to alexin. The proof of this supposition, which incidentally brings out the fact that absence of hemolysis in the case in point in no way disagrees with our interpretation, may be experimentally shown in the following manner: Corpuscles that have been subjected to horse serum and subsequently to a strong agglutination by heated bovine serum will resist hemolysis on the subsequent addition of both horse serum and bovine serum, in other words, when subjected to a mixture that is highly hemolytic for ordinary corpuscles. The agglutinated corpuscles, then, have a greater resistance than the normal corpuscles.

In short, the hemolysis due to these three substances is a phenomenon dependent on the manner in which the various factors are added, and its mechanism is so delicate as to be easily destroyed by varying the experimental conditions.

The reverse of this condition may be produced also, that is to -say, an active hemolysis accompanied by a slight agglutination. To increase this hemolytic tendency a small amount of guinea-pig alexin is added to the mixture of bovine serum plus horse serum. 
For example, hemolysis is much more rapid in a mixture of 1 c.c. of 5 per cent guinea-pig blood plus 0.3 of a cubic centimeter of heated bovine serum plus 0.3 of a cubic centimeter of horse serum plus 0.3 of a cubic centimeter of fresh guinea-pig serum than when the guinea-pig alexin is omitted. In the first instance the agglutination is little or none, whereas in the second it is strong.

Hemolysis and agglutination, therefore, are not inseparable and interdependent, but are two distinct results of a single phenomenon, namely, the absorption of the colloid by sensitized and alexinized corpuscles.

\section{Conclusions.}

1. As we know from Ehrlich and Sachs' experiment, guineapig corpuscles are hemolyzed by a mixture of fresh horse serum and heated bovine serum ( 56 degrees), but they resist hemolysis when they are treated first with bovine serum, and the horse serum is subsequently added. The interpretation of Ehrlich and Sachs, which supposes that the sensitizer in bovine serum will not unite with corpuscles until it has first combined with the alexin, is inexact. In the first place the more powerful and necessary sensitizer is not in the bovine serum, but in the horse serum. Both these sensitizers, moreover, act as do other sensitizers in that they do not need alexin in order to unite with corpuscles. In their interpretation, moreover, Ehrlich and Sachs make no mention of an essential factor which gives rise to the remarkable and peculiar appearance of the hemolysis in question.

2. This factor is a substance present in bovine serum that resists heat to 56 degrees and may be kept for some time. It is colloidal in nature, and is doubtless some albuminous substance which has the property of uniting with corpuscles that are laden with sensitizer and alexin and which remains free in the presence of normal or of simply sensitized corpuscles. This explains why the guinea-pig corpuscles not only are destroyed by a mixture of the two sera, but the reason of their agglutination in voluminous masses.

3. The absorption of this colloid by sensitized and alexinized corpuscles is probably due to molecular adhesion due to a change in the corpuscles brought about by the two substances with which they have been treated. If both sensitizer and alexin are present, 
corpuscles of any kind, and even from the same individual that furnishes the colloid, give the phenomenon.

4. The presence of this colloid cxplains the peculiarities that have been noted by Ehrlich and Sachs. It is, moreover, most evident in the experiments which we have detailed for the purpose of outlining the mode of action of the various substances that intervene in the agglutination and hemolysis under consideration.

5. Inasmuch as Ehrlich and Sachs' interpretation is false, it is evident that the single argument that would seem to prove Ehrlich's thesis of a separate complementophilic atom complex in the sensitizer must be rejected. And, accordingly, the terms "amboceptor" and "complement" should be abandoned as erroneous. 


\section{ON THE NATURE OF OPSONINS.*}

By J. G. SLEESWIJK (Leyden).

In a previous article I have reported some experiments bearing on the function of the so-called opsonins in cellular immunity. $\dagger$ I demonstrated a thermolabile substance in frog serum which appears indispensable for phagocytosis of the anthrax bacillus.

I have since continued my studies on this subject for the purpose of answering the familiar question: Are the opsonins individual substances sui generus, or are they identical with known immune bodies? I may refer to a few of the many publications on opsonins which are of particular importance in this connection.

It may be stated at once that my work has led to the conclusion that the non-specificity or, better, non-autonomy of the opsonins seems to me fully established.

The subject, indeed, is far from new. Metchnikoff and his pupil Bordet in his studies on preventive sera, $\ddagger$ and later Savtchenko $\S$ with hemolysis, and indeed all investigators that have worked with antisera, have noted their favoring influence on phagocytosis. The explanations of this influence have, however, varied: some have thought that the bacteria are prepared for the action of an intracellular or an extracellular alexin by specific sensitizers, whereas Metchnikoff assumes the existence of stimulins with a direct influence on the leucocytes.\|

For a long time attempts have been made to find specific substances whichactasintermediaries between bacteria and phagocytes, since we know that there are preventive sera which have not the

* Ueber den Bau der Opsonine. Cent. f., Bakt., I. Orig., XLVI, 1908, 513.

$\dagger$ Annales de l'Institut Pasteur, December, 1907.

$\ddagger$ On the mode of action of preventive sera, p. 81 .

§ Savtchenko. Annales de l'Institut Pasteur, 1902.

I| Contrary to the opinion of certain investigators (Sauerbeck, Leishman), Metchnikoff still supports his stimulin theory. Compare Lubarsch and Ostertags' Ergebnisse, 1907. 
slightest bactericidal action in vitro, but act particularly as stimulants to phagocytosis.

Denys and Leclef* showed that this was the case with antistreptococcus serum, and Neufeld and Rimpau $\uparrow$ later corroborated these findings for the streptococcus and extended them to the pneumococcus. They called these substances, which they supposed to exist in the immune sera and which they believed to act favorably on phagocytosis, bacteriotropins.

But even before that time Wright, working with Douglas and several other collaborators, had perfected a special technic by which it was possible to study the relations of phagocytes to bacteria during the process of phagocytosis. He demonstrated in the blood and in other body fluids substances which he called opsonins. These substances prepare the bacteria for phagocytosis by becoming fixed upon them. They are, in general, thermolabile, greatly increased in the immune sera, and specific.

These opsonins, which are considered individual substances by some, are by others believed to be identical with the sensitizers or with the alexin. Recently Levaditi $\ddagger$ with two collaborators, Inmann and Koessler, assumed an intermediate position in that he believes the opsonins of normal serum to be identical with alexin and the opsonins of specific sera to be identical with the sensitizers. Neufeld and Hüne $\underline{\S}$ maintain, on the other hand, a distinctive position for their bacteriotropins. Muir and Martin, $\|$ in a series of experiments, using a technic which partially corresponds to that of my experiments (fixation of complement), have shown a marked similarity between alexin and opsonin.

Let us now consider what our own investigations have taught us. I wish first to report on the character of the opsonins in normal sera. In my first experiments I employed frog leucocytes, since I knew that after three washings with physiological saline solution they lose completely their power of ingesting anthrax bacilli, and that fresh serum will reactivate them by the fixation of the serum opsonin upon the bacteria. Furthermore, I had previously con-

* La Cellule, 1895.

$\dagger$ Deutsche med. Woch., 1904, and Zeitschr. f. Hyg. u. Infektionskrank. 1905.

‡ C. R. Soc. de Biologie, April and May, 1907.

$\S$ Arb. a. d. Kaiserl. Gesundheitsamte, 1907.

|| Brit. Med. Journal, December, 1906. 
vinced myself of the fact that fresh frog serum produces completely hemolysis of bovine blood corpuscles previously treated with an inactivated specific serum (rabbit $>$ bovine). If, then, the anthrax opsonin of frog serum were identical with the alexin, the latter, after having been fixed on bacteria, should no longer be capable of hemolyzing sensitized red blood corpuscles. The entire experiment with the controls was arranged as follows:

A. Into each of 6 very small tubes a drop of thick emulsion of anthrax bacilli (24-hour agar culture) was placed, together with $\frac{1}{20}, \frac{1}{4} 0, \frac{1}{80}, \frac{1}{6} \frac{1}{6}, \frac{1}{3} \frac{1}{2}$ and $\frac{1}{6} 0$ of a cubic centimeter of fresh frog serum. These were properly mixed, left for one-half hour in contact and then centrifugalized.

B. In 6 similar tubes containing each 0.5 of a cubic centimeter of a 5 per cent suspension of bovine blood corpuscles saturated with hemolytic sensitizer was placed one drop of each supernatant fluid from the tubes in " $\mathrm{A}$ "'series.

C. Equal volumes of the washed bacilli from "A" series were mixed with an emulsion of thrice-washed frog leucocytes. A comparison was then made of the degree of phagocytosis in "C," which may begin at once, with the degree of hemolysis which appears in "B" after considerable time.

D. As a control, 6 tubes were arranged containing each 0.5 of a cubic centimeter of the same emulsion of sensitized blood corpuscles as in " $\mathrm{B}$," and to each is added one drop of equal volume containing, respectively, $\frac{1}{2} \sigma, \frac{1}{4} \delta, \frac{1}{8} \sigma, 1 \frac{1}{6} \sigma, \frac{1}{2} \sigma$ and $\frac{1}{4} \overline{0}$ of a cubic centimeter of fresh frog serum which was not previously treated with anthrax bacilli. The result of this experiment is as follows:

1. The intensity of phagocytosis in " $\mathrm{C}$ " decreases from tube 1 to 6 . In No. 1 the phagocytosis is very marked, in No. 6 none is apparent. This is evident, since the bacilli fixed less opsonin as the serum dilution increased in "A."

2. In "B," hemolysis appears in none of the tubes. The reason for this might be that the dilution of serum in "A " was too great to show any appreciable alexin action. This, however, is not so, because:

3. In " $\mathrm{D}$ " series there is hemolysis in the first four tubes, which grows progressively less, and in the last two there is none. 
these latter cases the serum dilution was actually too great.) This experiment tells us, therefore:

(a) That the alexin was fixed at the same time with the opsonins; (b) that the intensity of the action of opsonin and alexin decreases equally with increasing serum dilution. The parallelism between the action of opsonins and alexins is therefore striking.

I have tried the reverse experiment in order to convince myself whether a normal serum which has previously been brought together with a suitable amount of sensitized blood corpuscles, in order to bind the complement, has lost its opsonin together with its alexin. It might be that the hemolysis, which occurs after addition of fresh alexic serum to sensitized blood corpuscles, might influence the later phagocytosis experiment with this serum and check its opsonic action. This consideration led me to employ fresh horse serum, the alexin of which, as is well known, becomes fixed upon sensitized blood corpuscles readily without causing hemolysis. Furthermore, I had previously convinced myself that horse serum exerts a pronounced opsonic effect when added to anthrax bacilli and washed frog leucocytes. The technic of the experiment is as follows:

Tube A contains 2 e.c. of a 5 per cent emulsion of bovine corpuscles (thrice washed) and 0.2 of a cubic centimeter of inactivated specific hemolytic serum (rabbit $>$ bovine).

Tube B contains the same amount of blood as "A" and 0.2 of a cubic centimeter of physiological saline solution.

The tubes are properly mixed and allowed to stand a quarter of an hour. The tubes are then filled up with saline solution, shaken, centrifugalized and all the supernatant fluid carefully removed with a pipette. To the sediments in the two tubes is then added 0.2 of a cubic centimeter of physiological saline solution and 0.1 of a cubic centimeter of fresh horse serum. They are shaken and the mixtures allowed to stand for 2 hours. They are then centrifugalized, and the supernatant liquids are employed for the further tests. It is evident that if the quantities have been properly chosen (and such is the case here) all the alexin of the horse serum in tube " $\mathrm{A}$ " has been bound by the sensitized blood corpuscles, whereas, in tube "B" it is still free in the fluid. As a definite control we may employ a phenomenon which has given rise to a dispute 
between Ehrlich and Sachs* on the one hand and Bordet and Gay $\dagger$ on the other in regard to the explanation of the phenomenon of complement deviation. We do not wish here to enter into this theoretical controversy, but merely mention the fact that the bringing of diluted washed guinea-pig blood in contact with inactivated bovine serum and fresh horse serum in suitable quantities produces rapid and very pronounced agglutination (later hemolysis), for which phenomenon the alexin of the horse serum is necessary.

We now set up two tubes ( $a$ and $b$ ), each of which contains 0.2 of a cubic centimeter of physiological saline solution and 1 drop of a 1-10 dilution of washed guinea-pig blood and half a drop of inactivated bovine serum. To mixture " $a$ " is added a drop of the liquid from the tube "A," while, to the similar mixture "b," 1 drop of the liquid from tube " $\mathrm{B}$ " is added. In mixture " $\mathrm{b}$ " the blood corpuscles are distincily agglutinated in about 10 minutes. In " $\mathrm{a}$ " the agglutination is totally absent and remains so. Fluid "B" contains, therefore, free alexin; "A," however, does not.

Let us inquire, now, what the condition of the opsonic power of these two fluids is. For this purpose the mixtures indicated are prepared, using in each case equal quantities of the several components (1 drop of each in a hollow ground slide protected from the air by a cover glass). After a contact of 30 minutes smears are prepared. The account of one of my experiments gives the following results:

1. Washed frog leucocytes An emulsion of anthrax bacilli Physiological saline

2. Washed frog leucocytes Emulsion of anthrax bacilli Fluid from tube "A"

3. Leucocytes Anthrax bacilli Fluid from tube "B"

4. Leucocytes Emulsion of typhoid bacilli Physiological saline solution

5. Leucocytes

Emulsion of typhoid bacilli Fluid "A"

6. Leucocytes

Emulsion of typhoid bacilli Fluid "B"
Result, no phagocytosis.

28 per cent of the leucocytes counted engaged in active phagocytosis.

78 per cent of the leucocytes show phagocytosis.

Minimal phagocytosis.

19 per cent of the counted leucocytes are more or less filled with typhoid bacilli.

83 per cent of the leucocytes show phagocytosis.

* Studies on Immunity, Ehrlich-Bolduan, John Wiley and Sons, p. 209.

$\dagger$ See p. 363. 
This experiment shows, therefore, that the opsonin disappear to a great extent from the serum together with the alexin.

I should like to cite a final experiment upon the binding or fixation of alexin in support of the conception of the identity of normal opsonin and alexin. It is well known that rabbit blood is strongly hemolyzed by fresh dog serum. If suitable doses are used, the rabbit blood corpuscles will fix all the normal sensitizers and all the alexin of the dog serum. This fact is made use of in the following experiment:

Tube 1. 0.2 of a cubic centimeter of normal defibrinated rabbit blood is washed three times with physiological saline solution. After the third washing all the supernatant fluid is removed with a pipette. To the centrifugalized blood corpuscles is added 0.2 of a cubic centimeter of fresh dog serum and 2 drops physiological saline solution. They are properly mixed and allowed to remain in contact 2 hours.

Tube 2. 0.2 of a cubic centimeter of a thick emulsion of anthrax bacilli (24-hour agar culture) plus 0.2 of a cubic centimeter of fresh dog serum and 2 drops of physiological saline solution, mixed, and left in contact 2 hours. After 2 hours both tubes are centrifugalized. In tube No. 1 a marked hemolysis has taken place. As a control to determine the content of alexin in the fluid of tube 2 , the following three tubes are made:

A. 0.5 of a cubic centimeter washed rabbit blood; 1 drop of fresh dog serum.

B. 0.5 of a cubic centimeter washed rabbit blood; 1 drop of fluid from tube 2 .

C. 0.5 of a cubic centimeter washed rabbit blood; 1 drop of physiological saline solution.

Contact, 2 hours.

Result: A. Hemolysis.

B. Faint hemolysis, distinctly less than in A.

C. No hemolysis.

The hemolysis in " $\mathrm{A}$ " is naturally less than in tube 1 because in the latter tube equal parts of blood and serum had been brought together and the dilution of the serum in " $\mathrm{A}$ " is therefore much greater. The important point is that the weaker hemolysis in " $\mathrm{B}$ " proves that in tube 2 a fixation of alexin on the bacteria has taken 
place. The control with the fluid from tube 1 can, of course, not be made up because hemoglobin has already been dissolved there. The following opsonin experiments were furthermore carried out:

1. Washed dog leucocytes

Anthrax bacilli

Fresh dog serum

2. Leucocytes

Anthrax bacilli

Fluid from tube 1

3. Leucocytes

Anthrax bacilli

Fluid from tube 2

4. Leucocytes as in 1,2 and 3

Bacilli from tube 2 washed once

No serum; merely some saline solution.

Very marked phagocytosis.

Weak phagocytosis.

Slight phagocytosis.

Very pronounced phagocytosis.

The opsonin has, therefore, in great part disappeared from the dog serum (2 and 3), together with the alexin, having become fixed in tube 1 to the blood corpuscles (hemolysis) and in tube 2 to the bacteria (4).

All these facts lead us to the conviction that in the normal serum the parallelism between the action of the alexin and the opsonin goes so far that they can no longer be considered as different, but must be regarded as identical. This conclusion agrees very well with the frequently proven fact which has also been shown by me to hold for frog serum, namely, that normal opsonin is thermolabile.

I* have previously pointed out the difference between the opsonin and the agglutinin in frog serum for anthrax bacilli in that they are destroyed at different temperatures (opsonin at $56^{\circ}$, agglutinin at $70^{\circ}$ ). A further proof is found in tubes 5 and 6 of the series on page 393. The differences in the degree of phagocytosis are particularly large, here, whereas the extracellular typhoid bacilli in the preparations of both tubes were slightly agglutinated by the horse serum.

In investigating the opsonins of specific sera I have employed hemolytic sera. Savtchenko $\dagger$ has previously shown that blood corpuscles that are loaded with specific hemolytic sensitizers easily fall prey to the leucocytes.

* Loc. cit.

$\dagger$ Annales de l'Institut Pasteur, December, 1907.

‡ One must remember that these experiments were performed with unwashed exudate leucocytes, and other influences cannot be excluded. 
Metchnikoff $*$ himself has accepted this view and ascribed to the fixateur in general an adjuvant rôle in phagocytosis. Later, after Wright's investigations had turned the discussion to the opsonin, there were endeavors made to demonstrate opsonins for blood corpuscles in specific hemolytic sera. Let us see if this was justifiable. In experiments with an exudate of macrophages from a rabbit I found that the washed leucocytes took up very actively bovine blood corpuscles which had first been treated with specific hemolytic sensitizer (inactivated rabbit $>$ bovine serum) and then with alexin (fresh horse serum), but that only sensitized, not alexinized, blood corpuscles were taken up by the leucocytes in less degree. Washed untreated blood corpuscles were not taken up by phagocytes. I repeated these observations with thricewashed dog leucocytes in the following manner:

1. Leucocytes.

Washed bovine corpuscles.

2. Leucocytes.

Sensitized bovine corpuscles.

3. Leucocytes.

Sensitized and alexinized bovine corpuscles.

4. Leucocytes.

Bovine corpuscles plus alexin.

Contact, 1 hour at 37 degrees. The blood of 2 and 3 was washed after each preparatory treatment with salt solution, whereby only those constituents of the serum which the corpuscles had fixed could develop any action, and all else was removed. The experiment gave the result that in 1 and 4 the phagocytosis is about nul. In 2 it is fairly strong, whereas in 3 there is hardly a macro- or microphage which does not contain one or more blood corpuscles. The free alexin (4) is not able, therefore, alone to stimulate phagocytosis, whereas it is able when the specific sensitizers are fixed to blood corpuscles to increase (3) their already well-marked action (2).

Similar experiments with another hemolytic serum (rabbit > goat and goat corpuscles) gave entirely corresponding results.

I have not yet observed a specific scrum which was hemolytic but not hemotropic, as reported by Neufeld and Topfer. $\dagger$ Our results, however, show a distinct similarity with the findings of

* Handb. der Pathog., Microorg., Bd. IV, Teil. I.

$\dagger$ Cent. für Bakt., Bd. XXXVIII, p. 456. 
Levaditi* on bacterial sera. We have then in our hemolytic sera also a thermolabile opsonin which becomes fixed to the blood cells and thus causes phagocytosis. It is at once suggested that here the opsonin may be regarded as identical with the specific sensitizer. One can, as a matter of fact, accurately remove the specific sensitizer from a hemolytic serum by previously treating it with the corresponding blood corpuscles and then prove that it has at the same time and in the same degree lost its opsonic power. Thus Wakelin Barratt $†$ in his experiments on the quantitative opsonic absorption by blood corpuscles from an inactivated hemolytic serum worked only with the specific sensitizers. If one adds a fresh specific serum to blood corpuscles or bacteria, a combined lytic and opsonic action by sensitizers and alexin is obtained, as in tubes 2 and 3 of the last experiment.

Let us return for a moment to our experiment with normal serum. We have seen that the opsonin was fixed by specific sensitizers with the alexin and was also destroyed by heat. There remain, however, in the normal sera after fixation or destruction of the opsonins (alexins) almost constantly a few opsonic effects (compare page 363 , tubes 2 and 5 ) which are much greater in the specific sera. And since we do not find any reason for assuming that the alexin in the normal serum becomes fixed to the bacteria without the intervention of sensitizers we have to assume, that the opsonic action either of normal or of specific sera is in the main part a combined action of sensitizers and alexin. In normal serum it is caused chiefly by the alexin. In the specific serum it is increased and brought about chiefly by the sensitizers. I concur, therefore, with Dean $\ddagger$ in the belief that normal as well as specific opsonins have a dualistic complexity.§

* C. R. Soc. de Biologie, April and May, 1907.

$\dagger$ Proc. Roy. Soc., 1905 and 1907.

$\ddagger$ Proc. Roy. Soc., 1905 and 1907.

$\S$ See, further, my dissertation, "Phagocytose en Opsoninen," Amsterdam, 1908 , where I explain more fully the nature and action of the opsonins. 


\section{ALEXIN ABSORPTION AND THE ANTAGONISTIC PROPERTY OF NORMAL SERA.*}

\section{By JULES BORDET AND FREDERICK P. GAY.}

The alexin fixation method has been used for many purposes. The method depends on the fact $\dagger$ that bacteria or red blood cells when treated with a suitable sensitizer acquire the property of fixing alexin, and of removing it from the surrounding fluid. By this method it was shown seven years ago $\ddagger$ that:

(a) Animals vaccinated against bacteria usually produce specific sensitizers, whatever be the organism employed.

(b) And, consequently, although many bacteria resist bacteriolysis by specific immune serum it is not owing to a lack of active substances in the serum, but on account of resistance on the part of the bacteria.

(c) Fixation of alexin in the presence of immune serum may be used as a means of diagnosing bacteria and is of even more general applicability than is agglutination, for a sensitizing action is often present when agglutination is absent.

A year later Gengou§ demonstrated that sensitizers appear after immunization with substances other than bacteria and corpuscles; he found that the serum of an animal immunized against an amorphous albuminous substance (casein, fibrinogen, alien serum, etc.) sensitizes the specific substance in question, and so endows it with the property of absorbing alexin.

The subsequent applications of this method and the technic employed are too well known to require mention. The results obtained, however, may be considerably affected by certain factors

* L'absorption de l'alexine et le pouvoir antagoniste des sérums normaux. Annales de l'Institut Pasteur, XXII, 1908, 625.

† Bordet, Hemolytic sera, etc., p. 186.

$\ddagger$ Bordet and Gengou, The existence of sensitizers etc., p. 217.

$\$$ Gengou, On the sensitizers of sera active against albuminous substances, p 241. 
that have not always been as carefully considered as they should have been. One of these influences, which we wish to consider in particular, is the amount of normal salt solution that is used in the mixtures. Variations in this factor are evident in many experiments on hemolysis, and such variations, we believe, explain certain phenomena that have been noted by Pfeiffer and Friedberger and by Sachs, to which they have referred as the antagonistic property of normal sera. Sachs offers a rather complicated explanation of this property, which, as we shall later see, appears to us incorrect.

When fresh normal serum (alexin) is mixed with a suitable sensitizing serum (heated to 55 degrees) and the substance under consideration, usually red blood cells or bacteria, to determine alexin fixation, the cells employed are usually added as a suspension in salt solution. The amount of this solution, however, varies; in dealing with red blood corpuscles many workers use a considerable dilution, for example, a 5 per cent suspension, and a relatively large volume of this suspension is therefore employed.

It has frequently been noted by experimenters that hemolysis in salt solution is very rapid. A given quantity of corpuscles is generally hemolyzed much more rapidly by a given dose of alexin and sensitizer when the liquid that serves as a medium is salt solution than when it is normal, and supposedly inert, heated serum.* On comparing normal inert serum with salt solution, we find that the latter favors hemolysis, or, more correctly speaking, that the normal serum inhibits hemolysis; it is to be noted that this difference is very marked only when the corpuscles are not strongly sensitized. To what is the antagonistic effect due? As both Pfeiffer and Friedberger (bacteriolysis) and Sachs have shown, the normal serum does not act by weakening the sensitization; nor directly on the corpuscles or the bacteria. We shall presently consider Sachs' explanation, which supposes that the serum owes its inhibiting effect to the presence of normal amboceptors that

* Müller (Central. für Bakt., XXX, 1901) was, we believe, the first to note this fact in particular. It has been more recently considered by other investigators, and in particular by Muir and Browning (Jour. of Hygiene, VI, 1906), and by Liebermann and Fenivessy (Peters. Médic. Chirug. Pressen, 1907), etc. We have already mentioned (see p. 383.) Klein's researches and our own on horse serum, in which it was shown that alexin is better absorbed by corpuscles when salt solution is present. 
monopolize the alexin. As Muir and Browning have correctly stated, the serum is antagonistic because it prevents the fixation of the alexin on the sensitized cells, and this antagonism is overcome only when the avidity of the cells for the alexin is very marked, namely, when the cells are strongly sensitized or when the absorption of alexin is favored by the addition of salt solution.

Sachs' experiment, which deals with the antagonistic action of normal rabbit serum against the hemolysis of bovine corpuscles treated with a small amount of sensitizer (the serum of a rabbit immunized against bovine blood) in the presence of alexin (fresh guinea-pig serum) is a suitable one for the study that concerns us. It will be well to consider our experiment somewhat in detail in order to deal with Sachs' explanation of a similar experiment in which normal rabbit serum was also employed.* The following experiment shows that moderately sensitized corpuscles are hemolyzed by alexin if the medium in which they are suspended contains a relatively large amount of salt solution, whereas they remain intact or are affected only very slowly, if the surrounding fluid contains less salt solution and a correspondingly increased amount of normal rabbit serum (heated to 56 degrees).

Tube A contains 0.6 of a cubic centimeter of physiological solution ( $\mathrm{NaCl}, 0.9$ per cent); Tube B, 0.3 of a cubic centimeter of salt solution plus 0.3 of a cubic centimeter of normal rabbit serum (56 degrees for one-half hour). To each tube is added 0.05 of a cubic centimeter of alexin (fresh normal guinea-pig serum) and 0.3 of a cubic centimeter of salt solution containing 10 per cent of moderately sensitized bovine blood. $\dagger$

Tubes $\mathrm{A}$ and $\mathrm{B}$ are placed in a thermostat (35 degrees). Hemoly-

* Rabbit serum is used, as it is only faintly sensitizing for ox corpuscles. It is evident that if the normal serum has a distinct sensitizing effect on the corpuscles under consideration it cannot be considered as an inert medium. Theoretically there is perhaps no absolutely inert serum, but its sensitizing effect on a given bacterium or alien red blood cell is frequently so slight as to be imperceptible, as is the case in the present instance.

$\dagger$ This blood is prepared as follows: To 1 c.c. of bovine blood (previously washed to remove all serum) is added 9 c.c. of salt solution, and 0.1 of a cubic centimeter of serum ( 56 degrees) from a rabbit immunized against bovine blood. After shaking and allowing contact for 20 minutes the tube is centrifugalized and the supernatant fluid removed. To the sediment of corpuscles 10 c.c. of salt solution is then added. 
sis is complete in A in half an hour. The corpuscles in B are intact after 3 hours; slight hemolysis subsequently takes place. It is scarcely necessary to mention that control tubes $\mathrm{A} T$ and $\mathrm{B} \mathrm{T}$, identical with $\mathrm{A}$ and $\mathrm{B}$ respectively, but containing nonsensitized bovine blood, are likewise prepared; in these mixtures there is no hemolysis.

It is evident, then, that a small amount of normal serum inhibits hemolysis distinctly; further experiments show that this antagonistic effect varies in intensity with the increase of heated normal serum and the decrease of salt solution.

The question arises as to how intense this antagonistic power in normal serum is. We can also determine whether normal serum combats one or both the factors of hemolysis (alexin and sensitizer). We find that the serum shows a relatively retarding effect on alexin even if the dose of it is relatively high. For example:

Mixture A contains: 0.6 c.c. of salt solution,* 0.05 c.c. guinea-pig alexin, and 0.2 c.c. of salt solution containing 40 per cent of sensitized bovine blood. $\dagger$ Mixture B contains the same amount of blood and twice as much alexin, but 0.6 c.c. of normal rabbit serum, 56 degrees, in place of the salt solution. Hemolysis is more rapid in $\mathrm{A}$ than in $\mathrm{B}$.

When corpuscles are heavily sensitized, however, the antihemolytic effect of normal serum (56 degrees) is less evident, although still distinct. In the following experiment even small doses of serum show distinct effects:

Tubes A and C. Each 0.7 c.c. of salt solution.

Tubes B and D. Each 0.4 c.c. of salt solution plus 0.3 c.c. of heated normal rabbit serum.

To $\mathrm{A}$ and $\mathrm{B}$ is then added 0.25 c.c. of salt solution containing 40 per cent faintly sensitized ox blood ( 1 part of rabbit antibovine sensitizer to 10 parts of blood); to tubes $\mathrm{C}$ and $\mathrm{D}$ is added the same amount of blood sensitized with three times as much sensitizer. To each of the four tubes is then added 0.05 c.c. of guinea-pig alexin and the tubes are placed at 35 degrees. Hemoly-

* In these experiments 0.9 per cent $\mathrm{NaCl}$ is used.

$\dagger$ This blood is relatively well sensitized and is prepared as follows: To 1 c.c. of washed blood is added 9 c.c. of salt solution and 0.5 c.c. of rabbit-antibovine serum, 56 degrees. Contact, 1 hour. Centrifugalization and removal of the supernatant fluid. 2.5 c.c. of salt solution is added to the sediment. 
sis is complete in $\mathrm{C}$ in 12 minutes, and in $\mathrm{A}$ in 35 minutes. In $\mathrm{D}$ hemolysis is complete in 1 hour. B is not hemolyzed.

To what is the inhibiting effect of normal serum due? It may easily be shown that the serum does not act by suppressing the sensitization of the corpuscles. This has been demonstrated by our predecessors by the following simple experiment: In a mixture of moderately sensitized ox corpuscles, heated normal serum and guinea-pig alexin there is no hemolysis owing to the antagonistic power of the normal serum. On subsequent centrifugalization and decantation, however, the addition of salt solution containing a trace of alexin causes hemolysis, which shows that the corpuscles have retained their sensitization.

This result also shows that heated normal serum does not directly render the corpuscles refractory to hemolysis. One might suppose that such a serum contains "complementoids" that satisfy the affinities of the sensitized corpuscle and hinder the subsequent fixation of active alexin. The preceding experiment would, however, invalidate this supposition.

The normal serum, in addition to having no effect on the sensitization of the corpuscle, has also no neutralizing effect on the alexin. It does, however, oppose the fixation of the alexin on the sensitized corpuscles, and this explains the lack of hemolysis.

The proof that normal serum does not alter the alexin is given by the fact that hemolysis can be produced in an inhibited mixture of normal serum, alexin and sensitized corpuscles by adding salt solution; in other words, as already stated, the opposition to hemolysis depends on the concentration of normal serum. Muir and Browning in studying this fact have clearly shown that the alexin is not fixed on the corpuscles during the first phase of the experiment, when the mixture contains much serum and little salt solution, but is absorbed only on the addition of the saline solution. Similar results are obtained if, as we have performed the experiment, corpuscles before dilution in salt solution are sufficiently sensitized to produce their dissolution. Such an hemolysis, to be sure, naturally brings about an absorption of alexin, which, however, is only partial if suitable amounts are used and the sensitization is not too great; such absorption always remains less than when salt solution is added.

$$
*^{*} *
$$


What ideas concerning the mode of union of the alexin with sensitized corpuscles are suggested by these facts? One of us* has already expressed the opinion that this fixation of alexin is not a chemical combination, strictly speaking, between the alexin and a particular group of the sensitizer (complementophilic group of the amboceptor, according to Ehrlich), but represents simply a phenomenon of molecular adhesion (adsorption). This point of view was still further elaborated in $1906 \uparrow$ by the authors of the present article. We regard the union of the sensitizer and red blood cells as constituting a complex with more adsorption avidity for the alexin than the normal corpuscle: the alexin tends to precipitate on the sensitized corpuscle, the alteration of which is the more marked the greater the sensitization. How, from this standpoint, does the inhibiting serum act? It would seem to us that it holds the alexin in a state of more or less definite suspension in the medium, and gives it a more stable equilibrium; with salt solution, on the other hand, the equilibrium is more unstable, that is to say, the alexin condenses or precipitates more readily on those cells that attract it.

This idea seems to us to be in harmony with the observations of Gengou on eolloids and suspensions of inorganic precipitates. Gengout found that if washed red blood corpuscles are added to salt solution containing such an inert inorganic precipitate as barium sulphate in suspension, the cells and the precipitate clump and hemolysis occurs. But if a trace of serum is previously added to the precipitate, this phenomenon of agglutination and hemolysis does not occur. It was found that serum causes a dissociation of the particles of barium sulphate and gives it a milky appearance which delays the clarification of the fluid by sedimentation. And serum, then, inhibits the precipitation of alexin on the corpuscles just as it prevents the sedimentation of barium sulphate.

Citrate of sodium produces an effect on barium sulphate similar to the one caused by serum, and also changes it into a milky fluid and, as Gengou has found, deprives it of its property of agglutinating and hemolyzing red blood cells. And there is a still further analogy, in respect to the citrate, between the precipitation of

* Bordet, Hemolytic sera, p. 186, and Cytolytic sera, p. 228.

$\dagger$ Bordet and Gay, On the relation of sensitizers, etc., p. 363.

$\ddagger$ Gengou, Researches on the agglutination of red blood cells, etc., p. 312 . 
barium sulphate on corpuscles, and the fixation of alexin on sensitized cells. We have found that sodium citrate in suitable doses will protect red blood cells from hemolytic sera, and it may further be shown that it acts by preventing the fixation of the alexin on the sensitized cells.* The analogy between barium sulphate and alexin is evidently suggestive.

The following experiment may be cited as offering an adequate though somewhat homely comparison as to the mode of union of the alexin with sensitized corpuscles: Water rolls over the surface of a watch glass eovered with paraffin without adhering to it. If the water contains barium sulphate in suspension, the paraffin becomes wet in a few minutes and the drop spreads out over the surface, owing to the fact that the surface is covered, by molecular adhesion, with a thin white stratum of barium sulphate. This stratum is wet by water and will even resist rinsing, being removable only by friction. But if we use intead a suspension of barium sulphate that has been rendered milky by, citrate, no such phenomenon occurs; under such conditions the sulphate fails to cover the paraffin and the surface does not wet. In other words, the citrate prevents the precipitation of the sulphate on paraffin just as it does the precipitation of alexin on sensitized corpuscles.

We shall not consider the mechanism of this phenomenon at this point nor insist on the properties of the citrate in hemolysis, as these subjects have already been studied in detail by Gengou $\dagger$ in our Institute.

There are certain pertinent considerations that occur in this connection applicable to the methods based on absorption of alexin, and in a general way of service in studies on hemolysis and bacteriolysis.

In the first place the facilitating effect of a large amount of salt solution on alexin fixation should never be lost sight of. The

* After proving that sensitized blood corpuseles remain intact in a mixture of citrate and alexin, it may subsequently be demonstrated, after centrifugalization, that the supernatant fluid still contains alexin and is able to hemolyze new sensitized corpuscles; for this purpose a little calcium chloride is added to neutralize the eitrate. Control tubes show that a fixation of alexin does take place when no eitrate is added.

$\dagger$ Several observations, particularly as regards hemolysis by eel serum and by venom, have been published by Gengou in the Bulletin de la Société de Biologie (1907). See also p. 414. 
experimental method employed to demonstrate alexin fixation consists, as we know, of two phases: In the first phase the alexin is placed in contact with such cells as bacteria plus a serum " $\mathrm{A}$ " that is capable (or supposedly capable) of sensitizing these cells, that is to say, of conferring on them the property of fixing alexin. In the second phase one determines, by adding sensitized blood corpuscles, whether the alexin has disappeared from the fluid or remained free in it; in the latter instance the fixation takes place, not during the first phase (on the bacteria), but during the second phase (on the corpuscles). As a result, hemolysis occurs, and the conclusion is that serum " $\mathrm{A}$ " is not sensitizing, or, at best, only slightly so. But it is evident that, for experimental accuracy, the two phases of the experiment should be comparable in respect to the facility for alexin absorption. As we have seen, an excess of serum inhibits fixation and an excess of salt solution favors it; the effort, then, should be to maintain a constant proportion between salt solution and serum during the entire experiment. The sensitized corpuscles finally introduced are suspended in salt solution; it is evidently better to add the number of corpuscles desired, not by employing a large volume (1 c.c., for example) of a weak suspension of corpuscles, but a small volume of a thick suspension ( 0.1 c.c., for example). If, for example, the first mixture is: alexin, 0.1 c.c., plus bacterial suspension 0.3 c.c., and 0.3 or 0.5 c.c of the serum in which the presence of a bacterial sensitizer is to be determined, the fixation of alexin on the bacteria will be opposed by the large proportion of serum in the mixture and may be complete only in case the sensitizer is very strong. If much salt solution is added with the red blood corpuscles, the antagonistic effect is removed and a most minute trace of free alexin will produce hemolysis. And as the antimicrobial sensitizer has been handicapped by the hemolytic sensitizer it may easily escape detection. Or, indeed (and this error would seem to us to have been committed), * one might conclude that the alexin fixed by a given cell (bacterium, for instance) is not identical with the one fixed by a different cell (corpuscle), and might be led to agree with the erroneous hypothesis that the bacteriolytic alexindiffers from the

* We think that certain experiments of Moreschi that seem to indicate a plurality of alexins are open to criticism from this standpoint. (Berlin klin. Wochen., 1907, 1206); this investigator usually employs a large volume of salt solution in which to suspend his sensitized corpuscles (1 c.c.). 
hemolytic alexin. The numerous and varied applications of this method invariably show that the corpuscles used as an indicator of alexin fixation remain intact, whatever be the sensitized cells employed in the first phase of the experiment, provided the sensitizeremployed is sufficiently strong and the precautions mentioned aretaken in regard to the volume of salt solution. In a corresponding manner account should be taken of the respective potency of the two sensitizers which are successively used, in respect to the fixation of a given alexin. If the first is not very powerful and does not remove the last traces of alexin, the method maygive very varying results, depending on whether the corpuscles used as an indicator are moderately or strongly sensitized, for the traces of alexin may be sufficient to produce hemolysis in the latter case, whereas in the former instance, owing to an antagonistic effect of the serum which tends to disseminate the alexin, they may not. Two opposing forces of variable energy tend to take hold of the alexin and the result depends on the equilibrium established between them. With this idea in mind it is easy to show that, following contact with a large number of moderately sensitized corpuscles, a fluid containing alexin may subsequently act on similar corpuscles as if no active substance were present, but, on more highly sensitized corpuscles of the same species, as if still present. It will scarcely be claimed that the alexin used up by a feeble sensitization differs from the one taken by a greater sensitization. Such, however, is apparently the conclusion of Remy.* This investigator mixes sensitized typhoid bacilli with his alexin and finds that subsequently added corpuscles remain intact when sensitized by a serum of moderate activity, whereas they are destroyed if the serum with which they have been treated is very powerful; from this he concludes that the bacteriolytic alexin differs from the hemolytic alexin. $\mathrm{He}$ might equally well have concluded that there is an alexin particularly adapted for heavily sensitized corpuscles, since such corpuscles are hemolyzed in a fluid in which the same corpuscles, more weakly sensitized, remain intact. In fact, Remy has used a hemolytic serum that has a higher sensitizing power than his antityphoid serum.

* Contribution à l'étude des substances actives du sérum. Bull. de l'Académie de Médecine de Belgique, 1903. 
Similar instances have been met with in the studies on whoopingcough made by one of us with Gengou. The sera of three childrena brother and two sisters - suffering from the disease were tested on the same day and in the same amounts; as a general thing we used highly sensitized corpuscles to test for alexin fixation. In the control containing normal human serum, alexin and bacteria, hemolysis appeared in a few minutes; it took one-half hour and one hour respectively in tubes containing sera of two of the children; with the third serum there was no hemolysis. The three whooping-cough sera, then, were unequally active, and it was found that the most active came from the child that first fell ill and was then convalescent, while the weakest was from the one that was last taken with the disease and still showed marked symptoms. The result is quite natural, but if the sensitizing power were only moderate even in recovered children one might wrongfully be led to the conclusion that the alexin that affects the whooping-cough bacillus differs from the hemolytic alexin.

As a matter of fact the method is very exact only in the demonstration of powerful sensitizers, $*$ and is not applicable for the accurate titration of the activity of antimicrobial sera, or, at least, cannot be compared in exactitude to Ehrlich's methods employed in measuring the potency of antitoxins.

And indeed the originators of the method recommended it rather for the qualitative study of sera than for a quantitative evaluation of their activity.

\section{$*^{*} *$}

The researches of Pfeiffer and Friedberger $\dagger$ and of Sachs $\ddagger$ concerning the antagonistic properties (antibacteriolytic or antihemolytic) of normal sera may now be considered.

A few data in hemolysis will simplify the matter in hand. A

* It is not surprising, then, that in Moreschi's experiments (Berlin. klin. Woch., 1906 , p. 1244) the method failed to demonstrate sensitizing properties in the serum of a man who had received a single injection of killed tyhoid bacilli a few days previously.

$\dagger$ Pfeiffer and Friedberger, Deutsche med. Woch., 1905, Nos. 1 and 29; Centralblatt f. Bakt., XLI, 1906.

$\ddagger$ Sachs, Deutsche medicin. Wochen., 1905, No. 18; Centralblatt f. Bakt., XL, 1906. 
serum, whether normal or immune, may be endowed with sensitizing power for certain corpuscles and consequentlymay, in their presence, produce alexin fixation, although as serum it tends simply to inhibit such a fixation. These two forces in the same serum oppose one another and the antagonistic property will be the more evident the weaker the sensitizing property. Under such conditions it might well happen that there would be no hemolysis, in other words, it might appear as if no sensitizer were present. So, for example, it is generally considered that normal rabbit serum ( 56 degrees) does not sensitize bovine corpuscles; in a mixture of bovine corpuscles ( 0.05 c.c.), heated rabbit serum $(0.5$ c.c.), and a little guinea-pig alexin ( 0.1 c.c) there is indeed no hemolysis. But if the corpuscles are first subjected to rabbit serum, then centrifugalized, the supernatant fluid decanted, and an equal amount of normal salt solution added, followed by alexin,* we find that slow but complete hemolysis takes place. Normal rabbit serum, then, is sensitizing for bovine corpuscles, although weakly so.

Conversely, when corpuscles that are strongly sensitized by this serum are chosen, as Sachs has done, $\dagger$ there is no danger of losing sight of the antagonistic property. It is found, for example, that normal rabbit serum sensitizes goat corpuscles strongly. We mix 0.6 c.c. of rabbit serum ( 56 degrees) with 0.2 c.c. of salt solution containing 25 per cent of washed goat blood; half an hour later 0.05 c.c. of fresh guinea-pig serum is added. Hemolysis is nearly complete in one and a half hours at 37 degrees, whereas in a control with salt solution replacing the rabbit serum there is no hemolysis.

In this case the antagonistic property, although present, is concealed. Although it fails to prevent hemolysis, it does retard it notably. If we make a mixture of goat blood and rabbit serum like the preceding and, after contact, centrifugalize, remove the serum, and replace it with salt solution, we find that the addition of alexin will produce a much more rapid hemolysis; it is complete, indeed, in from 15 to 20 minutes. By removing the serumwe have eliminated the antagonistic property and hemolysis is accelerated. As is evi-

* A control is made at the same time with corpuseles that have not been treated with rabbit serum to which salt solution and alexin are added.

$\dagger$ Analogous results have been obtained in bacteriolysis by Pfeiffer and Friedberger. 
denced by this experiment, the antagonistic property inhibits the hemolysis produced by a normal sensitizer.*

Although the antagonistic power prevents hemolysis in a mixture of bovine corpuscles, normal rabbit serum, 56 degrees, and guineapig alexin, because the serum is only faintly sensitizing for the corpuscles, there is no inhibition when goat corpuscles are used, because they are powerfully sensitized by the rabbit serum. In the latter case, as we have just seen, the antagonistic property simply retards hemolysis. This effect may be shown in another way, as is evidenced by the experiments of Pfeiffer and Friedberger and of Sachs. In their experiments the property that opposes the antagonistic property, namely, the sensitizer, is removed. After treating normal rabbit serum (56 degrees) with a sufficient amount of goat corpuscles we have a fluid that is no longer sensitizing, but purely antagonistic; it will therefore protect moderately sensitized goat corpuscles from hemolysis.

If the sensitizer (whether in an immune serum or normal serum) is too strong, the antagonistic property will be overcome and hemolysis take place. The origin of the sensitizer is immaterial, but its strength is all-important.

This fact is quite conceivable in view of the fact that the sensitizer does not unite directly with the alexin. It is the sensitized corpuscle that unites with the alexin, and the greater the sensitization the better this union. The union with the sensitizer changes the properties of molecular adhesion in the corpuscle so that its avidity for the alexin is increased; in a similar manner the action of agglutinins on bacteria is to increase their reaction to the clumping effect of electrolytes. Any result produced by the sensitizer depends, not on its proper nature, but on its effect.

* This antagonistic effect is so distinct that goat corpuscles that have been sensitized by a small dose of rabbit serum and then suspended in salt solution are hemolyzed by alexin more rapidly than the corpuscles sensitized by twice as much serum when kept in this serum. For example: in each of four tubes is placed 0.05 c.c. of goat blood; to $\mathrm{A}$ and $\mathrm{B}$ is added 0.4 c.c. of normal rabbit serum, 56 degrees and to $\mathrm{C}$ and D 0.2 c.c. of the same serum. After 2 hours' contact $\mathrm{A}$ and $\mathrm{C}$ are centrifugalized, the supernatant fluids removed and 0.4 and 0.2 c.c. of salt solution added to the sediments in $\mathrm{A}$ and $\mathrm{C}$ respectively. To each of the four tubes is then added 0.1 c.c. of guinea-pig alexin. Hemolysis is complete in $\mathrm{A}$ in 15 minutes, in $\mathrm{C}$ in a half hour, in $\mathrm{B}$ in 50 minutes, and in $\mathrm{D}$ in a little over an hour. 
This, to be sure, is not the opinion of Sachs, who believes that there is inhibition to hemolysis when a specific serum is used, and not when a normal sensitizer like normal rabbit serum is employed. In harmony with Ehrlich's theory Sachs considers that normal sensitizers possess a complementophilic arm that is very avid of alexin (complement); even more so indeed, than is the corresponding arm in an immune sensitizer. When acted on by a normal sensitizer the corpuscles easily fix alexin even in presence of an excess of normal serum. But since a normal serum contains several normal sensitizers (Ehrlich and his pupils presuppose the existence of very numerous antibodies even in normal serum), these bodies take hold of the alexin present and, having a superior affinity for it, prevent its fixation on corpuscles even when they are sensitized by an immune serum. The antagonistic property therefore would be due to the presence in serum of certain normal sensitizers that have no affinity for the corpuscles employed, but monopolize the complement.

This theory is irreconcilable with the fact that the addition of salt solution suffices to attenuate the antagonistic effect of serum to a marked degree. The first premise of the hypothesis is incorrect; the antagonistic property is also present when a normal sensitizer is used, as we have just seen, and as the following experiment further evidences:

Two series of mixtures are prepared at the same time. The first series comprises eight tubes, each containing 1 c.c. of a 5 per cent suspension of washed goat corpuscles. To four of these tubes normal sensitizer, that is to say, normal rabbit serum, 56 degrees, is added in varying amounts $(0.4,0.2,0.1$ and 0.05 of a cubic centimeter); to the other four a specific sensitizer (serum of a rabbit immunized against goat blood) in doses of $0.01,0.005,0.003+$ and 0.0025 of a cubic centimeter.

One hour later the tubes are filled with salt solution centrifugalized, the supernatant fluids decanted, and 0.6 of a cubic centimeter of salt solution added to each sediment. To each tube is then added 0.05 of a cubic centimeter of fresh guinea-pig serum (alexin).

In the second series of tubes the same steps are taken except that, after sensitization, centrifugalization and decanting, 0.1 of a cubic centimeter of salt solution plus 0.5 of a cubic centimeter of 
heated rabbit serum deprived of its sensitizer by contact with goat corpusles is added.* The alexin is then added as to the first series.

Controls are also made without any sensitizer, either normal or immune, to prove the necessity of its presence for the production of hemolysis.

In the following table is shown the length of time in minutes required for complete hemolysis at $35^{\circ} \mathrm{C}$. The letters "M," "Sl" and "Nul" indicate that after several hours hemolysis is marked, slight or none, as the case may be.

OCCURRENCE OF HEMOLYSIS.

\begin{tabular}{|c|c|c|c|c|c|c|c|c|c|}
\hline \multirow{3}{*}{$\begin{array}{c}\text { Corpuscles sus- } \\
\text { pended in: }\end{array}$} & \multicolumn{8}{|c|}{ Corpuscles sensitized by: } & \multirow{3}{*}{$\begin{array}{c}\begin{array}{c}\text { Non-sen- } \\
\text { sitized cor- } \\
\text { puscles. }\end{array} \\
\text { Nul }\end{array}$} \\
\hline & \multicolumn{4}{|c|}{ Immune serum. } & \multicolumn{4}{|c|}{ Normal serum. } & \\
\hline & 0.0025 & 0.003 & 0.005 & 0.01 & 0.05 & 0.1 & 0.2 & 0.4 & \\
\hline $\begin{array}{l}\mathrm{NaCl} \text { sol }, 0.6 \mathrm{c} . \mathrm{c} \\
+ \text { alexin, } 0.05 \\
\text { c.c............. } \\
\text { NaCl sol., } 0.1 \mathrm{c.c} \\
+ \text { normal rab- } \\
\text { bit serum } \\
\text { minus sensi- } \\
\text { tizer, } 0.5 \text { c.c.+ } \\
\text { alexin, } 0.05 \text { c.c. }\end{array}$ & Sl. & $20^{\prime}$ & $15^{\prime}$ & $120^{\prime}$ & Nul & Sl. & M. & M. & Nul \\
\hline
\end{tabular}

In the mixtures containing salt solution but no antagonistic serum we may estimate the relative potency of the two sensitizers; we find, for example, that 0.005 of a cubic centimeter of immune serum affects the corpuscles as much as a relatively large dose $(0.2$ of a cubic centimeter) of normal rabbit serum, and that 0.01 of a cubic centimeter of immune serum sensitizes a little better than does 0.4 of a cubic centimeter of normal serum, and so forth. It is further to be noted that the antagonistic serum (deprived of sensitizer) retards hemolysis approximately equally in corpuscles sensitized to the same degree by normal or by immune serum.

* To the sediment of 5 c.c. of washed goat blood 5 c.c. of rabbit serum, 56 degrees, was added. A few hours later the mixture was centrifugalized and the supernatant fluid removed. 
Before Sachs' observations, Pfeiffer and Friedberger had studied the inhibiting effect of normal serum on bacteriolysis of the cholera vibrio. Their sensitizer, which was very active against the vibrio, was first removed by treating the immune serum with vibrios, followed by centrifugalization and decanting of the serum: the supernatant fluid so obtained, on injection into the peritoneal cavity of a normal animal together with vibrios sensitized by a moderate dose of anticholera serum, prevents bactcriolysis. Pfeiffer and Friedberger further noted that for the antagonistic power to be manifest a moderate sensitization only should be used. They have studied the conditions affecting the phenomenon with great care and reserve any decision as to its interpretation.

Sachs' findings in hemolysis are analogous to those of Pfeiffer and Friedberger. There can be no doubt that any explanation for one will serve for the other; the inhibiting property of certain constituents of serum on the fixation of alexin on sensitized blood cells, as opposed to salt solution, is doubtless applicable to bacteriolysis as well. A single objection occurs: when faintly sensitized vibrios are injected into the peritoneal cavity bacteriolysis does or does not take place, depending on whether antagonistic serum has or has not been previously added. But why is such serum necessary to protect the vibrios? Does not the peritoneal cavity contain a certain amount of an exudate which resembles the antagonistic serum at least more closely than it does salt solution? Why, then, does not this exudate inhibit bacteriolysis?

We find by experiment that peritoneal exudate, even when heated, has no more inhibiting effect on hemolysis* than salt solution. For example, a little peritoneal exudate from a rabbit was heated to 56 degrees. At the same time the serum of the same animal was obtained and treated in the same manner. One-twentieth of a cubic centimeter of moderately sensitized bovine blood (rabbit $>0 \mathrm{x}$ serum) is placed in each of several tubes, and 0.3 of a cubic centimeter of salt solution, normal serum or peritoneal exudate, respectively, is added; 0.05 of a cubic centimeter of fresh guinea-pig serum is then added to each tube. Hemolysis appears rapidly in the mixture containing salt solution and almost as soon in the one

* It seems that from the close analogies between bacteriolysis and hemolysis any conclusions referring to one are applicable to the other. 
with exudate, whereas it is very much retarded by the normal serum.

Peritoneal exudate, then, favors the demonstration of alexic activity. Thirteen years ago the "reactivation" of heated cholera serum by fresh normal serum was demonstrated by one of us. It was also shown at the time that bacteriolysis is due to the collaboration of the specific antibody and the alexin. The granular transformation of vibrios, which is indicative of bactericidal power, generally occurs more rapidly in the peritoneal cavity than in mixtures in vitro containing serum. The reason for this is simply that the antagonistic property of heated serum retards bacteriolysis more markedly in vitro than in vivo.

It is evident that the presence of an antagonistic property must have been a great source of error in many experiments and in particular in those based on the principle of specific absorption and those dealing with the multiplicity of active substances in a given serum; obvious examples of this error are present in experiments dealing with the discussion concerning the unity or multiplicity of alexins. 


\title{
XXII. A CONTRIBUTION TO THE STUDY OF MOLECULAR ADHESION WITH A CONSIDERATION OF ITS FUNC- TION IN VARIOUS BIOLOGICAL PHENOMENA.*
}

\author{
BY DR. GENGOU.
}

I.

For some years biologists and bacteriologists have studied both the inorganic and the organic colloidal substances. As we know the majority of the writers consider the sols (hydrosols, etc.), that is to say, the "solutions" of colloidal substances in fluid, as being not true solutions, but ultramicroscopic or microscopic suspensions of colloidal particles in the fluid. In addition to colloidal solutions we have fine suspensions, such as mastic, aqueous gum arabic, etc., which consist of fine particles in a fluid, with the difference that these particles are visible.

We have learned a great deal about colloidal solutions from studying these fine suspensions, inasmuch as both substances have certain properties in common. We believe also that certain studies of the chemical precipitates which sediment easily are bound to facilitate our knowledge of the various manifestations of colloidal substances. Inasmuch as colloids are probably very fine suspensions, it is likely that all transitions between them and the sedimenting chemical precipitates with large particles, exist. We must admit that all these substances in suspension have certain common properties. It is often easier to work with chemical precipitates than with colloidal solutions, and such a method is therefore advantageous in studying those properties which are common to both substances.

Among these common properties the one which we shall consider almost entirely is the power of adsorption. We have long known that certain solid substances in the form of a fine powder absorb

* This article is a résumé, which was kindly furnished by Dr. Gengou, of an article on the subject which appeared in the Archives Internat. de Physiologie, Vol. 7, Fasc. 1 and 2, 1908. 
substances in the form of gases or solutions (electrolytes, colloidal sols). These adsorption phenomena by solid bodies bear a definite relation to the considerable surface development of the particles of these substances. Colloids in the state of gels, which also have a large surface, are likewise endowed with energetic adsorption properties.* The laws of adsorption by solid bodies are the same as with colloidal gels.

The phenomena of molecular adhesion may begin between two substances of different natures and may also occur between the particles of the same substance, for example, the particles of an insoluble solid suspended in water. If increasing amounts of a suspension of barium sulphate in water are added to a constant volume of water, we find that the mixture becomes cleared of the powder with a rapidity which varies directly with the amount of barium sulphate added. Naturally this is due to the fact that the nearer the particles are to one another the more their mutual adhesion is facilitated and they form clumps which sediment more easily. The sedimentation of a substance which is insoluble in water is, as we know, facilitated by certain colloidal solutions and viscous fluids. $\dagger$ A few years ago Muller $\ddagger$ studied the inhibit-

* Van Bemmelen, Zeitschrift für Anorg. Chem., 1900, 23. This author has suggested the name of adsorption to designate the accumulation of gas or fluids in porous bodies or on the surface of non-porous bodies, and the word absorption for the instances in which the molecules of the adsorbed substance interchange with those of the adsorbing substance, for example, homogeneous solutions of gases, liquids or solids. The phenomena which we are about to consider deal with the adhesion of various substances to solid bodies suspended in a fluid, and we prefer to use the term adsorption.

$\dagger$ Lobny de Bruyn (Rec. des Trav. chim. des Pays Bas, 1900, vol. 19) has shown in particular that if solutions of salts which normally form an insoluble precipitate are added to gelatin the sedimentation occurs much more slowly. The reaction between these salts, however, takes place just as well under these conditions, for the rapidity of the reaction is as rapid in gels as in water.

(E. Cohen, Eder's Jahresbericht f. Photographie, 1895, cited by Hamburger, Osmot. Druck u. Ionenlehre, vol. 3, p. 89.

Rothland, Zeitschrift f. anorg. Chem., vol. 40; Spring, Bull. de l'Acad. Roy. de Belg., 1900, p. 515.)

Since this time several authors have applied the same method and similar - methods in preparing colloidal solutions of substances that are usually insoluble.

Paal and Amberger: Bericht d. deutschen chem. Gesellschaft, vol. XXXV and XXXVII; Paal and Voos, Ibid, vol. XXXVII; Lottermoser, Ueber anorg. Kolloide, Stuttgart, 1901; Guthier: Zeitschrift f. anorg. Chem., XXXII. Heinrich, Bericht d. deutsche chem. Gesellschaft, XXXVI; Garbowski: Ibid; Carey Lea, Ibid, XXIV.)

$\ddagger$ Bericht d. deutschen chem. Gesellschaft, 1904 . 
ing action of various stable colloids on the sedimentation of a colloidal solution of gold to which electrolytes had been added and thought that he found some relation between the viscosity and the intensity of its protecting power. Victor Henri and A. Meyer* did not think that the viscosity was a sufficient factor to explain the suspension of chemical precipitates by stable colloids.

We have studied the action of barium sulphate that has been washed and suspended in distilled water on the addition of various stable colloidal solutions or viscous fluids. Certain colloidal solutions agglutinate barium sulphate energetically, for example, a solution of starch or farina; certain organic fluids even when deprived of their cellular elements by centrifugalization (peritoneal or synovial fluid, nasal mucus, saliva) do the same thing; the agglutinating substance in these fluids would appear to be mucin.

The majority of stable colloids and viscous fluids, on the contrary, inhibit the sedimentation of barium sulphate. On shaking barium sulphate in aqueous solutions of sugar, glycerine, gum arabic, gum tragacanth, agar, gelatin, glue, dextrin, serin, pseudoglobulin, euglobulin or fibrinoglobulin, we find that the masses of barium sulphate break up into much smaller particles of apparently uniform size which have slight tendency to adhere to one another. As a result, we obtain much finer suspensions, which are more homogeneous and stable and, in the case of barium sulphate, are of milky appearance. These suspensions, when left to themselves, sediment very slowly.

It is only rarely that the action of such solutions on barium sulphate is due to their viscosity. Syrups composed of sugar or glycerine do act by viscosity; the more marked this viscosity is, the slower the sedimentation of the barium sulphate. All the other substances which we have just mentioned, however, as dissociating barium sulphate, do not act through their viscosity unless much more concentrated solutions than necessary are employed. Viscosity, then, functions only in a subsidiary manner. Thus we find that a gum solution of 0.03 per cent in distilled water which reacts with Ostwald's viscosimeter just as distilled water does, suffices, notwithstanding, to hold barium sulphate in suspension.

The mechanism of the suspension of barium sulphate becomes

* Revue gen. des Sciences, 1904. 
evident when the suspension which has been in contact with these various colloidal solutions is repeatedly washed. If this washing is kept up until no detectable amount of the colloid remains, we find that the treated suspension, when placed in distilled water, remains suspended as well as if the colloidal solution were still present. This fact is brought out very clearly on employing gum arabic, agar, gelatin, gum tragacanth, glue or pseudoglobulin. It seems reasonable to believe then, that bariuni sulphate, when treated with solutions of these substances, absorbs something from them and forms with the colloid a complex which is sufficiently stable to resist numerous washings with distilled water.* Such is not the case with the suspension that has been treated with sugar or glycerine syrup. A suspension treated in this way after washing becomes like untreated suspension; it takes nothing from these solutions which hold it in suspension through their viscosity.

Serum albumin, euglobulin and fibrinoglobulin also form complexes with barium sulphate; these complexes, however, withstand washing poorly.

$$
\text { *** }
$$

It is easily demonstrable that a complex between the insoluble barium sulphate and the colloids is formed: a weak solution of gum arabic treated with a sufficient quantity of barium sulphate and then freed of it by centrifugalization is no longer able to disseminate fresh barium sulphate.

If, on the other hand, a substance such as calcium phosphate, which is easily dissolved in acetic acid, is used instead of barium sulphate, the adsorbed colloid may be liberated from the complex after washing by dissolving the adsorbing substance; the colloid liberated in this manner is demonstrable by the fact that it is able to disseminate fresh barium sulphate.

When a washed complex, such as barium sulphate plus agar, is

* Substances other than barium sulphate, for example, animal charcoal, lead iodide, calcium oxalate, mercurous sulphate, manganese carbonate and copper oxide are held in suspension more or less well by gum arabic. Gum arabic, however, does not succeed in dissociating fine particles of kaolin, chromate of silver, sulphate of calcium and sulphate of zinc. It seems reasonable that such should be the case, since we are dealing with various powdered substances, the adsorbing power of which will evidently not be the same for the colloid under consideration. 
left for a long time at room temperature, that is, for several days, a small amount of the colloidal substance is demonstrable, after centrifugalization, in the supernatant fluid. Certain factors such as heat and the like accelerate the breaking up of these complexes. Thus we find that a complex of barium sulphate and gum arabic which has been washed, and has remained for several days at room temperature and has liberated very little of its colloid, liberates much more of it when boiled for a few moments.*

The adsorption of a colloid by an insoluble substance has long been known. The relation between this adsorption and the dissemination of insoluble substances by colloidal media is what gives interest to our data.

Although it may seem quite evident from what we have said that the facts observed are identical with adsorption phenomena, we have, nevertheless, endeavored to establish this identity more completely. Physicists have shown that if they add identical volumes of a fluid containing adsorbable substances in increasing concentration to a fixed quantity of the adsorbing substance, that the absolute amount of the substance adsorbed increases up to a certain point with the concentration, but not in direct proportion to it. It does not always form the same fraction of the quantity of adsorbable substance present. We find experimentally that the denominator of this fraction increases in proportion to the increase in concentration, so that the proportion of substance adsorbed relative to the amount of adsorbable substance employed, diminishes.

The same rule is true in the adsorption of gum arabic by barium sulphate; on the addition of increasing doses of gum arabic in a given volume to the same amount of this suspension, larger and larger amounts of the colloid are adsorbed; the fraction of the colloid adsorbed, however, diminishes in proportion to the total amount of colloid present.

In this relation we may recall the careful researches of Eisenberg and Volk, $\uparrow$ who observed similar effects in determining the amounts of agglutinin that a given amount of bacteria adsorbs when added to increasing doses of agglutinin in a constant volume. This fact

* Van Bemmelen has shown also that adsorption takes place better in the cold than at higher temperatures; in our instances we are dealing with an adsorption that has taken place in the cold and is partially broken up by heat.

† Zeitschrift für Hygiene, vol. 40. 
is one of those most strongly indicative of the relation which would seem to exist between adsorption phenomena and the reactions between agglutinins and bacteria.

The suspension of an inorganic powder in a colloidal medium begins, then, by adhesion of the colloid to the powder. We have just seen that certain colloids on uniting with barium sulphate agglutinate it. The two phenomena of agglutination and dissociation of barium sulphate by stable colloids, although apparently so different, are in fact due to the same mechanism, namely, the adhesion of the colloid to the suspension.

We have already found that a given colloidal substance in uniting with a given suspension may produce either its dissociation or its agglutination. This is particularly the case with such colloids as gelatin, the concentrated solutions of which harden in the cold. The dissemination or the agglutination of a suspension of barium sulphate by a 0.5 per cent solution of agar depends on whether the mixture is made at $50^{\circ}$ or at $16^{\circ} \mathrm{C}$. In both instances adhesion takes place. It is probable that weak solutions of agar at high temperature are composed of very small particles of the substance, which particles clump together as the temperature is lowered. This supposition indeed finds an experimental basis in the facts that Hardy* has noted. This author found that solutions of agar and gelatin are composed of granules, the volume and disposition of which depend on the temperature. The effect of agar, then, on barium sulphate depends on its physical condition or on the size of its particles, which latter fact depends on the temperature and the duration of time that the colloid has remained at this temperature.

The effect of cold on agar corresponds to the effect of heat on the protein substances of serum; these substances when unheated are in a colloidal condition which permits them to adhere to barium sulphate and to disseminate it; when larger proteid particles have been produced by heating the clumping of the sulphate follows. $\dagger$

These facts, we believe, may be compared with another phenomenon: if a fresh amount of barium-sulphate suspension is added to a complex of washed barium sulphate and colloid (for example, barium sulphate plus gum arabic), complete agglutination of the

* Proceedings of the Royal Society, 1900, LXVI.

$\dagger$ Gengou, p. 326. 
complex plus the fresh suspension takes place. The particles of the complex probably act on fresh barium sulphate just as voluminous particles of the gum would act on fresh barium sulphate, or, in other words, as do the granules of cold solutions of gelatin or the albuminous clumps produced in serum by heating.

$$
\text { *** }
$$

With the exception of the instances in which the viscosity of the fluid intervenes, the dissemination of suspensions in the solutions we have studied is due to a substitution of the adhesion of the particles among themselves for an adhesion of the particles of the suspension to particles of the disseminating colloid.

The dissemination of a washed suspension by colloidal solutions resembles the inhibition of the appearance or sedimentation of precipitates due to chemical reactions which might occur in such solutions. We think that the facts observed by Lobny de Bruyn and others in this connection, which have been made use of to obtain colloidal solutions of substances which are normally insoluble, are due to the fact that as soon as the chemical reactions which lead to the production of these substances within a stable colloid solution are finished, there is established, between the particles of these substances and the neighboring particles of the surrounding colloid, a complex which is similar to those that we have studied.

Colloidal solutions obtained in this way are therefore suspensions in a greater or less excess of stable colloid of complexes formed by the stable colloid with an insoluble chemical substance.

The dissemination of an insoluble salt by stable colloids in the form of a complex should apparently be compared with the homogeneous condition which colloids give to emulsions of oil. This phenomenon, as Quincke* has shown, is due to the fact that these colloids diminish the surface tension of the oil droplets for water by intervening between the droplets of oil and those of water. It is probable that the dissemination of barium sulphate in a colloidal medium is likewise due to the fact that the surface tension which exists between the suspension and water is lowered by the presence of the colloid on the surface of the particles of suspension that have absorbed it.

* Quincke, Wiedemann's Annalen, 1904, XXXVII. 
Some writers have concluded from Quincke's experiments that any substance which diminishes the surface tension of substance A for fluid B must be absorbed by A, and, on the other hand, that any substance that has been adsorbed must diminish the surface tension of the adsorbing substance. It is to be remembered, however, that in certain instances the mutual adhesion of two substances in fine suspension in a fluid results, not in a dissemination, but in an increase of surface tension. Thus the mutual flocculation of two unstable colloids of different electric charges indicates an increase of superficial tension resulting from the adhesion of particles of the two colloids, which fact Biltz* correctly compares with adsorption phenomena.

$$
*^{*} *
$$

We have endeavored to determine the reaction of our complexes of barium sulphate plus colloid to electrolytes. Such complexes as we have already mentioned may be removed from the colloidal solution in which they have formed, and resuspended in distilled water. We have stated that complexes washed in this manner react to electrolytes and flock out as unstable colloids do when these electrolytes are present. Alkalis, and particularly acids, have the same effect. It is evident that in this case we are dealing with an electrolytic effect and not with a phenomenon of plasmolysis due to concentration of the salt, inasmuch as such washed complexes do not flocculate when the salt is replaced by isotonic sugar solution; such a sugar solution may be employed in any concentration without flocculating the complex.

This flocculation is reversible, inasmuch as when the salt solution which flocculates is removed and replaced by distilled water, the complex recovers its original appearance and is again disseminated. Although a reversibility occurs in the agglutination of certain colloids and certain fine suspensions by means of salts of alkalis and of alkali earth, the fact should be mentioned in the present instance. We might suppose, for example, that in precipitating a complex of barium sulphate plus gum or the like, the electrolyte simply detaches the fixed colloids from the suspension, in other words, breaks up the complex and restores barium sul-

* Berichte der deutsche chem. Gesellschaft, 1904, XXXVII. 
phate to its original condition. The fact that the flocculation of the complex by sodium chloride is reversible proves, however, that this is not the explanation; it is the entire complex which is flocculated.

Flocculation, on the contrary, is lacking when the complex, barium sulphate plus colloid, has not been washed or when, after it has been washed, an excess of stable colloid is added. This latter colloid, then, would seem to "protect" complexes just as it protects an unstable colloid from flocculation by salts. This fact, we think, tends to validate the explanation of Beckhold, Girard-Mangan and v. Henri, Pauli, and others.* They propose to explain the mechanism of the protection of unstable colloids by stable colloids against precipitation by electrolytes. When we remove a complex of barium sulphate plus gum from the colloidal medium in which it has formed, by washing, it is very probable that we do not obtain it as it occurs in such a medium. If we accept the conception that has been proposed by Van Bemmelen for the phenomena of adsorption, and admit that the preparation of emulsions of oil in gum solution described by Quincke (that is, the covering of the oil droplets by a layer of gum), is similar to the mode of preparation of our complex, we may suppose that the particles of the complex, barium sulphate plus gum, are composed of a center formed by the barium sulphate around which the particles of gum are disposed in a homogeneous manner. The most distant of these gum particles are attracted only feebly by the barium sulphate and become detached in washing, which leaves us a complex poorer in gum than in its original condition. We may then logically imagine that on placing our washed complex in a fresh gum solution it becomes covered with fresh exterior layers of gum which have been removed by washing. The inhibiting effect of gum solution on the flocculation of the complex by sodium chloride may then be due to an increased thickness of gum and to the intervention of additional superficial layers on the complex. The following fact may be mentioned in support of this conception: if complexes of barium sulphate and gum are formed in increasing quantities of gum solution and subsequently washed in distilled water, we should obtain complexes of varying richness

* Cited by Aron, Bioch. Centralblatt, vol. 3 . 
in colloidal substance, the flocculation of which by salts should diminish in accordance with the amount of colloid present. This fact would seem to us to demonstrate that it is the stable colloid adhering to the powder which opposes in great part the flocking action of the electrolytes. The same fact also probably holds in the protection by a stable colloid against precipitation of an unstable colloid by salts.

\section{$*^{*} *$}

Apart from the flocculation of complexes by electrolytes, all the facts described to the present are due to the same cause, namely, a substitution of one form of adhesion for another. When a stable colloid transforms the unstable mixture of barium sulphate and water into a much more delicate and stable suspension, it is due to the fact that one adhesion (barium sulphate plus colloid) is substituted for another adhesion (barium sulphate plus barium sulphate). In order for this to occur the attraction of the suspension for the colloid must naturally be stronger than the attraction of the suspension for itself.

Likewise the fact that a stable colloid such as gum or serum disseminates barium sulphate by separating the mutual adhesion of the particles of barium sulphate resembles the fact that a stable colloid may inhibit the adhesion of barium sulphate to another colloid. Thus, in the presence of serum, starch solution and peritoneal fluid fail to agglutinate barium sulphate; the suspension adheres to the colloids of the serum and remains disseminated in spite of the presence of the agglutinating colloids. In the same way a stable colloid (gum) prevents the mutual flocculation of fresh barium sulphate with a washed complex of barium sulphate plus gum. In this instance the fresh suspension adheres to the free stable colloid and not to the washed complex.

II.

We have stated that barium sulphate may be held in fine suspension by substances other than stable colloids. Citrate of sodium, the inhibiting action of which on coagulation of the blood and milk is well known, will do this. The statements we have made about suspensions of powders by stable colloids render unnecessary any 
full description of the action of citrate. The dissemination of barium sulphate by the citrate does not increase, with a given dose of powder, with the concentration of the citrate. We have found, for example, that an amount of powder contained in eight drops of our barium sulphate solution, when added to 2 c.c. of fluid, is well disseminated if this fluid contains from 0.005 per cent to 0.1 per cent of citrate. Above this concentration the disseminating property of the solution diminishes, so that, in 20 or 30 per cent citrate, barium sulphate sediments almost as rapidly as in distilled water. This disseminating action may also occur with other substances that are insoluble in water, such as clay, tricalcium phosphate, animal charcoal, and olive oil.

We have found that this property in sodium citrate is due to its acid radicale. If increasing doses of citric acid ( 0.0016 per cent to 0.05 per cent in our experiments) are added to a given quantity of barium sulphate, distinct dissemination takes place in certain tubes, as indicated by a suspension of the powder. As with sodium citrate, the disseminating property is minimal with certain doses of citric acid and diminishes with stronger doses of it. These facts may be compared with the recent observations of Freundlich (Zeitschrift für phys. Chem., LVII, 4). This author found that charcoal (Blütkohle) remains for a long time in suspension in methylamine, dipropylamine, trimethylamine, pyridin, codein and picric acid; he found that this charcoal suspension has an optimum in the moderate concentrations of these substances. Oechsner de Coninck and Azalier* found that barium sulphate is retained in suspension by the chlorhydrate of methylamine, and that even when it is warm no chemical reaction occurs between this organic fluid and the suspension.

The disseminating action of the citrate is due to the fact that the suspension adsorbs it. We find, indeed, that on treating a weak solution of citrate with barium sulphate, the property of disseminating fresh barium sulphate is removed. And, what is more, if a uniform amount of barium sulphate is added to increasing doses of citrate, the absolute amounts of citrate fixed, increase, whereas the proportion of the citrate adsorbed varies relatively with the initial mass of the salt.

* Bull. de le Cl. des Sciences Acad. Roy. de Belg., 1907, No. 6. 
A complex is formed between the suspension and the citrate similar to that which occurs when barium sulphate is added to a colloidal solution (Section I). The dissemination of barium sulphate by sodium citrate corresponds with Spiros'* idea. This author thinks that the presence of electrolytes in small amount is necessary for the conservation of colloidal solutions. The salts capable of producing this effect would vary with the colloid under consideration; small amounts of alkali will sustain silicic acid and stannic acid in a colloidal condition; small amounts of acids act in the same manner on colloid ferric hydrate and on gelatin. Malfitano $\dagger$ has shown, also, that colloidal ferric hydrate filtered through collodium becomes more and more unstable as it loses. its electrolyte $(\mathrm{HCl})$; this writer believes that colloidal ferric hydrate formed by hydrolysis of a ferric salt is unable to remain alone in colloidal condition, and acquires such a condition only through adsorption of the ions, $\mathrm{Fe}$ or $\mathrm{H}$.

My own data may be compared with the facts that were mentioned by Arthus a few years ago. $\ddagger$ This writer found that if sodium citrate ( 0.5 to 1 per cent) is added to an emulsion of clay in water, the precipitating dose of the salts of alkalis or of alkali earths is considerably increased (20 to 28 times). "Although in the instance of calcium salts we may advance the hypothesis of a double interchange of salts leading to the production of calcium citrate and sodium chloride, no such hypothesis could be offered in the case of $\mathrm{NaCl}$. In this instance sodium citrate evidences an antagonism to sodium chloride, the precipitating salt; the citrate, then, may be regarded as endowed with a direct antiprecipitating property." This phenomenon is interesting when we consider the inhibiting action of the citrate on the coagulation of blood and milk, which is not accompanied by precipitation of calcium. Our researches show that the antiflocculating property of sodium citrate, observed by Arthus, is not due to some unknown obstacle to flocculation by sodium salts in general which this substance offers; we have indeed met with certain examples of flocculation by sodium chloride which are not inhibited by the citrate (in particular, flocculation of the

* Hoffmeister's Beiträge zur chem. Phys. u. Pathologie, Vol. 5.

† C. R. Acad. de Sciences de Paris, 1905.

$\ddagger$ C. R. de Soc. de Biol., 1902. 
iodide of starch). As a result of our experiments we conclude that the action of the citrate is directly on the flocculable substance itself and due to its adsorption by this substance.

The complex, barium sulphate plus citrate, can be washed in distilled water and resuspended in distilled water in the same way as the complex of barium sulphate plus a colloid; it remains in suspension. The stability of this complex, however, is far from being as great as complexes of barium sulphate with colloids, and we find that it must be washed with rather small volumes of water, since large amounts of water finally break it up and liberate the powder. The stability of the complex is, however, sufficient to allow us to study it by subjecting it to various influences.

When we submit a complex of barium sulphate plus citrate that has been washed and suspended in distilled water to the action of an electric current we find that it is directed feebly though distinctly toward the anode. This fact, which is much more distinct with a complex of barium sulphate and colloid, shows that the adsorption of an electrolyte may endow a suspension with a distinct electric charge. In this connection we may recall that Lottermoser* has shown that colloidal solutions of AgI may be obtained from $\mathrm{KI}$ and $\mathrm{AgNO}_{3}$ by means of an electric current which moves it either toward the negative or positive pole, depending on whether the initial mixture contains an excess of KI or an excess of $\mathrm{AgNo}_{3}$. The ions, $\mathrm{Ag}$ or I, in excess, bring about a colloidal state under these conditions (Solbildner, de Jordis), and endow the particles of AgI with a positive or negative electric charge.

Arthus, in a brief note on the study of sodium citrate, shows not only that it inhibits flocculation of clay by an amount of $\mathrm{NaCl}$ that is sufficient ordinarily to produce agglutination, but he also shows that this action of the citrate may be overcome by a larger amount of $\mathrm{NaCl}$. We have subjected the complex, barium sulphate plus citrate, to the action of electrolytes in the same way that we treated the complex of barium sulphate plus colloid. As in this latter instance, the complex, barium sulphate and citrate, is flocculated by a salt and sediments rapidly on the addition of a neutral salt, a base or an acid. 
We found that the flocculation of the complex, barium sulphate plus gum, by a salt, is reversible, that is to say, on the removal of the saline solution and suspension in distilled water, the disseminated condition comes back. In the same way the complex, barium sulphate plus citrate, floceulated by a salt, and after the removal of this salt suspended in distilled water, becomes disseminated again. There is then a distinct analogy between the complexes of barium sulphate plus colloid and the complex, barium sulphate plus citrate, in respect to flocculation by electrolytes. A reversibility of the flocculation of the citrate combination by means of a salt also occurs - at least within the limits of saline concentration which we have employed - on agglutinating the complexes by a soluble calcium salt in place of the sodium salt; the complex after the excess of calcium solution is removed when suspended in distilled water recovers its normal appearance. We have also noted that if barium sulphate, $\mathrm{NaCl}$, and sodium citrate are mixed together all at once, that the complex of barium sulphate plus citrate still forms; it is quite as readily flocculated by the sodium chloride present, but when washed and resuspended in distilled water it acts as if it had been formed without the presence of sodium chloride.

$$
\text { *** }
$$

To sum up, sodium citrate forms a complex with barium sulphate as with other insoluble substances which causes a fragmentation of the granules of the suspension into extremely small suspended particles; this results in the formation of a much more delicate and stable suspension of the sulphate. The same thing is true with clay. Arthus has, indeed, noted that the flocculation of clay by a sodium salt requires much more salt when citrate is present than when it is absent. The inhibition which citrate causes on the flocculation of a suspended substance in water by means of a salt may be made use of in detecting the formation of a complex between such a suspension and citrate. We may use this fact to advantage in dealing with a material which usually gives so fine a suspension in water that the addition of citrate produces no visible modification. Such is the case with calcium fluoride (fine suspensions of calcium fluoride in water which are almost colloidal may be obtained), and particularly with mastic. Both these substances, which are 
flocculated on the addition of a sodium salt, precipitate with much greater difficulty when citrate is present. This fact may be regarded as a result of the formation of complexes between the calcium fluoride or the mastic and citrate.

We may now consider the appearance of an insoluble substance, the particles of which undergo a much more marked dissemination than does barium sulphate when citrate is added. Inasmuch as the particles of such substances are extremely small; they may be more like the component particles of a colloidal solution.

We have known for some time that citrates of alkalis, particularly the insoluble citrates of calcium salts "dissolve" certain substances which are insoluble in water, it is in addition noted that the citrates of alkalis prevent precipitation of a copper, aluminium, or iron salt by a base. We think that this action of citrates of alkalis may be comparable to the action of sodium citrate and stable colloids on barium sulphate; it may be that in these cases the citrate forms a complex with the precipitate of hydrate as soon as it appears, so that the hydrate, instead of being flocculated, remains in colloidal solution.

We place a given amount of well-washed aluminium in increasingly concentrated solutions of citrate, and find, in a few moments, that cloudiness, caused by the aluminium, becomes more marked in certain tubes and that it then decreases and the fluid eventually becomes perfectly clear. We know that we are not dealing with an ordinary dissolution phenomenon, inasmuch as the hydrate gives a more marked cloud with the citrate than does ordinary aluminium, which is not the case with ordinary dissolutions. And what is more, no soluble citrate of aluminium has been described so far as we know.* But the reason for comparing a dissolution of aluminium with a suspension of barium sulphate in citrate is the corresponding action of such salts as sodium chloride in both instances. We have already noted that the complex, barium sulphate plus citrate, is flocculated on the addition of sodium chloride; in the same way we find the "solution" of aluminium in citrate becomes troubled when sufficient amounts of sodium chloride are added.

* In Wurtz' "Dictionary of Chemistry," only an insoluble citrate of aluminium transferred by an acid into a very soluble gummy product is mentioned. 
The opacity produced by sodium chloride may be due to a double decomposition: the sodium citrate could not give the insoluble substance, aluminium, with sodium citrate if it reacted chemically with sodium chloride, but could give only soluble substances. We must then be dealing with a flocculation of very small particles in limpid colloidal solution by sodium citrate; these particles must belong to the complex, aluminium plus citrate. This complex, then, would be analogous to the complex of barium sulphate plus citrate, but instead of being a fine suspension, as this complex, it would appear in water as an apparently colloidal limpid solution. Such a mechanism probably accounts for the inhibition to precipitation of aluminium salts by bases which citrate gives.

Notwithstanding, the reasons which seem to favor this opinion, we give this interpretation as an hypothesis only. The subject should be studied more carefully from a purely chemical standpoint.

We should like, however, to mention one more fact which is related to the phenomenon that we have just described. We know that the salts of aluminium, for example the sulphate, are coagulants and agglutinants. Alum flocks certain dyes, such as fuchsin, with ease. We have found that such a flocculation is completely inhibited by sodium citrate. This effect cannot be due to a precipitation of the agglutinating element from the solution, for we find that these salts show no precipitation after several days. It may be that the obstacle to the flocculation by alum produced by citrate is due to the dissemination of alum hydrate, which would remove its precipitating properties. This, to be sure, is only a hypothesis, the value of which must be determined only by more extensive experiments.

The inhibiting power of sodium citrate on the flocculation of certain substances by salts (Arthus) is better understood when we consider its disseminating effect on substances or elements which sediment spontaneously. In the same way as the suspension of insoluble substances by stable colloids is to be considered as the result of the formation of a complex between the powder and the colloid, so the disseminating action of sodium citrate on certain 
chemical precipitates is due to the production of a complex between the precipitate and the citrate. It is probable that the adsorbed citrate diminishes the surface tension between the particles of the adsorbed body and the surrounding fluid; it is owing to this fact, probably, that the modification brought about by the citrate in the physical condition of the adsorbing substance is similar to the inhibition by this salt to flocculation of adsorbing substances like clay or calcium fluoride by electrolytes. The inhibiting action of citrate on adhesion of particles of the adsorbing substance to other elements in colloidal solution, or in suspension, is also apparently due to the same fact. Citrate that has been adsorbed by barium sulphate can oppose, not only the reciprocal adhesion of the particles of this suspension, but also the adhesion between them and stable colloids of other substances in the same way as a stable colloid, A (serum), is able to inhibit the adsorption of another colloid, B (starch or gum). This is the way that citrate prevents the adsorption of gum arabic, mucin or of starch by barium sulphate; it likewise inhibits the adhesion and subsequent flocculation of fresh barium sulphate plus colloid. In like manner, the citrate prevents the adhesion and subsequent flocculation of calcium fluoride with indigo carmin; and finally, in a similar manner, it prevents, to a certain extent, the adsorption of eosin by animal charcoal.

These various examples suffice to show that the adsorption of citrate by calcium fluoride, barium sulphate, animal charcoal and the like may take the place of the adsorption of other substances by these insoluble bodies as well as the mutual adhesion of the individual particles of each one of these materials. The phenomenon is evidently due to the fact that the affinity of a given suspension for various substances (dyes, colloids and citrate) varies, and also to the fact that this suspension, which is attracted at the same time by two affinities of different intensity, naturally yields to the stronger one - in our experiments to its affinity for the citrate. The inhibiting effects of citrate are, then, simply the result of a struggle of affinities for barium sulphate between the citrate and the other substances, the adsorption of which, by the suspension, this citrate prevents. It is this principle of a struggle between two adsorption phenomena which we have employed in our study on the agglutination and dissolution of red blood cells by various substances. 


\section{III.}

The two preceding chapters have been given over to the consideration of adsorption phenomena which occur between substances neither of which is cellular, properly speaking. Certain of them, such as barium sulphate, calcium fluoride and the like, are insoluble inorganic substances; and others are stable colloids (gum and the like) or electrolytes (sodium citrate).

In the mutual adhesion between these substances which we have considered, one of them, the colloid, may be replaced by a cellular element. As we have previously shown,* barium sulphate, when mixed with red blood cells that have been washed free of all serum and suspended in salt solution, adheres to the corpuscles and produces agglutination in large clumps composed of suspension and corpuscles. The agglutination is followed by dissolution of the corpuscles when sufficiently large doses of barium sulphate are used.

We have here an adhesion phenomenon between two suspended substances which may be studied by subjecting it to the various influences which we have employed in dealing with other adhesion phenomena, as the one between barium sulphate and starch. We find here, too, that the agglutination of barium sulphate with corpuscles does not occur when a stable colloid like serum, or when sodium citrate, is present, any more than it does in the adhesion of barium sulphate with starch. We know from what we have seen in the preceding chapters what takes place under these conditions; it may be that another complex, namely, barium sulphate plus serum or barium sulphate plus citrate, takes the place of the complex, barium sulphate plus corpuscles, which occurs under normal conditions; in other words, one phenomenon of adhesion takes the part of another similar phenomenon.

We have already demonstrated that the particles of the complex, barium sulphate plus citrate, which remain separate in the solution in which they have been formed, without any tendency toward mutual adhesion, are agglutinated on the addition of a salt such as

* Gengou, C. R. Acad. Sciences, Paris, 1904. Landsteiner and Jagic noted the effect of colloidal silicic acid on corpuscles some time before (Wein. klin. Wochenschr., 1904, No. 3). Shortly after our communication, Madam GirardMangan and v. Henri (C. R. Soc. Biol., 1904) in turn studied this phenomenon very extensively with various colloids. 
$\mathrm{NaCl}$ or $\mathrm{CaCl}_{2}$. This salt increases the affinity of the particles in the complex for one another. In like manner, the agglutination of corpuscles with suspensions may be forced to appear even in a citrate medium on the introduction of sufficient amounts of $\mathrm{CaCl}_{2}$; and, what is more, if we use a solution of 7 per cent saccharose instead of 0.85 per cent salt solution as a medium, we may produce an agglutination of barium sulphate with corpuscles, which is prevented by the citrate, on the simple addition of $\mathrm{NaCl}$.

These experiments may all be repeated with other insoluble substances, as, for example, $\mathrm{CaFl}_{2}$ and mastic. Both these substances agglutinate washed red blood cells; the agglutination is prevented either by stable colloids or by sodium citrate, and this failure in agglutination is due to the fact that the adhesion of the insoluble substance with the corpuscles fails to occur. In the place of the complexes, $\mathrm{CaFl}_{2}$ plus corpuscles, or mastic plus corpuscles, the complexes, $\mathrm{CaFl}_{2}$ plus stable colloid or mastic plus citrate, occur. The addition of a salt like $\mathrm{CaCl}_{2}$ or $\mathrm{NaCl}$ causes the agglutination, which has been inhibited by the citrate, to appear, just as it provokes the agglutination of the corpuscles by barium sulphate in a citrate medium.*

* We have shown in Section II the inhibiting effect of sodium citrate on precipitation of fuchsin by alum. With every reserve as to the interpretation of this fact we have, nevertheless, insisted on the analogy which seems to exist between the function of the citrate in this instance and its action with suspensions. It is with a similar reserve that we mention the effect of the citrate on other phenomena of agglutination produced by alum. As we already know, Malvoz (Annales de l'Institut Pasteur, 1897) has shown that various chemical substances agglutinate emulsions of bacteria. Alum agglutinates typhoid bacilli well; we have also found that it agglutinates red blood cells and even dissolves them when a suffieient dose is employed. All these phenomena fail to occur when even a small amount of sodium citrate is present. Not only does agglutination fail to appear, but we have been able to show that the citrate prevents the adsorption of the alum by the bacteria or the red blood cells, which normally occurs when citrate is not present. We have also found that bacteria that have been agglutinated by moderate doses of alum, and then washed in distilled water, are well agglutinated on the addition of $\mathrm{NaCl}$ (this fact may be compared with the agglutination of bacteria by specific sera and with the observations of Beckhold (Zeitschrift für phys. Chem., Vol. 48) on the flocculation of bacteria by the salts of heavy metals). If we treat bacteria which have been mixed with alum in a citrated medium in the same way, the subsequent agglutination by $\mathrm{NaCl}$ fails to occur. It is evident, then, that the mode of action of the citrate in this instance seems comparable to its action on substances in aqueous suspensions (barium sulphate, calcium fluoride or mastic); but, owing to the fact that chemical reactions may take place between alum and eitrate, and thus prevent the manifestations produced by the alum, we think it better to reserve any interpretation of this fact. 
It should be noted, however, that the agglutination of corpuscles with insoluble bodies like barium sulphate by means of salts in a citrated medium is never so intense as when there is no citrate present. We think this is owing to the fact that the salts do not break up the complex of barium sulphate and thereby restore the suspension to its original condition, but endow the very particles of this complex with a certain amount of adhesive property. We have already seen that flocculation of the complex, barium sulphate plus citrate, by a salt is reversible, that is to say, that the complex recovers its normal appearance when the flocculating salt is removed. The salt, then, endows the particles of the complex with a mutual adhesive affinity (flocculation of the complex), or else with an adhesive affinity for other suspended substances (corpuscles). And, consequently, the agglutination of corpuscles with an insoluble substance in a citrated medium when a salt is present would naturally not be as great as the one produced by the same amount of salt when citrate is absent; this, indeed, is what we find to be true. The contrary would occur if the activating salt broke up the complex and restored the suspension to its normal condition.

The action of citrate on calcium fluoride, mastic and the like, as we have noted in Section II, is not immediately perceptible, as is the dissemination of barium sulphate. Citrate does, however, affect these various substances in a similar manner and prevents their adhesion to other substances, such as red blood cells, as we have already indicated. Calcium fluoride, an inorganic substance, is intermediary between barium sulphate, an inorganic substance that is disseminated by a citrate, and mastic, which is an organic substance that shows no immediate effects with citrate. From a consideration of mastic we may go on to the study of other organic bodies which in turn would seem to show no reaction to the citrate, but which are, however, influenced by it in a similar manner to calcium fluoride and mastic.

We find that we can continue our comparison of the adsorption phenomena that we have studied with similar phenomena in hemolysis produced by biological agents. We find that citrate of sodium inhibits hemolysis by eel serum, venom, lecithid and alexin in the 
same way as it prevents the adhesion of the particles of barium sulphate to one another or to suspended substances like corpuscles or to colloidal substances like gum arabic.*

It is evident that this inhibition of hemolysis by citrate is not due to a destruction of the biological hemolysins by this salt, inasmuch as we find they can be reactivated by the addition of an electrolyte. In the same way that we increased the adhesive properties of citrated barium sulphate on the addition of sodium chloride and calcium chloride so that the particles of the complex are able to adhere to one another and flock out, or to adhere to other elements and agglutinate them, so, on adding calcium chloride, we are able to reactivate the biological hemolysins in a citrated medium. In certain instances, as, for example, with venom, this reactivation may be produced by $\mathrm{NaCl}$. If the hemolysis of guinea-pig corpuscles by cobra venom is prevented in a sugar medium by a sufficient amount of citrate, the inhibition may be removed by adding a small amount of sodium chlorid to the mixture.

It might well be, however, that citrate prevents the action of the biological hemolysins by neutralizing the calcium salts which are necessary for their action. Such an interpretation would seem all the more admissible inasmuch as with certain of these hemolysins (venom, lecithid and alexin) the dissolution of the corpuscles is also lacking when oxalate or fluoride of sodium is added to the mixture. Inasmuch as it is generally supposed that the inhibition of coagulation of the blood and of milk, produced by these salts, is due to a neutralization of the calcium salts, it may well be supposed that the similar inhibition of hemolysis is due to the same reason.

Such a hypothesis, however, is not justified. In the first place we have to note that although citrate of sodium prevents hemolysis by eel serum, oxalate produces no such effect even when large amounts of isotonic oxalate solution are employed. It is therefore probable that the mechanism of the inhibition of hemolysis by the citrate is similar in the various other instances by biological hemolysins which have been mentioned.

From the facts which follow, and for other reasons, we are led to

* Bordet and Gay, see page 403, have also described similar facts in dealing with the inactivation of alexin by sodium citrate. 
believe that the inhibiting action is not due to a decalcifying power of the citrate, the oxalate or the fluoride. If it were so, we should expect to find a distinct parallelism between the intensity of the anticalcium property of the citrate or oxalate and their antihemolytic property. Such, however, is not the case. Sabatani (Archiv. Ital. de Biol., 1901, Vol. 36) has shown that if we regard the anticalcium power of the oxalate as 1 , the similar power in the citrate may be represented by 0.45 . According to this author the anticoagulating property of each of these salts is exactly parallel to their anticalcium property. This, however, is not true as regards their antihemolytic effect. We have found, indeed, that if we represent the antihemolytic action of the oxalate as 1 , the corresponding action of the eitrate would correspond to 3 as regards hemolysis by venom, to 1.45 in hemolysis with lecethid, and to 1.5 with alexin hemolysis.

We have found that if amounts of oxalate which are entirely sufficient to decalcify the serum are added to undiluted alexin or to concentrated venom, and allowed to remain with them for 24 hours, on removal of the calcium oxalate that has been formed, these mixtures, when suitably diluted, are quite as hemolytic as if no oxalate had been mixed with them.

Inasmuch as the citrate does not act as a decalcifying agent, we are forced to compare its action on animal hemolysins with its action on the insoluble substances which we have previously studied. To render the analogy complete it would be necessary, in order to produce an inactivation of the hemolytic agents in a citrated medium, that an inhibition to the adsorption of these substances by the corpuscles should exist. This we find to be the case: if in a citrated medium blood corpuscles and any one of the hemolysins which we have mentioned are mixed, the supernatant fluid subsequently removed may be shown on reactivation with $\mathrm{CaCl}_{2}$ to contain the hemolytic agent

It would seem, then, that sodium citrate prevents the action of eel serum, venom, lecithid and alexin on red blood cells in the same way that it prevents similar action by such substances as barium sulphate, calcium fluoride and mastic. We have seen that the inhibition of hemolysis by insoluble substances like barium sulphate, by means of citrate, is due to the formation of complexes such as 
barium sulphate plus citrate, calcium fluoride plus citrate, etc., in place of the complexes, barium sulphate plus corpuscles, and the like. It is probable, then, that hemolysis by biological agents fails, when citrate is present, because these substances form unions with the citrate similar to barium sulphate plus citrate, instead of attacking the corpuscles. We have seen that the action of salts like $\mathrm{NaCl}$ and $\mathrm{CaCl}_{2}$ on the complex, barium sulphate plus citrate, is reversible, and we have further seen that it is owing to this reversibility that this complex is not always so agglutinating for red blood cells when the salt is added as if the suspension in the complex had been restored to its normal condition by means of $\mathrm{NaCl}$ or $\mathrm{CaCl}_{2}$. This also we find to be true with biological hemolysins. These latter, when inactivated by citrate, can be reactivated by $\mathrm{CaCl}_{2}$ or even by $\mathrm{NaCl}$. This reactivation, however, does not always endow the mixture with as much activity as the same amount of hemolysin would have in a non-citrated medium. It would seem, then, as if the reactivating salts simply increase the affinity of the complex of hemolysin plus citrate for the corpuscles. This, to be sure, is the most plausible explanation of their activation of such complexes by $\mathrm{NaCl}$; it does not, however, authorize us at the present time to lay aside the possibility of a chemical neutralization of the citrate by $\mathrm{CaCl}_{2}$.

Apart from this question of the mechanism of reactivation by $\mathrm{CaCl}_{2}$, we may say that it would seem that the inhibiting effect of citrate on hemolysis by biological agents is due to the same mechanism as its influence on the various phenomena that have been studied in the preceding chapters. There is a very clear parallelism between these two classes of phenomena. As a result, we feel justified in concluding from our researches that the mode of union of biological hemolytic agents with red blood cells would seem to be an adsorption phenomenon which is comparable to the one that occurs between red blood cells (or the other substances which we have studied) and insoluble substances like barium sulphate or colloidal solutions like mastic. We have described these various phenomena of adhesion between red blood cells and insoluble substances, or biological hemolysins, to the present, as they occur in solutions of isotonic solutions of $\mathrm{NaCl}$ or in blood serum. The majority of these phenomena will also take place in a solution of saccharose as well; the hemolysis 
by eel serum and by alexin, however, does not occur under these conditions. We may leave aside hemolysis by alexin, inasmuch as it is known to be very much altered by dilution in a medium which is poor in salts. The failure of hemolysis by eel serum in a sugar medium is not, however, due to an alteration in the hemolysins, but to deprivation of salts. When we dialyze eel serum against distilled water we find the globulins which are precipitated by this treatment contain no hemolysin, but that this substance remains intact in the limpid portion of the dialyzed serum. We have found, moreover, that the reason this hemolysin does not dissolve red blood cells in a sugar medium is due to the fact that under such conditions it is not adsorbed by the cells. The addition of salt to such a mixture, however, brings about adhesion of the hemolysin to the cells, and their dissolution. When we compare the activating power of various salts, we find that they depend on the kation and that the salts of the alkali earths are much more potent than corresponding salts of alkali metals; the activating strength of the salts increases, then, parallel to their flocculating action on unstable negative colloids.

In this case, also, it would seem that the electrolytes increase the adhesive property of the particles of the hemolysin in eel serum for corpuscles by increasing their superficial tension in the same way that they increase the adhesive power of the particles of an unstable negative colloid for one another.* This evidently does not explain the action of the salts; we may, however, say that such salts increase the adhesive power of a suspended substance for other suspended substances as if they increased the affinity of the particles of such a suspended substance for similar particles. It would seem to us as difficult to explain the adsorption on the addition of salts, of substance A by substance B - and particularly of the hemolysin of eel serum by red blood cells - as a purely chemical phenomenon, as it is to explain the reciprocal adhesion between particles of a

* This phenomenon may be compared with the facts that have been mentioned by Nasse (Pflüg. Archiv. für Physiol., 1885, Vol. 37) and by Bayliss (Biochem. Journal, I). Nasse found that the adsorption of iodine by glycogen is increased by salts; Bayliss demonstrated that the coloring power of electronegative colloidal dyes for paper is increased by the kations and inhibited by the anions, and that the inverse is true with electropositive colloidal dyes. 
given substance, when salts are added, by the same method. This adhesive power, which the hemolysin in eel serum has only when electrolyes are present, would seem to exist normally in other substances, either for other suspended elements or for other particles of their own suspension.

This faculty of adhesion which is spontaneous with certain materials, and is brought about only when electrolytes are present in other materials, is combated by the citrate, which substitutes one adhesion for the other adhesions which these substances may show. The action of the citrate, its mechanism and the opposing effect of electrolytes are precisely the same whether we study them in distinct adsorption phenomena, such as the adsorption of one substance in colloidal solution (gum arabic), or in suspension (corpuscles) with an insoluble substance (barium sulphate), or when we study the hemolytic phenomena produced by the biological agents that have been studied by bacteriologists.

$$
\text { *** }
$$

It should be recalled in this connection that Bordet has long maintained that the neutralization of a toxin by an antitoxin is comparable to the phenomena of dyeing rather than to the ordinary chemical reactions expressed by equations. We cannot mention all the facts that serve to support this theory, in this place, but we may recall that certain experiments led Bordet to postulate that the dose of alexin, fixed by a given quantity of corpuscles, would vary according to the initial mass of alexin that was present. Certain other researches also led him to the conclusion that a given dose of anti-alexin will neutralize variable amounts of alexin in accordance with the manner in which the two substances are mixed. We meet with the same fact in considering the neutralization of certain toxins by their antitoxins; this fact is generally described as Danysz' phenomenon.

It was particularly on the basis of the facts concerned in the reactions of alexin with corpuscles on the one hand and with antialexin on the other, that Bordet formed his theory of the neutralization of toxins by antitoxins. It seems not without interest to compare this writer's conceptions with the conclusions which we have arrived at in this work, in so far as concerns the mode of 
reaction of the alexin on corpuscles. We have also been led to compare this reaction with the phenomena of molecular adhesion but arrived at this comparison in a somewhat different manner. Our conclusions, we believe, may also be considered as an additional experimental support for Bordet's theory. 


\title{
XXIII. THE PHENOMENA OF ADSORPTION AND THE CONGLUTININ OF BOVINE SERUM.*
}

\author{
BY DRS. J. BORDET AND OSWALD STRENG.
}

One of us has suggested and maintained for some time the opinion that the phenomena of union of antigens with antibodies in serum belong rather in the category of phenomena of molecular adhesion than among chemical reactions, properly speaking; in other words, they are apparently adsorption phenomena.

That the modifications on certain cells brought about by specific serum frequently resemble phenomena which have no relation to a chemical reaction both in their general appearance and in their course, is undoubted. Ten years ago one of us brought out the fact that hemolysis by an active serum obtained by immunizing animals against the blood of a foreign species resembles in certain respects the hemolysis produced by distilled water; in both instances the stromata persist and the hemoglobin is unaltered; when oval corpuscles are affected they become spherical and swell up before losing their coloring matter. A still more striking analogy was noted shortly afterward: the agglutination of bacteria by normal serum or specific serum takes place only in presence of a salt; and the flocculation which this electrolyte brings about in bacteria that have been previously treated with serum is very comparable to the effect produced by the same electrolyte on a suspension of clay or colloidal emulsions like mastic. This fact gave rise to the idea that we should doubtless be able to obtain, by immunizing animals, sera capable of flocculating finely suspended organic substances, or albuminoid substances like casein, and led to the discovery of "lactoserum," that is, the immune serum of animals that have been treated with injections of milk. $\dagger$

* Les phénomènes d'absorption et la conglutinine du sérum de bœuf. Centralblatt für Bakteriologie, 1st Abt. Orig. Vol. 49, 1909, p. 260.

$\dagger$ Bordet, p. 155. 
We recognize that these facts were indicative simply and were not an absolute demonstration. As concerns the phenomenon of agglutination, Bordet noted that it takes place in two distinct phases: during the first a complex is formed by the union of the agglutinin with the bacteria; in the second the salt precipitates this complex. In the analogous instances of clay flocculation we have to deal, then, with the second phase only and obtain no light on the mode of union of the agglutinin with the receptors of the bacteria.

The presumptions that we deduced from these preliminary observations led to researches on molecular adhesion which soon brought out serious experimental confirmations. In comparing the fixation of hemolysins on corpuscles with the adsorption of analin dye by filter paper, Bordet mentioned as an analogy that the corpuscles do not fix the active substances according to the law of definite proportions. Thus we find that a given volume of hemolytic serum destroys a larger amount of corpuscles when they are added to it in a single dose than when they are added in successive divided doses at relatively long intervals. If the second method is used it is evident that the first corpuscles become overladen with active principles and monopolize a larger amount than is necessary to hemolyze them, thus leaving less for subsequently added corpuscles. In the same way, if a leaf of filter paper is placed in a given amount of methylene-blue solution it takes a uniform color of a given intensity. But if a piece of the same size is first cut up into small pieces and these pieces introduced at intervals, we find that the first of them stain deeply and the last ones find no color left. The same experiments were made with the same result by Danysz with ricin and antiricin, by Bordet with alexin and anti-alexin, and by von Dungern with diphtheria toxin and antitoxin: the neutralizing dose varies depending on whether the toxin is added in a single or in several doses. The same conclusion may be drawn with agglutinins and bacteria, as Craw has shown. We do not wish to insist at this point on the obvious interest brought out by the fact that toxins unite with antitoxins in variable proportions.*

The analogies between phenomena brought about by sera and those due to substances which certainly do not act chemically,

* Consult on this subject the former article on the mode of action of antitoxins on toxins, p. 259. 
but by properties of adhesion, have been very instructive. The agglutination and hemolysis of red blood cells by barium sulphate (Gengou), by silicic acid (Landsteiner and Jagic), and similar facts brought out by researches of many writers on colloids, gave greater and greater importance to the effect of adhesion in the reactions between antisera and their antigens.

In hemolysis in particular, the phenomenon of alexin absorption by corpuscles laden with sensitizer (amboceptor) has given rise to most active controversies. The sensitizing theory of Bordet consists essentially in the conception that the sensitizer, which possesses in itself no particular affinity for the alexin, forms, by uniting with certain substances in the corpuscle, a complex endoryed with properties of molecular adhesion for the alexin which are not possessed by the normal corpuscle. The corpuscle that has been modified in this manner would seem to acquire the property of adsorbing alexin, of manifesting an avidity which may be compared to that which occurs in the fixation of fibrinogen or other albuminous substances by chemically inert particles (for example, barium sulphate) or to the union of diphtheria toxin with precipitates of calcium, etc. As we know, this point of view differs from the amboceptor theory of Ehrlich, in accordance with which this antibody owes its intermediary function to the fact that it possesses in its molecule two combining atom groups: one (complementophilic), which unites with the alexin, and the other (cytophilic), which combines with the corpuscle. We shall not take up at this point a general discussion of these theories, which one of us in collaboration with Dr. Gay has recently considered;* we may recall that no experiment has ever shown that sensitizers can unite with alexin in absence of antigens. Such a combination under certain conditions has been asserted by Ehrlich and Sachs, $\dagger$ as a result of their researches on the hemolysis of guineapig corpuscles by horse serum to which bovine serum has been added.

Bordet and Gay $\ddagger$ undertook two years ago the study of this instance of hemolysis and arrived at the conclusion that Ehrlich and Sachs' interpretation is inexact. Their conclusion, in turn, has

† See Studies on Immunity, Ehrlich-Bolduan, Wiley \& Co., p. 209.

$\ddagger$ See p. 363 . 
been objected to by Sachs' and Bauer*who maintain integrally the opinion that was advanced by Ehrlich and his collaborator. We feel led, therefore, to return to this question, which appears to us to present more interest than might at first be presumed, on the general problem of the action of sera and particularly on certain of their practical applications.

We may recall the facts briefly. Ehrlich and Sachs noted that guinea-pig red corpuscles remain intact in fresh horse serum, but are readily hemolyzed by a mixture of this serum with bovine serum that has previously been heated to 56 degrees, which fact gives rise to the supposition that there is a strong amboceptor in bovine serum which allows the corpuscles to fix horse alexin and be hemolyzed by it. But Ehrlich and Sachs found in addition that if the corpuscles are placed in contact with heated bovine serum and subsequently centrifugalized to remove this serum, and fresh horse serum is then added, no hemolysis appears. It would seem as if the bovine sensitizer required the presence of the horse alexin in order to become fixed on the corpuscles. A fact which seems to confirm this idea is that heated bovine serum that has been previously treated with a large quantity of guinea-pig corpuscles (which latter are then removed by centrifugalizing) does not act as if it had been deprived of this amboceptor. It is able, indeed, to form a hemolytic mixture with fresh horse serum, which destroys fresh guinea-pig corpuscles. At least this is Ehrlich and Sachs' interpretation of the experiment. This opinion is translated into words which conform better with their theories by saying that the cytophilic group of the bovine amboceptor is not able to react with the corpuscle unless the complementophilic group has previously satisfied its affinity for alexin. The mutual relation between corpuscle, alexin and sensitizer would seem, in the present instance to be unusual because, in dealing with the majority of hemolytic sera, we find that the corpuscles absorb the sensitizer without the presence of alexin.

Bordet and Gay in their study on this subject were led to quite different conclusions. Bovine serum possesses, in their opinion, distinct properties which are, however, quite different from those noted by Ehrlich and Sachs. According to Bordet and Gay, there

* Arbeiten ans dem K. Inst. für exper. Therapie, 1907. 
is present in bovine serum a particular substance which is neither an amboceptor, an agglutinin, nor an alexin, and which consequently differs from the active substances that have been previously recognized in studies on immunity. This substance has the property of combining with corpuscles that have already been laden with sensitizer and alexin, and this combination generally favors hemolysis.

Two preliminary ideas are necessary in order that the facts may be clearly understood. The first is that horse alexin is very weak in so far as producing hemolysis is concerned. Corpuscles that have been strongly sensitized by a heated immune serum show little or no hemolysis on the addition of horse serum.* Consequently, when horse alexin is used, an absence of hemolysis does not prove that the corpuscles concerned are not sensitized, that is to say, have not fixed the amboceptor.

The second point is, that fresh horse serum contains a relatively strong amboceptor for guinea-pig corpuscles which produces a good alexin fixation. The fact, then, that fresh horse serum does not destroy the corpuscles is not due to its lack of sensitizer, but because its alexin is incapable of hemolyzing. If this alexin is replaced by another, for example, if fresh guinea-pig serum is added to the mixture of guinea-pig corpuscles and horse serum, hemolysis takes place. We are forced, then, to admit in Ehrlich and Sachs' experiment, which consists in mixing fresh horse serum and heated bovine serum with guinea-pig corpuscles, the presence of two amboceptors, bovine and horse, both of which produce a sensitization, but either one of which suffices to produce the result. Either one of these amboceptors may indeed be used alone without changing the result of the experiment. This fact, to be sure, is scarcely acceptable to Ehrlich and Sachs, according to whom the amboceptor in their experiment is furnished entirely by the bovine serum. And when these authors find on treating bovine serum with guinea-pig corpuscles that the serum still retains its property of rendering horse serum hemolytic for new corpuscles, their conclusion is, as we have seen, that the bovine amboceptor has remained free in spite of contact with the corpuscles.

* For example, bovine corpuscles sensitized by rabbit antibovine immune serum. The hemolytic weakness of horse alexin is not the same with all species of corpuscles. 
The interpretation of Bordet and Gay, on the contrary, is the following:

The contact with the corpuscles has deprived the bovine serum of its amboceptor; this amboceptor, however, may be replaced by the sensitizer in horse serum, which latter serum also furnishes the alexin. Under these conditions the bovine serum, which still remains necessary for hemolysis, acts only on account of the peculiar substance which it possesses, which, as we have just seen, is neither amboceptor nor alexin, but endowed with the peculiar characteristic of allowing horse alexin to produce hemolysis.

We may, for the sake of clearness, explain this idea somewhat more fully before recalling the experiments which proved its exactness. This particular substance in bovine serum which resists heating to 56 degrees and which for simplicity Bordet and Gay called "bovine colloidal substance" does not react with normal corpuscles; it unites only with corpuscles that have been laden both with amboceptor and alexin.

This union or adsorption of the particular substance in question by sensitized and alexinized corpuscles is accompanied by visible manifestations; the corpuscles on uniting with this substance are energetically agglutinated and become, with certain exceptions which we shall later mention, more apt to hemolyze. This phenomenon of agglutination of the corpuscles into large clumps in Ehrlich and Sachs' experiment, although extremely characteristic, was apparently not noted by these investigators, who were more interested in the hemolysis. Heated bovine serum alone agglutinates guinea-pig corpuscles only feebly; horse serum agglutinates them better, but rather slowly. A mixture of the two sera, however, brings about in a very few moments an extremely marked clumping of large masses of corpuscles, which soon afterward lose their hemoglobin.

Bordet and Gay's explanation of this is then quite evident; the corpuscles in such a mixture fix first the bovine and horse amboceptors and then the alexin.* A certain amount of time is necessary for this to take place, but as soon as the corpuscles become laden with

* It would be very difficult to define the proportions in which each of these two sensitizers is fixed; it is, moreover, of little importance. The essential fact is to recognize that either of them will suffice to sensitize. 
these substances they are then in a position to unite with the bovine colloid, which clumps them and renders them more susceptible to hemolysis. The second assertion of Bordet and Gay, to which we have already referred, is that bovine serum presents no abnormality so far as its amboceptor is concerned. This sensitizer is able to unite with the corpuscles when the alexin is absent, contrary to the opinion of Ehrlich and Sachs. Heated bovine serum that has been subjected to a large amount of guinea-pig corpuscles still acts as if it had lost no essential element because its colloid has been retained. This is due to the fact that the corpuscles used to absorb the serum were not alexinized and consequently could not combine with the colloid. Such a treated serum, however, has lost its amboceptor. But inasmuch as this lack may be replaced by the sensitizer in horse serum, a mixture of bovine serum treated in this manner, of fresh horse serum and of guinea-pig corpuscles, acts almost exactly as when intact bovine serum is used.

It is necessary to explain these matters rather in detail, inasmuch as the mechanism in the experiment under consideration is rather complex and must be attentively considered. Many similar experiments have been offered by Bordet and Gay to demonstrate the function of this bovine colloid substance clearly. If we take washed bovine corpuscles and add to them a mixture of fresh horse serum and heated bovine serum, nothing occurs. But if, in a similar experiment, we use sensitized bovine corpuscles, we find that they become violently agglutinated and are then hemolyzed. This phenomenon is quite identical with the one in Ehrlich and Sachs' experiment on guinea-pig corpuscles. In both instances bovine serum is necessary; neither the bovine corpuscles nor the guinea-pig corpuscles are distinctly altered by the horse serum alone. In the experiment with bovine corpuscles, however, it cannot be asserted that the bovine serum acts as an amboceptor. It must certainly act on account of the particular colloidal substance. Since it is indispensable that such corpuscles should be sensitized and that alexin should be present, the only possible conclusion to be drawn is, that corpuscles that have been sensitized and alexinized are then capable of uniting with bovine colloid, which substance brings about clumping and hemolytic results. The objection may be raised, as indeed Sachs and Bauer have done in their article, 
that in this experiment with bovine corpuscles certain unexpected reactions, such as certain anticomplement effects, may take place between the bovine serum and the horse serum. This objection, however, can scarcely be maintained, inasmuch as the horse serum may be replaced by any other alexic serum without any change in the result. The experiment, indeed, becomes simpler if, as Bordet and Gay have done, we add simply fresh bovine serum to sensitized bovine corpuscles. Hemolysis appears, which is not surprising, but it is preceded - and this is the particular point to be noted - by an extremely energetic agglutination which characterizes the union of the colloidal substance. This phenomenon, moreover, is another indication of the phenomenon that has been previously noted. In the last-mentioned experiment bovine serum acts in two ways: both as a complement and as a colloid.*

May the somewhat vague name of colloidal substance be still employed to designate this active substance in bovine serum? Bordet and Gay made use of it only provisionally. As we know, this colloid is absorbed by various corpuscles provided they be sensitized and alexinized, and the union is indicated by a very evident phenomenon of agglutination in large masses. This colloidal substance should not be confounded with the ordinary agglutinins and yet should be designated with some name that suggests the fact that it produces agglutination. For this reason we propose, from now on, to give the name of "conglutinin" to this substance in bovine serum, and to refer to the agglutination which it produces as "conglutination."

\section{SECTION I \\ SaChs ANd Bauer's OBjections.}

Bordet and Gay naturally brought forward numerous proofs, which we cannot reproduce here, to support their interpretation. These proofs will be found in the previous article of 1906 . We wish at this time to offer certain new arguments, which, in addition to the previous ones, permit us to refute the objections that have been

* Muir and Browning, Journal of Hygiene, Vol. 6, page 20, noted, independently of Bordet and Gay, that rabbit-antibovine serum when added to bovine blood produces a marked agglutination of the corpuscles, in producing which the presence of alexin is necessary. 
offered by Sachs and Bauer, and also offer further information on the properties of the conglutinin.

No comparison between ordinary agglutinins and the conglutinin is necessary. The two substances are evidently different. Agglutinins have no need, for their action, of sensitized and alexinized corpuscles, as does the conglutinin. In the experiment that has just been mentioned, that is, the agglutination of sensitized bovine corpuscles by fresh bovine serum, it is evident that we are not dealing with an ordinary agglutinin. It is, however, interesting to compare the development of the two phenomena by adding bovine serum to two different species of corpuscles, one of which it agglutinates and the other of which it conglutinates. Guinea-pig and horse red blood cells, respectively, fulfill these conditions. We may consider, in the first place, the agglutinating effect: to two tubes containing 1 c.c. of salt solution and 0.1 of a cubic centimeter of heated bovine serum, we add 0.05 of a cubic centimeter of washed blood either from the guinea-pig or the horse. The guinea-pig corpuscles are not markedly agglutinated, those of the horse are rapidly agglutinated; in this case we are dealing with an ordinary agglutinin. We may then consider the conglutinin, that is to say, in a similar experiment we may employ fresh bovine serum instead of heated bovine serum. The horse corpuseles act just as they do with heated serum: there is rapid agglutination of a similar intensity, but no real conglutination appears.* The guinea-pig corpuscles show no immediate agglutination any more than with the heated serum. After 10 or 15 minutes conglutination appears and the corpuscles collect into large clumps which gradually hemolyze. It is to be noted that although a certain amount of time is necessary for the fixation of the alexin, the clumping by the conglutinin is much more marked than that by the ordinary agglutinin.

We may now consider Sachs and Bauer's objections:

(a) These authors consider Bordet and Gay's interpretation as inacceptable since it nęcessitates the admission of a new substance,

* This is due to the fact that they do not fix the alexin sufficiently, or, in other words, to the fact that the bovine serum is not sufficiently sensitizing for horse corpuscles. As Streng has shown in his memoir on the anticomplement (Zeitschrift für Immunitätsforschung, vol. 1), horse corpuscles are perfectly well conglutinated by fresh bovine serum when they have previously been sensitized by a specific immune serum. 
the conglutinin, in the bovine serum, which has never been described in other sera. But as we know, bovine serum is in itself rather unusual and why should not the effect that it produces be likewise so? Ehrlich and Sachs' theory, moreover, is also rather exceptional inasmuch as it presupposes that a sensitizer does not unite with the corpuscles when the alexin is absent.

(b) Sachs and Bauer seem to consider it axiomatic that the properties of agglutination and hemolysis cannot be attributed to a single substance. Gengou, as we know, has noted that a suspension of barium sulphate will agglutinate and hemolyze red blood cells.

(c) In their criticism of our work our contradictors concern themselves only with the hemolysis. They make no attempt to explain the marked clumping of the corpuscles and do not mention whether they consider our interpretation, at least in so far as this conglutination is concerned, as admissible.

(d) Sachs and Bauer insist strongly on the fact that heated bovine serum is capable of sensitizing guinea-pig corpuscles. We know this fact and have never denied it. We have simply said that in the Ehrlich and Sachs' mixture the bovine sensitizer is unnecessary on account of the presence of a similar stronger substance in horse serum.

(e) The activity of heated bovine serum is due to the fact, according to Bordet and Gay, that sensitized and alexinized corpuscles absorb the conglutinin. For example, if bovine corpuscles are sensitized with a specific immune serum from the rabbit, subsequently treated with horse alexin, and, after being carefully washed, heated bovine serum is added, this latter serum loses, if not all, at least part of its conglutinin and becomes much less able to form a conglutinating and hemolytic mixture with horse alexin either for bovine corpuscles or for guinea-pig corpuscles. Bordet and Gay have made this assertion. The experiment is, however, denied by Sachs and Bauer.

We are dealing here, not with an interpretation, but with a fact, and we insist on its accuracy. In the same way Sachs and Bauer say that guinea-pig corpuscles that have been treated with fresh horse serum, then washed and mixed with heated bovine serum, do not deprive this latter of its activity. We have repeated this experiment with different results; to be sure, we have taken pains to use a 
much larger quantity of alexinized guinea-pig corpuscles than they in order to exhaust the bovine serum. And we find that bovine serum treated in this manner becomes, if not absolutely inactive, much less active than before treatment.

In interpreting such experiments it is well to note that heated bovine serum acts in a very small dose. A trace of serum, for example, 0.01 of a cubic centimeter, on addition to a mixture of $0 . \bar{j}$ of a cubic centimeter of salt solution, 0.3 of a cubic centimeter of fresh horse serum and 0.05 of a cubic centimeter of guinea-pig corpuscles, produces aistinct conglutination followed by a certain amount of hemolysis. When we consider that bovine serum works in such small doses it is easy to conceive how difficult it is to deprive it entirely of its active substance, the conglutinin. To obtain such distinct results one should use much more blood than do Sachs and Bauer. It is also necessary, particularly in the experiments with bovine corpuscles, that the blood should be sensitized and alexinized sufficiently.*

The details of such an experiment with guinea-pig corpuscles follows:

In each of two tubes, $A$ and $B$, is placed 1 c.c. of washed guineapig blood. To tube $\mathrm{A}$ is added 20 c.c. of salt solution and 6 c.c. of horse serum. An hour later the corpuscles are washed, twice centrifugalized and the supernatant fluids decanted; 2 c.c. of salt solution plus 0.3 of a cubic centimeter of heated bovine serum is added to each sediment. An hour later the mixtures are centrifugalized and the supernatant fluids A and B decanted. Another mixture $(\mathrm{C})$ is prepared containing 2 c.c. of salt solution plus 0.3 of a cubic centimeter of bovine serum, 56 degrees.

In three tubes, "a," "b," "c," are placed 1 c.c. of each fluid, A, $\mathrm{B}$, and $\mathrm{C}$ respectively. To each tube there is then added 0.3 of a cubic centimeter of fresh horse serum and 0.05 of a cubic centimeter of washed guinea-pig blood, and the tubes are placed in the incubator. The result is, that in $S$ or 10 minutes "c" and " $b$ " show strong agglutination; there is scarcely visible agglutination in " $a$ " in 20 minutes. In a half hour, agglutination, very slight, and hemolysis, scarcely visible in "a," strong agglutination and hemoly-

* This is proved by the fact that such corpuscles show no agglutination and hemolysis in the mixture of heated bovine serum and horse alexin unless they are strongly sensitized. 
sis in "b," and strong agglutination and almost total hemolysis in "c."

(f) Sachs and Bauer take great pains to interpret, in harmony with their own ideas, Bordet and Gay's experiment, which shows that bovine corpuscles, provided they be sensitized, are conglutinated and hemolyzed by a mixture of fresh horse serum and heated bovine serum, that is to say, that they act just as guinea-pig corpuscles do. They conceive of horse serum as containing several complements, certain ones of which are neutralized by the bovine serum, whereas certain others are not; as we know, the Ehrlich school has no hesitation in multiplying the active substances in serum. We believe it quite useless to follow Sachs and Bauer in their hypotheses concerning the peculiarities of horse serum and the reason that in the experiment in question the horse serum may be replaced by any other alexin, for example, by bovine alexin itself, without any effect on the result.* As we have already noted, when sensitized bovine corpuscles are added to fresh bovine serum, the hemolysis is preceded by an intense and characteristic conglutination. The evolution of the reaction is absolutely the same in both instances, and it is therefore clear that the mixture of fresh horse serum, (alexin) and bovine serum, 56 degrees (conglutinin), acts exactly the same as does fresh bovine serum (conglutinin plus alexin). Certain of Sachs and Bauer's experiments, moreover, in this part of their article contain important experimental errors. $\dagger$

* Bordet and Gay have also described certain experiments in which guinea-pig alexin is employed. For example sensitized guinea-pig corpuscles are only slowly hemolyzed by a small dose of fresh guinea-pig serum. If a little bovine serum is added to this mixture, however, the hemolysis is markedly accelerated and energetic conglutination takes place.

$\dagger$ For example, in table 12 of their memoir, Sachs and Bauer say that heated bovine serum is anticomplementary for horse alexin, since it prevents the fixation of this alexin on faintly sensitized bovine corpuscles. We shall not here consider whether this conclusion is true or false. What is certain is that the deduction from the experiment is certainly incorrect. In judging the intensity of alexin absorption Sachs and Bauer place their corpuscles, on the one hand, in a mixture of horse alexin and salt solution, and,on the other, in a mixture of horse alexin with a certain amount of bovine serum. Sachs and Bauer seem to forget that, other things being equal, alexin fixation, particularly when the corpuscles are faintly sensitized, takes place better in a fluid containing much salt solution than in one containing heated serum; this serum, as we know, inhibits alexin fixation without acting in any truly anticomplementary manner. In the question in case the heated bovine serum may inhibit the fixation, not only of horse alexin, but 
Beginning with their theory that the bovine sensitizer fails to unite with guinea-pig corpuscles unless horse alexin is present, Sachs and Bauer presuppose that a mixture of heated bovine serum and horse alexin that has been prepared for some time, and in which this combination may have taken place, should hemolyze guineapig corpuscles more rapidly than a recently prepared mixture. They declare that this can be experimentally proved. We have repeated their attempts with different results. We have noted, as we shall later consider, that a mixture of fresh horse serum and heated bovine serum in suitable proportions is much less active when it has been kept for some time before the guinea-pig corpuscles are added.

Having examined Sachs and Bauer's objection, to close the debate we may relate certain experiments which prove in an irrefutable manner the accuracy of Bordet and Gay's interpretation. The reason that Ehrlich and Sachs' original experiment has given rise to so long a discussion is due to the multiplicity of the substances present and particularly to the fact that there are two amboceptors which can supplant one another. The obscurity is due to the fact that fresh horse serum contains a sensitizer as well as does the heated bovine serum. Let us suppose, for a moment, that horse serum contains alexin only. Under these conditions, on mixing the heated bovine serum (which, according to Bordet and Gay, is sensitizing and, in addition, contains the conglutinin) with horse serum an active mixture is obtained. We both agree on this point. But if we were to mix with such a horse serum containing alexin but no sensitizer. bovine serum, 56 degrees, which had been previously subjected to guinea-pig corpuscles, what should we obtain? Evidently, if Bordet and Gay's theory is correct, the mixture should be inactive because, although such a mixture contains conglutinin, it should have been deprived of all amboceptor. If Ehrlich and Sachs are correct, on the contrary, the mixture should be just as active, inasmuch as it should still, according to these authors, contain the bovine sensitizer which can unite and produce hemolysis only in presence of alexin.

It would therefore be of service to have horse serum which contains alexin but is unable to sensitize guinea-pig corpuscles. Such also of bovine alexin. We shall later refer to an experiment that proves this fact. In addition it may be mentioned that these facts have recently been considered in detail by Bordet and Gay (see article, p. 39S). 
a serum is easily obtained, and the experiments which have been outlined confirm Bordet and Gay's opinion entirely.

We know that the fixation of alexin on corpuscles is very markedly favored by the addition of normal salt solution; this fact is very evident in the case of horse serum. Klein was first to note that guinea-pig corpuscles mixed with fresh and undiluted horse serum fix little alexin, although they absorb it completely when a considerable amount of salt solution is added. The sensitizer, on the contrary, is well absorbed even when the serum is concentrated. Consequently, if we treat undiluted horse serum with an equal volume of washed guinea-pig corpuscles, we shall obtain a fluid which is rich in alexin but is deprived of sensitizer.

We find that such a serum produces the following effect:

(a) When mixed with intact heated bovine serum and guineapig corpuscles it produces the phenomenon of conglutination and hemolysis very clearly. This fact proves that the bovine sensitizer is the one which acts, as no other one is present.

(b) When mixed with heated bovine serum that has been previously treated with guinea-pig corpuscles it produces no phenomenon. This proves that such a mixture contains no sensitizer at all, and consequently condemns Ehrlich and Sachs' thesis, in accordance with which the bovine sensitizer should still be present. In control tubes, of course, it is proved that such heated bovine serum, when mixed with untreated horse serum, gives the phenomenon perfectly, in which case, of course, the sensitizer that acts is in the horse serum.

(c) When mixed with heated bovine serum that has been deprived of its amboceptor by contact with guinea-pig corpuscles this horse serum deprived of its sensitizer still gives the phenomenon if the corpuscles added are not normal, but have first been sensitized by contact with heated bovine serum and subsequently washed. This completes the demonstration of the previous experiment on the fixation of the bovine sensitizer by corpuscles in absence of alexin. The experimental details of these experiments follow. Materials are prepared as follows:

I. Fresh horse serum containing alexin but deprived of sensitizer. -2 c.c. of washed guinea-pig blood is added to 2 c.c. of horse serum. After contact for quarter of an hour the mixture is cen- 
trifugalized, the supernatant fluid decanted, and 6 c.c. of normal salt solution added.

II. Fresh horse serum deprived of both sensitizer and alexin.6 c.c. of salt solution plus 2 c.c. of washed guinea-pig blood is added to 2 c.c. of the serum. After contact for an hour and a half the mixture is centrifugalized and the supernatant fluid decanted.

III. Bovine serum (56 degrees) deprived of its sensitizer. -2 c.c. of salt solution plus 2 c.c. of washed guinea-pig blood is added to 2 c.c. of the serum. Contact for 1 hour, centrifugalization and decantation of the supernatant fluid.

IV. Intact bovine serum, 56 degrees.-2 c.c. of serum plus 4 c.c. of salt solution.

V. Intact horse serum.-2 c.c. of serum plus 8 c.c. of salt solution.

Mixtures are prepared as indicated in the following table from these fluids, and 0.05 of a cubic centimeter of normal guinea-pig corpuscles, or of corpuscles that have previously been treated with 0.3 of a cubic centimeter of heated bovine serum and subsequently carefully washed three times with a large volume of salt solution, is then added.

Mixtures in which the characteristic intense agglutination and hemolysis occurred are designated by the letters AH. In the other mixtures the corpuscles have shown no such modifications even after a considerable period of time.

TABLE I.

\begin{tabular}{|c|c|c|c|c|c|c|}
\hline \multirow{2}{*}{$\begin{array}{l}0.6 \text { c.c. of fluid con- } \\
\text { taining bovine serum. }\end{array}$} & \multicolumn{3}{|c|}{$\begin{array}{l}\text { Normal guinea-pig corpuscles, } \\
0.05 \text { c.c.; } 1.5 \text { c.c. of a fluid con- } \\
\text { taining horse serum. }\end{array}$} & \multicolumn{3}{|c|}{$\begin{array}{l}0.05 \text { c.c. of corpuscles sensitized } \\
\text { by bovine serum and washed. } \\
\text { Fluids contain } 1.5 \text { of horse serum. }\end{array}$} \\
\hline & $\begin{array}{c}\text { I. } \\
\text { Minus } \\
\text { sensitizer. }\end{array}$ & \begin{tabular}{|} 
II. \\
Minus \\
sensitizer \\
and alexin.
\end{tabular} & $\begin{array}{c}\text { V. } \\
\text { Intact. }\end{array}$ & $\begin{array}{l}\text { I. } \\
\text { Minus } \\
\text { sensitizer. }\end{array}$ & $\begin{array}{c}\text { II. } \\
\text { Minus } \\
\text { sensitizer } \\
\text { and alexin. }\end{array}$ & Intact. \\
\hline $\begin{array}{l}\text { III. Bovine serum } \\
\text { minus sensitizer }\end{array}$ & $\begin{array}{c}\text { No. } 1 \\
0\end{array}$ & $\begin{array}{c}\text { No. } 2 \\
0\end{array}$ & $\begin{array}{l}\text { No. } 3 \\
\text { AH }\end{array}$ & $\begin{array}{l}\text { No. } 10 \\
\text { AH }\end{array}$ & $\begin{array}{c}\text { No. } 11 \\
0\end{array}$ & $\begin{array}{l}\text { No. } 12 \\
\text { AH }\end{array}$ \\
\hline $\begin{array}{l}\text { IV. Intact bovine } \\
\text { serum .......... }\end{array}$ & $\begin{array}{l}\text { No. } 4 \\
\text { AH }\end{array}$ & $\begin{array}{c}\text { No. } 5 \\
0\end{array}$ & $\begin{array}{l}\text { No. } 6 \\
\text { AH }\end{array}$ & $\begin{array}{l}\text { No. } 13 \\
\text { AH }\end{array}$ & $\begin{array}{c}\text { No. } 14 \\
0\end{array}$ & $\begin{array}{l}\text { No. } 15 \\
\text { AH }\end{array}$ \\
\hline $\begin{array}{c}0.6 \text { c.c. salt solu- } \\
\text { tion............ }\end{array}$ & $\begin{array}{c}\text { No. } 7 \\
0\end{array}$ & $\begin{array}{c}\text { No. } 8 \\
0\end{array}$ & $\begin{array}{c}\text { No. } 9 \\
0\end{array}$ & $\begin{array}{c}\text { No. } 16 \\
0\end{array}$ & $\begin{array}{c}\text { No. } 17 \\
0\end{array}$ & $\begin{array}{c}\text { No. } 18 \\
0\end{array}$ \\
\hline
\end{tabular}


We may add that the previous sensitization of the corpuscles is of considerable importance in respect to the rapidity with which the conglutination and hemolysis appear. Thus we find that intense agglutination takes place in from 5 to 7 minutes in tubes 12,13 and 15 , in 10 minutes in tube 10 and in about 15 minutes in tubes 3,4 and 6 . The rapidity of hemolysis corresponds.

The contrast between tubes 1 and 10,1 and 4, and 1 and 3 is particularly to be noted.

Similar experiments made with fresh bovine serum give similar results. Inasmuch as alexin fixation follows the fixation of the sensitizer, by placing fresh bovine serum in contact with an equal volume of washed guinea-pig blood for a short period (about 10 minutes at room temperature) we obtain a fluid that is deprived of sensitizer but still contains an appreciable amount of alexin and of conglutinin. Serum treated in this manner has no effect on normal guinea-pig corpuscles, but it does hemolyze corpuscles that have been previously sensitized by heated bovine serum and subsequently washed, and the hemolysis in this case also is preceded by the characteristic conglutination. In the same way we find that a relatively small dose of fresh intact bovine serum, when added to guinea-pig corpuscles, conglutinates and hemolyzes them more rapidly if they have been previously sensitized and washed. For example, in two tubes, A and B, is placed 0.1 of a cubic centimeter of washed guinea-pig blood and 0.5 of a cubic centimeter of salt solution. To $\mathrm{A}$ is then added 0.5 of a cubic centimeter of heated bovine serum. A half hour later the corpuscles are washed, centrifugalized and the supernatant fluid decanted; 1 c.c. of salt solution and 0.1 of a cubic centimeter of fresh bovine serum is then added to each corpuscle sediment. In tube A conglutination takes place in 40 minutes and hemolysis in an hour; in B the phenomena occur in 1 and 2 hours respectively. It is certain, then, that guinea-pig corpuscles that have been subjected to bovine serum, 56 degrees, fix the sensitizer.

We may now recall in this connection the fact which we have already mentioned and which at first sight might seem contradictory to the preceding statements. If we prepare a mixture containing a large amount of salt solution with a little fresh intact bovine serum, the addition of sensitizer in the form of heated 
bovine serum may inhibit the hemolysis of guinea-pig corpuscles rather than favor it.* We found indeed that alexin fixation of the sensitized corpuscles takes place better when the fluid contains little serum and a large amount of salt solution. For example, we prepare two tubes, $\mathrm{A}$ and $\mathrm{B}$, containing each 0.8 of a cubic centimeter of salt solution plus 0.1 of a cubic centimeter of fresh bovine serum. To $\mathrm{A}$ we add 0.3 of a cubic centimeter of bovine serum, 56 degrees, and to both tubes 0.05 of a cubic centimeter of washed guinea-pig blood. Conglutination and hemolysis occur in tube $\mathrm{B}$ in 30 and 40 minutes respectively, in tube $\mathrm{A}$ in 40 and 60 minutes. In other words, the heated serum is more inhibiting as serum than it is useful as sensitizer, inasmuch as enough sensitizer is present in the fresh bovine serum. But if, instead of employing in this last experiment fresh intact bovine serum, we use serum that has been treated for about 10 minutes with an equal volume of guinea-pig blood, we obtain directly opposite results. Under such conditions the addition of a sensitizer is obviously necessary; and consequently we find that although the heated bovine serum ( 0.3 of a cubic centimeter), as serum, inhibits alexin fixation, it is necessary as containing sensitizer. An experiment modified in this manner shows in tube B no conglutination or hemolysis; the phenomena appear in A in $1 \frac{1}{2}$ and 2 hours respectively. It would seem to us in view of so many convincing facts that the thesis of Ehrlich and Sachs, which supposes that the bovine amboceptor can unite with the corpuscles only in presence of the alexin, can no longer be admitted. And what is more, we consider these authors' opinions on hemolysis as purely theoretical, in so far as they bear on the existence of a complementophilic group, partial anticomplements, multiplicity of complements, dominant and non-dominant complements, and the like.

\section{SECTION II.}

\section{Various Examples of Conglutination and the Mode} of Action of the Conglutinin.

Conglutination always appears when the cells added to fresh bovine serum or a mixture of heated bovine serum and alexin from another animal are sufficiently sensitized and consequently in a condition to absorb alexin. Whenever the normal sera employed

* See the previous footnote criticising certain experiments of Sachs and Bauer. 
in this mixture are not sensitizing for the corpuscles used, they must be treated with an appropriate specific immune serum. Such is the case in the experiment with bovine corpuscles. Many corpuscles, however, react sufficiently to the sensitizer in normal serum, particularly in bovine serum. Thus in Ehrlich and Sachs' experiment the guinea-pig corpuscles may be replaced by other red blood cells. For example, rabbit corpuscles act just the same as guinea-pig corpuscles and show conglutination, although somewhat less rapidly, and intense hemolysis. Human corpuscles react distinctly. In all these instances fresh bovine serum alone produces similar results.

Goat corpuscles act rather curiously on the addition of fresh bovine serum. They are energetically conglutinated, but are not hemolyzed. We should conclude from this fact that the bovine serum sensitizes goat corpuscles sufficiently to produce alexin absorption and bring about the action of the conglutinin, but that the alexin acts poorly in producing hemolysis, owing perhaps to the fact that the ox and the goat are closely related species.* In the phenomena under consideration, hemolysis is due neither to the alexin nor to the conglutinin alone, but to the combined effect of the two factors; the complex alexin-conglutinin does the hemolyzing, and we shall see presently that the conditions under which this complex is formed are of importance in respect to the strength of the hemolytic or the agglutinating property which it produces.

Why does the sensitized and alexinized corpuscle unite with the conglutinin? The absorption of alexin (complement) by a sensitized corpuscle is due, as we know, to a phenomenon of adsorption. But to what is this adsorption due? Neither the corpuscle itself nor the sensitizer alone shows any such attraction for the alexin. The corpuscle and the sensitizer when they unite, however, form a complex which is endowed with the new property of alexin absorption, owing doubtless to the fact that the sensitizer produces a change of physical condition in the corpuscle and sort of coagulation similar to that brought about by adding specific precipitating serum to an albuminous solution. This formation of a precipitate would affect the contact proper-

* Bovine alexin fails to hemolyze, or hemolyzes only faintly, goat corpuscles even when they are sensitized by a specific immune serum. 
ties markedly, and in particular would produce an avidity for alexin absorption. Thanks to the researches of Gengou and of Gay, we know that the albuminous substances of serum, of milk and the like, become avid of alexin when precipitins are added.

It does not seem, a priori, necessary that in each instance the same result should occur. In certain other cases the facility of different substances to clump together may be supposed to be due to the presence of such tendencies of adhesion in each separate substance. For example, if A, B and C clump one another it may be supposed that one of these elements, B for example, shows a property of adhesion both toward A and toward C, and may show an intermediary function in producing the complex.*

It would seem, in the case of conglutination, that alexin fills such an intermediary function. It seems to unite on the one hand with the sensitized corpuscle, as we already know, but it is also evident that there is some reaction between it and the conglutinin. To demonstrate this fact, we may take fresh horse serum, previously diluted with an equal volume of salt solution, and heated bovine serum diluted in the same manner. We add 1 c.c. of the diluted bovine serum to say 0.2 of a cubic centimeter of diluted horse serum. This mixture is left for a time at room temperature. After a while another similar mixture is prepared, and 0.05 of a cubic centimeter of washed guinea-pig corpuscles is then added to each of them. We find that the first mixture, in which a longer contact has taken place, has lost its property of producing conglutination and hemolysis almost entirely; in the second mixture the phenomena occur well.

The experiment may be repeated with modifications in the respective doses of each serum or in the duration of contact. In the doses we have given, a rather short contact suffices to diminish the activity of the mixture, even 10 or 15 minutes being sufficient to produce a distinct difference. As regards the dosage, it is found that the depressing effect of contact becomes less and less distinct as the quantity of the alexin is increased relative to the amount of heated bovine serum. For example, in a mixture of equal parts

* If this were so, B might well deserve the name of amboceptor, which Ehrlich has ineorrectly applied to sensitizers, with the reservation, however, that we are now dealing with tendencies to adhesion and not with purely chemical affinities. 
of the two sera diluted each with an equal amount of salt solution, the corpuscles react very much as they do in a fresh mixture.

Ehrlich's theories would feel no embarrassment in face of such a fact. To explain it, we may suppose that bovine serum contains several amboceptors, among which are some with no affinity for the guinea-pig corpuscles. Such amboceptors, if time were given them, would monopolize the alexin, would deviate it, and prevent it from uniting with the guinea-pig corpuscle. If the experimental result had turned out in the opposite way, the same theories would still be satisfied. It would suffice to say simply that the bovine amboceptor which combines with the guinea-pig corpuscles shows a greater affinity when it has had time to unite previously with alexin. As we have already seen, Sachs and Bauer have suggested this interpretation in similar experiments which prove to them in some inexplicable manner that a prolonged contact exaggerates the activity of a mixture instead of diminishing it.* As long as one has recourse to such purely hypothetical ideas, which may be varied and multiplied as one desires, it is clear that any experimental fact may be interpreted. The statement that the amboceptor has a complementophilic group is a hypothesis. The statement that the affinity of this group varies with different amboceptors is another hypothesis; the supposition that the cytophilic group becomes chemically more active when affinities of the complementophilic group are satisfied is a third hypothesis. A theory is of service only when used to coördinate real and demonstrated facts; it is of no value when it unites hypothetical facts produced, created and fashioned at the will of the theorist, with a single demonstrated fact.

The question of the mutual relations between the sensitized corpuscle, the alexin and the conglutinin should be subjected to additional researches in order to be quite clear. It seems, however, that to obtain the maximal effect of agglutination, and particularly of hemolysis, the order in which the three substances are added should be kept in mind. If the conglutinin and alexin are added in certain proportions and an interval allowed to elapse

* Sachs and Bauer's mixtures, to be sure, contained relatively more horse serum than ours. We have not, however, been able to produce their results even by following their experiment closely. 
before the corpuscles are added, only slight effects are produced. If the fixation of the alexin on the corpuscles is brought about, and they are then washed and subsequently subjected to the conglutinin, they are clumped but not hemolyzed.* The effects vary markedly, according to the modus operandi, although the substances concerned remain the same. This is not surprising, for we find similar examples throughout the phenomena of molecular adhesion. Toxin and antitoxin when mixed in constant proportions can, as we know, furnish complexes endowed with different properties depending on whether they are added to one another all at once, or in divided doses. In the serum diagnosis of syphilis, for example, the slightest details in the preparation of the liver extract is of importance. Sachs and Rondoni have found, for example, that their results vary in accordance with whether their concentrated alcoholic extract of liver is diluted with salt solution rapidly or slowly. It is conceivable, as these authors note, that the active substance may be carried to different states of colloidal division in accordance with these varying conditions, and that differences of a physical nature correspond to the various results in the energy of alexin adsorption.

The physical condition of the conglutinin would seem, likewise, to have a distinct influence on the result of the phenomenon. On dialyzing bovine serum, 56 degrees, we obtain by centrifugalization a supernatant fluid and a precipitate, the properties of which vary, although they both contain conglutinin. On adding sufficient sodium chlorid to reëstablish the primitive tonicity, we find that the supernatant fluid, when added to fresh horse serum, hemolyzes guinea-pig corpuscles better than it conglutinates them. $\dagger$ The precipitate gives the opposite result. When washed in distilled water and shaken in salt solution, this precipitate dissolves only partially; a cloudy fluid is obtained, which, on the addition of fresh horse serum, agglutinates guinea-pig corpuscles energetically, but hemolyzes them poorly. A mixture of the supernatant fluid and

* Several examples of this fact have been noted in the articles of Bordet and Gay and of Sachs and Bauer.

$\dagger$ In certain cases, however, this fluid may give agglutination without hemolysis as does heated bovine serum. This occurs when such a fluid is added to guineapig corpuscles that have been previously subjected to fresh horse serum and subsequently washed. 
the precipitate reproduces a mixture which is very like normal bovine serum.

In view of this relative dissociation of agglutination and hemolysis, it may be that the conglutinin is not a simple substance, or that it is a simple substance occurring in different physical conditions or conditions of greater or less solution in serum. If we like, we may regard it as a more or less fine colloid solution. This fact is evidently important to study. The fact of interest for the moment is, as we have brought out again in this article, that the hemolysis and conglutination by bovine serum follow a general law.

To summarize: The conclusions of the present article corroborate the ideas of Bordet and Gay of two years ago. The objections that have been offered to these ideas by Sachs and Bauer. have no foundation. Contrary to the opinions of Ehrlich and Sachs, bovine sensitizers unite perfectly well with corpuscles when the alexin is absent. The hypothesis of the existence of a complementophilic group in sensitizers does not agree with the facts. As for the special properties of bovine serum, they are due to a particular substance, the conglutinin, the principal characters of which are now clearly brought out. We may add that this substance is precipitated for the greater part by dialysis and seems to manifest a real affinity for alexin, and therefore tends to precipitate itself on sensitized and alexinized corpuscles. 


\section{SENSITIZERS FOR THE TUBERCLE BACILLUS.*}

\section{By J. BORDET AND O. GENGOU.}

One of us demonstrated in 1900 that if red blood cells or bacteria are added to a specific immune serum which contains a specific sensitizer, the cells become capable of absorbing the destructive substances in the serum (alexin). With this fact as a basis, Bordet and Gengou have described a method which allows one to prove the existence of a sensitizer in a given serum. By preparing a suitable mixture of typhoid bacilli, fresh normal human serum, and heated serum ( 55 degrees) of a convalescent from typhoid, it is found that the alexin of the normal serum is absorbed by the bacilli; this is proved by the fact that subsequently added sensitized corpuscles undergo no hemolysis. Consequently we may conclude that the serum of patients convalescent from typhoid contains a sensitizer that endows the typhoid bacillus with the power of fixing alexin. By this method we have endeavored to determine whether a guinea-pig can form an active sensitizer for the tubercle bacillus; our results follow:

Guinea-pigs injected with living human tubercle bacilli soon show generalized tuberculosis and produce no sensitizer. A negative result is obtained in any stage of their disease. If the guineapigs receive a subcutaneous inoculation of the avian tubercle bacillus, on the contrary, $\uparrow$ for two or three times they resist infection, and soon form a sensitizer in their blood which has the property of provoking alexin fixation when added to the bacillus. It is interesting to note that this sensitizer shows an equal activity with the human tubercle bacillus; the same amount of sensitizing serum will fix the same amount of alexin with the same volume

*Les sensibilisatrices du bacille tuberculeux: Comptes Rendus de l'Académie des Seiences, vol. 137, 1903, 351.

$\dagger$ Our culture of avian bacillus was derived from pigeons and had been grown for some time on glycerinated potato. 
of either human or avian bacilli. In other words, the serum obtained following an injection of avian bacilli does not give a means of distinguishing the two races of tubercle bacillus.

If guinea-pigs are given injections of a mixture of human tubercle bacilli, killed by heating to 70 degrees, and sensitizing serum, followed at the end of 2 weeks by another similar mixture containing simply dried bacilli, we find that the animals have become more resistant than controls to the living human tubercle bacillus. Such treated animals, on receiving an injection of this organism, survive considerably longer than do the controls, but when killed about 3 months after injection, their organs are found filled with tubercles, notwithstanding; in other words, the rapidity of the disease is simply checked. The serum of such animals tested at this period shows the presence of a sensitizer. The sensitizing property, then, although not quite useless, is incapable of preventing the evolution of the disease. Guinea-pigs that have been treated simply with injections of human bacilli killed by heating to 70 degrees, followed by dried bacilli, also acquire a sensitizing property in their serum, but, as we have already known for some time, their resistance to the living bacillus has not been remarkably increased. 


\title{
XXV. NEW CONTRIBUTION TO THE STUDY OF SEN- SITIZERS FOR TUBERCLE BACILLI.*
}

\author{
BY DR. O. GENGOU.
}

Bordet and Gengou† showed that guinea-pigs inoculated with virulent human tubercle bacilli die without forming any sensitizers for this bacillus, but if, on the contrary, they receive an injection of living avian tubercle bacilli, for which organisms they are usually resistant, they form sensitizers that act not only on the avian bacillus, but also on the human bacillus. Later on, Dembinskit stated that the living human bacillus produces no sensitizer either in the rabbit or in the pigeon, and he concludes "that the production of sensitizers for tubercle bacilli does not depend on the greater or less resistance of the animal employed against these bacilli," as Bordet and Gengou believed, "but is related to the type of bacilli employed."

In the course of studies on experimental tuberculosis, we have had occasion to control Dembinski's results. Since guinea-pigs succumb to an inoculation of virulent human bacilli without producing sensitizers, we used killed human bacilli (heated for onehalf hour to 65 degrees or for 5 minutes to $100^{\circ} \mathrm{C}$.). For comparison we gave other guinea-pigs injections of avian bacilli killed in the same manner. Similar injections were also made in rabbits. The animals were all immunized on three successive occasions by subcutaneous injections at intervals of 3 weeks; they were bled 2 or 3 weeks after the last injection.

We determined the presence of sensitizers in the serum of these animals by the Bordet-Gengou method based on the characteristic

* Nouvelle contribution à l'étude des sensibilisatrices des bacilles tuberculeux. Comptes Rend. de la Soc. de Biol., LVIII, 1906, 218.

$\dagger$ Gengou, p. 462. Comptes Rendus de l'Académie des Sciences.

$\ddagger$ Dembinski, Société de Biologie, 1901 . 
property of sensitizers to fix alexin on the cell for which the sensitizer is specific. If a given serum contains an active sensitizer for the tubercle bacillus, the alexin will be fixed by this bacillus, and subsequently introduced sensitized red blood cells will remain intact, owing to the absence of free alexin.

A résumé of our results is given in the following table. Apart from control tubes, * the composition of which is too complicated to write out in detail, each experiment comprises a tube that contains 0.1 of a cubic centimeter of guinea-pig alexin, 0.6 of a cubic centimeter of heated serum and 0.2 of a cubic centimeter of an emulsion of fresh human, avian, or bovine bacilli, as the case may be. Three hours later 0.1 of a cubic centimeter of sensitized goat corpuscles is added to each tube. $\dagger$

TABLE I.

1. Serum of guinea-pig injected with human T. B. $65^{\circ} \ldots$

2. Serum of guinea-pig injected with human T. B. $100^{\circ} \ldots$

3. Serum of guinea-pig injected with avian T. B. $65^{\circ} \ldots .$.

4. Serum of guinea-pig injected with avian T. B. $100^{\circ} \ldots$.

5. Serum of rabbit injected with human T. B. $65^{\circ} \ldots \ldots$.

6. Serum of rabbit injected with human T. B. $100^{\circ} \ldots \ldots$.

7. Serum of rabbit injected with avian T. B. $65^{\circ} \ldots \ldots \ldots$

8. Serum of rabbit injected with avian T. B. $100^{\circ} \ldots \ldots . . \pm \pm| \pm| \bar{\perp} \mid \overline{-}$

Fixation with emulsion of T. B. bacilli.

$+=$ Complete fixation; strong sensitizer

$\pm=$ Partial fixation; weak sensitizer

$-=$ No fixation; no sensitizer

As a result of our researches, we find that human or avian bacilli killed by heating to 65 degrees or 100 degrees stimulate the formation in guinea-pigs of sensitizers active against the various mammalian tubercle bacilli. This stimulation of sensitizer formation

* Bordet and Gengou, p. 217.

$\dagger$ In these experiments washed goat corpuscles are sensitized by an equal volume of rabbit-antigoat serum, and after contact for a quarter of an hour again washed in salt solution; the corpuscles are then suspended in a double quantity of 0.85 per cent salt solution. 
is also possible in a rabbit, particularly with human bacilli that have been heated to 100 degrees, but, as is evident from the table, it is more difficult to produce.* The facts that we have observed, however, as regards the rabbit, and particularly in the guineapig, prevent our adherence to Dembinski's opinion, who thinks in regard to the rabbit and the pigeon that "the injection of killed bacilli, whether human or avian, produces no sensitizer in their blood."

We feel justified in concluding from our results that, contrary to Dembinski's opinion, the production of antituberculous sensitizers does not depend on the type of bacilli injected. The human bacillus as well as the avian bacillus, and acid-resisting bacilli in general, may give rise on injection in the rabbit, and particularly in the guinea-pig, to sensitizers that are active for the various types of mammalian tubercle bacilli, as we shall later show. It may be, however, on the other hand, that the facility with which these antibodies are formed depends on the animal species employed; and the guinea-pig would seem to yield them more readily than does the rabbit, or, according to Dembinski, the pigeon.

We have been able to confirm the other results of this writer, who found that antituberculous sensitizers are just as active when heated human or avian bacilli (one-half hour to 65 degrees) are employed.

* The slight activity of the serum from the majority of our vaccinated rabbits may be due simply to insufficient immunization; we are, for the moment, engaged in a study along this line. 


\title{
XXVI. A CONTRIBUTION TO OUR KNOWLEDGE OF ANTI- TUBERCULOUS SENSITIZERS.
}

\author{
BY DR. GENGOU.
}

Since Bordet's researches on the mechanism of acquired immunity, our knowledge of the sensitizers (Ehrlich's amboceptors) has come to be more and more definite. These substances, which appear in the blood of inoculated animals during the course of immunization, are, as we know, specific and have as a peculiar property the power of fixing the alexin on that substance against which the animal that furnishes the sensitizers has been immunized. The alexin of normal serum alone has little or no tendency to become fixed on bacteria or red blood cells; a bacterium or red blood cell which has been affected by its specific sensitizer-has, on the contrary, the power of fixing a large amount of alexin, which then disappears from the surrounding fluid. Certain bacteria, as the cholera vibrio, show a morphological change as a result of this alexin fixation brought about by the specific sensitizer; they form granules and then dissolve. Sensitized blood cells are easily hemolyzed by alexin. Many forms of bacteria show no marked morphological change through the influence of sensitizer plus alexin. But even these bacteria, when they have been treated with the sensitizer, show the property, as do the more susceptible organisms, of fixing the alexin. This property, then, of fixing alexin on a cell is generalized and fundamental property in every sensitizer. Through this property, Bordet and Gengou, $\uparrow$ in 1900, were able to demonstrate the presence of sensitizers in certain active sera. These authors showed that the sera of animals that have been immunized against the typhoid bacillus, the plague bacillus and the like, as well as sera from patients who have recently recovered from typhoid

* Zur Kenntnis der antituberkulösen Sensibilisatoren. Berliner klin. Wochenschr., 1906, p. 1531.

$\dagger$ Page 217. 
fever, contains specific sensitizers, inasmuch as any one of these sera has the property of fixing alexin in the presence of the specific organism against which it was formed; this property is not possessed by any one of the sera under normal conditions.

Since then I have used the same method* to demonstrate specific sensitizers in the serum of animals which had received injections of albuminous substances such as milk, albumin, fibrinogen or alien serum. An albuminous solution on the addition of its specific antiserum acquires the property of fixing the alexin.

The technic employed is as follows: The bacteria or the albumin in question is mixed with a small amount of alexin and the antiserum in which we are endeavoring to prove the presence of a sensitizer. A few hours later sensitized red blood cells are added to this mixture. These cells, which, as we know, are very susceptible to alexin, served to indicate whether the alexin which was added in the first place has remained free or been absorbed by the albuminous bodies. If the bacteria or the albuminous substance has been sensitized by the serum that is being investigated, it absorbs the alexin and subsequently added corpuscles remain intact, as no free alexin is present.

In this communication I also make use of this method, which was first employed by Bordet and myself in 1901, to demonstrate the presence of sensitizers in the serum of animals that have been immunized with various acid-fast bacilli and for the purpose of showing by such sensitizers the relations between saprophytic bacteria and those which are pathogenic either for cold-blooded or warm-blooded animals.

Very little work has been done with antituberculous sensitizers. Bordet and I $\dagger$ have been able to show that guinea-pigs immunized with avian tubercle bacilli form sensitizers which are active against both human and avian tubercle bacilli.

Dembinski noted in 1904 that rabbits and doves which have received injections of avian bacilli form sensitizers against these organisms, but no such sensitizers following the injection of human tubercle bacilli. I have questioned certain of this author's conclusions. $\ddagger$ Wassermann and Bruck $§$ have recently employed

* Page 241. $\dagger$ See p. 462.

$\S$ Wassermann and Bruck, Deutsche med. Wochenschr., 1906.

$\ddagger$ See p. 464 . 
the Bordet-Gengou method to demonstrate the presence of antituberculous sensitizers in tuberculous patients who have received tuberculin.

I have carried out my experiments on guinea-pigs which have been immunized with the following acid-fast bacilli: the homogeneous culture of tuberculosis of Arloing*, obtained from Lille, bacillus of fish tuberculosis, the acid-fast butter bacilli of Rabinowitsch the acid-fast grain bacilli, No. I, and Tobler bacilli, I, II and V, and also the Timothy bacillus and a bacillus from horse dung. Cultures of these organisms were grown on glycerin potato without any particular reference as to their age and were dried at 37 degrees for 24 hours in a vacuum. The bacilli were then ground up and used at various periods, subsequently, for injection. Five milligrams of powder shaken up in salt solution was used for each injection in the guinea-pigs. Three such injections were given at intervals of 3 weeks in order to give the animals time for the abscesses, which frequently follow, to heal. The guinea-pigs were bled 14 to 21 days after the last injection and the separated sera as well as the normal serum employed for control were heated to 56 degrees for half an hour. Sensitizers were then demonstrated by the Bordet-Gengou method, a description of which is given in their first article. $\dagger$ It may be noted here simply that in all these experiments six parts of the serum to be tested were mixed with one part of guinea-pig alexin and two parts of an emulsion of bacilli. This mixture of bacilli is 1 c.c. in volume and contains 80 milligrams of bacilli from a fresh culture. + Three hours later one part of sensitized goat blood was added to each tube.§ In the following table the results of these experiments are given. This table shows

* These bacteria were kindly given us in part by Dr. Binot of the Pasteur Institute, Paris, and in part by Dr. van Steenberghe of the Pasteur Institute in Lille, to whom I wish to express my indebtedness.

$\dagger$ I have demonstrated to my own satisfaction that the traces of potato which of necessity must be present in the experimental test tubes with the bacilli, or which are injected into the animal during the immunization have no effect on the result.

$\ddagger$ I have found that it is better to use the fresh cultures instead of the dried bacilli; the dried powder frequently of itself absorbs alexin.

$\S$ In all my experiments one part of washed goat blood was placed in contact for one quarter of an hour with one part of heated immune serum and subsequently washed, and mixed with two volumes of 0.85 per cent salt solution. 
that, in general, the injection of acid-fast bacilli in guinea-pigs, whether they be saprophytic or pathogenic for cold-blooded animals, or of the homogeneous culture of Arloing, gives rise to sensitizers which are active not only against the homologous bacteria, but also against other acid-fast bacteria, whether saprophytic or pathogenic. These sensitizers are particularly active against the bacilli of human, bovine or avian tuberculosis. There are certain exceptions, however, to this rule which are brought out by my experiments. Acid-fast Tobler No. I has no sensitizer for acidfast Tobler No. V; Bacillus Tobler No. II has no sensitizer for fish tuberculosis, and Tobler No. V none against avian tuberculosis. I have not studied these exceptions any further, but have contented myself with calling attention simply to the general uniformity of this immunity against tubercle bacilli, which was formerly pointed out by Klemperer in guinea-pigs following the injections of saprophytic acid-fast bacilli.

It seems to me wise, before drawing any conclusions from my results as to the relationship between the different acid-fast bacilli, to continue these experiments and particularly to follow more closely the time of occurrence of the antibodies in the immunized animals. 
KNOWLEDGE OF ANTITUBERCULOUS SENSITIZERS.

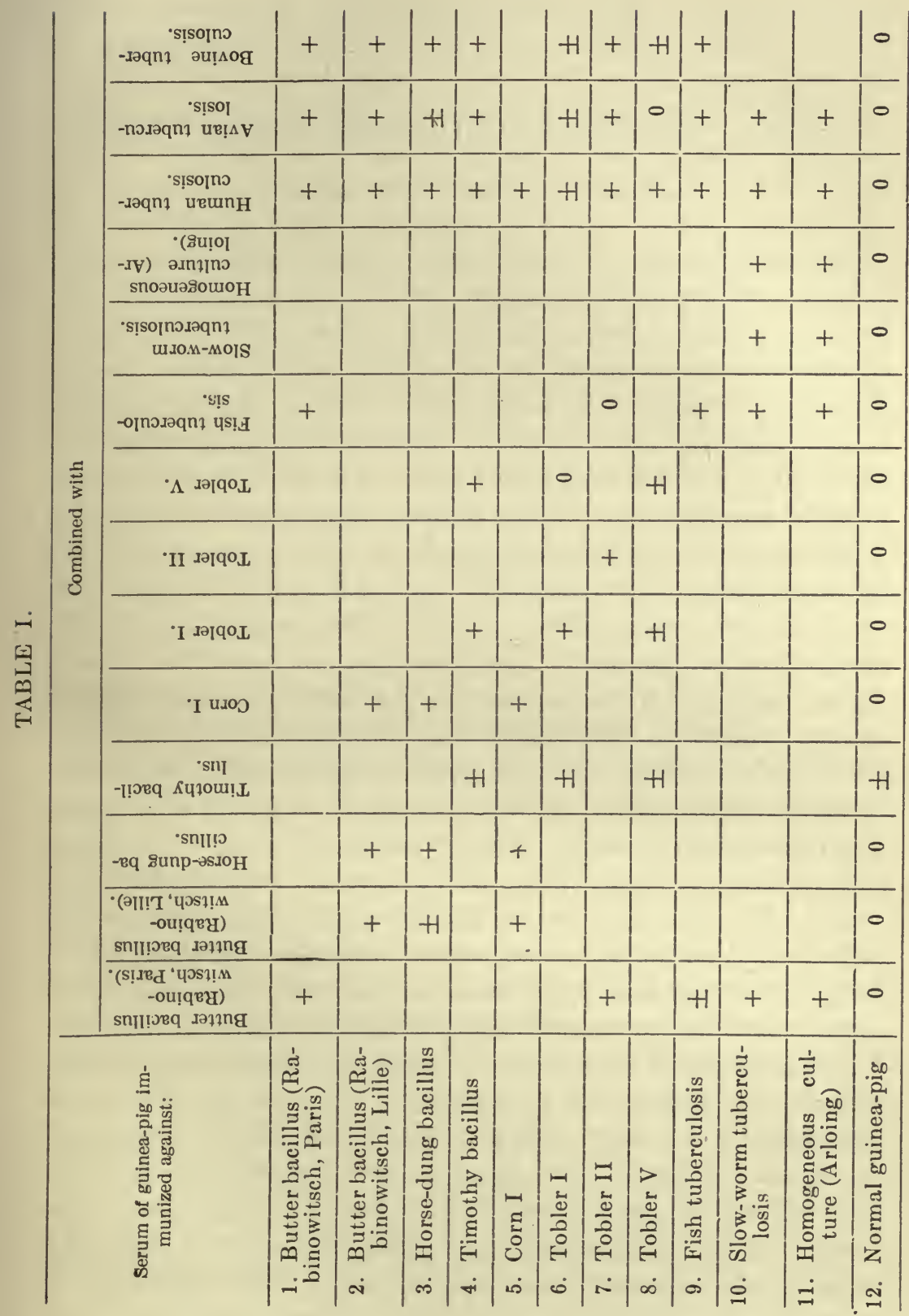




\title{
XXVII. THE BACILLUS OF WHOOPING-COUGH.*
}

\author{
By DRS. J. BORDET ANd O. GENGOU.
}

The bacteriology of whooping-cough has been studied to a considerable extent during the last twenty years. Many bacteria have been isolated from the sputum and described as the true etiological factor in this disease; we believe, however, that none of these micro-organisms that have been grown by our predecessors is identical with the one we have obtained, which latter organism, on account of the logical proofs that we have been able to find, must be considered as the true parasite that has been sought for.

The failure of other bacteriologists, as well as our own failures for nearly six years, during which time we have studied whoopingcough, are easily explicable in view of certain circumstances which may be briefly mentioned.

As every one knows, the success of attempts to isolate an unknown pathogenic agent depends on the presence of a sufficient number of the organisms in the product that is studied and on the fact that these organisms shall not be lost in the midst of innumerable non-specific bacteria. These conditions of relative abundance and purity of culture of the specific organism are not easily met with in the majority of cases of whooping-cough, except in the beginning. And, what is more, even during this beginning period only that part of the expectoration which comes from the region in which the bacteria are most active should be used; in other words, the products from the depths of the bronchi expectorated during the periodic accesses of the disease. This exudate during the characteristic "whoop" is white, thick, and rich in leucocytes; it contains the bacilli of whooping-cough in considerable numbers and in favorable cases in almost pure culture. At the same time the child of ten expectorates a more transparent mucous and sticky secretion which contains less cells and many fewer specific bac-

* Le microbe de la coqueluche. Annales de l'Institut Pasteur, vol. 20, 1906, p. 731 . 
teria and numerous other extraneous organisms. This latter form of expectoration should be avoided in making cultures. If the expectoration is examined on successive days, it is found that the leucocytic exudate contains fewer and fewer specific bacilli and that phagocytosis occurs more frequently than at first. Although the accesses of whooping-cough continue numerous and characteristic, the richness of the culture becomes less. It may be that the organism still persists in the tissue of the bronchi, but, at any rate, it is certain that the number of organisms eliminated by expectoration decreases notably. This later exudate is less suited for growing the organisms, and, what is more important still, microscopical preparations of it evidence this fact clearly; to one who has studied the previous earlier expectorations, the subsequent less favorable ones show clearly the importance of this bacillus and its function. The relativeiy short duration of the favorable period for obtaining cultures of the whooping-cough bacillus, the unequal distribution of the bacillus in the sputum; and, furthermore, the difficulty in obtaining the secretions at the proper moment from infants, all render the study difficult. Although the research is difficult even when dealing with pure cases of whooping-cough, it becomes even more so when the disease is complicated by nonspecific colds, bronchitis, bronchopneumonia, etc.; in other words, when other bacilli of respiratory affections are mixed with those that cause the disease. The sputum obtained from children at home is therefore evidently much more suitable for study than materials obtained in the hospital.

The necessary conditions for success in such a research, as well as the causes of error that may throw an investigator off the track, will appear more clearly if we give a short résumé of our own attempts. Incidentally we shall mention certain non-specific bacteria that we have frequently met with which may be confounded with the true bacillus and to which other bacteriologists have drawn attention.

Our first researches on whooping-cough began in 1900. At this time a child, B, aged 5 months, subsequent to contact with children that were beginning to cough and in whom the diagnosis of whooping-cough was later determined, suffered an attack of typical whooping-cough. The health of this child had hitherto been perfect. She had never had even the slightest disease of the 
respiratory tract. One of us who followed her case carefully was able to obtain a whitish shred of exudate that was not mixed with saliva which was projected by the whoop in the first characteristic crisis. The microscopical examination with Kühne's carbolated blue stain showed that this rich leucocytic exudate contained an enormous number of little ovoid bacilli which were somewhat elongated at times, but frequently so short as to resemble a micrococcus; in general the aspect was rather constant, the stain a pale blue, and the outline of the bacillus and particularly its poles were frequently more deeply stained than the center. This organism was found scattered indiscriminately among the cells and was at times within cells. Some of the larger organisms frequently showed a little point toward the center that appeared to be walled off; the majority of the bacteria were separate, but some of them occurred in pairs, end to end. Gram's stain was negative. The growth of the organism was so abundant and the culture was so pure that it seemed reasonable to admit some causal relation between this organism and the appearance of whooping-cough in a child whose bronchi had not previously been affected. All attempts to cultivate the organism, however, were unsuccessful. The exudate, when grown on ascites-agar (ascites fluid mixed in equal parts with melted agar) or on agar moistened with human or rabbit blood, gave only a few colonies of unimportant cocci. Such media, however, are very well suited to grow delicate bacteria; on blood agar, in particular, the inoculation of sputum from cases of grippe gives an abundant growth of the influenza bacillus. The sputum on subsequent days showed a progressive diminution in this bacillus. In short, although microscopical examination showed many organisms, culture was unsuccessful.

During the following years we made a bacteriological examination of a number of whooping-cough sputa in Brussels, the most part of which we collected in hospitals. We usually used the culture medium which we have found very useful in growing delicate bacteria, particularly those bacteria which we have studied in our work on the flora of the respiratory tract.* The majority of these

* The culture medium is prepared in the following manner: 100 grams of sliced potatoes are added to 200 c.c. of 4 per cent glyeerinated water. This is steamed in the autoclave and, on separating the fluid, a concentrated glycerinated extract of potato is obtained. To 50 c.c. of this extract is added 150 c.c. of 6 per cent 
sputa were from cases of whooping-cough that were not studied in the beginning, but were well advanced, and they contained, therefore, a rich and varied flora.। We paid particular attention, obviously, to the study of those bacteria that we had observed so abundantly in a pure state in the single case in 1900 which we have mentioned.

We found, indeed, in greater or less numbers, small ovoid bacteria which were faintly stained and were quite similar to those we formerly observed. The most frequent organism that we found, however, was still smaller, better colored and either separated or in clumps and frequently somewhat long or even filamentous. This organism, which occurred in almost every case in abundance, grew well on our medium; it gave, indeed, in the majority of cases, the greatest number of colonies. These colonies were bluish or grayish, slightly elevated in the center, rather diaphanous, particularly at the edges, and almost transparent in young cultures, and resembled drops of dew. Microscopically the organism was small, did not stain by Gram, was usually short and delicate, appeared frequently as a dot, and in certain cultures, particularly, showed a marked tendency to pleomorphism. Certain individual bacteria were somewhat thicker and swollen and others showed curious involution forms which stained faintly or unequally. This organism, although it does not grow on agar or ordinary bouillon, grows very well in the presence of hemoglobin. It was easy to determine that we had to deal with an organism which was identical, or at least analogous, with the one discovered by Pfeiffer in influenza and which other bacteriologists have noted in whooping cough and even considered as the specific bacillus-an opinion which is reasonable enough when we consider its frequency. The work of Jochmann and Krause,* giving a very exact description of this bacillus and mentioning its need of hemoglobin, had just salt solution and 5 grams of agar. This mixture is melted in the autoclave and the warm fluid is placed in test tubes, 2 or 3 c.c. to a tube. The tubes are then sterilized. Sterile defibrinated rabbit blood, or preferably human blood, is then obtained. To each melted agar tube an equal amount of blood is added, the mixture well shaken and the tubes slanted and cooled. This medium allows the growth of such delicate organisms as the meningococcus, gonococcus, the influenza bacillus and, as we shall presently see, the whooping-cough bacillus. As the media contains no pepton it is not very good for the growth of putrefactive saprophytes.

* Zeitschrift für Hygiene, 1901, Bd. 36. 
appeared at that time. This description, indeed, agrees perfectly with our own. We found as well as these authors that this organism was present in large numbers in almost pure culture (with the exception of a few pneumococci) in the pus of the smaller bronchi at an autopsy of a child who died of bronchopneumonia following whooping-cough. Such facts evidently seemed significant.

In studying this problem, we naturally had to compare this bacillus as it appeared in our cultures, or in the newer cases which we had, very carefully as regards morphology, with the organism which we found in our first case in 1900 and which seemed incontestably to be the true parasite. Such a comparison, however, did not settle our doubt. This latter organism evidently belongs in the group of small bacteria that stain poorly, as well as the influenza bacillus which has already been cultivated. The appearance of the two organisms is not, however, identical. If we consider the bacteria that we found in the exudate in 1900 as typical, they are of more regularly ovoid form, and, as a general rule, somewhat larger and with a more constant deeply stained center. Might it not, however, seem reasonable that a given bacillus in an artificial medium should not appear identical with one observed in a pathological specimen? In short, the two bacteria might be considered identical, and the opinion that a bacillus similar to the influenza bacillus has an etiological function might well be accepted, particularly when it has been claimed by the bacteriologists to whom reference has been made.

There are three grave objections, however, to this hypothesis. In the first place the bacillus in the typical exudate of 1900 did not grow in blood agar, in which medium the influenza bacillus would eertainly have grown readily. Then we have not been able to demonstrate any particular properties against this latter organism in the serum of children that have recovered from whoopingcough, in spite of repeated attempts. Other bacteriologists must also have obtained similar negative results, for no mention is made in the work of Spengler, of Jochmann and Krause and others, of specific properties in the blood of recovered children. And finally we found that this organism was present not only in whooping-cough, but also in the various respiratory affections in the adult as well as in children, in cases of grippe, bronchitis and 
bronchopneumonia as a complication, in various diseases, and also frequently in a pure state in simple coryza. These findings agree with the facts of certain of our predecessors, notably with those of Elmassian.

In short, this organism, which resembles the one described by Pfeiffer as the cause of influenza, is certainly not the cause of whooping cough. We have this year been able to isolate a specific bacillus, since we have had at our disposition favorable cases, among which the one that furnished our first successful culture may be mentioned in some detail. It was a male child of two months, nursed by its mother, in excellent health, who had been contaminated by a neighbor's child who later on showed a most characteristic case of whooping-cough. The child began to cough and the characteristic paroxysms soon came; during one of these paroxysms a white bit of exudate which was extremely rich in leucocytes was coughed up. This exudate contained enormous numbers of a micro-organism which was identical with the one we had seen several years before and in a similar condition of purity and abundance.*

This exudate was shaken up in salt solution so as to form various dilutions and these dilutions were inoculated on our medium. The surface of those tubes that had been inoculated with a relatively concentrated dilution, in which, therefore, the specific organism was present in large numbers, showed, instead of a uniform growth at the end of 2 days, only a few contaminating colonies (some coccus that occurs in the saliva). The platinum loop, however, removed, from the part of the surface of the culture which appeared to be sterile, a large number of the organisms that were being sought for. It appeared as if multiplication had actually taken place but was too slight to give rise to visible colonies. $\dagger$ This organism, implanted on a second tube, grew much better, giving rise to a definite line of implantation, and the culture then became luxuriant. Morphologically the identity of this organism with

* On successive days the quantity of bacteria progressively diminished. The paroxysms continued for about 3 weeks and the child got well after complications.

$\dagger$ At times we have found that certain individual micro-organisms give rise to distinctly visible colonies in 2 or 3 days even in the first culture. This, however, is the exception. 
that present in the exudate was not only wholly satisfactory, but as complete and absolute as possible.*

The cultural comparison of this organism with the influenza bacillus shows that the two are essentially different. On blood media the whooping-cough bacillus grows much less readily during the first generation, but much more luxuriantly subsequently. It grows a little more slowly. Its growth is whitish and thicker and without the bluish, diaphanous appearance of the influenza growth. It does not show as great a need of hemoglobin as does the influenza bacillus, but when grown subsequently on colorless ascites-agar it develops in a white, oily, and moist layer which becomes so opaque that in 2 or 3 days it is almost as thick as a culture of typhoid bacillus on ordinary agar. $\dagger$ The whoopingcough bacillus has less tendency to pleomorphism and involution. It does, to be sure, become smaller during several generations on our blood-culture medium, but it soon regains its primitive form on being inoculated in a liquid medium and then reinoculated on solid media. Although it fails to grow on the usual media sterilized in the autoclave, such as agar, gelatin and ordinary bouillon, it develops well in such liquid media as 1 per cent glycerinated bouillon with equal parts of rabbit blood or serum; although under these conditions its form frequently changes. $\ddagger$

Although our researches on the subject are not finished, it is probable that the organism secretes substances that produce local effects rather than general intoxication, that is to say, which provoke an irritating and even a necrotizing action. The organism when injected subcutaneously or intraperitoneally in the guineapig is fatal only in very large doses. On injecting a little of the

* We use, as a stain, carbolated toluidin blue prepared in the following manner: Toluidin blue (Grübler), 5 grams; alcohol, 100 c.c.; water, 500 grams; complete solution is allowed and then 500 grams of 5 per cent carbolated water is added. The stain is filtered 1 or 2 days later. This blue is preferable to ordinary earbolated methylene blue.

$\dagger$ These remarks naturally refer only to the organism that has been accustomed to growing on artificial media; it is by no means sure that the organism will grow in the first generation on ascites-agar.

$\ddagger$ This fact separates it usually from the other bacteria that grow on ordinary media noted by certain observers (Afanassiew, Czaplewski and Henzel, Vincenzi, Manicatide, Leuriaux, etc.), and are demonstrable in quite pure whooping-cough sputa. There is no need of considering these organisms in detail. 
whooping-cough exudate which contains numerous organisms, in the eye of the rabbit, only very slight growth occurs; the aqueous humor remains limpid and white and intense weeping takes place, together with an excessive conjunctival congestion. Injection of a small amount of pure culture produces the same lesions, the gravity of which is surprising in view of the fact that there is little or no multiplication of organisms. If such a course goes on in the bronchi, it is easy to understand the accesses and their persistence even when the growth of the organism decreases.

The authenticity of this bacillus as the causal agent in whoopingcough rests in large part on the circumstances in which it is found, namely, the excessive growth of the organism in pure culture during the initial period of diseases in young children who have never before been ill. The principal argument, however, in favor of this organism appears to us to be furnished on studying the specific properties of the serum. The serum of individuals that have never had whooping-cough, or who have had it a long time before, does not agglutinate the bacillus even in low dilution. The sera of children that have recently recovered from the disease has a moderate agglutinating property which is constant and evident. The most remarkable fact, moreover, is the intensity of sensitizing property in such sera. To demonstrate it we have used our accustomed method of alexin fixation.

The essentials of this method which we demonstrated in 1901 and employed to demonstrate the presence of sensitizers in many immune sera, are well known.* It has since been employed by numerous experimenters - Lesourd, Lambotte, Fassin, Cohen, and more recently by Wassermann and Bruck. $\dagger$ One of us established in $1900 \ddagger$ that specific sensitizers which are active against bacteria or red blood cells endow the element which they attack with the new property of absorbing alexin energetically. If a mixture of alexin (fresh animal serum), of the substance under consideration, and of a suitable sensitizer (that is, immune serum heated to 56 degrees), is prepared in suitable proportions it is found that after a certain period the alexin has disappeared from the fluid. Con-

* See p. 217.

$\dagger$ The historical account of our method, by Wassermann and Bruck, of which they have made considerable use, is remarkably brief.

$\ddagger$ See p. 186. 
sequently, if sensitized corpuscles are subsequently introduced into the mixture, they undergo no hemolysis. Certain other mixtures, the list of which will be found in our previous articles, give the necessary control; in one of them in particular it is proved that if normal serum, which is not sensitizing, is used in the first mixture, instead of the specific serum, there is no delay in the hemolysis of the subsequently added corpuscles.

If we employ the serum of children that have recently recovered from whooping-cough in such a test as this, the result is very demonstrative. In our first experiment we employed the serum of three different children who had recovered from two weeks to a month previously, and as controls the sera of three normal individuals.

These different sera were heated to 56 degrees and were mixed in doses varying from 0.1 to 0.3 of a cubic centimeter with 0.05 or 0.01 of a cubic centimeter of fresh human or guinea-pig serum (alexin) plus 0.2 of a cubic centimeter of an emulsion of the whooping-cough bacillus (a 24-hour culture suspended in salt solution).* Four hours later, after remaining at room temperature, a little well-sensitized goat blood was added to each tube. Hemolysis took place in a few moments in the tubes containing normal serum, but the corpuscles remained intact, even on the following day, in those which contained whooping-cough serum. The sensitizing property, then, of the serum from a case of whooping-cough is very energetic even in so small a dose as 0.1 of a cubic centimeter. It is scarcely necessary to mention that the serum of such cases does not fix the alexin unless the whooping-cough bacillus is present; the other necessary controls are also made.

The organism resembling the influenza bacillus acts no differently with whooping-cough serum than it does with normal serum. Such an experiment shows not only that this latter organism has nothing to do with whooping-cough, but serves to separate the two bacterial species from one another. $\dagger$ In other experiments the same results were obtained. We tested the sera from two different

* The experiment may be done with either of these alexins, but the guinea-pig alexin is, in general, more favorable for hemolysis and therefore better to use.

† It should be noted that the influenza bacillus has in itself a eertain property for absorbing alexin without the presence of any sensitizer. We have already stated that the whooping-cough bacillus does this only when whooping-cough serum is present. 
children of the same age (4 years), both of whom were convalescent, one of whooping cough and the other of bronchopneumonia following scarlet fever. As a result, 0.2 of a cubic centimeter of the serum in the first case sensitized the whooping-cough bacillus and produced alexin absorption. The subsequently added sensitized corpuscles remained intact, whereas hemolysis took place in 5 minutes in the mixture containing the other serum.

As we regard the facts in the etiology of whooping-cough as well established, we shall hope to announce soon the results of attempts at serumtherapy or active immunization which we have made. It may be mentioned in this connection that the injection of a cubic centimeter of culture, killed by heating to 62 degrees, * produces no unpleasant symptoms in man.

* Heating to 55 degrees suffices to kill the organism. 


\section{AN ADDITIONAL NOTE ON THE WHOOPING- COUGH BACILLUS.*}

By DRS. J. BORDET AND O. GENGOU.

It has seemed to us well to give certain additional information concerning our study of the whooping-cough bacillus in order to facilitate the work of other bacteriologists who are interested in the subject.

Our researches during the last year have fully confirmed our conviction as to the authenticity of the organism we described at that time as the causal agent in whooping-cough. We shall not repeat the arguments which we gave in our former article, but will simply add that we have in every instance found a strong sensitizing power in the sera of children that have recovered from whooping-cough.

A culture medium which we have already described - a mixture of rabbit blood and agar containing a little glycerinated extract of potato - still seems to be the best for isolating the organism. $\dagger$ In growing the organisms the most important fact is that the whooping-cough bacillus, at least in the first culture, grows very slowly; at least 2 days in the thermostat is necessary for the appearance of colonies, and these may remain very small unless the medium is most carefully prepared. Little or no growth takes place if the medium has become dried or if enough contaminating organisms of rapid growth are mixed with the colonies and monopolize the nutrient properties of the medium. If these unfavorable con-

* Note complémentaire sur le microbe de la coqueluche. Annales de l'Institut Pasteur, Vol. 21, 1907, p. 720.

$\dagger$ The following observation is of some importance in obtaining a growth. The defibrinated blood added to the fluid agar should be very carefully mixed with it by shaking. The blood is of greater specific gravity than the agar, and, unless they are carefully mixed, the upper part of the medium is composed of agar that contains very little blood, and therefore when the tubes are slanted their surfaces offer a very unfavorable culture medium. 
ditions are avoided, colonies may be obtained even in the first culture, which, although not close together, show a good growth at the end of the third day, and /are distinguishable by their whiteness, their projection and their clearly circumscribed outline.

Certain sputa which are particularly favorable, when suitably diluted and inoculated on the culture medium have given us almost pure cultures, including 100 to 200 times as many whooping-cough colonies as colonies of other bacteria.

We have, as well as other authors, mentioned the frequency of a bacterium similar to that described by Pfeiffer as the cause of influenza, in the sputum of whooping-cough,* and we have also mentioned the fact that the presence of these organisms offers a serious obstacle to the isolation of the true parasite of whooping-cough. They usually form numerous colonies which develop rapidly. These organisms are not agglutinated by the serum of a horse immunized against the whooping-cough bacillus, although this serum agglutinates the latter organism energetically; the serum may therefore be used as a ready and infallible means of differentiation. Microscopically these organisms are frequently rather difficult to distinguish from the real whooping-cough organism. $\dagger$ A few successive cultures on blood media suffice to distinguish the two organisms with certainty. When the whooping-cough bacillus is inoculated on the surface of blood agar in a delicate streak, the

* We might better say, identical with, rather than similar to. We have cultivated for some time parallel cultures of bacteria of this sort coming from cases of whooping-cough and the typical influenza bacillus which was kindly given us by Dr. Cohen, who obtained it from Pfeiffer's laboratory. Comparison of these cultures shows no perceptible difference between the organisms.

$\dagger$ This is particularly true in the first generation of cultures. Certain indications on this point may be useful. On suspending in water to make a stained preparation, the influenza bacilli give an emulsion with a tendency to spontaneous agglutination, so that in drying on the slide they are often clumped in small masses (see, for example, Fig. 1, plate 9, in Jochmann and Krause's article, Zeitschrift für Hygiene, 1901); in preparations of whooping-cough the organisms are better separated. After several successive cultures on our medium, the influenza bacillus often assumes larger shapes, which are frequently swollen and twisted; the mean size increases and is frequently greater than that of the whooping-cough organism. Cabolated blue stains color the influenza bacillus much more intensely than they do the whooping-cough organism. We may recall that transplanting on ascites-agar serves as a good method for distinguishing the two organisms; the whooping-cough bacillus grows on it slowly as a white streak; the influenza bacillus grows very slightly, although it does grow somewhat. 
growth of organisms thickens, and in 2 or 3 days projects distinctly from the surface. But although it becomes thicker it does not broaden, so that its sides are distinctly steep. The influenza bacillus, when grown in the same way, gives a much wider streak, has a festooned outline which slopes gently, and is moist and glistening. The whooping-cough growth, moreover, is whiter and never blackens the underlying blood medium, whereas the influenza bacillus frequently does so.* When the whooping-cough bacillus is looked at with transmitted light the growth of organisms appears as a pale line which stands out from the adjacent part of the culture medium which has not been touched by the inoculation. This clarification of the medium is due to the fact that the organisms have hemolyzed the adjacent corpuscles and so diminished the opacity of the nutrient substratum.

We have already insisted on the fact that the abnormal forms which occur frequently with the influenza bacillus in the form of clubs or long filaments with a tendency of swelling, with irregularity of aspect and poor staining, are rare in the whooping-cough bacillus, which keeps its appearance of a small cocco-bacillus invariably. The size of this cocco-bacillus, particularly in old cultures, may be extremely reduced. We are speaking now of cultures on a solid medium; the cultures on liquid media, which we shall discuss in a few words, show more distinct pleomorphism; the dimensions of the organisms become more variable, their staining more unequal, and they are frequently larger and less ovoid in shape.

Liquid cultures grow well, provided we take into account the necessity of atmospheric contact, which is very marked in the whooping-cough bacillus. We find, indeed, that the organism grows only under the best conditions of aerobiosis. When grown in a test tube containing a suitable nutrient fluid of several centimeters in depth, and maintained in a vertical position, the growth of the organism is slow and difficult. When the tube is placed horizontally, however, a cloud soon appears, covering the increased

* These influenza organisms are obtained from cases of whooping-cough and agree absolutely in all their characters with the organisms described by many of our predecessors and notably by Jochmann and Krause. We may recall that for a long time our attention was attracted by these organisms, which are constantly present in the sputum of whooping-cough. 
surface. It is therefore well to make cultures in a flask with a large flat bottom and to employ not more than a centimeter deep of fluid. An excellent culture medium is made by a mixture of 1 per cent pepton bouillon containing 1 per cent glycerin and equal parts of horse serum, the latter preferably heated for three-quarters of an hour to 57 degrees. Under these conditions the organism grows almost without clouding the fluid; in 4 or 5 days the bottom of the flask is covered with a whitish, slightly viscous, and rather thick sediment. The supernatant fluid becomes more cloudy, and the deposit less coherent if rabbit serum is employed instead of horse serum, as the latter has a certain agglutinating property for the organism.

If, instead of using normal horse serum (57 degrees) for the culture medium, we use the serum of a horse that has been immunized against the organism (also heated to 57 degrees), growth still takes place. The bacteria, however, are more agglutinated and show a more abnormal appearance; they grow in the form of strepto-bacilli, or may even appear as streptococci in long chains, their appearance, in short, being quite abnormal and resembling in no particular the morphology on solid media.

Immunization of a horse gives rise to an extremely agglutinating serum.* If a two-day growth of culture on blood agar is suspended in 2 or 3 c.c. of salt solution, and the organisms allowed to diffuse in the suspension without much agitation, we obtain a cloudy fluid of homogeneous and almost colloidal appearance. This emulsion is agglutinated by traces of the active serum; for example, agglutination takes place on the addition of 0.002 of a cubic centimeter of serum to 1 c.c. of the emulsion. Too great an excess of agglutinin inhibits the phenomenon; the whooping-cough bacillus, indeed, may serve as a good example to study this inhibiting power of an excess of serum, which has already been noted by various observers.

The various strains of whooping-cough bacilli obtained from different cases of the disease agglutinate variably. We find, for example, that our horse serum agglutinates the organism that was

* Our horse received in $\mathbf{1 5}$ successive injections, for the most part subcutaneously, but some intravenously, a total of about two liters and a half of rich fluid culture (glycerinated bouillon and horse serum). 
used to immunize the animal less than it does another organism from a different case of whooping cough.

We have hoped to be able to make a serum diagnosis of whooping cough by the simple and practical agglutination method. IVe have found, unfortunately, that the serum of children suffering from or convalescent of whooping cough shows very inconstant agglutinating properties. An agglutinating property is frequently distinctly manifest, although never very intense; it is often, however, entirely lacking. There are many sera which, although not agglutinating, are distinctly sensitizing. As we have already mentioned, the alexin-fixation method always gives very marked positive results. The two properties of agglutination and fixation are therefore quite separate in this disease. In this connection we may recall that the serum from the convalescent case of typhoid fever, with which we made our first attempts at alexin fixation (1901), was also strongly sensitizing without being agglutinating, and we have frequently noted this fact since that time. Various other observers have also noted the lack of necessary parallel between agglutination and bactericidal power.

We have already noted the lesions (excessive irritation and clouding of the cornea) which follow the injection of a sputum containing the organism in pure state, or of a culture of the organism in the anterior chamber of the rabbit's eye. Certain remarkable phenomena are also to be noted on injecting the organism in the guinea-pig's peritoneal cavity, and similar effects take place in the rabbit's peritoneal cavity if larger doses are employed. For this purpose an emulsion of culture from blood agar 2 or 3 days old is better than the less virulent fluid cultures. 1.5 to 2 milligrams of bacteria, weighed in a moist condition, causes death on the following day or on the second day. We have to deal here, not with an infection, for the bacteria show no marked increase in the peritoneal cavity; at autopsy only a few of the organisms are found in the exudate, and frequently all of them are phagocyted by the large number of leucocytes present. Intoxication phenomena, however, are very marked, and are evidenced by the appearance of petechix in the peritoneal wall, and also at times in the pericardial cavity, and by an extreme congestion of the cardiac blood vessels. Fatty degeneration of the liver is also found. The most apparent symp- 
tom that the animal shows before death is marked dyspnœa, which appears very early and may be accounted for by the pleural exudate. A subcutaneous injection causes an edema.

An emulsion of culture suspended in this way, and then killed by toluol or by heating to 56 degrees for half an hour, when injected into the peritoneal cavity of guinea-pigs, also causes death, with phenomena of intoxication and particularly with pleural exudation. It does not, however, give rise to petechiæ and larger doses are necessary than of the living organism. The serum of the immunized horse, although strongly agglutinating, has only slight antitoxic effect. Very large doses are necessary to neutralize the bacterial emulsion so that it may be injected into the peritoneal cavity without producing injury. It may be added that we have only a single immunized horse, and therefore we must be somewhat reserved in speaking of the properties of the immune serum, as these properties may be due to the individual animal employed. It is, however, very likely that horses immunized against the whooping-cough bacillus are similar to those that furnish antityphoid serum, the serum of which, although showing certain properties, such as agglutination very markedly, are not extremely antitoxic. This latter property we shall endeavor to increase. The results obtained by treating children suffering from whooping-cough with the serum in question, although at times distinctive, have not yet been sufficiently marked. 


\section{THE ENDOTOXIN OF THE WHOOPING-COUGH BACILLUS.*}

\section{BY DR. BORDET.}

In the recent studies on whooping-cough with Dr. Gengou we have succeeded in obtaining an endotoxin from the organism which we isolated some time ago, and which is the cause of this disease.

We already possess considerable knowledge concerning the endotoxins of bacteria. Certain bacteria diffuse the poisons that they form readily, as, for example, the tetanus bacillus and B. diphtherix; other bacteria, as the typhoid bacillus, retain the toxin within their bodies. Certain particular proceedings, then, are necessary to remove these active substances from the bacterial bodies. No absolute line of demarcation can indeed be drawn between diffusible toxins and endotoxins, and there are all degrees in the ease with which the poisons leave the bacterial bodies and appear in the surrounding fluid, depending on the organism under consideration.

In immunizing animals with an endotoxin, it is desirable to obtain it in a clear solution without the presence of the bacterial bodies themselves. In many instances it is of importance to immunize against the poison, particularly, and the presence of the bacterial bodies themselves gives a useless and often unpleasant complication. Too great amounts of bacteria wear out the animals, lead to suppuration, and, what is more, the immunity against the toxin is more easily obtained when the bodies that form it are not present; it would seem to diffuse better throughout the animal body and to stimulate more those cells which have to do with the elaboration of antitoxins. For example, we find in the case of the typhoid bacillus that a subcutaneous injection of the bacteria themselves produces no anti-endotoxin; the endotoxin must first

* L'endotoxine coquelucheuse. Bulletin de la Société Royale des Sciences Médicales et Naturelles de Bruxelles, No. 7, 1908. 
of all be separated in order to produce its antibody. In previous articles we have insisted on the fact that the rapid death of guineapigs that have received an injection of a sufficient number of living whooping-cough bacilli in the peritoneal cavity is due to an intoxication from the poison that comes from the bacteria, and not to an infection, properly speaking. The bacteria injected do not increase to any extent in vivo, and do not invade the animal body; they are retained at the point of inoculation, and are frequently phagocyted. At autopsy, however, considerable lesions are found and in particular a marked peritoneal exudate of hemorrhagic character, abundant disseminated ecchymoses, and pleural effusions which are so extensive as to produce asphyxia, and are the immediate cause of death. All these phenomena may be produced by almost perfectly limpid extracts containing endotoxin which we have obtained by employing. Besredka's method as described for typhoid, plague and dysentery endotoxins. We inoculate the whooping-cough bacillus on our blood-agar medium, and in making this medium we prefer horse blood in order to obtain large amounts of media readily; we leave the cultures in the incubator for 3 days, and then remove the layer of bacteria with a glass rod and suspend them in a small amount of salt solution. The very thick emulsion obtained in this way is dried in a vacuum at $37^{\circ} \mathrm{C}$. in the presence of caustic potash. The dried bacteria are then ground in a mortar with the addition of a little dried sterilized salt. Enough distilled water is then added to this powder to bring the tonicity of the solution to normal ( 0.75 per cent). This emulsion is left until the following day, energetically centrifugalized, and the supernatant fluid which is decanted is almost limpid.

This fluid, so far as suspended materials are concerned, contains sodium chloride in relatively large amount and bacterial substance in proportionately small amount. And yet this fluid on intraperitoneal injection kills a guinea-pig in doses of 0.5 and even 0.25 of a cubic centimeter, with the phenomena that we have just mentioned.

Intravenous injection in a rabbit of 1 or 2 c.c. of endotoxin is usually fatal, killing in less than 24 hours. At autopsy renal hemorrhages, acute fatty degeneration of the liver and a marked hemorrhagic condition of the suprarenal capsules are found. In respect to this latter symptom, as well as in its tendency to pro- 
duce pleural effusions, the whooping-cough toxin shows certain analogies with diphtheria toxin.

The most interesting observations, however, are furnished on subcutaneous inoculation. Guinea-pigs that have received an injection under the skin of 0.5 and even 0.25 of a cubic centimeter of endotoxin show a very marked edema, which frequently becomes hemorrhagic at the point of inoculation; the surrounding area rapidly takes a dark color without any tendency to suppuration. A rapid extensive necrosis of the skin follows and breaks down, leaving a large ulcer. This local effect is much more marked than are the generalized symptoms on subcutaneous inoculation; in horses, ulcers a decimeter square may be produced; a marked wasting is always noted.

The demonstration of these properties in the whooping-cough toxin seems to us to round out satisfactorily the ideas which we previously had as regards the pathogenesis of whooping-cough. The production by the bacillus of an extremely irritating poison which is capable of producing necrosis of the epithelial lining of the bronchi where the organism multiplies will explain the characteristic symptoms of the disease and the appearance and persistence of the violent accesses.

The fact that whooping-cough is not generally a very serious disease, in spite of the toxic power of the causative bacillus, is easily understandable in view of the fact that the organism does not tend to produce a generalized infection.

The whooping-cough bacillus is unfortunately rather difficult to handle on account of its remarkable instability on heating, which prevents sterilizing it. The grinding up of the bacteria is necessarily done in the open air, and it is impossible, therefore, to avoid dust and consequently contamination of the toxin. Sterilization such as is employed to eliminate contaminating organisms rapidly destroys our toxin, or at least weakens it very markedly. On heating to 55 degrees for a short time the endotoxin solution becomes opaque and almost inoffensive.

The use of chloroform, toluol and thymol, which ordinarily are so useful in conserving toxins without harming them, weaken the whooping-cough toxin very much, and render it almost useless. We have been foreed, therefore, to keep our bacilli ground with 
salt in a dry condition, and to add distilled water just before using them. In this way the poison may be kept for some time, although we have noted that even when kept from moisture it slowly loses its power.

The serum that we formerly obtained by injecting animals with living cultures has no power to neutralize the endotoxin. We are now engaged in an attempt to obtain an antitoxic serum by injecting endotoxin instead of bacteria. 


\section{RESEARCHES ON AVIAN DIPHTHERIA.*}

BY DR. BORDET.

Dr. Fally and I have recently been studying the diphtheria of hens. This disease has long interested bacteriologists on account of certain analogies that it shows to human diphtheria. Although the acute and rapid course of the disease in man differs from that in fowls, where it is slow in evolution and chronic, it was formerly believed that the relation between the two diseases was very close. This supposed relation was formerly frequently used to explain epidemics of human diphtheria as of avian origin. This hypothesis, however, has not been borne out by the pathologist.

The bacillus which causes human diphtheria is not met with in these hens. Nor does antidiphtheritic serum, as Gratia and Lienaux have shown, have any effect as a preventive or a cure in avian diphtheria. The specific bacteria in these two diseases are certainly different.

Many organisms have been described as causing the diphtheria in hens and pigeons. It is to be noted, however, that none of these organisms that have been mentioned produces the disease with its slow, capricious, and irregular course, nor the lesions which disappear in one place and reappear in another, and show a gravity and duration that varies with the individual. Avian diphtheria has not, in the majority of cases, the appearance of a disease which tends to generalize and become a septicaemia.

The technic that has been employed by many observers has been of a nature designed to bring out organisms that cause septicaemias. Those who have studied it have frequently inoculated healthy animals with false membranes. At times a generalized infection was produced and an organism isolated at autopsy from the heart's blood. The chances are, in such cases, that the organ-

* Recherches sur la diphtérie aviaire. Bulletin publié par la Société royale des sciences médicales et naturelles de Bruxelles, June 3, 1907. 
ism obtained was the cause of an accessory infection, and simply associated with, but not the specific cause of, the disease.

It has seemed to us, therefore, necessary on account of the tendency of the disease to remain localized, to isolate the organism from the morbid product itself rather than attempt a purification of culture in the animal body. A microscopical examination of a bit of false membrane sufficed, however, to convince us that this product is very unsuitable for purposes of direct isolation. The membrane, indeed, is swarming with different bacteria, the separation of which would be a difficult and probably fruitless task. The buccal cavity contains, as we know, under normal conditions, many bacterial species, and these ordinary organisms are still more increased when lesions are present. It seemed better, then, to produce the specific infection in some region of the body which is better protected from saprophytes, and the nictitating membrane in the hen's eye seems to answer this requirement. A thread that has been wet in water in which a fragment of false membrane has been suspended is passed through the nictitating membrane. On the following day this thread is withdrawn, and the irritation due to trauma disappears. Three or four days later a grayish point on the nictitating membrane appears at the point of inoculation.

This point then becomes red and thickened, and soon shows a typical diphtheritic lesion; a marked edema is also noticeable about the eye. At this time, that is, about the eighth day, the nictitating membrane is cut out and washed in sterile salt solution, and then ground up in a few drops of the same solution. The suspension obtained in this way is inoculated on the glycerinated-potato-bloodagar medium which Gengou and I have employed in growing the whooping-cough bacillus. This suspension when inoculated on the buccal membrane of a normal hen produces typical diphtheria. The surprising thing is that microscopical examination with a stain of carbolated toluidin blue or with the Giemsa stain shows no definite bacteria; a few granulations, and at times somewhat elongated spots, are seen, which are so small as not to be distinguishable from cell débris. The culture tubes inoculated with this emulsion are incubated for 3 or 4 days. Only a very small number of colonies is evident after this period; and these colonies 
on account of their rarity are obviously due to contaminating organisms. It would seem that the surface of the culture medium, which had been smooth and shiny, was uniformly roughened between these colonies, but the change is so very slight that it might easily be overlooked. Even with a magnifying glass a layer of bacterial growth could not be asserted to be present. But if a drop of water is inoculated with a scraping from this surface, with great care in avoiding the colonies of contaminating organisms, and this water used to inoculate the buccal mucosa of a normal hen, the disease is produced.

This same fluid on microscopical examination shows an enormous number of little granular elements which are at times slightly elongated about 0.0002 of a millimeter in size, and are most frequently united in compact zoöglea masses.

The culture may be transplanted and retransplanted indefinitely.

The layer of bacterial growth that is formed is never thick enough to be very evident. It is evidenced simply by a darkening and roughening of the surface. At times, however, very luxuriant cultures are obtained which show extremely small colonies or a distinct outline limiting the area covered with growth. The Giemsa stain is the best to demonstrate the organism.

This organism, together with the one causing peri-pneumonia of cattle, is probably the smallest that has ever been grown. It generally reproduces the disease in a somewhat attenuated form, to be sure, particularly when the buccal mucosa is inoculated. The white plaques that appear in 2 or 3 days often heal rapidly, but also frequently reappear later in the same place, and then become more extensive. These lesions, although somewhat benign, are of typical and undoubted specificity.

Certain hens are refractory. Inoculation on the nictitating membrane is always severe and always followed by characteristic symptoms, such as edema of the eye, and thickening and redness of the third eyelid, which shows the chronic nature of the disease. To demonstrate our infecting experiment on the nictitating membrane more conclusively, and to avoid any objection that this infection is simply due to a contagion, or a natural propagation of the disease in the laboratory, and not an artificial infection, we have passed a thread through each nictitating membrane in a nor- 
mal hen. One of these threads was wet with a culture, and the other was sterile; on the following day these threads were drawn out. The disease appeared only in the eye that received the infected thread. We have hitherto dealt only with the diphtheria in hens, but we propose to study a similar disease found in pigeons very soon, and to endeavor to determine whether it is due to the same organism as the one described. 


\section{A GENERAL RÉSUMÉ OF IMMUNITY.}

\section{By Professor Jules Bordet.}

THE majority of the articles which my friend and collaborator, Dr. Gay, has been so kind as to translate and bring together in this volume, bear on the general subject of immunity and have all more or less a single trend. The logical connection between these articles will be quite evident to the reader, who will have grasped my general conception of the mechanism of immunity and of the mode of action of sera so far as we can understand them in the present stage of development of science. I may content myself, then, in this final chapter with certain additional explanations.

I should have been pleased to conclude this volume with a synthetic view of the entire subject, or a general theory capable of coördinating the many facts that have been acquired. But in spite of the results that have been obtained by an army of investigators for many years, I must admit that such an attempt seems to me at the present day both rash and of questionable value. It is rash, on account of the great gaps that still exist in our knowledge of immunity. In spite of the numerous data which we possess, it is as yet impossible to offer a coherent whole or an harmonious and complete system; many of the facts which we have, cannot, as yet, be classed according either to relations or consequences. Anyone who should attempt at the present day to penetrate the mystery which shrouds the numerous problems of immunity by reasoning alone would be sure to fall into error. Immunity, like other biological sciences, does not permit overgeneralizing and adventurous theories. Immunity is scarcely in a shape to permit profitable deduction, since deduction in its endeavor to penetrate too rapidly and deeply into the unknown, loses immediate contact with established facts. Are not discoveries frequently unexpected? Do not experimenters find that their researches overthrow expectations which seem quite reasonable, and is it not evident that logic totters 
when it would force facts? What is perhaps the most obscure and important question with which immunity confronts us? Evidently the question of the specificity of immunization as shown in the specificity of sera. The solution of this question, however, is probably wholly unsuspected. Anyone who should attempt to furnish a solution at the present moment, or to give a distinctive explanation as a result of reasoning on the subject, would necessarily be led to support it by undemonstrated facts. It is better, then, to seek for the truth without wishing to define it before we have found it.

My objection to too generalized conceptions do not in the least prevent me from recognizing the ingenuity and the genius of those who, like my esteemed colleague Ehrlich, have proposed a general interpretation of specificity. Ehrlich's theory has influenced too many minds, has become too generally known on account of the well merited reputation of its author, not to deserve thorough consideration. My own impression of the theory has not been favorable, and it has seemed my duty to combat it. Its principal fault to my thinking is that it is not, strictly speaking, a theory, but rather an assertion of a certain number of undemonstrated facts. According to Ehrlich, antibodies are produced as follows: in the first place the antigen when introduced into the animal body meets with a substance with which it unites. So far we all agree. It is quite certain that foreign substances which lead to the formation of antibodies are taken up by the tissues and produce a reaction with certain substances in the body to which, for convenience, the name of receptors may be given. The body restores these destroyed receptors by producing new ones identical with the original. But, according to Ehrlich, these new receptors are over-produced to such an extent that they flow over into the fluid of the body, retain their essential property of uniting with the antigen, and are then to be designated as the antibodies of the serum. In short, the antibody is identical with the receptor affected by the antigen. To draw such a conclusion is, however, to affirm a fact that has never been demonstrated. Wholly different hypotheses might as legitimately be offered. We might suppose, for instance, that the body of the animal that is being immunized, instead of reproducing old receptors in large amount without changing them, builds up substances 
which in their character and cellular appearance resemble but are not completely identical with pre-existent principles. These new substances, as a result of elaboration and change, have become endowed with special properties, notably a more marked affinity for the specific antigen in question. In view of the wonderful faculty of adaptation of organisms and their inexhaustible resources, in view, for example, of what one sees (as Landsteiner and Reich have done) in Oscillaria that have been submitted to the influence of certain luminous rays and which take specifically the complementary color to that possessed by these rays (Engelman and Gaidukow), such an hypothesis would be in no way irrational. Both conceptions, then, are a priori equally possible to defend. Why should we choose one of them and condemn the other before awaiting the result of experimentation? Such solutions given prematurely to obscure problems are all the more useless, as with all their apparent detail they fail to answer definite important questions. In fact these over-produced receptors are but vague names. Where are they to be found? Are they, before immunization, enclosed within cell protoplasm or do they swim freely in the blood? More exactly, we are aware that in the blood of normal animals there are normal antibodies, the protecting power of which is in general slight but nevertheless detectable, such substances as agglutinins, sensitizers and even antitoxins. It is just as logical to suppose that bacteria or poisons, when injected into a normal animal, react with these normal antibodies. Are these normal antibodies really the same receptors which are subsequently overproduced to form the specific antibody that is characteristic of the immunity that has been acquired? Or are the receptors in whom this function resides other than the normal antibodies which are present in the blood; should we, in other words, seek for them in the protoplasm of the nerve cell, for instance, when dealing with diphtheria toxin? Ehrlich's theory, to be sure, harmonizes with either alternative, but it would scarcely seem that it is ever indicated which of the two should be accepted.

In certain other respects Ehrlich's theory has apparently been more precise. It attempted, at a stage when the data were still very limited, to interpret the mode of action of antibodies with antigens, here it seems to me that it has exercised a perturbing influence on 
the progress of knowledge, and has really hindered the free development of investigation. In offering explanations which seem definitive, and schemata which satisfy the experimenter and appease his curiosity, Ehrlich's theory has come to make certain problems, which have scarcely been touched upon, regarded as worked out. It must be admitted that its partisans have seemed chiefly preoccupied with justifying it, and it would seem as if their efforts were directed rather toward defending the theory than controlling it. And the guiding thought, to my thinking fatal, which they have endeavored to enforce, is the constant attribution to the molecule of the antibody of separate atom groups for each of the phenomena to which the antibody gives rise. To each manifestation that has been observed there must be a corresponding molecular group or even a new molecule. Such a method is favorable to the theory, inasmuch as each influence as noted finds material evidence in a special group which becomes a sort of sub-stratum for it. Its use, however, is doubtful for the same reason, for it gives too easily the appearance of an explanation, when in reality it establishes no definite relation between the phenomena that have been observed, since it satisfies itself in symbolizing these manifestations by independent groups, each of which appears as a resting place for such and such a property. These groups are simply evoked by the theorist as he wishes and their very existence in each and every case is far from sure. When we say that an agglutinin which unites with bacteria and clumps them produces these results because it possesses two groups in its molecule, one of which combines and the other of which agglutinates, it facilitates explanation, and seems, indeed, and in this its danger lies, to resolve the question entirely, but it is probably inexact. In the first place, it is not, strictly speaking, the agglutinin which agglutinates, but rather, as I showed in 1899, the salt. There are antigens which, when united with their antibodies, form complexes that have the characteristic of being flocculable by electrolytes.* It is the complex which agglutinates and there is no reason to localize the cause of agglutination in a

* In this respect there are analogies between bacteria laden with agglutinin and colloidal complexes, not only in respect to the action of salts but also to the influence of electricity. According to Neisser and Friedemann, the complex mastic-gelatin is flocculable by the electric current just as bacteria that have fixed agglutinin are. 
molecule of the antibody rather than in one of the antigen. The hypothesis of a functional group in the molecule of the agglutinin is all the more doubtful inasmuch as it is not the only substance which can render bacteria sensitive to the flocculating action of salts. Bacteria that have absorbed iron, uranium, or aluminium compounds are subsequently flocculable by salts (Neisser and Friedemann, Bechhold, Gengou); silicic acid is similar in its action (Landsteiner and Jagic).

It has been found that agglutinins when heated may keep the property of uniting with bacteria although they lose the property of agglutinating them (Michaelis, Eisenberg and Volk, Bail). And to explain this fact it has been said that under these conditions the agglutinin loses its agglutinating group but keeps its combining group.* Certain bacteria act in the same way as agglutinins. The typhoid bacillus, when heated to 80 degrees, still fixes agglutinin but is not agglutinable (Weil). $\dagger$ An aqueous solution of agar, so diluted as to be only slightly viscuous at room temperature, agglutinates barium sulphate suspended in water. Heating such a solution destroys this property without affecting the adsorbing property: under these conditions it produces the opposite effect, namely, disseminates the particles of barium and gives a milky appearance to the fluid (Gengou). Can we say here that by heating this solution we have caused it to lose its agglutinating molecular group? As Porges has already stated, the hypothesis of the existence of such a group in the antibody molecule, has no foundation; he found on studying the effect of heat on the agglutinating power of the albuminous substances of serum for mastic emulsions, that he could obtain entirely similar results to those that have been noted for agglutinins and bacteria. $\ddagger$ Any change in the physical properties of the constituents of the complex, agglutinin-bacterium, and particularly as regards the degree of colloidal stability, may influence the state of equilibrium of this complex in respect to the surrounding fluid, and on this state of equilibrium the phenomenon of agglu-

* It is well known that precipitins give similar results (Kraus and von Pirquet).

$\dagger$ This same author has shown that B. typhosus, which under normal conditions is feebly agglutinated by gelatin, loses this property when heated to 80 degrees.

$\ddagger$ Neisser and Friedemann and Bechhold have contributed interesting results on the effect of gelatin, serum and the like, on the agglutination of mastic by electrolytes. 
tination depends. In short, the essential phenomenon with agglutinins, as with other active substances in sera, is its union with the antigen; as far as the agglutination itself, which follows this union, is concerned, it is only a secondary phenomenon on which we cannot depend in considering agglutinins as functionally different in molecular structure from the other antibodies. Such remarks on agglutinins also apply to precipitins or the sensitizers which Ehrlich endows with a complementophilic group. It is particularly because this investigator's theory suggested an artificial classification of the antibodies, that it appears to me to have exerted a harmful influence on scientific progress.

Everyone agrees, naturally, that the numerous antibodies which the study of immunity has brought to our knowledge and which are active on such different elements as bacteria, cells, toxins, and the like, should not be considered as identical, inasmuch as they may be distinguished as regards specificity, or, in other words, since they unite with different antigens. But in addition to this incontestable difference of specificity, Ehrlich has imagined another one which is more far reaching and which deals with the molecular structure of the antibody. Indeed, he classes these antibodies in accordance with their molecular structure into three genera; antitoxins with a single combining group; agglutinins and precipitins with a single combining group but with an additional functional group which brings about agglutination or precipitation; and finally sensitizers which have two combining groups in their molecule, uniting on the one hand with the cell that is affected and on the other with the alexin (complement), and hence the name of amboceptor.

In every instance according to this classification the phenomena observed are attributed to special properties in the antibody and never to those in the antigen. As a matter of fact, these phenomena should be related, not as regards antigen or antibody considered separately, but as regards the complexes which result from their union, and it is evident that the special properties of the antigen must affect markedly and perhaps to a preponderating degree, the qualities of such complexes. I have just mentioned this idea as applied to agglutination, and I have frequently defended it in respect to sensitizers. The sensitizer, on uniting with the cell 
which it affects, forms with it a complex endowed with avidity for alexin, a complex which, in other terms, manifests properties of adsorption which neither of its constituents alone possesses. Just as the union of agglutinins with bacteria produces in them a remarkable sensitivity to the agglutinating effect of electrolytes by modifying their property of molecular adhesion, in a similar way sensitizers confer on their antigens a similar modified property of adhesion, namely, alexin adsorption. Such, indeed, is my conception of sensitization, and I shall later return briefly to a discussion of it in considering objections that have been raised to it.

Nothing authorizes us to separate the antibodies with which we have been dealing by such sharp damarcations as to attribute to them such different constitutions or as to suppose that the cause of all these observed manifestations is due to changes in their molecule, without paying any attention to the nature of the antigen. Antibodies of widely divergent appearance present, then, marked relationship to one another, and the separations that have been raised by classifying them in accordance with hypotheses dealing with the general structure of their molecule, are imaginary. In my opinion, antibodies, whatever their nature, act very much alike; but the effects that they produce differ with the antigen in question and the characteristics which, on account of its own nature, it can produce as soon as it unites with the appropriate antibody. It is evident that this point of view, in addition to being better in harmony with fact, presents also the marked advantage of producing a greater unity in our conception of the properties of sera, since it does not necessarily recognize various families of antibodies, but limits itself to noting the infinite variety of the antigens.

$$
\text { *** }
$$

It is not for me to defend my work, which is here offered in its entirety to the judgment of the scientific world. But I may be permitted to characterize my method of research by saying that I have yielded as little as possible to the inspiration of theory; and for this reason, moreover, no general conception of obscure questions will be found in the present article. Like every other observer, I have offered certain hypotheses, but they scarcely merit this name, for they are so little removed from the facts observed; they are rather a transcription of impressions gathered from the results of labora- 
tory experimentation. At the risk of being considered by some readers as not possessing a sufficiently generalizing mind, I must admit that I have been led to make my most important discoveries by yielding tractably to the impulse of facts, by letting myself be moved by my data without attempting to discipline them or subject them to systematic ideas of my own. This is quite evident to one who follows the evolution of my researches. For example, I have frequently been asked how I came to discover the law of bacteriolysis (that is to say the collaboration of two substances, alexin and sensitizer), or how, following that deduction, I had the idea of immunizing animals against the blood of an alien species or against milk (hemolytic sera and precipitins for albuminous substances), which enabled me to demonstrate the idea that the organism, by employing the same mechanism and by the same proceedings, immunizes itself against elements that differ markedly from bacteria; that is to say, the production of antibacterial antibodies simply represents the application, in the struggle against infective agents, of a faculty which the animal would have possessed even if contagious disease had not existed. I may be permitted then to note how these researches have been related to one another, and it will be evident that no guiding theory has been necessary.

Pfeiffer had just discovered lysis of the cholera vibrio in the peritoneum of guinea-pigs, and Metchnikoff had shown that, contrary to Pfeiffer's opinion, this phenomenon occurs in vitro as well as in the living animal; all that was necessary was to mix a little of the peritoneal exudate from a normal guinea-pig with cholera vibrios to which a trace of cholera serum had been added. I wondered whether the exudate could be replaced by fresh defibrinated blood from a normal animal. I found that it could; vibrios mixed with it in the presence of cholera serum showed granular transformation. The question then was whether it was the cells or the serum in the defibrinated blood which produce this effect, and experimentally I found that the serum was the important substance and that neither red nor white corpuscles were necessary. I could definitely discard the idea of cellular participation. But why was fresh normal serum necessary? The cholera serum, as we then employed it in experiments, usually came from a stock that had been kept for some time, or, as frequently happened, had been heated to 60 degrees. 
It was quite reasonable to presume that freshly obtained, unheated cholera serum should act both as an immune serum and as fresh normal serum; and this, indeed, was what happened. Under these conditions it alone produced metamorphosis of the cholera vibrio. When heated to 55 degrees it lost its bacteriolytic power, but recovered it on the addition of fresh normal serum. Such was the demonstration of the two substances, the collaboration of which is necessary to produce bacteriolysis, - the sensitizer or preventive substance, thermostable, specific, and characteristic of immune serum, and the alexin destroyed at 55 degrees, - non-specific, and present in practically the same amount in the sera of normal and of vaccinated animals, as may be shown by proper mensuration. While carrying out these experiments (1895), it was noted that cholera serum immobilizes and agglutinates cholera vibrios in clumps; this was the first instance of agglutination of a bacterial culture on the addition of a small dose of immune serum. It was further found that this agglutination would still occur, even with cholera serum that had been heated to 60 or 65 degrees, from which fact it was evident that the agglutinating property is separate from the bactericidal property. It became obvious at once that one might use this test which is so easy to perform in vitro, in the diagnosis of the cholera vibrio, instead of the in vivo method which Pfeiffer discovered. This marked the introduction in bacteriology of serum diagnosis in vitro.

As may be seen, the idea of a participation of two substances in bacteriolysis is not, properly speaking, a theory, for there is nothing hypothetical about it; it is simply a literal translation of observed facts, particularly the experiments of "reactivation" which have just been mentioned. Following is an account of the discovery of hemolytic sera:

In my experiments in bacteriolysis I frequently used as an immune serum, the serum of a goat that had been immunized against the cholera vibrio, and as alexin (complement), fresh normal guineapig serum. It frequently happened that the latter contained a certain number of red blond cells and I found that these were agglutinated and would be agglutinated even when mixed with normal goat serum. I had, moreover, already noted that neither motility nor vitality was a necessary condition for the agglutination of bacteria; cholera serum agglutinates vibrios that have been killed by 
chloroform very well. Since the bacteria then are passive agents, red blood cells may be similarly considered. I had further noted (and this fact was likewise mentioned at about the same time by Gruber and Durham), that normal sera (and particularly certain of them such as horse serum), frequently show an agglutinating property for various bacteria (for example, V. cholerae, B. typhosus, B. tetani). This constitutes a second relation between bacteria and red blood cells, inasmuch as the latter are also frequently agglutinated by normal sera of alien species. It then occurred to me, naturally enough, that if immunization against bacteria increases the agglutinating property toward a given organism to a considerable extent over that in the normal animal, we might hope to obtain a similar result with red blood cells. By immunizing an animal of species A with the blood of species B, we should obtain in animal A, a powerful agglutinin for the corpuscles of $\mathrm{B}$. With this purpose in mind I vaccinated guinea-pigs against rabbit blood. When I saw that specific hemolysis also occurred under these conditions, the analogy of which with bacteriolysis was most obvious, I had simply to repeat the experiments that I had already performed with cholera serum and cholera vibrios with hemolytic sera and corpuscles. The law of two substances was completely verified, and its significance and importance became more evident.

My researches on "lacto serum," that is to say an immune serum which precipitates milk, as well as the ideas which I obtained concerning, first, the mechanism of agglutination, and later, the nature of the reactions between antibodies and antigens, which, according to my idea, are probably to be classed in a category of adsorption phenomena, also began quite independently of any abstract conception. On examining test tubes which contained bacteria agglutinated by sera, I had been struck with the resemblance between their appearance and the flocking out of chemical precipitates, particularly of the insoluble salts coming from a double decomposition. It is a current notion that flocculation is favored by electrolytes, and the influence of the latter had been particularly studied in respect to the sedimentation of clay and the agglutination of emulsions of mastic. A study as to whether the agglutination of bacteria also necessitated the presence of salts was obvious, and as we know, such a necessity was experimentally proved. It was found that bacteria 
which had been affected by the agglutinin, act subsequently precisely like inert chemical particles. In other words, bacteria, on uniting with the agglutinin, give a coagulum which is flocculable by electrolytes. But could we not go further and say that any easily coagulable substance will give the same results? Should we not obtain an agglutinating serum following the injection, not of formed elements like bacteria, but of particles of amorphous organic substances? Milk casein, which occurs in a colloidal condition in the form of extremely minute particles, is suitable to prove this point. Animals that were immunized against milk gave a serum which precipitated casein and consequently agreed with the observations which Tchistowitch had just made on the formation of a precipitate on adding eel serum to its antitoxin.

The flocculation of milk casein by the corresponding immune serum established a new connection between the phenomena of agglutination and coagulation which Duclaux had previously asserted to be closely related. Adsorption of the agglutinin modifies bacteria in respect to their properties of adhesion with the surrounding fluid, and this modification is evident by their susceptibility to electrolytes. But how does the agglutinin unite? We might conceive of a single molecular contact, of an adsorption of the antibody by the antigen similar to the adsorption of anilin dye by filter paper. I tested antibodies from this standpoint, and in particular did the experiment which demonstrated that a given dose of hemolytic serum destroys variable amounts of corpuscles in accordance with their addition in a single or in several doses. It would take me too far afield to discuss this experiment in greater detail, an experiment which, as we know, has been employed by various investigators with similar results, particularly in dealing with antitoxins. Such are the purely experimental results which first suggested to me the ideas that I have since defended concerning the mode of action of antibodies, without being guided by any theoretical leaning in one direction more than another.

When we have hemolytic sera which are evidently toxic, it is quite natural to attempt to obtain antitoxins to them; the effect of such an antiserum on the hemolytic serum can then be split up into several factors and it is found to contain both an antisensitizer and an anti-alexin. From another standpoint, inasmuch as the alexin 
either hemolyzes or bacteriolyzes, it is necessary to determine whether it is used up in its action, and whether the surrounding fluid becomes inactive for new sensitized cells, whether corpuscles or bacteria. By such experiments the fixation of the alexin, and at the same time its functional unity, were determined - but here let me stop in this review of the evolution of my researches. It is quite useless to go further, as my single desire has been to show that it is not I, myself, who has created or even chosen my ideas; they have rather been forced upon me by facts, by the logical induction which, so to speak, is the inevitable result of experimentation, and by the immediate deductions from it. I have limited myself to being an experimenter and very little of a theorist, and the accuracy of the opinions which I have defended is, I think, best guaranteed by this fact. Certain of my conclusions which have been attacked for a long time are now beginning to gain the approbation of investigators. Such, for example, is the idea of a functional unity of alexin (complement), and the idea that sensitizers possess no complementophilic group but form a complex with the antigen which manifests adsorption properties for alexin. It would seem well, perhaps, to consider briefly the present status of these two subjects of discussion, and to sum up the arguments for and against them.

\section{$*^{*} *$}

Alexins derived from animals of different species are not all endowed with the same properties; they are particularly to be distinguished in that they are not all equally toxic and equally apt to produce hemolysis and bacteriolysis. I do not need to insist further on this point, inasmuch as it has been accepted by nearly all observers. The researches of Streng, which were done at our Pasteur Institute, demonstrated the real existence of anti-alexins which had been contested by certain authors, and still further showed the varying toxicity of different alexins.

But is there a single alexin in a given serum? Without citing in detail all the arguments in favor of it which have been offered in my articles, I wish again to insist that this problem should be considered exclusively from the standpoint of function. Our knowledge of the constitution of the alexin is too obscure to permit our determining whether the alexin in a given serum is chemically one or more substances. The important point to know is 
whether a given alexin reacts with various cells such as bacteria and corpuscles. In 1895 I thought to outline the process of immunization as follows:

"The bactericidal substance (alexin) is the same whatever be the bacterium in question. In animals vaccinated against certain infections the energy of the bactericidal substance is more markedly evident against a particular bacterium, owing to the effect of the preventive substance (sensitizer) which varies in each case and the nature of which depends on the micro-organism used for immunization. It is owing to the intervention of this particular substance, the specific antibody, that the animal body directs its destructive power against a particular infective agent."

Is this statement still accurate in spite of the affirmations to the contrary as regards a multiplicity of alexins which have come from Ehrlich in particular? At that time I expressed in a definite manner the opinion which was later confirmed by a study of hemolytic sera, that the animal body does not contain a series of alexins, some of which act in the presence of certain sensitizers and are thus employed in struggling against certain bacteria, while others are better adapted to work with other sensitizers to combat other bacteria. On the contrary it is the same weapon in each instance, a single alexin which reacts now against one and now against another bacterium, owing to the specificity of the sensitizer. Several of my articles, particularly the one on cytolytic sera in 1901, gave the experimental foundation for this conception, and I may content myself simply with recalling them.

If this conception were incorrect, if, for example, the hemolytic alexin differed from the bacteriolytic alexin, how would Gengou and I have been able to devise an invariable method for demonstrating anti-bacterial sensitizers, namely, the fixation of the alexin by sensitized bacteria, as shown by using sensitized red blood cells as a reagent? Would this method have been universally accepted as it has been? Should we have been able to use it as an argument in favor of the authenticity of our organism of whooping cough? If the conception, which is the basis of this method, were false, what confidence would remain in the serum diagnosis of syphilis? Notwithstanding, the contrary thesis of a multiplicity of alexins in a given serum met with the support of many investigators a few years ago. 
I believe this is owing to the fact that in interpreting experiments insufficient attention was paid to two essential points, the first of which is the antagonistic power of serum, and the other that a given alexin does not destroy all cells with equal facility. Gay and I have given over an entire article to the study of the antagonistic property of serum, and have called attention to the fact (which was likewise brought out by the researches of Muir and Browning in the same direction) that blood corpuscles, particularly. when feebly sensitized, may remove only a small amount of the alexin from a surrounding fluid, even when they are present in large numbers. The same idea applies, of course, to bacteria. Such a fact destroys all the value of the so-called "elective complement absorption method." The fact that in the presence of a sensitized cell a certain portion of the alexin may remain free in the surrounding fluid does not in any way prove that this non-absorbed alexin differs from that which has been fixed. This remark also applies to experiments in which weakly sensitizing sera are used (normal sera for example), experiments that have been frequently used to demonstrate a functional multiplicity of the alexin.

In the second place, when we note that a small dose of a given alexic serum suffices to destroy sensitized corpuscles of species A, whereas a larger amount of the same serum is necessary to destroy sensitized corpuscles B, we are not authorized to conclude that two different alexins are present, each one appropriate for only one of these two species of corpuscles, and that one of these alexins exists in larger amount than the other. In accordance with the work of Muir and Browning, and of Gay especially, we must conclude that there is only one alexin, but that corpuscles differ in the amount of this substance which they require for hemolysis.

It is further to be noted that the partisans of a functional multiplicity of the alexin have since modified their original conception to a great extent. They now admit that various amboceptors from a given animal species have a uniform structure in so far as the complementophilic group is concerned, which is equivalent to saying that they absorb the same alexin. If we are to admit, then, that various hemolytic or bacteriolytic sensitizers produce fixation of the same alexin on various cells which they affect, we evidently admit that these alexins may be used indifferently in hemolysis and bacterioly- 
sis, in other words, we admit the unity of the alexin at least functionally, if not chemically. In conclusion, then, I think that the opinion which I expressed in 1895 on the mechanism of acquired immunity in accordance with which the animal body would seem to direct the same weapon, the same alexin against various foreign cells, owing to the presence of a specific sensitizer, is gradually receiving the united agreement of scientists.

In so far as concerns the mode of fixation of alexin, the divergent opinions of Ehrlich and Morgenroth, and myself, are well known. According to these authors, the sensitizers have a complementophilic group. I have already previously mentioned my own opinion; I think that avidity for alexin logically resembles agglutination or sensitivity to the flocculating action of salts. Taken alone, neither the bacteria, or at least the great majority of them, nor the agglutinin is affected by salt, but the complex which they form is agglutinable by this electrolyte. In the same way neither the amboceptor nor the antigen in question has alone any manifest affinity for the alexin, but they form by their union a complex which can absorb alexin, in other words, which has particular properties of adhesion. As a result, as I have already noted, there is no fundamental difference between sensitizers and such other antibodies as antitoxins. There is no such thing as an amboceptor; all antibodies on the contrary are "uniceptors." There exist, however, antigens which, by uniting with the suitable antibody, give complexes which can adsorb alexin, whereas other antigens have no such property. I do not think it is necessary for me to return to the experiments that $I$ have described in my articles which show that there is no means of determining any direct affinity of sensitizers for alexin in absence of the antigen, and consequently there is no reason for admitting the existence of a complementophilic group.* Other experimenters have brought out similar facts which lead to the same conclusions. Thus, according to Frouin and Muir and Browning, on passing serum through certain filters it is found that the sensitizers pass through whereas the alexin is retained.

* This is true, not only as regards sensitizers that act on corpuscles or bacteria, but also, as Gengou has shown, as regards those that affect albuminoid substances; in this case also fixation of alexin takes place only when both the antibody and the antigen are present. 
In a similar manner these same English authors have recently found that sensitizers filter equally well whether mixed or not with a strong dose of complement, which latter substance, as already mentioned, does not succeed in getting through. Ehrlich and Morgenroth, to be sure, had already made similar observations which corroborate this idea that the alexin and the sensitizer exist side by side in serum without being combined. I established that participation of the antigen is always necessary in alexin absorption. Partisans of the complementophilic group now admit that such a conception is true in at least the majority of cases; they say, in fact, that the energy of affinity of the complementophilic group is frequently increased as a result of a union of the sensitizer with the antigen. This amounts essentially to accepting the notion, to my opinion the only important one, that fixation of alexin is subsequent to the formation of the complex, antibody-antigen. And what is more, the hypothesis of a complementophilic group is in distinct disagreement with certain of Muir's experiments. This author found that blood corpuscles that had fixed the sensitizer and had been saturated with alexin could subsequently, by diffusion, lose a certain amount of their sensitizer, although they retain the complement, and what is. more, in this instance they lose as much sensitizer as if they had not absorbed complement. Consequently it is in no way through the mediation of the sensitizer that the alexin attaches itself to the corpuscles; if this were the case the removal of the sensitizer would necessarily imply that of the alexin, which, as we have just mentioned, does not leave the corpuscles.

It is clear that if it had been proved that the sensitizer fixes the alexin without the presence of the antigen in even a single instance, the idea of a complementophilic group should be accepted. Ehrlich and Sachs, as we know, thought that they had found this decisive example in dealing with the hemolysis of guinea-pig corpuscles by a mixture of heated bovine serum and fresh horse serum. But the researches I carried out with Gay are also known, researches which I have subsequently continued and in accordance with which Ehrlich and Sachs' interpretation is completely inaccurate. These researches have been criticised by Sachs and Bauer, which accounts for my reconsideration of the subject in collaboration with Dr. Oswald Streng. Streng and I were able to prove that Sachs and 
Bauer's objection is without foundation, and we have defined still further the properties of that remarkable substance which we formerly called bovine colloid but which we now call conglutinin, the intervention of which explains the peculiarities of this particular instance of hemolysis. Without going into details as to this latter work (which may be found in the Centralblatt für Bakteriologie, Vol. 49, second Heft), I may content myself with noting that, contrary to Ehrlich and Sachs' opinion, the bovine amboceptor is in no way abnormal but agrees with the general law, which is that the sensitizer does not act alone in absorbing alexin, but only the complex, corpuscle-sensitizer. Streng has since continued the study of bovine serum (see Centralblatt für Bakteriologie, 1909). This author has made some very interesting observations by employing with the bovine serum, bacteria instead of corpuscles, which, in so far as the theory of the action of serum is concerned, agree perfectly with the experiments on corpuscles which we had performed. My studies with Gay on the antagonistic power of serum, and Gengou's numerous experiments on the phenomenon of adsorption, make more evident the idea that fixation of alexin is in reality an adsorption phenomenon. The striking analogies so far as the action of the citrate of sodium is concerned, between the fixation of alexin or other hemolysis on the one hand and the adsorption of certain chemical precipitates on the other, are very suggestive and it is unnecessary to insist on them further. Which of the arguments that have been offered in favor of a complementophilic group of the sensitizer still remain? In the first place the existence of complementoids. On heating certain alexins very carefully (for example toward 52 degrees), we find that their hemolytic energy is much decreased; but alexins that have been altered in this manner can still be absorbed by sensitized corpuscles. As would be naturally expected, such corpuscles charged with complementoids, that is to say an altered, or perhaps better, affected, alexin are thenceforth incapable of fixing active alexin for the simple reason that their capacity for alexin adsorption is not without limits. These corpuscles, having been saturated with weakened alexin, subsequently refuse the hemolytic alexin and consequently remain intact. Gay has carefully explained this phenomenon in his article on complementoids. What relation indeed have these 
substances with the theory of a complementophilic group? If it had been found that the sensitizer could absorb complements without the presence of antigen, the theory would have been fortified, but no such thing happens. As with intact alexin, so alexin that has been altered by a graduated temperature is absorbed, not by the sensitizer alone, but only by the complex, corpuscle-sensitizer; in other words, the general law holds.

As regards the arguments furnished by a study of cobra venom and lecithin, there is nothing to prove that they are applicable to sensitizers and alexin. The researches of Kyes and of Neisser and Friedemann have shown clearly that the venom, although in itself unable to destroy certain corpuscles, does hemolyze them when added to lecithin; in other words, the complex, venom-lecithin is remarkably active. But what reason is there to suppose that sensitizer and alexin necessarily act as do venom and lecithin? There would seem, on the contrary, no parallel between them. The union of venom and lecithin, which may perfectly well be a simple adsorption phenomenon, is easily demonstrable experimentally, whereas no such union between sensitizer and alexin is evident. What is more, corpuscles that resist the action of venom alone but are hemolyzed by the complex of lecithin and venom, are unable to fix venom when lecithin is not present, whereas we have seen that the sensitizer in hemolytic serum can always be absorbed by the corpuscles in the absence of alexin.

Experiments on antisensitizers, and particularly those which I published in 1904, have been made use of by Ehrlich and Sachs as favoring the existence of a complementophilic group. I found that sensitized corpuscles, when mixed with a suitable antisensitizer, lose their power of absorbing alexin; in other words, the antisensitizer really cures the corpuscles since it annuls the effect that the sensitizer has produced. From this observation Ehrlich and Sachs concluded that the antisensitizer unites with a complementophilic group of the amboceptor and consequently satisfies its affinities. According to these authors this interpretation is the only one which is compatible with the fact that the antisensitizer drives out the sensitizer from the corpuscle. It is, indeed, true, as I noted in my article, that the antisensitizer unites with the corpuscle-sensitizer complex but does not eliminate the latter substance. It simply 
causes the complex to lose its affinity for alexin. Adsorption phenomena frequently show similar precipitations of various substances on one another; for example, if we wash a rather thick suspension of barium sulphate over a firm paraffin surface, we find that the paraffin becomes covered with a delicate white layer of sulphate which adheres and resists washing. Let us now place a suspension of red blood cells on such a paraffin surface that has been covered with barium sulphate, and then wash with salt solution: we find the corpuscles adhere to the sulphate with which the paraffin is covered; the surface gradually takes a reddish color which resists washing in salt solution. The paraffin adsorbs the sulphate which in its turn adsorbs the corpuscles.* It is evident that in such an instance the phenomenon may be expressed by saying that the sulphate functions as amboceptor between the paraffin and the corpuscle.

But are we always obliged to conclude that when a property becomes manifest or disappears it is owing to the integrity of or an alteration in a definite atom group? When a sensitized corpuscle that has been disintoxicated by antisensitizer fails to fix alexin, must we suppose that the complementophilic group of the sensitizer is thenceforth saturated? Such an interpretation, in truth, cannot be reconciled with fact; experiment shows us that a given antisensitizer, for example the one furnished by guinea-pigs immunized against rabbit serum, neutralizes all the sensitizers from the same animal species (in this case the rabbit), but has no effect on sensitizers from other animal species. Studies on the fixation of alexin show that the sensitizers of different animals make use of the same alexin in bringing about either hemolysis or bacteriolysis; if we were to use Ehrlich's terminology we should be forced consequently to conclude that they have identical complementophilic groups. According to Ehrlich's interpretation they should all be neutralized by the same antisensitizer, and such is not the case. We find, moreover, that sensitized corpuscles treated by a suitable antiserum subsequently resist, whatever alexin be employed. And what is more, the phenomenon of alexin fixation appears more and more, as has been evidenced in the preceding pages, to be an adsorp-

* Gengou's experiments, as is well known, have shown that barium sulphate possesses the property of precipitating itself on red blood cells and forming clumps with them. 
tion phenomenon which does not depend on a strictly defined atom group. It is probable that the properties of molecular adhesion in a corpuscle-sensitizer complex may be modified by the addition of a third element, the antisensitizer, and that the tendency for alexin adsorption will disappear correlatively. In the same way the tendency of barium sulphate to fix itself on corpuscles disappears when the particles of this substance have been previously adsorbed by gum, by certain albuminoids, or by sodium citrate.

And finally the recent researches of Dr. Oswald Streng have shown that the anti-alexins act in the same way as the antisensitizers. Horse alexin becomes fixed easily on guinea-pigs' corpuscles, but inasmuch as it is only slightly hemolytic it does not destroy them. These corpuscles, which have fixed alexin, are henceforth capable of giving a reaction with the conglutinin of bovine serum; when mixed with this serum (previously heated to 56 degrees) they are energetically agglutinated. If we take, as Sachs did, guinea-pig corpuscles laden with horse alexin, and treat them with anti-alexin, that is to say, heated serum from a rabbit immunized against horse serum, and then test them with bovine serum, we obtain no conglutinin reaction. The anti-alexin, then, has neutralized the alexin which has been fixed to the corpuscles and has cured them by causing the property which the alexin produced, namely, that of adsorbing conglutinin, to disappear. And yet the alexin that has been fixed with corpuscles has no free group, and so far as it is concerned no explanation similar to the one proposed for sensitizer and antisensitizer by Ehrlich and Sachs can be accepted.

$$
*^{*} *
$$

Inasmuch as we have no explanation of specificity we must put aside for the moment the question as to why such and such an antibody unites with such and such an antigen. But even if the underlying reason for this combination escapes us, we can at least endeavor to determine how this combination is brought about, that is to say, can trace its development. Attempts, therefore, have been made to define the characters of the reaction and to determine in what category of phenomena it belongs. The reader doubtless knows the opinions which have been ofered on this subject. Certain authors thought that this reaction should be likened to chemical phenomena, properly speaking, as they exist either between substances endowed 
with an energetic affinity for one another, in which case the reaction is complete and gives rise to a relatively stable compound (Ehrlich), or between substances with weak affinities, in which case the combination is only partial and distinctly reversible; a state of equilibrium in other words would be established between the fraction of the antigen which remains free and the one which enters into combination (Arrhenius and Madsen). According to other authors, and I believe I was the first to offer this opinion, the union of the antibody with the antigen depends on what is called molecular adhesion or contact affinity, in other words should be classed in the category of adsorption phenomena.*

In this connection it is well to define the limit of the debate and avoid misunderstandings. The phenomena of adsorption are frequently contrasted with true chemical phenomena, but it is not the function of the biologist to define the frontiers of physics and chemistry. What is more, this task is no easy one, since the most authoritative physical chemists, as Van Bemmelen, Nernst and many others, themselves consider that there are all transitions between data of adsorption and those of true chemical combination. It is, then, superfluous for me to defend myself again, as I have already done in one of my articles, against criticism that has been raised against me on the ground that I deny definitely any chemical character to the union of antibody and antigen by classing them among adsorption phenomena. When in discussing the action of sera one mentions, indeed, as an example, the dyeing of a piece of filter paper by an anilin dye, it is not the purpose to find out whether

* Inasmuch as it is not possible to give a complete historical account at this time, I may confine myself to eiting what Ulrich Friedemann has published in the beginning of his remarkable work, "Ueber die Fallung von Eiweiss durch andere Kolloïde und Ihre Beziehungen zu den Immunkörperreaktionen," an historical account which deals with the first articles bearing on the relation of adsorption to immunity.

"Das Studium der Kolloîde hat bereits vielfache Aufschlusse über die physikalisch-chemischen Vorgänge bei der Immunitätsreaktionen gegeben. Die Verbindungen der Immunkörper wurden mit den Adsorptionverbindungen der Kollö̈de vergleichen (Bordet, Landsteiner und Jagic, Biltz, Zaugger, Much und Liebert, Pauli), während sich eine bemerkenswerte Ähnlichkeit zwischen den Fallungsreaktionen der Immunkörper (Agglutination und Präzipitation) und den Gelbildungen und Präzipitations-erscheinungen in Kolloïdaler Lösungen und feiner Suspensionen herausstellte. (Bordet, Bechhold, Neisser und Friedemann, Biltz, Landsteiner und Jagic, Henri und Mitarbeiter, Gengou.)" 
this dyeing is a physical or chemical phenomenon, but simply to determine whether there are analogies in the mode of reaction between the paper and the dye on the one hand, and antibody and antigen on the other.

Our knowledge of adsorption owes much to the study of colloids. Certain authors, indeed, who agree with me in recognizing the great importance of molecular adhesion in serum reactions, have become accustomed to say that the union of the antibody with the antigen represents a reaction between two colloids. It seems to me a little rash to define so clearly and I should prefer to speak of this union as an adsorption phenomenon. Adsorption, indeed, is evident, not only between two colloids but also between a colloid or suspended particles on the one hand and a substance in true solution on the other, and although it is very probable that antibodies are really colloids we cannot with certainty say as much of the antigens. It may be remarked in respect to colloids that too much importance has frequently been attached to the appearance of agglutination as an indication of the formation of a complex. The agglutination is a secondary phenomenon as Neisser and Friedemann, Bechhold, Biltz, Henri and Girard Mangin, and others have correctly stated, and its manifestation depends in large measure on certain conditions such as the relative proportions of the two substances concerned, temperature, presence of electrolytes, etc. The principal and essential fact is the affinity of adhesion produced by the union, but such a union may give rise quite as well to dissemination, that is, a more stable and perfect condition of emulsion, as to the formation of flecks; in his researches on adsorption by chemical precipitates, Gengou has insisted on this fact and shown that slight physical modifications of the substances concerned without any chemical change may give rise to dissemination instead of clumping, or the reverse. Porges has noted similar facts.

Can certain characters, such as reversibility or the rapidity of reaction, be called upon to prove whether the union of two substances belongs or not in the category of adsorption phenomena? In this respect we should note, particularly, that the affinity of adsorption varies greatly in different cases and these differences of intensity are evident by the more or less rapid unicn and the more or less stable resulting complex. As Van Bemmelen has mentioned, 
the power of adsorption varies with each adsorbing substance and each adsorbed substance. It depends, certainly, on physical qualities, but also on the chemical nature of the substances concerned, and would appear to us more variable still if we could measure by delicate methods in each instance. Certain colors act on substances like paper more rapidly than others, and the subsequent decoloration of the paper in a large volume of water is sometimes easy and sometimes difficult. In the adsorption of albuminous substances by inorganic colloids all degrees of reversibility are found. In serum reactions a similar irregularity occurs. Certain antibodies act very rapidly and others require more time to unite with their antigens and the complexes obtained are dissolved with very unequal facility. As is evident, particularly from the researches of Morgenroth, that the neutralizing of diphtheria toxin by antitoxin is much slower than was at first believed. Certain complexes, such as those formed by agglutinins or sensitizers with bacteria or corpuscles, or by certain toxins with their antitoxins, are, if not completely, at least distinctly, reversible. In this relation I may recall the work of Hahn and Tromsdorff, Landsteiner, Morgenroth, Muir, Madsen, Otto and Sachs. But it should be noted that, in general, the complexes of antibody-antigen, especially when they have been standing for some time, manifest a very imperfect reversibility, distinctly less than Arrhenius and Madsen's thesis would demand. In the same way, in general, adsorption phenomena give rise to incompletely reversible complexes. As we know the study of the neutralization of toxins by antitoxins has brought out the fact, particularly owing to the important researches of Ehrlich, that it is frequently impossible to prepare mixtures which are exactly neutral. It is likewise known, so far as diphtheria toxins and antitoxins are concerned, that Ehrlich explains this fact by supposing that the toxin contains in reality several poisons. The same phenomenon, however, occurs in instances where there is no reason for supposing that such a complexity in the toxic fluid exists. In such instances there is a priori no reason for agreeing with the thesis of Arrhenius and Madsen in accordance with which the reaction is incomplete and leaves a certain amount of the toxin free, or with my own theory in accordance with which the molecules of toxin and antitoxin may unite in variable proportions according to the respective quantities of the two 
substances in the mixture. A comparison with a process of dyeing is very apt; the molecules of the toxin would "stain" more or less deeply by the antitoxin molecules, and the complexes that result in the various instances are less toxic in proportion as they contain more antitoxin and less toxin. I brought out this point of view clearly in 1903 and discussed all the details of it so that no further insistence on the subject is necessary. It may be added that this interpretation has met with the support of various writers, among whom may be mentioned Grassberger and Schattenfroh, Biltz and Pauli, who have accepted it in its entirety.

The essential point of difference between these different conceptions evidently lies in attributing a different composition to the mixture of toxin and antitoxin and particularly to such mixtures as contain too small a dose of the antidote to bring about complete neutralization. In order to simplify it we may choose a toxic fluid which contains a single poison and add to it a relatively small amount of suitable antitoxin. In such a case are we to suppose that the mixture includes both well neutralized toxin and an excess of a free and intact toxin, or are we rather to believe, as I thought, that the antitoxin is shared by the totality of the toxin present, but saturates it only partially in the same way that a small amount of dye does a large quantity of the substance to be dyed, by spreading over the whole of it and staining it faintly? The complex thus obtained, which is weak in antitoxin, would still have certain toxic properties and would act like toxon.

But how, it may be objected, can we determine with certainty whether the toxic activity of such a mixture is due to a certain amount of intact toxin or the presence of a partially saturated complex? It is here that we must distinguish very carefully in the effects of a poison between quality and quantity. I may recall the observations which I offered in 1903 that showed that a hemolysin may manifest toxicity in two different ways; when employed in a small dose without being in any way affected by an antitoxin, it hemolyzes a small amount of corpuscles rapidly, but owing to the fact that it is not present in sufficient quantities it cannot destroy many of them. On the other hand, a large dose of the same hemolysin to which a little of its antitoxin has been added and which has been transformed thereby, according to my idea, into an 
incompletely saturated complex, can hemolyze a large amount of corpuscles but produces hemolysis very slowly, even if a very small amount of the corpuscles is added. It would seem, then, as if such a mixture included a strong dose of poison, the activity of which is distinctly depressed owing to the addition of a little antitoxin, and the result of the experiment is in no way compatible with the idea that such a mixture contains, in addition to perfectly neutralized hemolysin, a certain excess of perfectly intact hemolysin. But there is another way of proving that an antigen can unite with the antibody in variable proportions and so form complexes of varying constitution in accordance with the amounts of each substance present. It may be shown that such complexes do not react in the same way to certain agents such as heat. It is perfectly evident from the observations of Eisenberg and Volk, that the union of the agglutinin with the agglutinable substance of bacteria takes place in variable proportions. Landsteiner and Jagic have confirmed this idea by showing that the complexes obtained, which differ in the relative proportions of each constituent, show different resistance to heat.* The brilliant researches of Grassberger and Schattenfroh on the toxin of symptomatic anthrax, are very instructive in this connection. When we mix a certain dose of the toxin, either with little or with much antitoxin, we obtain complexes of toxin-antitoxin, which vary, as is shown by their varying reaction to heat. It is to be noted in the first place that the toxin in question is thermolabile, whereas the antitoxin resists heat much better. The complexes containing a large amount of antitoxin enjoy the properties of this substance, that is to say, they resist heat much better than do complexes which are weak in antitoxin; these latter mixtures are decomposed at a given temperature, and the poison is destroyed so that the antitoxin may be recovered. These authors have further shown themselves partisans of the idea that antitoxin unites with toxin in variable proportions. They have been able to frove that their poison absorbs much more antitoxin than is necessary to destroy its entire toxicity and forms a stable complex

* I noted in 1896 (Mode of action of preventive sera), that heating toward 50 degrees brings about a real disagglutination of the substanees which have been agglutinated. This is due to the fact, as Landsteiner and his collaborators have shown, that the complex is dissociated. What is more, the ease of this dissociation varies with the amount of agglutinin present. 
with it. They have also found that dilution renders neutralization much more difficult and that different mixtures are obtained depending on whether they mix the toxin and the antitoxin, after diluting them, or dilute the toxin-antitoxin mixture. This fact is evidently not in favor of the Arrhenius-Madsen theory, according to which the same state of equilibrium should exist in both instances owing to reversibility, and the same fraction of the toxin of necessity remain free.

The fact which lends significance to the researches of Grassberger and Schattenfroh, and which prevents their applying Ehrlich's interpretation, is that the toxic fluid which they employ contains in reality a single poison; there is no reason for assuming the existence of toxoids, inasmuch as the toxic power of the poison shows itself constantly parallel to its neutralizing power for antitoxin.

Among the facts which have most contributed to strengthen the idea of a union in variable proportions (an idea which is so highly compatible with the adsorption theory) and which, moreover, have shown that the complexes obtained frequently manifest only a very incomplete reversibility, there should be mentioned in particular those which have been observed by employing the method which I used for hemolysins in 1900 and which may be called "the method of addition of the antigen to the antibody in single or in divided doses." A given volume of hemolytic serum may destroy more or less corpuscles in accordance with their introduction in one or several doses separated by sufficient intervals. As we know, other authors have since performed similar experiments with the same results, employing antitoxin and toxin in place of hemolysin and corpuscles (Danysz, von Dungern, and Sachs) or using bacteria and agglutinins (Craw). It seems to me proper in this connection to mention the comparison with dyeing phenomena, and on this point I agree entirely with Craw. If a large piece of filter paper is placed in a certain volume of sufficiently diluted dye it takes a uniform shade of intensity; if, on the other hand, the same sized piece of paper is cut up in pieces and added in fragments, the first pieces are stained deeply and the last find no color left. In the same way, on adding toxin to antitoxin in divided doses the last portions of the poison cannot be neutralized as the first are supersaturated with antitoxin. When the entire mixture is made at 
once, on the contrary, the antitoxin is spread over all the toxic molecules and a complex is obtained which contains an even proportion of the antidote, and which, consequently, is not as fatal as even a small dose of free toxin.

It is an extraordinary thing that, so far as precipitins and its precipitable antigens are concerned, this interpretation is not contested, even by such writers as von Dungern, who are, in general, favorable to Ehrlich's ideas. As Halban and Landsteiner, Eisenberg, von Dungern, and Müller, in particular, have seen, if we mix a precipitable serum "A" with its appropriate precipitating serum "B," an excess of the precipitable substance inhibits the occurrence of a precipitate. For example, it is found that in a mixture of one volume of serum "A" with one volume of serum "B," a cloudiness appears, but a mixture composed of two volumes of " $\mathrm{A}$ " and one volume of "B," remains translucent. The almost obvious explanation is that the molecules of "A" are not susceptible to precipitation unless they have fixed a sufficient number of molecules of "B." Such, however, is the condition in the first mixture. In the second mixture the molecules of "B" are shared among all the molecules of "A" which are in too great a number to permit their perfect separation and consequently they are not precipitated. In accordance, then, with the proportions of constituent parts different complexes may be obtained, and this fact comes out quite clearly in the experiment of the addition in a single or in divided doses. If we add one volume of "B" to two volumes of "A," the mixture remains limpid, even after a considerable time. If, on the other hand, we add one volume of "B" to one volume of "A," a precipitate immediately appears. When the precipitate is completely formed we may add another volume of " $\mathrm{A}$ " so as to make the mixture correspond to the preceding one. The precipitate does not disappear, but remains indefinitely intact and so we have obtained two mixtures composed of the same sera in the same proportions but presenting entirely different aspects.

Does not this experiment offer most satisfactory analogies with the one on coloring filter paper with a dye to which I have just referred? The objection may be raised that, although true as far as precipitins are concerned, it may not be applicable to antitoxins. Such an objection, however, would be to deny any character of 
unity in the laws which govern the action of antibodies; it would be to distribute them into divisions, separated by impassable barriers, and I have already mentioned in speaking of Ehrlich's classification how inacceptable such a system is. Certain antigens are toxic and certain others have a greater tendency toward precipitation than others; such differences between the antigens, however, do not prevent us from supposing that their antibodies are very similar and that their action is subject to the same interpretation in the various instances.

A phenomenon which has justly attracted the attention of experimenters is the increasing stability of complexes of antibody-antigen following their formation. It would seem as if these complexes, which are easily dissociable in the beginning, subsequently become consolidated in some manner so as to resist decomposing influences better. Facts of this sort have been noted by Landsteiner and Jagic with agglutinins, and by von Dungern, Sachs, and Otto and Sachs with the antitoxins of certain poisons such as the toxin of botulismus, arachnolysin and the like. Facts of this nature, it seems to me, should be interpreted in accordance with the adsorption theory. As Nernst has noted, certain dyes as they unite more and more intimately with the object which they stain, lose correspondingly their adhesion for the surrounding fluid; when they have become fixed they show a distinct tendency to become insoluble. It seems that we may treat as an analogy the fact that various albuminous fluids treated by such agents as alcohol give precipitates which redissolve easily in water when they have been recently formed, but become more and more insoluble when kept. The adhesion which unites the albuminous molecules with one another and gives them a solid state becomes preponderating and opposes the dissemination which the contrary effect, the adhesion for water, would tend to produce. The consolidation of complexes of antibody and antigen is apparently a phenomenon of the same class.

The quantities of antibodies which antigens can fix depending on their concentration, have been determined particularly with agglutination phenomena by the important researches of Eisenberg and Volk. The greater the concentration of the antibody the more the fixation of the antigen, but the quantity fixed does not increase so 
rapidly as the concentration; the antigen loses its avidity for antibody in proportion to the amount of it that has been fixed. This, according to general opinions, is the way in which adsorption phenomena act.

It is evident from an examination of these facts that the presiding force in the union of antigens with their antibodies is the same as the one which produces the reactions that have been grouped under the name of adsorption phenomena. But is this force or affinity of adsorption sufficiently elective to permit one to attribute to it alone serum reactions, the most striking characteristic of which is their remarkable specificity? It has become more and more certain that adsorbing substances manifest toward adsorbable substances which are mixed with them, tendencies of attraction of very unequal intensity and consequently the affinity of adsorption is similar to a true chemical affinity; both, in other words, are elective. In both instances a struggle may take place between two substances for the possession of a third. In the same way fixation of one substance on another may be inhibited by a monopoly of the first by a third substance which thus protects the second. For example, the albuminous substances of blood protect red blood cells from soap (Meyer), or even against the hemolysin of eel serum (Frouin); sodium citrate protects corpuscles from the agglutinating and hemolytic effect of barium sulphate (Gengou), and so on. The lecithin which is present in bovine serum is probably united with another substance, without doubt of an albuminous nature, for if it were not so agglutination would take place in this serum itself.* In all instances a real struggle occurs between adsorption affinities; similar facts have also been found with animal charcoal. Substitutions may also be noted. Barium sulphate manifests avidity for mucin and for sodium citrate. When mixed with lecithin it clumps in large masses, and on adding sodium citrate to them they rapidly dissolve and the mucin is liberated (Gengou), in other words, the sulphate prefers citrate to the mucin.

Certain substances in the category of lipoids which may be ob-

* As we know, Toyosumi found that bovine serum has the power of agglutinating an aqueous emulsion of lecithin. Sleeswijk, and I have found that this is also true even with an extract (by means of methyl alcohol) of the lecithin from dried bovine serum. 
tained as Landsteiner, Pick and other scientists have done by extracting corpuscles or bacteria in colloidal suspension, are interesting from the standpoint of the selective action of serum. Sleeswijk and I have recently studied the action of hen serum on the lipoids extracted from red blood cells by methyl alcohol and subsequently made into the form of an emulsion in salt solution. We found, for example, that hen serum agglutinates the lipoids extracted from rabbit corpuscles energetically, but has very little effect on those from bovine corpuscles. The agglutination of an emulsion of lipoids is certainly an adsorption phenomenon. In this case adsorption affinity, of varying intensity in accordance with the substances employed, may certainly be stated as the cause of the differences observed when a given serum is mixed with different bacteria or corpuscles. And, moreover, the adhesion properties are a function not only depending on the chemical constitution but also on the physical state of the substances. It is easily conceivable, then, that the aptitude of the antigen to react with the antibody may disappear when affected by slight alterations or simple physical modifications. It is easy to understand, then, why, in Obermeyer and Pick's experiments, the serum obtained by immunizing animals against certain albuminous substances differed from the serum obtained by immunizing animals against the same substances previously heated. In brief, although an explanation of the specificity of sera is not yet clearly evident, we have at least the right to conceive of it as harmonious with the conception of adsorption affinity as the essential factor.

$$
*^{*} *
$$

This important problem of specificity must appeal to all bacteriologists and I have recently begun to study it experimentally with my collaborator, Sleeswijk. Inasmuch as our researches are not quite finished, I may content myself with mentioning certain facts. The problem of specificity may, obviously, be regarded from two different aspects. When, for example, we study, an antibacterial serum we wonder, on the one hand, why the serum is specific, and on the other hand, why the organism allows itself to be specifically affected. In other words, both the antibody and the affected element must be considered. I cannot deal here with the second half of the question. 
We know how to recognize a given bacterium by means of a specific serum. But is the character which this organism possesses of reacting with the appropriate serum constantly present? May it not disappear when the organism has been subjected to certain exigencies? What, for example, is the effect of change in culture medium? As early as 1896 Metchnikoff and I noted that the cholera vibrio as a result of certain vicissitudes, such as remaining with leucocytes, comes to resist the agglutinating effect of cholera serum markedly, and since that time many analogous instances have been collected. The remarkable work of Grassberger and Schattenfroh on symptomatic anthrax gives most interesting information on this point. These authors have found that a serum that is capable of agglutinating the organism when it is cultivated in a given medium, has scarcely any agglutinating effect on it when grown in a different medium.

We could understand this fact by supposing that the organism has, on account of certain vital reactions, succeeded in resisting the effects of the agglutinin, although it has kept its property of combining with it; in other words, we would be dealing with the simple phenomenon of adaptation to a harmful effect by the formation of a refractory condition. But as we shall see, the modification is still more profound. As a matter of fact, owing to certain conditions of life, the organism may completely lose the antigen, which combines with the antibody and which is its principal characteristic. In other words, the distinctive sign in serum diagnosis may be lost. As far as the action of the serum is concerned, the organism thenceforth acts as if it belonged to a different species, which proves distinctly that specific sera (or at least the agglutinins) do not act primarily on those substances of the bacteria which are essential to their life and characteristic of their particular species, but on certain of the accessory substances of the organism which may occur, but the existence of which is in no way part of the fixed hereditary character, which gives the living organism its particular appearance and autonomy.

The whooping-cough bacillus is very suitable for a study of this sort. We find that this organism is very variable, not only as regards the appearance of its growth, but, more important still, as regards its antigen, in accordance with the conditions under which 
it has been grown. It develops readily on the medium that is rich in defibrinated blood, as already described by Gengou and myself in our first article on whooping cough. It may be taught to grow on ordinary agar, in which instance it gives a thick and rather coherent layer. The two varieties of organisms obtained in this manner, although coming from a single original colony, give rise, on immunizing animals, to two different sera. We may consider the serum of a rabbit that has been immunized against the organism grown on ordinary agar. It is found that the serum agglutinates these organisms energetically, but has no clumping effect on a culture of whooping-cough bacillus on the other medium containing defibrinated blood. On the other hand, if we test the serum of a rabbit that has been immunized against the organism grown on blood media, we find that it agglutinates both races of bacteria. A careful study of this phenomena brings out the fact that two definite agglutinins affecting different antigens are present in different proportions. One of these antigens, which is present in large amount in the organism that has been developed on blood, is not to be found in the organisms grown on agar. To demonstrate this antigen we take a small amount of the serum and mix it with an excess of bacteria grown on agar. A few hours later we centrifugalize and decant the supernatant fluid; we find that this fluid no longer affects the same bacteria but has retained entirely its agglutinating effect for the other strain cultivated on defibrinated blood.

Horses immunized against the organism grown on blood $*$ furnish a serum with an extremely marked agglutinating property for this strain of organism, but practically no active agglutinin for the organism grown on agar. The serum, when treated with even a considerable amount of these latter organisms, even after prolonged contact, is found to have lost none of its specific effect for cultures grown on defibrinated blood. The effect on these latter cultures, then, is due to an antibody which does not find a suitable antigen in bacteria of the same species grown in a different culture medium.

The two strains that we have considered may be distinguished from other standpoints on which I shall not insist for the moment, particularly as regards their sensitivity to the antibodies of normal sera. I may add, however, that the reaction of alexin fixation

* Horse blood is used for these cultures. 
does not serve to differentiate them as clearly as agglutination does. This fact may be compared with the observations of various authors in accordance with which the reaction of fixation does not show as strict a specificity as the reaction of agglutination.

In so far as the production of antigens which are sensitive to agglutinating antibodies is concerned, we may conclude that the culture medium may be of very great importance. It may still further be shown that these two strains of organisms may be transformed into one another very rapidly by changing the culture medium. Thus, following two successive generations on blood medium, the organism that has been previously cultivated on agar recovers the faculty of producing the antigen which is characteristic of blood cultures and consequently becomes agglutinable by the serum of a horse that has been immunized against such cultures.

I cannot go further into these researches, the details of which would exceed the limits of this short summary, but the little that I have said suffices to justify experimentally the opinion which is also expressed in the work of Grassberger and Schattenfroh, that cultures of bacteria developed on culture media which differ too much from the body fluids (for example, grown on bouillon or agar sterilized in the autoclave), are not suitable, perhaps, to be employed in immunizing animals for the production of therapeutic sera. It is, perhaps, possible that bacteria grown on these two artificial culture media fail to form all the antigens which they produce in the animal body during infection and which would be affected by suitable antibodies.

\section{***}

I may conclude this brief résumé at this point. Although I have discussed in some detail the properties of sera, I have not taken up at all the essential topic of phagocytosis. The importance of this phenomenon in the defence of the animal body, which was so much combated fifteen years ago, has no need, at the present day, of emphasis. We have long since passed the time when the exact observations and the decisive experiments of my former master and present friend, Elie Metchnikoff, met with warm but often superficial opposition from those scientists who were too exclusively preoccupied by the antibacterial properties of the body fluids. Many facts which have long been known, but the significance of 
which has not been appreciated, have been confirmed and re-studied now that there is a generalizing acceptance of the value of phagocytic defense. And this is particularly the case with the numerous facts which we owe to Metchnikoff. Such is the case also with certain of the facts that have been mentioned in various articles of this volume, particularly as regards negative chemiotaxis, the phenomenon of adaptation which bacteria employ to protect themselves against phagocytosis, and the visible index of which consists in the appearance of a capsule, the manner in which leucocytes act with certain poisons, toxins, or alkaloids like quinine, and the like. The fundamental importance of phagocytosis is to-day universally admitted and is moreover evidenced by the large number of articles which deal with means of computing the intensity of this phenomenon in normal, infected, or immunized animals. 



\section{INDEX OF AUTHORITIES QUOTED.}

Afanassiew, 478.

Amberger. See Paal.

Aron, 422.

Arrhenius and Madsen, 295, 516, 518, 521.

Arthus, 425, 426, 427, 429.

Aschoff, 245.

Azalier. See de Coninck.

Bail, 238, 500.

Baratt, 397.

Bauer. See Sachs.

Bayliss, 437.

Bechhold, 422, 432, 500, 516, 517.

v. Behring, 77.

van Bemmelen, 415, 418, 422, 516, 517.

Besredka, 489 .

Biltz, 421, 516, 517, 519.

Binot, 223, 469.

Bordet, Charles. See Massart.

Bordet, 208, 241, 242, 243, 246, 248, $252,257,258,333,334,336,358$, $363,365,389,398,403,438,441$, $467,516$.

Bordet and Gay, 393, 403, 434, 442, $443,445,446,447,448,449,451$, 452, 453, 460, 461, 509, 511, 512.

Bordet and Gengou, 245, 246, 248, 346 , 398, 462, 464, 465, 467, 468, $493,508,527$.

Bordet and Sleeswijk, 524, 525 .

Bordet and Streng, 511.

Bredig, 314.

Browning. See Muir.

de Bruyn, 415, 420.

Buchner, 25, 51, 136, 153, 188, 213, 219, 235, 242, 277.

Buxton, 366.

Camus and Gley, 176, 197, 281.

Cantacuzène, 19, 35.
Canthack, 24.

Charrin and Roger, 18, 88.

Cherry. See Martin.

Cohen, 479, 483.

Cohen, E., 415.

de Coninck and Azalier, 424.

Craw, 441, 521 .

Czaplewski and Henzel, 478.

Danysz, 35, 438, 441, 521.

Dean, 397.

Dembinski, 464, 466, 468.

Denys, 25.

Denys and Leclef, 120, 123, 125, 133, 390.

Denys and Marchand, 118.

Dieudonné, 256.

Dineur, 147, 148, 150.

Douglas. See Wright.

Duclaux, 153, 154, 157, 158, 506.

Dunbar, 60, 77.

v. Dungern, 211, 441, 521, 522, 523.

Durham, 105. See also Gruber.

Dujardin-Beaumetz, 219.

Dyer and Madsen, 278, 279.

Ehrlich, 22, 33, 259, 264, 266, 267, $279,337,340,383,403,410,458,497$, $498,501,508,514,516,518,521,522$.

Ehrlich and Morgenroth, 161, 190, 191, $210,215,229,230,231,232,234$, $235,237,240,242,243,253,288$, 294, 298, 302, 303, 333, 334, 336, $364,365,366,368,371,510,511$.

Ehrlich and Sachs, 340, 366, 368, 369, $370,372,374,377,378,379,380$, $381,382,383,384,385,387,388$, $393,442,443,444,445,446,449$, $452,453,456,457,461,511,513,515$.

Eisenberg. See Kraus. 
Eisenberg and Volk, 262, 263, 418, 500, $520,522,523$.

Elmassian, 477.

Englemann and Gaidukow, 498.

Errera, 1.

Fally, 492 .

Fassin, 479.

Fenivessy. See Liebermann.

Fischer, 189.

Ford, 248, 290, 291, 294.

Fraenkel and Sobernheim, 46, 79, 88, 134, 168, 206, 304.

Frasey, 221.

Freundlich, 424.

Friedberger. See Pfeiffer.

Friedemann. See Neisser.

Friedemann, U., 516.

Frouin, 510, 524 .

Gaidukow. See Englemann.

Gameleia, 1.

Garbowski, 415.

Gay, 345, 366, 367, 442, 458, 509, 512. See also Bordet.

Gengou, 346, 347, 348, 356, 358, 398, 403, 404, 431, 442, 449, 458, 500, $510,512,514,516,517,524$. See also Bordet.

Girard-Mangin and Henri, 313, 314, $316,318,319,323,330,442,431,511$.

Gley. See Camus.

Grassberger and Schattenf roh, 519, $520,521,526,528$.

Gratia and Lienaux, 492.

Gruber, 81, 85, 90, 91, 97, 101, 102, $143,144,146,148,149,150$.

Gruber and Durham, 93, 95, 118, 136, 505.

Grünbaum, 253.

Guthier, 415.

Hahn and Tromsdorff, 518.

Hamburger, 415.

Hamburger, F., 251, 253, 256.

Hammersten, $25^{n}$

Hankin, 24, 25.

Hardy, 323, 419

Heinrich, 415.
Henri. See Girard-Mangin.

Henri, Lalou, Mayer and Stodel, 313, 330, 516.

Henri and Mayer, 328, 416.

Henzel. See Czaplewski.

Hüne. See Neufeld.

Inmann. See Levaditi.

Issaef, 33, 48, 52, 88, 100, 120 . See also Pfeiffer.

Ivanhoff, 88.

Jagic. See Landsteiner.

Jochmann and Krause, 475, 476, 483.

de Jordis, 426.

Klein, 383, 399, 453.

Klemperer, 470.

Koessler. See Levaditi.

Kolle, 223.

Kossel, 176, 197, 281.

Kraus, 145, 146, 148, 159, 362.

Kraus and Eisenberg, 304, 308.

Kraus and Lipschïtz, 283.

Kraus and v. Pirquet, 500.

Kraus and Seng, 150.

Krause. See Jochmann.

Kyes, 513.

Kyes and Sachs, 367.

Lalou. See Henri.

Lambotte, 479.

Landsteiner, 211, 384, 518.

Landsteiner and Jagic, 312, 314, 431, $442,500,516,520,523,525$.

Landsteiner and Reich, 498.

Lea, 415.

Leblane, 251.

Leclainche and Vallé, 256.

Leclef. See Denys.

Leishmann, 389.

Lemoine. See Linossier.

Lesourd, 479.

Leuriaux, 478.

Levaditi, 397.

Levaditi, Inmann and Koessler, 390.

Liebermann and Fenivessy, 399.

Lienaux. See Gratia. 
Linossier and Lemoine, 253.

Lipschütz. See Kraus.

Lipstein, 357.

Lipski, 10.

Lottermoser, 415, 426.

Madsen, 283, 518. See also Arrhenius, Dyer.

Malfitano, 425.

Malvoz, 158, 244, 432.

Manicatide, 478.

Marchand. See Denys.

Marmorek, 13, 104, 112, 116, 117.

Martin and Cherry, 204, 259.

Martin. See Muir.

Massart, 76, 103.

Massart and Charles Bordet, 3, 11, 12.

Mayer. See Henri.

Mertens, 256.

Mesnil, 10, 35, 98.

Metchnikoff, 1, 2, 7, 9, 18, 19, 24, 25, $32,34,51,57,68,74,79,81,83$ $85,86,88,90,98,100,103,105$, $120,130,134,140,162,186,197$, 206, 207, 208, 211, 241, 242, 307, $389,396,503,526,528,529$.

Meyer, 524.

Michaelis, 500.

Mijers, 246, 251.

Moreschi, 366, 405, 407.

Morgenroth, 261, 274, 275, 297, 298, 299, 300, 301, 355, 358, 518. See also Ehrlich.

Morgenroth and Sachs, 278.

Moro, 253.

Much and Liebert, 516.

Muir, 367, 511, 518.

Muir and Browning, 334, 399, 400, 402, 447, 509, 510.

Muir and Martin, 390.

Muller, 415.

Müller, 399, 522 .

Nasse, 437

Neisser, 238, 240.

Neisser and Friedemann, 320, 323, 499, $500,513,516,517$.

Neisser and Wechsberg, 299, 356, 357, $362,366$.
Nernst, 516, 523.

Neufeld and Hüne, 390.

Neufeld and Rimpau, 390.

Neufeld and Topfer, 396.

Nicolle, 145, 146, 148.

Noguchi, 367.

Nolf, 251.

Nuttall, 253.

Obermeyer and Pick, 525.

Otto and Sachs, 518, 523.

Paal and Amberger, 415.

Paal and Voos, 415.

Paltauf, 147, 148.

Pasquale, 27.

Pauli, 422, 516, 519.

Perrin, 312.

Petruschy; 117.

Pfeiffer, 19, 27, 34, 43, 45, 56, 57, 58, $60,63,77,78,81,82,83,85,86,87$, 91, 94, 95, 97, 102, 134, 136, 137, 149, 161, 206, 207, 208, 212, 213, 214, 223, 241, 475, 477, 483, 503, 504.

Pfeiffer and Friedberger, 283, 285, 294, 301, 304, 305, 351, 359, 399, 407, 408, 409, 412.

Pfeiffer and Issaef, 59.

Pick. See Obermeyer.

Porges, 500, 517.

Quincke, 420, 421, 422.

Remy, 406.

Rimpau. See Neufeld.

Roger, 130, $146 . \quad$ See also Charrin.

Rondini. See Sachs.

Rothland, 415.

Roux, 79.

Roux and Vaillard, 277, 278, 296.

Sabatini, 435.

Sachs, 340, 345, 351，352，353，359, $399,400,407,408,409,410,412,515$, 521, 523. See also Ehrlich, Kyes, Morgen roth and Otto.

Sachs and Bauer, 443, 446, 447, 448, $449,450,451,452,456,459,460$, 461,511 . 
Sach and Rondini, 460

Salimbini, 100.

Samoiloff, 10.

Sanarelli, 79, 120.

Sauerbeck, 389.

Savtchenko, 181, 389, 395.

Schütze, 256, 304. See also Wassermann.

Seng. See Kraus.

Shattenfroh. See Grassberger.

Sleeswijk. See Bordet.

Sobernheim. See Fraenkel.

Sollbildner, 426.

Spengler, 476.

Spiro, 425.

Spring, 415.

van Steenberghe, 251, 469.

Stern, 253.

Stodel. See Henri.

Streng, 448, 507, 511, 515. See also Bordet.

Tchistowitch, 148, 175, 246, 249, 255, 257.

Topfer. See Neufeld.
Toyosumi, 524.

Tromsdorff. See Hahn.

Trumpp, 149, 150.

Vaillard. See Roux.

Vallé. See Leclainche.

Vincenzi, 478.

Volk. See Eisenberg.

Voos. See Paal.

Wassermann, 189, 226, 246, 281, 290, 291, 294.

Wassermann and Bruck, 468, 479.

Wassermann and Schütze, 253.

Wechsberg. See Neisser.

Weil, 500.

Werigo, 11, 12, 13.

Widal, 222.

Woronin, 11, 12.

Wright and Douglas, 390.

Zaugger, 516.

Zuelzer, 256. 


\section{INDEX OF SUBJECTS.}

NOTE.-The numbers printed in BOLD-FACED type refer to pages on which the topic is specifically

\section{Absorption -}

compared with adsorption, 415.

in variable proportions, 263 .

Alexin, and the antagonistic property of normal serum, 398.

(see also Fixation Reaction).

Absorption Experiments -

as producing condition of equilibrium, 238.

showing multiplicity of normal agglutinins, 161.

Cause of error in, 383, 509.

Technic of, 171.

Adaptability -

of bacterial cultures in the body of immunized animals, 1.

Failure of, of cultures in vitro, 75 .

\section{Adsorption -}

between cells and chemical precipitates, 431.

between non-cellular substances, 414 et seq, 514 .

compared with absorption, 415.

Relation of, to concentration, 418 .

The phenomena of, 414, 516 et seq.

The phenomena of, and the conglutinin of bovine serum, 440 .

(see also Molecular Adhcsion).

Agar, compared with gelatine as a culture medium 23,29 .

\section{Agglutination -}

as a phenomenon of molecular adhesion, $93,144,154,158,163,419,517$.

as cause of error in bacteriolytic experiments, 152.

compared with coagulation, 153, $157,163,182506$.

compared with dissociation, 325 , 432.

compared with fixation reaction, 486 . of bacteria, $65,88,90,96,99,100$, $134,504$.

(see also under each micro-organism and under Antimicrobial Sera).

of casein particles by lacto-serum, $150,155$.

of clay suspensions by $\mathrm{NaCl}, 153$.

of killed bacteria, 93 .

of red blood corpuscles, 134, 165, 312 (see also Hemagglutination).
Agglutination - Continued.

Accuracy of, as a means of diagnosis of bacteria, 96 .

Artificial resistance of bacteria to, 98 .

Division of, into two phases, 158, 163.

Dissociation of, by chemical precipitates, 313, 318, 322, 324.

Effect of, on morphology of bacteria, $91,92,93,149$.

Effect of endorcorpuscular salts on, $313,314,316$.

Effect of heat on, 93.

Effect of mechanical motion on, 150.

Effect of salt on, 151 .

Inhibition of, by serum, 313, 318, $321,485$.

Inhibition of, by sodium citrate, 432 .

Mechanism of, 142.

Relation of, to immunity, 99, 102.

Relation of, to motility of bacteria, $91,143,147,183$.

Theories of, $91,143,145,147,149$, $163,499$.

\section{Agglutinins -}

compared with conglutinins, 448 .

compared with precipitins, 149.

for bacteria, 149 .

for red blood corpuscles (see Hemagglutinins).

in general, 91, 500 .

Absorption of, by cells, 161, 262, 520 .

Comparison of, in normal and immune sera, 181, 290.

Effect of heat on, 96, 181 .

Identity of normal and immune, 290.

Multiplicity of, in normal serum, $161,240$.

Relation of, to opsonins, 395 .

Relation of, to precipitins, 149.

Relation of, to preventive substance, 97.

Relation of molecular adhesion to, $158,163,419$.

Specificity of, 97 .

Variations in, to a given micro-organism, 527.

Albuminous Substances -

Sensitizers of sera active against, $\mathbf{2 4 1}$, $246,257,301$.

Specificity of sensitizers for, 253. 
AlexIn (syn. Bactericidal Property; Bactericidal Substance; Complement (Ehrlich), q.v.) -

absorption and the antagonistic power of serum, 398.

in normal serum, 89, 101, 135, 170.

Action of, 149, 161, 174, 189 .

Action of, on its proper corpuscles, $173,187,198,333$.

Antitoxin for (see Anti-alexin).

Comparison of combining and toxic properties of, 340 .

Death of infection not due to lack of, 226.

Effect of decalcification on, 435 .

Ehrlich's phenomenon in union of, with anti-alexin, 268, 271.

Evidence for multiplicity of, 237, 238.

Justification of term, 187.

Mode of union with anti-alexin, 268.

Pfeiffer's conception of, 213.

Reactivation of heated serum by, $137,138$.

Relation of, to opsonins, 390 .

Relation of, to sensitizing substance, $172,214,229,234,239,334,357$, 363, 368, 387, 403, 440, 511.

Union of, with sensitized cells, 196, $261,337,365$.

Unity of, in a given serum, 140, 189 , $211,216,219,228,235,382,405,507$.

Variations in, from different animals, 173, 187, 198, 333, 507.

Alexin Deviation (syn. Neisser-Wechsberg Phenomenon; Complement Deviation) -

due to serum precipitates, 355,358 .

in hemolysis, 299, $35 \%$.

Incorrect explanations of, 352,366 .

\section{Alexin Fixation -}

by serum precipitates, 346.

on sensitized cells, 190, 219, 230, 234, $244,261,363,365,398,510$.

Antagonistic effect of normal serum on, 351, 352, 398, 402, 410, 509 .

Effect of sodium citrate on, 404, 512 .

Inhibition of, by antisensitzer, 287.

Relation of normal amboceptors to, 399.

(see also Fixation Reaction; Molecular Adhesion).

\section{Alum -}

Agglutination of bacteria by, 432 .

Amboeeptor (Ehrlich and Morgenroth syn. for Sensitizing Substance), 230, $233,340,363,388,453,459,510$.

Normal, as cause of non-fixation of alexin, 399 .

Amphophiles -

in peritoneal exudate, $33,54$.

Importance of, in phagocytosis, 10.
Antagonistic Property of Normal Serum, see Normal Serum.

Anthrax Bacillus, see Bacillus Anthracis.

Anti-Agglutinins -

in antihematic sera, 176, 205.

Reaction of normal and immune agglutinins to, 290.

Anti-Alexin, 198, 448.

Action of, on alexin, 203, 204, 205, $216,268,519$.

Antihemolytic and antibactericidal effect of, 201, 216.

Effect of heat on, 199.

Ehrlich's phenomenon in mixtures of, and alexin, 268, 271.

Equilibrium between, and alexin, 274 .

Specificity of, 200, 202, 216.

Anti-Antibodies, 280, 306 (see Antisensitizers; Anti-agglutinins).

Antibactericidal Effect, see under Antialexin; Antihemotoxic Serum; $A n$ tilytic Properties of Normal Serum.

\section{Antibodies -}

Classification of, 501 .

Relation of, to receptors, 296, 301, $305,497$.

Relation of origin of, to their action, 280.

Union of, with antigen, 440, 501, 506. $515,523$.

(see Preventive Substance; Immune Bodies; Sensitizing Substance; Precipitins; Agglutinins; Amboceptors).

Anticholera Serum (as type of Bacteriolytic Sera).

Action of, on cholera vibrio, 22, 29, $44,47,65,173$.

Agglutination of vibrios by, 65,97,103.

Analogies between, and hemolytic sera, 140 .

Diagnosis of vibrios by, 60,94 .

Effect of, on leucocytes, 51 et seq.

Effect of, on chemiotaxis, 52, 80 .

Effect of heat and conservation on, $47,58,59,65,66,69$.

Formation of specific precipitates with, 145.

Preventive substance in, 51 et seq.

Specificity of, 94, 97.

(see also V. cholera; Pfeiffer's Phenomenon).

Anticorpuseular Serum (syn. Hemolysin; Hemolytic Scrum; Hemotoxin).

Antigens, 250, 523.

Accessory nature of, 526 et seq.

Union of, with antibodies, 440, 501, $506,515,523$.

Antihematic Serum (syn. Hemolytic Serum, q. v.)

Antitoxic properties of, 175, 185, 186 , 197 (see also Antihemotoxic Serum). 
Antihematic Serum - Continued.

Effect of, on blood corpuscles, 167.

Fixation of properties of, by specific red blood corpuscles, 171 .

Properties of, 166.

Antihemolytic Serum, 281 (syn. Antihemotoxic Serum, q. v.).

Antihemotoxic Serum, 186, 197.

Dual nature of, 198, 281 (see Antisensitizer; Anti-alexin).

Antihemolytic and antibactericidal nature of, 201, 281.

(see also under Hemolytic Serum; Antihematic Serum).

Antihuman Serum, Reactions of, with human products, 256.

Antilytic Properties of Normal Serum, see Normal Serum.

Antimicrobial Sera -

On the existence of sensitizing substances in the majority of, $\mathbf{2 1 \%}$.

(see also Anticholera Serum; Antistreptococcus Serum; also under each Micro-organism).

Antisensitizers, 198.

considered as solution of receptors, 297.

to normal sensitizers, 285 .

Action of, on sensitizer, 286, 287, 513 .

Conception of, by Ehrlich and Morgenroth, 301.

Effect of normal serum on action of, $288,293$.

Effect of unfavorable media on action of, 296.

Inhibition of alexin fixation by, 287 .

Preventive and curative action of, 283.

Properties of, 280, 283.

Unity of, active against sensitizers from a given animal species, 290 .

Antistreptococcus Serum -

Agglutination of streptococci by, 118.

Contribution to the study of, 104 .

Effect of, on streptococci in vitro, 117.

Effect of, on streptococci in vivo, $121,131$.

Preventive action of, 116 .

Relation of, to delayed phagocytosis, 127.

(see also Streptococcus).

Antitoxin -

Action of, on toxin, 204, 259, 295, 518 .

Ehrlich's phenomenon with toxin, mixtures, 264.

Nature of toxin, union, 260 et seq.,267.

Origin of, from toxin (Buchner), 51 .

Union of, with toxon, 267.

(see also Diphtheria Antitoxin; Toxins).
Antituberculous Sensitizers, see under Bacillus Tuberculosis.

Aqueous Humor -

in vaccinated animals, 73 .

Absence of preventive substance in, $73,289$.

Anthrax bacillus in, 7, 26 .

Bactericidal property of, $26,29,71$.

Areola -

formed about resistant streptococci, 106, 109.

Relation of, to negative chemiotaxis, 113.

Relation of, to virulence, 113.

Attenuation of Bacteria, 3 .

Avian Diphtheria, 492.

Bacteriology of, 492, 493.

Pathogenesis of, 494.

Relation of, to human diphtheria, 492.

Avian Tuberculosis, see Bacillus Tuberculosis.

\section{Bacillus Anthracis -}

in the aqueous humor, 7, 26 .

Antiserum for, 221.

Effect of heated rat serum on, 181 .

Effect of rabbit serum on, 26.

Opsonins for, 391 et seq.

Phagocytosis of, 35,36 .

Phagocytosis of, in relation to opsonins, $39 \mathrm{C}$.

Sensitizers for, in antiserum to, 221.

Bacillus Coli-

Agglutination of, 91 .

Phagocytosis of, 35.

Bacillus of Danysz, Phagocytosis of, 35 .

Bacillus Dlphtheriæ, 10.

Phagocytosis of, 35.

Bacillus of Hog Cholera -

Antiserum against, 52.

Phagocytosis of, 35.

Bacillus Influenza -

Etiological relation of, to whooping cough, 475.

Reactions of, with sera of whoopingcough patients, 480 :

Bacillus Muscoides, Phagocytosis of, 36 .

Bacillus Pestis, Sensitizers for, in antiserum to, 219.

Bacillus Proteus -

Phagocytosis of, 14, 36.

Pfeiffer's phenomenon with, 224.

Sensitizers for, in antiserum to, 223.

Bacillus Pyocyaneus, Phagocytosis of, 37.

Bacillus of Swine Plague, Sensitizers for, in antiserum to, 221.

Bacillus Typhosus -

Agglutination of, 91, 181, 500 .

Diagnosis, of, 95 . 
Bacillus Typhosus - Continued.

Opsonin for, 393.

Phagocytosis of, 35.

Sensitizers for, in antiserum to, 221.

Bacillus Tetani, 10.

Agglutination of, 91, 92, 99 .

Bacillus Tuberculosis -

Action of various mammalian types of, in producing sensitizers, 464 .

Relation between types of, as regards sensitizers, 467 .

Sensitizers for, $462,464,467$.

Baclllus Vermicularls -

Phagocytosis of, 36.

Bacillus of Whooping-cough (BordetGengou), 473, 482, 488.

Action of antiserum to, 483, 485 . 487.

Antigens of, 526 .

Comparison of, with B. influenzæ, 483.

Cultural characteristics of, 478,482 , 484.

Endotoxin of, 487, 488, 490.

Isolation of, $474,477,482$.

Morphology of, 474, 478, 484.

Pathogenicity of, 478, 486.

Reactions of, with sera of whoopingcough patients, 479,482 .

Stain for, 478.

Bacteria -

Action of preventive substance on, $90,225$.

Adaptation of, in immunized animals, 1.

Agglutination of, 65, 88, 90, 134. (see also under each micro-organism).

Agglutination of, by alum, 432 .

Agglutinins for, 149.

Artificial resistance of, to agglutination, 98.

Attenuation of, 3.

Chemiotaxis by, 3, 4, 11 .

Diagnosis of, by agglutination, 96 .

Increase in pathogenicity of, 2, 6 .

Modified cultures of, 2 et seq.

Selection of, by phagocytes, 6,16 .

Bactericidal Immunity, 48.

Hankin's theory of, 24.

Denys' theory of, 25.

Bactericidal Properties (of body fluids), 17 et seq., 20.

in immune serum, 42, 46, 50, 68, 70 .

in normal serum, 26, 45, 46, 68, 69, $70,75,78,201$.

in relation to the number of leucocytes, $24,30,32,84$.

in serum compared with peritoneal exudate and with plasma, 21, 27.

of aqueous humor, $26,29,71$.

of edema fluid, $27,29,31,71$.
Bactericidal Properties - Continued.

of phagocytes, 24, 33, 36 .

of plasma, 27.

of serum as due to coöperation of two substances, 89, 100, 135 .

Absence of, in intestinal transudate, 74.

Comparison of potency of, of normal and immune sera, $45,69,75,78$, 177, 201.

Effect of heat on, 67, 72 .

Effect of infections on, 67, 72 .

Effect of injections of normal serum or bouillon on, $31,32,67,87$.

Method of determining, of a fluid, 20.

Non-specificity of, in immunc sera, $42,46,50,68$.

(see also Bactericidal Substance; Alexin).

Bactericial Substances (of body fluids) -

Absence of, in edema fluid, 71, 72 .

Absence of, in intestinal transudate, 73.

Localization of, during life, 82 .

Origin of, of serum, 24, 30, 32, 41, $68,84,90$.

Unity of, in normal and immune sera, 45, 69, 75, 78, 201.

(see also Bactericidal Properties; Alexin).

Bacteriolysis, see Pfeiffer's Phenomenon.

Bacteriolytic Experiments, Cause of error in, 152.

(see also Pfeiffer's Phenomenon).

Bacteriotropins, syn. Opsonins.

Barlum Sulphate -

Agglutination and hemolysis of blood by, $313,315$.

Complexes of, with colloids, 420,421 , 500.

Complexes of, with sodium citrate, 425.

Dissemination of, by sodium citrate, 424.

Dissociating effect of colloids on, $416,419$.

Bordet's Theory of Immunity, 208, 215, 442,496 et seq.

Bordet's Theory of Agglutination, 143, 163.

Bordet's Theory of Toxin-Antitoxin Union, 266, 439, 519.

Body Fluids -

Bactericidal Theory of, 17 et seq.

(syn. Humoral Theory of Immuntty).

\section{Bouillon -}

Effect of injections of, on bactericidal property of blood, $31,32,67$, 87.

Effect of injections of, on streptococcus infection, 107, 112, 122. 
Bovine Serum -

Hemolysis of guinea-pig blood by, 368 et seq., 443 et seq.

Hemagglutination by, 370 .

Conglutinin of, see Conglutinin.

Calcium Chloride, Antagonism to action of, by sodium citrate, 425,434 .

Calcium Fluoride -

Action of sodium citrate on, 427, 432.

Agglutination and hemolysis of red blood corpuscles by, 313, 315, 432 .

Flocculation of, by electrolytes, 315 .

Chemical Precipitates, see under Precipitates.

Chemlotaxis -

by bacteria in vivo, $3,4,11$.

by bacterial products, 52 .

by preventive serum, 52,80 .

Effect of anticholera serum on, 52 , 80.

Effect of, on phagocytosis, 11 et seq.

Negative, 4, 5, 11 et seq., 13 .

Negative, by streptococci, 13, 15, 106, 112, 113.

Relation of negative, by streptococcus to an areola, 113.

Chloroform, Effect of, on phagocytosis, 23.

Cholera Vibrio, see Vibrio Cholerce.

Cholera Infection, 48.

in rodents, 74 .

Course of, 48 .

Phagocytosis in, 9, 19, 24.

(see also under Vibrio Choleræ).

Clay, Agglutination of suspensions of, 153.

Coagulin, 154, syn. Precipitin.

Coagulation -

as a phenomenon of molecular adhesion, 153.

compared with agglutination, 153, $157,163,182,506$.

\section{Colloids -}

Action of, compared with hemolysins, 404, 517.

Complexes of, with barium sulphate, $420,421,500$.

Definition of, 414 .

Dissociating effect of serum due to, 324.

Dissociation of suspensions with, 419 .

Flocculation of, by electrolytes, 313 , $421,422$.

Protection of unstable, by stable, 330 .

Suspension of chemical precipitates by, 312.

Colloidal Substance of Bovine Serum (Bordet and Gay), syn. Conglutinin.
Colon Bacillus, see Bacillus Coli.

Complement, syn. (Ehrlich and Morgenroth) for Alexin, 233, 299, 363.

Suitable, 367, 388.

(see also under Ehrlich's Theory of Immunity).

Complementoids (Ehrlich and Morgenroth), 402.

On the existence of, $333,340,512$.

Gay's interpretation of, 341,345 .

Nature of, according to Ehrlich, 340.

(see also under Ehrlich's Theory of Immunity).

Conglutination, 447.

as a phenomenon of molecular adhesion, 376, 379.

Examples of, 456 .

Necessity of sensitizer and alexin in, 370,374 et seq.

Relation of, to increased hemolysis, 456.

Conglutinin of Bovine Serum, 376,387 , 440, 444, 512 (Bordet and Streng), syn. Colloidal Substance of Bordet and Gay.

compared with agglutinin, 448.

Effect of, on goat corpuscles, 457 .

Effect of, on sensitized and alexinized red blood cells, 373 et seq., 444 .

Effect of dialysis on, 460 .

Mode of action of, 456 .

Potency of, $381,450$.

Proofs of accuracy of conception of, 380 et seq., 447 et seq.

Culture Media -

for bacillus of whooping-cough, 474 .

for streptococcus, 104.

(see under Agar; Gelatine).

Cytolytic Sera -

Theories of, 186.

Mode of action of, 228.

Denys' Theory of Immunity, 25.

Dineur's Theory of Agglutination, 147.

Diphtheria Antitoxin, Effect of, on leucocytes, 10, 37, 77 .

Diphtheria Bacillus, see Bacillus Diphtherice.

Diphtheria of Fowls, see Avian Diphtheria.

Diphtheria Toxin, effect of, on phagocytosis, 37.

Dissociation -

of agglutination caused by chemical precipitates by serum, 313, 318, $322,324$.

of suspensions with colloids, 419 .

Dissolution of Red Blood Corpuscles, 134, 165; (see Hemolysis).

Dog Red Blood Corpuscles, Fragility of, 179. 


\section{Dog Serum -}

Antiserum to, 248, 249, 251, 255, 258.

"Complementoids" in, 340.

Effect of heat on, 340, 345.

Normal hemagglutinins in, 178.

Normal hemolysins in, 179, 340 .

Precipitin for, 249.

Dyeing Phenomena, Fixation of sensitizing substances regarded as, 194, $216,230,262$.

\section{Edema -}

in vaccinated animals, 73 .

Absence of bactericidal substance in, $27,29,31,71,72$.

Means of producing, 27, 29.

Preventive substance in, 38 et seq., 73.

Eel Serum, Hemolysis by, 437.

Egg Albumin -

Antiserum to, 248.

Precipitins for, 248.

Sensitizers to, in antiserum to, 248, 254.

Ehrich's Phenomenon (of union of toxin with antitoxin), 264.

in union of alexin with anti-alexin, 268, 271.

Ehrlich's Theory of Immunity, 294, $296,305,333,352,363,388,410$, $442,459,497,523$.

(see also under Amboceptors; Complements; Complementoids; Receptors.)

Electrolytes -

Effect of, on agglutination of red blood corpuscles by chemical precipitates, 314 et seq., 321 .

Flocculation of colloids by, 313,421 , 422.

Endothelium, Function of, in Pfeiffer's phenomenon, 57.

Endotoxin -

of whooping-cough bacillus, 488 .

Pathogenicity of whooping-cough, 489.

Preparation of, 488, 489.

Eosinophiles, 10.

Origin of bactericidal substance in, 24.

Exudate, see Peritoncal Exudate.

\section{Fibrinogen -}

Antiserum to, 249, 250.

Precipitins for, 255.

Sensitizers in antiserum to, 249, 255.

Fixation Reaction (Bordet-Gengou), syn. Reaction of alexin fixation, 189,243 , 398.

with lactoserum, 251, 253.
Flxation Reaction-Continued.

Applicability of, to diagnosis, 407 $462,464,467,479,486,508$.

Comparison of, with agglutination 486.

Comparison of, with precipitation 249, 252, 255, 256.

Criticism of deduction drawn from, $245,405$.

Demonstration of sensitizers by, 218 , $245,250,348,467$.

Specificity of, 222, 253.

Technic of, 219, 247, 404 et seq., 469 .

(see also under Alexin Fixation).

Floceulation of Colloidal Complexes, $313,421,422$.

Frog Serum, as alexin and opsonin, 391 et seq.

Gelatin, compared with agar as culture medium for vibrios, $23,29$.

Gels, 414 et seq.

Goat Red Blood Corpuscies, Effect of conglutinin on, 457.

Goat Serum, Hemagglutination by, 92, 178.

Gruber's Diagnosis by Agglutination, 95.

Gruber's Theory of Agglutination, 91, 143,149 .

Guinea-pig Serum -

Action of, on bacteria, 96 .

Action of, on alien blood corpuscles, 178.

Normal hemolysins in, 179.

Potency of alexin in, 188.

Hankin's Theory of Immunity, 24.

Hemagglutination (Agglutination of red blood cells), 65.

by chemical precipitates, 312,431 .

by products of cholera vibrio, 92 .

Effect of electrolytes on, by chemical precipitates, 314,321 .

Effect of, on morphology of red blood cells, 93.

(sce Hemagglutinins; Conglutination).

Hemagglutinins, 370 .

Artificial, 137.

Effect of dilution on, 174 .

Effect of heat on, 136, 138.

Normal, 65, 92, 136, 178, 370; (hemagglutinins of normal serum).

Passive transfer of, 168.

Relation of normal, to hemolysins, 179.

(sec Agglutinins; Anti-agglutinins; Hemagglutination).

Hemolysins, syn. Hemolytic Serum; Antihematic Serum; Anticorpuscular Serum; Hemotoxin.

compared with colloids, 404, 517 . 
Hemolysins - Continued.

Activity of, due to two substances, 184 .

Analogy of, with bactericidal substance, 140, 167, 177.

Effect of heat on, 138.

Mode of union of, with cell, 436 .

Normal, 179, 237, 340, 367.

Reactivation of artificial, by normal serum, 138.

Specificity of, 139, 171 .

Toxicity of, 139.

(see Hemolysis, Hemolytic Serum, etc.).

Hemolysis, 134, 165, 186, 333.

by chemical precipitates, 313 .

by normal serum, 136; (see under Hemolysins).

by specific serum in vivo, 139 .

by venom, $367,433,513$.

Alexin deviation in, 299, $35 \%$.

Effect of heat on, 169.

Inhibition of, by sodium citrate, $404,433$.

Relation of conglutination to, 456 .

Effect of salt solution in experiments in, $296,301,335,341,383,399$, $451,453,456$.

Hemolytic Serum -

and its antitoxins, 186.

produced by injecting stromata, 194 .

Antitoxin to, 197; (see Antihemolytic Serum).

Data concerning, 229.

Precipitins in, 148.

Reactivation of, by normal serum, 138.

Toxicity of, 197.

Union of, with corpuscles in varying proportions, $194,260,521$.

(see also Hemolysin; Antihematic serum).

Hemotoxin, syn. Hemolytic serum, Hemolysin.

Hemosensitizer or Hemosensitizing Substance; (see under Sensitizing substance for blood corpuscles).

Hen Serum -

Hemagglutinins in, 136, 178.

Hemolysins in, 179.

Hog Cholera Bacllius, see under $B a-$ cillus of.

Horse Serum -

Hemagglutinins in, 92, 178, 370 .

Agglutination of bacteria by, 96, 99, 100.

Alexin of, 367, 368, 371, 379, 444.

Relation of opsonin to alexin in, 392.

Sensitizer in, $372,379,444$.

Immune Bodies, syn. Sensitizing Substances.

Single nature of hemolytic, 333.
Immune Serum, syn. Preventive Serum. $q . v$.

compared with normal serum, 45, $69,75,78,177,201$.

Action of, due to two substances, 46,89 .

Agglutinins of normal and, 290.

Bactericidal substance in, $42,46,50$, 68,70 .

Effect of heat on, 46, 59, 66, 69 .

Inhibition to agglutination by excess of, 485 .

Opsonins of, 396.

Specificity of, 525.

Immunity -

Active, 75, 103.

Bactericidal, 48 .

Mechanism of, 75.

Passive, 79, 99, 101, 102, 303, 304.

Relation of agglutination to, 99, 102 .

Relation of leucocytes to, 9 .

Theories of, 18, 24, 25, 206, 208, 212, 280, 294, 303, 366.

(see also under Ehrlich's Theory of Immunity and Bordet's Theory of Immunity).

Immunized Animals, Adaptability of bacterial cultures in the body of, 1 .

\section{Infections -}

Death of, not due to lack of alexin, 226.

Effect of, on bactericidal properties of serum, 67, 72 .

Mixed, due to phagocytic selection, 16.

(see Cholera Infection; Streptococcus Infection).

Intestinal Transudate, Absence of bactericidal substance in, 73 .

Lactic Acid, Effect of, on phagocytosis, 12.

Lactoserum, 246.

Action of, compared with rennin, 155.

Fixation reactions with, 251, 253.

Flocculation of casein by, 155, 506 .

Precipitins of, 156, 253.

Sensitizers of, 253.

\section{Leucocytes -}

of rabbit and guinea-pig in streptococcus infection, 114.

Artificial lowering of, in blood, 30 .

Effect of anticholera serum on, 51 .

Effect of bacterial products on, 51, $53,120$.

Effect of diphtheria antitoxin on, $10,37,77$.

Origin of bactericidal substances in, $24,32,75,78$. 
Leucocytes - Continued.

Relation of, to bactericidal power of serum, 24, 30, 32, 41, 68, 84, 90.

Relation of, to immunity, 9.

Relation of, to preventive power of serum, 38, 41.

Relation of, to Pfeiffer's phenomenon, 71, 85 .

(see also Phagocytes; Amphophiles; Macrophages; Microphages).

\section{Macrophages -}

in exudate caused by diphtheria antitoxin, 37.

in streptococcus infection, 128, 130.

Origin of granules in, 38 .

\section{Iastic -}

as a reagent for blood serum, 347 .

Agglutination of blood corpuscles by, 432.

Dissemination of, by sodium citrate, 424.

Microphages, in streptococcus infection, 14,128 .

Modifled Cultures, (of bacteria), 2 et seq.

Molecular Adhesion, 414.

Alexin fixation as a phenomenon of, $365,403,514$.

of conglutinin, 376,379 .

Agglutination in relation to, 93, 144, $154,158,163,419,517$.

Coagulation in relation to, 153 .

(see Absorption; Adsorption).

Negative Chemlotaxis, see under Chemiotaxis.

Nelsser-Wechsberg Phenomenon, see under Alexin Deviation.

Nictitating Membrane, as means of purifying cultures of avian diphtheria, 493.

Nicolle's Theory of Agglutination, 145.

Normal Serum -

compared with immune serum, 45, $69,75,78,177,181,201,290$.

Bactericidal property in, $26,45,46$, $68,69,70,75,78,201$.

Action of, with preventive serum in Pfeiffer's phenomenon, 58, 66, 103, 135.

Agglutination of alien blood by, 65, $92,136,178,181,290,370$.

Antagonistic effect of, on alexin fixation, 351, 352, 398, 402 et seq., $410,509$.

Alexin in, 89, 101, 135, 170.

Action of, on antisensitizers, 288, 293.

Effect of injection of, 31, 32, 67, 87 .

Multiplicity of agglutinins in, 161, 240.
Normal Serum - Continued.

Opsonins of, 390 .

Reactivation of hemolytic serum by 138.

Sensitizers in, 184, 237, 240, 288 $372,379,444$.

Suppression of cure of antisensitizer by, 288.

\section{Opsonins -}

of frog serum, 391 .

of immune sera, 396.

of normal sera, 390 .

Effect of, on sensitized red blood corpuscles, 395.

Nature of, 389.

Relation of, to agglutinins, 395.

Relation of, to alexin, 392.

Relation of, to phagocytosis, 389 et seq.

Relation of, to sensitizers, 397 .

Paltauf's Theory of Agglutination, 147.

Pathogenicity, Increase of, in bacteria, 2,6 .

Peritoneal Exudate, in infections with Streptococcus, 110, 124; (see also Streptococcus).

in infections with V. Massaouah, 40. Bactericidal power of, 21.

Method of producing, 33.

Non-antagonism of, to alexin fixation, 412.

Stains for study of phagocytosis in, 33.

Pfelffer's Phenomenon (syn. Bacteriolysis), 56.

in vivo, 57,83 .

in vitro, $48,57,58,83$

with Vibrio Choleræ, 58-60.

with Vibrio Metchnikovi, 64.

Combined action of preventive and normal serum in, 58, 66, 103, 135.

Comparison of, in vitro and in vivo, $214,413$.

Diagnosis of vibrios by, 44, 59, 61,94 .

Factors concerned in, 65.

Pfeiffer's explanation of, $57,85,95$, 207, 212.

Relation of endothelial cells to, 57 .

Relation of leucocytes to, 71,85 .

Stains for demonstrating, 63.

Pfelffer's Theory of Immunity, 212, 215.

Phagoeytes -

Bactericidal properties of, 24, 33, 36 .

Effect of chemiotaxis on, 11 et seq.

Selection of bacteria by, 4, 6, 16 .

Tactile reaction of, 12 .

(sce also Leucocytes; Phagocytosis).

Phagocytic Crisis in Streptococeus Infection, $124,127$. 
Phagocytic Theory of Immunity, 18, 25; (see also under Immunity).

Phagocytosis, 9, 10, 22, 24, 528.

as produced essentially by amphophiles, 10.

of various bacteria (see under each micro-organism).

in cholera infections, 9, 19, 24.

in vaccinated animals, 11.

in vitro, 33.

in streptococcus infection, 14, 108, $128,130$.

Delayed, in streptococcus infection, $124,127$.

Effect of chemiotaxis on, 11 et seq.

Effect of chloroform on, 23.

Effect of diphtheria toxin on, 37 .

Effect of lactic acid on, 12.

Relation of, to immunity, 99, 206.

Relation of opsonins to, 389 et seq.

Stains for, 22, 33.

Phagolysis in streptococcus infection, 105.

Pigeon Serum, Hemagglutinins in, 178.

Plague Baclllus, see Bacillus Pestis.

Plasma -

Bactericidal properties of, 27.

Production of artificial, 27.

(cf. Edema).

Polymorphonuclear Leucocytes, see $M i$ crophages.

Precipitins (precipitating serum), 175, $184,205,246,251,347,522$.

for albuminous derivatives, 251 .

for dog serum, 249.

for egg white, 248.

for fibrinogen, 255.

in lactoserum, 156, 253.

for rabbit serum, 249.

Comparison of, with agglutinins, 149.

Comparison of reaction of, with coagulation, 157.

Comparison of reaction of, with fixation reaction, $249,252,255,256$.

Effect of heat on, 157.

Specificity of, 253, 255.

Technic of reaction of, 175,349 .

Precipitinogen (precipitablesubstance)in insufficiently washed blood, 347 .

Effect of heat on, 157.

Preclpitates -

Chemical precipitates:

Hemagglutination by, 312, 431 .

Hemolysis by, 313 .

Relation of colloids to, $\mathbf{3 1 2}, 414$.

Adsorption by, 414, 431 .

Complexes of, with sodium citrate, 424, 429.

Dissociation of agglutination caused by, $313,318,322,324$.
Precipitates - Continued.

Serum precipitates:

in anticholera serum, 145.

as explaining antagonistic property of normal serum, 353.

in hemolytic serum, 148.

Alexin deviation due to, $\mathbf{3 5}$.

Action of, on sensitizing substance, 349.

Alexin fixation by, 346.

Specificity of, 149 .

Preventive Serum, (syn. Immune Serum) -

Combined action of, with normal serum, 58, 66, 103, 135.

Chemiotaxis by, 52 .

Mode of action of, 81.

(see Preventive Substance; Pfeiffer's Phenomenon).

Preventive Substance (syn. Immune Body; Sensitizing Substance) -

in anticholera serum, 51 et seq.

in aqueous humor, $73,289$.

in edema fluid, 38,73 .

Chemiotaxis by, 52,80 .

Effect of heat on, 46, 68, 88, 134.

Effect of, on bacteria, 90, 225.

Effect of injection of, 79 .

Origin of, in serum, 54, 162.

Relation of leucocytes to, 38, 41 .

Relation of, to agglutinins, 97 .

Specificity of, $48,60,78$.

\section{Rat Serum -}

Hemagglutinins in, 92, 178.

Effect of heated, on B. anthracis, 181.

Rabbit Serum -

Antagonistic effect of, 351, 400 .

Bactericidal action of, 26.

Hemagglutinins of, 92, 178.

Hemolysins of, 179,367 .

Precipitins for, 249.

Reactivation of antirabbit serum by, $138,188,333,334$.

Sensitizers for, 249.

Specific agglutinins in, 182.

Receptors (see Ehrlich's Theory), 363.

Antisensitizers as solution of, 297.

Relation of, to formation of antibodies, 296, 301, 305, 497.

Red Blood Corpuscles -

Agglutination of, see Hemagglutination.

Agglutination of, by chemical precipitates, 312.

Agglutination of, by mastic, 432 .

Difficulty in washing, 347.

Dissolution of, see Hemolysis.

Electric charge of, 313.

Effect of agglutination on morphology of, 93 . 
Salt Solution, Action of, in hemolytic experiments, 296, 301, 335, 341, 383, 399, 451, 453, 456.

Selection, Effect of phagocytic, in increasing virulence of bacteria, 4 , 6,15 .

\section{Sensitized Blood Corpuseles -}

Action of alexin on, 196, 337, 365 .

Action of alexin plus conglutinin on, 374 et seq.

Action of opsonins on, 395.

Effect of washing on, $172,185$.

Action of antisensitizer on, 283, 287.

Fixation of alexin on, 190, 219, 230, $234,244,261,363,365,398,510$.

Suppression of cure of, by antisensitizer, 288.

Sensitizing Substance, (syn. Preventive Substance; Immune Body; Sensitizer; Amboceptor).

as characteristic of specific immune serum, 160, 161, 187.

against albuminous substances, $\mathbf{2 4 1}$, $246,257,301$.

for bacteria (see under various Microorganisms).

for egg white, 248,254 .

for fibrinogen, 249, 255 .

for milk, 253.

for serum, 241; (also under various Scra).

in antimicrobial sera, $21 \%$.

in normal sera, 184, 240, 288, 372, $379,408,444$; (see also under Hemolysins).

Absorption of, by antigen, 160, 171, 191, 229.

Absorption of, by stromata of red blood cells, 193.

Action of antisensitizer on, 285, 286, $287,513$.

Action of precipitates on, 349 .

Action of, on heated blood cells, 337.

Antitoxin to; (see Antisensitizer).

Demonstration of, by fixation reaction, 218, 245, 250, 348, 467; (see also Fixation Reaction).

Effect of dilution on, 174 .

Effect of heat on, 174, 183, 185.

Fixation of, considered as similar to a dyeing process, 194, 216, 230, 262.

Mode of action of, $172,174,436$.

Multiplicity of partial, (Ehrlich and Morgenroth), 235, 302, 333.

Relation of alexin to, 172, 214, 229, $234,239,334,357,363,368,387$, $403,440,511$.

Relation of opsonins to, 397.

\section{Serum -}

of vaccinated animals, 8,56 .

Detection of, by mastic, 347.

Properties of specific, 159.

(see Normal Serum; Immune Serum; Anticholera Serum; Antistreptococcus Serum; also under various Microorganisms).

Silicic Acid, Agglutination of blood corpuscles by, 313 .

\section{Sodium Citrate -}

Action of, on calcium chloride, 425, 434.

Action of, due to acid radicle, 424 .

Antiflocculating action of, 404 .

Complexes of, with chemical precipitates, 424, 429.

Dissemination of chemical precipitates by, 424 .

Inhibiting effect of, on agglutination, 432.

Inhibiting effect of, on alexin fixation, 404, 512 .

Inhibiting effect of, on biological hemolysins, 404, 433.

\section{Stains -}

for bacillus of avian diphtheria, 492 .

for bacillus of whooping-cough, 478 .

for Pfeiffer's phenomenon, 63.

for phagocytosis, 22, 33 .

for streptococcus infection, 106, 123, 129.

Stimulin, 79.

as identical with opsonin, 389 .

\section{Streptococcus -}

Agglutination of, 91, 92, 118.

Areola formed about resistant, 106, 109.

Culture medium for, 104.

Destruction of, by leucocytic extract, 120.

Effect of age on virulence of, 112, 123 et seq.

Negative chemiotaxis by, 13,15 , $106,112,113$.

Phagocytosis of, 37.

(see Antistreptococcus Serum; Streptococcus Infection).

\section{Streptococcus Infection -}

in the guinea-pig, 104, 114.

in the rabbit, 109, 114 .

Delayed phagocytosis in, 124, 127.

Exudate in, 110, 124.

Phagolysis in, 105.

Phagocytosis in, 14, 108, 128, 130.

Portal of entry in, 116, 132.

Mechanism of cure in, 108.

Relapse or re-infection in, 109,113 115, 129.

Stains for studying, 106, 123, 129. 


\section{Stromata -}

Agglutination of, by chemical precipitates, 318 .

Fixation of sensitizing substance by, 192, 216.

Production of hemolytic serum by injection of, 194 .

\section{Tetanus Bacillus, see B. Tetani.}

\section{Toxins -}

of symptomatic anthrax, 520 .

Mode of action of antitoxins on, 259, 295, 518.

Susceptibility to, in animals with lowered resistance, 278.

(sec Antitoxin; Diphiheria Toxin; Endotoxin).

Toxolds, 279.

\section{Toxons -}

Arrhenius and Madsen's conception of, 295.

Avidity of, for antitoxin, 267.

Bordet's conception of, 278, 295.

Ehrlich's conception of, 267.

Tubercle Bacillus, see Bacillus Tuberculosis.

Typhoid Bacillus, see Bacillus Typhosus.

Typhold Fever, Fixation reaction in convalescents from, 222.

\section{Vaceinated Animals -}

Edema fluid in, 73.

Aqueous humor in, 73.

Serum of, 8, 56.

Phagocytosis in, 11.

Bactericidal substance in, $45,69,75$, 78, 201.

(see also Immunized Animals).

Venom, Hemolysis by, 367, 433, 513.

Vibrios, Diagnosis of, by Pfeiffer's phenomenon, 44, 59, 61, 94.

(see V. Cholerce, V. Metchnikovi and the like).
Vibrio Choleræ, 17, 18, 22, 27, 31 .

Agglutination of, $65,90,91,96,97$, $100,102,103$.

Antiserum to, 22, 29, 44, 47, 65, 74 .

(see also Anticholera Serum).

Artificial resistance of, to agglutination, 98.

Diagnosis of $42,59,60,94$.

Effects produced by products of, 51 , 92.

Pfeiffer's phenomenon with, 59, 60.

Phagocytosis of, 2, 9, 19, 21, 22, 34, 100.

Vibrio Massaouah, 21, 27.

Antiserum to, 21, 28, 30, 38, 43.

Chemiotaxis by, 52 .

Effect of anticholera serum on, 95 .

Effect of culture products of, on leucocytes, 53 .

Exudate in infections due to, 40.

Vibrio of Deneke, 42.

Vibrio Finkleri, 42.

Vibrio Metchnikevi, 1 et seq., 17.

Antiserum to, 44, 49.

Pfeiffer's phenomenon with, 64 .

\section{Virulence -}

Increase in, of bacteria by selection, $4,6,15$.

Relation of an areola to, 107.

Relation of negative chemiotaxis to, 113.

Whooping-cough, 407.

Bacillus of, 472 ( $q . v$.).

Difficulty of isolating specific cause of, 472 .

Occurrence of influenza bacillus in, $475,483$.

Pathogenesis of, 490 .

Zwischenkörper, (Ehrlich and Morgenroth) syn. for sensitizing substance of normal serum, 230, 232. 



\title{
Short-title Catalogue
}

OF THE

\section{PUBLICATIONS}

\author{
OF \\ JOHN WILEY \& SONS \\ NEW YORK \\ LONDON: CHAPMAN \& HALL, LIMTED \\ ARRANGED UNDER SUBJECTS
}

Descriptive circulars sent on application. Books marked with an asterisk $\left(^{*}\right)$ are sold at net prices only. All books are bound in cloth unless otherwise stated.

\section{AGRICULTURE-HORTICULTURE-FORESTRY.}

Armsby's Principles of Animal Nutrition...................... $\$ 400$

Budd and Hansen's American Horticultural Manual:

Part I. Propagation, Culture, and Improvement...........12mo, 150

Part II. Systematic Pomology...................... 150

Elliott's Engineering for Land Drainage............................ 150

Practical Farm Drainage. (Second Edition, Rewritten)..........12mo, 150

Graves's Forest Mensuration.............................. 400

Green's Principles of American Forestry.......................... 150

Grotenfelt's Principles of Modern Dairy Practice. (Woll.)............

* Herrick's Denatured or Industrial Alcohol .................. 400

Kemp and Waugh's Landscape Gardening. (New Edition, Rewritten. In Preparation).

* McKay and Larsen's Principles and Practice of Butter-making ......8vo, 150 Maynard's Landscape Gardening as Applied to Home Decoration........12mo, 150

Sanderson's Insects Injurious to Staple Crops................ 150

Sanderson and Headlee's Insects Injurious to Garden Crops. (In Preparation).

* Schwarz's Longleaf Pine in Virgin Forests................. $12 \mathrm{mo}, 125$

Stockbridge's Rocks and Soils.................................. 250

Winton's Microscopy of Vegetable Foods.................. 750

Woll's Handbook for Farmers and Dairymen...................... 150

\section{ARCHITECTURE.}

Baldwin's Steam Heating for Buildings.................... 250

Berg's Buildings and Structures of American Railroads................ 5 to, 50

Birkmire's Architectural Iron and Steel..................... 8 vo, 350

Compound Riveted Girders as Applied in Buildings................ 8 vo, 200

Planning and Construction of American Theatres................

Planning and Construction of High Office Buildings...............

Skeleton Construction in Buildings......................... 300 
Briggs's Modern American School Buildings.................8vo, Byrne's Inspection of Materials and Wormanship Employed in Construction.

$16 \mathrm{mo}, 300$

Carpenter's Heating and Ventilating of Buildings................. 8 vo, 400

* Corthell's Allowable Pressure on Deep Foundations................12mo, 125

Freitag's Architectural Engineering........................... 350

Fireproofing of Steel Buildings........................... 250

Gerhard's Guide to Sanitary Inspections. (Fourth Edition, Entirely Re-

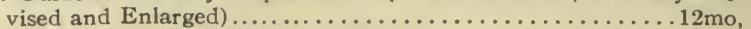

Moder 2 Baths and Bath Houses............................. 300

Sanitation of Public Buildings....................... 150

Theatre Fires and Panics............................. 150

Johnson's Statics by Algebraic and Graphic Methods............... 8 vo, 200

Kellaway's How to Lay Out Suburban Home Grounds............ 8 vo, 200

Kidder's Architects' and Builders' Pocket-book...............16mo, mor., 500

Merrill's Stones for Building and Decoration.................... 8 vo, 500

Monckton's Stair-building........................... 4 to, 400

Patton's Practical Treatise on Foundations..................... 500

Peabody's Naval Architecture..........................8vo, 750

Rice's Concrete-block Manufacture.................................... 200

Richey's Handbook for Superintendents of Construction ....... 16mo, mor. 400

Building Foreman's Pocket Book and Ready Reference. . 16mo, mor. 500

* Building Mechanics' Ready Reference Series:

*Carpenters' and Woodworkers' Edition............16mo, mor. 150

* Cement Workers' and Plasterers' Edition................

* Plumbers', Steam-Fitters', and Tinners' Edition...16mo, mor. 150

* Stone- and Brick-masons' Edition..............16mo, mor. 150

Sabin's House Painting. ................................... 100

Siebert and Biggin's Modern Stone-cutting and Masonry...........8vo, 150

Snow's Principal Species of Wood........................... 3 vo, 350

Towne's Locks and Builders' Hardware....................18mo, mor. 300

Wait's Engineering and Architectural Jurisprudence............... 8 vo, 600

Sheep, 650

Law of Contracts................................... 300

Law of Operations Preliminary to Construction in Engineering and Archi-

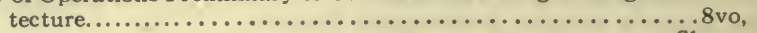

Wilson's Air Conditioning........................... 150

Worcester and Atkinson's Small Hospitals, Establishment and Maintenance, Suggestions for Hospital Architecture, with Plans for a Small Hospital.

$12 \mathrm{mo}, \quad 125$

\section{ARMY AND NAVY.}

Bernadou's Smokeless Powder, Nitro-cellulose, and the Theory of the Cellulose

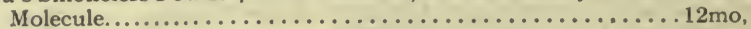

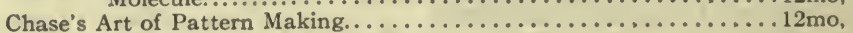

Screw Propellers and Marine Propulsion................... 8 vo,

* Cloke's Enlisted Specialists' Examiner......................... 8vo,

Gunner's Examiner...................................

Craig's Azimuth................................

Crehore and Squier's Polarizing Photo-chronograph................ 8 vo,

* Davis's Elements of Law..............................

* Treatise on the Military Law of United States............ $8 \mathrm{vo}$,

DeBrack's Cavalry Outpost Duties. (Carr.)................

* Dudley's Military Law and the Procedure of Courts-martial...Large 12mo,

Durand's Resistance and Propulsion of Ships..................... 8 vo,

* Dyer's Handbook of Light Artillery......................... 12mo,

Eissler's Modern High Explosives. .....................

* Fiebeger's Text-book on Field Fortification..................... $12 \mathrm{mo}$,

Hamilton and Bond's The Gunner's Catechism.................... 18mo,

* Hoff's Elementary Naval Tactics........................ 8 vo,

Ingalls's Handbook of Problems in Direct Fire...............

* Lissak's Ordnance and Gunnery............................ 
* Ludlow's Logarithmic and Trigonometric Tables................... . 8vo, \$1 00

* Lyons's Treatise on Electromagnetic Phenomena. Vols. I. and II..8vo,each, 600

* Mahan's Permanent Fortifications. (Mercur.).......... 8vo. half mor. 750

Manual for Courts-martial........................................ 150

* Mercur's Attack of Fortified Places............................... 200

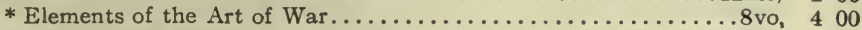

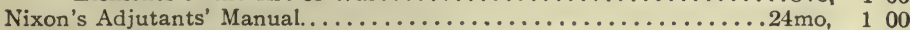

Peabody's Naval Architecture . . . . . . . . . . . . . . . . . 8vo, 750

* Phelps's Practical Marine Surveying..................... 250

Putnam's Nautical Charts............................ 8vo, 200

Rust's Ex-meridian Altitude. Azimuth and Star-Finding Tables.......8vo, 500

Sharpe's Art of Subsisting Armies in War................. 18mo, mor, 150

* Tupes and Poole's Manual of Bayonet Exercises and Musketry Fencing.

$24 \mathrm{mo}$, leather,

* Weaver's Military Explosives.......................... . . . . .

Woodhull's Notes on Military Hygiene............................... 150

\section{ASSAYING.}

Betts's Lead Refining by Electrolysis...................... 8vo,

Fletcher's Practical Instructions in Quantitative Assaying with the Blowpipe.

16 mo, mor.

Furman and Pardoe's Manual of Practical Assaying. (Sixth Edition. Re-

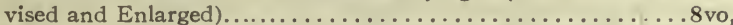

Lodge's Notes on Assaying and Metallurgical Laboratory Experiments..8vo,

Low's Technical Methods of Ore Analysis.................. . . .

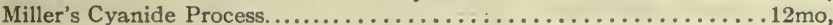

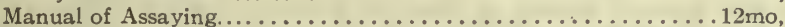

Minet's Production of Aluminum and its Industrial Use. (Waldo.)...12mo,

O'Driscoll's Notes on the Treatment of Gold Ores.................... . .

Ricketts and Miller's Notes on Assaying.................... . . . .

Robine and Lenglen's Cyanide Industry. (Le Clerc.) . . . . . . . . . . . . 8vo,

Ulke's Modern Electrolytic Copper Refining. . . . . . . . . . . . . . . 8vo,

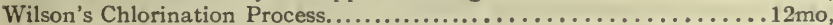

Cyanide Processes., ............................... 12mo,

\section{ASTRONOMY.}

Comstock's Field Astronomy for Engineers.................... 8vo,

Craig's Azimuth.................................

Crandall's Text-book on Geodesy and Least Squares.............. 8 vo,

Doolittle's Treatise on Pracical Astronomy.......................8vo,

Hayford's Text-book of Geodetic Astronomy...................

Hosmer's Azimuth............................. mor. mor.

Merriman's Elements of Precise Surveying and Geodesy............ 8 vo,

* Michie and Harlow's Practical Astronomy.................

Rust's Ex-meridian Altitude, Azimuth and Star-Finding Tables......8vo,

* White's Elements of Theoretical and Descriptive Astronomy........12mo,

\section{CHEMISTRY.}

* Abderhalden's Physiological Chemistry in Thirty Lectures. (Hall and

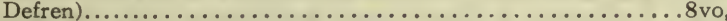
* Abegg's Theory of Electrolytic Dissociation. (von Ende.)........... 12mo, Alexeyeff's General Principles of Organic Syntheses. (Matthews.)......8vo, Allen's Tables for Iron Analysis........................ Armsby's Principles of Animal Nutrition..................... 8 vo, Arnold's Compendium of Chemistry. (Mandel.).............. Large 12mo, Association of State and National Food and Dairy Departments, Hartford Meeting, 1906................................ 8vo, Jamestown Meeting, $1907 \ldots \ldots \ldots \ldots \ldots \ldots \ldots \ldots \ldots \ldots . . . \ldots$. 
Austen's Notes for Chemical Students.

Baskerville's Chemical Elements. (In Preparation).

Bernadou's Smokeless Powder.-Nitro-cellulose, and Theory of the Cellulose

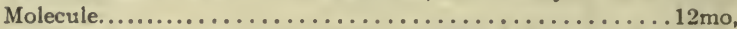

Biltz's Introduction to Inorganic Chemistry. (Hall and Phelan). (In Press). Laboratory Methods of Inorganic Chemistry. (Hall and Blanchard).

* Blanchard's Synthetic Inorganic Chemistry.................. 12mo,

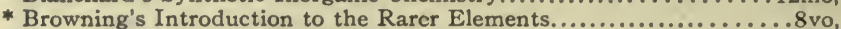

* Claassen's Beet-sugar Manufacture. (Hall and Rolfe.).............. 8vo, Classen's Quantitative Chemical Ana!ysis by Electrolysis. (Boltwood.).8vo,

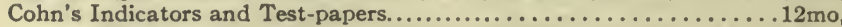

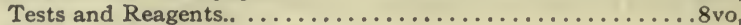

* Danneel's Electrochemistry. (Merriam.). .................... 12mo,

Dannerth's Methods of Textile Chemistry..................... 12mo,

Duhem's Thermodynamics and Chemistry. (Burgess.)..................

Effront's Enzymes and their Applications. (Prescott.)............ 8vo,

Eissler's Modern High Explosives.......................... . . . . . .

Erdmann's Introduction to Chemical Preparations. (Dunlap.).......12mo,

* Fischer's Physiology of Alimentation. .......................... 12mo,

Fletcher's Practical Instructions in Quantitative Assaying wlth the Blowpipe.

Fowler's Sewage Works Analyses.......................... 12mo,

Fresenius's Manual of Qualitative Chemical Analysis. (Wells.)........8vo, 500 Manual of Qualitative Chemical Analysis. Part I. Descriptive. (Wells.) 8vo, 300 Quantitative Chemical Analysis. (Cohn.) 2 vols............8vo, 1250 When Sold Separately, Vol. I, \$6. Vol. II, \$8.

Fuertes's Water and Public Health...................... 12mo,

Furman and Pardoe's Manual of Practical Assaying. (Sixth Edition, Revised and Enlarged.)...................... . . . .

* Getman's Exercises in Physical Chemistry................. . . . . 12mo, Gill's Gas and Fuel Analysis for Engineers..................... 12mo,

* Gooch and Browning's Outlines of Qualitative Chemical Analysis.

Large $12 \mathrm{mo}$

Grotenfelt's Principles of Modern Dairy Practice. (Woll.)........... 12mo,

Groth's Introduction to Chemical Crystallography (Marshall). . ...... 12mo,

Hammarsten's Text-book of Physiological Chemistry. (Mandel.)..... 8vo,

Hanausek's Microscopy of Technical Products. (Winton.)............8vo,

* Haskins and Macleod's Organic Chemistry................... 12mo,

Hering's Ready Reference Tables (Conversion Factors)............ 16mo, mor.

* Herrick's Denatured or Industrial Alcohol.................... . 8vo,

Hinds's Inorganic Chemistry........................... . . . . . .

* Laboratory Manual for Students........................ 12mo,

* Holleman's Laboratory Manual of Organic Chemistry for Beginners.

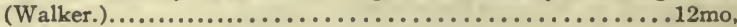

Text-book of Inorganic Chemistry. (Cooper.).................8vo,

Text-book of Organic Chemistry. (Walker and Mott.).............. . . . . . .

* Holley's Lead and Zinc Pigments............................. 12mo, Holley and Ladd's Analysis of Mixed Paints, Color Pigments, and Varnishes.

Large $12 \mathrm{mo}$,

Hopkins's Oil-chemists' Handbook. . . . . . . . . . . . . . . . . . . . 8vo,

Jackson's Directions for Laboratory Work in Physiological Chemistry. . 8vo, Johnson's Rapid Methods for the Chemical Analysis of Special Steels, Steel-

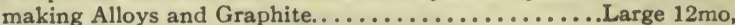

Landauer's Spectrum Analysis. (Tingle.)..................... 8vo,

* Langworthy and Austen's Occurrence of Aluminum in Vegetable Products, Animal Products, and Natural Waters.............. 8vo,

Lassar-Cohn's Application of Some General Reactions to Investigations in

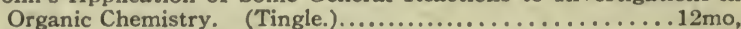

Leach's Inspection and Analysis of Food with Special Reference to State

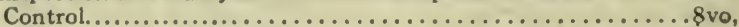

Löb's Electrochemistry of Organic Compounds. (Lorenz.)............8vo, Lodge's Notes on Assaying and Metallurgical Laboratory Experiments..8vo,

Low's Technical Method of Ore Analysis........................8vo,

Lunge's Techno-chemical Analysis. (Cohn.)..................... 12mo,

- McKay and Larsen's Principles and Practice of Butter-making.........8vo, Maire's Modern Pigments and their Vehicles..................12mo, 
Mandel's Handbook for Bio-chemical Laboratory................. 12mo, \$1 50

* Martin's Laboratory Guide to Qualitative Analysis with the Blowpipe

Mason's Examination of Water. (Chemical and Bacteriological.).....12mo, 125 Water-supply. (Considered Principally from a Sanitary Standpoint.)

8vo,
Mathewson's First Principles of Chemical Theory................. 8vo, Matthews's Laboratory Manual of Dyeing and Textile Chemistry. . . . . 8vo,

Textile Fibres. 2d Edition, Rewritten.................. 8vo,

* Meyer's Determination of Radicles in Carbon Compounds. (Tingle.)

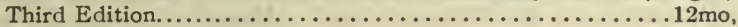

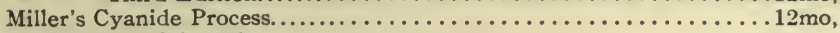

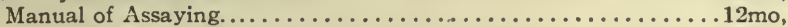

Minet's Production of Aluminum and its Industrial Use. (Waldo.)...12mo,

Mixter's Elementary Text-book of Chemistry................. 12mo,

Morgan's Elements of Physical Chemistry..................... 12mo,

Outline of the Theory of Solutions and its Results............12mo,

* Physical Chemistry for Electrical Engineers.................. 12mo,

Morse's Calculations used in Cane-sugar Factories.............. 16mo, mor.

* Muir's History of Chemical Theories and Laws................ 8vo,

Mulliken's General Method for the Identification of Pure Organic Compounds.

Vol. I. Compounds of Carbon with Hydrogen and Oxygen. Large 8vo,

Vol. II. Nitrogenous Compounds. (In Preparation).

Vol. III. The Commercial Dyestuffs. (In Press).

O'Driscoll's Notes on the Treatment of Gold Ores...................8vo,

Ostwald's Conversations on Chemistry. Part One. (Ramsey.)......12mo,

Part Two. (Turnbull.)....12mo,

Owen and Standage's Dyeing and Cleaning of Textile Fabrics..........12mo,

* Palmer's Practical Test Book of Chemistry. .....................12mo,

* Pauli's Physical Chemistry in the Service of Medicine. (Fischer.)..12mo,

Penfield's Tables of Minerals, Including the Use of Minerals and Statistics

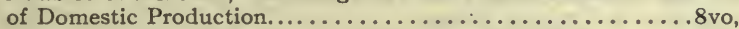

Pictet's Alkaloids and their Chemical Constitution. (Biddle.)........8vo,

Poole's Calorific Power of Fuels........................... 8vo,

Prescott and Winslow's Elements of Water Bacteriology, with Special Reference to Sanitary Water Analysis..................... 12mo,

Richards and Woodman's Air, Water, and Food from a Sanitary Stand-

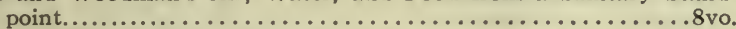

Ricketts and Miller's Notes on Assaying.................... . . . .

Rideal's Disinfection and the Preservation of Food.................8vo,

Sewage and the Bacterial Purification of Sewage............... 8 vo,

Rigg's Elementary Manual for the Chemical Laboratory.......... . . . . 8vo,

Robine and Lenglen's Cyanide Industry. (Le Clerc.). . . . . . . . . . . 8vo,

Ruddiman's Incompatibilities in Prescriptions...............

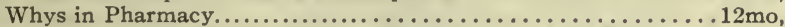

Ruer's Elements of Metallography. (Mathewson). (In Press.)

Sabin's Industrial and Artistic Technology of Paint and Varnish. ..... .8vo,

Salkowski's Physiological and Pathological Chemistry. (Orndorff.).....8vo,

Schimpf's Essentials of Volumetric Analysis.................... 12mo,

Manual of Volumetric Analysis. (Fifth Edition, Rewritten).... .8vo,

* Qualitative Chemical Analysis. . . . . . . . . . . . . . . . . 8vo,

Smith's Lecture Notes on Chemistry for Dental Students. . . . . . . . . . . 8vo,

Spencer's Handbook for Cane Sugar Manufacturers..............16mo, mor.

Handbook for Chemists of Beet-sugar Houses.............

Stockbridge's Rocks and Soils.........................

Stone's Practical Testing of Gas and Gas Meters................... . . . . . . .

* Tillman's Descriptive General Chemistry.................... 8vo,

* Elementary Lessons in Heat . . . . . . . . . . . . . . . . . . . . . .

Treadwell's Qualitative Analysis. (Hall.).................... . . . . . . .

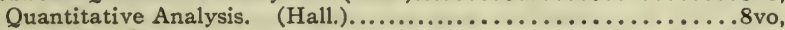

Turneaure and Russell's Public Water-supplies......................

Van Deventer's Physical Chemistry for Beginners. (Boltwood.)......12mo,

Venable's Methods and Devices for Bacterial Treatment of Sewage..... .8vo, Ward and Whipple's Freshwater Biology. (In Press.)

Ware's Beet-sugar Manufacture and Refining. Vol. I..............8vo, 
Washington's Manual of the Chemical Analysis of Rocks.............8vo, \$2 00

* Weaver's Military Explosives............................ 8vo, 300

Wells's Laboratory Guide in Qualitative Chemical Analysis...........8vo, 150

Short Course in Inorganic Qualitative Chemical Analysis for Engineering

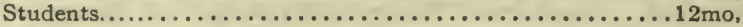

Text-book of Chemical Arithmetic....................... 12mo,

Whipple's Microscopy of Drinking-water......................

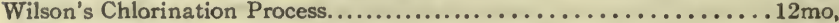

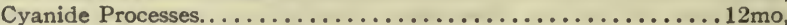

Winton's Microscopy of Vegetables Food...................... 8vo,

Zsigmondy's Colloids and the Ultramicroscope. (Alexander)...Large 12mo,

\section{CIVIL ENGINEERING.}

\section{BRIDGES AND ROOFS. HYDRAULICS. MATERIALS OF ENGINEER-} ING. RAILWAY ENGINEERING.

Baker's Engineers' Surveying Instruments.................... 12mo, Bixby's Graphical Computing Table............... Paper $19 \frac{1}{2} \times 24 \frac{1}{4}$ inches. Breed and Hosmer's Principles and Practice of Surveying. Vol. I. Elementary Surveying............................. 8 vo,

Vol. II. Higher Surveying......................... 8 vo * Burr's Ancient and Modern Engineering and the Isthmian Canal. .... 8vo, Comstock's Field Astronomy for Engineers....................8vo, * Corthell's Allowable Pressure on Deep Foundations .............12mo, Crandall's Text-book on Geodesy and Least Squares............... . . . .

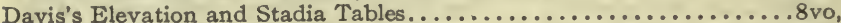

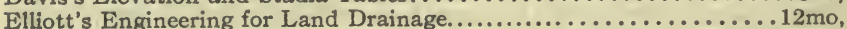
Practical Farm Drainage. (Second Edition Rewritten.)........12mo, * Fiebeger's Treatise on Civil Engineering..................... . 8 vo, Flemer's Photographic Methods and Instruments.................. 8 vo, Folwell's Sewerage. (Designing and Maintenance.)................ . 8vo, Freitag's Architectural Engineering.......................... . . . Goodhue's Municipal Improvements........................ . . . . . . . . . . . . * Hauch and Rice's Tables of Quantities for Preliminary Estimates... 12mo, Hayford's Text-book of Geodetic Astronomy.................. 8vo, Hering's Ready Reference Tables (Conversion Pactors)...........16mo, mor.

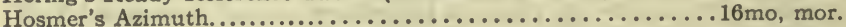

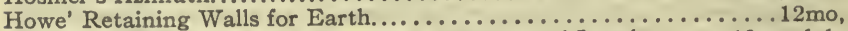
* Ives's Adjustments of the Engineer's Transit and Level. . . . . . . . . . . . . . . . . bds. Johnson's (J. B.) Theory and Practice of Surveying. ......... Large 12mo, Johnson's (L. J.) Statics by Algebraic and Graphic Methods............ . 8vo, Kinnicutt, Winslow and Pratt's Purification of Sewage. (In Preparation).

* Mahan's Descriptive Geometry.......................... . . . . . Merriman's Elements of Precise Surveying and Geodesy. . . . . . . . . 8vo, Merriman and Brooks's Handbook for Surveyors. . . . . . . . . . . . . . . . . mo, mor.

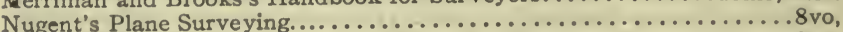

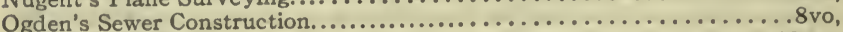

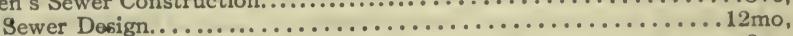
Parsons's Disposal of Municipal Refuse...................... 8vo,

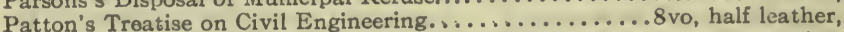
Reed's Topographical Drawing and Sketching................... . to, Rideal's Sewage and the Bacterial Purification of Sewage............. . . . . . . Riemer's Shaft-sinking under Difficult Conditions. (Corning and Peele.).8ro, Siebert and Biggin's Modern Stone-cutting and Masonry............ 8vo, Smith's Manual of Topographical Drawing. (McMillan.)...............

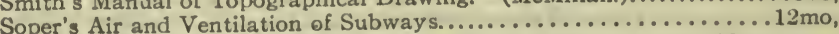
* Tracy's Exercises in Surveying. . . . . . . . . . . . . . . 12mo, mor.

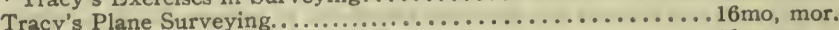

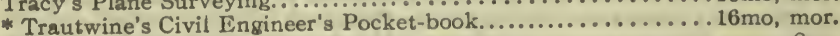

Venable's Garbage Crematories in America. ...................... . . . . . . . Methods and Devices for Bacterial Treatment of Sewage...........8vo, 
Wait's Engineering and Architectural Jurisprudence..............8vo, \$6 00

Sheep, 650

Law of Contracts................................. 8 vo, 300

Law of Operations Preliminary to Construction in Engineering and

Architecture. .............................. 500

Sheep, 550

Warren's Stereotomy-Problems in Stone-cutting................ 8 vo, 250

* Waterbury's Vest-Pocket Hand-book of Mathematics for Engineers.

$2 \frac{7}{8} \times 5 \frac{3}{8}$ inches, mor. 100

Webb's Problem's in the Use and Adjustment of Engineering Instruments.

16 mo, mor. 125

Wilson's Topographic Surveying.......................

\section{BRIDGES AND ROOFS.}

Boller's Practical Treatise on the Construction of Iron Highway Bridges..8vo,

* Thames River Bridge...................... Oblong paper,

Burr and Falk's Design and Construction of Metallic Bridges.........8vo,

Rois'se Lines for Bridge and Roof Computations................8vo, 300

Foster's Treatise on Wooden Trestle Bridges...................4to, 500

Fowler's Ordinary Fopndations............................ 8 ro, 350

Greene's Arches in Wood, Iron, and Stone......................

Bridge Trusses........................................ 250

Roof Trusses................................................. 125

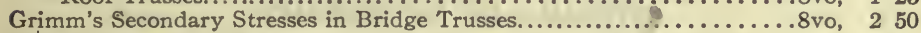

Heller's Stresses in Structures and the Accompanying Deformations... .8vo, 300

Howe's Design of Simple Roof-trusses in Wood and Steel.............8vo. 200 Symmetrical Masonry Arches............................8vo, 250 Treatise on Arches............................... 8vo, 400

Johnson, Bryan and Turneaure's Theory and Practice in the Designing of Modern Framed Structures................................ 4to, 1000

Merriman and Jacoby's Text-book on Roofs and Bridges:

Part I. Stresses in Simple Trusses...................... 2 vo, 250

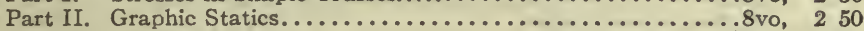

Part III. Bridge Design.......................... 8 vo, 250

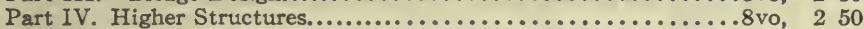

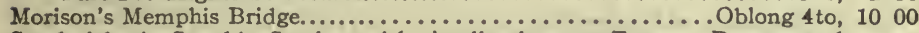

Sondericker's Graphic Statics, with Applications to Trusses, Beams, and

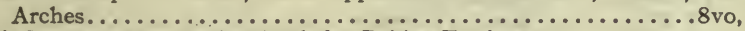

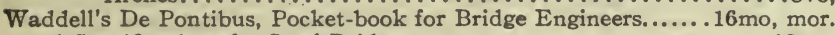

* Specifications for Steel Bridges...............................

Waddell and Harringtoon's Bridge Engineering. (In Preparation.)

Wright's Designing of Draw-spans. Two parts in one volume..........8vo,

\section{HYDRAULICS.}

Barnes's Ice Formation. .............................

Bazin's Experiments upon the Contraction of the Liquid Vein Issuing from

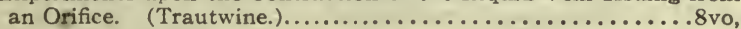

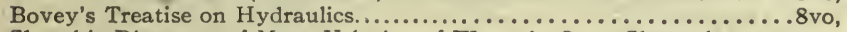
Church's Diagrams of Mean Velocity of Water in Open Channels.

Hydraulic Motors Oblong 4to, paper,

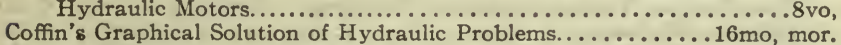

Flather's Dynamometers, and the Measurement of Power............12mo,

Folwell's Water-supply Engineering. . . . . . . . . . . . . . . . . . .

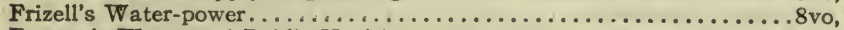

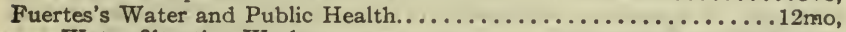

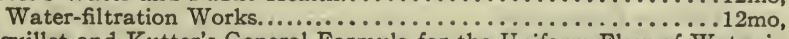

Ganguillet and Kutter's General Formula for the Uniform Flow of Water in Rivers and Other Channels. (Hering and Trautwine.).....8vo, 
Hazen's Clean Water and How to Get It............................ $12 \mathrm{mo}, \$ 150$

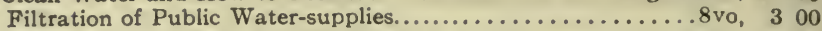

Hazelhurst's Towers and Tanks for Water-works................ 8vo, 250

Herschel's 115 Experiments on the Carrying Capacity of Large, Riveted, Metal

Conduits............................................ 200

Hoyt and Grover's River Discharge...................... 8vo, 200 Hubbard and Kiersted's Water-works Management and Maintenance.

* Lyndon's Development and Electrical Distribution of Water Power.

Mason's Water-supply. (Considered Principally from a Sanitary Stand-

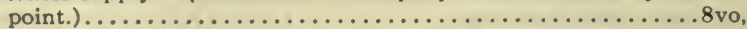

Merriman's Treatise on Hydraulics........................... . . . . .

* Molitor's Hydraulics of Rivers, Weirs and Sluices............... . 8vo,

* Richards's Laboratory Notes on Industrial Water Analysis. . . . . . . . 8vo,

Schuyler's Reservoirs for Irrigation, Water-power, and Domestic Watersupply. Second Edition, Revised and Enlarged........ Large 8vo,

* Thomas and Watt's Improvement of Rivers..................

Turneaure and Russell's Public Water-supplies................... 8vo,

Wegmann's Design and Construction of Dams. 5th Ed., enlarged......4 to,

Water-Supply of the City of New York from 1658 to $1895 \ldots . . . \ldots .4$ to,

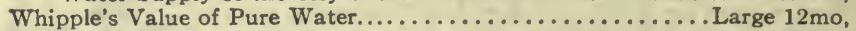
Williams and Hazen's Hydraulic Tables. . . . . . . . . . . . . . . . . . . . 8 vo,

Wilson's Irrigation Engineering.........................

Wood's Turbines....................................... . . . . .

\section{MATERIALS OF ENGINEERING.}

Baker's Roads and Pavements........................ 8vo, 500

Treatise on Masonry Construction.............................. 500

Black's United States Public Works.................... Oblong 4to, 500

Blanchard's Bituminous Roads. (In Press.)

Bleininger's Manufacture of Hydraulic Cement. (In Preparation.)

* Bovey's Strength of Materials and Theory of Structures.............8vo, 7 50

Burr's Elasticity and Resistance of the Materials of Engineering........ 8vo, $7 \quad 50$

Byrne's Highway Construction........................... 8vo, 500

Inspection of the Materials and Workmanship Employed in Construction. $16 \mathrm{mo}, 300$

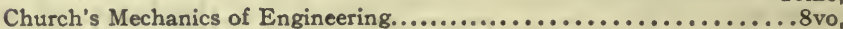
Du Bois's Mechanics of Engineering.

Vol. I. Kinematics, Statics, Kinetics................. Small 4to,

Vol. II. The Stresses in Framed Structures, Strength of Materials and Theory of Flexures................................................ 1000

* Eckel's Cements, Limes, and Plasters........................8vo, 600 Stone and Clay Products used in Engineering. (In Preparation.)

Fowler's Ordinary Foundations........................... 8vo, 350

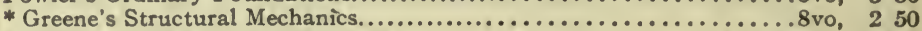

* Holley's Lead and Zinc Pigments.................... Large 12mo, 300

Holley and Ladd's Analysis of Mixed Paints, Color Pigments and Varnishes. Large $12 \mathrm{mo}$,

Johnson's (C. M.) Rapid Methods for the Chemical Analysis of Special Steels, Steel-making Alloys and Graphite................. Large 12mo,

Johnson's (J. B.) Materials of Construction................. Large 8vo,

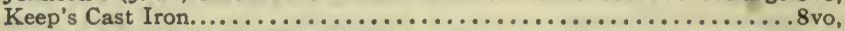

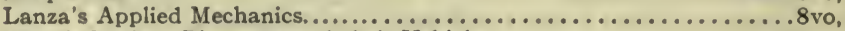
Maire's Modern Pigments and their Vehicles....................12mo, Martens's Handbook on Testing Materials. (Henning.) 2 vols...... . 8vo, Maurer's Technical Mechanics............................. . . . . . Merrill's Stones for Building and Decoration.................... . . . . . . Merriman's Mechanics of Materials...................... . . .

* Strength of Materials............................. 12mo,

Metcalf's Steel. A Manual for Steel-users.................... 12mo,

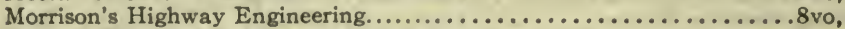
Patton's Practical Treatise on Foundations.................... . . . . . Rice's Concrete Block Manufacture.......................... . . . . . 
Richardson's Modern Asphalt Pavements....................8vo, \$3 00

Richey's Building Foreman's Pocket Book and Ready Reference.16mo,mor.

* Cement Workers' and Plasterers' Edition (Building Mechanics' Ready

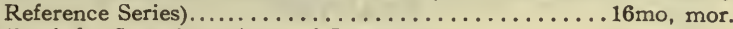

Handbook for Superintendents of Construction...........16mo, mor.

* Stone and Brick Masons' Edition (Building Mechanics' Ready

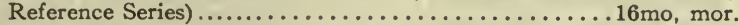

* Ries's Clays: Their Occurrence, Properties, and Uses............... . . . .

* Ries and Leighton's History of the Clay-working Industry of the United

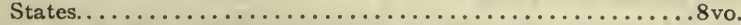

Sabin's Industrial and Artistic Technology of Paint and Varnish....... 8vo,

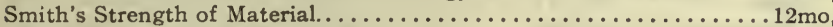

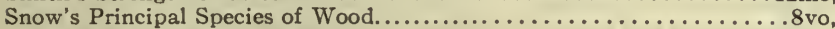

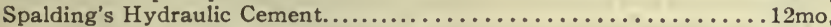

Text-book on Roads and Pavements....................... 12mo,

Taylor and Thompson's Treatise on Concrete, Plain and Reinforced....8vo,

Thurston's Materials of Engineering. In Three Parts..............

Part I. Non-metallic Materials of Engineering and Metallurgy... .8vo,

Part II. Iron and Steel..........................

Part III. A Treatise on Brasses, Bronzes, and Other Alloys and their

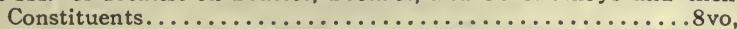

Tillson's Street Pavements and Paving Materials................8vo,

Turneaure and Maurer's Principles of Reinforced Concrete Construction.

Second Edition, Revised and Enlarged...............

Waterbury's Cement Laboratory Manual. . . . . . . . . . . . . . . . 12mo,

Wood's (De V.) Treatise on the Resistance of Materials, and an Appendix on

the Preservation of Timber.....................

Wood's (M. P.) Rustless Coatings: Corrosion and Electrolysis of Iron and

Steel.........................................

\section{RAILWAY ENGINEERING.}

Andrews's Handbook for Street Railway Engineers...... $3 \times 5$ inches, mor.

Berg's Buildings and Structures of American Railroads.............

Brooks's Handbook of Street Railroad Location.............16mo, mor.

Butts's Civil Engineer's Field-book.....................16mo, mor.

Crandall's Railway and Other Earthwork Tables..................8vo,

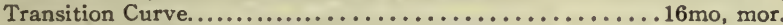

* Crockett's Methods for Earthwork Computations............... . 8vo,

Dredge's History of the Pennsylvania Railroadd. (1879)............... Papes

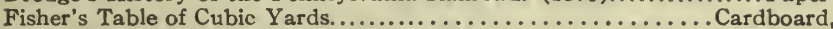

Godwin's Railroad Engineers' Field-book and Explorers' Guide..16mo, mor.

Hudson's Tables for Calculating the Cubic Contents of Excavations and Fmbankments..............................

Ives and Hilts's Problems in Surveying, Railroad Surveying and Geodesy

$16 \mathrm{mo}$, mor.

Molitor and Beard's Manual for Resident Engineers...............16mo,

Nagle's Field Manual for Railroad Engineers.................16mo, mor.

* Orrock's Railroad Structures and Estimates.....................8vo,

Philbrick's Field Manual for Engineers............................ mor.

Raymond's Railroad Engineering. 3 volumes.

Vol. I. Railroad Field Geometry. (In Preparation.)

Vol. II. Elements of Railroad Engineering. . . . . . . . . . . . 8vo,

Vol. III. Railroad Engineer's Field Book. (In Preparation.)

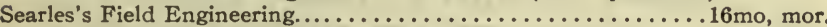

Railroad Spiral..............................mo, mor.

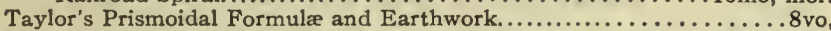

* Trautwine's Field Practice of Laying Out Circular Curves for Railroads. $12 \mathrm{mo}$, mor.

* Method of Calculating the Cubic Contents of Excavations and Embankments by the Aid of Diagrams....................

Webb's Economics of Railroad Construction........................ . $12 \mathrm{mo}$,

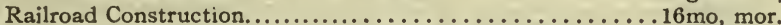

Wellington's Economic Theory of the Location of Railways.....Large 12mo,

Wilson's Elements of Railroad-Track and Construction.............12mo, 


\section{DRAWING.}

Barr's Kinematics of Machinery.......................... 8 vo, $\$ 250$

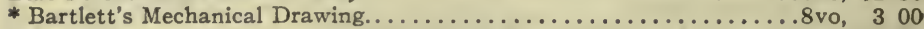

* " " " Abridged Ed....................... 150

Coolidge's Manual of Drawing.......................................... paper, 100

Coolidge and Freeman's Elements of General Drafting for Mechanical Engi-

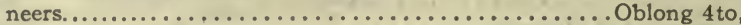

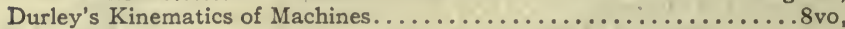

Emch's Introduction to Projective Geometry and its Application..... .8vo,

French and Ives' Stereotomy.............................

Hill's Text-book on Shades and Shadows, and Perspective............8vo,

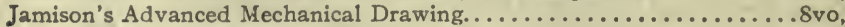

Elements of Mechanical Drawing......................

Jones's Machine Design:

Part I. Kinematics of Machinery...................... 1 vo, 150

Part II. Form, Strength, and Proportions of Parts.............8vo, 300

Kimball and Barr's Machine Design. (In Press.)

MacCord's Elements of Descritpive Geometry.................8vo, 300

Kinematics; or, Practical Mechanism.............................. 500

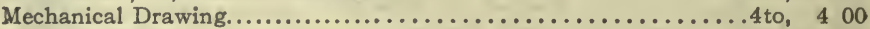

Vclocity Diagrams.................................8vo, 150

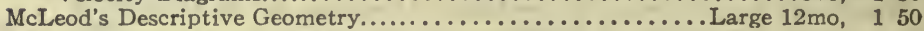

* Mahan's Descriptive Geometry and Stone-cutting.............8vo, 150

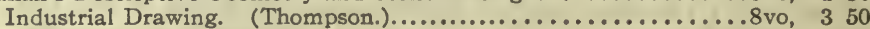

Moyer's Descriptive Geometry............................8vo, 200

Reed's Topographical Drawing and Sketching...................4to, 500

Reid's Course in Mechanical Drawing.......................8vo, 200

Text-book of Mechanical Drawing and Elementary Machine Design..8vo, 300

Robinson's Principles of Mechanism........................ 8vo, 300

Schwamb and Merrill's Elements of Mechanism...................8vo, 300

Smith (A. W.) and Marx's Machine Design......................8vo, 300

Smith's (R. S.) Manual of Topographical Drawing. (McMillan).......8vo, 250

* Titsworth's Elements of Mechanical Drawing............. Oblong 8vo, 125

Warren's Drafting Instruments and Operations................. 12mo, 125

Elements of Descriptive Geometry, Shadows, and Perspective.....8vo, 350

Elements of Machine Construction and Drawing...............8vo, 750

Elements of Plane and Solid Free-hand Geometrical Drawing.....12mo, 100

General Problems of Shades and Shadows.................. 8vo, 300

Manual of Elementary Problems in the Linear Perspective of Forms and

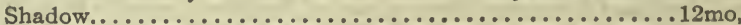

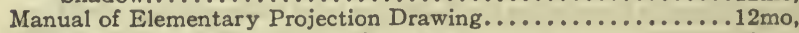

Plane Problems in Elementary Geometry................... 12mo,

Problems, Theorems, and Examples in Descriptive Geometry.......8vo,

Weisbach's Kinematics and Power of Transmission. (Hermann and

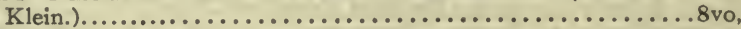

Wilson's (H. M.) Topographic Surveying.................... . 8vo,

* Wilson's (V. T.) Descriptive Geometry........................ . 8vo,

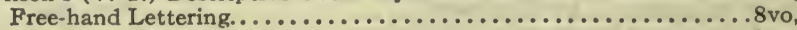

Free-hand Perspective.............................. 8vo,

Woolf's Elementary Course in Descriptive Geometry........... Large 8vo,

\section{ELECTRICITY AND PHYSICS.}

* Abegg's Theory of Electrolytic Dissociation. (von Ende.)........12mo, Andrews's Hand-book for Street Railway Engineering.....3 35 inches, mor. Anthony and Brackett's Text-book of Physics. (Magie.)..... Large 12mo, Anthony and Ball's Lecture-notes on the Theory of Electrical Measure-

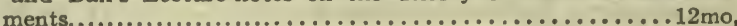

Benjamin's History of Electricity..................... 
Betts's Lead Refining and Electrolysis..................... 8vo, $\$ 400$ Classen's Quantitative Chemical Analysis by Electrolysis. (Boltwood.).8vo, 300

* Collins's Manual of Wireless Telegraphy and Telephony...........12mo, 150

* Mor. 200

Crehore and Squier's Polarizing Photo-chronograph................8vo, 300

* Danneel's Electrochemistry. (Merriam.............................. 125

Dawson's "Engineering" and Electric Traction Pocket-book....16mo, mor. 500

Dolezalek's Theory of the Lead Accumulator (Storage Battery). (von Ende.)

$12 \mathrm{mo}, 250$

Duhem's Thermodynamics and Chemistry. (Burgess.)............ 8vo, 400

Flather's Dynamometers, and the Measurement of Power..........12mo, 300

Getman's Introduction to Physical Science. . . . . . . . . . . . . . 12mo,

Gilbert's De Magnete. (Mottelay).............................8vo,

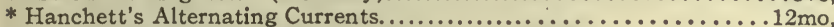

Hering's Ready Reference Tables (Conversion Factors).........16mo, mor.

* Hobart and Ellis's High-speed Dynamo Electric Machinery... . . . . . 8vo,

Holman's Precision of Measurements..................... . . . . Telescopic Mirror-scale Method, Adjustments, and Tests....Large 8vo,

* Karapetoff's Experimental Electrical Engineering................ . 8vo, Kinzbrunner's Testing of Continuous-current Machines............. . 8vo,

Landauer's Spectrum Analysis. (Tingle.)................... 8vo, 300

Le Chatelier's High-temperature Measurements. (Boudouard-Burgess.) 12mo, 300

Löb's Electrochemistry of Organic Compounds. (Lorenz)..............8vo, 300

* Lyndon's Development and Electrical Distribution of Water Power. .8vo, 300

* Lyons's Treatise on Electromagnetic Phenomena. Vols, I and II. 8vo, each, 600

* Michie's Elements of Wave Motion Relating to Sound and Light.... .8vo, 400

Morgan's Outline of the Theory of Solution and its Results.........12mo, 100

* Physical Chemistry for Electrical Engineers..................... 150

* Norris's Introduction to the Study of Electrical Engineering........ 8vo, 250

Norris and Dennison's Course of Problems on the Electrical Characteristics of Circuits and Machines. (In Press.)

* Parshall and Hobart's Electric Machine Design..........4to, half mor, 1250

Reagan's Locomotives: Simple, Compound, and Electric. New Edition.

Large $12 \mathrm{mo}, 350$

* Rosenberg's Electrical Engineering. (Haldane Gee-Kinzbrunner.). 8vo, 200 Ryan, Norris, and Hoxie's Electrical Machinery. Vol. I.............8vo, 250 Schapper's Laboratory Guide for Students in Physical Chemistry.....12mo, 100

* Tillman's Elementary Lessons in Heat.....................8vo, 150

Tory and Pitcher's Manual of Laboratory Physics................................... 200

Ulke's Modern Electrolytic Copper Refining....................8vo, 300

\section{LAW.}

* Brennan's Hand-book of Useful Legal Information for Business Men. $16 \mathrm{mo}$, mor.

* Davis's Elements of Law................................ 8vo,

* Treatise on the Military Law of United States............... . . . . . * Dudley's Military Law and the Procedure of Courts-martial.. Large $12 \mathrm{mo}$,

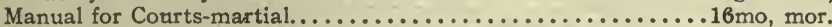
Wait's Engineering and Architectural Jurisprudence............... 8vo,

Sheep,

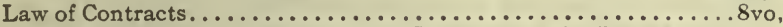
Law of Operations Preliminary to Construction in Engineering and

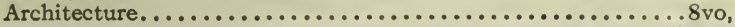

\section{MATHEMATICS.}

Baker's Elliptic Functions...............................

Briggs's Elements of Plane Analytic Geometry. (Bôcher)......... 12mo, 
Byerley's Harmonic Functions. . . . . . . . . . . . . . . . . . 8vo, \$1 00

Chandler's Elements of the Infinitesimal Calculus...............

* Coffin's Vector Analysis. ........................... 12mo, 250

Compton's Manual of Logarithmic Computations..............12mo, 150

* Dickson's College Algebra........................................ 12mo, 150

* Introduction to the Theory of Algebraic Equations... . . Large 12mo, 125

Emch's Introduction to Projective Geometry and its Application... . .8vo, 250

Fiske's Functions of a Complex Variable...................8vo, 100

Halsted's Elementary Synthetic Geometry................... 8vo, 150

Elements of Geometry.......................... 8vo, 175

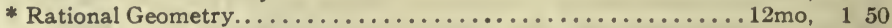

Hyde's Grassmann's Space Analysis. . . . . . . . . . . . . . . . . . . 8vo, 100

* Johnson's (J. B.) Three-place Logarithmic Tables: Vest-pocket size, paper, 15

100 copies, 500

* Mounted on heavy cardboard, $8 \times 10$ inches, 25

10 copies, 200

Johnson's (W. W.) Abridged Editions of Differential and Integral Calculus. Large $12 \mathrm{mo}, 1$ vol. 250

Curve Tracing in Cartesian Co-ordinates.................12mo, 100

Differential Equations.............................. 100

Elementary Treatise on Differential Calculus............................. 12mo, 150

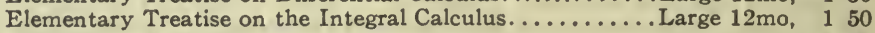

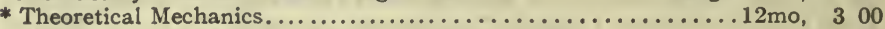

Theory of Errors and the Method of Least Squares............. 12mo, 150

Treatise on Differential Calculus..................................... 300

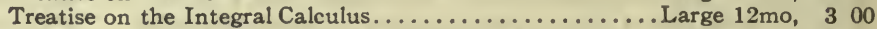

Treatise on Ordinary and Partial Differential Equations... Large $12 \mathrm{mo}, 350$

Karapetoff's Engineering Applications of Higher Mathematics.

(In Preparation.)

Laplace's Philosophical Essay on Probabilities. (Truscott and Emory.).12mo,

* Ludlow and Bass's Elements of Trigonometry and Logarithmic and Other

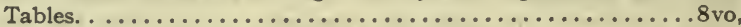

* Trigonometry and Tables published separately. ............ Each,

* Ludlow's Logarithmic and Trigonometric Tables. . . . . . . . . . . . . . . . 8vo, Macfarlane's Vector Analysis and Quaternions. . . . . . . . . . . . . . . . . . .

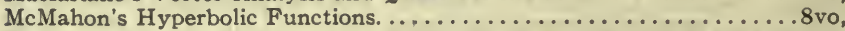
Manning's Irrational Numbers and their Representation by Sequences and

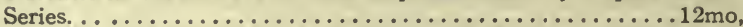

Mathematical Monographs. Edited by Mansfield Merriman and Robert

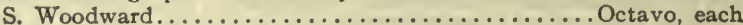

No. 1. History of Modern Mathematics, by David Eugene Smith.

No. 2. Synthetic Projective Geometry, by George Bruce Halsted.

No. 3. Determinants, by Laenas Gifford Weld. No. 4. Hyper-

bolic Functions, by James McMahon. No. 5. Harmonic Functions, by William E. Byerly. No. 6. Grassmann's Space Analysis, by Edward W. Hyde. No. 7. Probability and Theory of Errors, by Robert S. Woodward. No. 8. Vector Analysis and Quaternions, by Alexander Macfarlane. No. 9. Differential Equations, by

William Woolsey Johnson. No. 10. The Solution of Equations, by Mansfield Merriman. No. 11. Functions of a Complex Variable,

by Thomas S. Fiske.

Maurer's Technical Mechanics. ........................8vo,

Merriman's Method of Least Squares. .................... . . . .

Solution of Equations. .............................

Rice and Johnson's Differential and Integral Calculus. 2 vols. in one.

Elementary Treatise on the Differential Calculus. ......... Large $12 \mathrm{mo}$,

Smith's History of Modern Mathematics. . . . . . . . . . . . . . . . 8vo,

* Veblen and Lennes's Introduction to the Real Infinitesimal Analysis of One

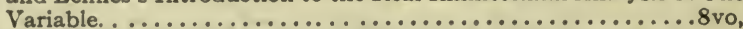

* Waterbury's Vest Pocket Hand-book of Mathematics for Engineers.

Weld's Determinants. . . . . . . . . . . . . . . . . . . . . . . . . 8vo,

Wood's Elements of Co-ordinate Geometry. . . . . . . . . . . . . . . . 8vo,

Woodward's Probability and Theory of Errors. . . . . . . . . . . . . . . 8vo, 


\section{MECHANICAL ENGINEERING.}

\section{MATERIALS OF ENGINEERING, STEAM-ENGINES AND BOILERS.}

Bacon's Forge Practice. .......................................... $\$ 150$

Baldwin's Steam Heating for Buildings............................. 250

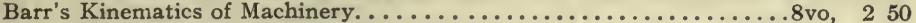

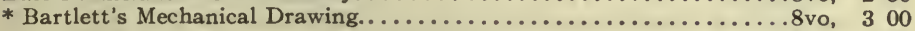

* " " " Abridged Ed...............8vo, 150

* Burr's Ancient and Modern Engineering and the Isthmian Canal. . . .8vo, 350

Carpenter's Experimental Engineering. ........................ 600

Heating and Ventilating Buildings..................... 8 vo, 400

Clerk's Gas and Oil Engine. (New edition in press.)

Compton's First Lessons in Metal Working................12mo, 150

Compton and De Groodt's Speed Lathe. . . . . . . . . . . . . 50

Coolidge's Manual of Drawing.................................. paper, 100

Coolidge and Freeman's Elements of Geenral Drafting for Mechanical Engineers. . . . . . . . . . . . . . . . . . . . .

Cromwell's Treatise on Belts and Pulleys. ................... 12mo,

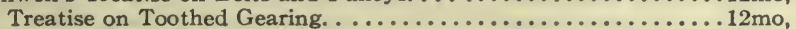

Dingey's Machinery Pattern Making.......................... 12mo,

Durley's Kinematics of Machines. . . . . . . . . . . . . . . . . . . . . . . . . . . . . . .

Flanders's Gear-cutting Machinery. ........................... $12 \mathrm{mo}$,

Flather's Dynamometers and the Measurement of Power. .........12mo,

Rope Driving. . . . . . . . . . . . . . . . . . . . . 12mo,

Gill's Gas and Fuel Analysis for Engineers. . ................... 12mo,

Goss's Locomotive Sparks. . . . . . . . . . . . . . . . . . . . . . 8vo,

Greene's Pumping Machinery. (In Preparation.)

Hering's Ready Reference Tables (Conversion Factors). . . . . . 16mo, mor.

* Hobart and Ellis's High Speed Dynamo Electric Machinery. . . . . . .8vo,

Hutton's Gas Engine. ............................ 8vo,

Jamison's Advanced Mechanical Drawing. .................. . . . Elements of Mechanical Drawing.......................8vo,

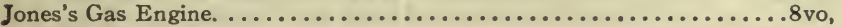
Machine Design:

Part I. Kinematics of Machinery..................... 8vo,

Part II. Form, Strength, and Proportions of Parts. . . . . . . . . 8vo,

Kent's Mechanical Engineer's Pocket-Book. ....................... mor.

Kerr's Power and Power Transmission. . . . . . . . . . . . . . . . 8vo, Kimball and Barr's Machine Design. (In Press.)

Levin's Gas Engine. (In Press.)........................ 8vo, Leonard's Machine Shop Tools and Methods. ...................8vo,

* Lorenz's Modern Refrigerating Machinery. (Pope, Haven, and Dean)..8vo,

MacCord's Kinematics; or, Practical Mechanism.................8vo,

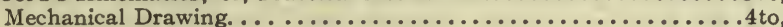

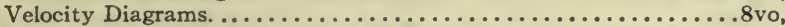

MacFarland's Standard Reduction Factors for Gases. . . . . . . . . . . . 8vo,

Mahan's Industrial Drawing. (Thompson.). . . . . . . . . . . . . 8vo,

Mehrtens's Gas Engine Theory and Design......................... 12mo,

Oberg's Handbook of Small Tools. ............................... 12mo,

* Parshall and Hobart's Electric Machine Design. Small 4to, half leather,

Peele's Compressed Air Plant for Mines. . . . . . . . . . . . . . . . 8vo,

Poole's Calorific Power of Fuels. . . . . . . . . . . . . . . . . . . 8vo,

* Porter's Engineering Reminiscences, 1855 to $1882 \ldots \ldots \ldots \ldots \ldots \ldots \ldots$. . . . . . . . . .

Reid's Course in Mechanical Drawing. . . . . . . . . . . . . . . . 8vo

Text-book of Mechanical Drawing and Elementary Machine Design.8vo,

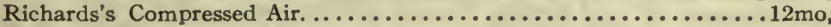

Robinson's Principles of Mechanism. . . . . . . . . . . . . . . . . . . 8vo,

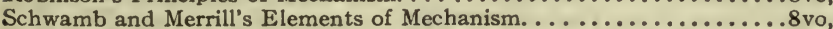

Smith (A. W.) and Marx's Machine Design. . . . . . . . . . . . . . . 8vo,

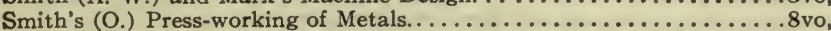
Sorel's Carbureting and Combustion in Alcohol Engines. (Woodward and

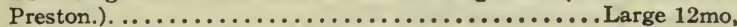

Stone's Practical Testing of Gas and Gas Meters.................. 
Thurston's Animal as a Machine and Prime Motor, and the Laws of Energetics.

Treatise on Friction and Lost Work in Machinery and Mill Work. . 8vo, 300

* Tillson's Complete Automobile Instructor. . . . . . . . . . . . . 16mo, 150

* Titsworth's Elements of Mechanical Drawing.............. Oblong 8vo, 125

Warren's Elements of Machine Construction and Drawing. .........8vo, 750

* Waterbury's Vest Pocket Hand-book of Mathematics for Engineers.

$2 \frac{7}{8} \times 5 \frac{3}{8}$ inches, mor. 100

Weisbach's Kinematics and the Power of Transmission. (Herrmann-

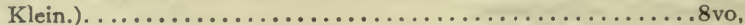

Machinery of Transmission and Governors. (Hermann-Klein.). .8vo,

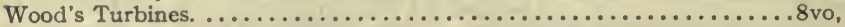

\section{MATERIALS OF ENGINEERING.}

* Bovey's Strength of Materials and Theory of Structures. . . . . . . . .8vo, Burr's Elasticity and Resistance of the Materials of Engineering. . . . . .8vo, Church's Mechanics of Engineering. . . . . . . . . . . . . . . . . . 8vo,

* Greene's Structural Mechanics. . . . . . . . . . . . . . . . . . . . 8vo,

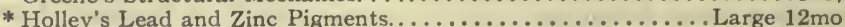
Holley and Ladd's Analysis of Mixed Paints, Color Pigments, and Varnishes.

Large $12 \mathrm{mo}$,

Johnson's (C. M.) Rapid Methods for the Chemical Analysis of Special Steels, Steel-Making Alloys and Graphite.......... Large 12mo, Johnson's (J. B.) Materials of Construction. . . . . . . . . . . . . . . 8vo, Keep's Cast Iron. . . . . . . . . . . . . . . . . . . . . . . 8vo, Lanza's Applied Mechanics. ............................ . . . . . .

Maire's Modern Pigments and their Vehicles. ................. 12mo, Martens's Handbook on Testing Materials. (Henning.). ........... 8vo, Maurer's Techincal Mechanics. ......................... . . . Merriman's Mechanics of Materials....................... . . . .

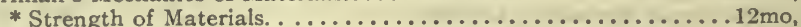

Metcalf's Steel. A Manual for Steel-users..................... 12mo,

Sabin's Industrial and Artistic Technology of Paint and Varnish. . . . .8vo,

Smith's ((A. W.) Materials of Machines. . . . . . . . . . . . . . . 12mo,

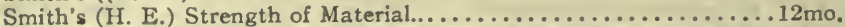

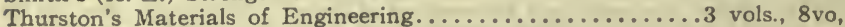

Part I. Non-metallic Materials of Engineering, ............. . . . . .

Part II. Iron and Steel. . . . . . . . . . . . . . . . . . . . . . .

Part III. A Treatise on Brasses, Bronzes, and Other Alloys and their

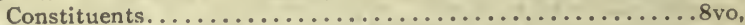

Wood's (De V.) Elements of Analytical Mechanics. .............. 8vo,

Treatise on the Resistance of Materials and an Appendix on the

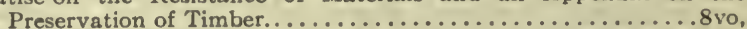

Wood's (M. P.) Rustless Coatings: Corrosion and Electrolysis of Iron and

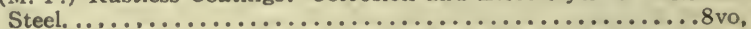

\section{STEAM-ENGINES AND BOILERS.}

Berry's Temperature-entropy Diagram..................... 12mo, Carnot's Reflections on the Motive Power of Heat. (Thurston.).....12mo,

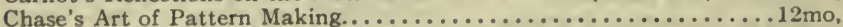
Creighton's Steam-engine and other Heat Motors.............. . . . . Dawson's "Engineering" and Electric Traction Pocket-book....16mo, mor. Ford's Boiler Making for Boiler Makers. . . . . . . . . . . . . . . . 18mo, * Gebhardt's Steam Power Plant Engincering. . . . . . . . . . . . . . 8vo, Goss's Locomotive Performance. . . . . . . . . . . . . . . . . . . . . . . 8vo, Hemenway's Indicator Practice and Steam-engine Economy... . . . . . 12mo. Hutton's Heat and Heat-engines. . . . . . . . . . . . . . . . . . . . Mechanical Engineering of Power Plants. . . . . . . . . . . . . . 8vo,

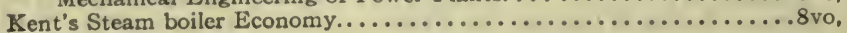


Kneass's Practice and Theory of the Injector. . . . . . . . . . . . . 8 vo, $\$ 150$

MacCord's Slide-valves. ..............................8vo, 200

Meyer's Modern Locomotive Construction. .....................4to, 1000

Moyer's Steam Turbine. ............................ 8vo, 400

Peabody's Manual of the Steam-engine Indicator. .............. 12mo, 150

Tables of the Properties of Steam and Other Vapors and Temperature-

Entropy Table. ............................ 8vo,

Thermodynamics of the Steam-engine and Other Heat-engines. . . 8vo.

Valve-gears for Steam-engines....................

Peabody and Miller's Steam-boilers. .................... 8 vo,

Pupin's Thermodynamics of Reversible Cycles in Gases and Saturated Vapors.

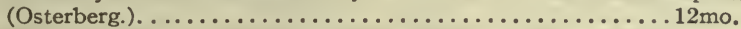

Reagan's Locomotives: Simple, Compound, and Electric. New Edition.

Large $12 \mathrm{mo}, 350$

Sinclair's Locomotive Engine Running and Management. . . . . . . . 12mo, 200

Smart's Handbook of Engineering Laboratory Practice. . . . . . . . . 12mo, 250

Snow's Steam-boiler Practice. ......................... 8vo, 300

Spangler's Notes on Thermodynamics. .................... 12mo, 100

Valve-gears. .......................................... 250

Spangler, Greene, and Marshall's Elements of Steam-engineering. . . . . 8vo. 300

Thomas's Steam-turbines. .......................... 8vo, 400

Thurston's Handbook of Engine and Boiler Trials, and the Use of the Indi-

cator and the Prony Brake....................

Handy Tables.....................................

Manual of Steam-boilers, their Designs, Construction, and Operation 8vo,

Manual of the Steam-engine............................ 8vo.

Part I. History, Structure, and Theory. . . . . . . . . . . 8vo,

Part II. Design, Construction, and Operation............. . . . . .

Steam-boiler Explosions in Theory and in Practice............. Wehrenfenning's Analysis and Softening of Boiler Feed-water. (Patterson).

Weiso, 400
8vo 00 .

(Du Bois.)..........8vo. 500

Whitham's Steam-engine Design. ............................. 500

Wood's Thermodynamics, Heat Motors, and Refrigerating Machines. . .8vo, 400

\section{MECHANICS PURE AND APPLIED.}

Church's Mechanics of Engineering. . . . . . . . . . . . . . . . . . 8vo,

Notes and Examples in Mechanics. ....................... . . . . . . . . .

Dana's Text-book of Elementary Mechanics for Colleges and Schools .12mo, Du Bois's Elementary Principles of Mechanics:

Vol. I. Kinematics. ...................

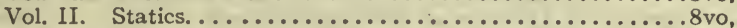

Mechanics of Engineering. Vol. I. ............................

Vol. II ......................................... 750

* Greene's Structural Mechanics. ................................ 250 James's Kinematics of a Point and the Rational Mechanics of a Particle.

Large $12 \mathrm{mo}, 200$

* Johnson's (W. W.) Theoretical Mechanics. . . . . . . . . . . . . . . . 12mo,

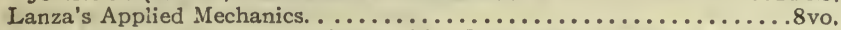

* Martin's Text Book on Mechanics, Vol. I, Statics................ 12mo, * Vol. II, Kinematics and Kinetics.12mo.

Maurer's Technical Mechanics. ......................... 8 vo.

* Merriman's Elements of Mechanics. . . . . . . . . . . . . . . . . 12mo,

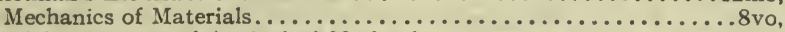

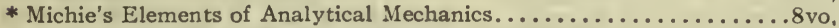

Robinson's Principles of Mechanism. . . . . . . . . . . . . . . . . . . . . .

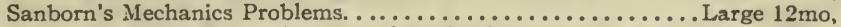

Schwamb and Merrill's Elements of Mechanism. . . . . . . . . . . . . 8 vo,

Wood's Elements of Analytical Mechanics. . . . . . . . . . . . . . . . .

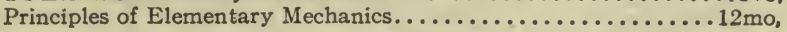




\section{MEDICAL.}

* Abderhalden's Physiological Chemistry in Thirty Lectures. (Hall and Defren.)....................................... $\$ 500$ von Behring's Suppression of Tuberculosis. (Bolduan.)..........12mo, 100 Bolduan's Immune Sera. . ............................. 150

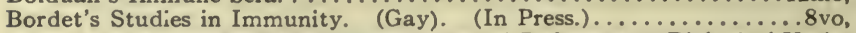
Daven port's Statistical Methods with Special Reference to Biological Varia-

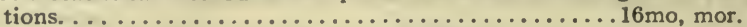

Ehrlich's Collected Studies on Immunity. (Bolduan.). . ......... 8vo,

* Fischer's Physiology of Alimentation..................... $12 \mathrm{mo}$, de Fursac's Manual of Psychiatry. (Rosanoff and Collins.)....Large 12mo, Hammarsten's Text-book on Physiological Chemistry. (Mandel.). ... 8vo, Jackson's Directions for Laboratory Work in Physiological Chemistry..8vo, Lassar-Cohn's Practical Urinary Analysis. (Lorenz.)............. 12mo, Mandel's Hand-book for the Bio-Chemical Laboratory...........12mo, * Pauli's Physical Chemistry in the Service of Medicine. (Fischer.)..12mo,

* Pozzi-Escot's Toxins and Venoms and their Antibodies. (Cohn.). 12mo, Rostoski's Serum Diagnosis. (Bolduan.).......................... Ruddiman's Incompatibilities in Prescriptions...............

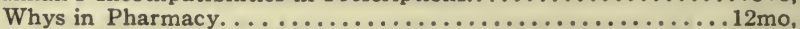
Salkowski's Physiological and Pathological Chemistry. (Orndorff.) ....8vo,

* Satterlee's Outlines of Human Embryology. ................. 12mo, Smith's Lecture Notes on Chemistry for Dental Students........... 8 vo,

* Whipple's Tyhpoid Fever. ..................... Large 12mo, Woodhull's Notes on Military Hygiene.......................

* Personal Hygiene. . . . . . . . . . . . . . . . . . . . . . Worcester and Atkinson's Small Hospitals Establishment and Maintenance, and Suggestions for Hospital Architecture, with Plans for a Small Hospital. ., ..............................12mo.

\section{METALLURGY.}

Betts's Lead Refining by Electrolysis. .................... 8 vo, Bolland's Encyclopedia of Founding and Dictionary of Foundry Terms used

in the Practice of Moulding. .......................

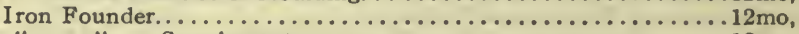
Supplement.........................

Douglas's Untechnical Addresses on Technical Subjects. .......... Goesel's Minerals and Metals: A Reference Book...............

* Iles's Lead-smelting. . ..................................... Johnson's Rapid Methods for the Chemical Analysis of Special Steels, Steel-making Alloys and Graphite.................... 12mo,

Keep's Cast Iron. .................................. Le Chatelier's High-temperature Measurements. (Boudouard-Burgess.)

$12 \mathrm{mo}$,

Metcalf's Steel. A Manual for Steel-users..................... 12mo. Minet's Production of Aluminum and its Industrial Use. (Waldo.). . 12mo, Ruer's Elements of Metallography. (Mathewson)................. Smith's Materials of Machines. .............................. Tate and Stone's Foundry Practice............................ Thurston's Materials of Engineering. In Three Parts. ........... Part I. Non-metallic Materials of Engineering, see Civil Engineering, page 9.

Part II. Iron and Steel. . .....................

Part III. A Treatise on Brasses, Bronzes, and Other Alloys and their Constituents.................................

Ulke's Modern Electrolytic Copper Refining................. West's American Foundry Practice.........................

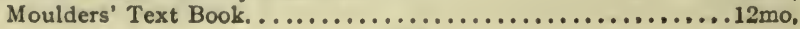




\section{MINERALOGY.}

Baskerville's Chemical Elements. (In Preparation.).

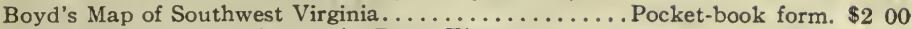

* Browning's Introduction to the Rarer Elements..............8vo, 150

Brush's Manual of Determinative Mineralogy. (Penfield.).........8vo, 400

Butler's Pocket Hand-book of Minerals. ......................... mor. 300

Chester's Catalogue of Minerals.................... pvo, paper, 100

Cloth, 125

* Crane's Gold and Silver. ........................... 5 vo, 500

Dana's First Appendix to Dana's New "System of Mineralogy". . Large 8vo, 100

Dana's Second Appendix to Dana's New "System of Mineralogy."

Large $8 \mathrm{vo}$

Manual of Mineralogy and Petrography........................... 200

Minerals and How to Study Them. ....................... 150

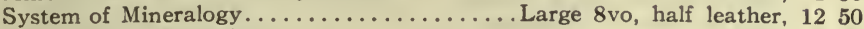

Text-book of Mineralogy. ........................... 400

Douglas's Untechnical Addresses on Technical Subjects. . . . . . . . 12mo, 100

Eakle's Mineral Tables. ............................... 125

Eckel's Stone and Clay Products Used in Engineering. (In Preparation).

Goesel's Minerals and Metals: A Reference Book.............. mo, mor. 300

Groth's Introduction to Chemical Crystallography (Marshall).......12mo, 125

* Hayes's Handbook for Field Geologists.................16mo, mor. 150

Iddings's Igneous Rocks. . . . . . . . . . . . . . . . . . . . . 8vo, 500

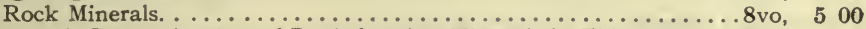

Johannsen's Determination of Rock-forming Minerals in Thin Sections. 8vo,

With Thumb Index 500

* Martin's Laboratory Guide to Qualitative Analysis with the Blow-

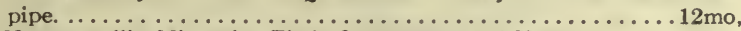

Merrill's Non-metallic Minerals: Their Occurrence and Uses. ......... . . . . . . . .

Stones for Building and Decoration. . . . . . . . . . . . . . . . . 8vo,

* Penfield's Notes on Determinative Mineralogy and Record of Mineral Tests.

8vo, paper

Tables of Minerals, Including the Use of Minerals and Statistics of

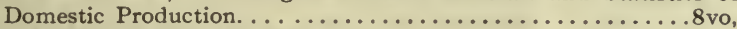

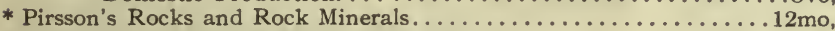

* Richards's Synopsis of Mineral Characters. . . . . . . . . . . . . 12mo, mor.

* Ries's Clays: Their Occurrence, Properties and Uses. . . . . . . . . . . . . 8vo,

* Ries and Leighton's History of the Clay-working Industry of the United

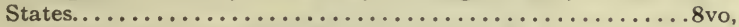

* Tillman's Text-book of Important Minerals and Rocks........... . . 8vo,

Washington's Manual of the Chemical Analysis of Rocks. . . . . . . . . . . 8vo,

\section{MINING.}

* Beard's Mine Gases and Explosions. . . . . . . . . . . . . . Large 12mo,

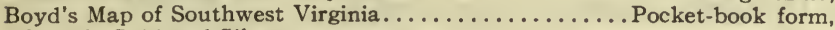

* Crane's Gold and Silver. ............................ . . . . .

* Index of Mining Engineering Literature................. . . 8vo,

* 8vo, mor.

Douglas's Untechnical Addresses on Technical Subjects. . . . . . . . . 12mo,

Eissler's Modern High Explosives....................... . . . . .

Goesel's Minerals and Metals: A Reference Book...................... mor.

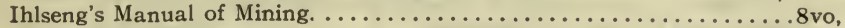

* Iles's Lead Smelting. . . . . . . . . . . . . . . . . . . . . . . . . . 12mo,

Peele's Compressed Air Plant for Mines. . . . . . . . . . . . . . . . . 8vo,

Riemer's Shaft Sinking Under Difficult Conditions. (Corning and Peele).8vo,

* Weaver's Military Explosives. . . . . . . . . . . . . . . . . . . 8vo,

Wilson's Hydraulic and Placer Mining. 2d edition, rewritten. . . . . . . . .

Treatise on Practical and Theoretical Mine Ventilation ... . . . . 12mo, 


\section{SANITARY SCIENCE.}

Association of State and National Food and Dairy Departments, Hartford

Meeting. 1906............................... 8vo, $\$ 300$ Jamestown Meeting, 1907........................ 300

* Bashore's Outlines of Practical Sanitation. .................. 125 Sanitation of a Country House.......................... 100 Sanitation of Recreation Camps and Parks. .................... 100

Folwell's Sewerage. (Designing, Construction, and Maintenance.)....8vo, 300 Water-supply Engineering. .......................... 400

Fowler's Sewage Works Analyses. . . . . . . . . . . . . $200 \ldots \ldots \ldots \ldots$

Fuertes's Water-filtration Works........................... 250

Water and Public Health. ............................... 150

Gerhard's Guide to Sanitary Inspections....................mo, 150

* Modern Baths and Bath Houses.......................... 300

Sanitation of Public Buildings............................ 150

Hazen's Clean Water and How to Get It. . ......................... 12mo, 150

Filtration of Public Water-supplies. .................... 300

Kinnicut, Winslow and Pratt's Purification of Sewage. (In Preparation.)

Leach's Inspection and Analysis of Food with Special Reference to State

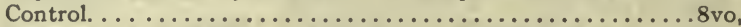

Mason's Examination of Water. (Chemical and Bacteriological)....12mo, Water-supply. (Considered principally from a Sanitary Standpoint).

* Merriman's Elements of Sanitary Enigneering. ................8vo, 200

Ogden's Sewer Design. ............................... 2 mo, $^{2} 00$

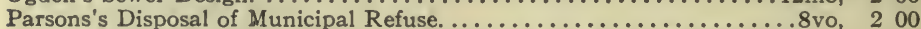

Prescott and Winslow's Elements of Water Bacteriology, with Special Reference to Sanitary Water Analysis. ...................

* Price's Handbook on Sanitation. .................................

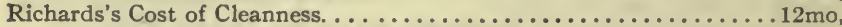

Cost of Food. A Study in Dietaries. ....................

Cost of Living as Modified by Sanitary Science............ 12mo,

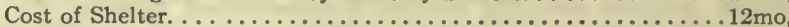

* Richards and Williams's Dietary Computer................... 8vo,

Richards and Woodman's Air, Water, and Food from a Sanitary Stand-

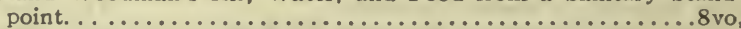

* Richey's Plumbers', Steam-fitters', and Tinners' Edition (Building Mechanics' Ready Reference Series)............16mo, mor.

Rideal's Disinfection and the Preservation of Food. . . . . . . . . . . 8vo,

Sewage and Bacterial Prification of Sewage. ..............

Soper's Air and Ventilation of Subways...................

Turneaure and Russell's Public Water-supplies................

Venable's Garbage Crematories in America. ....................

Method and Devices for Bacterial Treatment of Sewage........8vo,

Ward and Whipple's Freshwater Biology. (In Press.)

Whipple's Microscopy of Drinking-water................... 350

* Typhoid Fever. ........................................ 12mo, 300

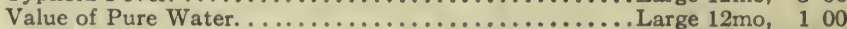

Winslow's Systematic Relationship of the Coccacex............. Large 12mo, 250

\section{MISCELLANEOUS.}

Emmons's Geological Guide-book of the Rocky Mountain Excursion of the International Congress of Geologists.............. Large 8vo.

Ferrel's Popular Treatise on the Winds. ...................

Fitzgerald's Boston Machinist. .............................

Gannett's Statistical Abstract of the World...............24mo,

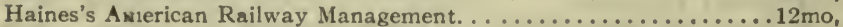

* Hanusek's The Microscopy of Technical Products. (Winton)......8vo, 
Jacobs's Betterment Briefs. A Collection of Published Papers on Organized Industrial Efficiency. ................. $\$$ vo, $\$ 50$

Metcalfe's Cost of Manufactures, and the Administration of Workshops..8vo, 500 Putnam's Nautical Charts. .......................... 8vo, 200

Ricketts's History of Rensselaer Polytechnic Institute 1824-1894.

Large $12 \mathrm{mo}, 300$

Rotherham's Emphasised New Testament. ............... Large 8vo, 200

Rust's Ex-Meridian Altitude, Azimuth and Star-finding Tables.......8vo, 500

Standage's Decoration of Wood, Glass, Metal, etc..............12mo, 200

Thome's Structural and Physiological Botany. (Bennett).........16mo, 225

Westermaier's Compendium of General Botany. (Schneider). . . . . . . 8vo, 200

Winslow's Elements of Applied Microscopy........................ 150

\section{HEBREW AND CHALDEE TEXT-BOOOKS.}

Gesenius's Hebrew and Chaldee Lexicon to the Old Testament Scriptures. (Tregelles.)........................ Small 4 to, half mor, 500

Green's Elementary Hebrew Grammar........................... 12mo, 125 




RETURN TO the circulation desk of any University of California Library or to the

NORTHERN REGIONAL LIBRARY FACILITY Bldg. 400, Richmond Field Station University of California Richmond, CA 94804-4698

ALL BOOKS MAY BE RECALLED AFTER 7 DAYS

- 2-month loans may be renewed by calling (510) 642-6753

- 1-year loans may be recharged by bringing books to NRLF

- Renewals and recharges may be made 4 days prior to due date.

DUE AS STAMPED BELOW

\section{JUN 161999}




\section{9}
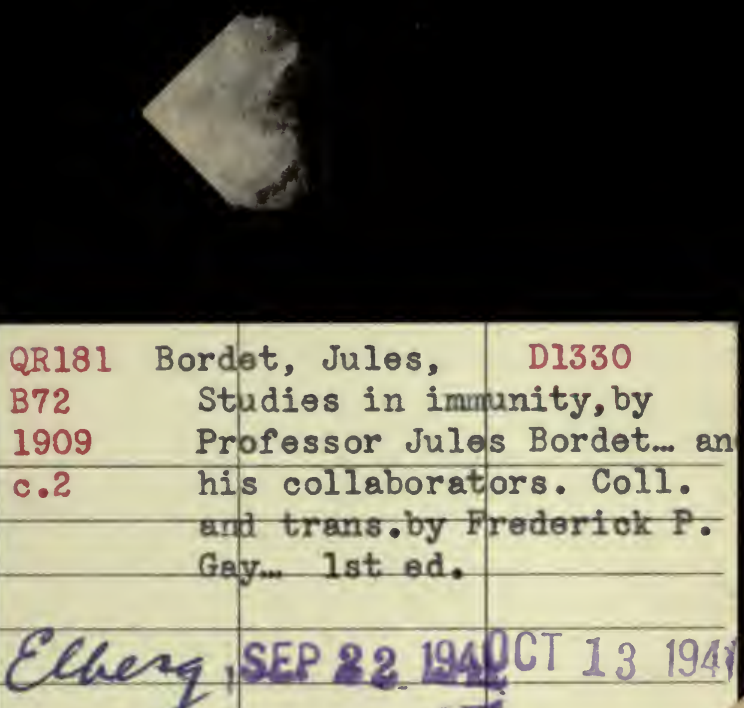

B72 Studies in immunity, by

1909 Professor Jules Bordet... an

c.2 his collaborators. Coll. and trans.by Frederiok P. Gay.m 1st od.

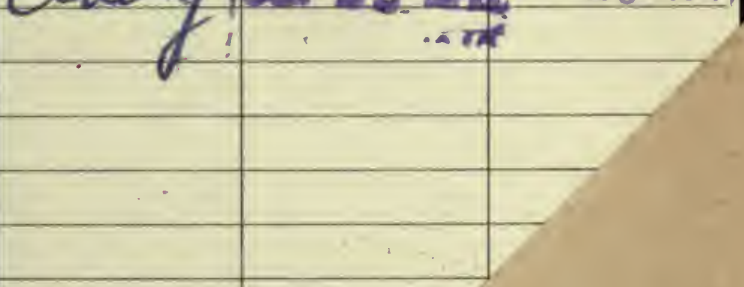




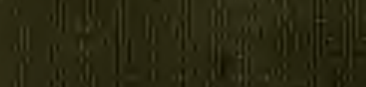

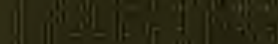

I.

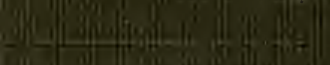

$\int_{0}$

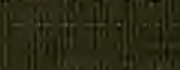

(1)

(1)

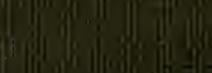

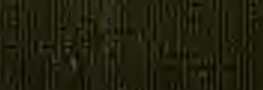

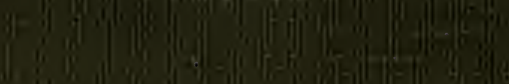

